
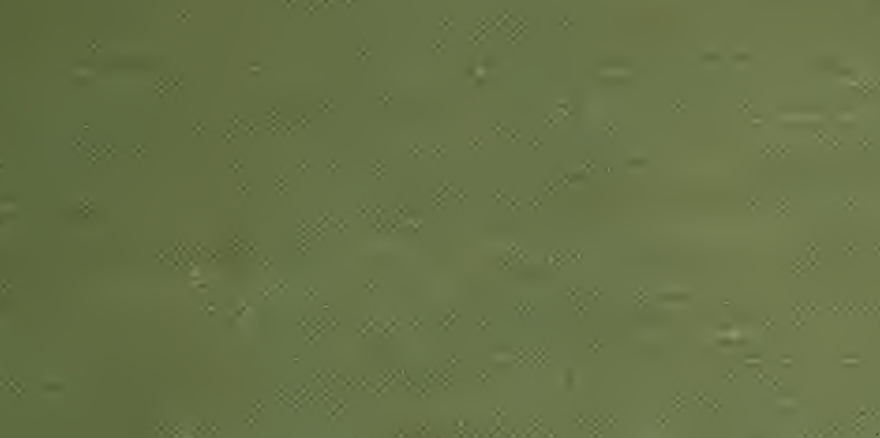

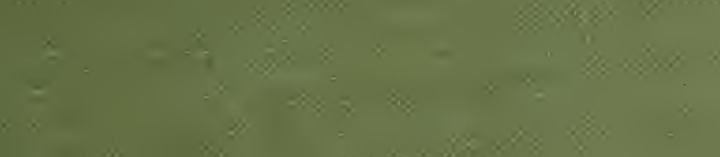

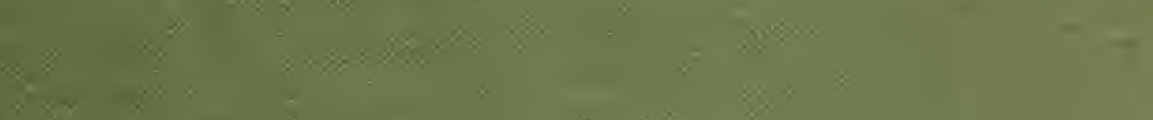

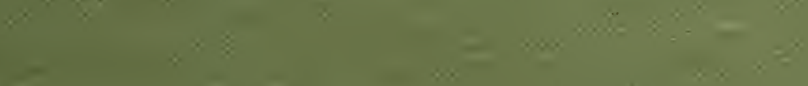

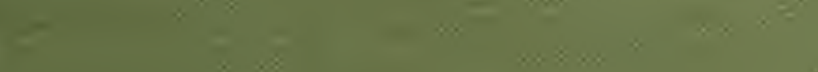

$x,-x=2 \times$

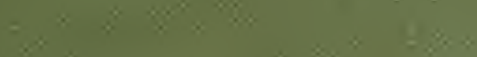

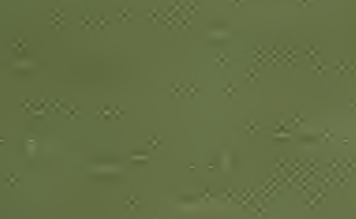

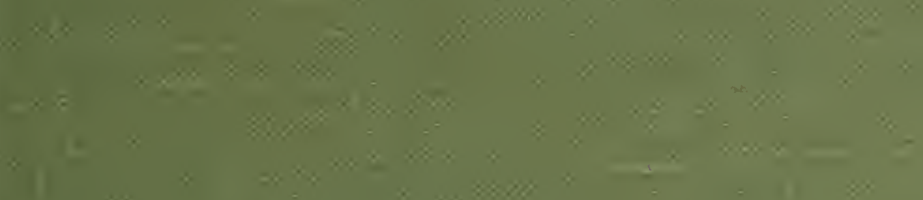

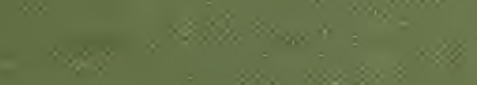

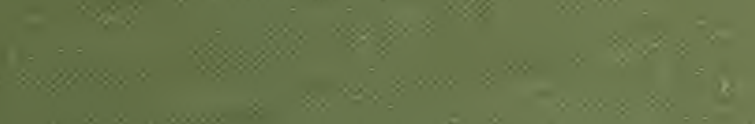

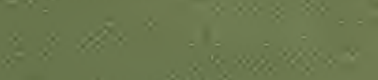

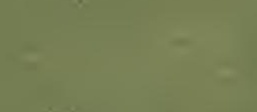





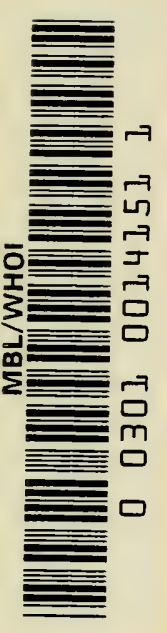





\section{A MANUAL of the}

\section{ASPERGILLI}

\section{By}

\section{CHARLES THOM}

Collaborator, Northern Regional Research Laboratory, Formerly Principal Mycologist, Bureau of

Plant Industry, U.S. Department of Agriculture, Washington, $D$. C.

$$
\text { and }
$$

\section{KENNETH B. RAPER}

Senior Microbiologist, Fermentation Division, Northern Regional Research Laboratory, Bureau of A gricultural and

Industrial Chemistry, U.S. Department of

Agriculture, Peoria, Illinois

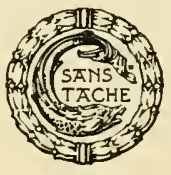

BALTIMORE

THE WILLIAMS \& WILKINS COMPANY 1945 
COPYRIGHT, 1945

The Williams \& Wilkins Company

Made in the United States of Amerca

Published May, 1945

Reprinted January, 1951

Composed and Printed at the IVAVERLY PRESS, INC.

for

THE WILLIAMS \& WILKINS COMPANY

Baltimore, MD., U. S. A. 


\section{CONTENTS}

Preface....................................... vii

Part I. General Discussion.......................... 1

Chapter I. Historical Introduction..................... 3

Chapter II. Classification, Generic Diagnosis, and Synonymy .... 6

Chapter III. Morphology and Description................ 10

Chapter IV. Cultivation and Examination................ 31

Chapter V. Preservation of Cultures................... 50

Chapter VI. Variation......................... 63

Part II. The Manual Proper........................... 79

Chapter VII. The Use of the Manual................... 81

Chapter VIII. The Aspergillus clavatus Group............... 92

Chapter IX. The Aspergillus glaucus Group............... 100

Chapter X. The Aspergillus fumigatus Group.............. 148

Chapter XI. The Aspergillus nidulans Group ............. 155

Chapter XII. The Aspergillus ustus Group............... 171

Chapter XIII. The Aspergillus flavipes Group.............. 179

Chapter XIV. The Aspergillus versicolor Group............. 183

Chapter XV. The Aspergillus terreus Group.............. 195

Chapter XVI. The Aspergillus candidus Group............. 206

Chapter XVII. The Aspergillus niger Group ................ 214

Chapter XVIII. The Aspergillus wentii Group ............. 241

Chapter XIX. The Aspergillus tamarii Group............ 250

Chapter XX. The Aspergillus flavus-oryzae Group.......... 259

Chapter XXI. The Aspergillus ochraceus Group............. 273

Part III. Reference Material .......................... 287

Chapter XXFI. Topical Bibliography ................... 289

Chapter XXIII. General Bibliography................... 319

Chapter XXIV. Check List of Species and Genera .......... 331

Chapter XXV. Accepted Species, Varieties, and Mutations...... 360

INDEX 363

\section{1}





\section{PREFACE}

Aspergillus as the name for a genus of molds dates back to Micheli (1729), but it was not until the middle of the 19th Century that the Aspergilli began to be recognized as active agents in many decay processes, as occasional causes of human and animal disease, and as fermenting agents capable of producing valuable biochemical products. Various taxonomic efforts were made. DeBary, Fresenius, van Tieghem, and others described the particular species that they used. Bainier described in brief inadequate terms all the Aspergilli he found. Wilhelm and Wehmer reviewed the literature, described and figured the forms they knew; these were few in number, but the work was done so well that it fixed group types. In 1926, Thom and Church brought all of this material together in a monograph. The increased study devoted to the Aspergilli in recent years shows some of their groupings to be inadequate. In addition, a large amount of new material has accumulated. The Aspergilli have become increasingly important as responsible agents in a number of industrial fermentations. Many of them are being found capable of producing antibiotic substances and their possible use in this field will undoubtedly be exhaustively explored. For these reasons, the need for a manual for those who wish to identify Aspergilli under observation, without regard to the historical aspects of the group, has become increasingly apparent.

This book is definitely a manual, not a monograph. It is based upon comparative study of thousands of strains of Aspergilli in culture. Representative strains giving the range of morphology and biochemical activity in each species are maintained in the permanent collection of the Northern Regional Research Laboratory. Consistent efforts have been made to obtain the organisms actually used by authors who have put forward new nomenclature. The manual thus seeks to present under species names only living cultures known to the authors, although it seemed advisable to make a few additions based upon literature. The species included are arranged as far as possible into natural groups which bring together aggregates of strains or species agreeing in important morphological characters. For the most part, physiological or biochemical information, if available, indicates related activities within these groups. The names selected for use appear to be taxonomically correct. A large number of names are necessarily rejected. If known to belong to some unidentifiable member of a group, or believed from literature to be correctly placed there, each of these names is accounted for in the discussion of the group. If the information available does not justify allocation to some species or species 
aggregate, the name will be found in the check list with any information at hand. Large gaps in our information about the Aspergilli still exist. Some of these are pointed out in the text. The great activity of the present day will undoubtedly render any arrangement of the Aspergilli obsolete sooner or later, but it is believed that the classification put forward here is at least temporarily practical.

Recognizing that any species name for an Aspergillus appearing at any time in the literature may at some future time become important for some unanticipated reason, an alphabetical check list giving each of the names found, the author, the date, and the place of publication has been included. An index reference to page in the manual, or the method of disposal of the name, is added to bring the material to its greatest usefulness as a ready reference.

Two types of bibliography are presented: a general bibliography, alphabetical to author's name and sub-indexed as to date of publication when necessary, includes authors of species and other investigators whose work is cited in the text. In addition, a topical bibliography is presented. Although incomplete, it is hoped that the latter will assist greatly in the search for special literature on particular subjects. Believing that the more recent literature on these subjects will generally be of the greatest interest and value, the material is presented chronologically. Duplication between the two bibliographies may or may not occur.

A manual, if it is to facilitate the identification of these molds by the actual worker in the laboratory, must present descriptive and illustrative material in as simple form as seems consistent with sound scholarship. From our present point of view, the describer of a mold must know that mold in fruiting form under the microscope as known to the early mycologists, and.know it also in the culture tube. It must be isolated as a pure culture and its life history and reactions followed out upon laboratory media. Its definite place in some one of the aggregate species or groups of Aspergilli should be thoroughly established; then, by careful study and comparison proper nomenclature should not prove difficult.

This manual, then, seeks to serve two purposes: (1) to provide the worker encountering an Aspergillus with means for its identification, and hence to open to him the whole literature of the group, as well as the particular species; and (2) by enumerating all forms found in the literature, and indicating their proper allocation, to guide the user of that literature in the interpretation of names found in his reading but not known to him in nature, in culture, or in exsiccati.

The authors acknowledge the cooperation of Dr. Johanna Westerdijk, Dr. F. H. van Beyma, and their colleagues at the Centraalbureau voor Schimmelcultures, at Baarn, Holland, in the free exchange of cultures and 
information. Professor Ph. Biourge and Dr. Paul Simonart at Louvani put their entire collection at our service after preparing and demonstrating their interpretations in their own laboratory. Dr. Raoul Mosseray sent his extensive series of variants in the Aspergillus niger group as accumulated from the Belgian Congo. Dr. Adalbert Blochwitz made many comments and criticisms in his numerous letters. Professor Harold Raistrick and Mr. George Smith submitted all strains reaching their laboratory with full notes on their own interpretations. Cultures and photographs, some of which are used in this manual, have been furnished by Messrs. John and Edward Yuill. Series of cultures have been received from Drs. Marie B. Morrow and J. J. Taubenhaus in Texas, Drs. Roberta Ma and Y. K. Shih in China, Drs. G. Kita, R. Nakazawa, J. Hanzawa, and K. Oshima in Japan, Drs. G. R. Bisby and G. A. Ledingham in Canada, and Dr. H. Macy in Minneapolis, as well as individual strains from many correspondents. The laboratory collection owed much of its completeness to the punctilious workmarship and painstaking scholarship of Dr. Margaret B. Church.

In the preparation of the manuscript outstanding contributions have been made by Dorothy F. Alexander who prepared the line drawings and assisted generously in the checking and proofreading of the textual material; by Mr. Roland IT. Haines, Photographer of the Northern Regional Research Laboratory, who made all the color pictures, as well as many of the black and white photographs; and by Miss Nancy Brant who typed the manuseript in its final form.

The authors are indebted to the Chas. Pfizer and Company Inc., Brooklyn New York, for underwriting the cost of reproducing the natural color photographs.

Administratively, the preparation of this manual was made possible by the vision of Dr. O. E. May, Chief of the Bureau of Agricultural and Industrial Chemistry, Mr. H. T. Herrick, Director of the Northern Regional Research Laboratory, and Dr. Robert D. Coghill, Chief of the Fermentation Division of the Northern Regional Research Laboratory, who have developed the industrial exploration of the biochemical utilization of the fungi over many years.

The Authors 

PART I

GENERAL DISCUSSION 



\section{Chapter I}

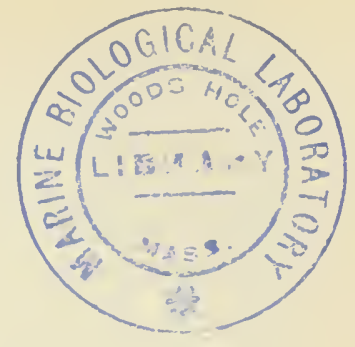

\section{HISTORICAL INTRODUCTION}

Historically, the Aspergilli, as a part of moldiness of things, have always been a factor in man's environment, but for ages were brushed away as white, yellow, green, red, or black mold, with or without any attempt at interpretation. After the development of the microscope, men began to see structure. Micheli (1729) distinguished conidiophores and heads. He noted that the heads were rough, the spore chains or columns producing an uneven surface, hence he gave the name Aspergillus (rough head). He then marked with Latin phrases his sketches of differently colored moldy substances, for example, Aspergillus capitatus ochroleucus, probably some strain of Aspergillus ochraceus; Aspergillus capitulo pulla for a black form, etc. Other authors followed, using much the same terminology, but without illustrations definite enough to give knowledge of the structure of the heads. Thus, Haller, in 1742, put what appears to have been Sporodinia into the genus as A. ramosissimus, etc. There is just about enough certainty in the use of $A$. albus, $A$. niveus, A. capitulo pulla, A. purpureus, etc., to justify the continued use of the name Aspergillus after taking out of the aggregate the extraneous material thrown into it by the very scanty microscopic examination given by the early mycologists.

Persoon (in the 1790's) threw the Aspergilli into his polyglot concept, Monilia, based upon the production of spores in chains resembling strings of beads. He made no record concerning their origin. Then Link in 1809 went back to Micheli and based his rejection of Monilia upon the specification that these chains of spores must have their origin in a "head" (capitulum)

Link failed to examine that head closely enough to keep out questionable forms although we know that in describing $A$. glaucus he had under his microscope one of that group as it was found, then and now, upon partly dried herbarium specimens. Correct interpretation of the structure of this head appears first in the work of Corda who began, about 1828, to publish his studies of fresh material, as seen under his microscope. Up to about 1850 each worker was prone to look at his predecessor's descriptions and figures, and either assign whatever he had to another man's species, or conclude that each specimen he had was new and add another group of names. Montagne complained (1856) that none of the descriptions written before Corda were identifiable, while some of us are equally uncertain of our ability to interpretMontagne. 
DeBary's laboratory in the early 1850's seems to have introduced sufficient culture of the molds found to form the beginning of a permanent literature. This started with the recognition that the yellow perithecia, called Eurotium herbariorum by Link, which developed among the heads of Aspergillus glaucus upon his herbarium specimens, were actually borne upon the same mycelium (fig. 7). Fresenius, Cramer, Wilhelm, and Brefeld in Germany followed. Raulin and van Tieghem in France developed the fermentation of the tannins in gall nuts to gallic acid in the 1860's with comparative study of other molds as a corollary. In 1880, in Paris, Bainier began publishing his studies of molds as they appeared in pharmaceutical products. He was followed by Gueguen, the Sartorys, and others in France, and somewhat later by Biourge in Louvain, Belgium.

Wehmer, in Hanover, began publishing his biochemical studies in 1891, which led him to develop his more pretentious monograph published in 1901. Blochwitz undertook to develop his "system" early in the new century, but the World War delayed its publication until 1929. Meanwhile, Thom and Church, beginning about 1910, had published The Aspergilli as a taxonomic monograph in 1926. Aspergilli were listed in cryptogamic floras, lists, manuals, and special papers of many kinds over the whole period, but critical discussions were few.

In somewhat over 200 years, an enormous mass of Aspergillus literature has accumulated. Justice to the writers at each stage in the development of our information calls for an analysis of the conditions which surrounded its development. Practically all of the early literature was microscopical: the worker confined his study to specimens brought in from natural sources, each of which was often assumed to be typical of some species. Each worker used the microscope that he had at hand and the technique of study already known to him. Life histories and comparative examination of material from many sources were disregarded. Publications appeared as parts of floristic studies of particular regions, as reports of organisms found in particular lesions of man or animals, or as observed in special industrial connections. After DeBary's group began to study organisms in comparative culture, the number of publications began to increase rapidly. By 1929-1930 Tamiya and Morita were able to cite 2,424 titles of papers which, in some way, concerned the Aspergilli, in their published Bibliographie von Aspergillus, 1729 bis 1928. A mathematical analysis of this literature was published by Tamiya in 1931. Referring to his table 1, 71 titles appeared in the 125 years before DeBary's 1854 paper; 73 appeared in the next 18 years preceding Brefeld's 1872 papers; 236 in the next 19 years just preceding Wehmer's oxalic acid reports in 1891 . All of this may be called the period of physiological morphology. The remaining two thousand, published between 1891 and 1928, represent the 
pure culture period. This may equally well be called the biochemical period.

The taxonomic part of this literature was scattered through several languages and represented many schools of nomenclatorial thought. The man who had seen only three or four Aspergilli found no difficulty in separating them. Each used his own descriptive terms-adequate for his purpose but useless to the next man with different species. Saccardo just published them all. Critical analyses were not available.

In The Aspergilli (1926) as a monograph, Thom and Church sought to bring together all of this taxonomic literature, as published before that date, and to present a critical opinion as to the proper relationship of the species described, whether retained in the genus or placed elsewhere. Some 350 names were thus accounted for, but the actual number of species accepted as known in culture or probably determinable from existing literature was given as 69 (p. 252). These were more or less arbitrarily considered in 11 groups. In undertaking to account for all the described forms, it was deemed advisable to include, in the various groups discussed, many forms whose published descriptions were inadequate for positive identification, but complete enough to indicate their affinities with known sections of the genus. Citation of these species in the older literature might, therefore, be traced to group relationship, and in that way, correlated with more recent studies of the same or related organisms. In addition, certain names were listed as entirely unidentifiable and certain other forms as belonging to other genera.

Various other proposals for this purpose have been made. Blochwitz in 1929 published his long-delayed "System und Phylogenie," with interpretations and proposals for grouping quite different from those of Thom and Church. Neill (1939) reduced the species recognized to the larger aggregates, paying little attention to details of head and spore formation. George Smith (1938), seeking industrial utility, simplified his descriptions and introduced many photomicrographs. He discarded the literature for the most part and undertook to guide the worker to the larger groups which could be located principally by color and shape of head, as shown by his figures. Dodge (1935) keyed all species whose names appear in medical literature, from their descriptions but without studying them in culture.

In 1939, Biourge prepared a manuscript analysis of the genus for the Third International Microbiological Congress in New York. His associate, Dr. Simonart, came to represent him, but left because of the war. The paper was not presented but was transmitted to us because return to the author was impossible. Biourge died somewhat later. His scheme of classification prepared in his last years is not presented because it contains many things too bizarre to do justice to a man who for many years was a master workman, as well as a valued friend. 


\section{Chapter II \\ CLASSIFICATION, GENERIC DIAGNOSIS, AND SYNONYMY}

Class: Ascomycetes

Order: Plectascineae

Family: Aspergillaceae

Genus: Aspergillus

Class: Fungi Imperfecti

Subclass: Hyphomycetes

Order: Mucedineae

Family: Mucedinaceae

Subfamily: Aspergilleae

Genus: Aspergillus

The above classification follows Engler and Prantl. Changes in the names of class, order, and family appear in various proposals without essential differences in placement. G. W. Martin would replace the names Plectascineae with Eurotiales, Aspergillaceae with Eurotiaceae, and Aspergillus with Eurotium in the plea that the first name applied to the ascosporic form determines the generic usage. Since the group has too many common characters to be split to advantage, and since the non-ascosporic forms vastly outnumber the ascosporic, it is better to forget Eurotium along with the technicality. In this arrangement, the name Aspergillus appears in its proper place among the ascosporic fungi. It also appears among the Hyphomycetes properly keyed to facilitate the identification of organisms obviously related but which do not produce ascospores as far as known.

\section{GENERIC DIAGNOSIS}

There is progressive need for broadening the application of the name Aspergillus to include organisms whose structures, as determined in culture by microscopic study, point to membership in specific natural groups. There is need for analysis of the question whether the whole group shall be retained as Aspergilius or further divided into more closely related entities, such as Eurotium of Link, Aspergillopsis of Spegazzini, Diplostephanus of Langeron, Sterigmatocystis of Cramer, and perhaps others. There are so many arguments for keeping them in a single group that the characterization of the genus Aspergillus used by Thom and Church in 
1926 has been emended and introduced here. This is followed by brief considerations of the other more significant synonyms.

Aspergillus Micheli, in Nova Plantarum Genera, p. 212, Plate 91. 1729. Compare Link, in Obs. p. 16. 1809; Corda, in Icones Fungorum

4:31, Tab. VII, fig. 94. 1840; and Thom and Church, in The Aspergilli, p. 4.1926.

Vegetative mycelium consisting of septate branching hyphae, colorless, bright colored, or in a few forms slowly becoming brown in localized submerged areas, or producing brown crusts, or sclerotia ; conidial apparatus developed as conidiophores and heads from specialized, enlarged, thickwalled hyphal cells (the foot-cells) producing conidiophores (stalks) as branches approximately perpendicular to the long axis of the foot-cell and usually to the surface of the substrata in or upon which they are borne; conidiophores unseptate or septate, usually enlarging upward and broadening into elliptical, hemispherical, or globose fertile vesicles bearing fertile cells or sterigmata either parallel and clustered in terminal groups, or radiating from the entire surface; sterigmata either in one series only, or as a primary series, each bearing a cluster of two to several secondary sterigmata at the apex; conidia varying greatly in color, size, shape, and markings, successively cut off from the tips of the sterigmata by crosswalls (not produced by budding), and forming unbranched chains arranged into radiate (globose) heads or packed into columnar masses; perithecia found in certain groups only, unknown in most species, cleistocarpic, thin-walled, producing asci and ascospores within a few weeks; sclerotia regularly found in some strains, occasionally found in other strains, and not found in other and closely related strains, mostly globose or subglobose, composed of polyhedral thick-walled cells.

Eurotium Link, in Obs. p. 31, Taf. 2, fig. 44. 1809.

Synonym: Mucor herbariorum Wiggers, in Primitiae Florae Holasticae as No. 1158. 1780. See also DeBary, in Bot. Ztg. 12: 425. 1854 .

The yellow perithecia suspended in networks of hyphae above or at the surface of his badly dried herbarium specimens were taken by Wiggers (1780) as the basis of Mucor herbariorum. Link (1809) recognized the bodies as ascosporic, hence segregated them under the generic name Eurotium. Then in 1854 DeBary published proof that these perithecia were borne upon the same mycelium as the asexual $A$. glaucus fruits among which they developed. He then called each of his Aspergilli, Eurotium Aspergillus followed by the specific name, whether ascosporic strains were known or not. In spite of technicalities invoked by some to bolster the 
use of the name Eurotium for all Aspergilli, or failing in that, for all ascosporic strains, most workers have accepted the numerical predominance of the non-ascosporic strains as ample reason for the general use of the name Aspergillus. For practical purposes, Eurotium is not used here.

Sterigmatocystis Cramer, in Vrtljschr. Naturf. Gesell. Zurich Jahrg. 4, Heft 4, p. 325, Taf. II, figs. 1-15. 1859.

Cramer, in 1859, published his study of a black Aspergillus from the human ear. Since the fruiting head differed from that of Fresenius' A. fumigatus by showing a primary series of sterigmatic cells radiating from the vesicle, each bearing a crown of several sterigmata, which in turn each bore a chain of spores, he made this character the basis of his new genus Sterigmatocystis. Cramer's name has been accepted by many workers, but was rejected by Wehmer, Thom, and others on the proof that such use would separate strains obviously related in such a group as Aspergillus flavus and its allies, and even among the black Aspergilli studied by Cramer himself. The additional name serves no useful purpose as an aid to identification; hence is not recognized here.

Euaspergillus Ludwig, in Lehrbuch des niederen Kryptogamen p. 258. 1892 .

The proposal to apply a separate generic designation to all Aspergilli producing sclerotia would take out the groups typified by $A$. candidus, A. niger, A. wentii, A. tamarii, A. flavus, and A. ochraceus. No one has followed Ludwig.

Aspergillopsis Spegazzini, in An. Mus. Nat. Buenos Aires Ser. 3, 13: 434. 1911.

The black-spored Aspergilli were described as dematiaceous, hence separated from all the other groups. No practical reason for accepting this proposal has been offered.

Diplostephanus Langeron, in Compt. Rend. Soc. Biol. Paris 87: 343-345. 1922.

Under this proposal ascosporic Aspergilli with the double series of sterigmata would be separated with $A$. nidulans Eidam as type. No technical application of nomenclatorial rules justifies the complications introduced.

In addition to the above names proposed to cover blocks of species with particular characters in common, a series of names have been used 
by various authors for individual species: Alliospora for a black form; Ascophora nigrans for A. niger; Aspergiliopsis Sopp for an unidentified organism; Cladosarum for Yuill's mutant of A. niger; Dimargaris for some white forms; Emericella and Inzengaea for A. variecolor; Mucor as a place to assign A. herbariorum; Sartorya for a possible ascosporic A. fumigatus. These names are cited in the check list but contribute nothing to this study of the group as a whole.

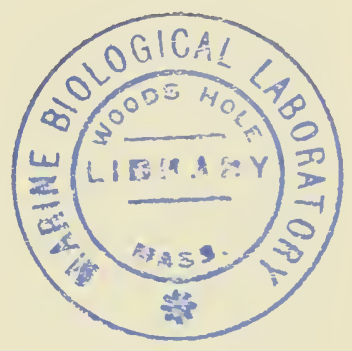




\section{Chapter III MORPHOLOGY AND DESCRIPTION}

\section{INTERPRETATION OF PUBLISHED DESCRIPTIONS}

Basic Assumptions: In interpreting the descriptions of Aspergilli in a literature covering a long period of time, certain assumptions, although not always justified, form a working hypothesis for presumptive identification. Conidiophore walls and conidial walls are assumed to be colorless and smooth, unless color or markings are either figured or described. Perithecia and sclerotia are assumed to be lacking unless the presence of such structures is specifically noted. Colors are assumed to apply to the general color scheme of the colony, unless specifically applied to the conidia, ascospores, or other details by the describer. Whereas colony coloration may arise from an admixture of conidial structures and varying amounts of vegetative hyphae, colored or uncolored, together with perithecia or sclerotia in greater or lesser numbers, it is assumed to result from the massing of conidial heads unless otherwise stated.

Difficulties encountered in interpreting descriptions based upon color are less for the worker with a growing culture before him than for the one handling descriptive literature alone, since the presence of white, green, yellow-green, brown, or black heads is readily distinguished with a handlens, even though sparingly produced upon a colony in which another color predominates as in many members of the A. glaucus group, or in A. flavipes. The color of the conidial heads is often made the primary basis of species description.

Extent of Study: Interpretation of descriptive literature accompanied and supplemented rather than preceded the study of great numbers of cultures so that the groups established are based upon the actual handling of thousands of cultures representing hundreds of forms of Aspergillus handled during a period of more than thirty years. Many of these were studied on natural substrata before their isolation. In addition, examination of exsiccati from several large herbaria, while more or less unsatisfactory as to detail in identification of species, furnish confirmatory evidence of the soundness of the groupings proposed.

Types: For a few of the specific names in use today, the type strain has been definitely maintained in culture. For most series, selection of a morphological entity to give a concrete concept back of the use of a name becomes a matter of critical judgment. For the purposes of this manual, an attempt has been made to base the use of the individual name upon the 
morphological picture most frequently encountered, rather than upon a selected strain assumed to be, but not known to be, the one first described.

Descriptive terms: For purposes of description, a standardized use of terms has been adopted. Great diversity is encountered in the literature in various languages. Even translated into Latin, Saccardo never homogenized the terms so that succeeding descriptions upon the same page use descriptive terms in the same sense. To make comparison with existing literature more convenient, the usages defined in the following pages will cover the morphology as definitely as possible and indicate the usages found in the older literature. To serve as a basis for the collection, interpretation, and presentation of pertinent information regarding Aspergilli to be studied, a guide sheet of the type used by the authors is presented in the introductory portion of the manual proper (p. 82).

\section{THE ASPERGILLUS COLONY}

Since few of the Aspergilli regularly produce perithecia and ascospores, a basis for identifying the majority of the molds of this group as they are actually encountered in nature and in culture must be found in the description of the colonies and in the details of morphology found in the sporebearing structures available.

The vegetative mass of most Aspergilli consists of submerged mycelium from which only fruiting hyphae rise above the surface. Such colonies suggest a field of ripening grain, in which conidiophores and ripening heads predominate, and have been described as velvety (the German term used is rase) from their appearance in many species. Some species produce a more or less aerial felt (floccosity) of branching and interlacing hyphae bearing conidophores. This is characteristic of certain strains of the $A$. versicolor and $A$. fumigatus groups upon Czapek's solution agar and other culture media commonly employed, and it is normally one of the most striking characters of $A$. wentii under laboratory cultivation (fig. $1 \mathrm{~B}$ ). Many strains of the A. glaucus group form long streamers of hyphae hanging from meat stored in cool, damp rooms and certain of these retain this character in laboratory culture. The character of the surface growth is a diagnostic characteristic which is usually fairly reliable under reasonably uniform conditions of culture.

In describing the Aspergillus colony, cognizance should be taken of such factors as age, rate of growth, temperature of incubation, and the composition of the substratum. Provided with this information, subsequent investigators can intelligently interpret their cultures in terms of species previously described.

Many species and strains of Aspergillus often produce conspicuously zonate colonies. Most commonly, these take the form of fairly regular 

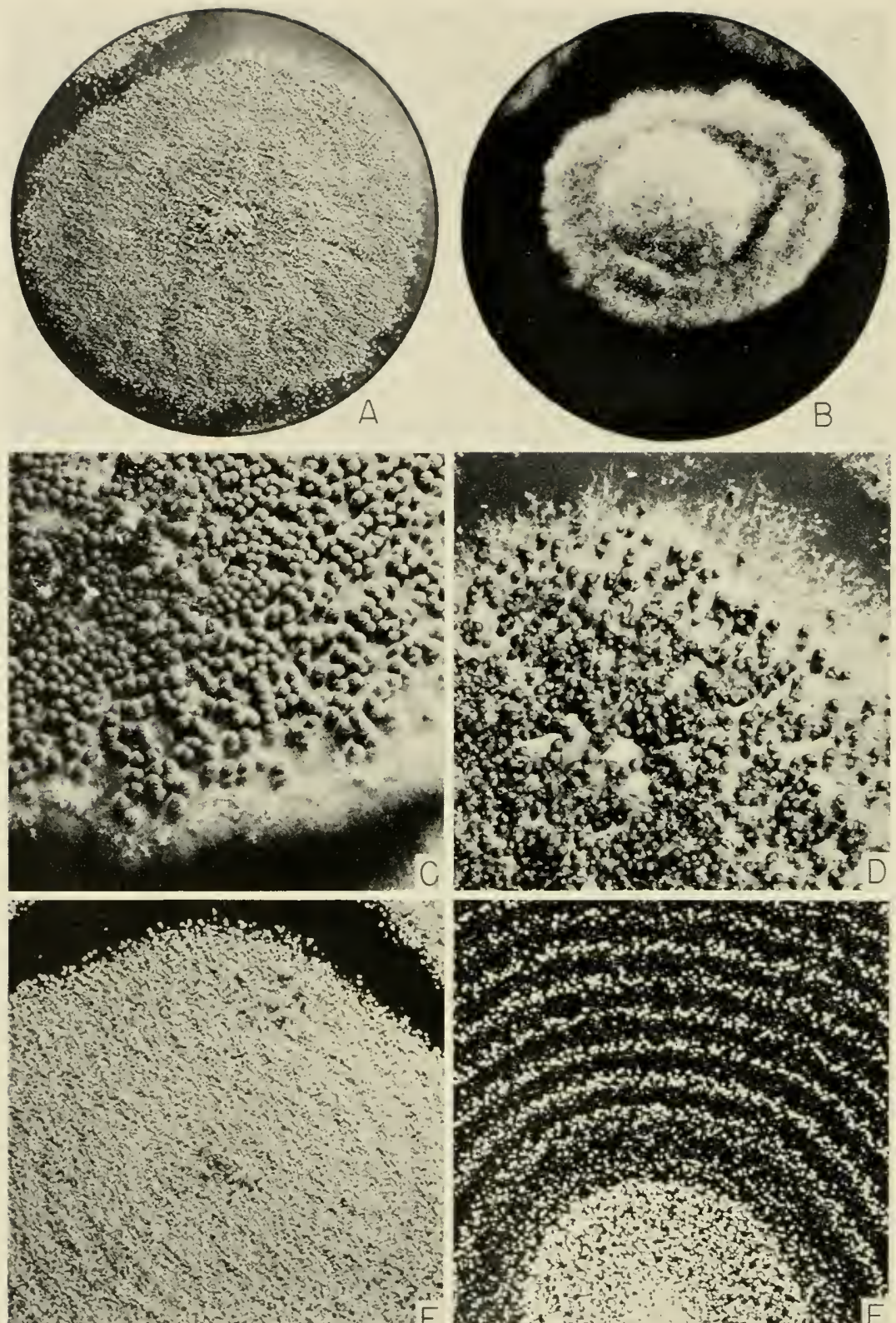

(A)

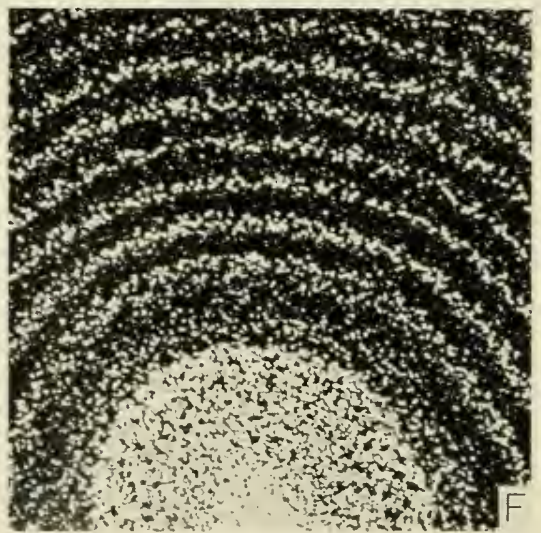

Frg. 1. Colony types in the Aspergilli. A, Aspergillus parasiticus NRRL No. 465, heavy sporing, non-floccose colony on Czapek's solution agar, room temperature, 10 days, $\times 2$. B, Aspergillus wentii NRRL No. 375, light sporing, floccose colony grown under the same conditions, $\times 2$. C, Aspergillus variecolor NRRL No. 1954 characterized by the production of abundant, large perithecia, $\times 3$. D, Aspergillus alliaceus NRRL No. 315 characterized by abundant black sclerotia, $\times 2$. E, Asper. gillus ochraceus NRRL No. 408 on Czapek's solution agar, showing heavy sporing, essentially azonate colony, $\times 2 . F$, The same on hay infusion agar, strongly zonate, $\times 2$. 
concentric zones and result from an increased production of conidial structures periodically during the development of the colony (fig. $1 \mathrm{~F}$ ). The exact conditions and factors responsible for their appearance has not been determined, but they obviously develop, in some way, as a response of the fungus to its environment. Particular strains cannot generally be described as either zonate or azonate, since the same organism exhibits both characters at different times and under different conditions. In contrast to concentric zones, certain species in laboratory culture characteristically develop conidial heads in localized areas only. This is particularly true of members of the $A$. glaucus group, and the $A$. sulphureus series, with conidial heads normally more concentrated at the margins of slant tube cultures.

Coremia, in the broader sense of ropes of hyphae anastomosing and trailing upon or near the surface of a substratum, occur more or less commonly in Aspergilli of certain groups; but as specialized erect aggregations of conidiophores (Stilbum-like), such structures are described and figured only for $A$. vitellina of Ridley. Since Ridley apparently described his form only as collected upon a natural substratum, the actual development of a specialized structure in this form remains doubtful. Ridley's figure could be repeated many times by roughly drawing masses of conidiophores bursting through the otherwise unbroken surface of a rich nutrient substratum, such as seeds of cereals, producing a dense cluster of conidiophores.

\section{COLOR}

The most striking character of an Aspergillus colony is usually its color production. This takes two general forms: (1) color in the aerial parts, including hyphae, conidiophores, heads, and conidia; (2) colors appearing in the substratum, and representing the specific response and effect of the organism upon particular media. The former is universally used in the characterization of species, while the latter usually furnishes additional pertinent data. In our study of the Aspergilli, citations of color have been made according to the terminology employed by Ridgway since the publication of his "Color Standard and Nomenclature" in 1912. This terminology is used consistently in the present manual.

\section{Group Color}

There is a characteristic range of colors for each group (or collective species) of Aspergilli, with a much narrower range in successive cultures of the particular species or strains. The coloring substance may be deposited in the conidia only, as in A. flavus; in the conidial wall, and more or less present in the sterigmata, vesicle, and upper part of the conidiophore 
as in $A$. niger; or the heads may be uncolored or nearly so, while the outer layers of the conidiophore wall may be colored as in $A$. flavipes and $A$. ochraceus. In some species of the $A$. glaucus group, the colony color is at first green with the development of conidia, then predominantly yellow to ferrugineous from ripening perithecia. Again, in other species of the same group, the walls of the conidiophores and, more particularly, aerial hyphae become encrusted with granules that are characteristically yellow in the young colony, but become reddish or ferrugineous in age. Such colonies are at first predominantiy yellow with green heads inconspicuous on a yellow background and later become rusty red or brown.

In the A. flavus group, Saito (ivo7) followed by Thom and Church (1921, p. 115) found that cultures with brighter shades of green when subjected to a vapor of ammonia would lose the green color and assume the somber yellow shades of variant members of the same group, and that this reaction was reversible, since the vapor of acetic acid would restore or even intensify an orignal green shade. The experiments pointed to the hiypothesis that the wilue range of shades produced by mixtures of yellow and green in the A. flavus-oryzae group may be attributed to racial limitations, strain by strain, in the range of hydrogen-ion concentration produced by metabolism. When a carbohydrate fermentable by the particular species is present, an acid reaction is promptly produced in the growing colony; as growth progresses alkaline products are also produced. The colony color in this series, therefore, reflects first the intensity of the initial acidity as shown by the intensity of the green color reached. The persistence of this shade or its subsequent reduction or entire disappearance to leave a somber yellow or finally brown colony, represents the balancing of the two activities. As a result, certain strains of this group, if grown on Czapek's solution agar, are quickly and very persistently deep green, others become particular shades of green, which fade to yellow and finally some of them to brown, and a few forms produce no true green color but assume a somber yellow with the first development of conidia.

Color changes in the conidia have not been satisfactorily worked out. In the $A$. niger group, the shade of yellow to purple-brown or black scems to be a strain or race character little influenced by handling which is not destructive of the racial entity. Each race seems to reach a fixed quantitative limit in the secretion of the coloring substance, thus reducing the most conspicuous diagnostic character among closely related forms to a quantitative rather than a qualitative basis of separation.

\section{Color in Conidial Walls}

The conidial walls may be smooth but carry sufficient coloring matter in diffused form to give the characteristic colony color. Within such 
series as A. fumigatus, $A$. nidulans, and $A$. ochraceus, however, strains or races may be found which vary from conidial walls smooth or nearly so, to walls bearing echinulations or even traceries apparently produced by aggregation of color substance into spinules, or bars between the outer and inner walls of the cells. Although smoothness and echinulation have received much weight in descriptive literature, observations such as the above would indicate that this character should only be used to separate nearly related strains in the same group, rather than as a group character.

Literature on the nature of color in Aspergilli seems to begin with Linossier's study of aspergilline as produced by A. niger in 1891; this was followed more recently by Quilico, and Quilico and Di Capua in 1933. Disregarding the record of observations only, more pretentious work appeared when Bainier and Sartory undertook to use color production to separate members of the A. glaucus group about 1910 to 1912 . Their experiments supplemented observations of colonies checked against a color chart, with a routine series of solubility and precipitation tests. Blochwitz (1929-1935) followed by using a series of routine solubility tests against all species producing bright colors but failed to coordinate his tests to show the relation of test to culture medium and conditions and to age of the culture studied. Later Gould and Raistrick (1934) and Raistrick, Robinson, and Todd (1937) studying the A. glaucus group, extracted and defined the colors found, but again failed to follow the transformations in the color of the particular species during the course of colony development. Until someone correlates color determination and composition more closely, color observations will continue to be useful accessory data which must be related to the age of the colony and to the composition of the medium as closely as possible to have value.

\section{Colors in the Substratum}

Production of bright colors in the substratum is frequent among the Aspergilli. The color produced by any species or race in any medium is dependent first on the ability of the mold to elaborate the particular product, and second on the presence of the necessary building material in the substratum. A mold may grow well upon a particular medium without discoloring it; a transfer from this colony to another substratum may turn the second medium red or yellow.

Color in the substratum is the result of the particular Aspergillus acting upon the particular medium under a certain range of temperature. Most of the species of Aspergillus, if they produce any color, produce from a trace to abundant yellow in the early stages. This may persist, or give place to shades of orange, red, or purple. In some cases, the color fades out as the colony becomes older. In descriptive work, progressive changes 
in intensity of color, or the presence or absence of color in different substrata, make the use of closely defined shades or intensities of color in the substratum an unreliable means of characterizing cultures.

Observations of colors produced in thè substratum remain, however, very conspicuous and exceedingly useful accessory characters which aid in the placing of species. The describer must bear in mind that such color reactions are confirmative, not absolute characters in separating species.

The final difficulty in dealing with color as a separating character rests in the loose use of color names, which is only partially corrected by the use of color standards. Comparison of the same culture by different individuals introduces very considerable discrepancies which become serious when a specific descriptive name or number from one of these standards is introduced into a technical description. In general, a series of observations giving a range of colors for a species is less liable to introduce errors of subsequent identification.

\section{MORPHOLOGY}

From the time of Micheli, the name Aspergillus (literally, rough head) has been used for molds with a conidiophore or stalk and spore-bearing head (capitulum).

\section{The Head}

The first structure observed in a detailed study of the colony is the spore-bearing head. The color, shape, size, and arrangement of such heads are characteristic of the species and to a lesser extent of the groups to which they belong (figs. 4 and 5). Wehmer (1901) roughly grouped his Aspergilli into Microaspergilli and Macroaspergilli on the basis of the size of the fruiting parts. Thus, A. fumigatus, A. nidulans, A. sydowi, and $A$. versicolor would represent Microaspergilli, while $A$. niger, $A$. clavatus, A. ochraceus, $A$. wentii, and $A$. tamarii would be readily classed as Macroaspergilli. The distinction breaks down when great numbers of forms are studied, but the comparative size of heads and conidiophores remains a useful adjunct in description. The heads in certain species show a consistent range of measurements and form, as in A. fumigatus and $A$. nidulans which have heads of small diameter forming columnar masses (fig. 4). Similarly, characteristic heads of A. niger, A. ochraceus, or $A$. wentii are globose and large (fig. 5). In other species, notably in $A$. flavus or $A$. candidus (fig. 60), several sizes and shapes of heads are regularly found in the same colony. The range of size and shape, however, remains characteristic. The observation of many heads in the colony, and preferably in many separate cultures, forms a better basis for description of sizes and measurements than limited observation. Further description 


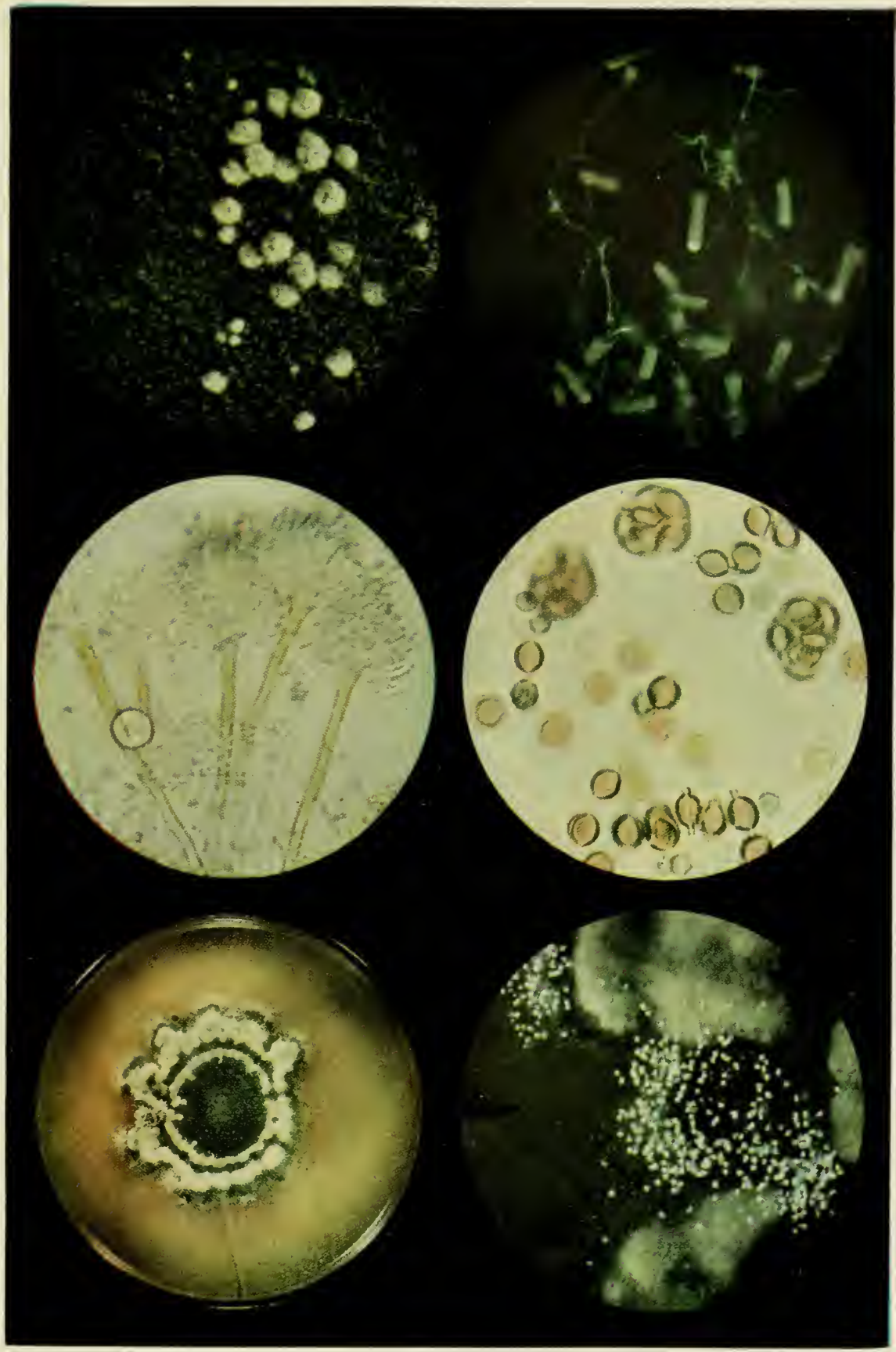

Plate I

A-D, Aspergillus nidulans (Eidam) Wint., NRRL No. 193: A (upper left), portion of colony showing developing perithecia and abundant conidial heads; $B$ (upper right), conidial heads showing typieal color and columnar form, $\times 60 ; C$ (center left), photomicrograph of conidial heads showing brown-walled conidiophores and green conidia, $\times 500 ; D$ (center right), ascospores, red in color and showing two equatorial ridges when seen in profile, $\times 1200$. E and $F$, Aspergillus janus Raper and Thom, NRRL No, 17s;: $E$ (lower left), single colony showing crowded short-stalked green heads in central area and long-stalked white heads in localized marginal areas, the yellow-white, floceose areas being composed largely of hülle cells; $F$ (lower right) portion of same colony somewhat enlarged, $X 8$. (Color photographs by llaines, Northern Regional Research Laboratory. Reproduced through co-operation of Chas. Pfizer \& Co., Inc.) 

of the structure of the head is given in the more logical order, following the discussion of the stalk or conidiophore.

\section{The Foot-Cell}

The first step toward conidium formation in the Aspergilli is the differentiation of certain cells (the foot-cells) in the mycelium for propagative purposes. These cells become larger, thick-walled, and each usually bears a single conidiophore as a branch, perpendicular to the long axis of the cell (fig. $2 \mathrm{~A}$ ) and usually about midway between the ends of the cell. In age these cells frequently become fantastically curved and twisted with their connection to regetative hyphae inconspicuous but usually still determinable. These foot-cells are commonly submerged in the substratum, although there are a number of strains of the $A$. glaucus, $A$. fumigatus, A. versicolor, and especially of the A. flavus-oryzae groups in which the conidiophores arise in this way from aerial hyphae. In A.effusus the foot-cells are frequently long and several of them connected together to form whole hyphae bearing considerable numbers of very short conidicphores (fig. $71 \mathrm{~B}_{3}$ ), hence their differentiation from the sterile or regetative cells is less easily determined. Failure to recognize the foot-cells as present in the Aspergilli led Ferdinandsen and Winge (1920) to describe $S$. dipus, using the foot-cell as the principal diagnostic character of the species. The presence of such a differentiated foot-cell is proposed as an arbitrary character to be used in separating certain depauperate forms of Aspergilli, which approach the structure and appearance of the monoverticillate Penicillia (Citromyces), from the Penicillia. Organisms which lack the typical Aspergillus head with its conidiophore and especially its footcell may be best classified elsewhere.

\section{The Conidiophore or Stalk}

The erect, perpendicular branch from the foot-cell constituting the conidiophore usually enlarges upwards toward the apex at which it dilates more or less definitely to form the vesicle (fig. $2 \mathrm{C}-\mathrm{E}$ ). The section of the conidiophores from the foot-cell to the base of the conidial head is measured and reported in describing species.

The conidiophore in some groups is not only septate, but each cell is sufficiently distinct to justify the term articulate which is frequently encountered in the older descriptions. In our experience in examining specimens, articulate conidiophores are found only in the A. glaucus group. In most Aspergilli the unity of the whole conidiophore is fairly accentuated. Septa, if present, are thin, fragile, and inconspicuous; the whole conidiophore is enclosed by continuous characteristically thickened walls without conspicuous nodes as an evidence of septation. 
Thickening of the conidiophore wall may be uniform or may be greater at the base, thinning to negligible toward the apex. Two general sections

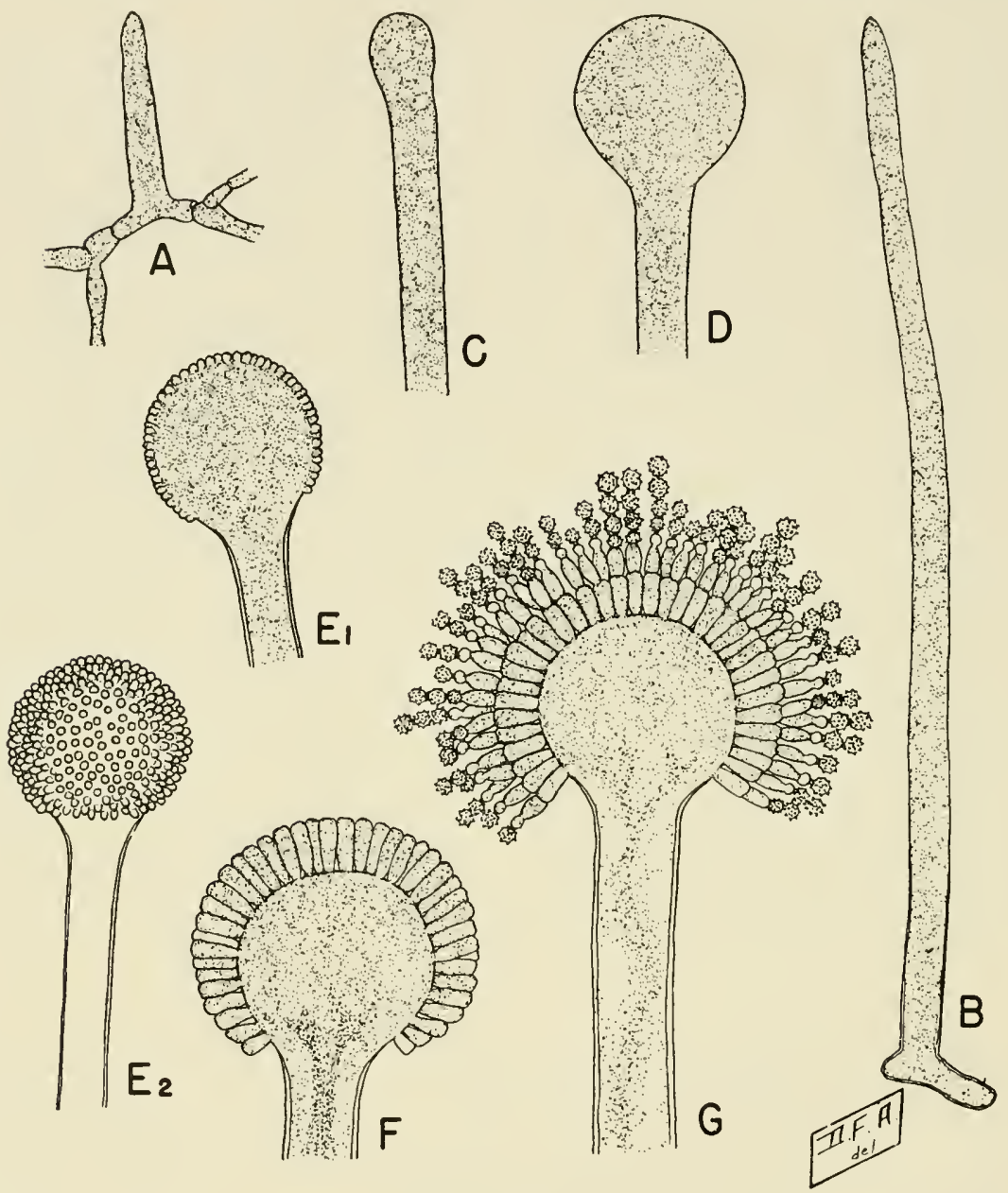

FIG. 2. Development of the conidial apparatus in Aspergillus niger. A, Foot cell bearing young conidiophore as a vertical branch. $B$, Developing conidiophore, $\times 172$. $C$ and $D$, Development of the vesicle by swelling of the terminal portion of the conidiophore, $\times 265 . \quad E_{1}$ and $E_{2}$, Vesicle in optical section and surface view showing early development of primary sterigmata, $X 265$. $F$, Later stage in development of primary sterigmata, $\times 265$. $G$, Young fruiting head showing secondary sterigmata bearing chains of conidia, $\times 265$.

based upon the character of the thickening of the conidiophore wall are fairly readily distinguished, although the careful use of high magnification is occasionally necessary to separate certain strains. In the first, the 
outer surface of the wall is free from pits, warts, or roughenings, hence is ealled smooth; its strueture is difficult to differentiate; the mass of the cellwall appears homogeneous or nearly so under the microscope and does not absorb the ordinary protoplasmic stains. In some speeies the inside
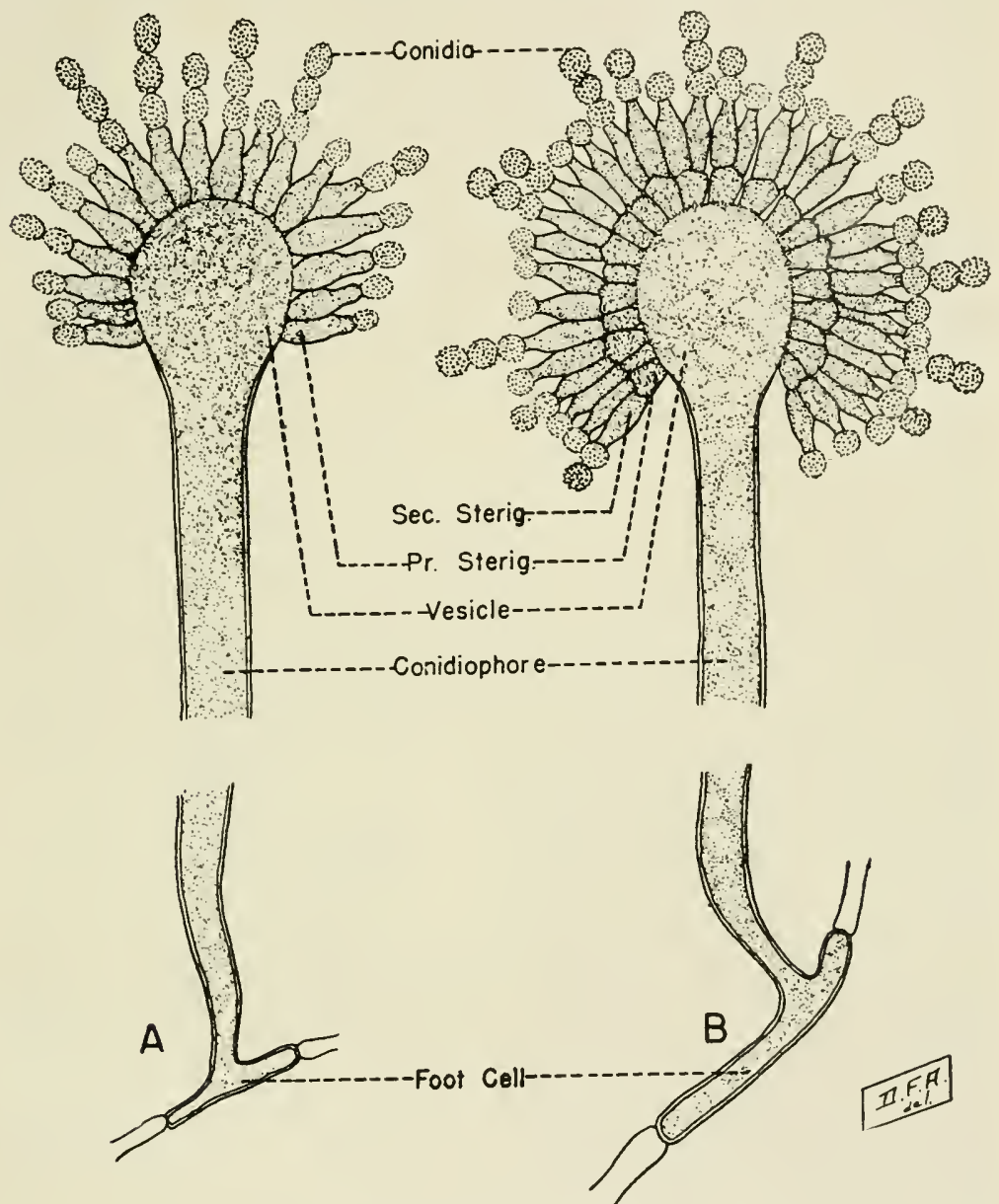

FIG. 3. A, Aspergillus niveo-glaucus, NRRL No. 127, typical head showing only one series of sterigmata, $\times 575$. B, A. versicolor, NRRL No. 239 , typical head showing sterigmata in two series, $\times 1200$.

surface of the wall shows irregular elumps or uneven thickenings. When broken, many of these conidiophores show uneven or jagged ends like a broken glass tube; in the $A$. niger group the broken ends split like bundles of laths (fig. $64 \mathrm{~B}$ ), giving a possible elue to the method of their formation. In the second section, the wall appears dotted or pitted, or as interpreted 

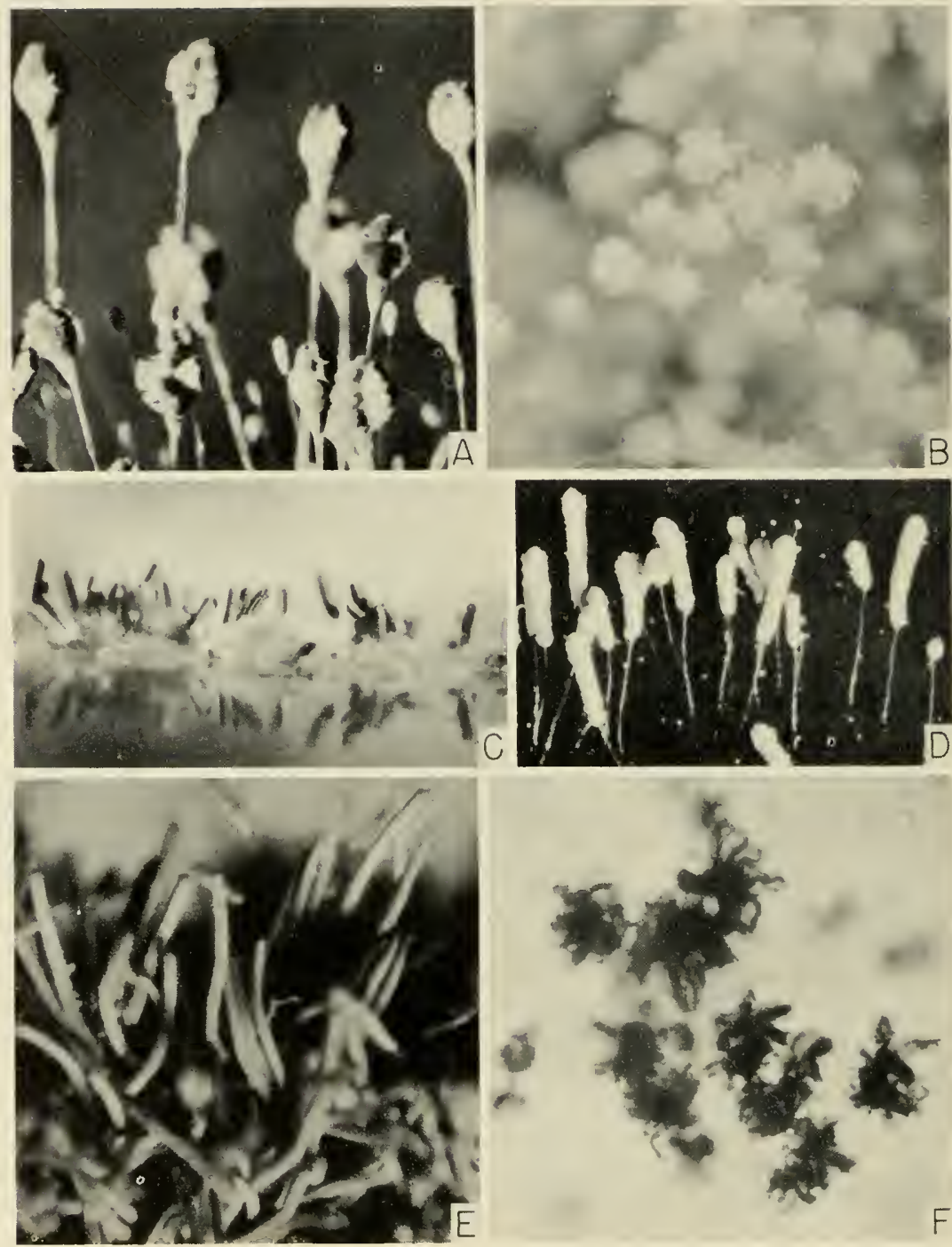

FIG. 4. Conidial heads; group types. A, Aspergillus clavatus group: conidial heads of $A$.giganteus, NRRL No. 10 , showing typical clavate form, $\times 15$. B, Aspergillus glaucus group: heads of $A$. niveo-glaucus, NRRL No. 127, showing characteristic radiate pattern, $\times 35 . C$, Aspergillus nidulans group: heads of $A$. nidulans showing typical short-columnar form, $\times 35$ (Photograph by Edward Yuill). D, Aspergillus flavipes group: heads of $A$. flavipes, NRRL No. 1959, typically barrelform, or loose columnar as shown, $\times 18$. E, Aspergillus terreus group: A. terreus, NRRL No. 265, heads columnar, of uniform diameter throughout, of ten becoming quite long as shown, $\times 22$. F, Aspergillus ustus group: A. ustus, NRRL No. 1974, heads typically loose and radiate as shown, under certain conditions approaching columnar, $\times 22$. 

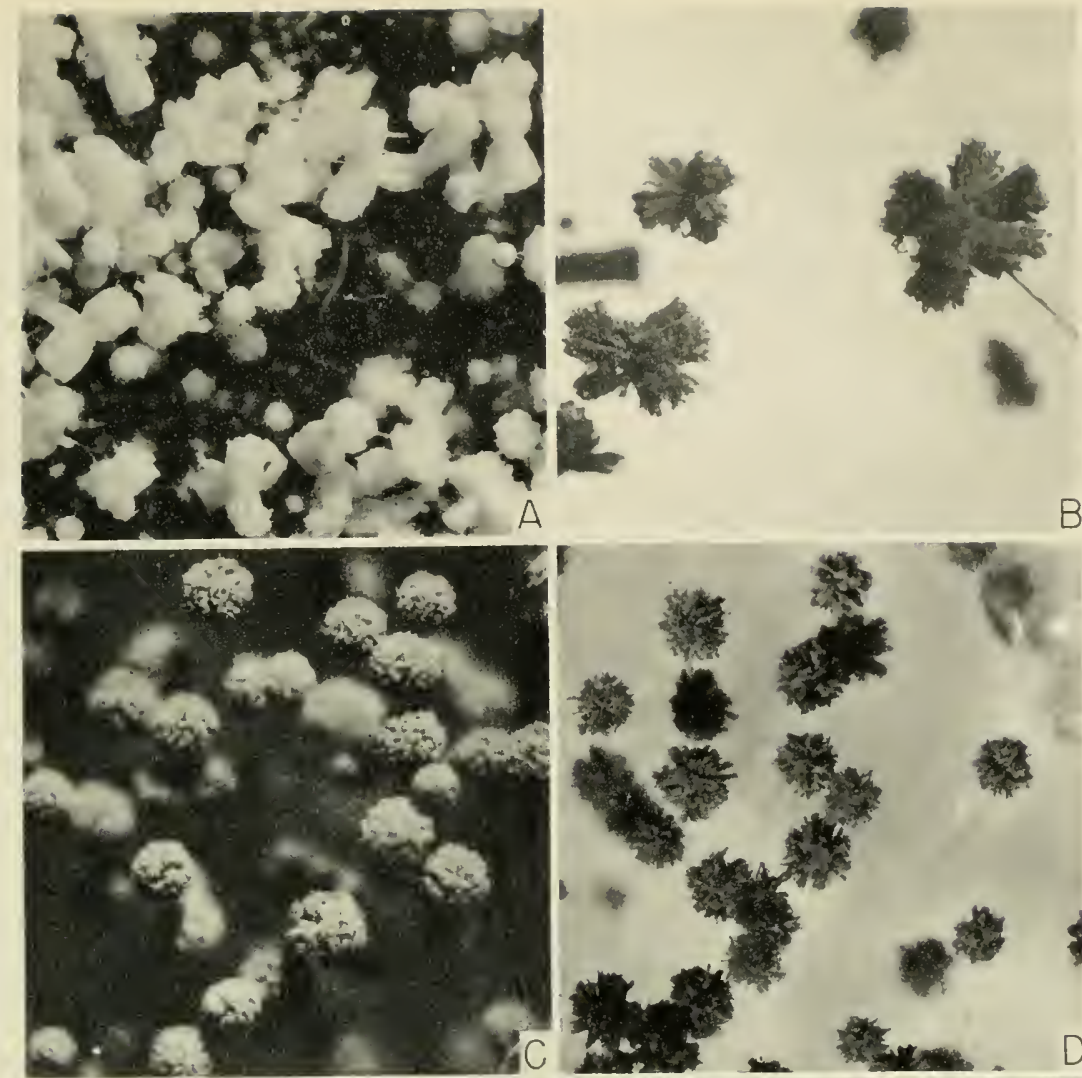

B

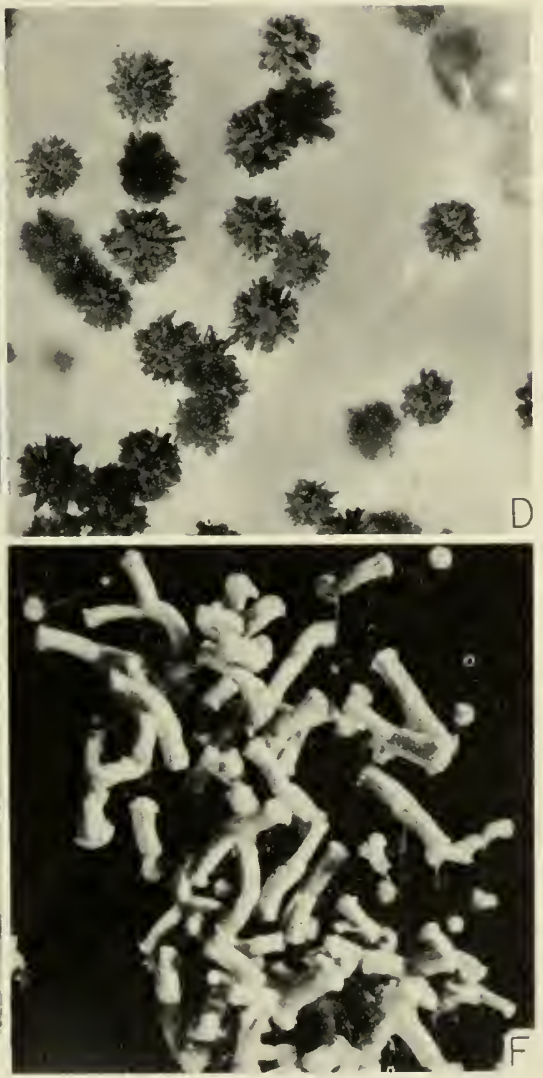

FIG. 5. Conidial heads; group types. A, Aspergillus candidus group: $A$. candidus, NRRL No. 308, showing characteristic mature heads of different dimen. sions, $\times 18$. $B$ and $C$, Aspergillus niger group: $B$, strain NRRL No. 67, showing typical mature heads, $\times 30 ; C, A$. niger mut. schiemanni, heads typically globose as shown (Photograph by Edward Yuill) $\times 30$. D, Aspergillus wentii group: typical globose heads of A. wentii, NRRL No. $397, \times 18 . \quad E$, Aspergillus flavus-oryzae group: A. flavus, NRRL No. 1957, typical mature heads, $\times 18 . F$, Aspergillus ochraceus group: A. ochraceus, NRRL No. 398, typical mature heads splitting into divergent columns of conidia, $\times 18$. 
with low magnification, often rough or echinulate (e.g., A. flausu-oryzae group). The secondary thickenings in such conidiophores appear to have been laid down around protoplasmic areas, which, for a time at least, maintain contact with the primary wall outside. The size and abundance of the pits in the mature conidiophore wall differ with the species, but in a general way correspond with the rate of withdrawal of the protoplasmic mass from its primitive connection with the original outer wall. In some of the species in both groups, warts, or superficial and usually more or less hemispherical concretions are found on the outer surfaces of the conidiophore wall, sometimes few and scattered widely, again fairly numerous, but always unevenly distributed (e.g., A, ochraceus group). These warts, or concretions, appear to be deposits of excreted substance, possibly due to the evaporation of the numerous drops or globules of liquid abundantly visible upon the young and growing conidiophores.

The color of the conidiophore wall may be homogeneous, or the layers may differ markedly in shade. In a number of the groups the entire wall is hyaline. In certain other groups the outer layer is yellow as in A. ochraceus and $A$. flavipes, or it may be some shade of green, brown, or avellaneous. In some species the whole wall is colored for all or part of its length. No explanation of these color differences is available, except possibly the varying concentration of aspergilline (Linossier, 1891) in the upper part of the conidiophores of members of the $A$. niger group, as well as in the conidial heads.

\section{The Vesicle}

The conidiophore is usually much larger toward the apex than at the point of origin. At the base of the head a further dilation occurs more or less abruptly to produce the vesicle (blase, of the German mycologists). This vesicle is globose, hemispherical, elliptical, or long clavate in various groups of the Aspergilli and furnishes an enlarged surface for the attachment of spore-bearing cells. The lumen of the vesicle is continuous with that of the upper part of the conidiophore; a septum near the base of the head is occasionally, but only rarely, seen (see Corda, A. mucoroides for description), and has not been regularly found in any species.

\section{Sterigmata (Compare Fig. 3)}

The conidia-bearing surface, represented by the fertile area of the vesicle, is closely covered by the simultaneous development of a layer of cells, the sterigmata, each in a general way perpendicular to a point on the fertile surface of the vesicle. In figure $3 \mathrm{~A}$ a single layer of such cells is shown, each of which produces an unbranched chain of conidia. In figure $3 \mathrm{~B}$ each of the first series of cells, or primary sterigmata, bears two to several 
cells, the secondary sterigmata, forming a crown, or verticil, at the apex. Each of the sccondary sterigmata bears one chain of conidia. The mechanism shown in figure $3 \mathrm{~B}$ would produce several times as many chains of conidia as figure $3 \mathrm{~A}$. Such a head as figure $3 \mathrm{~B}$ would be compact, whereas figure $3 \mathrm{~A}$ would represent a loose head.

In figure $3 \mathrm{~A}$ the cells in the single layer, each producing a chain of spores, are in the strict sense the sterigmata. In figure $3 \mathrm{~B}$ cells of the first layer, or primary sterigmata, produce verticils of cells, the secondary sterigmata, each of which is in the strict sense a sterigma. The cells are usually characteristic in size and shape for series of closely related species. Where there are both primary and secondary series, the primary sterigmata are essentially supporting cells and vary much more in size and shape than do the secondary sterigmata. For this reason they are more useful in species diagnosis than the secondary series.

Various usages are found in the literature. The primary sterigmata are often called basidia. The secondary sterigmata are called phialids because they have somewhat the shape of the pharmacists' phial (vial). In translating descriptions into the Latin, Saccardo apparently followed the describers rerbatim, hence used no consistent terminology. We find the primary sterigmata as sterigmata, basidia, „or pseudobasidia, and the secondary series as sterigmata, pseudosterigmata, ramuli ("ramulis sporiferis") or even rami (branches); all of these usages have been homogenized here into "primary and secondary sterigmata."

\section{Conidium Formation}

The actual spore-producing cell, or sterigma, is definitely specialized. It ordinarily consists of an essentially cylindrical body, which, after reaching a length more or less uniform for the species, narrows into a spore-producing tube whose diameter is fairly uniform within the species. Elongation is thenceforth confined to this spore-producing tube. The nuclei in the sterigmata divide and one of each pair of daughter nuclei passes into the tube; cell division follows. Parallel with the repeated division of the sterigma nucleus, the tube continues to elongate rapidly, successively cutting off new sections and pushing the older cells outward. Each such chain of spores typically consists then of series of equal sections cut from one tube or tip of a sterigma and each carries a daughter nucleus derived directiy from the active nucleus of the sterigmatic cell at the base of the chain. No further divisions occur among the cells in the chains. Such chains of ten contain several hundreds of spores, or conidia, each of which is theoretically at least exactly like the rest, hence fully capable of propagating the species (fig. 6). 


\section{The Conidium, or Spore}

The conidia are thus specialized propagative cells, asexual in origin, produced by a complex cellular fruiting structure. This consists (1) of a foot-cell connected with the vegetative mycelium and usually imbedded in the moist substratum, (2) of a conidiophore, or stalk, rising more or less vertically into the air to a distance typical of the species and enlarged at the apex to form the vesicle which is the central unit, and (3) of a dilated head consisting of one or two series of cells, the outermost of which are specialized for the purpose of producing chains of cells (the conidia), each equally capable of carrying the genetic factors necessary to propagate the species.
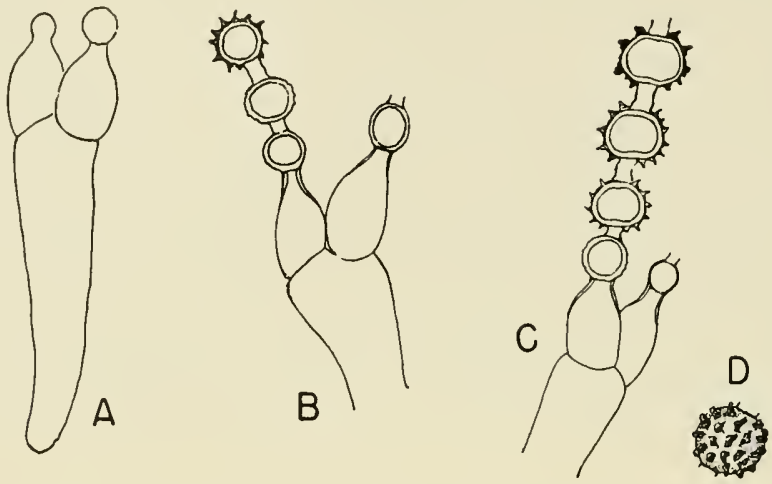

FIG. 6. Camera lucida sketches showing progressive stages in conidium formation, $\times 700$. $A$, Initiation of conidium formation. $B$, Secondary sterigma bearing a chain of three conidia, the outermost developing characteristic roughenings by the deposition of coloring matter between the outer (thin) and inner (firm) wall. $C$, Sterigma bearing a chain of conidia in which differentiation of the outermost spore is complete. $D, \mathrm{~A}$ single mature conidium seen in surface view. See discussion on page 24 .

After the conidium is separated by a septum from the mother cell or sterigma, it remains so attached ${ }^{1}$ as to draw nutrients from the parent cell at first, while it assumes the size and shape characteristic of the species, then it lays down within its original, or primary wall, a secondary cell wall whose color, texture, and marking are those of the species. The secondary wall completely separates this spore from the parent cell. (fig. 6). Exact uniformity is not attained. An occasional cell fails to develop; some differences in size are usually evident; markings, while characteristic in nature and general pattern, are not always identical as to details. For descriptive purposes, size ranges are therefore more important than exact measurements and the nature of the markings found are more important than the relative

${ }^{1}$ Buller (Researches on Fungi V, Chapter II, 1933) discusses the primary septum as having a central pore through which connections are maintained. 
number and dimensions of such. Such conidia may be very thin-walled, delicate and readily destroyed, or firm-walled, almost impervious to stains and able to retain their vitality for many years. They are extremely small, light, and float readily in air currents. In many species, the outer layer of the spore wall absorbs water slowly, hence such spores tend to float in currents of fluid or to develop as mycelia covering the surfaces of liquid media. Molds being typically aerobic, normal colonies develop only on the surface of the substratum where oxygen is abundant and their spores can be discharged directly into the air. Spores developing under submerged conditions in the absence of adequate oxygen produce fragmentary and defective mycelia only.

\section{The "Connective"}

Descriptive literature often cites the presence of a "connective" or "disjunctor", a "bridge" between conidia in the chain. This is sometimes present, again absent, in the same microscopic preparation, and when seen, it appears as a short space between spores, bridged by transparent cell walls. This is exactly what it is. Cells cut off from a cylindrical tube may swell and assume subglobose form without breaking their area of contact, or, in the swelling and rounding up process, they may partially or completely break that contact leaving the original cell wall of the tube, within which they developed, as a bridge across the open space (fig. $6+\mathrm{C}$ ). The critical examination of the developing cells in thousands of preparations have failed to justify interpretations which assume the degeneration of every alternate cell, or fantastic fusions in the production of conidia. The observation of connectives is, therefore, ordinarily worthless because morphologically it means nothing, and it is not justified by successive studies of the same species.

\section{Endogenous Conidia}

A spore is described as endogenous if it is formed within a tube or cell wall of a previously existing cell. It may be extruded through a tube. That tube may be used once only or many times. The critical factor is the formation of a cell or spore within an existing specialized sporebearing organ and its extrusion from that body through a fixed tube. In Aspergillus, the tip or tube of the sterigma elongates, a cylindrical section is cut off carrying the tube wall and the septum at each end as the primary wall of the spore itself. Within that primary wall the spore as an entity rounds itself up to characteristic form, deposits or lays down its own wall with whatever coloration or markings may be typical of the species. The primary wall may remain separate and distinct and in the ripe spore be visible under the microscope, it may be blended with the secondary wall, 
or it may not be determinable on the ripe conidium by ordinary examination. In the sense of the definition above, no endogenous conidia appear in Aspergillus. In cases, the primary walls are seen to break away if ripe spores are mounted in fluid, of ten carrying with them the granular materials which impart the characteristic marking to the spore, hence leaving the wall of an $A$. niger spore smooth (A. luteo-niger Lutz).

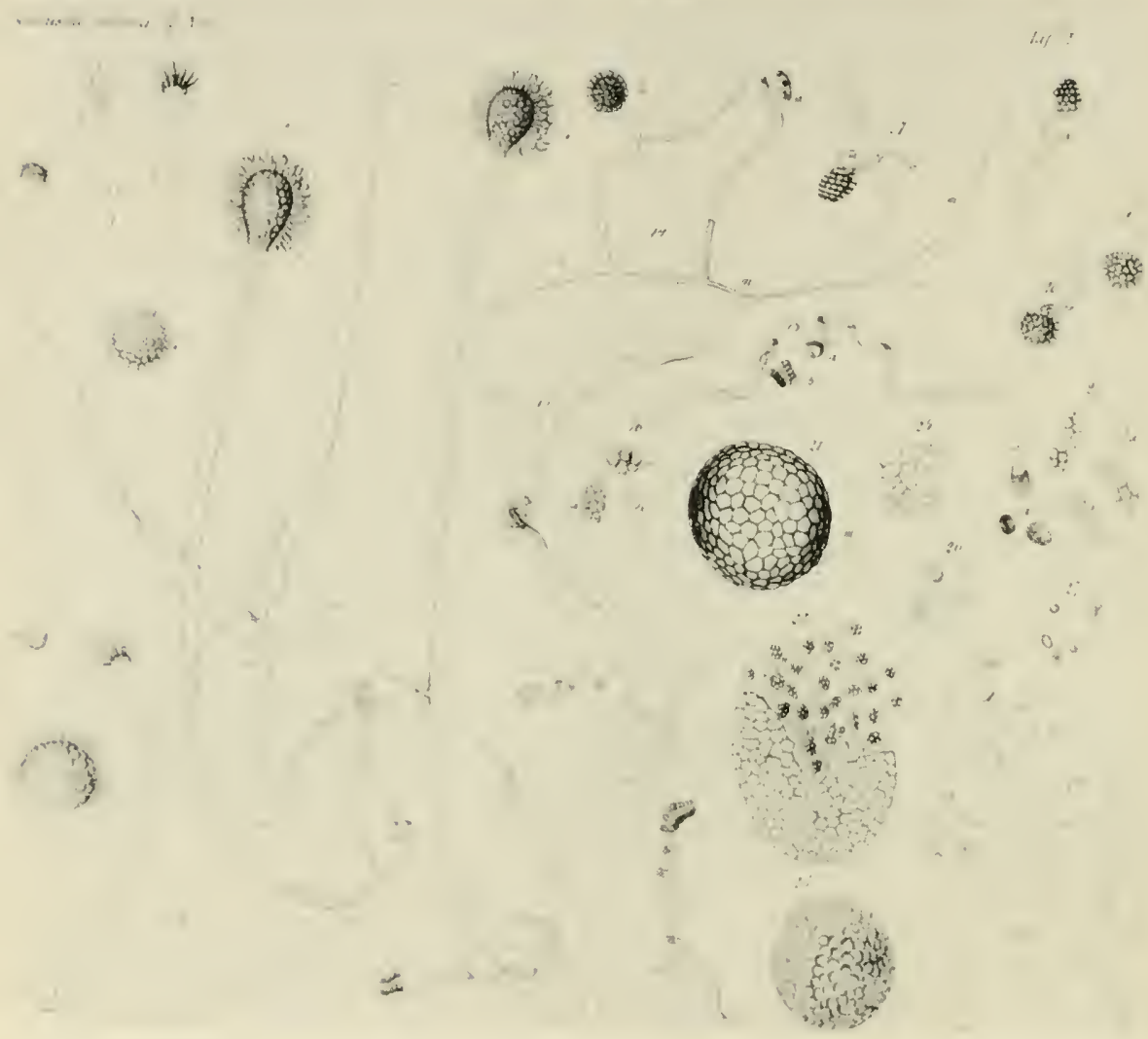

FIG. 7. The development and relationship of Aspergillus glaucus and Eurotium, after DeBary, $18 \tilde{1}$.

\section{Perilhecia}

The general morphology of the perithecium of the A. glaucus group (Eurotium) was described and figured by DeBary $(1854,1870)$, while that of $A$. nidulans was described by Eidam soon thereafter (1883). DeBary described the yellow to orange or ferrugineous perithecia as from 90 to $300 \mu$ in diameter, without ostiole and without specialized appendages (fig. 7). Thesc perithecia are borne, above the surface of the substratum 
and are never more than loosely hung in networks of hyphae; they are often very abundant and dominate the color of the colony. Perithecium formation is favored by abundance of assimilable carbohydrate, but may or may not be completely suppressed by its replacement with nitrogenous products; transfers from the same culture have developed colonies yellow from perithecia when grown on sugar solution, or dense green without sign of perithecia upon a peptone medium or a piece of leather.

The perithecium of Aspergillus arises from a branch which coils in various manners in the different species to become the ascogone, as figured by Dangeard from forms reported as E. herbariorum, A. flavus, A. fumigatus, $S$. ochracea, $S$. nidulans, by Fraser and Chambers for $A$. herbariorum, and by Dale (1909) for $A$. repens. No fertilization process was demonstrated in those studies. Dangeard (1907) reported the cells in the fruiting apparatus as multinucleate in $E$. herbariorum, but to be mononucleate in the other species figured. In general, however, the development of an ascogone, followed by the development of the perithecial wall and accessory cell masses from vegetative hyphae below the ascogone, has been roughly described and figured for the group represented by A.glaucus and A.nidulans but interpretation of the other specific names used by Dangeard is doubtful, even for grouping, on the basis of the descriptions and figures given.

Henrard (1934), working with 15 "species" of Aspergilli, reports that all of them are "sexually homothallic." He summarized the literature by saying that homothallism in the Aspergilli has been affirmed by Kniep (1928) for A. repens; by Schwartz (1928) for A. ruber, A. repens, four other strains of "A. glaucus", and four strains of A. nidulans; by Blochwitz (1932) for six strains in the $A$. glaucus group; and by Greene (1933) for A. fischeri.

So far then, as present information goes, heterothallism cannot be assumed to account for the great variability of the Aspergilli.

Perithecia are regularly found in most of the species of the A. glaucus group and in $A$. fischeri, which is closely related to $A$. fumigatus, and in a series of closely related forms in the $A$. nidulans group. They are not sporadic or dependent upon unknown conditions, but are regularly produced in media which are adequately supplied with sugars and the salts in routine use. Their presence in A. wentii and A. citrisporus was asserted by Thaxter (personal communication) without producing either material, or description of the ascospores. Dangeard (1907) claimed to have found ascospores within the sclerotia of $A$. niger but failed to describe them. Diligent search over many years has failed thus far to confirm either statement. Still, it may be assumed that such sclerotia may be the homologue of structures which under some conditions might become perithecia. 


\section{The Ascospore}

So far as fully described, the ascospore of Aspergillus follows the general type shown in the figures and deseription of DeBary. In the course of its development, the secondary thickening of the cell-wall develops in the form of two symmetrical valves suggesting the arrangement found in the shell of a bivalve mollusk, such as the hard clam (Venus mercenaria). The ripe ascospore is commonly shaped as a double convex lens with the valves more or less closely in contact at the edges. A series of variations upon this basic patteln oceur and characterize particular species (figs. 27, 34 , and 43 ). If the exospore is smooth and the margin of the valves is not marked by folds or ridges, figure $27 \mathrm{~A}$ characteristic of $A$.repens appears; if the exospore is rough in the absence of marginal folds, figure $27 \mathrm{D}$ characteristic of $A$. amstelodami results; if the exospore is rough and the margin of the valves bear folds or ridges, figure $43 \mathrm{C}$ characteristic of $A$. rugulosus and A. fischeri is produced. An extreme development of the marginal folds is seen in $A$. variecolor, figure $43 \mathrm{D}$.

When such an ascospore germinates (fig. 8A), the figure first shown by DeBary (1854) develops. Exactly the same type of germination is shown by $A$. nidulans, in which as the spore swells, the valves first separate at one edge, then parting completely, remain on opposite sides of the germinated spore conspicuously identifiable by the bright purple-red color of the ralves (Thom and Church, 1926).

\section{Hitlle Cells}

Mature perithecia were described for A. nidulans by Eidam (1883) but without giving attention to their origin. In his description, Eidam pictured a loose network of hyphae more or less completely surrounding the perithecium containing large numbers of "Hülle" cells, terminal or intercalary cells which swell and become vesiculose, elliptical or almost globose, then develop very heavy thick walls almost obliterating the cell lumen (fig. 49). These cells were later noted by Dangeard (1907) who designated them as chlamydospores, but failed to present evidence of function as propagative cells. These cells are abundant in connection with perithecia in all strains of the $A$. nidulans group, but are lacking in A. unguis which does not produce perithecia. Cells of the same general character appear in sterile masses of hypae in strains of the A. ustus and A. flavipes groups (fig. 49), and in some strains of the $A$. versicolor group, while thick-walled, septate hyphae at least suggestive of hülle cells appear in Aspergillus carneus in the A. terreus group (fig. 49F).

In the A . nidulans group hülle cells, "Eidamsche blasen," or chlamydospore (terms found in the literature) are always produced in connection with perithecium formation (fig. 42 ). In the A. flavipes, $A$. ustus, and $A$. 

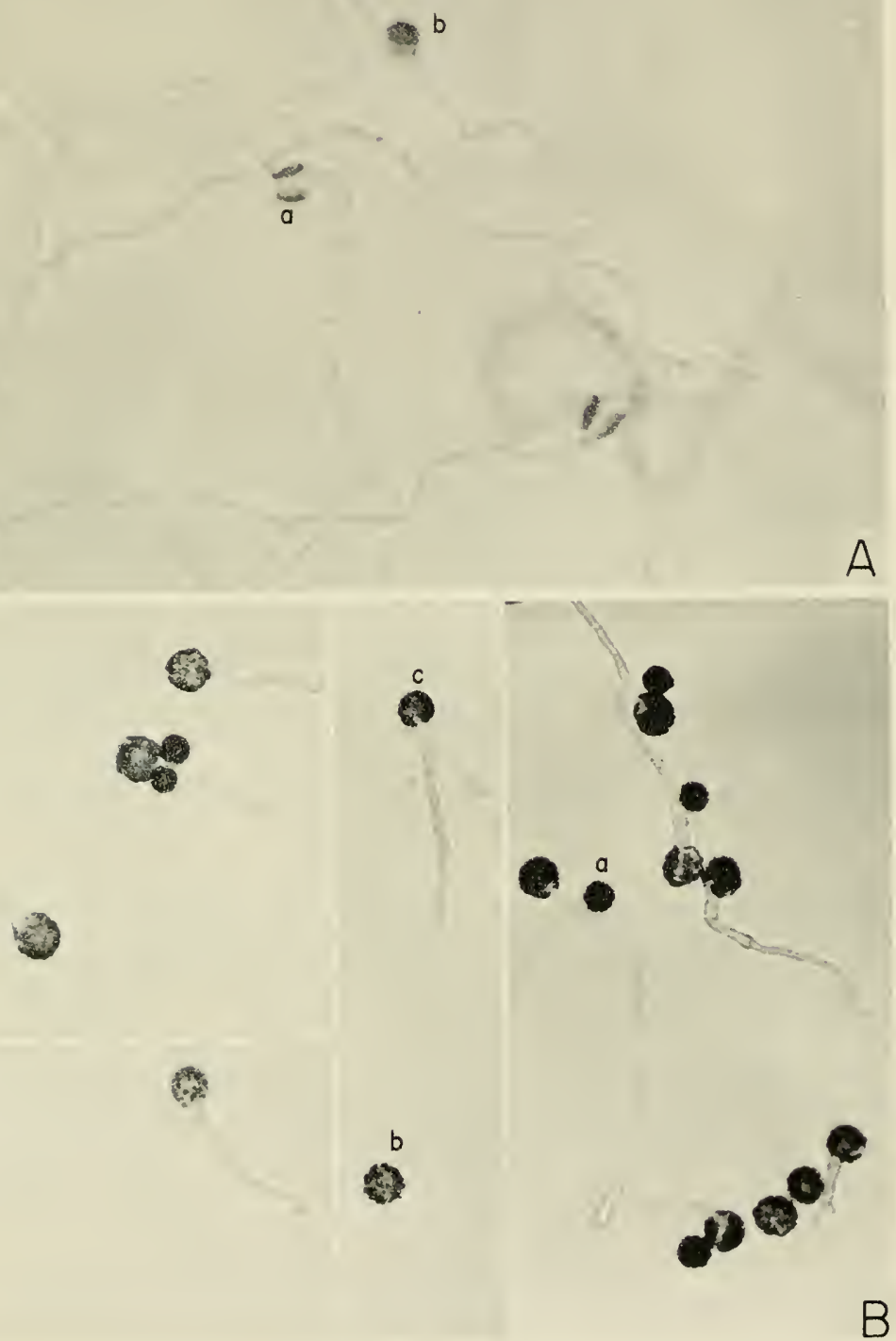

FIG. 8. Spore germination. A, Germinated ascospores of Aspergillus nidulans, $\times 750: a$, ascospore in profile showing how the two valves comprising the spore wall are pushed apart as the spore content enlarges and the sporeling develops; $b$, the same in face view. $B$, Germinating conidia of Aspergillus niger, $\times 600: a$, ungerminated spore; $b$, spore in early stage of germination; $c$, germinated spore showing rapidly elongating sporeling. 
versicolor groups, they occur in scattered aggregates of varying conspicuousness, although no perithecia have been found. Occasionally, as in one of the A. flavipes group, foot-cells and sterile cells, which appear to be conidiophores ending in points instead of heads, appear. These aborted conidiophores vary from nearly full length to vestigial. From the study of such material it is believed that Eidam's hülle cells represent aborted conidiophores surrounding the perithecia of $A$. nidulans and they may be indicative at least of a vestigial precursor of perithecium formation when they occur elsewhere.

Hülle cells do not, however, accompany the perithecia of either the $A$. glaucus group or $A$. fischeri. In the former, the perithecia are smoothwalled and naked; in the latter, they are surrounded by a loose network of sterile but unspecialized hyphae.

In $A$. panamensis (Raper and Thom, 1944) aborted conidiophores commonly occur, but no hülle cells have yet been observed. In $A$. unguis (Thom and Raper, 1939), which is one of the A. nidulans group lacking only the ascosporic phase, the long, sterile, thick-walled "spears" suggest homologous structures. To summarize, hülle cells are specialized structures that normally occur in certain groups of the Aspergilli. They are of somewhat questionable origin and, in our experience, are not known to serve any functional purpose. Schwartz (1928) figures the hülle cells of $A$. nidulans as capable of germinating and thus acting as reproductive cells. Since the cells of different groups are fairly characteristic, they often provide valuable diagnostic group and species characters.

\section{Sclerotia}

Definitely hard masses with characteristic surface marking and coloration, and consisting of thick-walled parenchyma-like cells occur in several groups of Aspergilli. Such structures have not been seen in the groups typified by $A$. clavatus, $A$. glaucus, A. fumigatus, $A$. nidulans, $A$. ustus, $A$. versicolor, or $A$. terreus. On the other hand, they develop regularly in certain members of the $A$. candidus, A. niger, A. wentii, A. tamarii, A. flavus-oryzae, and in the $A$. orchaceus groups; in other members of these same groups grown under similar conditions, no such structures have been found. In occasional strains of $A$. flavipes dark hyphal masses are seen, but these are not sufficiently compact to be considered true sclerotia. The development of sclerotia has not been followed in sufficient detail in any species to fix their genetic significance, but the specialized, characteristic structure of the sclerotia of many species lends color to the report of ascospore formation by Dangeard (1907), although no one else seems to have been able to verify such development. Environmental factors are known to influence sclerotium development, but these have not been cárefully analyzed. 


\section{Chapter IV}

\section{CULTIYATION AND EXAMINATION}

An Aspergillus occurring upon a natural substratum may frequently be identified as to group from the original material if it belongs to one of the large and well-marked groups. Among members of the same group, however, differences in the nature and composition of the substratum produce marked contrasts in colony appearance, quantity of growth, coloration, measurements of fruiting structures, and in the appearance of conidiophores and conidial masses. Many Aspergilli can be identified as to group from dried herbarium materials, but the process of drying generally changes the colors materially and renders the hyphal masses so fragile that the morphological details necessary for identification inside a particular group are of ten obliterated or made difficult to interpret. Although accurate placing of such materials is sometimes possible, a much more satisfactory identification may be reached by transferring fresh material to culture media of known composition, followed by purification of the cultures so that they present single species or strains for intensive study.

Two types of material for identification are regularly encountered by one undertaking a study of the Aspergilli: (1) the culture already isolated by another worker, and (2) the moldy substratum with its natural flora of micro-organisms of ten representing several or many species, including miscellaneous molds, bacteria, actinomycetes, and even protozoa and other forms. In either case, the final decision as to the proper name of an Aspergillus must usually be sought in fresh cultures made by the one responsible for identification.

Classification within the genus has become so dependent upon observation in pure culture that the whole subject of laboratory cultivation, including favorable substrata, culture making, and culture handling needs to be considered.

\section{CULTURE MEDIA}

Pure culture upon known substrata is almost essential to the identification of Aspergilli. Since the morphological responses to diverse nutrients, and especially to the stimulus of mixtures of other molds and bacteria growing with any particular species, is great, the study of each strain or species in pure culture in media of known composition is practically necessary.

Aside from Raulin's group in Paris, most of the early culture work with Aspergilli was carried through upon so-called natural media. DeBary and 
Brefeld used decoctions of horse dung variously diluted and stiffened with gelatin. Much of the European work was done with brewery wort; Mazé (personal communication, 1904) used extract of white beans; potato and carrot decoctions have been widely used; Bainier grew his molds and described many of them upon sticks of licorice root; others preferred plugs of potato or of carrot, string beans, etc. The primary aim was to obtain an optimum growth of the mold under observation, rather than to analyze its relation to the substratum or furnish comparative data to distinguish it from other members of a series.

An optimum culture substratum for comparative study of the Aspergilli needs to contain the necessary chemical elements in the form of pure but assimilable salts, supplemented by carbohydrates of such structure as to be available to the largest number of species, and purchasable in pure form in the chemical trade. Supplementary and often very important information must be sought from variations in the proportions of nutrients used, or in the introduction of widely different substances in replacement of particular components of media already used. The Aspergilli as they are isolated from nature are not very dependent upon vitamins or growth-promoting substances. For cultivation upon solid substrata, agar is almost universally employed as a gelling agent.

When the study of a large number of molds is undertaken, comparison of these molds under controlled and reproducible conditions of growth become essential. Foremost among the conditions which must be standardized is the culture medium, or substratum. Various authors have proposed standardized and reproducible formulae which in their experience have provided uniform cultures over long periods, hence are of value for comparative studies. A series of such media are presented.

\section{Czapek's Solution Agar}

As a routine medium for comparative work, the following formula originally adapted from Czapek (1902, 1903) by Dox (1910) has been widely used. Minor variations in quantities apparently do not affect the reactions. Cultural information given in this manual is obtained from growth upon media produced by this formula unless otherwise specified.

Czapek's solution ąar:

Water. $1,000 \mathrm{cc}$.

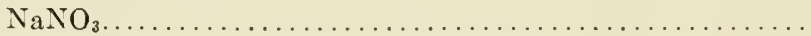

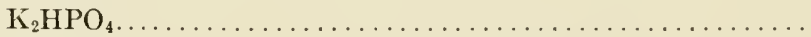

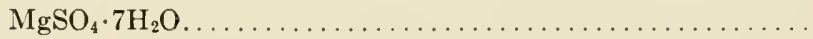

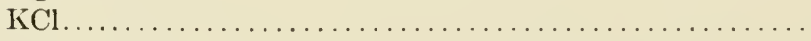

$\mathrm{FeSO}_{4} \cdot 7 \mathrm{H}_{2} \mathrm{O}$.

3.0 grams

1.0 gram

$0.5 \mathrm{gram}$

0.5 gram

Sucrose (Cube or other good commercial grade).

$0.01 \mathrm{gram}$

Agar.

30.0 grams

15.0 grams 
To reduce caramelization the sugar is added just prior to final sterilization.

Czapek's solution agar is not offered as an optimum substratum for any particular species, but as a mixture approximately neutral in reaction, which is readily made in any laboratory in fairly uniform manner, and which permits moderately vigorous growth of nearly all of the saprophytic Aspergilli. The quantities of mycelium and conidia produced by many forms are much greater upon other media, but for comparative study, a moderate growth of the majority of the species is more useful than the great mass of mycelium and conidia which are readily obtained by using enriched substrata.

Since the purpose of the Czapek formula is to insure the presence of the chemical elements required in quantities sufficient to support good growth, it is frequently modified as to quantities and nutrients introduced. For some Aspergilli such as the A. glaucus group, the addition of 20 percent or even 40 percent of sucrose has proved useful. Ammonium nitrate is sometimes substituted for sodium nitrate but with the loss of information as to whether the mold utilizes the ammonia or the nitrate, or both. Dextrose is commonly substituted for sucrose. The monobasic potassium phosphate prevents precipitation of certain components in sterilization, but it produces an acid instead of a neutral medium, hence complicates many pieces of work. The introcluction of peptone or yeast extract increases the sporulation of some forms. These and other changes are made, however, by investigators who still refer to Czapek's solution as the basis of their work.

Biourge, in his monograph of Penicillium (1923) and in his unpublished Manuscript of Aspergillus (1939), put much emphasis upon the method of preparing the neutral Raulin medium which he used for the growth of the colonies analyzed in making his species diagnoses.

\section{Neutral Raulin's Solution-Dierckx-Biourge}

1. Magnesium carbonate............................. gram

Tartaric acid. .

0.71 gram

Triturate in a mortar with a few drops of distilled water and add quickly to a flask of distilled water; make up to $100 \mathrm{ml}$.

2. To a liter flask with 800 to $900 \mathrm{ml}$. distilled water add:

Sucrose. . . 46.60 grams

Ammonium nitrate

2.66 grams

Ammonium phosphate.

0.40 gram

Potassium carbonate.

0.40 gram

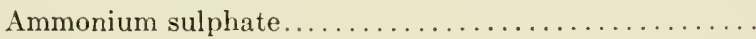

C.16 gram

Zinc sulphate.

0.04 gram

Iron sulphate

0.04 gram

3. Add 66 to $67 \mathrm{ml}$. of the magnesium tartrate solution (1) to the mineral saltsucrose solution (2) and make up to $1,000 \mathrm{ml}$. with distilled water. 
In his detailed study of the A. niger group, Biourge's pupil, Mosseray (1934a), gives his simplified Raulin's solution as follows:

Water, distilled

Sucrose

Tartaric acid' $\mathbf{1}$.

Magnesium carbonate ${ }^{1}$.

Ammonium nitrate 2 .

Potassium carbonate 2

Ammonium phosphate $\left(\mathrm{NH}_{4}\right)_{2} \mathrm{HPO}_{4}{ }^{1}$

Ammonium sulphate ${ }^{1}$.

Iron sulphate ${ }^{1}$ (cryst.)

Zinc sulphate ${ }^{1}$ (cryst.).

Agar-agar ${ }^{1}$.
$1,000 \mathrm{ml}$.

50 grams

0.40 gram

0.250 gram

0.250 gram

0.40 gram

0.40 gram

0.20 gram

0.05 gram

0.05 gram

20.00 grams

Sterilize $120^{\circ} \mathrm{C}$. for 20 minutes.

\section{Steinberg's Solution}

Steinberg in the course of many years' investigation of a single strain of Aspergillus niger (NRRL No. 334: Thom No. 4247) developed a basic formula for testing other phases of the nutrition of his mold. It is called the "dibasic optimum" solution and carries mannitol instead of sucrose, sodium nitrite instead of ammonium nitrate, with the addition of sufficient sulphuric acid to obtain any desired reaction. Interpolations into this solution offer many possibilities.

\section{Steinberg's "dibasic optimum" (Thom and Steinberg, 1939)}

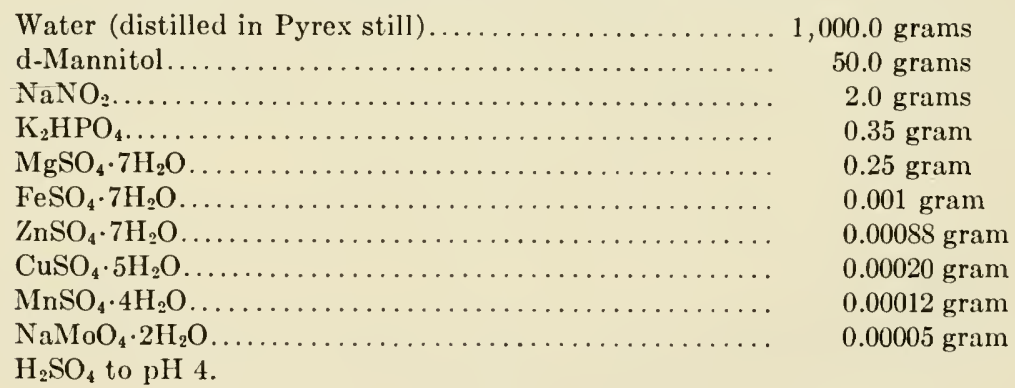

A number of so-called natural substrata, including malt extract and hay infusion agars, are very useful in the study of the Aspergilli. A great majority of species sporulate more freely upon malt extract than upon Czapek's solution agar (fig. 9), and for this reason it is very useful where large quantities of spores are desired. This medium is, however, less diagnostic than Czapek's solution.

1 Merck reagents.

${ }^{2}$ Kahlbaum reagents. 
Malt Extract Agar (Blakeslee's Formula, 1915)

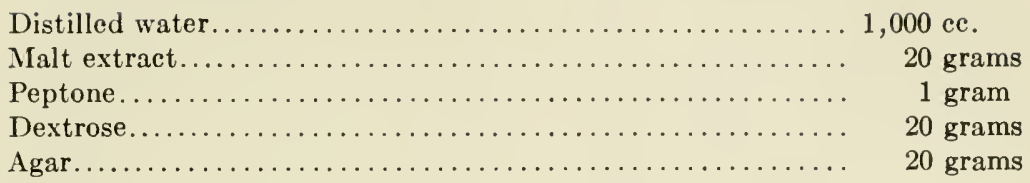

Add dextrose just prior to final sterilization. Conidial structures are generally more numerous and are often borne on shorter conidiophores, and there is an almost complete absence of coloration in the substratum. Except in a few isolated cases, coloration of the spore heads themselves is not materially altered. While the production of exudate in the form of drops is not characteristic of many of the Aspergilli, it can be generally said that droplet formation on malt agar is much less than upon Czapek's solution agar.

Hay infusion agar is very useful in the isolation of Aspergilli from nature. A 1:10 suspension of soil in sterile water is streaked on hay infusion agar plates and incubated for one week to 10 days. Isolations are then made from individual fruiting structures with the aid of a low-power binocular.

\section{Hay Infusion Agar}

Distilled water............................ 1,000 cc.

Decomposing hay......................... 50 grams

Autoclave for 30 minutes at 15 pounds. Filter.

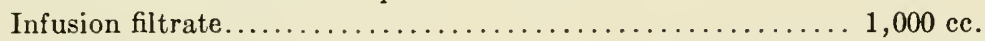

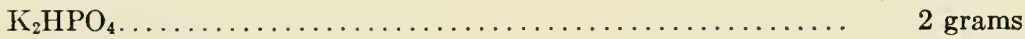

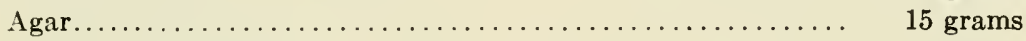

Adjust $\mathrm{pH}$ to $6.2 \pm$

No Aspergillus makes a luxuriant growth upon this medium, but a great variety of forms make a limited development. Furthermore, such fruiting structures as are produced are generally characteristic of the different species present. It thus constitutes a very favorable substratum with which to analyze and isolate the Aspergilli occurring in soils or other natural substrates. The medium is likewise useful for securing limited sporulation of certain forms, such as Aspergillus sparsus, which fruit very sparsely upon Czapek's solution agar.

Czapek's solution agar enriched with peptone or corn steeping liquor is often very useful. For example, in the cultivation of members of the glaucus group, the addition of a limited amount of peptone greatly increases the production of conidia, while addition of a small amount of corn steeping liquor (e.g., 0.2 percent) increases the growth of most forms without markedly affecting the character of the resulting colonies. 

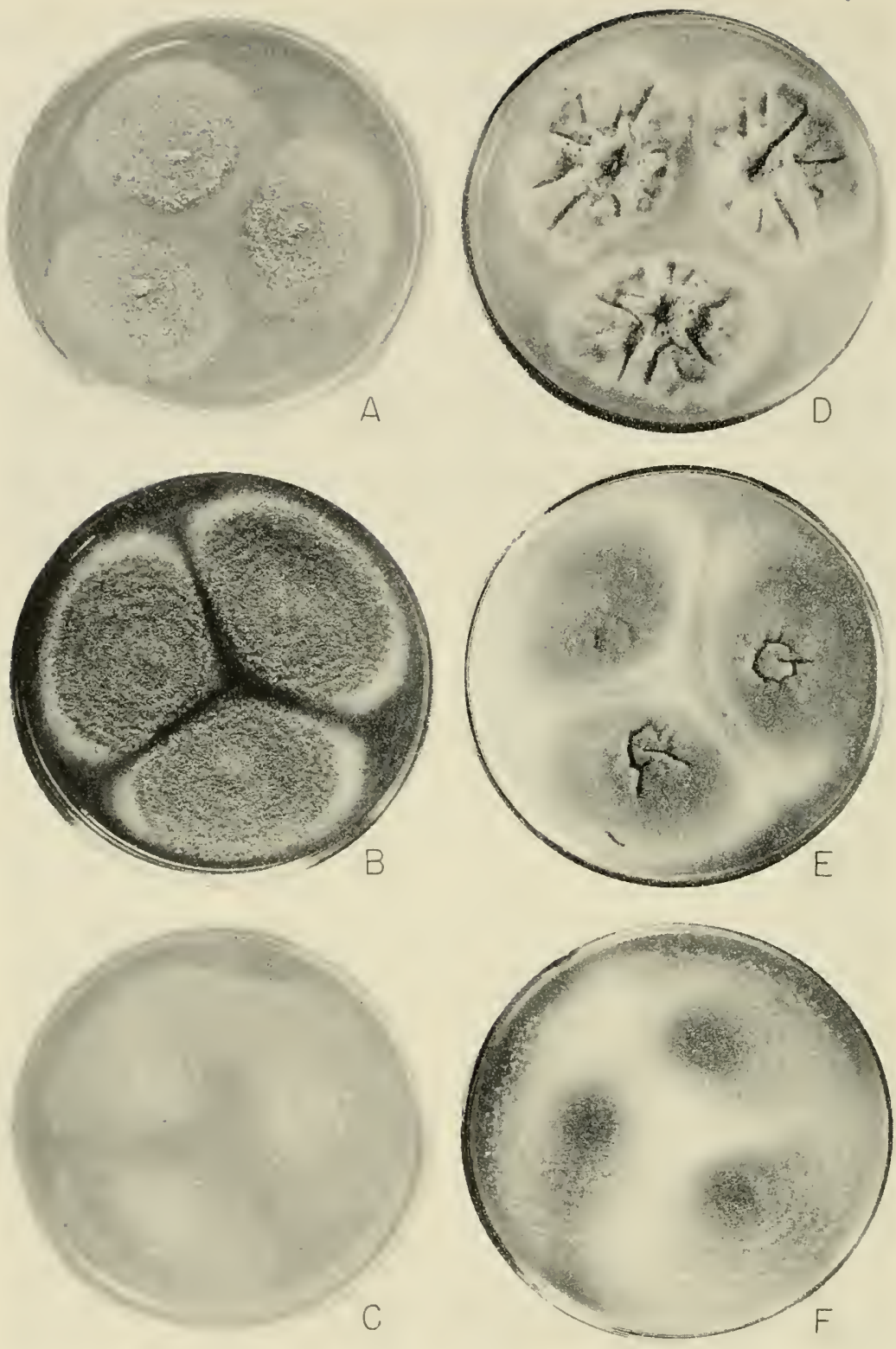

FIG. 9. Influence of substratum. A-C, Aspergillus variecolor, NRRL No. 212, growing upon Czapek's solution, malt extract, and hay infusion agars, respectively; 10 days; room temperature. Note particularly the heavy development of perithecia upon malt and the very sparse development of the same upon hay infusion agar. $D-F$, Aspergillus caespitosus, NRRL No. 1929, growing upon the same media under similar conditions. Upon Czapek's solution agar $(D)$ dark hülle cell masses develop along with a limited development of conidial structures; upon malt $(E)$ and hay infusion $(F)$ agars hülle cell masses are lacking and conidial structures are very abundant. 


\section{SPORULATION MEDIA}

In addition to the ordinary handling of molds in the laboratory, exploration of their biochemical potentialities of ten necessitates the production of considerable quantities of spores. Whereas some of the media listed above may be employed for this purpose, it is generally advisable to employ so-called "sporulation media." Requirements of different species and strains vary and one frequently has to develop special solutions to meet the needs of particular organisms under study. Such media may be used either as liquid substrata or as solutions solidified with agar. In either case the objective is the same: to secure the maximum production of spores with the production of as little regetative mycelium as possible. ${ }^{3}$

A number of sportulation media for use with different molds have been developed by Dr. A. J. Moyer. Three of these will be cited, while additional formulae may be found in the papers published by members of the Fermentation Division, Northern Regional Research Laboratory.

\section{Sporulation medium for A. niger (Moyer, Wells, Stubbs, Herrick, and May, 1937)}

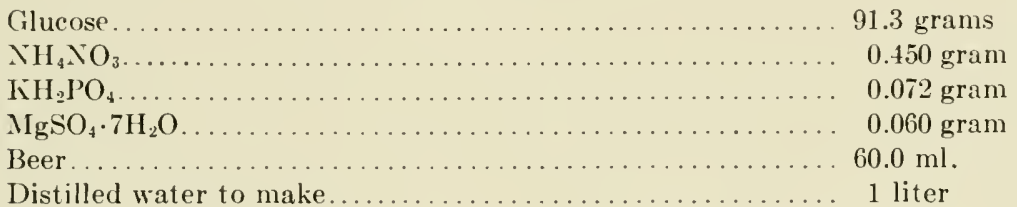

This solution can also be used as a solid medium by the addition of 0.5 gm. per liter $\mathrm{CaCO}_{3}$ and $30.0 \mathrm{gm}$. per liter agar. The above solution was subsequently modified as follows (Gastrock, Porges, Wells, and Moyer, 1938):

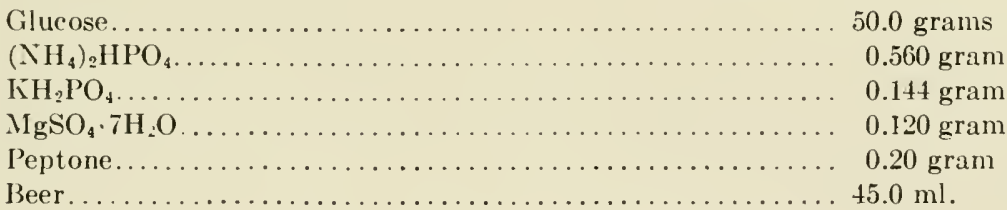

Distilled water to make........................ 1 liter

${ }^{3}$ For the surface inoculation of nutrient solutions in small flasks or other containers, dry spores in quantity can be removed from agar surfaces and floated on the liquid surface by means of a $5 \mathrm{~mm}$. loop. To inoculate from a liquid culture, t.le usual procedure is to remove a small portion of the heavily sporing mat, transfer this to the solution, and dislodge the spores by vigorous agitation. Spores from either type of culture can be suspended in water and used as inoculum in submerged, or shaken, cultures. The addition of sodium lauryl sulfonate to the suspending water in a concentration of $1: 10,000$ aids greatly in securing uniform spore suspensions. 
Sporulation medium for A. flavus (Moyer, Personal communication)

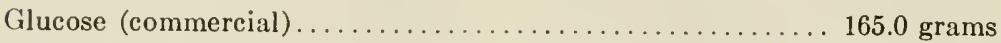

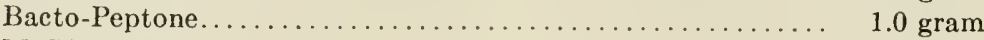

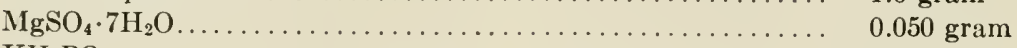

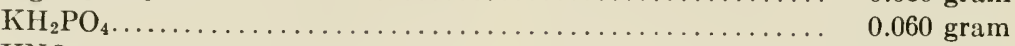

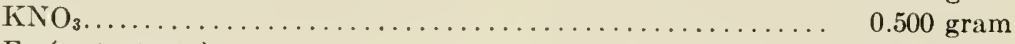

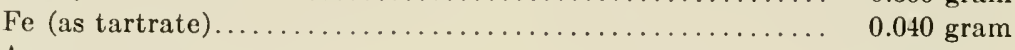

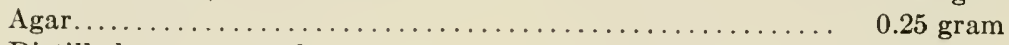

Distilled water to make.................... 1 liter

The above solution can be used as a solid medium by increasing the agar concentration to $30.0 \mathrm{gm}$. per liter. (The small amount of agar included in the basic formula is added solely for the purpose of increasing viscosity.) Incubation should be at $22^{\circ}$ to $24^{\circ} \mathrm{C}$. This factor is critical for maximum spore production with the kojic acid producing strain, NRRL No. 484 (Thom No. 3538), for which the medium was developed. This medium can also be successfully employed for spore production in $A$. niger by incubating cultures at $30^{\circ} \mathrm{C}$.

Abundant sporulation of many strains and species can be secured by cultivation upon bread, whole cereal grains, or various types of milled products of the same. Bread, if used, should not contain proprionates or other mold inhibitors. The material to be inoculated should be moist but in no sense wet, and special precautions must be taken to insure that the grain or bread is properly sterilized before being used. The use of grain as a substratum for molds dates back to prehistoric time in the fermentation industries of the Orient where rice was, and still is, commonly used to produce the "koji," or inoculum, used in the alcoholic and soya fermentation industries of that area. In its classic usage, the molds cultivated were mostly members of the $A$. flavus-oryzae group, but experience has shown that this general type of medium can be used to advantage to secure heavy sporulation of many other forms. From such material, series of surface fermentation flasks or other vessels can be uniformly inoculated by various means involving aspiration of spores, or the direct transfer of heavily spore-laden particles. Spore suspensions can be prepared and used for seeding submerged cultures

\section{TYPES OF CULTURE}

Cultures for grouping and identification of the Aspergilli should be grown in petri dishes. At the same time an adequate number of slanted tubes should be inoculated and held in reserve as an uncontaminated stock culture. Colonies so situated in the petri dish that they can be viewed directly under low magnifications with the compound microscope are necessary to supply a clear picture of the structure and course of development of mycelium and fruiting parts. Such colonies can be obtained by several 
procedures, some of which have been developed for special tests, or for individual mold problems. Practices useful in bacteriology are of ten applicable to the problems of the mycologist. Seleetion of a procedure which satisfactorily provides the information necessary to describe an Aspergillus calls for diseussion of a series of procedures eommonly in use. Only in that way may we show why some of these are adapted to the problems of identifying an Aspergillus while others fail, for specified reasons, to furnish important observations.

\section{Spot Inoculations}

Over long periods and in the hands of many investigators some type of mass conidial inoculum has given dependable and reproducible results. The most common method of transfer and, on the whole, probably the most satisfactory one for maintaining a strain of Aspergillus, as well as other molds, is the removal from the stock culture of a variable mass of conidia, fruiting structures, or vegetative hyphae with some sort of needle or loop and the transfer of this material to selected positions on fresh medium. Practices differ. With the Aspergilli, colonies so placed as to permit radiate development from the point of inoculation are most satisfactory for study. Where there are two or three colonies to the plate, these eventually reach into each other's zones of influence. They may blend and become indistinct, or inhibit each other and leave sterile bands between the colonies. Both types of eulture furnish useful data. Usually, the line where two colonies approach and partially or completely inhibit each other hastens fruiting in the adjacent margins and permits favorable examination with the compound microscope. The opposite margin of the same colony, unaffected by competition, furnishes at the same time the normal and symmetrieal growth which is typical of the speeies. The one-colony plate usually provides the rnost striking exhibit of the species, but the 2- or 3-colony plate is the most generally useful (fig. 10).

Once an Aspergillus has been obtained in pure culture, the most effective way to insure that plates will contain 1, 2, or 3 colonies, as desired, is to suspend a quantity of spores in melted agar at approximately $45^{\circ} \mathrm{C}$., allow this to solidify, and then transfer small quantities of the gelled suspension to the surface of plates or agar tubes in the positions where the colonies are desired. With the Aspergilli, as with all of the molds which produce dry spores, it is often very difficult to secure placement of colonies at selected positions, and only at such positions, without the use of some type of wetted inoculum.

In the routine examination of Aspergilli, where it is not essential that the number of colonies be limited to 3 or less, satisfactory transfers have been made by using a sharp nichrome wire as an inoculating needle and selecting 
the inoculum from a colony in a petri dish by working under a $10 \times$ pocket magnifier, or under a binocular microscope of the Greenough type carrying similarly magnifying lenses. Material to be removed can be exactly located in the parent colony. It is found possible in dealing with most Aspergilli (1) to remove conidia from a single head, (2) to remove one or more heads borne upon a single hypha at the margin of the colony, or (3) to select regetative hyphal tips from a single mycelial sector. It is usually possible to avoid (1) heads and conidia of other species or strains, (2) foreign mycelia, and (3) bacterial contaminations present in the substratum. Purity in repeated culture over many years has been possible by this procedure.
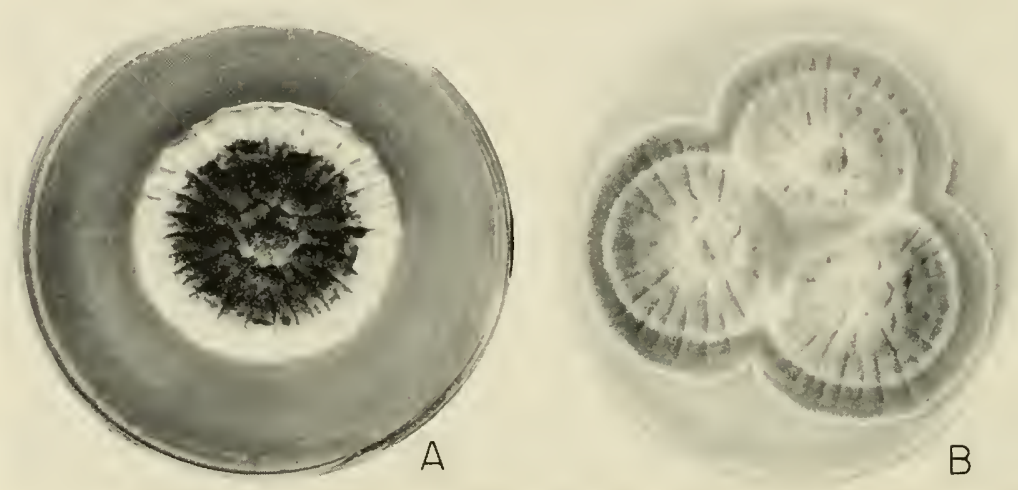

FIG. 10. Single and three-point cultures of Aspergillus foetidus on Czapek's solution agar, room temperature, 10 days, $\times \frac{1}{2}$ approximately. Note that spore production extends to the colony margins in the central triangle of ifigure $B$, and almost to the outer colony margins as well. In figure $A$, sporulation is limited to the central area only. Figure $B$ is favorable for direct examination with the low power objective of the compound microscope; figure $A$ is not.

\section{Dilution Cultures}

If dilution cultures are to be used, the suspension of conidia should be sufficiently dilute so that the individual petri dish will show not more than six, but preferably not over three, colonies. Such dilutions are difficult to gauge and of ten result in some plates crowded with numerous colonies while others contain none. The colony locations are not under control. In general, the dilution of spores in successive water blanks with the subsequent plating of aliquots from such dilutions has not been found satisfactory.

An alternate technique involves the introduction of one loopful of inoculating material into a tube of melted agar which is then rolled or shaken, 
and a loopful removed to a second tube, followed by the same manipulation a third or fourth time. The higher dilutions are then poured into petri dishes where colonies develop. This method has all the faults of the water blank dilution technique. The procedure has been widely used but has not been followed in our study of the Aspergilli. Blakeslee (1915) and other mycologists have prepared such dilutions in bottles by slowly rotating the bottle in a cold water or ice bath, thus allowing the agar to congeal in a thin layer against the glass surface. This practice has little to recommend it: (1) the resulting colonies could be isolated more readily from a petri dish, and (2) it is basically impractical to make satisfactory observations regarding the character and structure of a mold colony, or the gross features of its fruiting structures, when observations have to be made through the curved and non-uniform walls of a glass bottle.

\section{Smears and Streak Cultures}

The practice of smearing a suspension of mold spores over the whole plate, or the whole length of a slanted agar tube, results in a growth of mycelium which covers the entire surface and usually produces a greater mass of conidia. But individual colonies are usually unidentifiable in such preparations, hence they are of little value in providing those critical details of colony habit, coloration, and texture necessary for identification and classification. In general, mycelium derived from different conidia of the same strain will intertwine without inhibition, and even anastomose, if they come into contact in the early stages of growth; for example, before any sign of conidium production appears. If the spacing of the spores is great enough to permit the establishment of small fruiting colonies before such contact is made, the colonies frequently do not converge and complete coverage of the surface of the agar with the production of maximal quantities of conidia does not occur. Closely-placed seeding of spores is useful to obtain the largest possible supply of conidia; but to study the normal characters of a species, individual colonies must be allowed to develop without interference by others of the same or different species.

Streak cultures can be employed to advantage in freeing one mold from another, or from other contaminating organisms. By touching a sterile, moistened needle to a single conidial head or other selected fruiting surface of limited extent, and subsequently streaking this repeatedly across the surface of an agar plate, isolated colonies of the desired species can usually be obtained. Occasionally it is desirable to streak two plates in succession in order to secure a satisfactory separation of colonies. In any case the spore source should be selected with great care and the use of a low-power binocular microscope or pocket magnifier is recommended. When this method is employed, it is desirable to reisolate from these individual colo- 
nies as soon as possible after sporulation begins. For freeing one species or strain of Aspergillus from another, or from a contaminating Penicillium, streaking upon Czapek's solution agar usually gives satisfactory results. The method finds its greatest usefulness in separating comparatively slowgrowing molds, such as the Aspergilli, from such very rapidly growing forms as the Mucoraceae, Trichoderma, etc. The critical step in this procedure is to isolate the slow growing Aspergilli during the first two to three days before the whole plate is overrun by the spreading, faster growing forms. If the contamination is bacterial in nature, malt extract agar or some other medium of more acid reaction should be substituted. Diluting the spores in water before streaking is not recommended since this often tends to disperse the contaminating organism more than it does the desired form; this is especially true with bacterial contaminations, actinomycetes, and other minute forms.

\section{Single Spore Cultures}

The isolation of cultures from single spores is a time-honored technique with many mycologists, and with many workers it is considered a "must." When properly employed, there is much to recommend this practice. There are also certain dangers inherent in this procedure, hence we believe it worth while to consider the subject at some length and to analyze both its advantages and its limitations. As generally employed, the primary objective of single spore isolations is to secure cultures of unquestionable purity. If the operation is skillfully performed and adequately verified, there can be no doubt but that the resulting colony will represent a pure culture of a single species or strain. The continued purity and physiological stability of such cultures, however, cannot be taken for granted. There is no substitute for vigilance, and the culture thus isolated must be kept under critical observation.

As a means of purifyng a culture, i.e., separating it from foreign forms, the single spore method undoubtedly has its place; but it cannot be recommended as a means of preserving the morphological and physiological stability of a particular strain. One has only to study the reports of Stakman and his associates to realize that monospore selection and isolation is not a touchstone to strain stability. In fact, they have ably exploited the technique to show just the opposite. While we do not have an accumulation of data on the Aspergilli and related genera which can compare with that on the smuts and other pathogenic fungi, we do have enough information to know that one of the best methods of obtaining change in some of the Aspergilli is to make a series of successive monosporous isolations. For every species and strain there is apparently a normal range of natural variation, and by isolating single spores it is often possible to secure certain 
progeny which represent essentially the outside limits of such variation. This is worth while since it offers one means of securing strains which may prove more useful than the parent culture for some particular purpose, industrial or otherwise. The single spore method can be of real value in purifying a culture. It can be of real value as a means of "dissecting" a culture. But once a promising strain has been discovered and its purity is established, perpetuation by the transfer of masses of conidia is the best safe-guard for preserving, in a constant condition, its morphological and physiological characteristics.

Some of the techniques by which such single spore isolations can be made follow:

(1) Serial Dilution: Spores are thoroughly suspended in water and the resulting suspension is subsequently diluted in sterile water blanks in steps of $1: 5$, or $1: 10$. One cubic centimeter aliquots from two or three selected dilutions, depending upon the density of the original suspension, are added to sterile petri dishes. Melted agar at approximately $45^{\circ} \mathrm{C}$. is then added to these plates, which are rotated to secure uniform mixing of the still liquid agar and the diluted spore suspension. The plates are then incubated at room temperature and isolates are made from plates showing a limited number of colonies which are uniformly separated. Using this technique, one cannot be certain that any particular colony results from a single spore, but if the original suspension was properly prepared, one can feel sure that more then 95 percent of the colonies resulted from single spores. More uniform suspensions of spores can be obtained by adding to the suspending medium some suitable detergent or aerosol. For this purpose we have successfully used sodium lauryl sulfonate in concentrations of $1: 10,000$ or $1: 100,000$ without apparent harmful effect upon the molds under study. The dilution method of securing "single spore" isolations is more rapid than any other, and for many purposes it is quite satisfactory.

(2) Selection and Removal of Individual Spores: Where the investigator wishes to be positive that every colony results from a single spore, it is necessary to employ some technique combining actual microscopic examination with some device for the mechanical removal of selected spores. The various types of micro-manipulators are well-suited for this work and, with sufficient practice, single spore isolations can be made quite satisfactorily with any of these. Dilute spore suspensions in water are mounted on the undersurface of a cover slip supported by a glass chamber open at either one or both ends. With the aid of mechanical controls and a micro-pipette, a single spore is withdrawn from the suspension and ejected upon a suitable substratum where the spore develops into a mature colony.

Single spores can likewise be removed by mechanical cutting devices such as those described by LaRue (1920), Keitt (1915), and Lambert (1939). 
A comparatively thin spore suspension is spread on an agar surface, and single well-separated spores are located with the microscope. A small cutting device, mounted on a holder screwed into the nose-piece of the same microscope, is lowered into the agar and a small block bearing the selected spore is removed. This is subsequently transferred to a suitable culture surface where the colony develops.

At the Northern Regional Research Laboratory we have employed a somewhat different and simpler method. A thin spore suspension is spread evenly over the surface of a firm agar gel that has been specially filtered to remove all particulate matter. This is incubated overnight and the spores allowed to germinate. On the following day, well-separated sporelings are

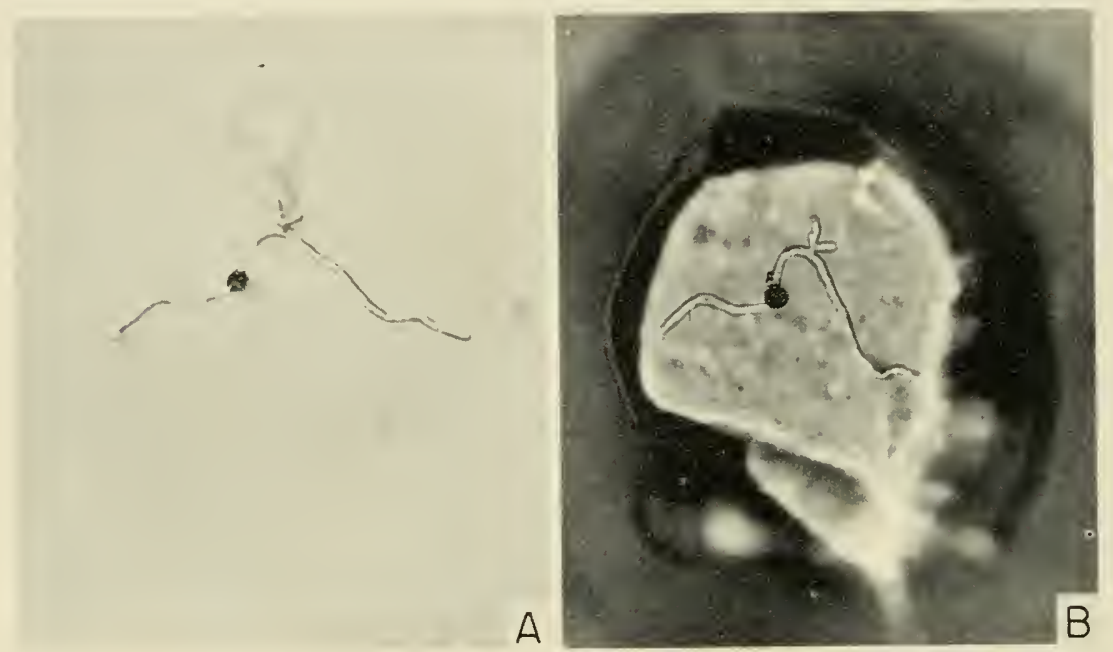

FIG. 11. Single spore isolation. A, A single, well-isolated, germinated conidium of Aspergillus niger. B, The same removed on a small agar block and transplanted to a fresh agar plate as described in the text, p. $44, \times 300$.

located with the aid of a microscope and their positions marked on the under-surface of the culture dish. These are then checked with a $8 \mathrm{~mm}$. objective and $10 \times$ or $15 \times$ oculars to insure that there are no other ungerminated spores in the same area. Using a wide-field binocular of the Greenough type and very small micro-scalpels fashioned out of platinumiridium wire (B and S gauge 22 or 24), small agar blocks on which the spores spores rest are transplanted to fresh agar plates. Each of these agar blocks is then examined again with the $8 \mathrm{~mm}$. objective to insure that the selected spore has been transplanted. An experienced worker can isolate from 25 to 30 spores within a period of an hour. Photographs showing essential steps in this technique are presented in figure 11. 


\section{Hanging Drop Cultures}

The drop of culture fluid inoculated with conidia and hanging from a slide or cover glass into a closed chamber can be incubated and examined readily. It furnishes information as to the percentage of conidia which are viable, the changes which occur in the germinating spore, such as swelling, bursting along definite lines, germination from specialized germ-pores, or branching of the germ tube, but descriptions of fruiting structures in such hanging drops are worthless in the study of the mold colony or normal fruiting habits of the species.

\section{TEMPERATURE}

The great majority of Aspergilli grow well and sporulate abundantly at temperatures of $23^{\circ}$ to $26^{\circ} \mathrm{C}$. For this reason, most cultures can be incubated on laboratory tables or shelves, and it is not necessary to give special consideration to incubation. There are, however, certain exceptions. 'The large-spored members of the Aspergillus glaucus group, such as A. echinulatus and $A$. niveo-glaucus, grow more rapidly and fruit more abundantly at $20^{\circ}$ than at $24^{\circ}$ to $25^{\circ} \mathrm{C}$. (Thom and Raper, 1941). This temperature response is especially marked in $A$. medius: at $18^{\circ}$ to $20^{\circ} \mathrm{C}$. growth is rapid, abundant large conidial heads are produced, and numerous perithecia are developed; at $25^{\circ} \mathrm{C}$. and above, growth is restricted, few and smaller conidial heads are developed, and only occasionally perithecia are produced (fig. 12). On the other hand, the very abundant small-spored members of the $A$. glaucus group, such as $A$. repens, $A$. chevalieri, and $A$. amstelodami, grow rapidly and fruit abundantly at $30^{\circ} \mathrm{C}$. (Thom and Raper, 1941). In $A$. janus (Raper and Thom, 1944), two different types of conidial heads are produced and the ratio of these types is strongly influenced by temperature (fig. 12): at $18^{\circ}$ to $20^{\circ} \mathrm{C}$. almost all heads are white with clavate vesicles and are borne upon long conidiophores; at $30^{\circ} \mathrm{C}$. almost all heads are dark green with globose vesicles and are borne upon short conidiophores (see species description, p. 187).

When grown upon suitable media and incubated at $20^{\circ} \mathrm{C}$. in the presence of light or alternate light and darkness, Aspergillus giganteus produces large heads on long conidiophores ranging up to 5 or even $10 \mathrm{~cm}$. In similar cultures incubated at $30^{\circ} \mathrm{C}$. heads are smaller and conidiophores are uniformly short, rarely exceeding $1 \mathrm{~cm}$. in length, whether the cultures are exposed to light or incubated in total darkness. A.terreus, A. carneus, and A. fischer $i$ thrive at temperatures up to $35^{\circ} \mathrm{C}$., while $A$. fumigatus grows well at $45^{\circ}$ or even $50^{\circ} \mathrm{C}$. In all of these forms growth is more rapid and sporulation more abundant at $30^{\circ} \mathrm{C}$. than at normal laboratory tempera- 

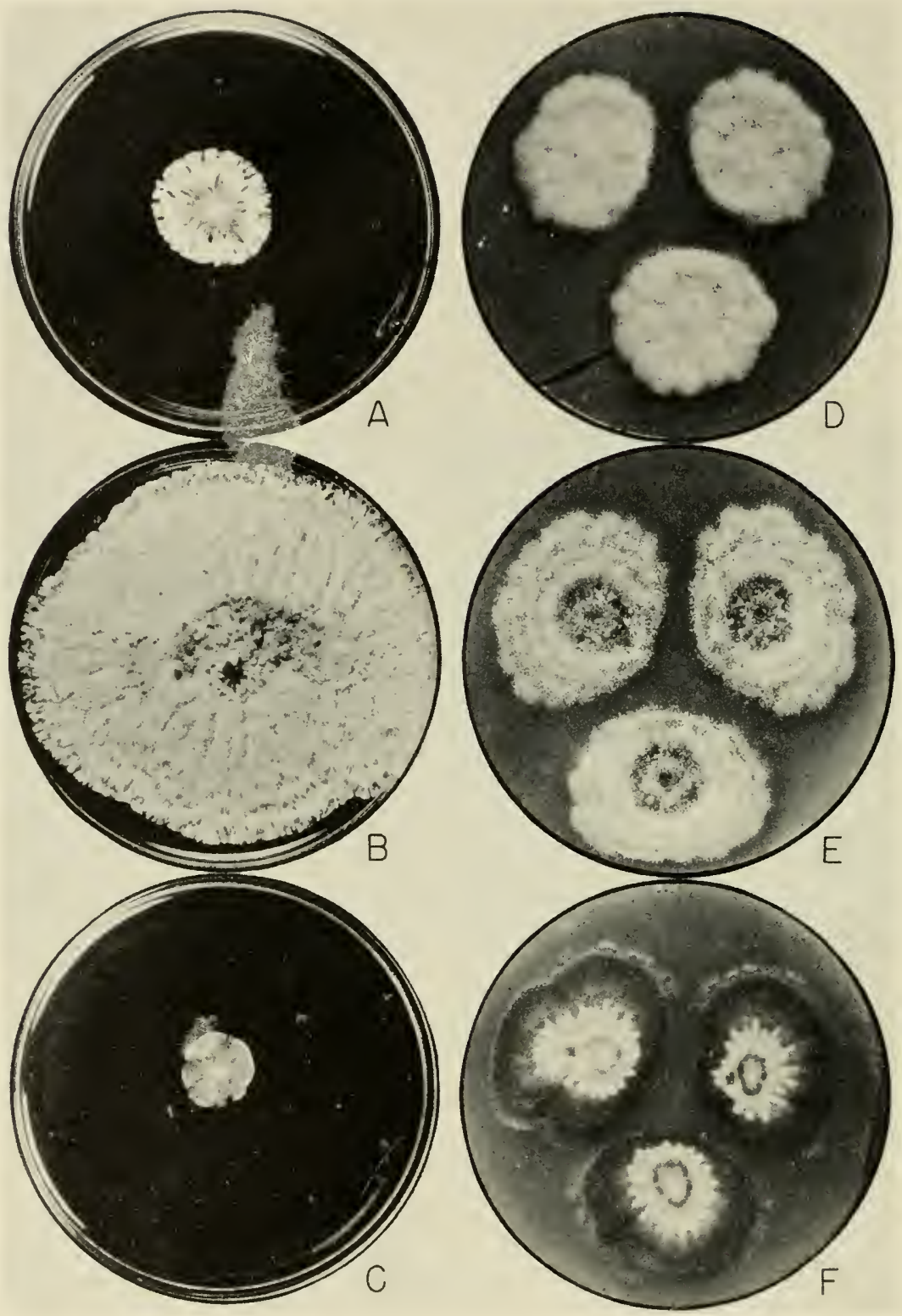

FIG. 12. Influence of temperature upon growth and sporulation in Aspergillus medius, NRRI، No. 124, and A spergillus janus, NRRI No. 1787. $A, B$, and $C$, $A$ spergillus medius, 17 days at $12^{\circ} \mathrm{C}$., $20^{\circ}$ to $22^{\circ} \mathrm{C}$., and $30^{\circ} \mathrm{C}$, respectively; note maximum growth and limited sporulation at $20^{\circ}$ to $22^{\circ} \mathrm{C}$. D, E, and $F$, Aspergillus janus, 3 weeks at $20^{\circ} \mathrm{C}$, $24^{\circ}$ to $25^{\circ} \mathrm{C}$, and $30^{\circ} \mathrm{C}$., respectively; note evidence of white heads only at $20^{\circ} \mathrm{C}$, , abundance of white and presence of green heads at $24^{\circ}$ to $25^{\circ} \mathrm{C}$, and green heads only at $30^{\circ} \mathrm{C}$. Temperature is the only variable. 
tures of $24^{\circ}$ to $25^{\circ} \mathrm{C}$, although wholly typical cultures are produced at both temperature levels.

In the production of spores for biochemical investigation (see p. 37) and in the conduct of such studies themselves, temperature is often very important. In work of this kind, it is essential to determine the optimum temperature for each organism and process, and then to control this within a narrow range in order to secure consistent and reproducible results.

Temperature is known to be a critical or limiting factor only in a few species, but its effect in these is sufficiently pronounced that its influence should not be disregarded in any case.

\section{CULTURE TOOLS AND EQUIPMENT}

\section{Transfer Needles}

In making transfers, we have used with satisfaction for many years a No. 20 or No. 22 B. and S. gauge nichrome wire thrust into a slender brass or stainless steel tube so that only about 15 to $20 \mathrm{~mm}$. are exposed. (The tubing employed is of the type used in temperature controls of mechanical ref rigerators and other thermostatically controlled devices.) The point of this wire is then ground down to the sharpness and smoothness of a needle. This instrument can be heated to redness in the flame a great many times with only the occasional necessity of resharpening. It thus has many advantages beside cheapness: it is firmer and takes a better point than platinum; it withstands sterilization in the flame, which promptly destroys the usefulness of steel; and it can be made any size or length to suit the workman's purposes or preferences.

\section{Loops}

Loops of various dimensions are very useful and can be inserted into handles made from small brass or stainless steel tubing as noted above. These, too, can be fashioned from nichrome wire, but it has been our experience that loops made of platinum-iridium wire possess certain marked advantages. While these are not as rigid as nichrome loops, they are much more rigid than loops of pure platinum, and they can be re-heated indefinitely without corroding. Loops of this type will be found especially useful in making mass inoculations, such as the seeding of large tubes and plates for the production of spores to be used in various types of experimental work.

\section{Mounting Fluid}

In the microscopic examination of the Aspergilli it is often satisfactory to mount conidia, heads, or other structures in water. It is more generally 
satisfactory, however, to use some mounting fluid of a composition designed neither to swell nor plasmolyze the tissues to be observed. Such a mounting fluid was developed by Amann as early as 1896 and has been used by mycologists, quite generally, for many years. Its composition is as follows:

Carbolic Acid Crystals (c.p.)...................... 20.0 grams

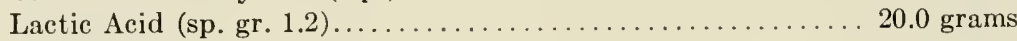

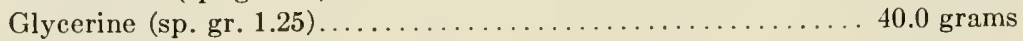

Distilled water............................... $20.0 \mathrm{cc}$.

The carbolic acid crystals are first liquefied by heating in a water bath.

The mounting fluid is normally used without the addition of any dye, since in the diagnosis of the Aspergilli the natural colors of conidiophores, conidia, etc., are very important. If it is considered desirable to stain the tissues under observation, it is possible to incorporate into the lactophenol solution some coloring substance such as cotton blue, eosin, or some other aniline dye. In making mounts of the Aspergilli, it is profitable to wet the material first with 70 percent alcohol to drive off air bubbles, and then quickly add a small drop of the lactophenol prior to the placement of the cover glass.

\section{Incubators}

Almost all of the Aspergilli grow well and sporulate abundantly at laboratory temperature. There are, however, certain exceptions to this general rule (see p. 45), and for this reason it is desirable to have available an incubator which can be regulated at temperatures below that of the laboratory and others covering different ranges up to $37^{\circ} \mathrm{C}$., or even $50^{\circ} \mathrm{C}$. The type of incubator is not critical and almost any type of cupboard, or room, will prove satisfactory if the temperature can be controlled to within $1^{\circ} \mathrm{C} . \pm$, and if the air is neither excessively dry nor humid to the point where cotton plugs become moist upon continued exposure.

\section{Microscopes}

In the study and identification of Aspergilli it is essential to have at one's disposal a good-quality compound microscope. If possible, this should be provided with apochromatic lenses. In our experience we have found a 3 $\mathrm{mm}$. objective used in conjunction with either $10 \times$ or $15 \times$ oculars to give us magnifications and a degree of definition which is most satisfactory for the examination of conidial structures. While it is not absolutely necessary, it is desirable to have, in addition, a low-power, wide-field dissecting microscope covering magnifications from 10 to 40 diameters. In the absence of such a microscope a high-quality pocket magnifier provides a good substitute. 


\section{Photographic Equipment}

A limited amount of photographic equipment is a very valuable aid in the study of this or any other group of molds. A camera should be available which can be used in conjunction with a compound microscope, and to which low-power lenses can be attached directly. In addition to a goodquality lens producing pictures above and below natural size, a series of Tessar lenses which will provide magnifications of from 5 to 30 diameters is extremely useful. With the aid of these, details of colony structure can of ten be recorded which cannot be pictured with the lenses of the compound microscope and which are very difficult to deseribe adequately in words. With these low-power lenses it is possible to photograph types of fruiting heads, the relative abundance of conidial structures to vegetative mycelium, and the relationship between each of the above and sclerotia or perithecia when such structures are present. 


\section{Chapter V PRESERVATION OF CULTURES}

Any mold that is valuable because it has been used in fundamental research work, because it has been found useful in some industrial process, or because it is a significant agent in some destructive or pathogenic situation should be preserved to insure its identity for subsequent use or reference. Identification by description will take the careful worker to the group, species, and often to the variety as based upon morphology or some conspicuous character, but the reidentification from description of the exact organism used in a biochemical investigation or discovered in some ecological situation is generally impossible. Culture collections have, therefore, developed. The Centraalbureau voor Schimmelcultures (cited by them as C.B.S.) at Baarn, Holland, the National Type Culture Collection in London, and the American Type Culture Collection at Washington are well-known sources of such material. More recently established, but containing a greater number of industrially important molds, is the culture collection of the Northern Regional Research Laboratory (commonly cited as N.R.R.L.) at Peoria, Illinois. The Aspergilli are especially well represented, and contained in the collection are almost all of the species considered in this manual, together with records which check the identifying numbers of this collection against the records of the source collections* which were brought into it.

In undertaking a comparative study of the Aspergilli, or any other group of molds, it is essential to maintain in a viable state a large number of isolates representing diverse species and strains. By this means, it is often possible to interpret current isolates and accessions in terms of historic types and concepts. In maintaining such a collection of molds, the objective should be to preserve viability without growth or germination of spores during the storage period. By so doing, the user can reasonably expect to maintain his organism without variation, degeneration, or mutation. A number of techniques can be employed to advantage, the more useful of which will be considered in some detail with certain of their advantages and limitations noted.

* Currently contained in this collection are the molds formerly maintained by Thom and Church, and later by Thom and Raper, in the United States Department of Agriculture, Washington, D. C.; many of the Mueorineae maintained by Dr. A. F. Blakeslee for many years at the Carnegie Institution, Cold Spring Harbor, Long Island, New York; a large number of miscellaneous forms from the Harvard University Collection initiated by Prof. $R$. Thaxter and more reeently maintained by Dr. D. H. Linder; and limited numbers of eultures from many other collaborators. 


\section{Agar-Slant Method}

The method most generally employed for maintaining mold eultures, and the one which has been successfully used by the writers for many years, may be termed the agar-slant method. This involves the periodic transfer of spores from old agar slants, or plate cultures, to new agar tubes. The composition of the substratum is varied to suit particular requirements and groups of organisms. In our work we regularly employ the Czapek's solution agar. Few, if any, strains make their maximum growth on this medium, but it has been our experience that they maintain exceptionally well any characteristic morphological or physiological features which may characterize them. It is necessary to know the expected viability of all cultures to be maintained, and to gauge the intervals of transfer accordingly. With the Aspergilli, transfer every 8 to 9 months is sufficiently frequent for all species, with the possible exceptions of $A$. citrisporus and $A$. itaconicus, and a period of one year is not too long for most forms. In practice it is advisable to handle separately the few very short-lived species, and to set the regular period of transfer well within the known viability period of the remaining forms. Transfer of the general collection at least once each year insures a complete survey, within the yearly period, of all strains maintained. New tubes are inoculated at least in duplicate, while triplicate preparations afford a desirable margin of safety. The new cultures are incubated for 2 to 3 weeks, or until a good crop of spores has developed. Incubation at room temperature is suitable for most of the Aspergilli, although a few forms such as the large-spored members of the A. glaucus group sporulate more abundantly at $20^{\circ} \mathrm{C}$. The correctness of the cultures is then checked with a wide-field binocular or a $10 \times$ pocket magnifier, the plugs are poisoned to preclude any possibility of subsequent contamination, and selected tubes are placed in storage. Cultures can be stored for reasonable periods at room temperature; certain species will remain viable for many years at $24^{\circ}$ to $26^{\circ} \mathrm{C}$. The viability of most species is materially lengthened and the possibility of progressive variation reduced by storage at $2^{\circ}$ to $4^{\circ} \mathrm{C}$. (i.e., above any danger of actual freezing but sufficiently low to prohibit further growth and possible dissociation). Tubes of any desired size may be employed. We have found lipless tubes 15 by $125 \mathrm{~mm}$. to be quite satisfactory since they provide adequate culture surface and at the same time require much less storage space than the larger tubes commonly in use. Each culture should be maintained at least in duplicate, with the different tubes of each pair stored in separate refrigerators. With the accidents and failures of refrigeration, the possible escape of toxic gases, or the possible ingress of contaminations that escape the usual inspection, the maintenance of not less than two complete series of strains is a necessary precaution. If natural conditions, such as temperature and relative hu- 
midity, are favorable as at Baarn, Holland, refrigeration may be dispensed with, but watchfulness against invasion by mites becomes more important. Additional slants should be prepared for cultures which are frequently used.

Agar slant cultures are convenient for use, easily examined and compared, and easily replaced (fig. $13 \mathrm{~A}$ ). They are, however, easily contaminated when handled carelessly. Uneven drying subjects the culture to

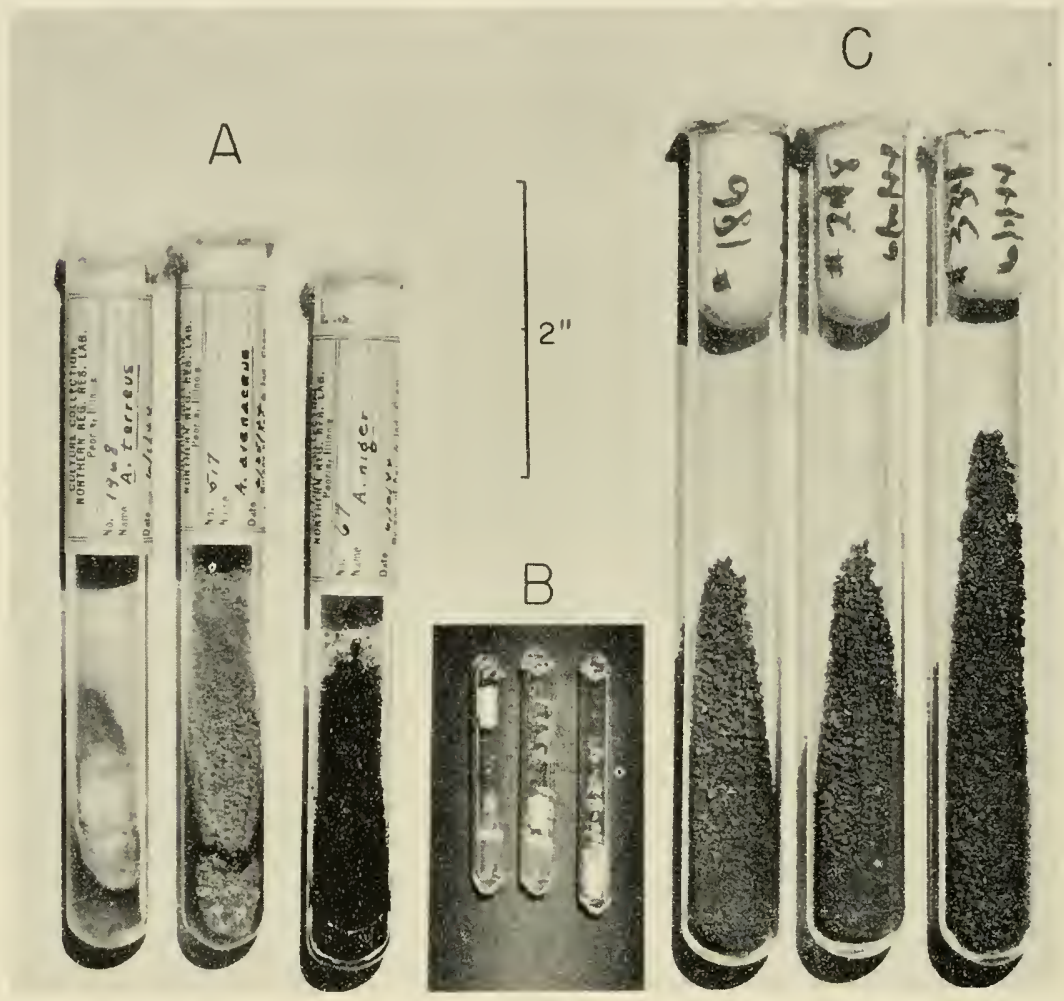

FIG. 13. Methods of maintaining stock cultures as discussed in the text. $A$, Cultures growing on agar slants. $B$, Cultures preserved in lyophile form; note the compact, chalky pellets formed by the dried serum in which the spores are suspended. $C$, Cultures preserved in dry soil.

extremes of contrast in concentration of media and metabolic end products, between the thin edge of the slant and the heavier mass in the bottom of the tube. Variations (apparent or real) often appear in the stored cultures and these may be propagated in subsequent transfers. As a safeguard, stock cultures should be grown in petri dishes after several transfers in tubes, thus making possible more complete examination to maintain purity and typical morphology. 


\section{Preservation in Lyophile Form}

Studies now in progress at the Northern Regional Research Laboratory indicate that many, if not all, of the Aspergilli can be successfully maintained in a dried state for extended periods. Viability tests for a number of species including A.terreus, A. niger, A. oryzae, A. flavus, and A. ttaconicus have been made at $3 \frac{1}{2}$ years; while a much greater and wider variety of forms has been tested at 20 to 24 months. Positive results have been obtained with all cultures tested, although comparatively few colonies developed from certain strains of $A$. niger, $A$. flavus, and the large-spored members of the A. glaucus group. Observations are being continued and in time information will be obtained as to the feasibility of employing this as the principal means of maintaining a collection of molds. It is known that many bacteria, especially staphylococci, streptococci, and pneumococci can be successfully preserved for periods up to 16 to 18 years (Elser, Thomas, and Steffen, 1935; Swift, 1937). Wickerham and Andreasen (1942) have presented evidence covering a period of one year which suggests the practicability of applying the method to the yeasts. Such information as we have to date regarding the molds seems to indicate that the method may prove of great significance in two ways: first, as a means of prolonging viability, and second, as a means of preserving in viable form spores of a particular "generation," or other selected origin, which can be used in comparative tests over a period of many months or even years.

The drying technique employed at the Northern Regional Research Laboratory is essentially like that described by Wickerham and Andreasen (1942) and may be briefly summarized as follows:

Employing aseptic techniques throughout, the spores from selected cultures are suspended in sterile beef, or horse serum. The resulting suspension is then dispensed into small cotton-stoppered Pyrex glass tubes $6 \mathrm{~mm}$. by $100 \mathrm{~mm}$. that have been properly labeled with glass-marking ink. Approximately 0.05 to $0.1 \mathrm{cc}$. of the spore suspension is added to each tube by means of a long thin-necked pipette. Most of the cotton plug is burned away, and the remaining portion pushed down into the tube to prevent possible contamination during the drying process. The tubes are inserted in rubber sleeves on the manifold, as shown in figures $14 \mathrm{~A}$ and $\mathrm{B}$, and lowered into a freezing bath of carbon dioxide ice and methyl cellosolve at a temperature of approximately $-40^{\circ} \mathrm{C}$. The suspension is frozen almost instantaneously. The manifold is connected to a vacuum pump and evacuation and desiccation initiated. Water is removed from the system by the insertion of a water-trap immersed in a $\mathrm{CO}_{2}$-methyl cellosolve filled Dewar flask as shown in figure $14 \mathrm{~A}$, or in a column of drierite (anhydrous $\mathrm{CaSO}_{4}$ ) as shown in figure $1+\mathrm{B}$. After a few minutes the temperature of the bath 

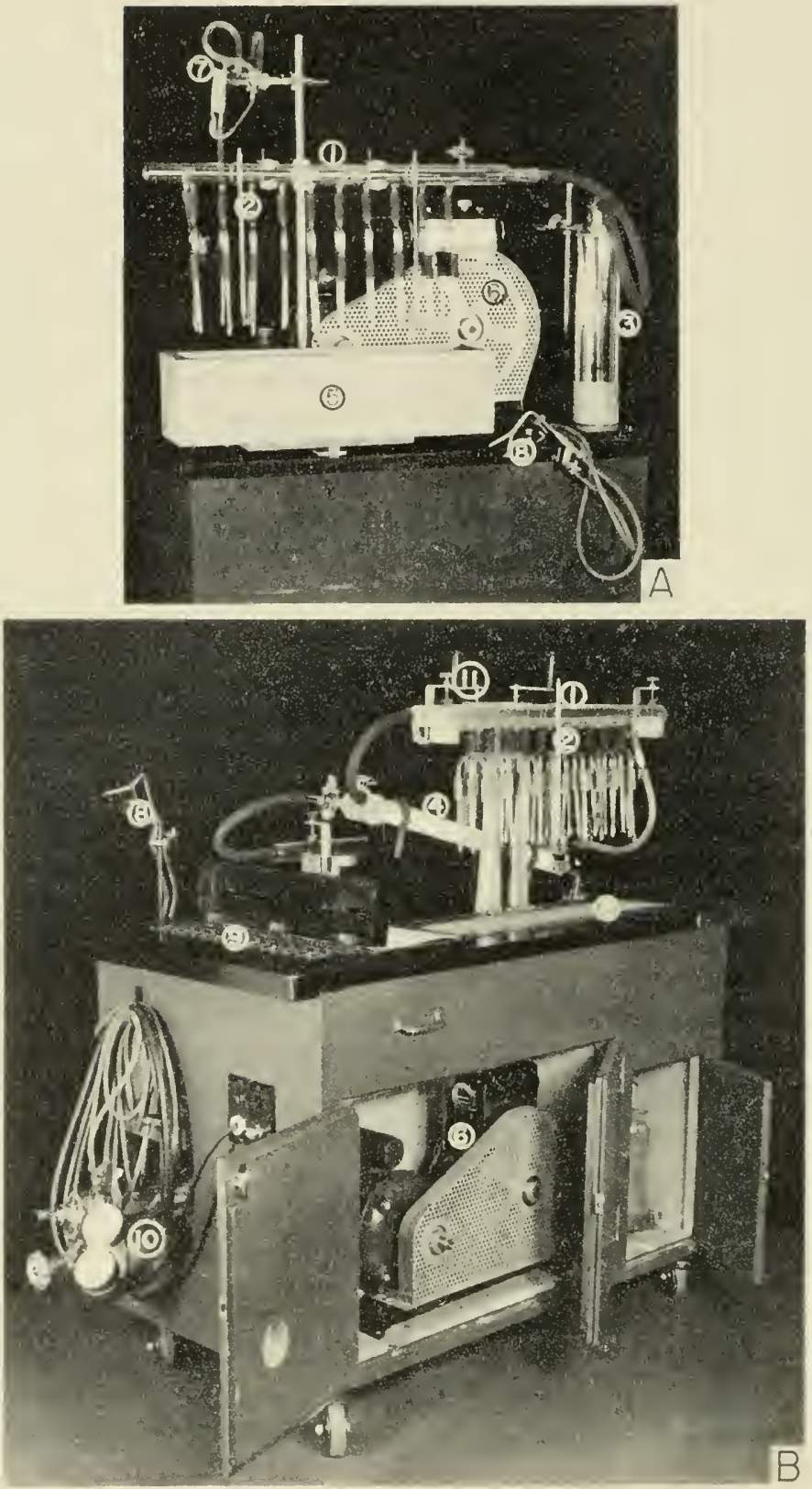

FIG. 14. Apparatus employed at the Northern Regional Research Laboratory for preserving microorganisms in lyophile form. A, Table model of thirty tube capacity, utilizing a trap immersed in a Dewar flask filled with $\mathrm{CO}_{2}$ ice and methyl cellosolve to collect the water vapor removed from the drying preparations. $B$, Larger, self- 
surrounding the tubes is raised to approximately $-5^{\circ} \mathrm{C} .{ }^{1}$ by supplying additional methyl cellosolve that has been precooled to approximately $0^{\circ} \mathrm{C}$. The bath is maintained at this level continuously until the preparations appear thoroughly dry. In drying, the serum contracts slightly to form a well-defined chalky pellet. When the pellets are apparently dry, the tubes are raised above the bath and evacuation is continued for one-half to threequarters of an hour at room temperature to insure as complete removal of water as possible, after which time they are sealed off with a gas-oxygen torch (fig. $14 \mathrm{~A}$ ). On the following day each tube is tested for the presence of a good vacuum by means of a high-frequency, spark coil tester, and only those tubes which show such a vacuum are retained. Tubes not maintaining a satisfactory vacuum are very rarely encountered in actual practice. Quadruplicate tubes are regularly made for each stock culture, and the finished preparations are stored in a refrigerator.

In recultivating the molds, the tubes are marked with a file scratch, surface-sterilized, and the tube broken inside a wrapping of sterile cotton. The content, which is in the form of a well-formed pellet (fig. $13 \mathrm{~B}$ ), is then dissolved in 1 to $2 \mathrm{cc}$. of sterile broth or water. This is streaked on agar plates and colonies are allowed to develop. New isolations can be made within a period of a few days. It is possible, of course, to go directly from the lyophile tubes into flasks or other cultures used in actual experiments, but generally speaking, much larger quantities of material would need to be processed.

The feasibility of preserving molds in lyophile form over long periods has by no means been proved, but results to date are very encouraging. Should it be found that spores of molds, like bacterial cells, can be kept viable by this method for many years, it will prove ideal as a means of pre-

${ }^{1}$ Wickerham and Andreasen in 1942 governed the temperature at which the suspension was dried by adjusting the level of the tubes above a bath which was kept at a very low temperature. Subsequent to this, Wickerham developed the procedure outlined above.

contained and portable unit of sixty tube capacity (designed by Dr. L. J. Wickerham) which utilizes a column of anhydrous calcium sulphate ("drierite") to collect water vapor removed from drying preparations. $A_{1}$ and $B_{1}$, Glass manifolds; $A_{2}$ and $B_{2}$, Thermometers; $A_{3}$, Dewar flask containing water vapor trap immersed in $\mathrm{CO}_{2}$ ice and methyl cellosolve; $B_{4}$, Column of drierite; $A_{5}$ and $B_{5}$, Freezing bath containing $\mathrm{CO}_{2}$ ice and methyl cellosolve in which preparations are immersed for quick freezing and subsequent temperature control; $A_{6}$ and $B_{6}$, Vacuum pump; $A_{7}$, Vacuum guage (mounted at opposite end of apparatus shown in figure $B$ ); $A_{8}$ and $B_{8}$, Gas-oxygen torch for sealing off dried preparations; $B_{9}$, Terminals on which finished tubes are mounted to be tested for presence of good vacuum by means of a high frequency spark coil tester (not shown); $B_{10}$, Oxygen tank; $B_{11}$, Screw lift for raising and lowering manifold and attached tubes (In figure $A$, manifold is raised and lowered manually and locked into position by means of a wing bolt). 
serving large culture collections. It possesses certain marked advantages:

(1) There is no possibility of contaminants entering the sealed preparations.

(2) The investigator recultivating the molds starts with the actual spores contained in the original suspension.

(3) The space required for storage of a large number of lyophile preparations is much less than for any other type of culture (fig. $13 \mathrm{~B}$ ).

\section{Preservation in Soil}

Soil has been successfully employed as a means of preserving vigorous stock cultures over long periods. As early as 1918, Barthel (Cent. Bakt. II, 48: 340-49. 1918) reported the successful maintenance of yeasts and bacteria in this medium and modifications of this technique are now employed in many laboratories for preserving bacteria. Greene and Fred in 1934 compared cultures of various molds preserved for two years in soil with the same strains continuously maintained on malt extract and malt extract-potato-glucose agars and on bread. In their experience, soil preparations were most satisfactory, and Professor Elizabeth McCoy (personal communication) has recently reported cultures of $A$. sydoui preserved in this manner to be viable after nine years. Since the publication of Greene and Fred's work, the soil method has been rather generally used by the Wisconsin group as a means of preserving valuable stock strains of molds. Furthermore, it is known to have been successfully employed during the past two years by a number of laboratories to maintain cultures of penicillinproducing molds in a high and uniform state of productivity. The soil substrate used by Greene and Fred was prepared as follows:

"To air-dried orchard loam soil (Miami silt loam) sufficient water is added to bring it to a moisture content of about 20 percent. The soil is then transferred in convenient amounts (about 5 grams on a dry basis) to ordinary half-inch $(1.27 \mathrm{~cm}$.) culture tubes. The tubes are plugged with cotton and given four 3-hour sterilizations at 15 pounds per square inch $(1 \mathrm{~kg}$. per sq. $\mathrm{cm}$.) pressure on alternate days, and tested for sterility by addition of yeast-water-glucose broth to tubes selected at random. The tubes are then inoculated with 1 ce. of a heavy spore or mycelium suspension of the desired mold and kept at room temperature. That there is appreciable growth and sporulation on the soil can usually be ascertained without difficulty by direct microscopic observation. While the addition of nutrient to the soil may bring about somewhat greater growth, it does not seem to enhance the keeping qualities of the cultures.

"It has been found possible to preserve on soil mold stocks used for large-scale growth-namely, Aspergillus fischeri, A. sydowi, and Penicillium chrysogenum-for over 2 years without loss of their essential and desirable characters. . . Moreover, the gross colony characters have remained much more constant than did those of the corresponding cultures maintained in the usual way on agar slants. The soil cultures 
can be recovered as required simply by streaking some of the soil particles on fresh agar slants.

"It is not in all cases advisable to depend on soil alone for the preservation of valuable stocks, but reserve stocks may without difficulty be prepared on soil, and the writers believe that in many instances soil will be found to be an excellent medium for maintenance, with a minimum of change over long periods of time."

During the past two years the soil method has been used at the Northern Regional Research Laboratory with but minor modifications of the technique cited above (fig. $13 \mathrm{C}$ ). Its principal advantages lie in the fact that (1) the viability of strains is apparently lergthened, and (2) from a single stock tube, opened with proper care, repeated cultures can be started simply by removing some of the soil particles to suitable substrata.

\section{Vegetable Substrata}

The oriental fermentation industries maintained their inoculating material as selected rice or soybeans upon which the mold had been grown under favorable conditions to produce maximum quantities of spores. This nutrient, dried and packaged, was stored and sold under the Japanese name "Koji". Samples examined after several years showed excellent viability.

Bainier was a pharmacist. He distributed licorice root in sections 5 to 10 $\mathrm{mm}$. in diameter and 5 to $8 \mathrm{~cm}$. in length in test tubes, sterilized them, and kept his cultures regularly for years upon them. Tested by us, the method was a very satisfactory laboratory practice. American mycologists have successfully used bean stems for the purpose. Apparently any organic material which provides frameworks of cellulose enmeshing sufficient nutrients to support mold growth without complete breakdown of the mass may be used.

\section{CONTAMINATION}

\section{Mixed Strains}

In the routine conduct of cultural work, contaminations of cultures of one species of Aspergillus by other species, or species of other genera, is very common. The conidia of most molds are exceedingly light and are carried freely in the air. Entire exclusion of such contamination is difficult. In dealing with contaminations, several problems arise and different procedures are possible. A colony of a single Aspergillus, well established, usually inhibits the growth of other species developing in the immediate vicinity. Even if invasion occurs, the effects are commonly so distinct as to leave little doubt as to the limits of the different forms. When, however, the contamination with spores or mycelium is carried in the inoculum and so placed as to germinate in intimate contact with the organism desired, (1) the species may sector out, and hence be easily recognized, or (2) the colony 
resulting may assume the character of either organism with the other present only as an inconspicuous, even unrecognizable, mycelium with dwarfed heads, yet continue present for a long period. In this way the retarded species may suddenly reappear in some later transfer upon media favorable to it. In other cases, the dominant species may grow and fruit in an erratic manner that is deceptive in suggesting a reaction to the medium $r$ other physical factors. Again, in less common cases, the two may grow nd fruit together without apparent inhibiting effects. This is the most lifficult form of mixture.

Mixtures are sometimes encountered in which mycelia, sterile under all zonditions tested, become so intimately mixed with the mycelium of an Aspergillus or Penicillium as to persist through many generations without apparent effect in the earlier states of growth of the Aspergillus, but develop as overgrowths of sterile hyphae in very old cultures. Many of these forms can be isolated, but they defy identification because of an absence of diagnostic characters. Such sterile mycelia may arise from the species studied, but unless such origin can be definitely proved, they must be regarded as contaminants. Great care is necessary in interpreting cultures producing sterile overgrowths, since the contaminating organism, or non-sporulating variant of the same strain, may induce marked changes in the physiological activity of the species supposedly pure.

\section{Secondary Growth}

In many species, part or all of the conidia produced by a colony germinate and cover the primary mycelium with more or less abortive hyphal growths, many of them unrecognizable unless traced by their origin. Again spores floating on the surface of a globule of transpired fluid may germinate, their hyphae interlace and a hollow ball surrounded by felted mycelium produce structures which probably account for reported perithecia without ascospores. Such overgrowths, being irregularly produced, interfere with one's judgment as to the whole character of the colony.

\section{Replacement by Other Species}

Other species of fungi invade mold cultures and some of them become so intimately associated with the mycelium and conidia of particular species that it is difficult to eliminate them by the ordinary method of transferring spores with a loop or wire to streaks or stabs. Proper dilution culture presents the possibility of elimination but demands careful examination and selection from the resulting colonies. The progressive replacement of a particular species, by invading organisms, is constantly encountered in examining cultures passed from laboratory to laboratory. The original organism may disappear without detection if the displacing species bears 
a superficial resemblance to it, or if no detailed examination of successive transfers is made by a worker really familiar with the specific characters of the stock strain. The physiological or biochemical investigator, obtaining such a culture assumed as correctly named, will be seriously misled in the interpretation of his experimental results. No culture should be used for such an investigation without being fully identified at the outset and having its proper appearance and reactions sufficiently studied to insure the reliability of the results during the progress of the work. In other words, before undertaking to do biochemical or physiological work, sound scholarship calls for adequate precautions in the study and identification of the original material supplemented by such mastery of its morphology and variability as will insure its maintenance in proper condition.

\section{Mold Discase of A. niger}

A mold disease of Aspergillus niger is common in cultural study. The colonies of the Aspergillus are overrun with an olive-green Penicillium belonging in the Biverticillium group close to Penicillium rugulosum Thom. This mold invades the mycelial felt, winds its hyphae within and about the conidiophores, and fruits in a radiating series of short-stalked penicilli surrounding the heads and upper halves of the conidiophores. This is beautifully illustrated in figure 15, made by Edward Yuill and sent to us by his brother John L. Yuill of Yorkshire, England. The black fruiting surface may be completely covered with the olive-green conidial masses of the Penicillium. If $A$. niger is grown in trays for acid formation, spots infected in this way may be killed and disintegrated, thus interfering with fermentation, while the infected areas may be seen to drop out when the blanket or felt of $A$. niger is lifted from the surface of the liquid. Other species when inoculated have been irregularly affected, some strains of the same species were attacked, whereas others were apparently immune.

\section{Bacteria}

Freedom from bacteria is essential to uniformity in the appearance and in the reactions of molds. Colonies infected with bacteria may be unchanged in character, but usually show marked physiological differences when compared with colonies free from contamination. Associative action may have important effects upon both organisms. Sartory (1920) discussed without identifying a species producing ascospores, but only when accompanied by a particular bacterial associate. Nevertheless, a symbiotic colony must not be allowed to masquerade as a pure culture representative of a species. The next contaminated colony of the same species of mold but with different bacteria may present a very different picture. These conditions have been met often enough to make the emphasis upon freedom from bacteria essential in study of this group. 


\section{Mites}

Mites are very common in rotting vegetables, especially in partly dried condition, in dried meat, products, in hard cheeses, and in organic soil masses. They are thus common associates of molds as they occur in nature,

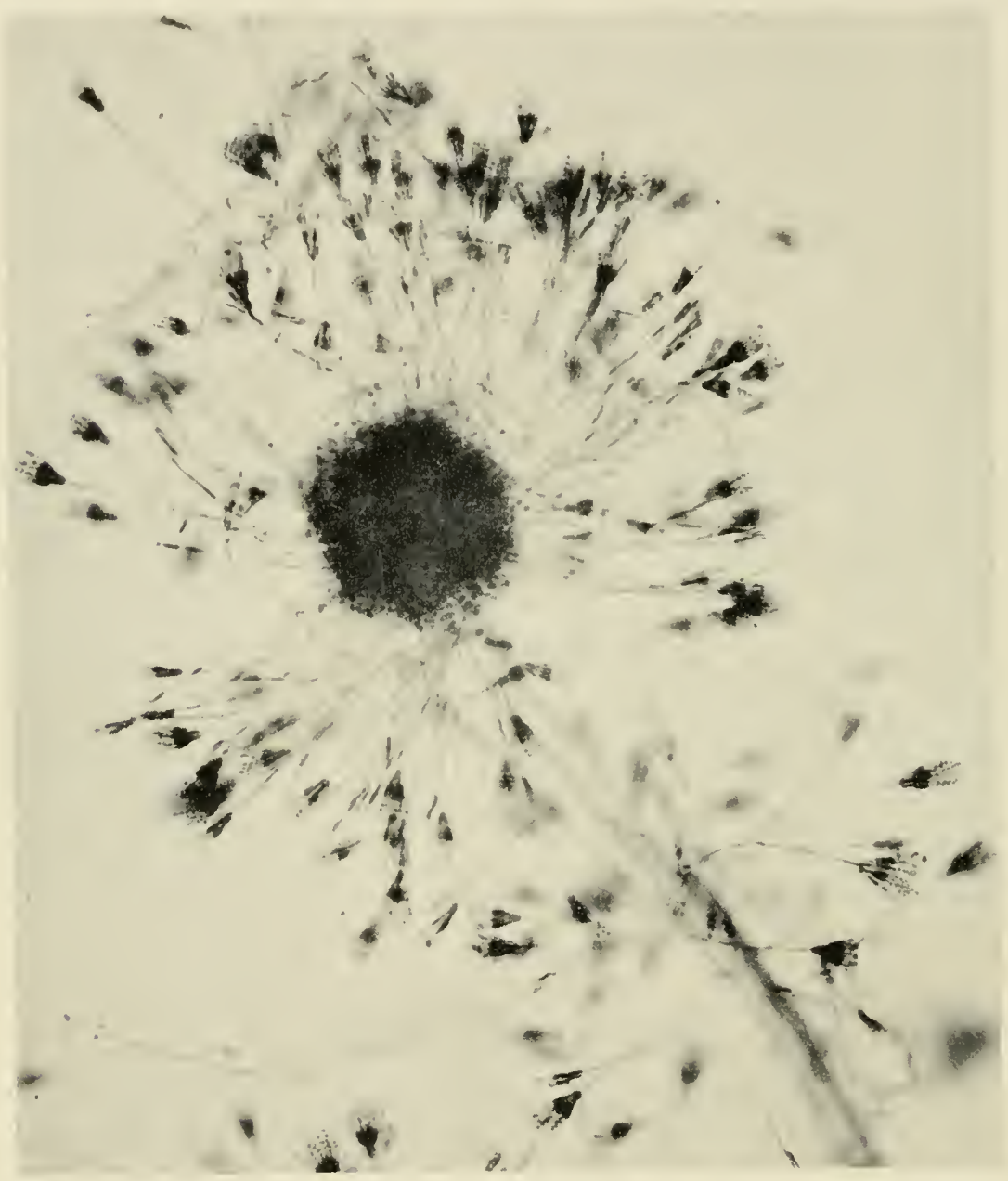

FIG. 15. Penicillium sp. parasitic on Aspergillus niger, $\times 165$. (Photograph by Edward Yuill)

hence the worker who handles moldy substrata, in isolating his organisms must constantly watch for them. In size, mites are commonly just about at the limit of visibility by the unaided eye. One accustomed to them will detect them readily; but until seen and the appearance of their depredations 
understood, they ean pass unnoticed for considerable periods by persons who are otherwise good culture workers. Mites will crawl from petri dish to petri dish, leaving behind them a trail of bacterial and mold contaminations, as well as streams of eggs which develop rapidly into more mites that actually destroy the colonies. Since mites have preferences as to food, some species are invaded and others avoided. To reach an attractive food supply a mite will frequently go through a cotton plug as ordinarily made and occasionally seems to get through even a paraffined plug. As a factor in the mixing of strains of molds in a laboratory collection, mites must not be ignored.

Similar mixing and contamination occurs frequently whenerer a laboratory becomes infested with ants, roaches, or other insects.

\section{Poisoning Cotton Plugs}

Mites: Entire elimination of mites by sanitary measures is possible but often not attained. As a preeaution in the preservation of stock cultures, some scheme of poisoning should be used. One of these formulas consists of dipping the tips of the cotton plugs in a solution of the following composition:

$95 \%$ alcohol $95 \mathrm{cc}$.

Bichloride of mercury ....................... $0.5 \mathrm{gm}$.

Glycerine............................. 5 ce.

Color with any aniline dye.

Care must be taken that the solution does not come in contact with the colony. The cultures must be allowed to derelop into typical colonies before poisoning. An antiseptie formula for the purpose needs alcohol to insure penetration of the plug, a poison to destroy the mites, glycerine to prevent the crystallization of the poison as the alcohol evaporates, and the dye to insure the destruction of the cotton plugs when removed from the tubes. In our own experience we have consistently made it a practice to wipe off the outside of all culture tubes with the above, or some other sterilizing solution, and to poison all plugs before cultures are replaced in or added to the collection of stock cultures.

Molds: Under humid laboratory conditions, cotton plugs, especially if made from the absorbent type of cotton, absorb moisture. Careless handling in preparation and care of such plugs often adds enough nutrients to support growth. Steam sterilization tends to distribute nutrients. Dirt, bacteria, and molds fall from the air upon the exposed portion of the plug. Handling detaches spores from the colony within so that both ends of the plug are commonly well seeded. Spore germination, therefore, may begin at either or both ends. Outside molds may grow through and drop into the 
culture, or the culture itself may grow out through the plug and contaminate other cultures or experiments. Surface sterilization of the outside of the tubes and poisoning the plugs takes care of molds, as well as mites.

Spraying: Oily sprays, as selected fractions from petroleum, available from commercial sources, even kerosene, distributed with a "gun" that produces a mist penetrating and filling all cracks, crevices, open spaces among apparatus or furniture and clouding the whole atmosphere of the laboratory, have been found effective in carrying down mold spores and bacteria from the air and ridding the laboratory of mites, insects, and vermin.

\section{PRESERVATION OF DRIED SPECIMENS}

Mold cultures lose many of their characteristic and diagnostic features upon being dried. Nevertheless, dried herbarium specimens serve a useful purpose in preserving type material which might otherwise be lost. Details of morphology are often difficult to establish from such material, but group characteristics are preserved and over-all colony appearances can be recognized after many years. The retention of culture tubes or petri dishes containing such dried specimens constitutes a reasonably satisfactory means of preservation, and the material contained therein approximates as nearly as is possible the cultural picture of the growing colony. Glass tubes and dishes, however, are cumbersome and easily broken, hence may prove unsatisfactory if frequent handling is necessary. For many years we have employed an alternate technique with generally satisfactory results. Representative portions of colonies grown in petri dishes are cut with a large cork borer, lifted out with a spatula, and dropped into paper pill boxes where they are allowed to dry. These can then be stored in larger boxes or attached to herbarium sheets for filing. The boxes should be provided with tight-fitting lids, and for greatest convenience should measure approximately $1 \frac{1}{2}$ inch in diameter. Aspergilli stored in this way prove useful in many comparative studies. They cannot, however, under any condition, take the place of carefully handled living cultures. 


\section{Chapter VI}

\section{VARIATION}

The Aspergilli are a variable and mutable group of fungi. They are characterized by great diversity and variability as they are isolated from nature, and an increasing amount of evidence shows that they can be made to vary, or mutate, in the laboratory by subjecting them to a number of different imposed stimuli. Frequently the same types of mutants or variants ultimately result under both natural and artificial conditions. Nevertheless, it is believed desirable to consider somewhat separately variations and mutations resulting from natural causes and those resulting from imposed stimuli.

\section{Definition of Terms}

Before entering upon a discussion of variation, either natural or induced, it is important to define certain terminology which is to be employed. It is recognized that our definitions will not agree in all cases with those of earlier workers, nor do we expect that all subsequent investigators will accept those which we propose. If the meaning in the present discussion is clear, our purpose will have been served.

(1) The term mutant, or mutation, is used to designate a strain whose source is actually known and can be verified. Furthermore, it is limited to those substrains which originated as sharp breaks from parent cultures (usually interpreted as gene mutations), and in successive culture generations retain their distinguishing characteristics unaltered. This may or may not have a taxonomic connotation. Upon occasion it is used in essentially the same sense as "variety". To illustrate, A. nidulans mut. albus, A. fumigatus mut. helvola, A. niger mut. cinnamomeus, etc., are used as Latin names to designate forms which differ from the parent species in certain striking details. In other instances it is used to identify a type of change, rather than to designate a particular and isolated strain resulting from such change. It is considered correct to refer to artificially produced albino, yellow, and buff-colored strains of $A$. terreus as mutations (see p. 75 ), since they are constant in character and are known to have originated from a cinnamon-colored parent culture, wholly representative of the species; and we believe it represents good judgment to refrain from assigning Latin designations to each of them. The term mutation, then, refers to altered strains of constant character and known lineage, whether or not they are given Latin designations. 
(2) The term variant, or variation, is used loosely and reference to it in this manual is not, in all cases, entirely consistent. In general, however, it is applied to subcultures, or strains, arising through gradual change from well-defined strains of identifiable species. The characters of a variant, then, are not generally stable but subject to continued change and further variation. As used by us, the term has no taxonomic implication and can be considered essentially synonymous with the term "saltant" which is so commonly employed in reports on variation in the Fungi Imperfecti. Variants frequently appear as colony sectors, overgrowths, or other localized areas of changed appearance or texture. When isolated in pure culture, they may or may not retain their distinguishing characteristics.

\section{NATURAL VARIATION}

Cosmopolitan species and groups of Aspergilli show adaptability to wide ranges of environmental conditions. As these molds are isolated from nature, variation among the members of any species, series, or group is regularly encountered. Such variations commonly differ in degree rather than in basic characters, and one can distinguish a series of intergrading or bridging forms. Even striking isolates are often unmistakably allied with some well-defined species or group in this manner. Such different but intergrading forms arising in nature can be considered as natural variants. Natural variants of a similar kind can frequently be obtained in laboratory culture by selective isolation and cultivation from sectors or other areas of atypical growth, or by single spore isolations. Distinction must be drawn between differences in appearance, morphology, and habit of growth resulting from inherent differences between strains, and alteration in colony character in response to changes in the composition of the culture medium or other environmental factors. Rigid comparative culture is often necessary to distinguish between the two. For the present discussion, we are concerned with differences that are more fundamental than direct temporary responses to artificial stimuli (ecads); but the latter, unless carefully evaluated, may appear no less real. Rightly or wrongly, Blochwitz (1930, p. 247) comments that $A$. flavus, ${ }^{1}$ in specimens collected in the Botanical Garden at Buitenzorg, was called A. penicillopsis (Henn.) Rac.; in the Botanical Garden at Singapore, S. vitellina Ridley; in India, S. corolligena Massee; and in Columbia, A. delacroixii Saccardo.

Occasionally isolations are made from laboratory cultures which represent sharp "breaks" from the parent strain, and since they are constant in subsequent culture, they may be considered as true mutations. Representatives

1 The name of the original describer is only used for specimens or strains in culture which were definitely attributed to the describer. A. flavus, A. niger, A.terreus, etc., are series concepts as used here. 
of such natural mutations which originated in the absence of any artificially imposed stimuli are luill's A. fumigatus var. helvola (1939), A. nidulans var. albus (1939), and Cladosarum olivaceum (1938).

\section{Intra-strain Variation}

A certain amount of variation can be expected to occur in any given strain of Aspergillus. In certain species and strains this is very limited and cultures can be re-cultivated repeatedly upon a variety of media, and at different temperatures and $\mathrm{H}$-ion concentrations without eridences of risible change other than those resulting from the immediate effects of the altered environment. Many strains of Aspergillus niger are characterized by such comparative stability. Other species and strains are subject to continual variation with differences in character and rate of growth appearing rather abruptly as sectors or overgrowths, or gradually developing as a progressive alteration in the general aspect of the whole culture. By successive and selective subculturing, strains of $A$. alliaceus and A. ochraceus showing a marked difference in sclerotium production can be obtained. In like manner, strains of $A$. itaconicus Kinoshita can be secured which are almost completely sterile upon all media tested. The same is true of $A$. granulosus Raper and Thom, A. flavipes, etc.

Working with a strain of the ascosporic species, A. fischeri, Greene (1933) isolated 448 single spore cultures and among these found variant progeny of two main types: (1) cultures producing very large, scattered perithecia as opposed to the typical picture of many small perithecia, and (2) cultures producing conidial structures in profusion, but forming few perithecia and these tardily. The second type was fairly stable, whether derived from single ascospores or conidia. The first type was rariable, in some cases reproducing the characters of the variant parent, in others reverting to the character of the original stock culture.

Hansen (1938) and Hansen and Smith (1932) have studied many of the Fungi Imperfecti rather exhaustively and report that single strains of these fungi are basically composed of a mycelial (M) type and a conidial (C) type. By proper techniques the two forms can be separated and recombined at will. While it has not been explored as yet, the possibility exists that the same condition may prevail to a limited degree in the Aspergilli, and that this may account for a certain amount of the intra-strain variation encountered.

Back of variability in molds, many lines of discussion have been developed. Buller (1933) has frequently called attention to the unmeasured possibilities of nuclear and cytoplasmic disturbance from the commonly observed phenomenon of anastomosis. Vegetative hyphae belonging to the same mycelium (mycelium derived from a single spore), or different mycelia, 
throw out branches which fuse without showing any other sign which might suggest a sexual process either before or after fusion. Anastomosis is usually observable, if at all, in the rapidly growing area where many spores placed as an inoculum are developing into one colony. By transferring large numbers of spores from an old culture to a new one, most of the Aspergilli studied by us have shown fairly consistent repetition of colony characters and conidial morphology and have been maintained for a long time with little or no observable change. Other organisms handled in the same manner have not been successfully maintained with the morphology originally studied. Differences in behavior can be attributed to variability between strains.

\section{Intra-species Variation}

Variation within the species is very prevalent and is well marked in many cases. When a large number of isolates of any particular species or series is collected, one can regularly expect to find among them wide variations in color, amount of sporulation, and in their general habit of growth. Usually, however, such variation is graduated, and strains representing various intermediate steps between the extremes are to be expected. While it is by no means unique, we may use $A$. terreus as an example, since a very large number of strains belonging to this species have been isolated and observed in plate and tube cultures during the past two years (fig. 16). Colonies of the type strain, and of the great majority of isolations made from nature, are plane, cinnamon in color, very heavy sporing, with conidial heads arising directly from the substratum in an even and close stand. An occasional isolate is much brighter in color, approximating xanthine orange (Ridgway, $\mathrm{Pl}$. III), but in all other respects it is fairly typical. It is believed to represent a form such as that described by Blochwitz (1934) as A. boedijni, and in the present manual we have designated it as $A$. terreus var. boedijni. In each of the above cases, the production of abundant fruiting structures follows closely the advancing margin of the growing colony and there is little or no continued growth of mycelium except in the marginal area. Certain other strains are quite floccose; conidial heads are typical in form and color but are greatly reduced in number and are borne upon aerial hyphae. Shih (1936) was probably working with such a culture when he described the variety $A$. terreus var. floccosus. We feel that the forms are sufficiently distinct to warrant maintaining his variety. Still other strains possess abundant but fairly close-, rather than loose-textured mycelia and bear abundant but very pale buff-colored conidial heads. The vegetative mycelium in this form is bright yellow and for this reason we have designated it as $A$. terreus var. aureus $\mathrm{n}$. var. (see p. 198). If one should examine only the type strain and these three atypical forms, it is entirely probable that one 
would deseribe them as four distinct species. Actually, however, they are not sufficiently distinct to warrant specific rank, for they represent only extremes of variations along three divergent lines with numerous inter-
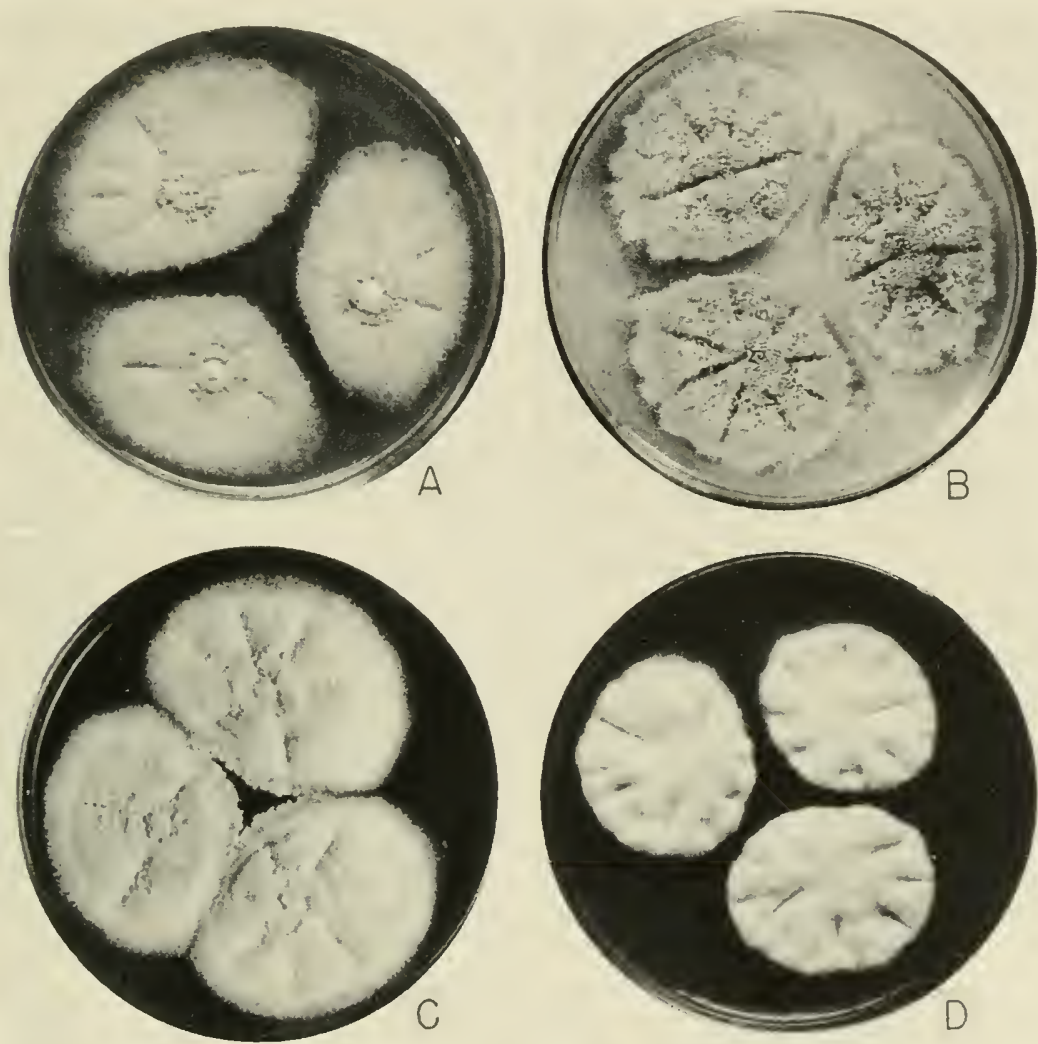

Fig. 16. Intraspecies variation in Aspergillus terreus. A, A. terreus, typical strain NRRL No. 265, characterized by heavy conidium production and colonies cinnamon in color. $B, A$. terreus var. boedijni, NRRL No. 680 , characterized by deeper colonies and conidial heads near xanthine yellow. C.A.terreus var. floccosus, NRRL No. 1921, characterized by loose floceose colonies and spore heads light pinkish-cinnamon in color. D, A. terreus var. aureus, NRRL No. 1923, characterized by yellow floccose colonies and comparatively few cream to buff-colored conidial heads.

grading strains aligning them, almost without interruption, with the typical form itself.

Another series of variants in our collection shows gradation from the usual radiate head and conidiophore of $A$. sydowi to the simple mono-verticillate penicilli of Penicillium restrictum Abbott. The tendency to form 
reduced conidial apparatus is observed in all of the strains of $A$. sydowi examined. Over a period of many years variants have exhibited all gradations in colony appearance from typical $A$. sydowi to the aspect of $P$. restrictum of Abbott except for the presence of an occasional conidiophore and head of $A$. sydowi. Repeated cultural tests exclude contamination. These variants occur in nature, they produce abundant conidia, and they undoubtedly maintain themselves successfully in the field.

Aspergillus fumigatus presents a somewhat similar condition. In this species, typical cultures produce heavily sporing, velvety colonies that are dark green in color and show almost no aerial mycelium. Other strains commonly isolated from nature produce very floccose colonies and bear comparatively few conidial heads (fig. 37). These heads, however, are typical in form and in dimension. Strains possessing this contrasting character are relatively stable in culture, but in this species, as in A.terreus, all degrees of intergradation are found between this floccose type and strains entirely typical of the species. The same story is repeated in other speries.

Appreciable variation can normally be expected among the isolates of any of the very abundant and cosmopolitan species. Such rariant strains, however, are the exception rather than the rule, since the great majority of isolates are quite typical of the species. Attention was called to this fact in our study of the A. glaucus group (1941).

When grown in comparative culture, strains successively isolated as representing a particular species usually show enough difference to give each strain a kind of individuality. Exact identity, point by point, is not expected. Such strain variation may be incidental and unimportant, or it may be correlated with activities which make one strain a valuable agent in an industrial process and the other worthless.

\section{Intra-group Variation}

Inside the different groups of Aspergilli one normally finds somewhat similar but wider variations than those seen among the strains constituting any particular species. To what degree species in nature have developed by mutations and by progressive variation can only be guessed. We do know, however, that the species within a group, like the strains or varieties within the species, are regularly bridged by intermediate forms which render it difficult to establish sharp and immutable lines of separation. One can almost cite it as a rule that the definiteness with which one regards a species is inversely proportional to the number of strains of that species which have been examined. Still species are necessary as guide-posts-as fixed points around which closely related organisms showing a certain but limited amount of variation can be grouped.

The Aspergillus flavus-oryzae group can be taken as illustrative of the type of variation to be expected within a group of the Aspergilli. Thom's 
culture No. 113 (NRRL No. $44 \overline{7}$ ) of A. oryzac, received from Baarn and believed to stem from Cohn's original strain, is a very floccose, loose-textured culture bearing comparatively few, small, light yellow to tan heads. Conidiophores are long, ranging up to 2 to $5 \mathrm{~mm}$., and are very thin-walled. There is only a trace of green even in individual heads, and in general aspect, the culture normally show's no green color. Aspergillus flan'us in its typical form is not floccose and is very heavy sporing. Conidiophores arise directly from the substratum in a close stand, are usually $1 \mathrm{~mm}$. or less in length, and are comparatively heary walled. Colonies are regularly in yellowgreen shades and range from light to comparatively dark green (see species description). A. parasiticus Speare goes even farther. Colonies are very dark green in color. Conidiophores usually range from 200 to $400 \mu$ in length, and sterigmata are typically in a single series, whereas they may be in a single or double series in $A$. oryzae and A. flavus. In the opposite direction, but markedly different from $A$. oryzae is $A$. effusus Tiraboschi. Typically this is very floccose and comparatively light sporing, with heads borne upon short conidiophores which arise from the loose aerial mycelium rather than from submerged mycelium in the substratum.

The cultural pictures of these species are fairly characteristic. Yet, it is practically impossible to take a large collection of 100 or more strains and separate them into these species with any degree of confidence or satisfaction. The difference between A. oryzae and A. flaus is bridged completely by a series of intermediate forms showing all degrees of variation between the two strains selected as typical. Nomenclature in this group is then further complicated by the fact that among the great collections of these forms obtained from the Orient, and designated $A$. oryzae, the majority of forms are somewhat intermediate between $A$. oryzae and $A$. flaus as depicted above. A similar series of intermediate forms bridges completely the gap between $A$. flavus and A. parasiticus. There is no sharp line of demarcation between any of these species, still they are not one and the same, and to attempt to lump these diverse forms together into one species, as Neill (1939) has done for the A. glaucus group, intensifies rather than reduces the difficulties encountered.

Intra-group variation is also particularly marked in the A. niger group. During an extended period of study and observation of molds in culture, Biourge, who was a discriminating collector, accumulated 63 strains of black Aspergilli which suggested sufficient individuality to be deemed worthy of further study. These were turned orer to Mosseray when he entered Biourge's laboratory. He assumed that he had before him all of the black Aspergilli possible to collect and, knowing that Biourge had selected each of them because it seemed to have some special character, he undertook a taxonomic study to define those characters and to organize them into a systematic presentation (1934a). His paper lists 35 species of 
which 25 were either described as new species or new combinations. His findings in $A$. niger are fairly illustrative of the same type of study in other groups (compare Thom and Currie [1916] for A. niger; Thom and Church [1921] for A. flavus.

Mosseray based his primary separations upon conidial sizes, shapes, and markings, while secondary and tertiary separations were based upon conidiophore lengths and the characters of colonies in tube cultures on Biourge's "Raulin-neutre gelose" - a variation of the classic Raulin solution. Biourge and Simonart demonstrated the entire series to one of us (C. T.) showing how wrinkling and granulation of mycelium, intensity and changes of secreted color, shades of color in the conidial area, lengths and proportions of conidiophores and heads, and their distribution over the mycelium gave to each strain an individuality which had been repeated in successive cultures over a considerable time. We raised just one question, "What would you do with the next thousand?"

The large majority of all isolations of black Aspergilli conform within a range of minor variation with the general van Tieghem concept,-i.e., black-brown colonies with conidiophores and heads giving the general structure and measurement of parts found in the classical description. Then, in contrast with these, there are shades of colony color from the coal black of $A$. carbonarius through shades of purplish-black to the brown of $A$. ferrugineus Fuckel or to the lighter shades of Schiemann's mutants (pp. 223-224). Some of them produce no colors in the substratum and reverse of the colony; some show yellow in traces; others are persistently deep orange, giving the whole a yellowish appearance. Or, again, the agar and mycelium may develop a red-brown or "mauve" shade of violet.

Conidiophores in the usual type of culture reach nearly enough the same length to give the effect of a field of grain. But their length may be quite short and the heads seem to be borne directly on the substratum, or they may be several millimeters in length with the heads borne well above the substratum and correspondingly large. Between these extremes, every variation can be found. Conidiophores may be scattered thinly over the vegetative mycelium, collected in a zone at the border, or crowded in the center.

The vegetative mycelium may grow as a flat felt (plane) or may be variously wrinkled, sulcate, or buckled. In a smooth or plane colony the mycelial cells seem to stop growing early - the colony extends only at the margin. In the plane colony intercalary growth (i.e., the formation of new cells in the filament, or the lengthening of the old cells), and the production of new branching ceases. Such mycelia ordinarily produce one crop of conidial heads, beginning at the center of the colony and progressively developing toward the margin until the medium is exhausted or some inhibiting factor paralyzes growth. Marginal growth in such a colony 
often shows longer and fewer conidiophores and larger heads than in the central area.

Within the group with the usual structures still recognizable, many variants with contrasting features appear. Heads with very long primary sterigmata, which are sometimes septate, appear in A.carbonarius (Bainier) Thom, A. pulchella Speggazini, or A. tubingensis Mosseray. The primary sterigmata may grow out into sterile filaments as in Mosseray's figure for A. ficuum (Reich.) Henn.; much more commonly, some primary sterigmata grow out as tiny conidiophores and produce little heads, often consisting only of a cluster of simple sterigmata and conidial chains. Thus, sterigmatic changes may run from the simple sterigmata of $A$. japonicus Saito, A. luchuensis Inui, or A. malvaceus Mosseray, where only some are double, to other species showing the widest range in length and arrangement.

Another group of variants show marked suppression of the ordinary structures expected. Strains in which conidiophore formation has been reduced or almost suppressed have been studied in continuous culture. Such colonies showed an occasional long conidiophore and large head, conforming to the $A$. niger pattern, produced at the end of the colony growth period. Meanwhile the mycelium was fully covered with irregularly branching hyphal elements bearing single sterigmata variously placed, groups of sterigmata, or penicilloid clusters of sterigmata each bearing a short chain of conidia showing the characteristic markings of the group. Transfers from the simplest form developed the complex or A. niger elements. Transfers from the large heads brought a recurrence of the reduced type of fruiting. No method of selection tried brought back the typical $A$. niger aspect, and cultures of this type appear to represent degenerate forms.

It would appear, then, that a general type or morphological picture when found dominant in large numbers of natural isolates can be regarded as typical for a species of Aspergillus. It is recognized that marked divergences from such types occur under the unrecorded stimuli of nature. Some of these forms succeed in establishing themselves as permanent elements of the microflora and thus become successful as species, or varieties. Others do not digress quite so markedly and thus constitute intermediate or bridging forms. At the same time marked changes can be induced by the application of artificial stimuli. Where the origin of such altered strains is known, they are commonly regarded as mutants. Were they isolated directly from nature and their previous history not known, it is probable that they would be considered as separate varieties or even species.

\section{Natural Mutation}

While most of the mutants which we recognize as such have originated in the laboratory as the result of certain artificially imposed stimuli (or 
drastically altered conditions of growth), and hence can properly be termed induced mutations, a number of well authenticated cases of natural mutation are known. In 1939 Edward Yuill described as A. fumigatus var. helvola a buff-colored mutant of this species isolated by him in 1937 (fig.
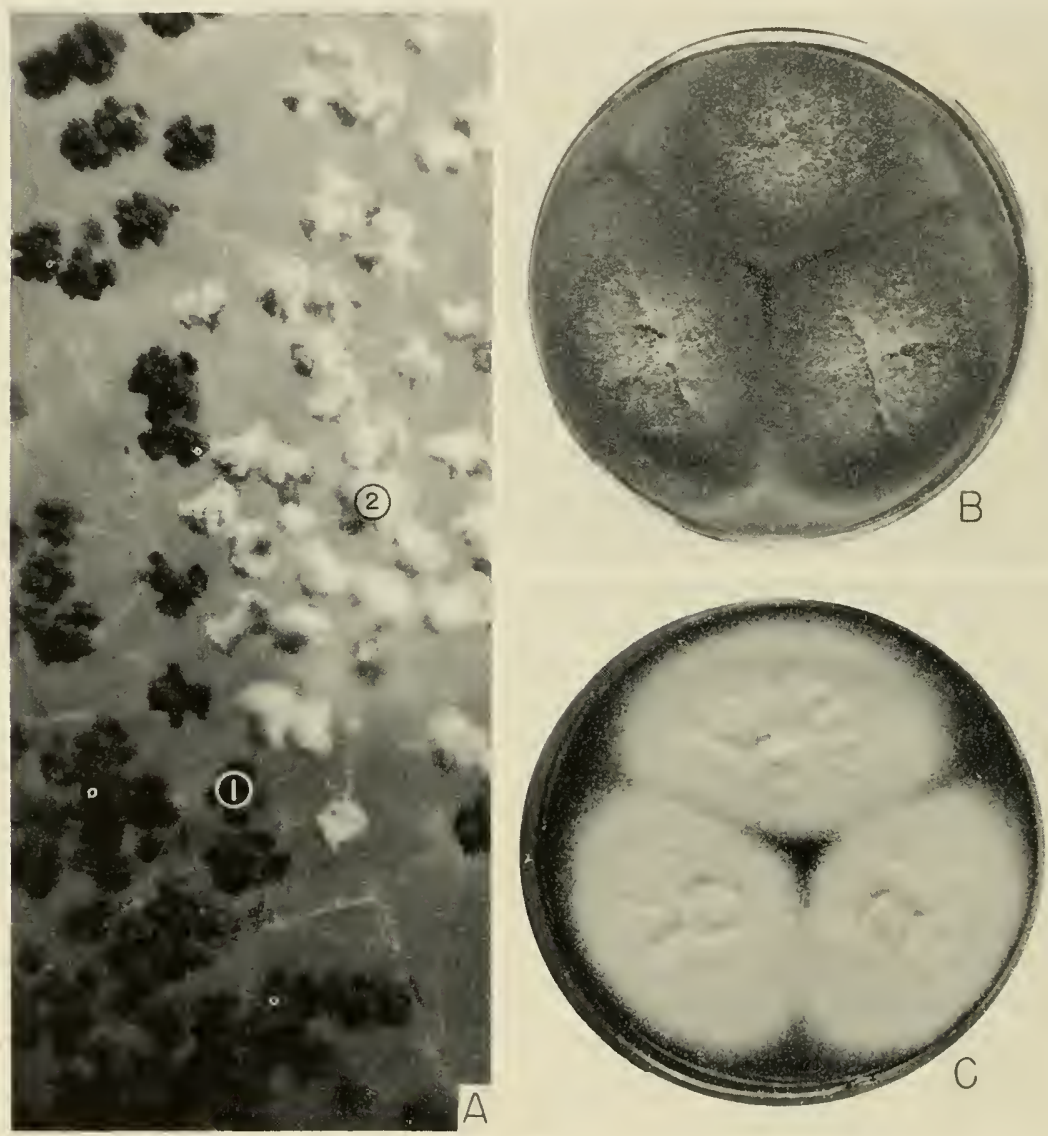

FIG. 17. Natural mutations. A, Portion of a colony of Aspergillus niger in which a tan spored mutation appeared as a $V$-sector: $A_{1}$, typical black head of parent strain; $A_{2}$, tan head of naturally occurring mutation; $\times 25$ approximately. $B$ and $C$, Typical strain of Aspergillus fumigatus and a naturally occurring mutation discovered and described by Edward Yuill as Aspergillus fumigatus mut. helvola. The mutations in both species have proved completely stable in continued culture.

$17 \mathrm{C})$. In the same report a white-spored mutant of $A$. nidulans, isolated in 1937 , was described as $A$. nidulans mut. alba. In both cases the mutants developed as natural phenomena without the application of any artificial stimuli-in the former case as a single head, in the later case as a group of heads, and in both cases from wholly typical strains (fig. 17). 

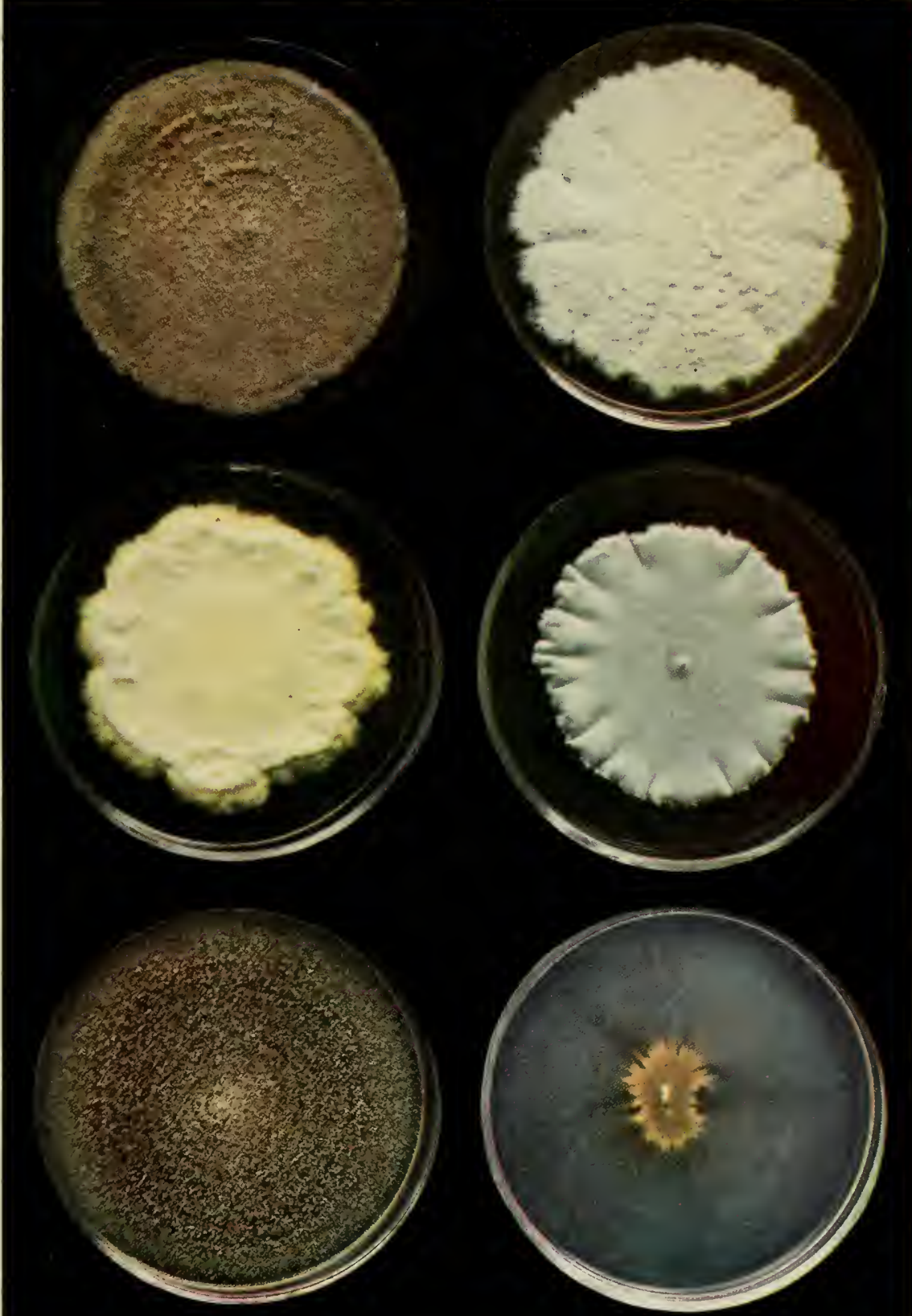

Plate II

Mutations in Aspergillus terreus Thom produced by irradiating conidia with ultraviolet light. Colonies grown upon Czapek's solution agar at room temperature for two weeks. A (upper left), Aspergillus terreus, NRRL No.265, unirradiated stock culture. $B$ (upper right), Albino mutant, producing white conidial heads but retaining the basic cultural and morphological characterjstics of the parent culture. (C (center left), floccose, yellow-white mutant producing few and atyvical conidial heads. $D$ (center right), Leathery mutant, producing tough, close-textured colonies and very few and atypical conidial heads. $E$ (lower left), Nitrate mutant, a form unable to use nitrate nitrogen, producing thin spreading colonies and few but entirely typical heads. F (lower right), Thiamin mutant, a form unable to produce thiamin, producing thin, spreading colonies and very few and atypical conidial heads, see text p. 75 . (Color photographs by Haines, Northern Regional Research Laboratory. Reproduced through co-operstion of Chas. Pfizer \& Co., Inc.) 

In studying a group of cultures three years ago, the authors noted a few tan heads in the form of a $\mathrm{V}$-sector in an otherwise typical black colony of A. niger (fig. 17A). Isolations from a tan head reproduced the mutant head characters, and repeated transfers of this strain have proved consistently stable over a period of three years. The mutant strain cannot be distinguished from $A$. niger mut. cinnamomeus ( $A$. cinnamomeus of Schiemann).

In dealing with natural as well as induced mutations, we have endeavored to limit the use of the term mutant to forms whose origin was definitely known. Blochwitz $(1934,1935)$ was not so precise. Under the name A. glaucus mut. alba he refers to a white-spored member of the A.glaucus group. He believed Aspergillus giganteus Wehmer to represent essentially a long-stalked $A$. clavatus, hence designated it $A$. clavatus mut. giganteus. This treatment may be justifiable. We believe, however, that until their origin from other and well-marked species can be proved, it is wise to continiue to recognize as species these very distinct forms that are isolated from and are able to maintain themselves in nature.

Cladosarum: The most striking variant, or mutant, ever described in the Aspergilli is Cladosarum olivaceum of Yuill and Yuill (1938). This appeared in a culture of $A$. niger growing on bread at $28^{\circ} \mathrm{C}$. (Personal correspondence). Its colony, conidiophores, vesicles, and primary sterigmata are those of Aspergillus. The secondary sterigmata, instead of producing conidia, thrust out cells which are essentially the same in morphology as the secondary sterigmata themselves; the same procedure is then repeated several times. Occasionally, however, a terminal cell changes and thrusts out several equal cells; in other words, it resumes the function of a primary sterigma. The new secondaries repeat the process of producing chains of cells each resembling the basal cell with the aspect of a sterigma not a conidium, and always with the youngest cell at the tip of the chain. In Aspergillus the sterigma which produces a chain of conidia always produces the new conidium at the base of the chain, shoving the next most recent farther out. Differing then from Yuill's interpretation, Cladosarum produces no conidia, however readily any cell detached from the mass may grow.

In Aspergillus the ordinary nuclear procedure in conidium formation involves mitosis in the sterigma actually producing the conidia. After each mitosis one daughter nucleus migrates through the tube into the new spore in which it "rests" until that spore begins to germinate. The other nucleus remains in the sterigma and repeats the process. This goes on until there may be a chain of 200 conidia-the oldest at the outer end, the newest directly attached to the sterigma.

In the absence of cytological study, one may offer the following hypothesis. In "Cladosarum" the nuclear procedure must be reversed. 
The same mitosis occurs. One active and one resting nucleus result, but the resting nucleus remains in the sterigma while the active nucleus moves into the newly forming cell. This determines the course of development. The active, multiplying nucleus is always in the newest cell formed.

Previously Barnes (1928) had stimulated a strain of the A. glaucus group (identified by us as $A$. amstelodami, 1941) by heat, and reported certain mutants which were deposited with Dr. Westerdijk at Baarn. Among them, under the designation "Creamy" (NRRL No. 143), a mold with the morphology of Cladosarum appeared. It was obviously derived from some $A$. glaucus strain and is, in so far as the writers are aware, the only other appearance of the Cladosarum structure ever discovered. Barnes does not appear to have recognized its contrasting structure.

Since no collector has reported this type of mutant in nature, it must either be very rare or be unable to maintain itself in a competitive environment. However readily such mutants may be maintained in the laboratory, they would rarely reach the second generation in nature on account of lack of spores. The name Cladosarum was thus applied to a defective organism (zoologically designated a "monster"), which does not become a component of any natural flora, hence taxonomically the name should be untenable.

\section{INDUCED VARIATION}

Striking mutations have been obtained from various species of the Aspergilli by subjecting them to artificially imposed stimuli. Schiemann (1912) was among the first to draw attention to the possibilities inherent in this approach. By subjecting a strain of $A$. niger to various concentrations of potassium bichromate, she was able to produce two striking mutations which she designated according to color, A. fuscus (= A. niğer mut. schiemanni of this manual) and $A$. cinnamomeus ( $=A$. niger mut. cinnamomeus ibid.), respectively. Both cultures have remained stable in our hands, and in various collections, throughout the 32 years since their original isolation. A third "mutation" designated A. niger var. altipes could not be distinguished from other strains of black Aspergilli isolated from naturethe parent strain was not seen. Working with a member of the A. glaucus group designated Eurotium herbariorum Wigg., Barnes in 1928 reported the production of a series of variations by exposing spores to heat. While there are reasons for questioning the correctness of some of Barnes' interpretations and conclusions (see Thom and Raper, 1941), there is evidence that he succeeded in producing a mutant which, in its habit of growth and in the character of the fruiting structures developed, bears a striking resemblance to a form subsequently isolated from $A$. niger by the Yuills (1938), and described by them as Cladosarum olivaceum, genus and species 
new. Galloway (1933) obtained marked variation in colonies of Aspergillus terreus by growing them upon media containing flour to which was added 0.003 to 0.005 per cent of salicylanilide.

Thom and Steinberg (1939), and Steinberg and Thom (1940a, 1940b) in a series of experiments, applied chemical stimulants to a strain of $A$. niger (Thom No. 4247: NRRL No. 334) which had been in the collection many years without noticeable change. From the cultures resulting, Steinberg picked out and purified for study all variants he could observe with the naked eye and with the aid of a handlens. In examining a fruiting area of a colony, general changes were not common; ordinarily an occasional head changed color, long or short conidiophores appeared in spots, gross malformation showed as areas of no fruit, or too much fruit, or color effects in the mycelium. These were picked out and grown in successive cultures. Expressed in terms of morphology, the most striking feature of the tested culture was disturbance of uniformity. The same types of changed aspect were reproduced many times. In general, they followed the same lines as have been described as present in the natural series selected by Biourge or in the collections of Mosseray at Brussels. In general, these changes were destructive in character and included large numbers of "injury mutants", or variants, which reverted in subsequent transfer to the original aspect of Steinberg's culture. Sodium nitrite was the most effective agent used. Some isolations, however, represented cleancut mutations. Strains of $A$. fumigatus with albino heads were obtained. Forms of $A$. niger with light brown to cinnamon-colored spore heads, essentially like those earlier obtained by Schiemann (1912), those obtained by Whelden (1940), and those subsequently obtained by Raper, Coghill, and Hollaender (see below) from members of the same group, were likewise isolated. These have remained stable in culture for the four years that they have been in our collection.

Whelden (1940) succeeded in obtaining a series of mutants in A. niger by bombarding conidia with low voltage cathode rays and subsequently isolating colonies which developed from such irradiated cells. Forms possessing heads in various brown shades, rather than black, were isolated, as well as one giant form with conidial structures appreciably larger than the parent. By means of ultra-violet irradiation of spores, Raper, Coghill, and Hollaender (in press) obtained mutants which produced tan-colored conidial heads but otherwise closely resembled the parent strain. In a more exhaustive study of $A$. terreus (Pl. II, A), the same investigators succeeded in isolating a number of striking and markedly different mutations. These included albino forms with colorless conidial heads (Pl. II, B); forms with pale buff-colored heads; yellow, yellow-white, floccose forms with few and smaller conidial heads (PI. II, C); forms producing very thin, sparsely 
sporing colonies; forms producing restricted colonies characterized by the production of an excessive amount of orange-brown exudate; and forms with leathery, close-textured colonies bearing very few conidial heads (Pl. II, D). Alterations in microscopic details commonly accompanied these changes in colony appearance (fig. 19). In addition to these morphological mutants, which were found to be stable when checked through ten successive transfers over a period of 12 months, various physiological mutants were also isolated. These included forms unable to utilize nitrate nitrogen (Pl. II, E) but able to grow and sporulate normally upon media containing ammonia nitrogen, and a form unable to synthesize thiamin (Pl. II, F). Upon Czapek's solution agar containing sodium nitrate and sucrose, each of these is strikingly different from the parent strain; upon
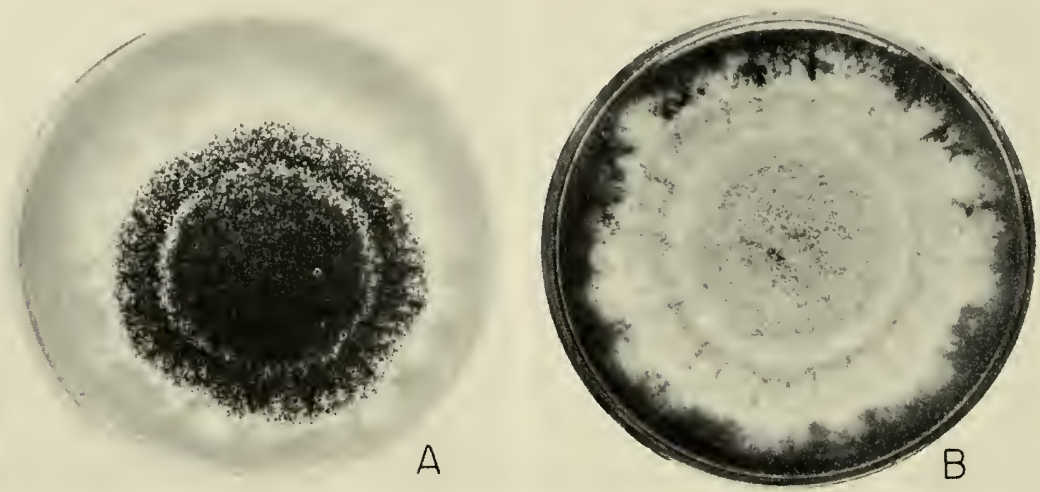

FIG. 18. Induced mutation. $A$ and $B, A$ spergillus niger group, strain NRRL No. $67: A$, parent culture growing on Czapek's solution agar, 10 days, room temperature; $B$, tan-spored nutation of same produced by ultraviolet radiation.

malt extract agar, which contains adequate amino nitrogen and thiamin, neither could be difierentiated from the parent (Raper, Coghill, and Hollaender, in press). Thus the need for comparative study and examination upon a variety of media is apparent, while the importance of such studies in reliable taxonomic work cannot be over-emphasized. In cultures of Aspergillus terreus resulting from irradiated spores the capacity to produce itaconic acid varied from zero in some isolations to levels somewhat above the parent culture in others. The majority of such isolations produced yields somewhat lower than the parent strain, many produced yields approximately equal to it, while a very few produced superior yields (Lockwood, Raper, Moyer, and Coghill, in press).

Based upon our own investigations and the published reports of other 
workers, certain observations of a summary eharacter can be made regarding variation in the Aspergilli:

1. Aspergilli include strains and species adapted to a very wide range of environmental conditions. Such conditions may influence materially the cultural and morphological characteristics of these molds.

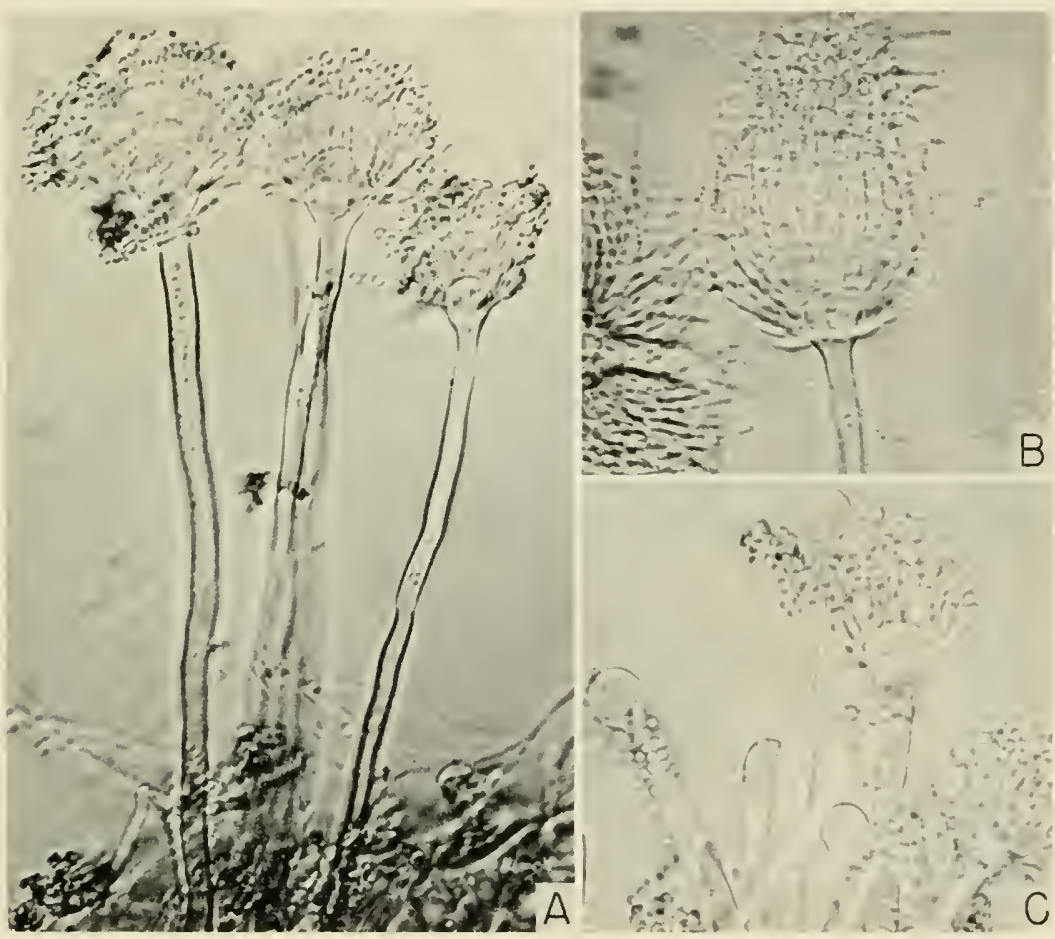

FIG. 19. Photomicrographs showing details of structure in the conidial heads of the parent strain, and in two selected mutations of Aspergillus terreus (NRRL No. $265)$ produced by ultra-violet radiation, $\times 600 \quad A$, Typical heads of non-irradiated parent strain. $B$, Mutation in which conidium formation is incomplete and cells adhere in long chains in liquid mounts. $C$, Mutation in which many fruiting structures develop vesicles but often fail to produce sterigmata and spores.

2. Under natural conditions, great numbers of variants appear along with occasional sharply separable forms, or mutants, which are definitely of species rank. The extent to which natural mutations may account for described species is a matter of conjecture.

3. The Aspergilli can be made to mutate in the laboratory by subjecting them to a variety of different excitants, or stimuli. Induced mutations may parallel some of those found in nature and deseribed as species. 
4. Particular species of the Aspergilli tend to mutate along certain definite lines, e.g., the production in $A$. niger of forms with tan to light brown spore heads (Schiemann, 1912; Steinberg and Thom, 1940; Whelden, 1940; and Raper, Coghill, and Hollaender, in press), and the production in .A. fumigatus of forms with colorless spore heads (Yuill, 1939; and Steinberg and Thom, 1940a). The type of mutant produced is not governed by the type of treatment given, although the number of mutations produced is strongly influenced by this factor.

5. Great variability in biochemical activity is encountered among strains isolated from nature, but these differences are rarely linked with specific morphological changes.

6. The Aspergilli can be made to mutate physiologically as well as morphologically by the application of various stimuli. Physiological mutations may conceivably be of tremendous importance in the development of improved strains for fermentation processes.

7. Despite natural variation, most strains of Aspergillus when subjected to critical transfer and maintenance under rigorous culture conditions can be kept for many years with constant colony appearance, stable morphology, and dependable biochemical activity. 


\section{PART II}

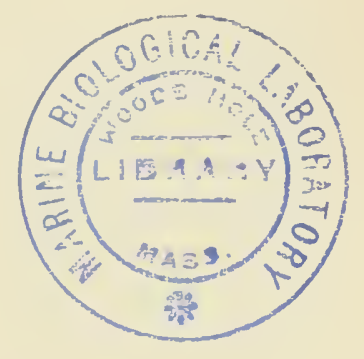

THE MANUAL PROPER 



\section{Chapter VII \\ THE USE OF THE MANUAL}

Since the purpose of this manual is to facilitate the identification of Aspergilli as they are isolated from nature and as they are encountered in the investigation of special problems, the procedures and considerations involved in the use of the manual must be discussed. The general morphology and structural details found in the spore-producing apparatus of the Aspergilli have been described and figured in Chapter III. Complexities in the specific combinations of these characters found in the examination of moldy material and in the isolated colonies of individual strains makes desirable a summary outline of the exact observations to be made in describing an Aspergillus. Such a descriptive sheet is presented as page 82. For practical use, a standard sheet of record paper is folded over on the left-hand margin for about $5 \mathrm{~cm}$. The column of observations desired is written upon this marginal fold; a fresh sheet of paper is slipped under the fold and the descriptive data are filled in, appearing exactly in the same order for each strain studied. A single glance at the sheet shows the discrepancies, if any, in the descriptive data obtained. With such a sheet properly filled out, the keys to groups and within groups facilitate the placement of the strain in its proper group first, then its allocation to species within the group.

Such descriptive sheets, to have comparative value in species diagnoses must present their data in standardized terms. It has, therefore, been necessary to define and illustrate the morphological terms accepted in this manual, and to indicate as synonyms in the chapters on morphology the usages of various describers of Aspergilli back over the 200 years since Micheli.

Identification from specimens: The field mycologist working with specimens collected and examined fresh or dried will of ten find completion of a technical description very difficult and some observations impossible. One with long acquaintance with the Aspergilli may place his specimen to the group or aggregate species correctly, but even such workers are frequently puzzled. If the organism is deemed important, the fresh or recently collected specimen should be taken to the culture laboratory to insure its isolation and preservation in pure form. The descriptive data at hand should then be checked and supplemented from the pure culture.

To identify an unknown Aspergillus, the worker needs pertinent data which will permit him to interpret his mold in terms of species already de- 
scribed-including the observations essential in a species characterization. For convenience such data may be indicated vertically upon a descriptive sheet which is elaborate enough to include observations that are regarded as useful. Measurements should be presented as ranges encountered in the examination of many units, not as exact and single measurements of individual cells or structures.

$\begin{array}{lc}\text { Aspergillus-identifying number or marks. } & \text { Primary sterigmata } \\ \text { Culture medium or natural substratum } & \text { measurements } \\ \text { Temperature of incubation } & \text { arrangement } \\ \text { Colony characters } & \text { color } \\ \text { Rate of growth } & \text { Secondary sterigmata } \\ \text { Texture } & \text { measurements } \\ \text { Mycelium } & \text { Conidia } \\ \text { submerged } & \text { color } \\ \text { floccose } & \text { measurements } \\ \text { color: above } \quad \text { reverse } & \text { markings } \\ \text { Heads } & \text { Perithecia } \\ \text { color } & \text { color } \\ \text { form } & \text { size } \\ \text { measurements } & \text { shape } \\ \text { Conidiophore } & \text { Ascospore } \\ \text { length } & \text { color } \\ \text { diamcter } & \text { size } \\ \text { wall: thickness } & \text { markings } \\ \text { markings } & \text { Sclerotia } \\ \text { color } & \text { color } \\ \text { Vesicle } & \text { size } \\ \text { shape } & \\ \text { size } & \\ \text { color } & \end{array}$

With such a descriptive sheet before him, the user of the manual finds that the Aspergilli have been arranged into a series of natural groups (fig. 20 ), each containing one to several species aggregates. Each group includes species with varieties, and at times mutants, having a series of essential characters in common. These groups have been arranged as nearly as possible in natural order, based upon the presence or absence of certain contrasting intergroup characters.

These major separating characters are usually evident and positive. Nevertheless, individual species are found in which certain of these characters are reduced to vestigial or apparently suppressed, yet which show so many characters allying them with a particular group that such placement is more logical than any other. Such species must sometimes be arbitrarily placed, and their possible affiliation with other groups indicated both in the discussion of the species and in the discussion of the related group. 


\section{Species}

The species concept in Aspergillus is very difficult to define in tangible terms. In this manual, the species names already in use have been preserved wherever possible. The actual material originally described under a particular species name (i.e., type material) exists for but a few species. If such material exists, it is more important as fixing one point, one individual strain in a series of intimately related variants, than tying the name to extremely definite morphology. If such material is not known, comparison of large numbers of strains in pure culture with authoritative descriptive information, supplemented by laboratory usages coming down from the original describer, usually fixes the series or form intended.

From such composite sources it is commonly possible to establish a fairly concrete morphological aspect based upon ranges of color, differences in structure, and variations in spore measurement which are repeated in great numbers of isolates. In such series of isolates, there are no sharp lines of demarcation when large numbers of strains are brought together. Within our concept, a single strain may show much of this variability within its colonies in culture, or it may reduce or suppress certain characteristics and intensify others. Such variants have often been given species rank by workers unaware of the existence of other variants completely bridging the gap between such forms and other members of the series. Great differences in biochemical activity may be shown by different strains with or without contrasting morphology. Nomenclature based upon an assumed correlation of a particular cultural aspect with industrial significance has been offered but has proved utterly unreliable in identifying an organism if lost, or in seeking a new strain to serve the same purpose.

Two contrasting tendencies in classification are always encountered. In the Aspergilli these may be represented by Mosseray (1934a) who found diagnostic marks to distinguish 35 species among 63 cultures of black Aspergilli in the collection of Biourge at Louvain. He later received many more variants and faced the question whether to try to describe them all or abandon the field. He admitted inability to write descriptions explicit enough to identify them all. In contrast, Neill (1939), disregarding ascospore measurements and markings, "lumped" all of the A. glaucus group into A. glaucus Link. Likewise, all of the black Aspergilli were considered as $A$. niger van Tieghem. Forms that he did not happen to recognize as belonging to one of his groups were discarded. These are extremes.

With abundant living material before him, the student of the Aspergilli can usually recognize as representative a reasonable number of forms which can be described in tangible specific terms. Commonly, forms which actually play a significant role in nature or in biochemical processes can be selected as the points around which such species descriptions are drawn. 
On the other hand, forms such as $A$.janus, $A$. itaconicus, A. lutescens, etc., while probably rare in nature, possess sufficiently distinctive morphology to warrant species reeognition irrespective of other considerations.

\section{Varieties}

The taxonomic term, variety, is used here to designate any homogeneous member of a speeies complex which carries most of the diagnostie characters of the speeies but maintains one or more clearly defined differences in particular characters. For example, variety alba is used for certain strains of particular species in which the eharacteristic color of that species is absent.

There is little agreement in the literature in the applieation of the term, variety; certain authors use the term to indicate their belief that one form with particular morphologieal eharacters had its origin from another. In such cases, the belief is hypothetical, not a matter of observation. Sometimes previously known species were merely moved to varietal standing without speeifying the eharacters upon which the decision was based. Such ehanges are reduced to synonymy or, if entirely unsupported, are oceasionally ignored in this manual. The term variety is only useful if definitely associated with a clearly defined variation in structures within an otherwise homogeneous series of strains.

\section{Mutations, or Mutants}

The term mutation, or mutant, is only recognized here for forms resulting from a sharp break in morphology (including eolor) from known struetures characteristic of a species, to a definitely altered and inherited contrasting structure. Obviously the only exeuse for the term in taxonomie usage is to designate the origin of the form studied. If the source of such a variant were unknown, the taxonomist would designate the form present as a variety or species, depending upon the nature and importance of the changes encountered. The inereasing number of studies in experimental evolution make reeognition of induced variation taxonomically necessary.

\section{New Species}

The discriminating collector will oceasionally find an organism markedly divergent in eharacters from any described form. Usually these divergences leave the organism readily recognized as a member of one of the great groups. If the differences in aspect and detail of structure separate such a form from the other deseribed members of the group, and if the form is found often enough to prove that it has a place in nature, description as a new species is warranted. Similarly, an occasional form, either by sup- 
pression or complete disappearance, loses the arbitrary diagnostic character which furnishes the basis for separating two adjacent groups. In such cases it has at times seemed more practical to add such a species to the group most nearly allied to it by general colony aspect, but to cross-reference it to the related group.

The detection and description of species hitherto unrecognized necessitates extensive review of the literature and restudy of available living cultures of at least one whole section of the great genus Aspergillus. Unless the one who encounters a form that he cannot recognize under names already in the literature is prepared to investigate his form adequately in relation to the whole genus, he should not describe his organism as a new species.

\section{Summarized}

Comparative study of the taxonomic literature brings out the need for a standardized series of morphological and descriptive terms into which the many usages introduced in the two centuries since Micheli can be translated. Variations in measurement are cleemed significant only if they exceed the common limits between closely related organisms and predominate in the preparations examined. Ranges in measurements are more significant than exact dimensions of either selected structures or areraged ralues based upon many measurements.

Merely quantitative variations are not recognized as warranting separation of species. For example, differences in the shade of color, or the intensity of a particular reaction, especially when other strains are found to fall between the "old" and proposed "new" species, are not regarded as species characters. Such proposed names either fall to varietal status or to synonymy.

The names not accepted here fall into several categories. (1) Many are listed as synonyms because they are believed to have been given to variants not recognizable by dependable and interpretable differences from other members of the same series. (2) Fantastic variants, or "monsters", appearing in culture may, like "Cladosarum", be maintained in the laboratory, but unless found perpetuating themselves in nature, clearly fall in the class of "natures experiments" which do not contribute to the permanent flora. Such names are not regarded as established. (3) Unidentifiable species-names appearing in the literature based upon structures or reactions regarded by the describer as unique, but whose identity is so completely lost in large collections among series of closely related strains as to make them unidentifiable by description-are listed in the check list without characterization. 


\section{SPECIES KEYS}

No general or comprehensive key to the species of Aspergillus is presented. Instead, a series of comparatively simple species keys are included in the discussions of the several groups. The recommended procedure in identifying an Aspergillus with the aid of this manual is first to determine its group relationship by means of one or more of the group keys presented below, then assign the culture more precisely to species by means of the intra-group species key for the particular group to which the form belongs.

\section{GROUP KEYS}

Three keys to the groups of Aspergilli are offered: The first is presented in the form of a diagram and presents the different groups in what we consider their natural order. Presentation of this key in graphic form, it is believed, will materially assist the user in grasping the various characters which ally and interrelate the different groups. Primary separation is based upon the number of series of sterigmata, whether single or double. Secondary separation is based upon the character of the conidiophore, whether rough or smooth. Tertiary separations are based upon the presence or absence of perithecia, hülle cells, and sclerotia, and upon the color of the conidiophore wall.

In assigning species to groups by means of this key, the transitional or intermediate character of certain species becomes strikingly apparent. Frr example, Aspergillus caespitosus possesses the brown conidiophore and conidial coloration of $A$. nidulans, furthermore, it produces clusters of irregular, thick-walled hülle cells; but the head is radiate, or only loosely columnar, and no perithecia or ascospores are produced. It is placed in the $A$. nidulans group with full recognition that it possesses certain characters which relate it to A. ustus. Aspergillus alliaceus is another form with intermediate characters. The conidiophore is uncolored and smooth when examined in liquid mounts (appearing finely roughened when examined dry), and the sclerotia are black, but the heads are essentially ochraceous in color. It is placed in the $A$. wentii group but shows unmistakable relationship to A. ochraceus. Aspergillus sparsus is a species of uncertain relationship. It possesses a conspicuously roughened, yellow conidiophore and globose head, and is placed in the $A$. ochraceus group; but the conidial heads show a greenish color which is not found in any other known member of this group, while the character of the conidiophore will not warrant placement elsewhere. The so-called "bronze series" in the A. tamarii group is transitional in the direction of $A$. flavus. Colonies are conspicuously green when young and retain a greenish tint for a considerable period, in contrast to $A$. tamarii which never shows true green and appears greenish only transiently when young. Such a list of intermediate species and forms 


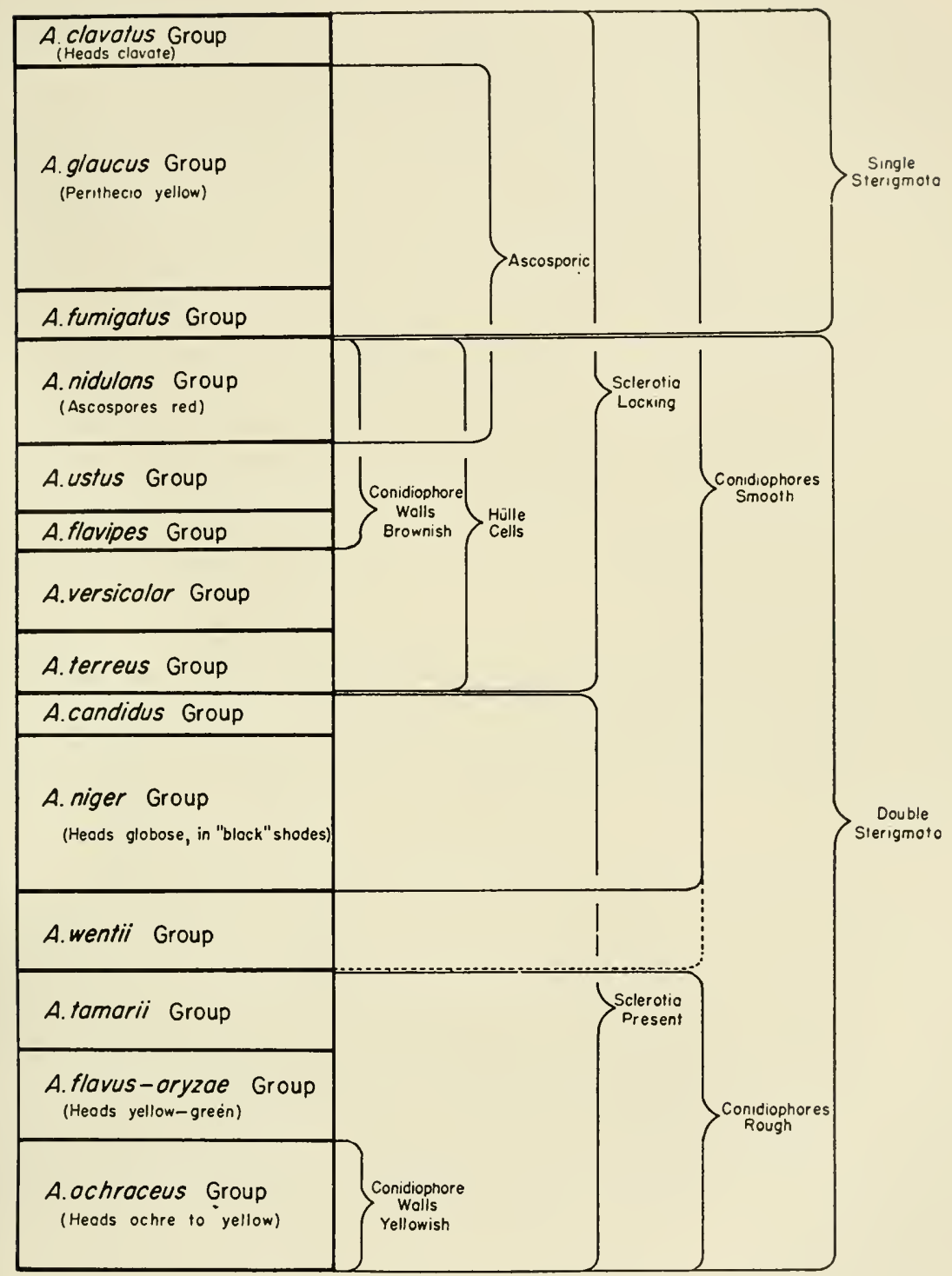

FIG. 20. Graphic representation of natural relationships among groups comprising the genus Aspergillus. The abundance of species in the different groups is roughly indicated by the size of the group boxes in the figure.

could be extended, but sufficient have been cited to indicate that the various groups, like the species which comprise them, often cannot be set apart by sharp lines of demarcation. 
In using this manual, and in studying the Aspergilli generally, it is important that the worker should realize that these organisms vary within the species, the species vary within the group, and to a lesser extent, the groups themselves vary within the genus. In other words, nature did not realize that we were going to write this manual when the various species and groups were being developed, hence not all of the forms one encounters will fit into the various compartments which have been constructed, although these are, on the whole, comparatively elastic. This can be illustrated in another way. If we completely disregard color, the genus Aspergillus, as depicted in figure 20, can be likened to the spectrum. There is a green region and a yellow region in the spectrum and the two regions are, on the whole, distinct. Furthermore, within each of these, certain fixed and definite lines can be identified. It is, however, extremely difficult, if not impossible, to say where the green region ceases and the yellow begins. So it is with the species and groups of the Aspergilli. There is a definite nidulans group and a definite ustus group, but the line separating the two is extremely tenuous.

We do not, in any sense, infer that the Aspergilli cannot be classifiedthat they cannot be separated into groups, species, and even varieties. This manual is evidence that they can. But we do wish to emphasize that we are dealing with living and variable organisms, and that in describing them, we should be as explicit as possible and still keep our concepts reasonably elastic.

\section{Key to Groups-Based Primarily Upon Color}

The second key is based primarily upon color and is entirely artificial in its construction. The various groups are separated by contrasting coloration, and closely related groups may appear widely separated in the key. In practice, such a key is very useful since color is the most obrious character of an Aspergillus, and since the species comprising a particular group, with but few exceptions, are characterized by variations in shade of color rather than differences in basic coloration. Presumptive assignment of the Aspergilli to groups can usually be made from this type of key which is based primarily on color supplemented by the use of a handlens or dissecting microscope.

A. Conidial heads in definitely green, blue-green, or yellowgreen shades in young fruiting colonies.......... B.

AA. Conidial heads lacking green colors (Greenish in excep-

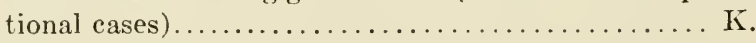

B. Conidial heads in green and blue-green shades....... C.

BB. Conidial heads in yellow-green shades............. flavus group 
C. Conidial stalks and heads coarse-heads clavate

A. clavatus group

CC. Heads not clavate.

D. Colonies mostly showing yellow perithecia and more or

less yellow and red hyphae.

A. glaucus group

DD. Colonies lacking yellow perithecia and more or less yel-

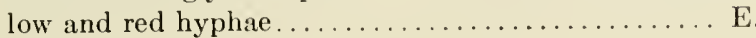

E. Colonies producing columnar spore masses......... F.

EE. Colonies producing radiate, globose, or hemispherical heads.

H.

F. Rapidly growing and spreading colonies.

FF. Slowly and restrictedly growing colonies

A. restrictus series

G. Conidial columns long, narrow

A. fumigatus group

GG. Conidial columns short and broad; perithecia usually

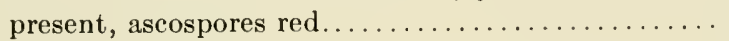

A. nidulans group

H. Heads radiate in blue-green, dull green, to pale tan or flesh-colored shades.

A. versicolor group

HH. Heads in some other color.

K. Heads in long compact columns, avellaneous to cinnamon, shading toward colorless through light flesh

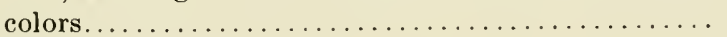

A. terreus group

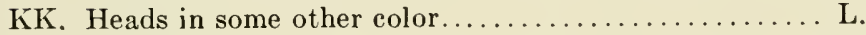

L. Colonies more or less floccose; heads in dull olive-grays

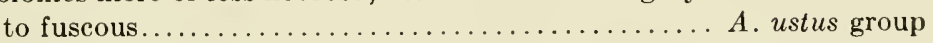

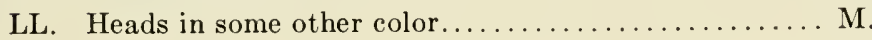

M. Young heads white or only slightly tinged in age..... N.

MM. Heads in some other color...................

N. Young heads white, usually in short columns, broadening at apex, of ten becoming avellaneous in age.... A. flavipes group

NN. Heads persistently white, larger heads definitely globose

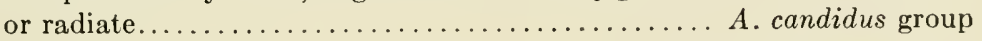

O. Heads in sulphur yellow to ochre shades............ ochraceus group

OO. Heads in some other color.................

P. Young colonies showing a greenish color passing into

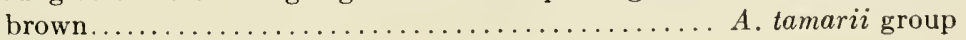

PP. Heads not showing greenish...............

Q. Heads in purple-brown to black shades............. A. niger group

QQ. Heads in yellowish-brown shades, orange to deep brown to umber color.

A. wentii group 


\section{Key to Groups-Based Primarily Upon Morphology}

The third key is based primarily upon morphology, with colony color employed as an accessory differentiating character. This key is also artificial in construction and is designed to separate the various groups by the simplest and most direct means possible. Groups naturally related may or may not appear in their proper sequence. With the data developed upon the descriptive sheet (p. 82), most of the Aspergilli can be traced to their proper placement in classification schemes by using the following key. Natural arrangement is disregarded and the same group is occasionally reached in different places in the key.

A. Species producing perithecia and ascospores......... B.

AA. Species not producing perithecia and ascospores...... D.

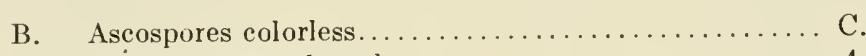

BB. Ascospores purple-red....................... nidulans group

C. Perithecia white to flesh color, enmeshed in a loose network of colorless hyphae................... fischeri

CC. Perithecia yellow to orange, naked, vegetative hyphae often showing red to orange granules.............. glaucus group

D. Conidial heads cylindrical-clavate; vesicles definitely

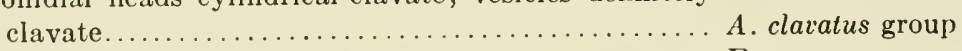

DD. Conidial heads not cylindrical-clavate........... E.

E. Colonies showing green or greenish color at some stages

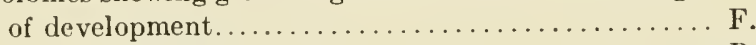

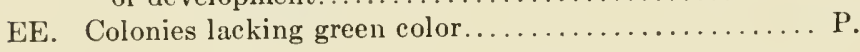

F. Conidiophore wall rough or pitted..............

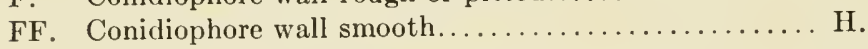

G. Colonies green or yellow-green to yellowish........ A. flavus-oryzae group

GG. Colonies greenish-brown when young, becoming rich brown or umber in age .................... A tamarii group

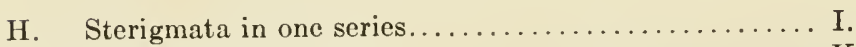

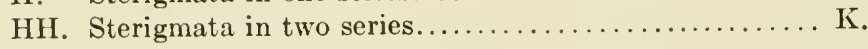

I. Conidia elliptical to pyriform...............

II. Conidia spinulose, 2.5 to $4 \mu$, globose; chains in compact columns.......................... fumigatus group

J. Colonies mostly showing yellow perithecia; sterigmata usually coarse... ...................... glaucus group

JJ. Colonies lacking perithecia; conidia in narrow columns. A. restrictus group 
K. Conidiophores in yellow-brown shades........... L.

KK. Conidiophores not colored................

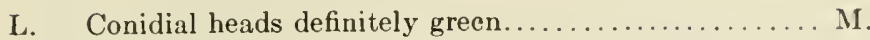

LL. Conidial heads greenish only when young, then in yel-

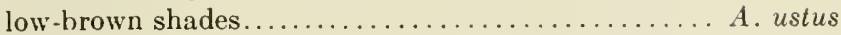

M. Ascospores produced-purple-red in color........... A. nidulans group

MM. Ascospores not produced................. $\mathrm{N}$.

N. Colonies showing irregularly clustered hülle cells......A. caespitosus

NN. Colonies not showing hülle cells: bright green, spreading $A$. unguis

O. Conidial area in dull green shades (sometimes partially or completely replaced by $\tan ) \ldots \ldots \ldots \ldots \ldots \ldots$ A. versicolor

OO. Conidial area in blue-greens..................... sydowi

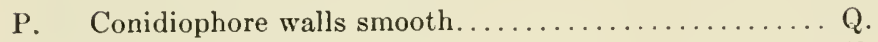

PP. Conidiophore walls rough................ $\mathrm{V}$.

Q. Conidiophore walls pale yellow in outer layer; heads white when young of ten becoming avellaneous in age. A. flavipes

QQ. Conidiophore walls colorless, or partially yellow-brown

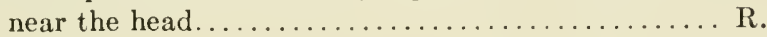

R. Conidial chains in solid columns, compact at base.... S.

RR. Conidial chains radiate at least in the larger and typical

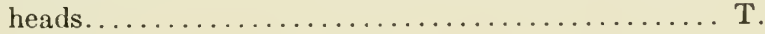

S. Conidial heads in avellaneous shades............... A. terreus

SS. Conidial heads flesh color (in pinkish shades)........ A. carneus

T. Heads white or tardily in yellowish shades............ candidus group

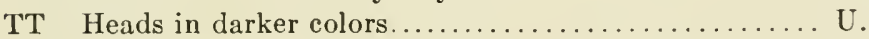

U. Conidial heads globose in purple-brown to black, (rarely

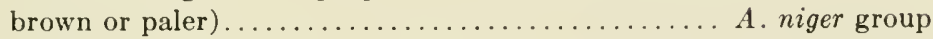

UU. Conidial heads globose in yellowish, yellow-brown, and dark brown shades...................... wentii group

V. Conidiophore walls rough, yellow; heads yellow to

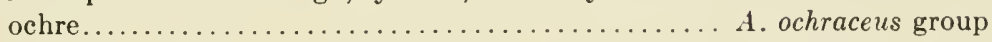




\section{Chapter ViII \\ THE ASPERGILLUS CLAVATUS GROUP}

\section{Outslanding Characters}

Conidial heads clavate ${ }^{1}$, large, pale blue-green.

Conidiophores generally coarse, smooth-walled, uncolored.

Sterigmata in one series.

Conidia elliptical, smooth, comparatively thick-walled.

\section{Group Key}

Conidial structures not exceeding $4.0 \mathrm{~mm}$. in length.

Aspergillus clavatus Desm.

Conidial structures often 1 to 5 or more $\mathrm{cm}$. in length.

Aspergillus giganteus Wehmer.

Aspergillus claratus Desmazieres, in Ann. Sci. Nat. Bot. (2) 2: 71, p. 2, fig. 4. 1834 .

Colonies upon Czapek's solution agar growing rapidly at $20-24^{\circ} \mathrm{C}$., plane or slightly furrowed, in certain strains tending to become floccose but generally characterized by a surface mycelial mat and abundant erect conidiophores up to $3.0 \mathrm{~mm}$. in length, bearing large, blue-green, clavate conidial heads evenly distributed or arranged in more or less well defined zones (Pl. III, A and Fig. $21 \mathrm{~A}$ ); reverse generally uncolored, but becoming browned in age in some strains; odor strongly foetid in some strains, not pronounced in others. Conidial heads clavate, large, commonly ranging from 300 to $400 \mu$ by 150 to $200 \mu$, in age splitting into 2,3 , or more divergent columns of compacted conidial chains (Fig. 21 B), approximately slateolive in color (Ridgway, Pl. XLVII). Conidiophores $1.5-3.0 \mathrm{~mm}$. in length, 20 to $30 \mu$ in diameter, comparatively thin-walled, smooth, colorless, gradually enlarging at the apex into a clavate vesicle which is fertile over an area up to 200 to $250 \mu$ in length and 40 to $60 \mu$ or more wide (Fig. $21 \mathrm{C}$ ). Sterigmata in a single series, varying in size from 2.5 to $3.5 \mu$ by 2.0 to $3.0 \mu$ at the base of the vesicle to 7.0 or 8.0 and occasionally $10 \mu$ by 2.5 to $3.0 \mu$ at its apex (Fig. 21 D). Conidia elliptical, comparatively

1 Aspergillus janus Raper and Thom (see page 187) is characterized by a smaller clavate vesicle in one of its conidial phases. It is hardly to be confused with the clavatus group, however, because of the whiteness of its conidial masses, the double series of sterigmata, and the intermixture of more or less abundant green $A$. sydowilike heads in cultures at room temperature. 
heavy walled, smooth, 3.0 to $4.0 \mu$ by 2.0 to $3.0 \mu$, ocasionally larger in some strains and irregular in others.
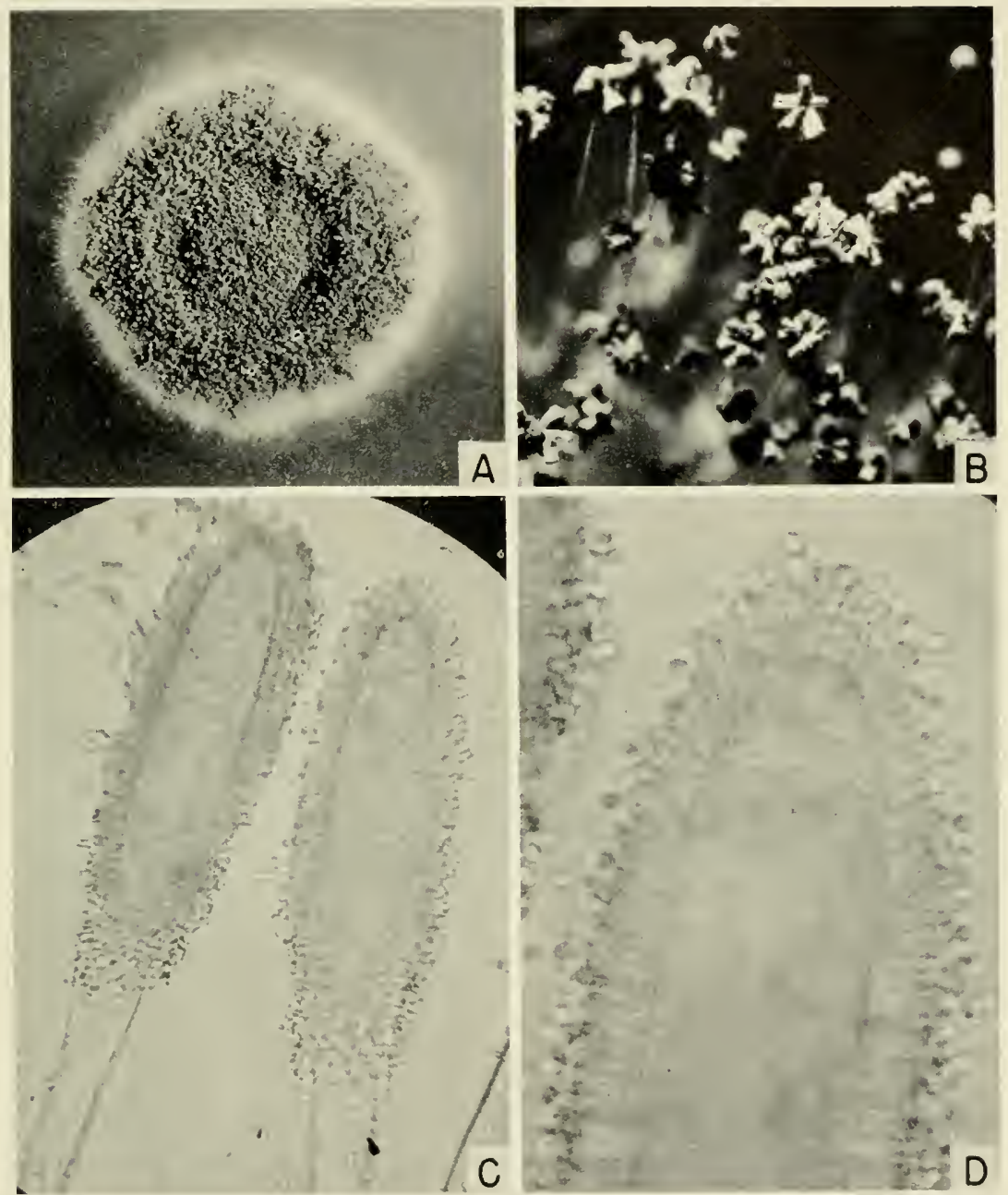

Fig. 21. Aspergillus elavatus Desm. A, Colony growing on Czapek's solution agar, 10 days, room temperature, $\times 1.3$ (Thom No. 5169). $B$, Conidial heads showing characteristic splitting in age, $\times 12$ (Strain NRRL No. 1823). $C$, Conidial heads showing characteristic clavate form of vesicle, $\times 180$ (Strain NRRL No. 6). $D$, Portion of a conidial head further enlarged, showing closely packed single series of sterigmata, $\times 600$ (Strain NRRL No. 6).

Cosmopolitan in distribution and especially common in soil, decaying vegetation, dung and other materials where active decomposition of nitrogenous materials is taking place. Cultures examined include numerous 
strains from various parts of the United States, together with isolations from China, British Guiana, Cuba, Panama and European sources.

The species description as presented is based upon a large number of closely related strains that have been examined, and is adequately represented by such specific strains as NRRL Nos. 2, 5, 8, and others.

Upon malt extract agar, details of morphology and colony characteristics may or may not conform with those listed above. For example, in such typical strains as Nos. 2, 5, and 8, conidial structures are generally more abundant upon malt than Czapek's agar and may average as much as 20 to 25 percent larger in size. In other strains such as Nos. 4 and 6 , a markedly different response is noted upon this medium. Conidial heads, although
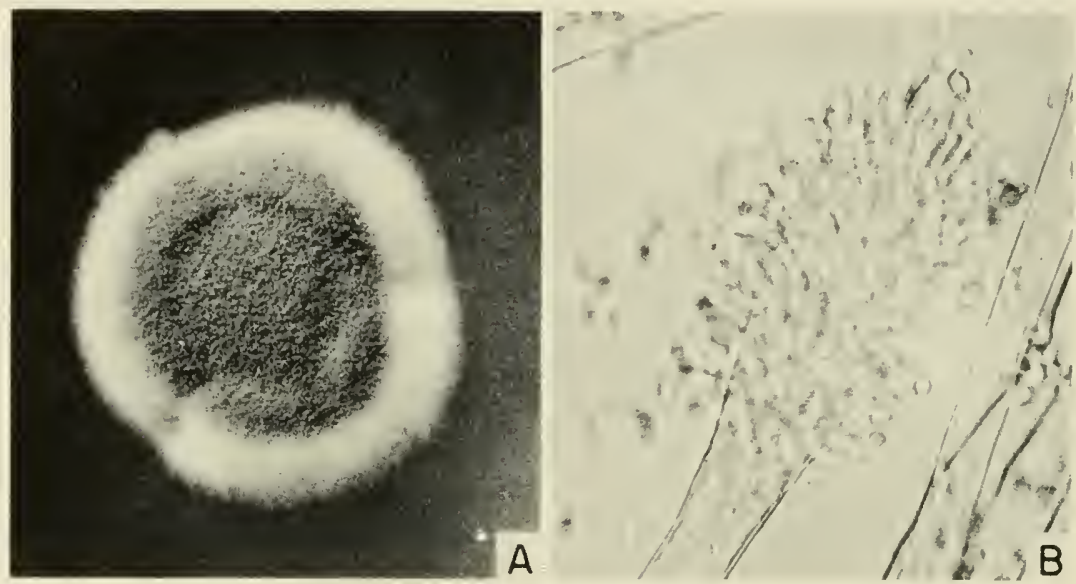

FIg. 22. Aspergillus clavatus Desm. (Strain NRRL No. 1: Thom No. 107). A, Colony growing upon Czapek's solution agar, 10 days, room temperature, $\times 1.3$. $B$, Conidial heads diminutive and somewhat atypical but showing characteristic clavate form, $\times 600$.

greatly increased in number, are much reduced in size, with conidiophores generally $1 \mathrm{~mm}$. or less in length bearing heads only 100 to $125 \mu$ long and proportionately reduced in diameter. In these latter forms, conidia are somewhat irregular in form and generally larger than in the more typical strains first considered.

Culture NRRL No. 1 (Thom No. 107) differs markedly from the species description in producing deeply floccose colonies (Fig. $22 \mathrm{~A}$ ) and comparatively few spore heads which are extremely variable in size. These range from very small fruiting structures (Fig. $22 \mathrm{~B}$ ) borne as branches upon aerial hyphae to structures arising from the substratum which are characterized by dimensions almost typical of the species. This culture is more nearly normal upon malt than upon Czapek's solution agar but is unique 
among the strains examined by us and must be considered somewhat atypical. It deserves particular attention because it was obtained from the Centraalbureau in Baarn in 1908 as Aspergillus clavatus Desm. and has undoubtedly been widely distributed by that organization-in fact, it is quite probable that it has been examined by more investigators than any other strain belonging to this group. We refrain from using it as a basis for the species description, however, despite its classic history, since the less floccose and more heavily sporing strains are so much more commonly encountered in nature.

Members of this species produce a strong alkaline reaction upon many culture media and this is usually associated with a strong foetid odor. For example, when grown upon Czapek's solution agar containing only $\mathrm{NaNO}_{3}$ as nitrogen and sucrose as a carbon source, the reaction of typical strains may reach pH 9.5 or even higher accompanied by a strong odor of trimethylamine almost approaching putridity. No other species of Aspergillus is known to react in this manner although some strains of A. flavipes give some suggestion of it. The ability to produce, and more particularly to withstand strong alkaline conditions undoubtedly accounts for the common occurrence of this species upon dung and other nitrogen rich substrata undergoing decomposition.

Aspergillus giganteus Wehmer, in Central. f. Bakt., etc., 2, Abt. 18, No. 13/15: 385 . 1907 .

Colonies upon Czapek's solution agar growing rapidly at $20^{\circ} \mathrm{C}$., characterized by an extensive surface and submerged vegetative mycelium and an early development of abundant conidiophores 2.0 to $4.0 \mathrm{~mm}$. high, followed by the subsequent development of less numerous conidiophores ranging up to several centimeters in length (Pl. III, B and Fig. $23 \mathrm{~A}$ ), the latter strongly phototropic and generally more abundant in marginal areas, commonly obscuring the more central mass of short conidiophores; colonies at first white, becoming pale blue-green as conidial heads mature; reverse dull tan, becoming brown in age; odor none to somewhat foetid in certain strains. Conidial structures varying greatly in dimensions and falling for the most part into two general size ranges: (1) conidiophores commonly 2 to $3 \mathrm{~mm}$., rarely exceeding $4 \mathrm{~mm}$. in height, bearing clavate heads 200 to $350 \mu$ in length; (2) conidiophores one to several centimeters in length, bearing heads up to $1 \mathrm{~mm}$. in length. The relative proportions of these head types is strongly influenced by environmental conditions, and specific strain characteristics. Conidial heads pale blue-green, in age splitting into 2 or more columns extending the length of the vesicle (Fig. $23 \mathrm{~B}$ ). Vesicles consisting of the expanded terminus of the conidiophore, ranging from 100 to $250 \mu$ by 30 to $50 \mu$ upon short conidiophores to 400 to $600 \mu$ by 120 to 
$180 \mu$ upon long conidiophores (Fig. $23 \mathrm{C}$ ). Sterigmata in a single series ranging from 3.0 to $4.0 \mu$ by 2.5 to $3.0 \mu$ at the base of the vesicle to 6.0 to
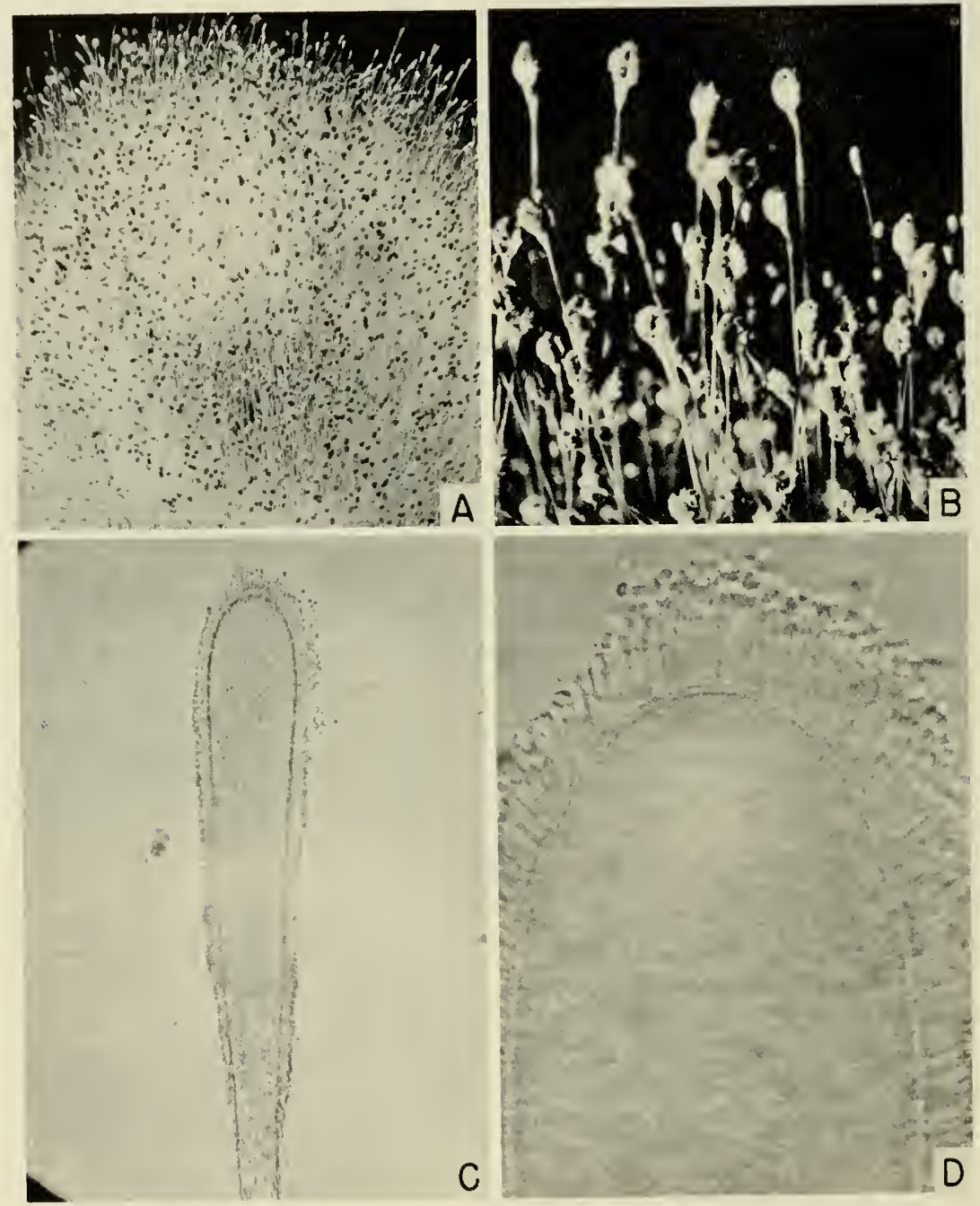

FIG. 23. Aspergillus giganteus Wehmer (Strain NRRL No. 10). A, Portion of colony on Czapek's solution agar showing characteristic long conidiophores and large clavate heads; 10 days, room temperature, $\times 1.3$. $B$, Portion of colony margin somewhat enlarged, $\times 11$. $C$, Single conidial head showing the very elongate vesicle characteristic of the species, $X 130$. $D$, Terminal portion of vesicle showing the closely crowded single series of sterigmata, $\times 600$.

$8.5 \mu$ by 2.8 to $3.5 \mu$ at the apex (Fig. 23 D). Conidia elliptical, thickwalled, smooth, 3.5 to $4.5 \mu$ by 2.4 to $3.0 \mu$. 


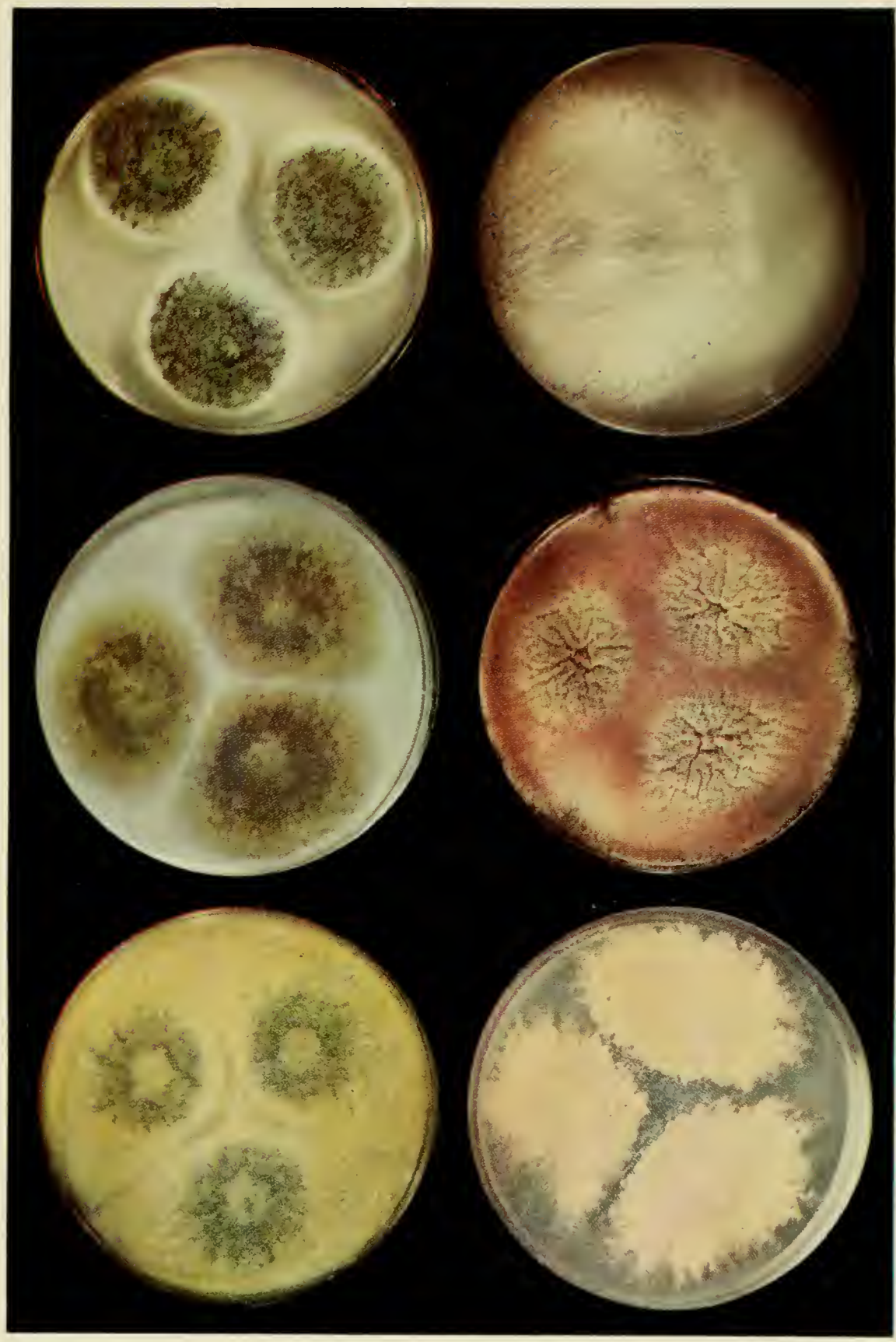

Plate III

A (upper left), As pergillus claratus Desm., NRRL No. s. B (upper right), Aspergillus giganteus Wehmer, NRRL No 10, incubated at $20^{\circ} \mathrm{C}$ in one-sided illumination. C (center left). Asnergillus repens (Cda.)
deBary, NRRL No. 20. D (center right), Aspergillus ruber Bremer, NRRL No. 54 . E (lower left), Aspergillus a nstelodami (Nang.) Thom and Church, NRRL No.90. F (lower right), Aspergillus niceo-glaucus Thom and Raper, NRRL No. 127. Figures $A$ and $B$ growing upon standard Czapek's solution agar with 3 percent sucrose; $C$ to $F$, growing upon Czapeh's solution agar with 20 percent sucrose. (Color photographs by Haines Northern Regional Research Laboratory. Reproduced through co-operation of Chis. Pfizer \& Co., Inc.) 

The above species description is centered upon strain NRRL No. 10 (Thom No. 5581.13A) isolated from Yucatan caves by Prof. F. A. Wolf (1938). Additional strains examined include isolations from Texas, Illinois, Mexico, Puerto Rico, and strain NRRL No. 1725 (Thom No. 138) received from Dr. Westerdijk in 1910 as A. giganteus Wehmer.

In the present treatment we include under the species name $A$. giganteus Wehmer all strains which produce conidiophores in excess of $1 \mathrm{~cm}$. in length. While this may appear somewhat arbitrary, it is done since members of the $A$. clavatus group seem to fall into two natural series: (1) those which never produce conidiophores in excess of 5 to $6 \mathrm{~mm}$. in length irrespective of environmental conditions or medium composition, and (2) those which regularly produce few to many very long-stalked fruiting structures under the usual conditions of laboratory cultivation and examination. To the first of these series is applied the species designation $A$. clavatus, to the second, A. giganteus.

Strains of $A$. giganteus, like those of $A$. clavatus differ materially in their growth and cultural appearances upon different culture media. More striking, however, is their response to light and temperature. This has been observed by Wehmer (1907), Wolf (1938) and others, and has been studied somewhat exhaustively by Webb (1942). Using the Wolf isolate, Webb found that the production of long conidiophores was favored by cultivation upon media containing from 1 to 10 percent sucrose and incubation at $20^{\circ} \mathrm{C}$. in the presence of light or darkness, whereas heavy conidial production and the development of short conidiophores were favored by incubation at $30^{\circ} \mathrm{C}$. in darkness. Different strains vary materially in their cultural appearance upon such standard media as Czapek's solution agar and can be roughly grouped into three sub-series as follows: (1) wholly typical strains consistently producing the cultural picture as defined for the species, (2) strains producing an unusually heavy crop of short-stalked conidial structures in colony centers, followed by the production in marginal areas only of long-stalked fruits typical of A. giganteus, and (3) rather sparsely growing strains in which there is a general admixture of short-stalked and scattered long-stalked fruits ranging up to 2 to $3 \mathrm{~cm}$. in length. The latter group is considered as possibly representing atypical and somewhat depauperate strains of the first. The second seems to constitute a consistent and fairly well-defined cultural entity, but does not differ from typical forms sufficiently to warrant separation as a variety.

The validity of the species $A$. giganteus has been questioned by some authors. Blochwitz (1929) regarded it as a mutation of $A$. clavatus and so designated it in his monograph of the Aspergilli. His view may be correct. It is our belief, however, that the species should be retained since forms producing the giant conidiophores noted by Wehmer are repeatedly 
isolated from nature, and since no evidence has been presented indicating that these larger forms arise directly from the smaller and more common forms. While it is true that conditions can be altered so that $A$. giganteus cultures suggest $A$. clavatus, no one has yet demonstrated that the reverse can be accomplished.

\section{Group Synonyms}

The following names have been proposed for specimens belonging to this group but without adequate data to warrant recognition as valid species:

A. clavellus Peck, in N. Y. State Mus. Nat. Hist. Rept. 34: 49, Pl. 2, figs. 1-5. 1881. Described from cooked squash in New York State. No data is presented which would warrant separation of this form from A. clavatus Desm.

A. westendorpii Sacc. and March, in Rev. Mycologique 7: 149, 1885, was listed from cow dung. Correctly assigned to A. clavatus by Lindau, in Deutsch. Krypt. Fl. Pilze 8: 152.1907.

A. fusco-cinereus Ellis and Morgan was the name attached to Morgan's jacket No. 674 , showing a very small, clavate aspergillus which has not been collected again, hence never cultivated. It was probably some member of this group.

A. pseudo-clavatus Purjewitch, in Schrift. Naturforsch. Gesell. Kiev 16 (2): 309, pl. 12, 1900; see also Sacc. Syll. 16: 1028. The organism in culture was reported as having both primary and secondary sterigmata in a small-sized "clavatus" type of head, and perithecia with ascospores which were not adequately described. Until somebody finds this organism again and reports its cultivation and more complete description, it will remain doubtful. (See Thom and Church, The Aspergilli, p. 100. 1926.)

\section{Occurrence and Economic Importance}

Members of the $A$. clavatus group are quite common in soils and decomposing materials characterized by a comparatively high nitrogen content. They appear to be common upon the dung of various animals and in the writers' experience have been isolated repeatedly from that of chickens. While the subject has not been adequately investigated, it is probable that the ability of members of this group to withstand strongly alkaline conditions enables them to operate successfully as agents of decomposition in situations where almost all other fungi are eliminated.

\section{Antibiosis}

Certain strains of $A$. clavatus produce substances in the substratum which are capable of destroying Staphylococcus and other microorganisms. Such activity was first reported by Weisner in March 1942 (Nature 149: p. 356) and subsequently by Waksman, Horning, and Spencer in August of the same year (Science 96: p. 202). To the active substance the latter investigators assigned the name clavacin and noted that it appeared similar to, if not identical with, that studied by Weisner (1942) to which the designation 
clavatin has since been applied. Additional studies by Waksman and his co-workers have further defined its action and enlarged the list of bacterial species inhibited (1942b and 1943). Hooper et al (1944) have demonstrated the identity of clavacin and patulin, a bactericidal substance obtained from Penicillium patulum by Raistrick and associates (1943) and reported to be of value in the treatment of the common cold.

Philpot (1943) reported the production of a penicillin-like substance by a strain of Aspergillus giganteus. In earlier tests by Wilkins and Harris (1942) this strain had been found to produce a substance active against Staphylococci. 


\section{Chapter IX}

\section{THE ASPERGILLUS GLAUCUS GROUP ${ }^{1}$}

\section{Outstanding Characters}

Perithecia generally present; yellow, globose to subglobose, thin-walled, suspended in networks of red or yellow hyphae.

Asci 8-spored, without definite arrangement; usually ripening in 2 to 4 weeks.

Ascospores lenticular, smooth or rough-walled, generally showing an equatorial line or furrow with or without flanking ridges or crests.

Conidial heads more or less abundant, radiate to somewhat columnar, typically in some shade of green.

Conidiophores smooth-walled, terminating in dome-like vesicles.

Sterigmata in one series, rather coarse.

Conidia elliptical to subglobose, uniformly and characteristically roughened.

\section{General Considerations}

Aerial hyphae encrusted with yellow, orange, or red granules are abundant in perithecial areas of most of the strains of the group. Both laboratory cultures and naturally moldy specimens frequently show this as their most conspicuous character, one which is readily recognized with the hand lens. In nature, molds of this group appear as patches of green, yellow, reddish, or reddish-yellow mold, depending upon the relative abundance of conidial heads, perithecia, and encrusted aerial hyphae, and especially influenced by the composition of the substratum.

Representatives of the $A$. glaucus group are universally distributed in nature and are significant in the incipient spoilage of many organic materials useful to man. They occur particularly upon products characterized by a high osmotic tension such as preserves, jams, cured meats, leather goods, improperly dried hay, moist grain, and soft woods stored under humid conditions. The classic habitat is improperly dried herbarium specimens.

The earliest references to any Aspergilli concern representatives of this group, for botanists early encountered them upon herbarium material. Micheli in 1729 used the generic name Aspergillus (rough head) for the conidial heads, characterized by divergent chains of spores, commonly present upon such specimens. Later in the century, Wiggers (1780) pro-

1 Abridged from: Charles Thom and Kenneth B. Raper, The Aspergillus Glaucus Group, U. S. Dept. of Agr., Misc. Pub. No. 426, Washington, D. C., September 1941. 
posed the name Mucor herbariorum for the yellow perithecia found mixed with the Aspergillus heads, which he regarded as a different mold. In 1809, Link designated the green heads Aspergillus glaucus and the yellow perithecia Eurotium herbariorum. Half a century later, DeBary (1854) proved that the Aspergillus heads and Eurotium perithecia were borne upon the same mycelium, hence were one fungus. Although it could be maintained that the name Eurotium (designating the perfect stage) should take precedence over Aspergillus (descriptive of the conidial apparatus), most recent authors have tended to go back to Micheli and use the name Aspergillus for the whole group because of the obvious relationship of many conidial forms for which no perithecia are known.

\section{Laboratory Cultivation}

The pattern and size of the ascospore, when present, is especially significant in describing species of the Aspergillus glaucus group. Nevertheless, the conidial apparatus and the vegetative mycelium of particular subgroups are so important that pure culture under known conditions is always desirable. The character of the colony, as well as the amount of growth, is strongly influenced by the culture medium, and it is only upon substrata characterized by a high osmotic tension that typical perithecia and conidial heads are produced. It should be noted, however, that characteristic heads and perithecia normally develop, although few in number, in situations where less concentrated media dry out rapidly, as at the edge of an agar slant. Colony comparisons for correct identification can best be made in Petri-dish cultures in which direct observation with the compound microscope is feasible. Incubation at $22^{\circ}$ to $25^{\circ} \mathrm{C}$. will permit the development of satisfactory colonies for descriptive study, although the optimum for certain species is above or below this range as will be noted in connection with these descriptions and in the general discussion on the influence of temperature on colony growth and development in the genus (p. 45). Colony descriptions are based upon 3-week old cultures, except as otherwise stated. For comparative culture the authors have followed Dale (1909) in using substrata containing high concentrations of sugar. The following formula is recommended:

Czapek's solution agar with 20 percent of sucrose

Sodium nitrate.

$3 \mathrm{gm}$.

Dibasic potassium phosphate..................... $1 \mathrm{gm}$.

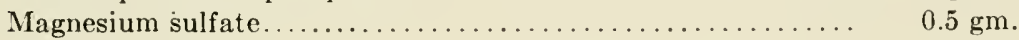

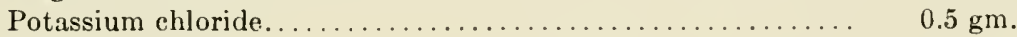

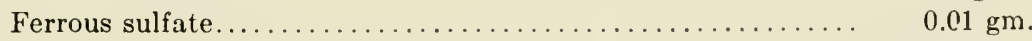

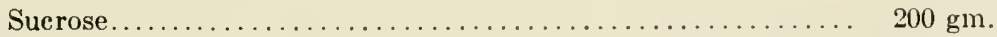

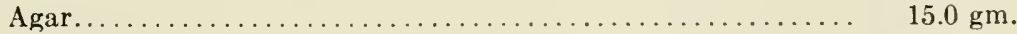

Dist. water........................ 


\section{Group Limits and Relationships}

Exceptional strains lacking perithecia but presenting conidial morphology clearly belonging to the $A$. glaucus group in its strictest sense are occasionally found. In addition, the $A$. restrictus series ( $A$. penicilloides series of George Smith, 1931) shows conidial morphology clearly related to the group, but differing markedly from the usual types in colony coloration and in the absence of perithecia. Thom and Church (1926) considered these latter types as representing intermediate forms between the Aspergillus glaucus and $A$. fumigatus groups. In the present treatment, we include them as non-ascosporic and for the most part diminutive forms sufficiently related to the ascosporic species to be included with them in the $A$.glaucus group.

Certain other groups of the Aspergilli present characters suggesting those of the "glaucus" group considered here. The ascospores of A. fischeri Wehmer (See A. fumigatus group) and of the A. nidulans group (which see; also Thom and Raper, 1939) in general resemble those of the $\boldsymbol{A}$. glaucus group, but the yellow perithecia suspended by yellow and red encrusted hyphae do not occur outside of this group.

Group Key (Based Primarily Upon Perithecia and Ascospores)

I. Perithecia present.

A. Ascospores lenticular, $6 \mu$ or less in long axis.

1. Ascospores with convex faces smooth (or nearly so).

a. Equatorial ridges lacking, furrow absent or showing only as a trace A. repens series

b. Equatorial ridges low and rounded, furrow broad and shallow

A. ruber series

c. Equatorial ridges thin and flexuous, crestlike (spore resembling

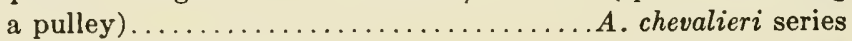

2. Ascospores with convex faces rough.......... amstelodami series B. Ascospores lenticular, $6 \mu$ or more in long axis

Large-spored species or the $(E$.) herbariorum series

II. Perithecia absent.

A. Colonies predominantly in yellow-orange to brownish shades.

1. Heads approximating those of $A$. repens.....A. argillaceus Biourge ${ }^{2}$

2. Heads proliferating, giving rise to many subheads

B. Colonies in dark green or blue-green shades.

A. proliferans G. Smith

1. Restricted, velvety, with short conidiophores and abundant columnar heads............................... restrictus series

2. Spreading, floccose, with long conidiophores and globose heads

A. itaconicus Kinoshita

${ }^{2}$ Culture distributed by Biourge as a new species. Believed to represent only a non-ascosporic strain of $A$. repens, hence not recognized as a valid species by the writers (see p. 111). 
THE ASPERGILLUS REPENS SERIES

Ascospores lenticular, mostly 4.8 to $5.4 \mu$ by 3.8 to $4.4 \mu$, smooth-walled, with equatorial area rounded or somewhat flattened and occasionally indented showing a trace of furrow, but without crests or ridges.

The Aspergillus repens series as based upon the ascospore described includes a great number of universally distributed strains which retain some cultural individuality. Consequently several of them have been described as species by earlier workers. From comparison of a great series of these forms, it seems necessary to bring together under the name $A$. repens (Cda.) DeBary, a very considerable number of forms (some of them regarded as species by others) in which the ascospore is typical for the group and the colony difference falls within lines of quantitative rather than qualitative variation.

The following key is offered as a means of separating culturally distinct strains or groups of strains:

A. Conidial heads large, borne above the surface layer of perithecia and enveloping hyphae.

1. Heads radiate, long-stalked................... repens (Cda.) DeBary

2. Heads columnar, short-stalked................. dierckxii Biourge ${ }^{3}$

B. Conidial heads small, enmeshed with the perithecia in a felt of sterile hyphae.

1. Felt orange-yellow, loose-textured, radially wrinkled

A. pseudoglaucus Bloch.

2. Felt yellow-buff, close-textured, plane or nearly so.....A. profusus Hann ${ }^{3}$

Aspergillus repens (Cda.) DeBary, in Abhandl I. Senkenberg. Natürf. Gesellsch. $7: 379.1870$.

Synonyms: A. glaucus var. repens Cda., Icones Fungorum 5: 53, Taf II, fig. 27. 1842.

A. scheelei Bain. and Sart., Soc. Mycol. de France, Bul. Trimest. 28: 257-262, pl. X. 1912.

A. $B$ var. scheelei Bain. and Sart., Soc. Mycol. de France, Bul. Trimest. 28: 262-267, pl. XI. 1912.

Colonies upon Czapek's solution agar (3 percent sucrose) restricted, plane or somewhat wrinkled, forming a rather compact felt (fig. $24 \mathrm{~A}_{1}$ ), with the marginal area near Scheele's green (Ridgway, Pl. VI) from developing heads, older areas yellow-green to greenish-gray and enmeshing large numbers of aborted perithecia producing few ascospores; normal perithecia found only when such colonies spread over the bare walls of the vessel. Reverse in shades of greenish-yellow at colony margin to deep maroon or almost black in older areas.

${ }^{3}$ Species name not recognized as valid by authors of this publication. 


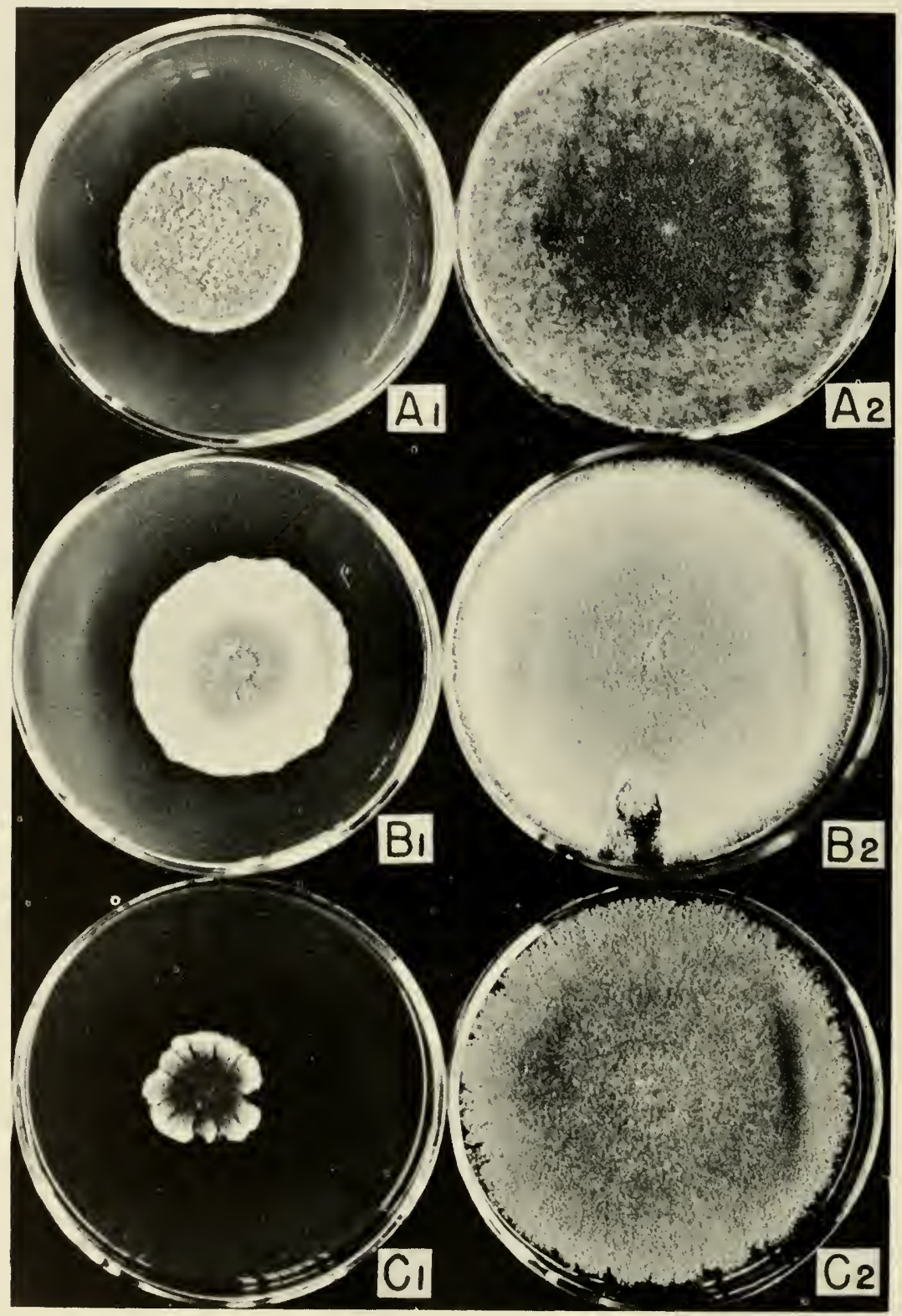

FIG. 24. Comparative growth of Aspergillus repens, A. chevalieri, and A. ruber upon two different culture media; three weeks incubation at room temperature. $A$, 1 and 2, A. repens, NRRL No. 17, upon (1) Czapek's solution agar (3 per cent sucrose), and (2) Czapek's solution agar with 20 percent sucrose. $B, 1$ and $2, A$, chevalieri, NRRL No. 78; and $C, 1$ and $2, A$, rubcr, NRRL No. 52, upon the same media in similar arrangement. 
Colonies upon Czapek's solution agar with 20 percent of sucrose spreading broadly and rapidly, plane or slightly wrinkled, orange-yellow, commonly characterized by broad zones of dull-green conidial heads (PI. III C, and fig. 25, A and B); surface growth consisting of loosely woven hyphae studded with orange granules enmeshing abundant yellow perithecia above
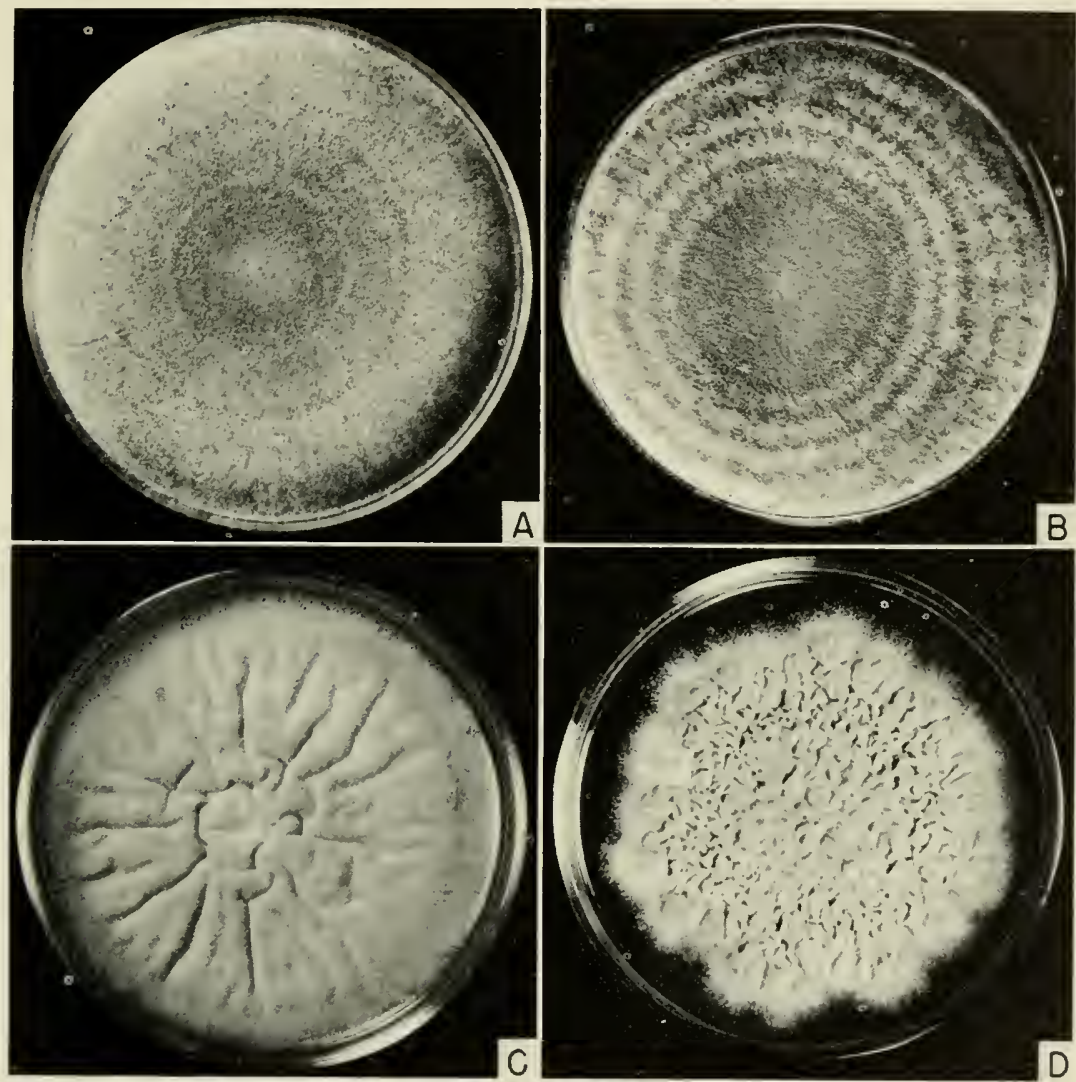

FIG. 25. Comparative growth of members of the Aspergillus repens series upon Czapek's solution agar containing 20 percent of sucrose; incubation at room temperature for three weeks. $A$ and $B$, Typical cultures of $A$. repens. $C, A$. pseudoglaucus, NRRL No. 40. D, A. pseudoglaucus, NRRL No. 45.

which project abundant conidial heads, the whole colony and especially the marginal areas and adjacent wall of the culture dish commonly overgrown by a loose aerial network of hyphae bearing conidial heads and scattered perithecia; reverse varying from yellow-orange to deep maroon.

Perithecia very abundant, borne in loose networks of yellow to orangered hyphae (fig. 26 A), yellow, spherical to subspherical, mostly 75 to $100 \mu$, 


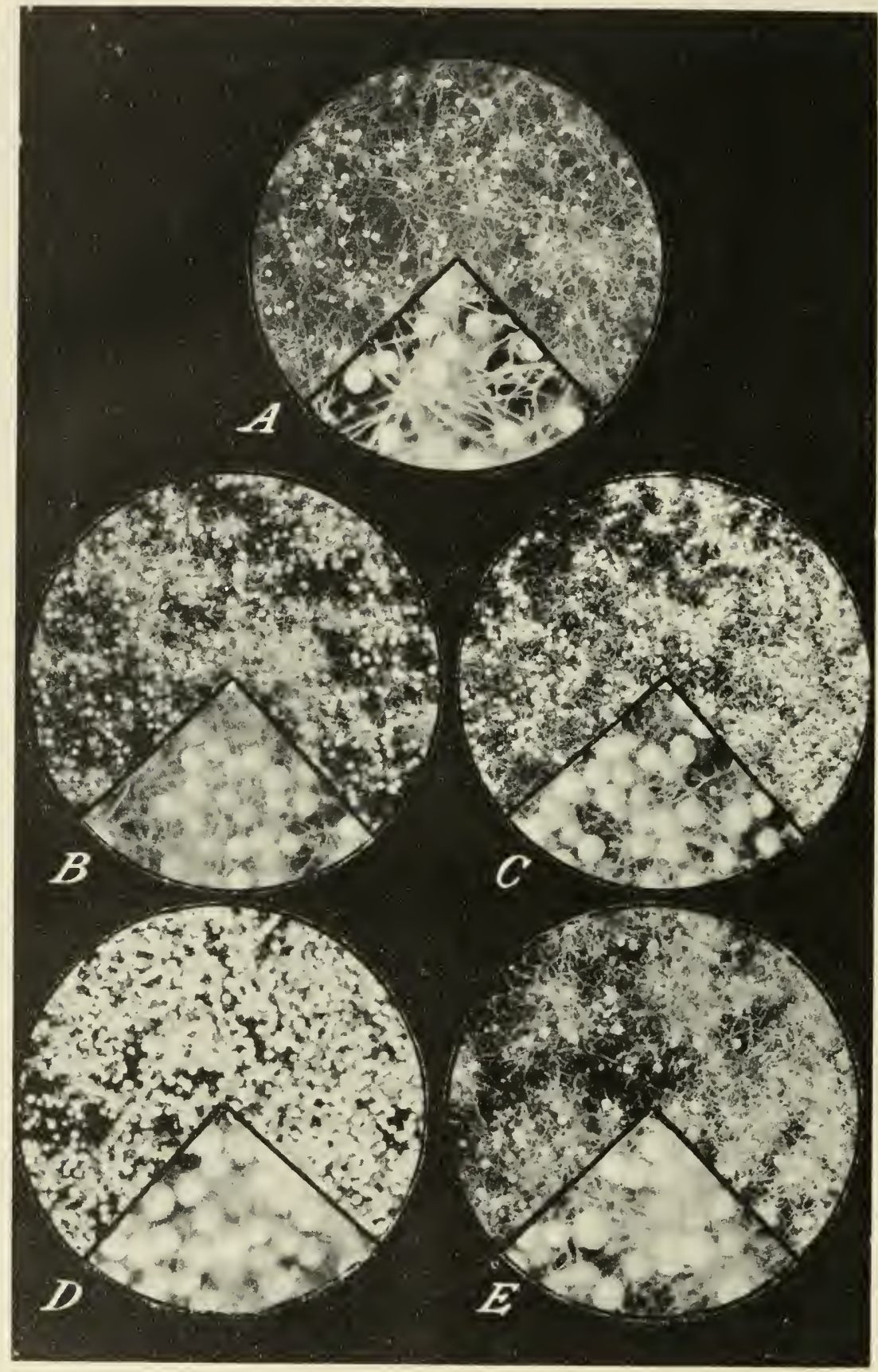

FIG. 26. Marginal areas of colonies of representative species of the Aspergillus glaucus group showing the relative size and arrangement of perithecia (conidial heads not in focus): $A, A$ spergillus repens; $B, A$. chevalieri; $C, A$. ruber; $D, A$. amstelodami; $E, A$. echinulatus. Figures $\times 10$; inserts $\times 30$. (Reprinted from Thom and Raper, "The Aspergillus glaucus Group," U.S.D.A. Mise. Pub. 426: 1-46. 1941.) 
occasionally up to $125 \mu$; asci 10 to $12 \mu$; ascospores lenticular, mostly 4.8 to $5.6 \mu$ by 3.8 to $4.4 \mu$, smooth-walled, with equatorial area rounded or somewhat flattened and occasionally indented showing a trace of furrow but without crests or ridges (fig. $27 \mathrm{~A}$ ). Conidial heads abundant, varying in different strains from 125 to $175 \mu$ in diameter; consisting of diverging chains of conidia radiating from a hemispherical vesicular apex of the conidiophore (fig. $28 \mathrm{~A}$ ); conidiophores smooth, mostly colorless, 500 to $1,000 \mu$ in length, broadening at the apex to a vesicular area, about 25 to $40 \mu$ in diameter; sterigmata in one series 7 to $10 \mu$ by 3.5 to $4.5 \mu$; conidia elliptical to subglobose, spinulose, mostly 5 to $6.5 \mu$.

Represented by cultures NRRL No. 12, No. 17, and more than a score of others included in this study. In this connection it should be noted that of 37 cultures examined in the present study that produced ascospores characteristic of the $A$. repens series, 29 produced colonies and microscopic details that place them in the species $A$. repens as described.

This description is manifestly broad enough to include strains approximating the description given by Bainier and Sartory for Aspergillus scheelei and Aspergillus $B$ var. scheelei (1912b). Evidently $A$. scheelei was thought by the describers to represent a species with somewhat larger ascospores showing a more definite furrow, whereas $A$ spergillus $B$ var. schcele $i$ was a strain with smaller ascospores almost without a trace of furrow. Both species were described as characterized by the production of a yellow pigment. In the authors' experience, a distinction based upon color is largely invalidated by variants bridging the whole range from yellow-orange to deep orange-red and even shades of brown when large numbers of strains of this series are compared in culture. Strains also vary slightly in the pattern of their ascospores, some rarely producing spores with a trace of furrow and others bearing a large proportion with such traces. But among spores of a single strain limited variation in this character is normaily encountered. Thus the presence or absence of a slight furrow, unless accompanied by significant differences in morphology or colony character, would not seem to justify specific descriptions in this series.

A strain designated as Aspergillus dierckxii, presumably by Biourge but thus far unpublished, was included in Gould and Raistrick's study of pigment production in the $A$. glaucus group (1934). As received from Raistrick's laboratory, this organism (NRRL No. 39) produces colonies showing no zonate arrangement of conidial heads. Further, heads are borne on shorter conidiophores than in typical $A$. repens and consist of columns of conidia rather than radiating chains. Little or no red color appears in the colonies or in reverse. Although no other strains showing exactly these differences have appeared in the authors' collection, separation as a distinct species is believed unwarranted. 

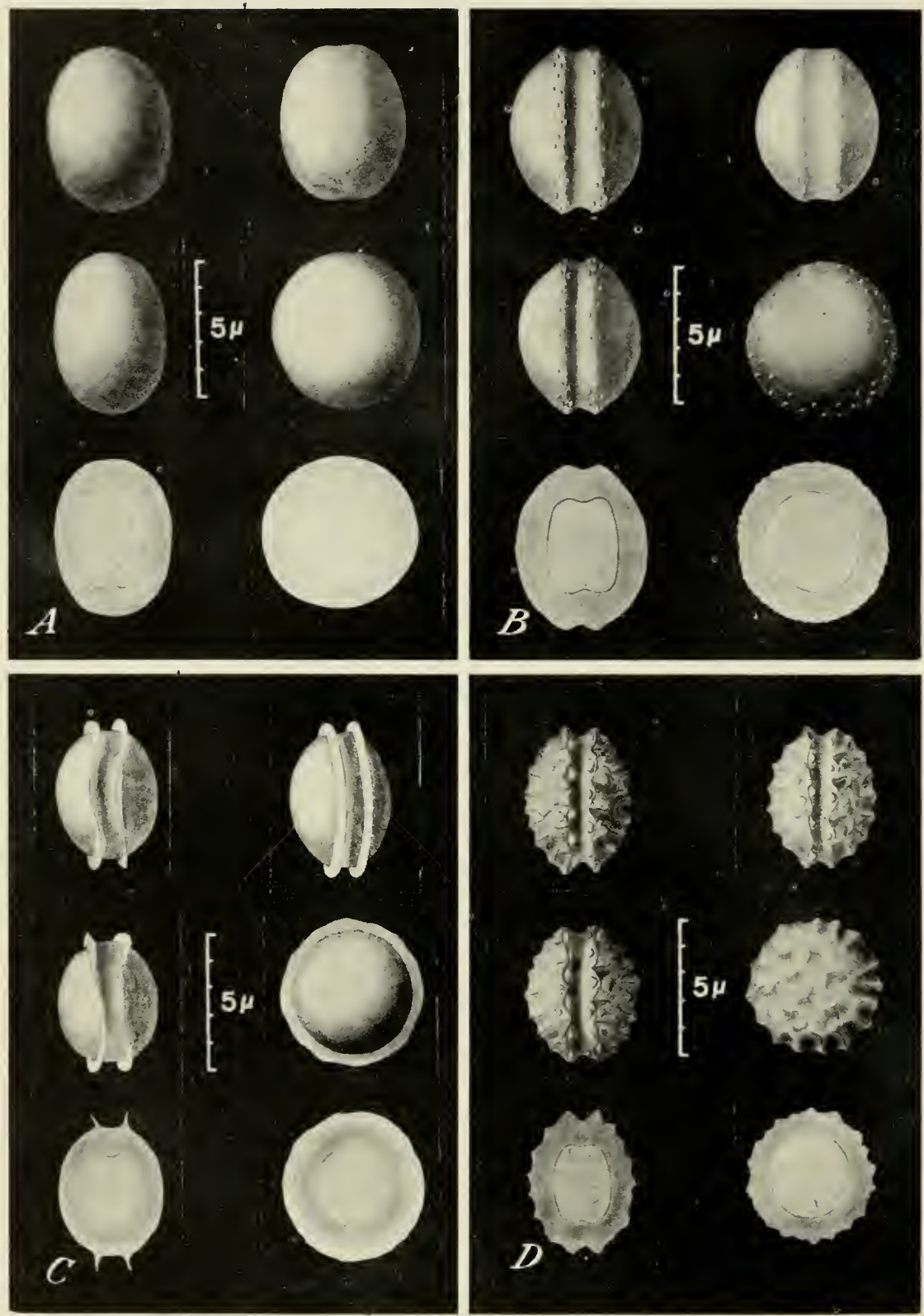

Fig. 27. Ascospores representative of the four small-spored series of the Aspergillus glaucus group. $A, A$. repens. $B, A$. ruber. $C, A$. chevalieri. $D, A$. amstelodami. In each species upper left and right and center left spores represent surface, profile views; center right, surface in face view; lower left, optical section in profile; and lower right, optical section in face view. (Reprinted from Thom and Raper, "The Aspergillus glaucus Group," U.S. D. A. Misc. Pub. 426: 1-46. 1941.) 

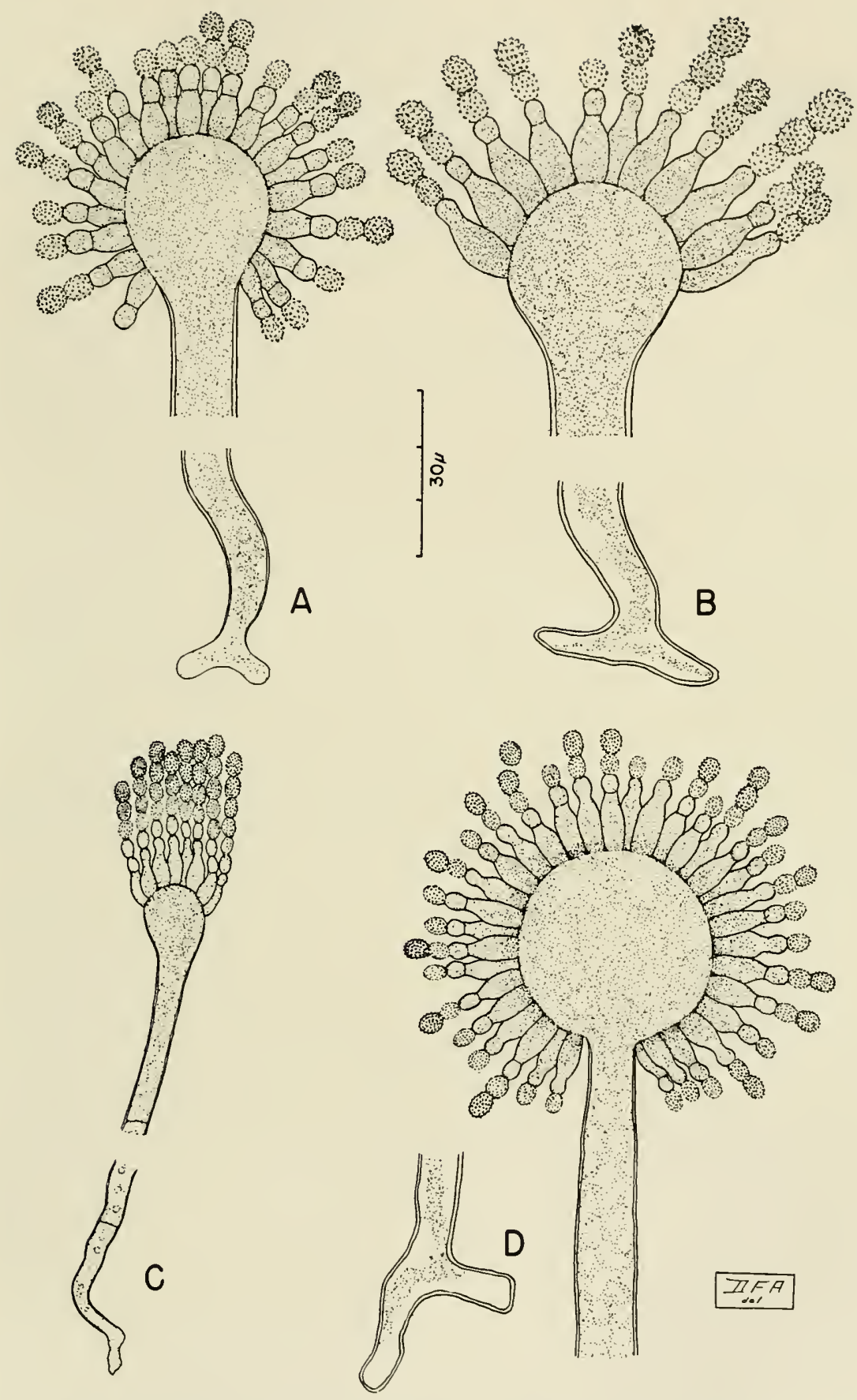

FIG. 28. Conidial structures in the Aspergillus glaucus group, $\times 750: A, A$. repens, NRIRI, No. $21 ; B, A$. echinulatus, NRRL No. 131; $C, A$. restrictus, NRRI No. 154 ; D, A. itaconicus, NRRL No. 161. 
Aspergillus pseudoglaucus Blochwitz, in Ann. Mycol. 27: 207. 1929; emend Thom and Raper, U.S.D.A. Misc. Publ. No. 426, p. 12.1941.

Colonies upon Czapek's solution agar (3 percent sucrose) restricted in growth, radiately wrinkled, yellow-green to shades of gray, consisting of a mixture of small conidial heads, young or aborted perithecia and more or less colorless hyphae; reverse orange at center becoming lighter toward the margin.

Colonies upon Czapek's solution agar with 20 percent of sucrose spreading, strongly wrinkled in a predominantly radiate manner, consisting of a felt of orange-encrusted hyphae enmeshing abundant perithecia, orange except at margin where yellow-green predominates from the presence of small conidial heads admixed with perithecia in the mycelial felt (fig. $25 \mathrm{C}$ ); reverse yellow becoming orange-brown or maroon in marginal areas.

Perithecia abundant, spherical to subspherical, mostly 60 to $80 \mu$ though occasionally $100 \mu$ in diameter, yellow, embedded in a felt of orange mycelium; asci 10 to $12 \mu$ in diameter; ascospores lenticular, 4.6 to $5.2 \mu$ by 3.6 to $4.0 \mu$, occasionally $5.6 \mu$ in long axis, smooth-walled, with equatorial region rounded or flattened, without ridges, and with furrow generally lacking though occasionally showing as a trace. Conidial heads few in number and generally submerged in the mycelial felt, small, mostly 50 to $75 \mu$ in diameter but occasionally up to $100 \mu$; conidiophores mostly 150 to $300 \mu$ in length, 5 to $8 \mu$ at the base, broadening to a terminal vesicle 12 to $20 \mu$ in diameter; sterigmata in a single series, 6 to $8 \mu$ by 3 to $4 \mu$; conidia subglobose, delicately spinulose, variable in size ranging from 5.5 to $7.5 \mu$ in diameter.

Represented in the NRRL collection by No. 40 received from Baarn as A. pseudoglaucus Blochwitz and No. 41 received from George Smith as A. fumigatoides Bain. and Sart.

There is reason to believe that the former culture is directly derived from Blochwitz's type. It becomes necessary therefore to emend the description given by him insofar as the measurements and markings of ascospores and conidia are concerned. Gould and Raistrick (1934) reported biochemical data upon a culture, No. A 38, received from Biourge as $A$. fumigatoides Bain. and Sart. (NRRL No. 41), which is identical with $A$. pseudoglaucus (NRRL No. 40) as sent to the authors by Westerdijk. Obviously the culture from Biourge is incorrectly named. It does not fit the species description nor the figures of $A$. fumigatoides (1909) in the size of its conidia, the character of its perithecial wall, or the pattern of its ascospores. The ascospores of $A$. fumigatoides are shown as roughened over their entire surfaces, as in $A$. fischeri, whereas those of No. 41 certainly belong in the A. repens series. Distinctive colony characters, however, maintained stably through many transfers, together with the size of the conidial apparatus, warrant separating $A$. pseudoglaucus from $A$. repens and maintaining it as a species. 
A strain, NRRL No. 45, received from Dr. B. O. Dodge and Miss Marjorie E. Swift, of the New York Botanical Garden, in 1931, is characterized by an intensely wrinkled colony and a further reduction in the size and number of conidial heads (fig. $25 \mathrm{D}$ ). Colonies are dull orange red and bear abundant perithecia enmeshed in a close felt of sterile encrusted hyphac. Obviously it should be considered with $A$. pseudoglaucus.

In cultures received from Baarn (NRRL No. 44) and from George Smith (NRRL No. 45) as A . profusus Hann (nomen nudum) there is a pronounced accentuation of the floccose habit already noted in $A$. pseudoglaucus. Upon 20 percent sucrose Czapek agar these cultures, which are obviously duplicates, produce spreading, plane or radiately wrinkled, floccose colonies consisting of a close felt of light tan to buff-colored hyphae, bearing occasional perithecia and widely scattered conidial heads. The perithecia are commonly embedded deep within the felt, whereas the conidial heads are most evident at the colony margin. Although the ascospores of these strains are definitely of the $A$. repens type, they are generally flattened along their equators and commonly show a trace of furrow. An occasional ascospore shows a minute roughness in the equatorial region. The differences observed do not seem to warrant perpetuating the name A. profusus, and in agreement with Dr. Westerdijk and coworkers (Centraalbureau List, 1939), the cultures have been assigned to A. pseudoglaucus.

Culture NRRL No. 46, received from Raistrick in 1923 as Aspergillus novus Wehmer (nomen nudum) bears ascospores duplicating those of the cultures just considered. This strain is of particular interest, because in rcutine transfers colonies of two distinct types commonly appear. One of these is predominantly floccose and suggests the colonies of the strains received as $A$. profusus. The other consists of a crowded surface layer of perithecia, which is thinly veiled by a loose felt of orange-red hyphae, and in its gross appearance, with the exception of its lighter color, is strongly suggestive of certain cultures of Aspergillus ruber. The authors agree with Wehmer (1901) and Blochwitz (1929a) that the species designation, Aspergillus novus, should be withdrawn.

A nonascosporic culture distributed by Biourge as Aspergillus argillaceus n. sp., was received upon two occasions from Prof. Raistrick's laboratory. From its appearance in culture and the morphology of its conidial structures this fungus would seem to represent a member of the Aspergillus repens series in which perithecial development has been wholly suppressed. Although it is questionable whether this fungus represents a true species, a brief description is given because of its inclusion in biochemical studies by Raistrick and coworkers (1939, 1934, 1937). Colonies upon Czapek's solution agar with 20 percent of sucrose spreading irregularly, consisting of a loose floccose felt of aerial hyphae and abundant conidial heads, pale yellow-green to clay color; reverse yellow.

Upon Czapek's solution agar 
(3-percent sucrose) colonies restricted, raised in center, thinning toward margin, consisting of abundant conidial heads and interlacing hyphae, buff to clay colored; reverse yellow to tawny. Conidial heads abundant, dull green, up to $200 \mu$ in diameter and commonly splitting into fairly well-defined columns, conidiophores up to $1,000 \mu$ in length. Conidia subglobose mostly 5.5 to $6.0 \mu$ but occasionally up to $7.0 \mu$ in long axis, spinulose.

\section{ASPERGILLUS RUBER SERIES}

Ascospores lenticular, 5.0 to $6.0 \mu$ by 4.0 by $4.8 \mu$, colorless, with broad, shallow furrow generally evident and flanked by low ridges, and with walls smooth except for minute roughness along the equatorial ridges.

This series includes a great number of strains showing variations in cultural appearance but producing ascospores of a limited size range and fairly well-defined pattern. For this particular study some 30 strains, received from various culture collections and contributors and selected from the isolations made in this laboratory over a period of many years, have been chosen for repeated culture and examination. Among these, many strains appear distinct, but their differences are commonly bridged by intermediate forms. Separation within the series, therefore, must be along one of the following lines-either (1) strains must be separated upon minor characters, such as differences in the intensity of pigmentation, slight variations in ascospore character, etc.; or (2) strains must be set off in broad and elastic subgroups, in some cases including large numbers which vary appreciably in detail. The second alternative is desirable, for the first can lead only to increased hairspliting and end in greater confusion than that which already exists.

Spieckermann and Bremer's designation Aspergillus ruber (1902) is assigned to the series, because its members are predominantly producers of an intense red pigment and bear ascospores of the general size and pattern described by these authors. The only other described fungus possessing a similar ascospore and characterized by its red color is Bainier and Sartory's Aspergillus sejunctus (1911b).

Although it is now quite impossible to say what particular fungus either pair of investigators had at hand, both are believed to have worked with members of the large series now under consideration. Aspergillus ruber is retained since its description is more adequate for the series in addition to being the prior species.

To accentuate cultural similarities and differences between the strains studied, a wide variety of culture media has been employed. Based upon their appearance in culture, the strains fall into a few well-defined subgroups (see figs. 29 and 30). The strains belonging to one of these seem 
to represent the fungus described by Spieckermann and Bremer (1902) and are at the same time most abundant in the entire series, hence are considered as typical of $A$. ruber (figs. $29 \mathrm{~A}$ and $30 \mathrm{~A}$ ). The general characters of the remaining subgroups are listed to show the extreme cultural variation
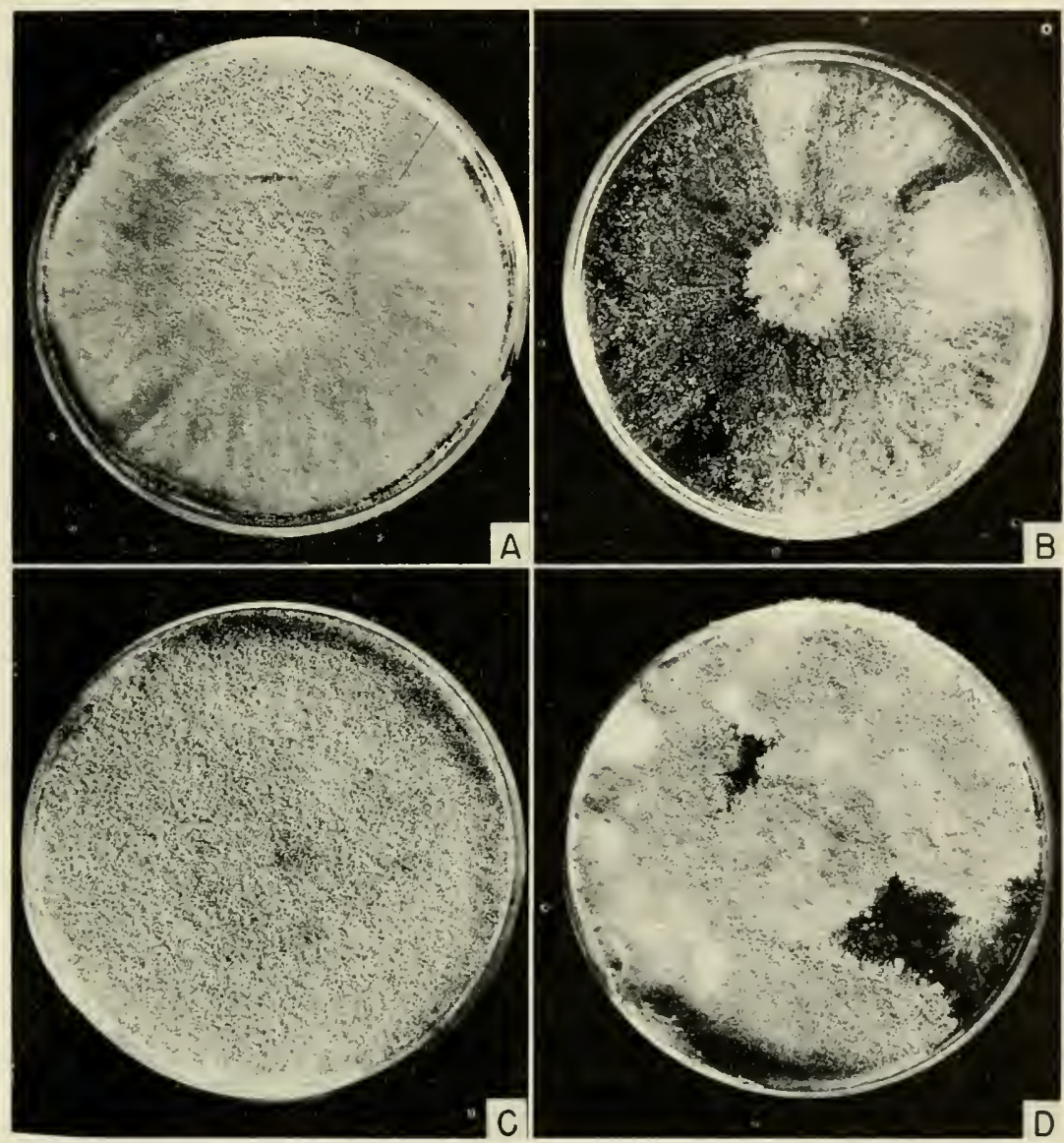

FIG. 29. Different colony types developed in the Aspergillus ruber series in three weeks at room temperature upon 20 percent sucrose Czapek agar: $A$, Typical $A$. ruber, NRRL No. $52 ; B$, NRRL No. 70 , characterized by thin colonies, with mycelium largely submerged; $C$, NRRL No. 65, colony floceose, bearing abundant perithecia and few conidial heads; and $D$, NRRL No. 75, deep floccose colony with very abundant conidial heads and only scattered perithecia.

that is to be expected within the series, but spccific names are withheld, because to perpetuate or propose such would multiply rather than clarify the confused nomenclature of this abundant and variable series of organisms. 
To illustrate more definitely the variation that occurs, the following outline of possible lines of separation is inserted:

A. Colonies predominantly perithecial.

1. Colonies red, perithecia abundant in a layer at the agar surface and overgrown by a felt of red encrusted hyphac

A. ruber (Spieck. and Brem.) Thom and Chureh, NRRI, Nos, 52, 49

2. Perithecia abundant in a loose, floccose overgrowth of red hyphae as well as in a layer at the agar surface

NRRL No. 65

3. Colonies thin, orange-red, perithecia abundant in old cultures and on very eoncentrated media............................. NRRL No. 70

B. Colonies predominantly conidial.

1. Colonies gray, heads long-stalked, perithecia few

NRRL No. 75

C. Colonies mixed conidial and perithecial.

1. Colonies orange-red and green, zonate, perithecia borne at the agar surface and piled in a loose network of superficial hyphae. NRRL No. 71

D. Colonies predominantly floccose, colonies red-brown, perithecia and conidial heads few...................... lovaincnsis Biourge, ${ }^{4}$ NRRL No. 76

\section{Aspergillus ruber (Bremer)}

Synonyms: A. ruber (Spieckermann and Bremer) Thom and Church, in The Aspergilli, 112. 1926.

Eurotium rubrum Bremer, in Zeitschr. f. Untersuch. d. Nahrung. und Genussmittel IV. 1901, p. 72, also in Die fettverzehr. Organismen in Nahr. u. Futtermitteln, Dissert. Munster 1902.

E. rubrum Spieckermann and Bremer, in Landw. Jahrb. 31: 81-128. 1902.

A. sejunctus Bain. and Sart., Soc. Mycol. de France, Bul. Trimest. 27: 361-367, pl. XI. 1911.

Colonies upon Czapek's solution agar (3 percent sucrose) more restricted, plane (fig. $24 \mathrm{C}_{1}$ ), orange-brown to red-brown in color; perithecia generally abundant though often abortive; conidial heads pea-green to olive-green, abundant in some strains, few and largely vestigial in others; reverse orangered to maroon.

Colonies upon Czapek's solution agar with 20 pereent of sucrose spreading rapidly and broadly in a regular manner or unevenly, plane, predominantly red, ranging from ferrugineous to moroceo red; perithecia very abundant, borne in a dense layer at the agar surface and largely concealed within and beneath a close-textured felt of red-encrusted hyphae; conidial heads projecting above the felt, pale gray-green to deep olive-gray, more or less abundant, and generally crowded near the center or seattered unevenly

${ }^{4}$ Species name not recognized as valid by authors of this publication. 
over the colony (Pl. III D; figs. $29 \mathrm{~A}$ and $30 \mathrm{~A}$ ); reverse in shades of dark red-brown.
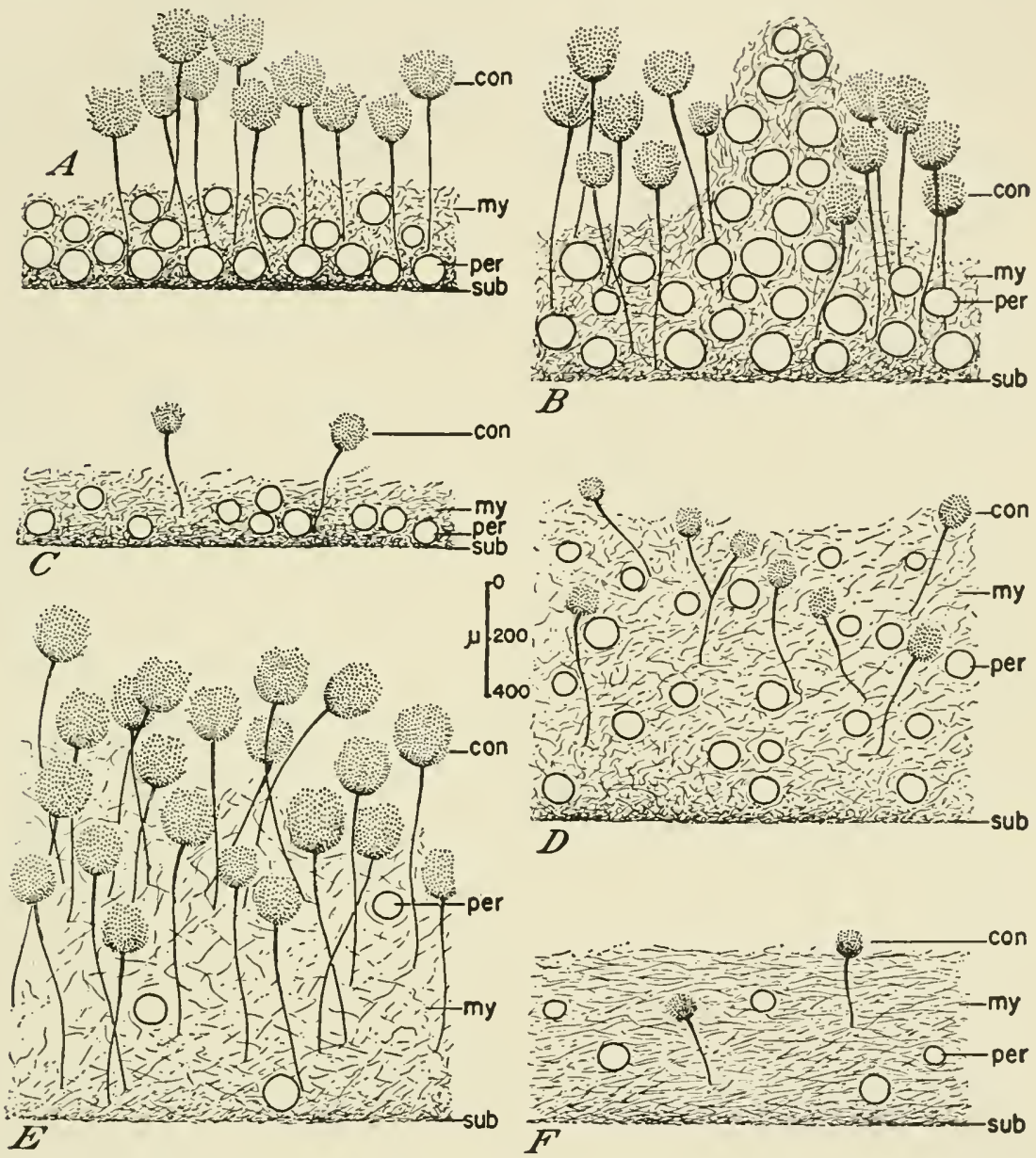

FIg. 30. Diagramatic representation of cross sections of different colony types in the Aspergillus ruber series developed at room temperature upon 20-percent sucrose Czapek agar, showing relative abundance and disposition of the conidial heads (con) and perithecia (per), and the amount and character of the mycelium $(m y)$ above the substratum $(s u b): A$, Typical colony of $A$.ruber as seen in NRRL No. $52 ; B-F$, atypica colonies as seen respectively in NRRL No. 71, No. 70, No. 65, No. 75 , and No. 76 . Scale approximate. (Reprinted from Thom and Raper, "The Aspergillus glaucus Group," U.S.D.A. Misc. Pub. 426: 1-46. 1941.)

Perithecia very abundant, largely enmeshed in a felt at the agar surface (fig. $30 \mathrm{~A}$ ), yellow to orange-red, spherical to subspherical, mostly 80 to $120 \mu$ though occasionally up to $140 \mu$ in diameter; asci 12 to $15 \mu$; ascospores lenticular, 5.2 to $6.0 \mu$ by 4.4 to $4.8 \mu$, with furrow generally evident as a broad and shallow depression around the spore equator, ridges low and 
often inconspicuous, walls smooth except for minute roughness along equatorial ridges (fig. 27 B). Conidial heads generally abundant, numerous in localized areas or scattered thinly over the colony, pale blue-green, radiate, 150 to $250 \mu$ in diameter; conidiophore smooth, colorless to orangebrown, 500 to $750 \mu$ in length, broadening to 14 to $16 \mu$ where it passes into the subglobose vesicular area of 25 to $35 \mu$ diameter; sterigmata in a single series 7 to $9 \mu$ by 4 to $5 \mu$; conidia elliptical to subglobose, closely spinulose, mostly 5 to $6.5 \mu$ in long axis.

A spergillus ruber is represented in this study by NRRL Nos. 52, 53, and others. Of 31 strains examined belonging to the whole series, 19 showed colonies and microscopic characters that place them within the species Aspergillus ruber as described above. Although the majority of strains belonging to the $A$. ruber series produce plane colonies as noted in the description, occasionally strains may produce colonies more or less wrinkled.

Culture NRRL No. 65 (figs. $29 \mathrm{C}$ and $30 \mathrm{D}$ ) represents a subseries of several strains that differ from the above not only in colony character upon 20 percent sucrose Czapek agar, as indicated in the preceding key, but also in their growth upon media of lower concentration. These grow slowly and poorly on Czapek (3 percent sucrose), potato-dextrose, and wort agars, producing small raised colonies of 1 to $2 \mathrm{~cm}$. in diameter bearing neither normal conidial heads nor perithecia.

Strain NRRL No. 70 (figs. $29 \mathrm{~B}$ and $30 \mathrm{C}$ ) produces abundant perithecia only on very dry areas of the substratum in old cultures or on media containing a sucrose concentration of 40 percent or more. In contrast to other strains, this fungus grows better upon media containing 4 percent agar than the usual 1.2 percent agar, further establishing its xerophytic character.

Strains such as NRRL No. 75 (figs. $29 \mathrm{D}$ and $30 \mathrm{E}$ ), occasionally encountered, are predominantly conidial and characterized by rampant hyphae bearing abundant conidial heads piled in floccose masses above the substratum and upon the edges of the culture dish or tube. They thus produce colonies markedly in contrast with the usual Aspergillus ruber concept. But the character of their ascospores, together with the occurrence of occasional sectors in colonies of these strains showing the usual mixture of perithecia and conidial hearls, relates them definitely with $A$. ruber.

Strain NRRL No. 71 (fig. 30 B) represents a subsection of the series in which conidial heads are abundant and generally arranged in fairly definite zones and patches with loose clusters of perithecia irregularly and conspicuously distributed among and above the grouped green heads. These strains are further characterized by somewhat larger perithecia than those of NRRL No. 52, being mostly in the range of 125 to $150 \mu$ in diameter, and by producing less red color in the colonies and in their reverse. 
Culture NRRL No. 76 (fig. $30 \mathrm{~F}$ ) is characterized by a close felt of red-brown hyphae, which completely covers the agar surface and in which scattered perithecia are borne. Conidial heads are scarce and largely confined to the colony margin. This strain, which was included in Gould and Raistrick's study of pigmentation in the Aspergillus glaucus group (1934), was received from George Smith under the name Aspergillus lovainensis and attributed to Biourge. Except for its dark color this fungus in culture bears a striking resemblance to one received from Baarn as Aspergillus profusus (NRRL No. 44), which showed similar floccose habits. However, the ascospores of the latter are smaller and less furrowed and are essentially smooth along the equatorial margin. The degree of relationship between the two is questionable.

In addition to the ascosporic strains definitely placeable in the series, George Smith has recently described $A$. proliferans in which the proliferation of the sterigmata has become so pronounced as to become the most conspicuous character, while perithecium formation has been suppressed. In colony characters, however, it belongs here. This strain diverges further from the type as described but may be arbitrarily placed here by the branching of its simplified heads and the size and markings of its conidia.

Aspergillus proliferans George Smith, in Brit. Mycol. Soc. Trans. 26(1/2): 26, Pl. III. 1943.

Colonies on Czapek's solution agar spreading very slowly, with growth at first largely submerged, then with matted floccose aerial mycelium, white changing to yellowish shades, sporing tardily, with conidial areas graygreen, reverse yellowish-brown; on wort agar growing slowly but better than on Czapek, with mycelium white then yellow and finally orange and tardy development of gray-green to gray conidial areas, becoming more deeply floccose in age especially at shallow end of the slope; reverse yellow; normal conidial heads loosely radiate; conidiophore smooth, thin-walled, usually with one or two septa, 4 to $14 \mu$ in diameter; vesicles occasionally almost globose, more frequently obconical or mere broadening of the ends of the conidiophores, up to about $20 \mu$ in diameter; sterigmata when normal, in one series, 8 to $11 \mu$ by 3.5 to $6 \mu$, often elongate, septate and bearing small secondary heads, frequently resembling heads of monoverticillate Penicillia, or with upper portion much swollen and appearing almost as very large, thick-walled conidia with long connectives, up to $20 \mu$ in diameter, with normal and swollen sterigmata often appearing in the same head; conidia globose or subglobose, rough, fairly dark-colored, 5 to $9.5 \mu$ in diameter; perithecia not found. (Species description after George Smit.1.) 
Aspergillus halophilus Sartory, Sartory, and Meyer (in Ann. Mycol. 28(3/4): 362363, PI. III. 1930) appears from the description based upon colonies grown upon licorice sticks, to have been some member of this general group. No cultures have been available for comparison, hence placement near A. proliferans of George Smith can be only tentative. If placement were to be based upon their figure 12 , it might be a species of Scopulariopsis.

\section{ASPERGILLUS CHEVALIERI SERIES}

Ascospores lenticular, mostly 4.6 to $5.0 \mu$ by 3.4 to $3.8 \mu$, occasionally up to $5.2 \mu$ in long axis, with walls smooth or slightly rough, with crests prominent, flexuous, often recurved, and with furrow conspicuous but consisting more of a trough between extended equatorial crests than a depression in the spore wall.

Strains belonging to this series show appreciable difference in colony character and to a limited degree in the surface markings of their ascospores. The ascospores of all, however, are characterized by their continuous, prominent equatorial crests which do not form an integral part of the spore wall, but extend well beyond the margin of the spore body proper. To use Mangin's exceedingly descriptive term, they are characteristically "pulley-form."

The following key will serve to differentiate groups of strains within the series:

A. Ascospore walls smooth.

1. Crests prominent, thin, flexuous, often recurved

2. Crests cvident, low, usually erect

A. chevalieri (Mangin) Thom and Church

A. chevalieri var. multiascosporus Nakazawa et $a^{5}$

B. Ascospore walls more or less roughened.

1. Crests thin, flexuous, of ten recurved; conidia roughened. A. oriolus Biourge ${ }^{5}$

2. Crests thicker, usually erect: conidia smooth

A. chevalieri var. intermedius, Thom and Raper

Aspergillus chevalieri (Mangin) Thom and Church, The Aspergilli, p. 11. 1926.

Synonym: Eurotium chevalieri Mangin, Ann. des Sci. Nat., Bot. (Ser. 9) 10 : 361-362, fig. 12.1909.

Colonies upon Czapek's solution agar (3 percent sucrose) restricted, plane, closely felted, bluish-gray in center, with typical heads and perithecia largely confined to marginal area (fig. $24 \mathrm{C}_{1}$ ); reverse maroon in center to orange at margin.

Colonies upon Czapek's solution agar with 20 percent of sucrose growing best at $30^{\circ} \mathrm{C}$. or above, spreading, plane to somewhat wrinkled in central

${ }^{5}$ Species name not recognized as valid by authors of this publication. 
area (fig. $31 \mathrm{~A}$ ) with abundant conidial heads in blue-green shades, distributed evenly over the whole surface or more crowded in localized areas, projecting above a layer of abundant perithecia enmeshed in orange-red hyphae at the agar surface; reverse in shades of orange-red to brown, more intense in center.

Perithecia abundant and closely enmeshed in a felt of orange-red encrusted hyphae (fig. $26 \mathrm{~B}$ ), mostly 100 to $140 \mu$, occasionally up to $150 \mu$, globose to subglobose, yellow to orange; asci 9 to $10 \mu$; ascospores lenticular, 4.6 to $5.0 \mu$ by 3.4 to $3.8 \mu$, with walls smooth, with equatorial crests prominent, thin and often recurved, and with furrow consisting more of a trough between parallel crests than an equatorial depression in the spore body
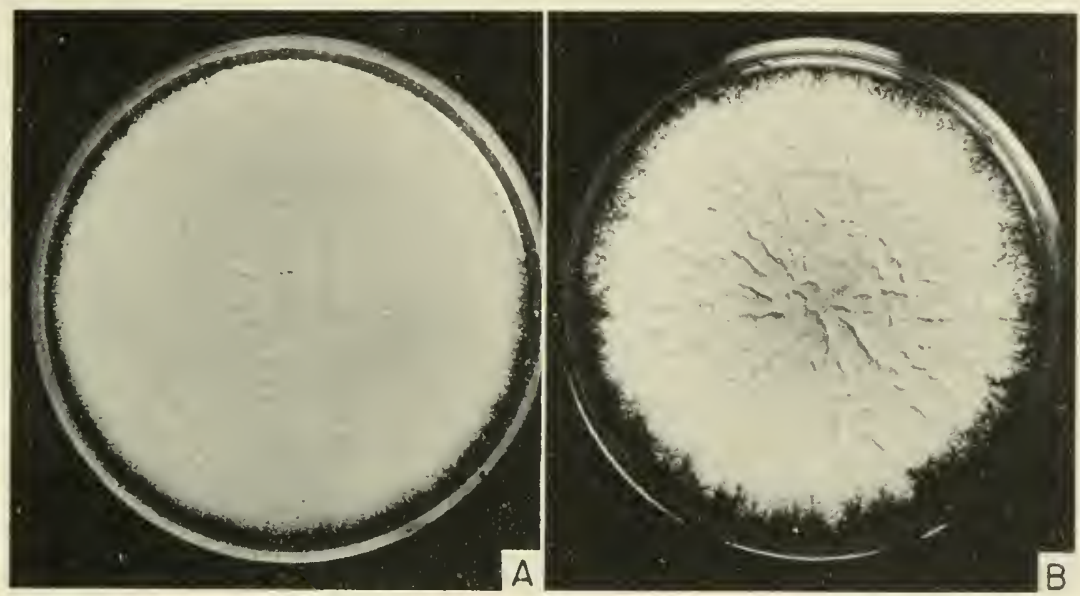

FIG. 31. Comparative growth of members of the Aspergillus chevalieri series in three weeks at room temperature upon 20 percent sucrose Czapek agar: $A$, Typical A. cheralieri, NRRL No. 78; $B, A$. cheralieri var. intermedius, NRRL No. 82.

(fig. $27 \mathrm{C}$ ). Conidial heads abundant, pale blue-green, appearing radiate from divergent conidial chains, mostly 125 to $175 \mu$ in diameter, occasionally larger; conidiophores mostly 700 to $850 \mu$ in length, enlarging to a resicular apex, somewhat globose, 25 to $35 \mu$ in diameter; sterigmata in a single series, closely packed, 5 to $7 \mu$ by 3 to $3.5 \mu$; conidia subglobose, spinulose, mostly 4.5 to $5.5 \mu$ in diameter.

Aspergillus chevalieri is represented in the present study by cultures NRRL Nos. 78, 79, and others. The species name is limited to strains bearing ascospores with smooth walls and prominent, thin equatorial crests because it is believed that these strains most nearly represent the organism described by Mangin (1909). When grown upon 20 percent sucrose Czapek agar, these strains are further characterized by their predominantly orange- 
red colonies, pale blue-green conidial heads, and dark-colored reverse. Within the series, different strains vary in the quantity of conidial heads produced, e.g., NRRL No. 79 regularly produces an abundance of heads, NRRL No. 78 relatively few.

Strains of this series are not so commonly encountered as are those of the $A$. repens, $A$. amstelodami, or $A$. ruber series. And within the series, strains that conform with the typical species description are relatively less numerous than in these other series. In the present study 14 strains belonging to the $A$. chevalieri series have been examined and only 6 , or less than half, are wholly representative of the species A. chevalieri.

The series as a whole seems to be relatively unstable, and certain strains and groups of strains appear transitional between this series and the $A$. repens series on the one hand and the A. amstelodami series on the other.

Culture NRRL No. 88 received from Baarn as the type of Aspergillus chevalieri var. multiascosporus Nakazawa, Takeda, Okada, and Simo points toward the $A$. repens series. Its ascospores have smooth walls, as in the typical strains of $A$. chevalieri, but bear low, erect crests in constrast to the thin, flexuous crests characteristic of this species. Spores lacking crests are occasionally seen and these closely resemble $A$. repens. The colony upon 20 percent sucrose Czapek agar is definitely of the character of $A$. chevalieri. Nakazawa and coworkers (1934) separated it from A. chevalieri because of its more floccose habit and its more abundant production of perithecia. The former character is evident in the authors' cultures, but perithecia are not produced more abundantly than in certain strains entirely typical of $A$. chevalieri.

Another variation from the typical species is seen in culture, NRRL No. 87 received from George Smith as Aspergillus oriolus and attributed to Biourge. The ascospores of this culture (and another that is in the NRRL collection, No. 81) have crests typical of $A$. chevalieri, but the spore walls are finely roughened over their entire surfaces. This character is suggestive of $A$. amstelodami, although the roughening of the wall is slight in comparison with that species. The colony upon 20 percent sucrose Czapek agar is essentially like that of typical strains of $A$. chevalieri but is less red in color and bears fewer conidial heads. Although these cultures can be distinguished from type they are not recognized as warranting separation.

The roughening of the ascospore wall is further accentuated in a group of four apparently similar strains, which it is believed are truly intermediate between $A$. chevalieri and $A$. amstelodami. Because their ascospores bear crests of the A. chevalicri type and hence appear "pulley-form" they are retained in the species. However, because they differ from typical strains in additional particulars, they are considered a new variety, namely Aspergillus chevalieri var. intermedius. 
Aspergillus chevalieri (Mangin) var. intermedius Thom and Raper, in U.S. D. A. Misc. Publ. No. 426, p. 21. 1941.

Colonies upon Czapek's solution agar with 20 percent sucrose differing from the species in texture and color, and presenting withal a picture intermediate between A. chevalieri and A. amstelodami (fig. $31 \mathrm{~B}$ ). Ascospores lenticular, mostly 4.6 to $5.2 \mu$ by 3.6 to $4.0 \mu$, occasionally $5.4 \mu$ in long axis, with walls roughened and with prominent equatorial crests. Conidial heads dull green, radiate to columnar, mostly 100 to $125 \mu$ in diameter, and up to $175 \mu$ in length; conidia elliptical to subglobose, smoothwalled, mostly 3 to $4 \mu$ in long axis.

Represented in this study by culture NRRL No. 82 which was received from George Smith as No. 107 and bore the following notation: "Isolated G. S. from cotton yarn, 1927. Close to A. chevalieri-differs in having smooth, small conidia and ascospores somewhat larger than type." Duplicated by three additional strains received from European sources.

Aspergillus chevalieri var. intermedius appear's to be transitional between the $A$. chevalieri and the $A$. amstelodami series. Such a view is supported (1) by the pattern of the ascospores, which shows both the extended and often recurved equatorial crests characteristic of $A$. chevalieri and the rough spore walls of $A$. amstelodami; and (2) by the coloration of the colony. Aspergillus chevalieri var. intermedius upon 20 percent sucrose Czapek agar becomes orange-yellow above and orange to light brown in reverse, $A$. amstelodami remains bright yellow with reverse uncolored, whereas $A$. chevalieri becomes red in the colony and reverse. The smoothness of conidia in $A$. chevalieri var. intermedius is a distinctive character and appears in neither $A$. chevalieri nor $A$. amstelodami. Although this variety from many points of view appears to be a hybrid, proof of such origin is lacking.

Aspergillus diplocystis (Sartory, Sartory, Hufschmitt and Meyer) Dodge, Med. Myc. p. 625. 1935. Syn. Eurotium diplocyste Sartory, Sartory, Hufschmitt and Meyer, in Compt. Rend. Soc. Biol. 104: 881-883. 1930. Not E. diplocystis B. and Br., Jour. Linn. Soc. 14: 55-56 Tab. 10. 1875.

Characterization: Colonies greenish-ycllow, becoming yellow from perithecia. Conidiophores erect, 50 to $100 \mu$ high, 3.1 to $3.7 \mu$ in diameter, membrane thick, hyaline. Sterigmata confined to a portion of head, 5 to $6.25 \mu$ by 1.5 to $2.5 \mu$. Secondary sterigmata small; conidia spherical, 2.25 to $3.1 \mu$ in diameter, slightly ellipsoid, green (tendre to cendré); sterigmata sometimes abortive and proliferous. Perithecia canary yellow asci 4 to $6 \mu$ by 5 to $7 \mu$, containing 8 ascospores which are ovoid, with a furrow and two crests, 1.5 to $2.5 \mu$ by 1.8 to $3.1 \mu$.

This description suggests an Aspergillus with the heads approximating $A$. nidulans and the perithecia of $A$. chevalieri. Ascospore measurements as reported are appreciably smaller than those of $A$. chevalieri or any other known species of Aspergillus. It was described from a case of onychomycosis from the thumb and the great toe. Tentatively placed in the $A$. chcvalieri series. The name is invalid because of $E$. diplocystis B. and Br. 1875 . 


\section{ASPERGILLUS AMSTELODAMI SERIES}

Ascospores 4.7 to $5 \mu$ by 3.6 to $3.8 \mu$, lenticular, colorless, with equatorial furrow conspicuous, broadly $V$-shaped and flanked by broad irregular ridges, with walls irregularly and unevenly ridged or roughened over the entire surface.

Included in this series are strains that differ greatly in colony appearance. However, their close relationship is demonstrated by the similarity in size and pattern of their ascospores and is further shown by the dark olive-green color of their conidial heads, the bright yellow color of their perithecia, and the absence of any red either in the colonies or their reverse.

The following key is designed to show the variation that occurs within the series and to offer a means of separating strains or groups of strains that are culturally distinct:

A. Colonies predominantly perithecial.

1. Conidial heads abundant in central area and often in concentric zones

A. amstelodami (Mangin) Thom and Church

2. Conidial heads widely scattered or lacking............. NRRL No. 113

B. Colonies predominantly conidial.

1. Perithecia widely scattered, superficial................ NRRL No. 111

2. Perithecia abundant in a felted layer above the conidial heads

A.montevidensis Talice and MacKinnon

C. Colonies very thin, perithecia and conidial heads widely scattered

NRRL No. 110

Aspergillus amstelodami (Mangin) Thom and Church, The Aspergilli, p. 113. 1926.

Synonyms: Eurotium amstelodami Mangin, in Ann. des Sci. Nat., Bot. (ser. 9) 10: 360-361. 1909.

E. repens var. amstelodami Vuill., Soc. Mycol. de France, Bul. Trimest 36: 131. 1920.

Colonies upon Czapek's solution agar (3 percent sucrose) restricted, 4 to $6 \mathrm{~cm}$. in diameter, plane or closely wrinkled, yellow to dull yellow-gray in color from abundant perithecia admixed with sterile hyphae and developing conidial heads; reverse uncolored, becoming tawny in age.

Colonies upon Czapek's solution agar with 20 percent of sucrose spreading, 8 to $10 \mathrm{~cm}$. in diameter, more or less wrinkled and zonate (PI. III E, and fig. $32 \mathrm{~A}$ ), perithecia very abundant and clustered in masses forming a dense layer at the agar surface (fig. 26 D), bright yellow in color, lending a characteristic appearance to the colony; conidial heads deep olive-green, abundant in colony center and scattered more or less unevenly over the whole surface, occasionally obscuring the layer of perithecia beneath. 
Reverse persistently yellow under perithecial areas, more or less green where conidial areas predominate.

Perithecia globose to subglobose, mostly 115 to $140 \mu$ in diameter, oceasionally up to $160 \mu$, not covered by or embedded within a felt of sterile hyphae (fig. $26 \mathrm{D}$ ); asci mostly 10 to $12 \mu$, 8-spored; ascospores lenticular,
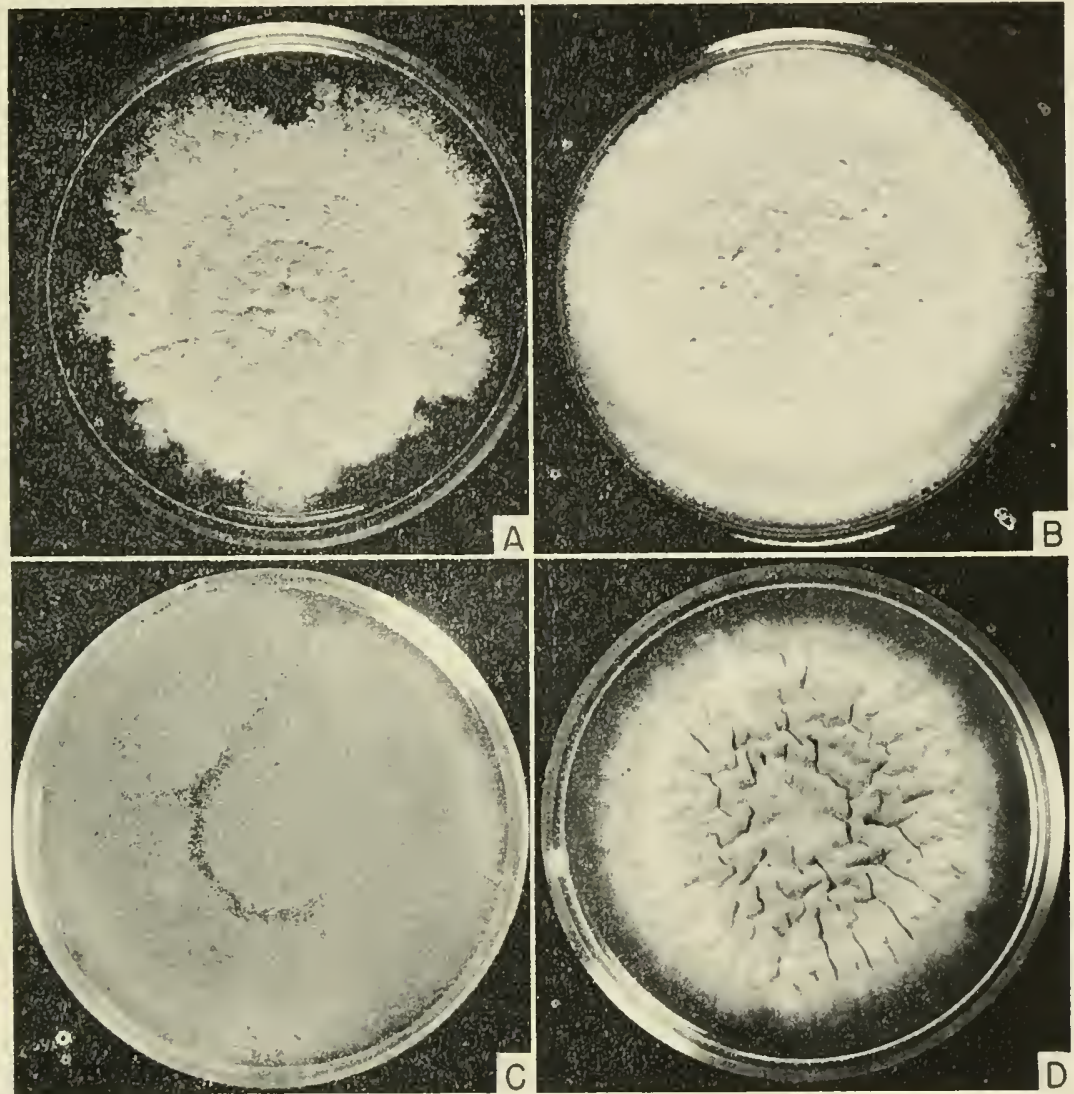

Fig. 32. Comparative growth of members of the Aspergillus amstelodami series: $A$, Typical $A$. amstelodami, NRRL No. $90 ; B$, NRRL No. 113 , a strain producing abundant perithecia and few conidial heads; $C$, NRRL No. 111, strain producing abundant conidial heads and very few perithecia; $D, A$. montevidensis, NRRL No. 108.

4.7 to $5.0 \mu$ by 3.6 to $3.8 \mu$, with prominent $V$-shaped equatorial furrow and broad irregular ridges, and with walls roughened over their entire surfaces (fig. $27 \mathrm{D}$ ). Conidial heads radiate-columnar, mostly 120 to $150 \mu$ in diameter, occasionally larger; conidiophores colorless to pale yellow-green, 275 to $350 \mu$ in length, broadening to 10 to $12 \mu$ in diameter below the vesicle; vesicle subglobose, 18 to $25 \mu$ in diameter; sterigmata about 5 to 
$6.5 \mu$ by 2.5 to $3.5 \mu$; conidia finely spinulose, subglobose, variable in size, ranging from 3.5 to $5.2 \mu$ mostly about $4 \mu$ in long axis.

Represented in the NRRL collection by cultures Nos. 89, 90, and many other:

Thirty-two eultures belonging to this series have been examined in the present study. Included in this number are the authors' own isolates from a wide variety of sources together with cultures contributed by collaborators in this country and abroad. Of these, more than three-fourths regularly produce colonies conforming with the above description of the species A. amstelodami. Although wide variation in colony character does occur within the serics, it is obvious that such variations are exceptional rather than commonplace. Aceordingly, it is not believed advisable to assign or create specific or varietal names for these variations although they differ markedly from the typical A. amstelodami in gross appearance. An exeeption to this policy has been made in the case of cultures received as $A$. monteridensis, for reasons that will be considered later.

As indieated in the preeeding key to the series, marked variation from the normal eultural character of $A$. amstelodami occurs along certain divergent lines.

Culture NRRL No. 113 (fig. 32 B) received from Baarn as Eurotium repens (C'da.) DeBary and Wor. var. amstelodami Vuill. (1920) represents a variation that tends toward an almost complete suppression of the eonidial phase with only an occasional small and atypical head present.

In the opposite direction, culture NRRL No. 111 (fig. 32 C) recently received from Bliss in California (isolated from date fruits) represents a variation that produces a dense stand of conidial heads and only occasional perithecia, these being borne above rather than below the layer of crowded conidial heads.

In contrast to both of the preceding, culture NRRL No. 110 isolated from an old shoe, produces an extremely thin, spreading colony that bears only widely scattered perithecia or conidial heads.

A fourth distinct variation is represented by culture NRRL No. 108 received in 1932 from Talice as A. montevidensis Talice and Mackinnon (1931). This fungus is characterized by an initially strong development of the conidial phase, and subsequently of perithecia in a felted overgrowth, which in the colony center more or less obscures the underlying conidial layer. Perithecia and conidial heads are somewhat smaller than in strains of $A$. amstelodami. Although this culture does not differ from A. amstelodami more widely than the variations previously noted, since it has an imputed pathogenie history and since it has been described and distributed widely under the name Aspergillus montevidensis, it is believed advisable to retain the name in association with this culture. Accordingly, the writers include the following emended description. 
Aspergillus montevidensis Talice and Mackinnon, in Soc. de Biol. (Paris)

Compt. Rend. 108: 1007-1009. 1931. emend. Thom and Raper,

$$
\text { U. S. Dept. of Agr. Mise. Pub. 426, p. 26. } 1941 .
$$

Colonies upon Czapek's solution agar (3 pereent suerose) restricted, radiate sulcate, with zonation evident toward the margin, central area showing coremia, perithecia few or lacking; reverse and agar very dark, almost black.

Colonies on Czapek's solution agar with 20 percent of sucrose, spreading, wrinkled and buckled (fig. 32 D), at first bluish-greon from massed conidial heads, with esutral area later becoming yellow from developing perithecia in a more or less tufted overgrowth of somewhat floccose mycelium; reverse in yellow-green shades to deep olive in colony center.

Perithecia abundant, of variable size and irregular shape with relatively few fertile asci and ascospores, late in developing, commonly 75 to $100 \mu$ in dianeter, occasionally larger; asei 10 to $12 \mu$ in diameter; ascospores lenticular, roughened, with broad and prominent furrow flanked by low acute and irregular ridges, mostly 4.8 to $5.2 \mu$ by 3.6 to $4.0 \mu$, occasional spores larger or smaller. Conidial heads very abundant, small, somewhat columnar, with few conidial chains, mostly 70 to $80 \mu$ wide, occasionally up to $100 \mu$; conidiophore up to 300 to $350 \mu$ long, frequently very short when borne upon the aerial mycelium, broadening to a hemispherical domelike vesicular area at the apex; commonly deep green or greenish-brown; vesicle mostly 15 to $20 \mu$ in diameter, occasionally larger or smaller; sterigmata in one series relatively short and thick, 6 to $7 \mu$ by 3 to $3.5 \mu$; conidia roughened, subglobose, commonly 4 to $5 \mu$ by 3 to $4 \mu$, occasionally $5.5 \mu$ diameter.

Type culture isolated by Talice and MacKinnon from the tympanic membrane of the human ear (1931). It is carried in the NRRL collection as No. 108.

\section{LARGE-SPORED SPECIES, OR THE HERBARIORUM SERIES}

Under Eurotium herbariorum Lk., Mangin includes all of the members of the group with ascospores more than $6.6 \mu$ in long axis (1909). In a general way this represents a very conmon usage in older literature beginning as far back as Corda in the 1830's. Because neither measurements nor markings of the ascospores were given, no one can fix the type of $E$. herbariorum. In general, the species in the large-spored group have both conidia and ascospores definitely larger than those in series already described. They beeome very conspicuous to the collector who finds the anomalous situation of an overabundance of published names and a dearth of isolations. Over a period of many years the scarcity of strains isolated in this laboratory which show ascospores larger than $7.0 \mu$ leads the authors to believe that such forms are definitely rare if not abnormal. This obser- 
vation is, in effect, confirmed by George Smith (1931). From textiles in particular he has isolated many small-spored strains but none with large spores. Possibly the present collection contains as many large-spored strains as it does because the authors have regarded them as curiosities, and for that reason retained them, whereas scores of strains of such common species as $A$, repens or $A$. amstelodami have been isolated and forthwith discarded.

In contrast to the small-spored forms, where complete duplication between large numbers of isolates is the rule, among the large-spored forms there is a marked tendency for each strain to present a somewhat different cultural picture, which is commonly coupled with differences in morphology. This would suggest that these forms are unstable and variable, but such a conclusion is refuted by their behavior in culture. To illustrate, culture NRRL No. 131, a strain of Aspergillus echinulatus, has for 20 years of continuous culture by the authors retained its distinguishing characters, similarly the single known strain of $A$. medius has been under observation in this and European laboratories for more than 40 years without appreciable change.

Thus the problem of assigning a relatively small number of quite distinct strains is presented. To describe each of them would merely add to the confusion already existing, hence they have been grouped somewhat, choosing either historic cultures that have become widely distributed or cultures of marked individuality as representing specific names. Homogeneity among the strains brought together is not claimed. The names A. glaucus and $E$. herbariorum are not identified with particular organisms in this discussion.

In setting apart a so-called "large-spored series" the authors do not, in any sense, wish to imply close relationship or genetic continuity within this subgroup. Species are grouped together primarily as a matter of convenience, and $(E$.$) herbariorum is selected as the series designation primarily$ because of Mangin's usage of this species name to cover all of the largespored forms.

A. Conidial heads green.

$$
\text { Key }
$$

1. Asci ripening within 2 to 4 weeks.

a. Ascospores 6.5 to $7.5 \mu$ in long axis

A. mangini n. comb.

b. Ascospores 7.5 to $8.5 \mu$ in long axis.

c. Ascospores 9.0 to $10.0 \mu$ in long axis

A. umbrosus Bain. and Sart.

A. echinulatus (Delacr.) Thom and Church

2. Asci ripening slowly, 2 to 3 months, colonies favored by 40 per cent sugar.

a. Ascospores with equatorial ridges and furrow........ A. medius Meiss.

b. Ascospores usually without equatorial ridges and furrow

A. carnoyi (Biourge) Thom and Raper

B. Conidial heads white................. niveo-glaucus Thom and Raper 
Aspergillus mangini (Mangin) n. comb.

Synonyms: Eurotium herbariorum ser. minor Mangin, Ann. des Sei. Nat., Bot. (ser. 9) 10: 365. 1909.

Aspergillus minor (Mangin) Thom and Raper, U. S. D. A. Misc. Publ. No. 426, p. 27. 1941.

Colonies upon Czapek's solution agar (3 percent sucrose) very restricted, attaining a diameter of only 1 to $2 \mathrm{~cm}$. in 3 weeks, irregular and wrinkled, cream colored to bluish-brown, conidial heads present or lacking, small perithecia present or lacking, mostly abortive; reverse uncolored to orangemaroon.

Colonies upon Czapek's solution agar with 20 percent of sucrose plane or somewhat wrinkled in the central area, spreading evenly, attaining a diameter of 8 to $10 \mathrm{~cm}$. in 3 weeks (fig. $33 \mathrm{~A}$ ), predominantly brick-red in color becoming maroon in age, perithecia abundant and borne in a close felt of red-encrusted hyphae at the agar surface, conidial heads few in number, projecting above the perithecial layer, generally distributed over the entire colony, but occasionally concentrated in localized areas; reverse in shades of deep red-brown.

Perithecia abundant, largely embedded in and obscured by a close mycelial felt at the agar surface, yellow to orange, globose to subglobose, mostly 100 to $120 \mu$ in diameter, occasionally up to $150 \mu$; asci 14 to $16 \mu$; ascospores lenticular, commonly 6.6 to $7.4 \mu$ by 5.2 to $5.8 \mu$ occasionally up to $7.8 \mu$ in long axis, finely roughened in the equatorial area, ridges low and rounded or pyramidal in section, furrow generally definite, shallow but often steep-sided, V-shaped.

Conidial heads few, generally scattered, projecting above the perithecial layer, pale blue-green in color, radiate, mostly 150 to $200 \mu$ in diameter, but frequently larger; conidiophores smooth, pale to dark brown, mostly 700 to $800 \mu$ in length, occasionally reaching $1 \mathrm{~mm}$. broadening to 15 to $18 \mu$ below the vesicular apex; vesicles subglobose, 30 to $40 \mu$; sterigmata in a single series, 8 to $10 \mu$ by 4 to $5 \mu$; conidia dull green, elliptical to subglobose mostly 6.0 to $7.5 \mu$ and frequently $8.0 \mu$ in long axis.

Represented by culture NRRL No. 117 isolated from an unpainted board, as type; and by several additional cultures isolated in this laboratory. Culture NRRL No. 115, received in 1937 from Oscar W. Richards, of the Spencer Lens Company, differs from the type by producing ascospores of somewhat smaller size and with less evident furrow and ridges. Thus, it may represent a strain transitional between Aspergillus mangini and $A$. ruber. However, it is not sufficiently different from A. mangini, either culturally or morphologically, to warrant its description as a separate species or as a distinct variety.

Mangin (1909), in his study of the group, found specimens in his collec- 


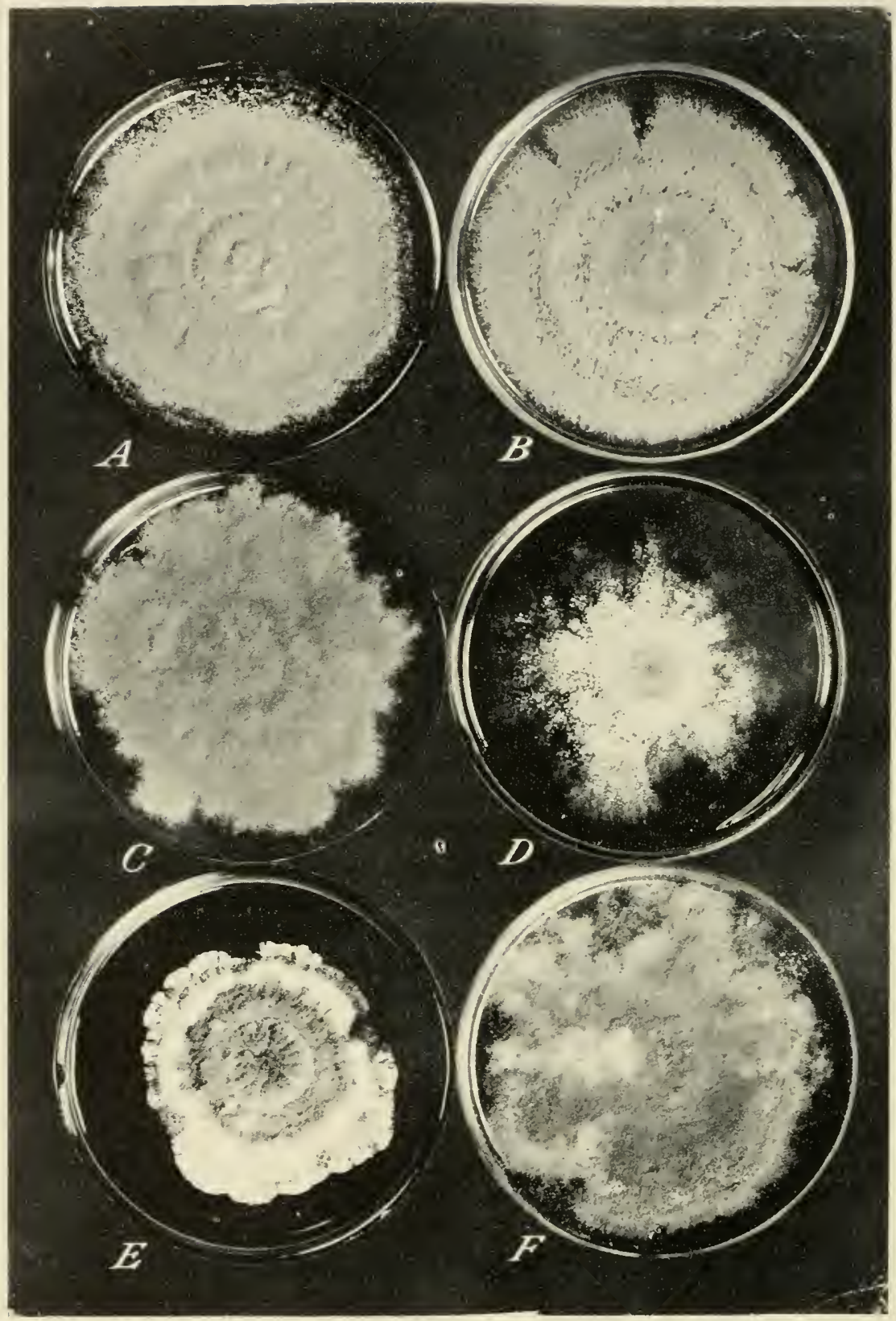

FIG. 33. Comparative growth of large-spored members of the Aspergillus glaucus group upon 20 percent sucrose Czapek agar at room temperature: $A, A$. mangini, NRRL No. 117,4 weeks. B, A. umbrosus, NRRL No. 120, 4 weeks. C, A. echinulatus, NRRL No. 131, 6 weeks. D, A. niveoglaucus, NRRL No. 127, 4 weeks. E, A. medius, NRRL No. 125, 6 weeks. F, A. carnoyi, NRRL No. 126, 4 months. (Reprinted from Thom and Raper, "The Aspergillus glaucus Group," U.S.D.A. Misc. Pub. 426: $1-46.1941$. 
tion with ascospores less than $7.5 \mu$ in long axis, yet larger than those of the small-spored forms which he described. Having no faith in any of the descriptions existing at the time, he called the aggregate Eurotium herbariorum series minnr. The cultures cited above have sufficient common characters to warrant the belief that they are variants of a common stock which may be constituted a species aggregate to which we apply the name Aspergillus mangini.

Aspergillus umbrosus Bain. and Sart., in Soc. Mycol. de France, Bul. Trimest. 28: 267-269, pl. XII. 1912.

Probable synonyms: A. mutabilis Bain. and Sart., in Soc. Mycol. de France, Bul. Trimest. 27: 458, pl. XVII. 1911. A. mollis Bain. and Sart., Soc. Mycol. de France, Bul. Trimest. 27 : 453, pl. XVI. 1911.

Colonies upon Czapek's solution agar (3 percent sucrose) very restricted, attaining a diameter of 0.5 to $1.0 \mathrm{~cm}$. in 3 weeks, raised, tufted, white to orange-red, bearing neither perithecia nor conidial heads; reverse colorless to orange-brown.

Colonies upon Czapek's solution agar with 20 percent of sucrose, plane or somewhat wrinkled, spreading evenly or irregularly (fig. $33 \mathrm{~B}$ ), reaching a diameter of 8 to $10 \mathrm{~cm}$. in 3 weeks, predominantly vinaceous red to orangebrown in color, consisting largely of a surface felt of sterile hyphae encrusted with orange-red granules enmeshing abundant perithecia, occasionally characterized by a loose floccose overgrowth bearing scattered perithecia, conidial heads pale blue-green, widely scattered and projecting above the perithecial layer; reverse in red-brown shades.

Perithecia abundant, yellow to orange, globose to subglobose, largely embedded in a felt of sterile red-encrusted hyphae at the agar surface, occasionally borne in a loose aerial felt, mostly 120 to $140 \mu$ in diameter, rarely up to $175 \mu$; asci 14 to $16 \mu$; ascospores lenticular, mostly 7.2 to $8.0 \mu$ by 5.6 to $6.4 \mu$, occasional spores up to $8.4 \mu$ in long axis, finely roughened to smooth in the equatorial areas, ridges low and generally rounded, furrow shallow, commonly V-shaped (fig. $34 \mathrm{~A}$ ); conidial heads few, scattered, projecting above the perithecial layer, pale bluish-green, radiate, compact, mostly 175 to $250 \mu$ in diameter; conidicphores smooth, colorless to brownish, 700 to $850 \mu$ in length, broadening to 15 to $20 \mu$ below the expanded, domelike vesicular apex; vesicle 25 to $40 \mu$ in diameter; sterigmata in a single series, 10 to $12 \mu$ by 4.5 to $6 \mu$; conidia pale green, elliptical to subglobose, spinulose, mostly 7 to $8 \mu$ in long axis, frequently larger.

Represented in the present collection by culture NRRL No. 120 received in 1925 from Dr. Florence A. McCormick, by European strains, and by several cultures isolated by the authors. 

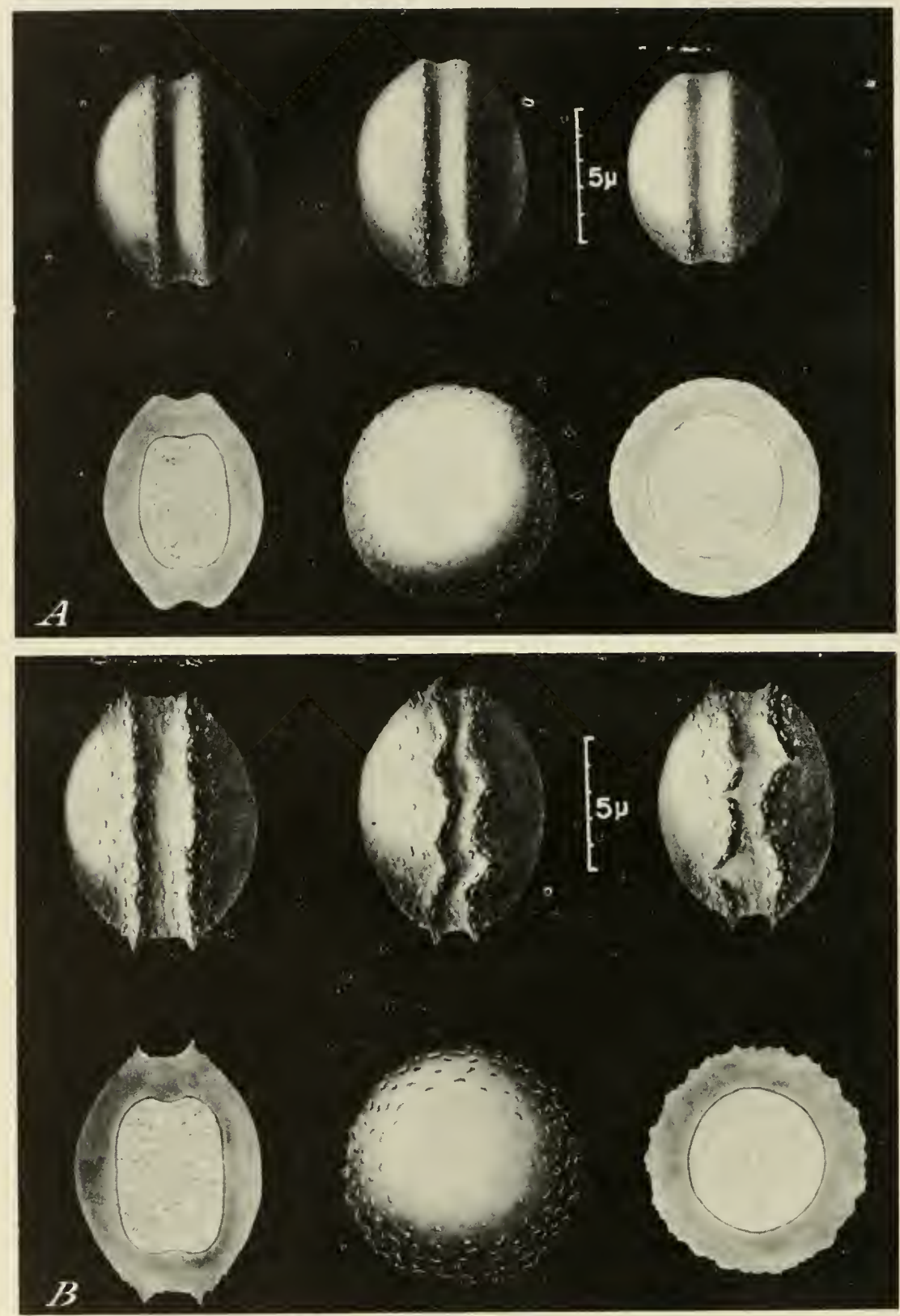

Fig. 34. Ascospores representative of $A, A$. umbrosus, and $B, A$. echinulatus. In each species upper left, right, and center spores represent surface profile views; lower left, optical section in profile; lower center, surface in face view; and lower right, optical section in face view. (Reprinted from Thom and Raper, "The Aspergillus glaucus Group," U. S. D. A. Misc. Pub. 426: 1-46. 1941.) 
Culture NRRL No. 123 contributed by Dr. Paul Simonart as an unnamed culture from the Biourge collection differs from the species as above described by consistently producing ascospores with walls entirely smooth, whereas in other characters the ascospores duplicate essentially those of NRRL No. 120. Further NRRL No. 123 produces colonies of lighter color than other strains under observation and may, in fact, represent a fungus comparable to that described as Aspergillus mutabilis by Bainier and Sartory.

Aspergillus umbrosus, A. mutabilis, and A. mollis were described by Bainier and Sartory (1911c, 1912b) primarily upon the basis of colony color (pigment production) and conidial apparatus, with the ascopsores of $A$. umbrosus recorded as slightly less in long axis ( 8.0 by $5.6 \mu$ ) than those of the other species $(8.4$ by $5.6 \mu$ ). After careful consideration of the three descriptions and detailed study of the strains in the authors' possession showing in general the ascospore described by Bainier and Sartory, it is believed that they had at hand three cultural variants of the same species. A. umbrosus is retained as the species designation, as it is believed that their description of this species more adequately pictures the cultural and morphological characters of the fungi under consideration than either of the earlier descriptions, which are left as probable synonyms.

Aspergillus cchinulatus (Delacr.) Thom and Church, The Aspergilli, p. 107. 1926.

Synonyms: Eurotium echinulatum Delacr., Soc. Mycol. de France, Bul. Trimest. 9: 266, pl. XIV, fig. III. 1893.

A. brunneus Delacr., in Bul. Soc. Mycol. France 9: 185, Pl. XI, fig. 9, 1893; was described as the conidial stage.

E. verruculosum Vuill., Soc. Mycol. de France, Bul. Trimest.

34: 83. 1918.

Colonies upon Czapek's solution agar (3 percent sucrose) very restricted, 1.5 to $3.0 \mathrm{~cm}$. in diameter after 4 weeks, marginal area blue-green from conidial heads and central portion reddish-brown from an overgrowth of sterile encrusted hyphae, perithecia lacking; reverse deep orange.

Colonies upon Czapek's solution agar with 20 percent of sucrose slowgrowing, plane or somewhat wrinkled, spreading irregularly, attaining a diameter of 7 to $8 \mathrm{~cm}$. in 4 weeks (fig. $33 \mathrm{C}$ ), commonly mottled in appearance due to the uneven distribution of green conidial heads above the underlying orange-red perithecial layer; conidial heads bottle-green, abundant, commonly crowded in localized areas but scattered thinly throughout the remainder of the colony; perithecia abundant and borne in a felt of hyphae encrusted with red granules at the agar surface, conspicuous where not obscured by massed green heads; reverse cinnamon to deep red-brown. 
Perithecia abundant, embedded in a looose felt of sterile red hyphae at the agar surface (fig. $26 \mathrm{E}$ ), yellow, globose to subglobose, mostly 125 to $150 \mu$ in diameter, and occasionally up to $175 \mu$; asci 18 to $22 \mu$; ascospores lenticular, mostly 9 to $10 \mu$ by 6.5 to $7.5 \mu$, occasionally up to $11 \mu$ in long axis, conspicuously roughened in the equatorial area, furrow pronounced, broad, ridges prominent and irregular (fig. 34 B).

Conidial heads densely crowded in localized areas and scattered throughout the remainder of the colony, bottle-green in color, radiate, consisting of relatively few, long, divergent chains of conidia, commonly 250 to $300 \mu$ in diameter but often larger or smaller; conidiophores smooth-walled, colorless to brown shades, commonly 700 to $850 \mu$ in length, occasionally in excess of $1 \mathrm{~mm}$., broadening from 5 to $7 \mu$ at the base to 15 to $20 \mu$ below the vesicular apex; vesicle 25 to $35 \mu$ in diameter, consisting of a domelike terminus of the broadening conidiophore; sterigmata in a single series, not crowded, bottle-shaped, 12 to $15 \mu$ by 5 to $7 \mu$; conidia elliptical, pyriform, or subglobose, echinulate, mostly 8 to $10 \mu$ in long axis, commonly larger or smaller, extremely variable.

Represented by NRRL No. 131 isolated in 1921 from figs received from California. A subeulture of this strain, forwarded by Miss Margaret Church about 1926, is maintained in the Centraalbureau; the two lines remain identical. No ascosporic stage has in the authors' experience been found in culture NRRL No. 133 received in 1937 from George Smith as A. echinulatus Delac., and obtained by him from Biourge, but its conidial development duplicates NRRL No. 131 and it is apparently correctly assigned. Da Fonseca's and the Centraalbureau's isolations of A. echinulatus maintained at Baarn produce somewhat smaller ascospores (8 to $9 \mu$ by 6.2 to $7.0 \mu$ ) and conidia than No. 131, but otherwise agree essentially with the species description as given above. Somewhat further reduction in ascospore size is seen in cultures NRRL No. 523 isolated from honey, and NRRL No. 137 received from George Smith and Raistrick as Aspergillus mongolicus Biourge (nomen nudum). Although these are less red in color than No. 131 and appear distinct in culture, the authors do not feel warranted in separating them as a species or variety, believing that they represent only variations from the general type designated as $A$. echinulatus.

Bainier and Sartory described $A$. disjunctus (1911b) and $A$. repandus (1911c) as vigorous species possessing ascospores 11 by $6 \mu$ and 11.2 by $5.6 \mu$, respectively. Authentic cultures of these speeies are not now available, but the deseriptions as published would seem to place them close to A. echinulatus.

\section{Probable Synonyms}

Several Aspergilli with ascospores ranging near that described for $A$. cchinulatus appear in the literature. Unfortunately, the details of asco- 
spore markings are not given and there is a dearth of data to identify them. Some of thesc names are given:

A. disjunctus Bainier and Sartory, in Soc. Mycol. France Bul. 27:346-368. Pl. X, XI. 1911. Ascospores described as 11.2 by $5.6 \mu$ with furrow and crests.

A. repandus Bainier and Sartory, in Soc. Mycol. France Bul. 27: 463. Pl. XVIII. 1911. Ascospores described as 11 by $6 \mu$ with furrow but no crests.

A. mencieri Sartory and Flament, in Compt. Rend. Soc. Biol. (Paris) 83: 1114-1115. 1920. Ascospores 10 by $4.7 \mu$ with furrow and crests.

A. godfrini Sartory and Roederer, in Assn. Française pour l'Avancement des Sciences, 42nd Session, Tunis 1913. pp. 601-603. 1914. Conidial stage only. This was growing at blood heat and warmer. Its general description suggests affinity with the large-spored species of the A. glaucus group. It has not been seen in culture by us.

Strains with the particular measurements reported for the three ascosporic species listed above have not come into our collection, yet presumably they and many more with minor variations may be found.

Aspergillus medius Meiss., in Bot., Ztg. 55: (337)-344, (353)-357. 1897.

Colonies upon Czapek's solution agar (3 percent sucrose) very restricted, 0.5 to $1.5 \mathrm{~cm}$. diameter in 6 weeks, tufted, consisting of a dense growth of yellow-brown hyphae bearing neither conidial heads nor perithecia; reverse in shades of yellow-brown.

Colonies upon Czapek's solution agar with 20 percent of sucrose at room temperature very slow-growing (optimum $20^{\circ} \mathrm{C} . \pm$ ), strongly wrinkled, tufted, irregular in outline, attaining a diameter of 5 to $6 \mathrm{~cm}$. in 6 weeks (fig. $33 \mathrm{E}$ ), colony center deep orange-red becoming ycllow to white at the margin, which is characterized by bundles (becoming branching columns at lower temperatures) of hyphae bearing dark-green conidial heads in the manner of loose divergent coremia; perithecia ripening very slowly, maturing ascospores in 2 to 3 months, mostly abortive; conidial heads relatively few (more abundant and larger at lower temperatures) and borne either in coremiform masses or scattered throughout the colony; reverse in shades of orange-maroon.

Perithecia scattered, mosily abortive, borne in a dense felt of orange-red hyphae, very slowly ripening, globose to very irregular in form, extremely variable in size, rarely attaining a diameter of $125 \mu$, containing very few mature ascospores; asci 18 to $20 \mu$; ascopsores sparingly produced, lenticular, mostly 8.8 to $9.6 \mu$ by 6.0 to $6.8 \mu$, occasionally $10 \mu$ in long axis, somewhat roughened in the equatorial region, furrow broad and shallow, ridges prominent, relatively thin and irregular.

Conidial heads deep-green, radiate, compact, and of two types: Small heads 100 to $150 \mu$ in diameter borne on loose coremiform columns, and larger heads 200 to $250 \mu$ in diameter often scattered throughout the colony, 
produced more abundantly at $12^{\circ}$ to $15^{\circ} \mathrm{C}$. than at room temperature; conidiophores colorless to brown, mostly 250 to $350 \mu$ in length, enlarging to 15 to $20 \mu$ below the vesicle; vesicle subglobose, mostly 30 to $40 \mu$ in diameter; sterigmata in a single series, crowded, short, 7 to $8 \mu$ by 4 to $5 \mu$; conidia green, globose to subglobose, finely echinulate, thick-walled, mostly 8 to $10 \mu$ in diameter, but frequently larger or smaller.

Represented in the NRRL colleetion by culture No. 124 which was received in $192+$ from Raistrick, who in turn reeeived it from the Centraalbureau. It is believed to be Meissner's original strain (1897). Subcultures of this strain are currently maintained at Baarn and by George Smith in London. The three lines remain identical as shown by parallel cultures during recent study.

This fungus is distinguished particularly by (1) its very slow growth upon 20-percent sucrose Czapek agar at room temperature, (2) its tardiness in produeing perithecia and especially in ripening ascospores, (3) its sparse production of ascospores, and (4) its formation of aerial hyphal bundles bearing conidial heads in loose coremiform fashion. Further, it grows much more rapidly upon Czapek agar containing 40 percent of suerose than upon that eontaining 20 percent, a difference in concentration which does not materially affect the growth rate of such vigorous species as $A$. repens and $A$. chevalieri. Growth is much more rapid at $20^{\circ} \mathrm{C}$. than at $28^{\circ}$ to $30^{\circ} \mathrm{C}$. (fig. 12). The fungus attains a more favorable form at the lower temperature, at which there is a heavier growth of mycelium, a more extensive development of aerial hyphal columns, and a greater production of conidial heads and perithecia.

Culturally this fungus is easily separated from all other species of the group, except possibly A. carnoyi.

Aspergillus carnoyi (Biourge) Thom and Raper, U. S. Dept. Agr. Misc. Pub. 426, p. 34. 1941.

Colonies upon Czapek's solution agar (3 percent sucrose) very restricted reaching a diameter of only 3 to $4 \mathrm{~mm}$. in 6 to 8 weeks, thin, white, bearing neither conidial heads nor perithecia; reverse colorless.

Colonies upon Czapek's solution agar with 20 percent of sucrose at room temperature extremely slow growing (optimum $18^{\circ}$ to $20^{\circ} \mathrm{C}$.), reaching a diameter of 7 to $8 \mathrm{~cm}$. in 6 to 8 weeks, irregular in outline, somewhat floccose, forming a deep felt, bearing abundant perithecia and scattered conidial heads (fig. $33 \mathrm{~F}$ ), orange-brown in central area to orange at margin from abundant peritheeia in a loose network of sterile hyphae encrusted with orange-red granules; reverse in orange-red shades.

Perithecia late in developing, abundant, yellow to orange, globose to subglobose, mostly 125 to $175 \mu$ in diameter but frequently larger or smaller, borne in a loose floccose felt of sterile brown hyphae; asei 16 to $18 \mu$, 
typically 8-spored, frequently with some or all spores aborted; ascospores lenticular, variable in size and pattern, mostly 7.2 to $8.4 \mu$ by 6 to $6.5 \mu$ but often larger (up to $9.0 \mu$ in long axis) or smaller (down to $6.5 \mu$ in long axis), generally smooth-walled but occasionally roughened in equatorial area, generally rounded but often flattened and occasionally indented, with ridges wholly absent or indefinite, and with furrow absent or present as a trace only.

Conidial heads sparsely produced, commonly scattered, dull gray-green, radiate, compact, mostly 150 to $200 \mu$ but often up to $250 \mu$ in diameter; conidiophores smooth-walled, colorless, long, commonly up to $2 \mathrm{~mm}$. in length, uniform in diameter, 12 to $18 \mu$, to just below the resicle; vesicle subglobose, 40 to $50 \mu$ in diameter and occasionally larger; sterigmata in a single series, crowded, bottle-shaped, 10 to $12 \mu$ by 5 to $6 \mu$; conidia globose to subglobose, echinulate, dull green, mostly 8 to $10 \mu$.

Species description based upon culture NRRL No. 126 received in 1937 as Aspergillus carnoyi Biourge from George Smith and by him earlier from Biourge. Presumably the culture is type, although Biourge's description of the species remains unpublished. The culture is distinct, not only differing in its colony character and in the length of its conidiophores but especially in the variable character of its ascospores. The majority of spores, although much larger, resemble those of $A$. repens, whereas spores with rough walls are occasionally produced. This culture is, therefore, somewhat of an exception to the general rule of constancy in ascospore pattern, and the variability of its spores affords one of the best characters for its identification.

Aspergillus niveo-glaucus Thom and Raper, U. S. Dept. Agr. Misc. Pub. 426, p. 35.1941.

Synonyms: A. glaucus mut. alba Bloch., Deut. Bot. Gesell. Ber. 50: 248-256. 1932.

A. glaucus var. albida. Speg., An. del Mus. Nac. de Buenos Aires 6: 332. 1899.

Colonies upon Czapek's solution agar (3 percent sucrose) very restricted, $1 \mathrm{~cm}$. in diameter after 4 weeks, white to cream, bearing abundant small conidial heads but no perithecia; reverse coloriess to yellow-brown.

Colonies upon Czapek's solution agar with 20 percent of sucrose slow growing, plane, spreading irregularly, 6 to $8 \mathrm{~cm}$. in diameter after 4 weeks (fig. $33 \mathrm{D}$ ), thinning toward the margin, with mycelium in shades of yelloworange becoming cinnamon brown in age, more or less obscured by abundant white heads, and with perithecia abundant, yellow (Pl. III F), embedded and irregularly clustered in a close felt at the agar surface; reverse yellow at margin to deep brown at colony center.

Perithecia abundant, yellow, globose, to subglobose, mostly 100 to $125 \mu$ 
in diameter, occasionally larger, commonly clustered, borne in an interrupted surface felt of buff to brown hyphae; asci 15 to $17 \mu$ in diameter; ascospores lenticular, mostly 7.2 to $7.8 \mu$ by 5.0 to $5.6 \mu$, smooth-walled
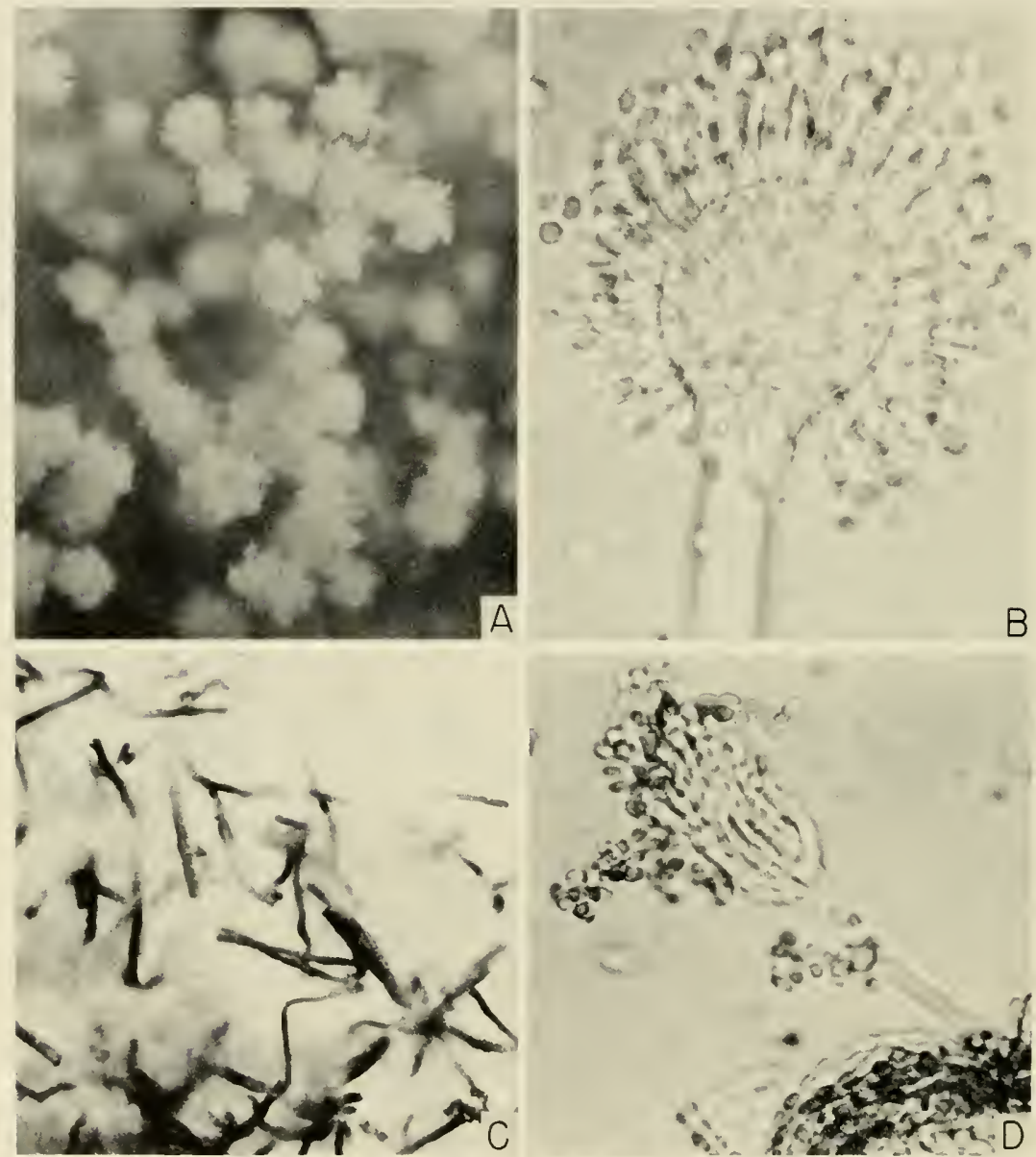

FIG. 35. Aspergillus glaucus group, conidial heads. $A$ and $B, A$. niveo-glaucus, NRRL No. 127: A, surface view showing loose, radiate character of heads, $\times 35$; $B$, photomicrograph of the same showing large, globose vesicle fertile over almost the entire surface, $\times 600$. $C$ and $D$, Aspergillus restrictus, NRRL No. 154: $C$, surface view showing long, columnar heads, $\times 120 ; D$, photomicrograph of the same showing characteristic small vesicle fertile on the uppermost surface only, $\times 600$.

except in equatorial area, furrows broad and shallow, ridges prominent, roughened, rounded or acute and often appearing ragged. Conidial heads abundant, white (fig. $35 \mathrm{~A}$ ), often becoming browned in age, radiate, mostly 250 to $300 \mu$ in diameter: conidiophores smooth-walled, colorless to brown, 
mostly 1,000 to $1,500 \mu$, rarely longer, broadening to 16 to $20 \mu$ below the vesicle; vesicle subglobose, 40 to $50 \mu$ in diameter (fig. $35 \mathrm{~B}$ ); sterigmata in a single series, crowded, 8 to $10 \mu$ by 3 to $4 \mu$; conidia elliptical to pyriform, colorless, spinulose, 6 to $8 \mu$ in long axis.

The eultural description is based upon NRRL No. 127 of this collection as type; this is duplicated by NRRL No. 130 received from Baarn in 1938 as Aspergillus glaucus Link mut. alba Bloch. Culture NRRL No. 128, received in 1935 from George Smith and Raistrick and by them from Biourge as A. albidus Speg., differs from type by consistently producing a greater quantity of conidial heads which are commonly of smaller size; the ascosporic stage of the two is indistinguishable. Apparently the name "albidus" is a manuscript use in which Spegazzini's variety (1899) has been raised to species rank, presumably by Biourge. Blochwitz' mutation albus assigned to $A$. glaucus is untenable, because no definite organism can be designated as A.glaucus. Furthermore, the specific name albus applied to an Aspergillus has already been used in another section of the genus.

It is possible that Blochwitz (1932c) is right in regarding this as a mutation, but there is nothing to indicate which particular large-spored form is the parent species. The strain maintains its identity in culture and hence must be regarded as a species. Yuill (1939), in contrast, has described white mutants of $A$. nidulans and $A$. fumigatus and has properly designated them as mutants, for they appeared in cultures under observation and are known to have been derived from typical chromogenic strains.

\section{THE ASFERGILLUS RESTRICTUS SERIES}

Thom and Church (1926) called this series the "Intermediate Forms" between the A. glaucus and the A. fumigatus groups. George Smith, wishing to emphasize the resemblance of the conidial apparatus to the monoverticillate Penicillia called the lot the A. penicilloides group (1931), thus suggesting Spegazzini's species as the typical member. However, the important relationship indicated by the structure of the conidial apparatus is not with Penicillium but with the A. glaucus group of which these forms appear to be merely reduced members. All of these forms, like many of the A. glaucus forms, grow characteristically under conditions of physiological drought-represented by their frequency upon mildewed textiles as studied by Smith (1928) and Galloway (1930) or in concentrated cane products as reported by Owen (1923). Similarly we have found them in many situations in which physiological drought is attained by physical dryness or osmotic concentration attained by the presence of high percentage of sugar or sodium chloride.

This natural relationship is on the whole better indicated by accepting Smith's A. restrictus as typical, and regarding the other known members 
of this series as allied species. To perpetuate an assignment to a subdivision entitled the Microaspergilli, as suggested by some authors, would complicate nomenclature without compensating values. The series is, therefore, keyed as a part of the great $A$. glaucus group.

Series diagnosis: Perithecia not found. Colonies growing weakly or restrictedly upon Czapek's solution agar, more freely upon wort agar, especially well on high concentrations of sugar or salt; green, dark green, grayish-green, to brownish-green in various strains and under varying conditions; surface growth consisting of conidiophores only, or of mycelial felts more or less buckled or heaped; conidiophores smooth, slender, more or less sinuous, septate; vesicles vary from convex or lens-like areas on the broadened apices of conidiophores to definitely ovate to globose enlargements, fertile over all or the upper fraction of such surfaces; heads mostly definitely columnar, less commonly radiate, hemispherical or almost globose, especially when young; sterigmata in one series, mostly closely packed over the fertile area, varying from 2 to $3 \mu$ by 5 to $6 \mu$ up to 3 to $4 \mu$ by 6 to $10 \mu$; conidia barrel-shaped to ovate, mostly in dark greenish shades, smooth or slowly becoming echinulate or roughened as in the A. glaucus group, commonly adherent into long chains which are packed into columns.

\section{Series Key}

A. Conidiophores broadening upward to produce a convex vesicular apex varying from 8 to $20 \mu$ in diameter, producing a long slender column of conidia

A. gracilis Bainier

B. Conidiophores broadening more abruptly, forming a more definite vesicular area.

1. Vesicles more convex, toward hemispherical.

a. Slime development evident. A. conicus Bloch.

b. Slime absent or not conspicuous A. restrictus G. Smith

2. Vesicles ovate to hemispherical, columns of conidial chains more or less conspicuous......................... penicilloides Speg.

Differences between the above may appear to be of somewhat minor character. Nevertheless, each of the sections accounts for sufficient literature to necessitate separate consideration.

Aspergillus gracilis Bainier, in Bul. Soc. Myc. France, 23: 92, pl. IX, figs. 11-14. 1907.

Colonies on Czapek's solution agar very slow growing, reaching a diameter of only a few millimeters in several weeks (fig. 36A), variously plane or convoluted or buckled with close textured mycelium at first white, then slowly green to very dark green, with radiating lines of vegetative mycelium about the denser area of the colony, growing somewhat better upon wort agar and upon Czapek's agar containing high concentrations of sugar, reverse in yellowish shades, conidial heads in columns up to 200 or $300 \mu$ long 
by 10 to 20 even $25 \mu$ in diameter, straight or twisted. Conidiophores mostly arise as very short branches of aerial hyphae up to 20 to $30 \mu$ long or less commonly up to 100 to $125 \mu$ even up to $250 \mu$ and gradually broadening toward the apex to a vesicular area which is very flat, dome-like, almost the effect of a truncated cone, 8, 10 to $20 \mu$ or more in diameter, bearing a single series of sterigmata, $6,8,10$ by 2 to $3 \mu$, or in particular strains growing out into little conidiophores producing secondary heads; conidia at first barrel form then subglobose about $3 \mu$ in long axis in Bainier's strain, progressively larger in related strains. No perithecia found.

The type culture has not been seen. Forms with approximately the morphology described, however, have appeared in strains NRRL No. 145 (Thom 4246) from moldy corn; Thom 4197.3 (culture lost) from Owen in
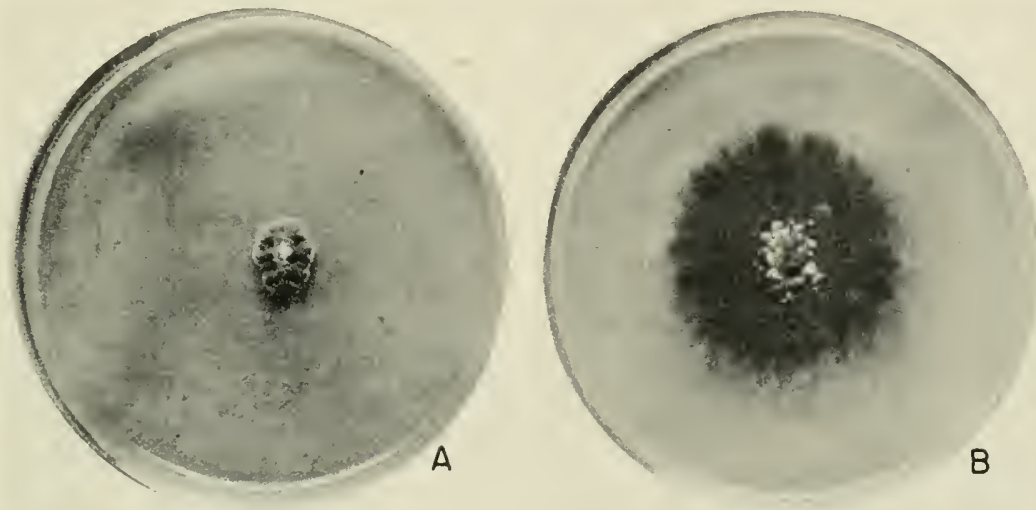

FIG. 36. A, Aspergillus gracilis, NRRL No. 145, on Czapek's solution agar with 20 percent sucrose, after incubation for 2 weeks at room temperature. $B, A$. restrictus, NRRL No. 154 , on Czapek's solution agar with 20 percent sucrose after 18 days at room temperature.

the sugar laboratory, New Orleans; and a strain from Thaxter appearing as a contaminant in a Papulaspora culture. A. gracilis may thus be assumed to represent a form occasionally found especially in very concentrated substrata. Biourge later contributed a culture, as type, of A. hypojanthinus originally described by him as Penicillium hypojanthinum Biourge (1923). Three other cultures from Biourge labeled P. (Microaspergillus) hickeyi, Microaspergillus albo-marginatus, and P. (M.) guegueni (figured in his monograph, Plate XX, but not described) appear in culture to be only minor variations of this general form. Later, Biourge sent his undescribed A. sartoryi. This grew more freely and showed the conidiophore and vesicle of $A$. gracilis; conidia were 6 to $7 \mu$ or greater in long axis, definitely rough and corresponded almost exactly with a culture received from George Smith 
as A. gracilis (NRRL No. 156). Whether further accumulation of strains will justify giving Biourge's proposed name, A. sartoryi, sectional or varietal status or merely emphasize the completeness of the series as showing great variations in structural detail is left uncertain. There does not appear to be any warrant for preserving $A$. gracilis var. exiguus Bainier and Sartory (1912 a). According to the description this variety differs slightly in physiological characters from $A$. gracilis Bainier.

A. conicus Blochwitz, in Dale, Ann. Mycol. 12: 38. 1914. Previously described by Dale as a Penicillium in Ann. Mycol. 10: 465. 1912.

See also Thom and Church "The Aspergilli" p. 125. 1926.

The outstanding character of this species is found by microscopic examination of colonies which become buckled or contorted from a close felting of mycelia. Relationship back toward the A. glaucus group is found in the sterigmata, and in the elliptical conidia. Heads differing little in microscopic structure from $A$. gracilis are found to be more or less completely submerged in dark green to almost black slime. Many strains have been collected from widely separated regions showing all gradations from a trace of slime only to complete submergence of the heads, particularly in old cultures.

Dale isolated the strain first described from English soil, and sent duplicate cultures labeled Penicillium sp. to Blochwitz and to Thom; Thom returned the very brief descriptive note without name as published by Dale in 1912. Later Blochwitz proposed by letter to her the name only, $A$. conicus, which was published by Dale in 1914 without further description. Later, in his own publication, Die Gattung Aspergillus (1929), Blochwitz denies the slime development as a character of his organism and redescribes the species in terms to make $A$. conicus cover the section of this group represented by Smith's $A$. restrictus (1931). Since the name was already in the literature for the slimy series which certainly appears in culture from widely separated places, it should stand as originally applied. Whether the slime disappears in some strains long kept on artificial media is not settled.

In 1928, Biourge contributed under the manuscript name $A$. cyanogencs a strain which reproduced the characters given by Thom and Church for $A$. conicus. This or a nearly related organism appeared as a contaminant in several of the cultures received from Biourge. The culture (Thom No. 4733.138) figured in his Monograph (1923) as No. 126 in Plate XXI, under P. glabrum Dale was another. Still another strain from Biourge, labeled A. viridans differed only in the delayed development of the slimy covering of the columnar mass of conidia. 


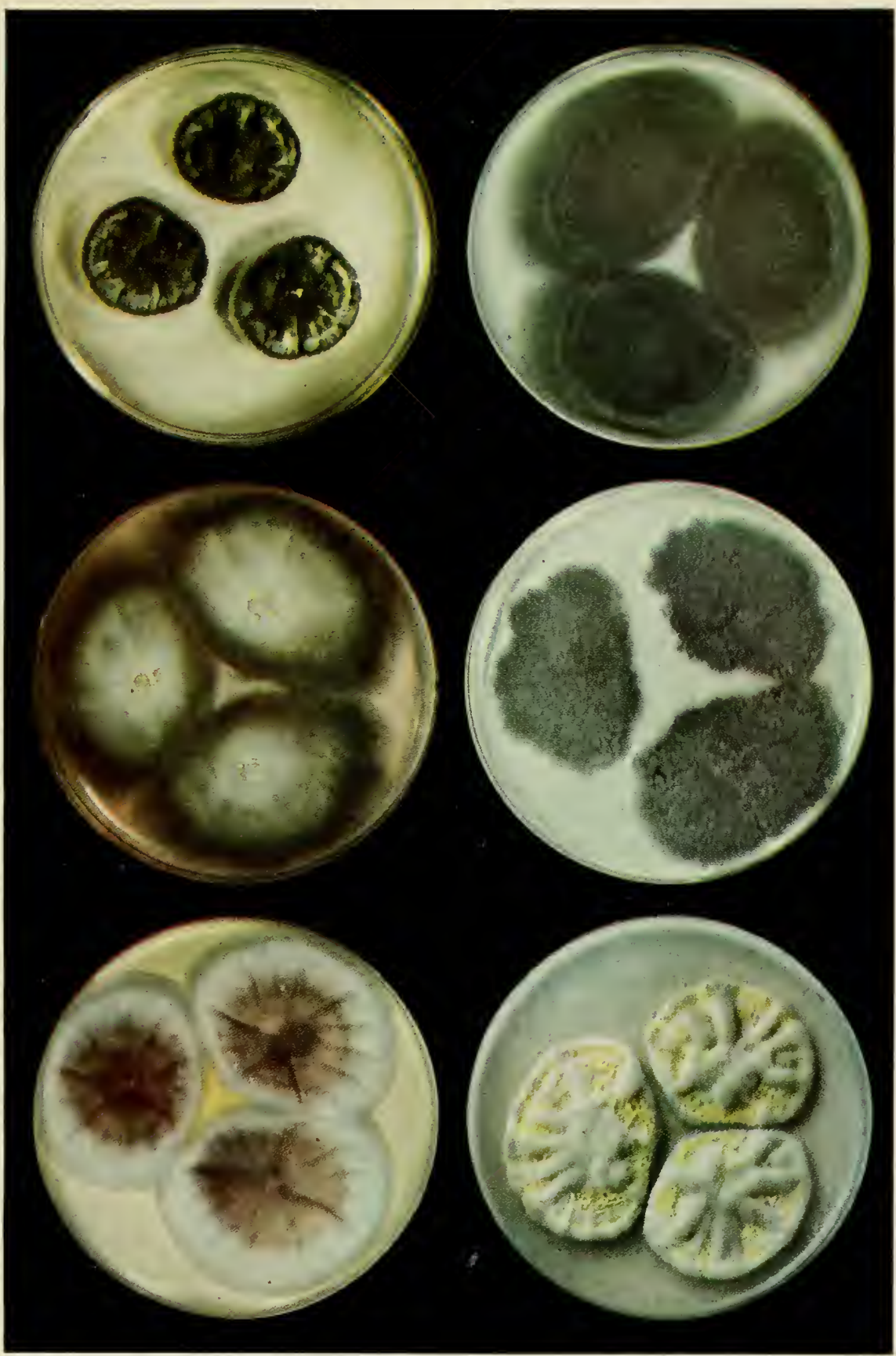

Plate IV

A (upper left), Aspergillus restrictus Smith, XRRL No. 154. $B$ (upper right), Aspergillus fumigatus Fresenius, NRRL No. 163. C (center left), Aspergillus nidulans (Eidam) Wint., NRRL No. 192. $D$ (center right ), Aspergillus variecolor (Berk. and Br.) Thom and Raper, NRRL No. 1954. E (lower left), Aspergillus ustus (Bain.) Thom and Clureh, NRRL No.2-s. F (lower right), Aspergillus flaripes (Bain. and Sart. Thom and Church, NRRL No. 295. Figure A growing upon Czupek's solution agar containing 20 perrent sucrose and 0.5 percent peptone; all other cultures growing upon standard Czapek's solution agar. Color photographs by Haines, Northern Regional Research Laboratory. Reproduced through co-operation of Chas. Pfizer \& Co., Inc.) 

Neill in his study of the Aspergilli of New Zealand (1939) applied the name A. caesicllus Saito (1904) to what was manifestly some strain near $A$. conicus Blochwitz, and then placed the whole group in that speeies.

\section{A. restrictus G. Smith, in Jour. Text. Inst. 22: T115, fig. V. 1931.}

\section{Species characterization by George Smith}

Colonies growing very poorly on Czapek agar; growing moderately well on wort agar, dark dull green, gradually turning grey or brownish-grey; reverse in some eultures uncoloured, in others green to dark green; surface velvety at first, becoming wrinkled and often aequiring a warted appearance (Pl. IV A and fig. $36 \mathrm{~B}$ ); heards forming long, compact, slender columns (fig. 35 C) up to $350 \mu$ by 20 to $30 \mu$ in diameter; conidiophores arising mostly from substratum but also as branches of aerial hyphac, commonly 50 to $100 \mu$, oceasionally $150-200 \mu$ long by 3 to $3.5 \mu$ in diameter, often with one or two septa, smooth, sinuous, uncoloured; vesicles flask-shaped 7.5 to $14 \mu$ in diameter; sterigmata in one series, borne on upper surface of vesieles only, 6 to $9 \mu$ by 2.5 to $3 \mu$ (fig. $28 \mathrm{C}$ ); conidia rough, spinulose, elliptieal or somewhat pyriform, often showing a distinct eonnective, dark greenishbrown, 4 to $6.5 \mu$ by 3 to $4 \mu$, mostly 4.5 to $6 \mu$ by 3 to $3.5 \mu$; perithecia not found. The young eonidia are hyaline and cylindrieal and almost appear to be segments of enormously elongated septate sterigmata. They gradually swell without inereasing in length, at the same time becoming pigmented, but even in old heads they adhere strongly together in columns of parallel chains; and mounts (fig. $35 \mathrm{D}$ ) made in lactophenol usually show eompact, twisted, columnar masses of ripe conidia, both attached to and separated from the heads.

It is evident from Smith's discussion of a large aceumulation of cultures, especially from the textile industry, that the usual colony-type in his experience is not the "conicus" type with its slime but the columnar head deseribed as $A$.restrictus and aceepted here as giving the most appropriate name to the series.

Among organisms belonging to this series, Smith isolated a single strain which differed from typical A. restrictus in "showing somewhat larger dimensions throughout and, more particularly, in the production of conidia up to $10 \mu$ or more in length," and to which he assigned the designation A spergillus restrictus var. B. (G. Smith, Jour. Text. Inst. 22: T115. Figs. IV, VI, and VIII. 1931). Upon examination by us, this culture (NRRL No. 148) was found to correspond fairly closely to his published description. However, we do not believe it is sufficiently distinct to warrant continued separation as a variety since other strains showing intermediate dimensions are encountered. 
Aspergillus penicilloides Spegazzini, in Rev. Agrar. Veter.

La Plata, p. 246. 1896.

Spegazzini's description was emended by Thom and Chureh (The Aspergilli, p. 126, 1926) then broadened by Smith (Jour. Text. Inst. 22, pp. $114-115,1931)$ as follows:

"Colonies growing fairly slowly on wort agar, rich dark green with paler edge, turning darker and duller, and finally becoming dirty greenish-grey overgrown with sterile hyphae; reverse brown, greenish-brown, and dark green in patches; surface much wrinkled and folded; heads globose when young, 40 to $70 \mu$ in diameter, becoming columnar, somewhat ragged, and up to $200 \mu$ long; conidiophores arising either from substratum or from aerial hyphae, smooth, thin-walled, 75 to $150 \mu$ long by 6 to $10 \mu$ in diameter; vesicles rather sharply marked off from conidiophores, pear-shaped to subglobose, 15 to $23 \mu$ in diameter, fertile over the upper half or two-thirds; sterigmata in one series, crowded, 8 to $10 \mu$ by 2.5 to $3.5 \mu$; conidia ovate, barrel shaped or nearly spherical, usually showing connective, rough, 3.5 to $5 \mu$ by 3.2 to $4 \mu$, with very dark colored walls."

Thom and Chureh had strain No. 4197.3 isolated from cane products in Louisiana by Owen and agreed to by Spegazzini; NRRL No. 151 (Thom No. 7), also from cane products, fits this deseription satisfactorily; as did also Biourge's strain labeled $A$. pertardus. Smith reports various strains from mildewed textiles.

It would thus appear that the vesicular area in the series varies from the curved apex of a clavate conidiophore as in $A$. gracilis, to a fairly welldefined hemispherical vesicle as in $A$. restrictus, and finally to an almost globose body as in $A$. penicilloides. All have so much in common with each other and with the A. glaucus group that their relationship as "degraded" mutants appears probable.

Aspergillus itaconicus Kinoshita, in Botan. Mag. Tokyo 45: 60-61. 1931.

Probable synonym: A. varians Wehmer, in Bot. Centralb. 80: 460-1. 1899; also in Wehmer Monogr. 77-79, Taf. I, fig. 1. 1899-1901.

Diagnosis from Kinoshita's organism obtained from Dr. Westerdijk. Colonies on Czapek's solution agar forming dense felts 1 to $2 \mathrm{~mm}$. deep, white or yellowish, ridged and irregular, with scattered long-stalked green heads upon dry areas and on the glass; fruiting more abundantly upon malt agar, and particularly upon Czapek's solution agar containing 20 percent sugar; reverse of colony and agar yellow to orange-reddish upon some media; heads large, light green, globose to radiate, breaking up easily under the coverglass; conidiophores smooth, colorless, 8 to $16 \mu$ or larger in diameter and up to several millimeters in length under some conditions, with 
walls 0.5 to $1.5 \mu$ in thickness and splitting lengthwise as $A$. niger when broken; vesicles 15 to $40 \mu$, globose or subglobose (fig. $28, \mathrm{D}$ ); sterigmata in 1 series 8 to $9 \mu$ by 1.5 to $2 \mu$; conidia more or less pyriform, 4.3 to $5 \mu$ by 3.5 to $4 \mu$, finely echinulate, of the $A$. glaucus type.

Diagnosis is drawn from culture NRRL No. 161 (No. 5344 of Thom) received from Westerdijk as the type of Kinoshita's A. itaconicus and agrees closely with the description given by Wehmer for $A$. varians (cf. Thom and Church, The Aspergilli, p. 127, 1926), not with Thom and Church's description of their culture No. 115 which was subsequently lost. A. itaconicus is so closely related in its physiological responses and in several of its structural characters to the $A$. glaucus group that it is placed as an extreme variant at the end of this whole group.

Kinoshita described his organism as a producer of itaconic acid (1931a) and detailed his experimental work (1931b). The culture as distributed to laboratories outside of Japan seems to conform to Kinoshita's description and to produce the acid, but in quantities too small for commercial development. More recently, Calam, Oxford, and Raistrick (1939) have recovered itaconic acid as a metabolic product of $A$. terreus. From an industrial point of view, this source appears to be far more promising.

\section{VARIATION IN THE ASPERGILLUS GLAUCUS GROUP}

The genetic history of the Aspergilli is an untouched field. Separation into large groups is easily made definite enough to include all but a few strains. Within these groups, variation is so great that differentiation of species requires critical examination and comparison of material, including extensive culture. Unwilling to undertake this, Neill (1939) disposed of the whole group with yellow perithecia by calling them all $A$.glaucus. On the other hand, Mangin (1909), with the same problem of variability before him, found the ascospores sufficiently distinctive and dependable to warrant proposing to separate $A$. amstelodami and $A$. chevalieri as separate species in the $A$.glaucus group, leaving certain aggregates admittedly inadequately studied. Bainier and Sartory (1911b, 1911c, 1912), working with members of these ill-defined species, used color production as the basis for separation. They cited ascospore measurements as incidental details in description. Because they appear to have had only a few strains in culture and to have described them all as new species, the task presented few difficulties to them; but unfortunately without the original cultures, no one has ever been able to identify their species with confidence. Raistrick and his colleagues (1934, 1937, 1938, 1939), using cultures named as received and including an unpublished series from Biourge, have given quantitiative figures as to pigment production and pigment mixtures for each culture listed by the name on the tube, without reporting comparative study of the morphology found. 
It is clear that wide mycelial or colony variations may be found in nature between strains that retain the ascospore characters of the species. Several such groups have been held by the authors for 30 years or more and furnish convincing evidence that Mangin was justified in using the ascospore as the stable and readily determinable integrating character. Some of these forms retain colony characters in fairly stable form through many transfers on many laboratory substrata and over a period of years. Others grown in various substrata in Petri-dish cultures show sectors or other irregularities in colony habit, which can be picked out and established as strain variants that maintain their special characteristics in continuous culture.

The possibility that variants of similar nature might be induced by chemical stimulation led Thom and Steinberg (1939) to select from the authors' collection certain strains that had remained fairly constant for many years. Of this group they subjected strain NRRL No. 90 of A. amstelodami, found apparently stable for 30 years, to extensive chemical stimulation (1940a). These experiments yielded two groups of effects: (1) A progressive reduction in the production of conidial heads and of perithecia, and (2) a great increase in the mass of vegetative mycelia. In no ease was spore production completely suppressed although reduced to inconspicuous quantity. The conidia and ascopsores when examined were found to have retained the size and markings characteristic for the species, whereas the mass of vegetative mycelium became excessive and formed a floccose or cottony mass entirely different in colony appearance from the original.

At this point they reversed the procedure and applied stimulants designed to reestablish spore production (1940b). As a result, the final cultures show abundant green heads with normal conidia and numerous perithecia with ascospores retaining the characters of the species. In routine laboratory examination these extreme variants would not suggest the original strain of A. amstelodami, although both types of rariants produce eonidia and ascospores typical of the species.

In 1928, Barnes studied the possibility of heat in inducing variations. He used a strain reported as "Eurotium herbariorum (Wigg) Link," which had been isolated and maintained in his laboratory for several years without apparent changes. Unfortunately, no description of his normal strain was given, but a strain received from Westerdijk as "Barnes' normal strain" proves to be identical with $A$. amstelodami (Baarn strain, NRRL No. 89; or strain No. 90 as used by Thom and Steinberg). No reasons were offered for the original identification.

Barnes described a series of experiments in which the spores of his organism were subjected to heat under varied conditions, then planted. From the resulting colonies he described 11 variants, 6 of which are available in the Centraalbureau. The authors' transfers of these have been 
checked against the deseriptions in Barnes' paper and obviously represent his isolations.

In the following list, his designations appear as quotations, followed by our identifications based upon careful eultural study:

"Flame" variant is A. ruber.

"Green flame" is A. repens.

"Blue conidial" is A. chevalieri var. intermedius.

"Creamy" is a pale yellowish strain of Yuills' genus Cladosarum.

"D Brown" is A. ustus.

"C Yellow" is A. amstelodami.

Of these, "C Yellow" (NRRL No. 112) shows the ascosporic pattern and differs little from Barnes' normal strain or the authors' A. amstelodami. "Flame" (NRRL No. 59), "Green flame" (culture discarded), and "Blue conidial" (NRRL No. 85) show ascospores of the glaucus group but differ markedly in pattern. Among large numbers of induced variations, changes in the characters of the ascospore have not been found during this study. "D Brown" (eulture discarded) produees no ascospores but develops the hülle cells and conidial heads characteristic of the Aspergillus ustus group. These four forms belong to ubiquitous species quite abundant as contaminants where plant material is handled. Such contamination is not satisfactorily excluded by the work reported.

"Creamy" (NRRL No. 143) presents a different problem. The possibility that this "Cladosarum" was actually derived from the "normal" $A$. amstelodami is not excluded. Proliferation of the sterigmata in the head of Aspergilli, especially among the $A$. glaucus lot, is very common. The branches produced sometimes are found sterile but usually become diminutive conidiophores with very small vesicles and groups of sterigmata produeing normal spores. The Yuills' Cladosarum olivaceum (NRRL No. 374) was found as a conspicuous variant or contaminant in their culture of $A$. niger (1938). The colony, conidiophore, vesicle, primary sterigmata, and initial secondary sterigmata are produced as in $A$. niger, then instead of chains of conidia with the newest or youngest conidia at the bases of the chains and eonnected directly with the sterigmata, chains of cells are produced that replicate the sterigmata; occasionally a chain is interrupted by one cell producing a group of new chains, thus acting as a primary sterigma.

These chains of cells lengthen not at the base as in Aspergillus but at the distal end. In spite of prolonged search, which shows that the cells toward the outer ends of such ehains lose definiteness as sterigmata, the authors cannot confirm the finding of a single terminal conidium on each chain as reported by the Yuills. This morphological picture is repeated by Barnes' "Creamy" strain, which must therefore be interpreted in terms of Yuills' genus. Only the two isolations are known thus far. Their failure to pro- 
duce true spores and their rapid loss of vitality observed in the present cultures lead to the hypothesis that they are both variants from Aspergillus and belong to the "monster" type of organisms that fail to survive in competitive environments. Rare occurrences of the "monster" type such as these can hardly be regarded as permanent members of the fungous flora, even though the individual can be kept viable by regular vegetative transfer. It is doubted, therefore, whether generic designation is warranted.

\section{Occurrence and Economic Importance}

The members of the $A$. glaucus group are among the most common molds on earth. Thoy are extremely abundant in nature, and live under all sorts of conditions, thriving in moist and dry situations. However, they are most conspicuous upon concentrated substrata, such as drying plant products of all kinds as they come from the field and reach storage just above the absolute low percentage of water for stability. They are equally commonplace in sweetened products, including jams, jellies, soft sugars, honey, soft candies; in salted products such as meats, pickles, etc.; in dried foods where preservation is a complex of sugars and acids; upon manufactured leather goods exposed to humid conditions; upon clothing and textiles stored in moist atmospheres; and upon soft wood inadequately cured or subjected to improper storage. Nevertheless they are frequently overlooked in routine culture because they grow poorly upon substrata commonly employed and soon become overgrown by bacteria and more rapidly developing molds. In following the deterioration of products by cultural procedures, these forms will appear with amazing frequency if care is taken to select media approximating the osmotic concentration of the substance examined.

Molds of this group herald incipient spoliage. As excess water increases, first other molds, then bacteria, appear and complicate the decomposition process. The presence of any of these organisms is evidence of the earliest stages of decomposition, and is generally followed by the invasion of other and more destructive molds and bacteria. Insofar as is known, products infected by these forms in pure culture are non-poisonous; but naturally occurring materials in which they appear, or even predominate, should be considered "suspect" because of the possible presence of toxigenic forms.

\section{Pathogenicity}

Members of the A. glaucus group have been reported in connection with various types of ailments of man and domestic animals. A. hageni of Hallier (1870) and A. repens have been occasionally reported as fruiting in the external canal of the human ear; A. montevidensis Talice and MacKinnon (1931) was isolated from a case of otomycosis; A. mencieri Sartory and Flament (1920) was described from sputum of a consumptive; Fonseca 
(1930) isolated, in Brazil, A. amstelodami from a case of mycetoma of the foot; and A. keratitis Ball was described as on the cornea (Thom and Church, The Aspergilli, p. 86, 1926). There is thus data enough to justify belief that an occasional strain of the A. glaucus group is found in connection with lesions in man. We know nothing of the manner of infection and have no evidence that these organisms appear in any consistent manner as pathogens. Quevedo's case in which A. maydis (1912) appeared as a cause of poisoning of horses reads plausibly; this, however, is rendered doubtful by the observations of other investigators who have reported in widely separated places such masses of mycelium and spores without encountering similar injury. A. fontoynonti of Guéguen (1909 and 1911) isolated from an abcess was not found pathogenic in subsequent animal experiments. Species which grow well at blood heat have possibilities of pathogenesis; fortunately, however, most members of the group do not grow at $37^{\circ} \mathrm{C}$. 


\section{Ch.pTtek X \\ THE ISPERGILLL FIMIGITLS GROLP}

\section{Outsknding Characters}

Conidial hosds colummar, in shatdes of green through dark green to fuligineus.

lesieles flask-shaped, typieally fortile orer the upper half.

sterigmata in one series, crowded.

Conidiophores smooth-walled, watully colored in shades of green.

Conidia globose, echimulate, green.

Norking with hug material from birds dying of aspergillosis, Fresenius, ahout 1sion, deseribed and figured the speeies. Aspergillus fumigatus so well that there has nerer been amy doubt as to the morphology of the mold present. The investigator of molds in enlture, howerer, quickly finds that this type of conidiophore and head charateterizes not a single pathogente strain hut a multitude of rariant forms that are abundant in soil, upon decaring regetation, and, in fact, wherever organic materials are undergoing eren the slightest acrobic deromposition. To all of these forms, collectively, the designation Aspergillus fumigatus group is applied. .

The group falls naturally into two series:

Cultures strietly conidial. varying from volvety to thecose . . . . . fumigaius series Cultures producing perithecia and ascospores, conidial development generally limited A. fischeri series

\section{THE ASPELGLLL'S FLMIGATLS SERIES}

Aspergillus fumigatus Fresenius, in Beitrage zur Mtykologie, p. S1, pl. 10, tigs 1-11. Frunkfurt, 1850-53. Thom and Church.

The Aspergilli, p. 129. 1926.

Colonies upon Czapek's solution agar spreading broadly over the substratum, in some strains strietly velvety (Pl. II B and fig. 3i A), in others more or les thecose with varying amounts of tufted acrial mycelium to deep felted or extremely flocese forms (fig. 3- B), white at first. becoming green with the derelopment of heads but varying eonsiderably in the final shade of green, often becoming dark green to almest black in age. Reverse and substratum, in some strains uneolored, in others showing varying amounts of yellow, or again passing orer in dark red shades in age. Conidial heads columnar, compact, varying in measurement from st rain to strain up to 400 by 50, but usually much shorter, ocensionally rery small. Conidiophores 

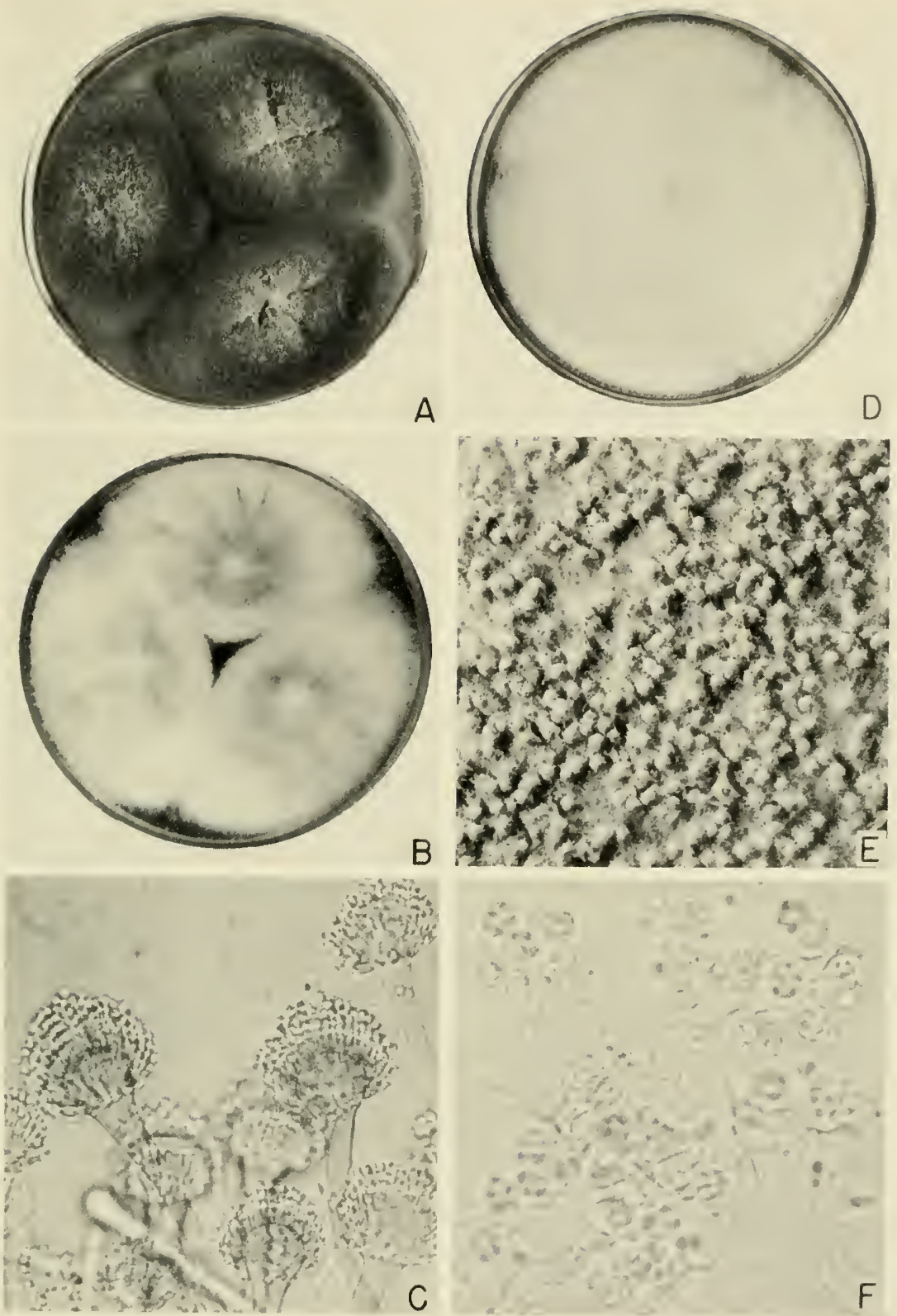

FIG. 37. Aspergillus fumigatus group; A-C, A. fumigatus: A, Typical, heavy sporing strain, NRRL No. 178, on Czapek's solution agar, 10 days, room temperature; $B$, Floccose, light sporing strain of same species, NRRL No. 171; and C, Photomicrograph of typical conidial heads showing characteristic form of vesicles and crowded sterigmata in a single series, $\times 500 . D-F$, Aspergillus fischeri: $D$, Strain NRRI, No. 186, growing upon Czapek's solution agar, characterized by very abundant perithecia and few conidial heads; $E$, Portion of a colony enlarged showing crowded perithecia more or less obscured by loose enveloping hyphae, $\times 6.0$; and $F$, Asci containing ascospores at various stages of maturity, $\times 500$. 
short, smooth, usually densely crowded, up to $300 \mu$ (in occasional strains up to $500 \mu$ ) in length by 2 to $8 \mu$ in diameter, frequently more or less green colored, especially in the upper part, arising directly from submerged hyphae or as very short branches from aerial hyphae, septate or unseptate, gradually enlarging upward and passing almost imperceptibly into the apical flask-shaped vesicles. Vesicles up to 20 to $30 \mu$ in diameter, usually fertile on the upper half only (fig. $37 \mathrm{C}$ ). Sterigmata in one series, usually about 6 to $8 \mu$ (varying from 5 to $10 \mu$ ) by 2 to $3 \mu$, crowded, closely packed with axes roughly parallel to the axis of the conidiophore. Conidia dark green in mass, echinulate, globose, mostly 2.5 to $3 \mu$ in diameter with extremes ranging from 2 to $3.5 \mu$. Sclerotia or perithecia are not found. The species grows well at temperatures up to $45^{\circ} \mathrm{C}$. or even higher, and is commonly present in compost and other material undergoing decomposition at high temperatures.

The species description presented is a composite rather than an exact citation of detailed data about one strain, but NRRL No. 163 (Thom No. 118) may be considered typical. Organisms coming within this series as described are world-wide in distribution and omnivorous in habit. They are regularly abundant in soil and in decomposing organic masses; they are recoverable from apparently sound cereals, corn, oats, wheat, etc.; they are encountered as pathogenes in the air passages of birds and occasionally as lung parasites of mammals, including man. One strain (NRRL No. 164) was named $A$. cellulosae by Hopffe (1919) because of its ability to break down cellulose, but when examined, it presented no morphologic differences from hundreds of common isolates from soil.

Efforts to induce perithecium formation by the conidial strains of A. fumigatus have been disappointing. Organisms maintained in culture over long periods and subjected to multitudes of transfers upon all sorts of substrata have failed to give any response suggesting perithecium formation.

Extremes of variation in different strains range from colonies characterized by crowded conidiophores rising vertically from submerged hyphae to a height of $300 \mu$, or perhaps at times $500 \mu$, then producing columns of conidia sometimes up to 400 by $50 \mu$, to very floccose forms in which spore formation is generally retarded and in which conidiophores develop as very short branches of aerial hyphae and produce short columnar heads. Many of these variants can be isolated and maintained in culture, thus they have been made the types of species by earlier workers. Others encountered upon unique substrata have been given specific names in the belief that the substratum relation was obligate. Yuill (1939) isolated a buff-colored mutant from a typical green strain and applied to it the designation $A$. fumigatus var. helvola (fig. $17 \mathrm{C}$ ). Shortly thereafter Steinberg and Thom (1940) likewise recovered an essentially uncolored mutant from a typical green strain. Both forms still remain unchanged in culture. 
If the taxonomist could seize a half dozen widely spaced variants and destroy those which bridge the gaps between, identification as separate species would be easy. This, however, becomes impossible when large numbers of isolates are cultivated and studied in comparative culture. In attempting to divide this great series into tangible entities, earlier workers have created a long list of species and, while we do not consider these to be valid, they are presented in alphabetical order with the places of description. It is believed that all of these should be regarded as synonyms of $A$. fumigatus Fres.

A. aviarius Peck, in N. Y. State Museum Rept. 44, p. 25, pl. 4, figs. 9-12. 1891 .

A. bronchialis Blumentritt, in Ber. Deutsch. Bot. Ges. 19: 442-446, Pl 22, figs 1-f. 1901; also ibid. 23: 419-427, Pl. 19, figs. 1, 3, 6, 7, 8, 19, 23.1909.

A. calyptratus Oudemans, in Arch. Neerl. Ser. II. 7:283. Tab. XIII. 1902.

A. cellulosae Hopffe, in Centralb. f. Bakt. etc., Abt. 83: 531-537. 1919.

A. desseyi Spegazzini, in Physis (Rev. Soc. Argentina Cien. Nat.) ViII: 115-117, 1 figure. 1925; review only seen, Rev. Appd. Mycol. 4: 542. 1925.

A. fumigatus var. alpha Sion and Alexandrescu, in Compt. Rend. Soc. Biol. (Paris) 64: 288-289. 1908. 1919.

A. fumigatus var. minimus Sartory, in Bul. Acad. Med. Paris 3 Ser. 82: 304-305.

A. fumigatus var. tumescens Blumentritt, in Ber. Deut. Bot. Ges. 23: 419-427, Pl. 19, figs. 5, 6, 18-21. 1905.

A. glaucoides Spring, in Bull. Acad. Sci. Belg. 19: 560-572. 1852.

A. lignieresi Cost. et Lucet, in Ann. Sci. Nat. Ser. 9, II: 119, tab. 5, fig. 18-23. 1905. 1853.

A. nigrescens Robin, in Histoire Naturelle des Vegetaux Parasites, p. 518, Paris.

A. pulmonum hominis Welcker, in Kuchenmeisters Parasiten II, p. 144. This is discussed and figured by Theodor von Dusch, in Virchow's Archiv. (n. f. 1) 11:561566. 1857, but no ground is given for separating it from A. fumigatus.

A. ramosus Hallier, in Zeitschr. Parsit. 2: 266-269, pl. 6, figs. 1-6. 1870.

A. syncephalis Gueguen, in Les Champignons parasites de l'homme et des animaux 299 pp., 12 pl. Paris, 1904.

A. virido-griseus Cost. and Lucet, in Ann. Sci. Nat. Bot. IX. 2: 140. 1905.

THE ASPERGILLUS FISCHERI SERIES

Aspergillus fischeri Wehmer, in Centralb. f. Bakt., etc., 2 abt., 18: 390-2, figs. 1-5. 1907.

Synonyms: A. fumigatus-ascosporic, see Thom and Church, in Am. Jour. Bot. 5: 91-92. 1918. See also Thom and Church, The Aspergilli, p. 132.1926.

Sartorya fumigata Vuillemin, in Compt. Rend. Acad. Sci. (Paris) 184, No. 3: 136-137. 1927.

The conidial form is strikingly similar to Aspergillus fumigatus. Colonies grow well upon Czapek's solution agar with conidial heads sparingly pro- 
duced at room temperature (fig. $37 \mathrm{D}$ ), but more abundantly at $37^{\circ} \mathrm{C}$. Conidial heads frequently small and generally of a lighter green color than those of typical A. fumigatus. Perithecia quickly and abundantly produced, in most strains dominating the colony appearance (fig. $37 \mathrm{E}$ ), commonly up to $300 \mu$ in diameter, not colored, or very pale salmon, with walls scarcely colored, consisting of a single layer of cells, crushing easily, covered by a loose network of uncolored sterile hyphae. Asci abundant,

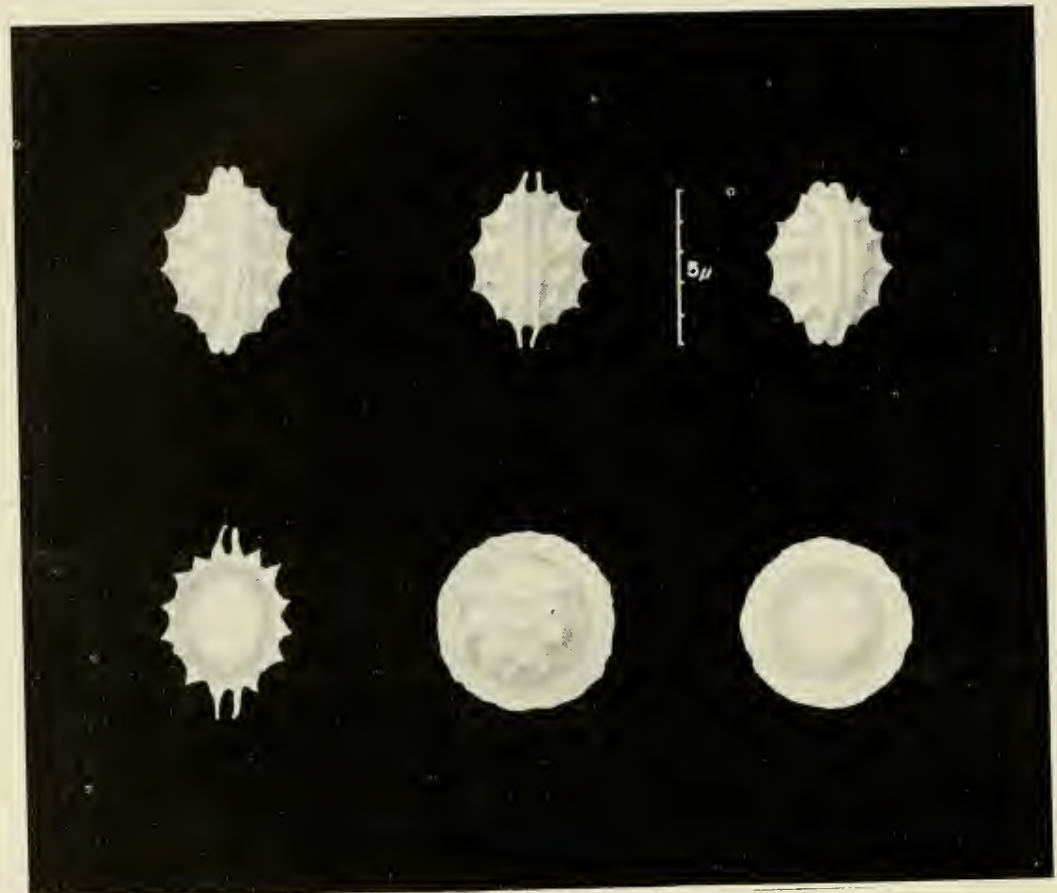

FIG. 38. Ascospores of Aspergillus fischeri, NRRL No. 181 (= Thom No. 4651.2). Upper left, center, and right represent surface, profile views; lower left, optical section in profile; lower center, surface in face view; and lower right, optical section in face view.

8-spored, filling the perithecium within a few days, 8 to $10 \mu$ by 10 to $12 \mu$, subglobose (fig. $37 \mathrm{~F}$ ), breaking down quickly to leare the perithecium full of ripe ascospores. Ascospores biconvex, uncolored, usually about 7 by $4 \mu$, consisting of a central body 5 by $4 \mu$, with two frilled equatorial banks about $1 \mu$ in width, roughened with echinulations or anastomosing bands on each convex surface (fig. 38), separating into two valves in germination.

Culture NRRL No. 181 (Thom No. 4651.2), received as type from Wehmer in 1923, is apparently identical with numerous isolations from American sources. Many strains have been seen, including a series from sputum of human cases showing lung involvement by X-ray examination. 
It is regarded as world-wide in distribution but seemingly not abundant anywhere. The species grows well at temperatures of $37^{\circ} \mathrm{C}$. and higher.

Some additional perithecial forms have been described with characters suggesting relationship with $A$. fischeri. However, these are inadequately known in culture and assignment here must remain somewhat provisional.

A. malignus Lindt, in Arch. Exp. Path. Pharmakol. 25: 257-271, fig. 1-11. 1889.

Lindt probably had before him some strain which corresponded closely with Wehmer's Aspergillus fischeri despite the fact that his description of the conidial apparatus offers sufficient contrast as to lead to question.

A. fumigatoides Bainier and Sartory, in Bull. Soc. Myc. France 25: 112, pl . 5. 1909.

While the describers believed this strain close to $A$. fumigatus, Thom and Raper (1941) found it necessary to place the organism studied under this name by Gould and Raistrick (1934) in A. Pseudoglaucus, although the original description and figures of Bainier and Sartory probably represented material close to, or identical with, A. fischeri.

Sartorya fumigata (Fres.) Vuill., in Compt. Rend. Acad. Sci. (Paris), 184(3): 136137. 1927.

Sartory, Sartory, and Meyer (1926) reported that a culture of A. fumigatus subjected to radiation produced an ascosporic form. Later this was designated by Vuillemin (1927) as a new genus Sartorya. Neither Vuillemin, nor Sartory, Sartory, and Meyer appear to have known the relation between A. fumigatus and A. fischeri although it was pointed out by Thom and Church in 1918. Since the material named Sartorya does not appear to have been distributed or fully described, Sartorya fumigata (Fres.) Vuillemin may be regarded as a synonym for $A$. fischer $i$ Wehmer and the generic name dropped.

It is believed probable that Aspergillus fischeri Wehmer represents the primary organism of this group and that from it the conidial form A. fumigatus developed as a species lacking entirely the ascosporic phase. The fact that $A$. fumigatus was described first merely reflects the much greater abundance of this species.

\section{Occurrence and Economic Importance}

Aspergillus fumigatus is an extremely cosmopolitan mold and occurs with particular frequency in soil containing appreciable organic materials, upon vegetable matter undergoing slow decomposition, and upon imperfectly dried, stored grains. The mold is an important agent in many decomposition processes, particularly at temperatures above $37^{\circ} \mathrm{C}$. Growing successfully at $45^{\circ}$ to $50^{\circ} \mathrm{C}$., within the lower reaches of thermophilic decomposition, it is able to operate within a range where most fungi are excluded. Whereas some forms have been described as very active agents of decomposition (e.g. A. cellulosae of Hopffe, 1919), their more significant role is believed that of forerunners of active bacterial decomposition on the one hand, and as slow destroyers of more resistant tissues on the other. Aspergillus fischeri, though much less abundant, may be found in situations generally similar to those yielding A. fumigatus. 


\section{Pathogenesis}

There is an extensive pathological literature which covers the occurrence of $A$. fumigatus in lesions of birds and mammals, including man, together with biochemical and animal experimentation. Such experiments have repeatedly proved that the organism is pathogenic to fowls confined in congested quarters in which moldy grain, straw, and other plant remains are abundant. Direct inoculation to the cornea in laboratory animals causes lesions characteristic for the species.

Infection of human beings occasionally appears, and observations seem to indicate that the patients generally have been exposed to air carrying large numbers of spores. Allergists have reported asthmatic conditions arising from sensitization to this species, and Bernton (1930) reports having successfully treated a patient by means of an extract prepared from the spores and mycelium of $A$. fumigatus. The occurrence of $A$. fischeri in cases grouped with $A$. fumigatus is clear indication that the pathogenic principle, whatever it is, is generally present in the group although it may vary in its intensity among different strains as indicated by workers such as Costantin and Lucet (1905).

Among organisms known to be, or believed to have been, pathogenic strains of $A$. fumigatus, the following named forms may be cited: $A$. gratioti, A. malignus, A. fumigatoides, A. virido-griseus, A. bronchialis, $A$. glaucoides, A. nigrescens, A. pulmonum hominis, A. ramosus, and A. aviarius (see p. 151). For a more complete discussion of this group in relation to disease in birds and mammals, the reader is referred to Thom and Church's The Aspergilli (1926) and Dodge's Medical Mycology (1935).

\section{Antibiosis}

Anslow and Raistrick (1938a) reported the production by Aspergillus fumigatus of a substance to which they applied the name, fumigatin, and in the same year (1938b) reported the species to produce a second metabolic product termed spinulosin, which they had previously isolated from Penicillium spinulosum. In 1942 fumigatin was further discussed by Oxford and Raistrick as a powerful agent against such bacteria as Bacillus anthracis, Escherichia coli, Salmonella typhi-murinum, Staphylococcus albus, S. aureus, Streptococcus viridans, and Vibrio cholorae. Also in 1942 Waksman, Horning, and Spencer reported an antibiotic substance, termed fumigacin, to be produced by A. fumigatus, and presented methods of differentiating this from fumigatin as studied by Raistrick and associates. Fumigacin was found to be both bactericidal and bacteriostatic in its action and to be effective in fairly high dilutions in inhibiting the growth of gram-positive cocci and bacilli; it was much less effective against the gram-negative members of the coli-aerogenes group. Both fumigatin and fumigacin have been found toxic to experimental animals. 


\section{Chapter XI \\ THE ASPERGILLUS NIDULANS GROUP'}

\section{Outstanding Characters}

Conidial heads short columnar, usually dark green with primary and secondary sterigmata.

Conidiophores smooth-walled, more or less browned, usually sinuate, commonly less than $200 \mu$ long and terminating in dome-like or hemispherical vesicles.

Conidia globose, echinulate, 3 to $4 \mu$ in diaméter.

Perithecia usually present; ascospores purple-red in color and characterized by equatorial bands.

Large, thick-walled, globose bodies, termed "hülle cells" (by Eidam), forming an irregular layer around the perithecia.

Aspergillus (Sterigmatocystis) nidulans was described by Eidam in 1883. Since that time the general type of organism covered by his diagnosis has become fairly well-known and certain striking characters have become recognized as defining a number of cosmopolitan strains or species, commonly referred to as constituting the Aspergillus nidulans group.

The hülle cells of Eidam, first described in A. nidulans, appear also in various transformations in the $A$. versicolor, $A$. ustus, and $A$. flavipes groups, together with other common characters indicative of close relationship. They do not appear in the A. clavatus, A. glaucus, or A. fumigatus groups which are characterized by single sterigmata, nor do they occur in the sclerotium forming groups, A. candidus, A. niger, A. wentii, A. tamarii, $A$. flavus, and A. ochraceus.

I. Ascospores present.

\section{Group Key}

A. Ascospores smooth-walled.

1. Equatorial ridges two in number.

a. Ridges 0.5 to $1.0 \mu$ wide, margin entire.

Conidial heads green.............. nidulans (Eidam) Wint.

Conidial heads white............. nidulans mut. atba Yuill

b. Ridges 1.5 to $1.8 \mu$ wide, margin entire

A. nidulans var. latus Thom and Raper

c. Ridges 3.0 to $4.9 \mu$ wide, margin dissected, starlike

A. variecolor (Berk. and $\mathrm{Br}$.) Thom and Raper

2. Equatorial ridges usually four in number

A. quadrilineatus Thom and Raper B. Ascospores rough-walled................ rugulosus Thom and Raper

1 Abridged from: Thom and Raper, The Aspergillus nidulans group, Mycologia, Vol. XXXI, No. 6, 653-669, Nov.-Dec. 1939. 
II. Ascospores lacking.

A. Hülle cells forming irregular masses, suggestive of sclerotia

A. caespitosus Raper and Thom

B. Hülle cells absent; heavy walled sterile hyphae present

A. unguis (Emile-Weil and Gaudin) Thom and Raper

Aspergillus nidulans (Eidam) Wint. in Rab. Krypt.-Fl. 1²: 62.1884.

Synonyms: Sterigmatocystis nidulans Eidam in Cohn, Beitr. Biol. Pflanzen 3: 392-411. pl. 20-22. 1883.

Diplostephanus nidulans (Eidam) Langeron, Compt. Rend. Soc. Biol. Paris, 87: 343-345. 1922.

Colonies upon Czapek's solution agar plane, spreading broadly, dark cress green (Ridgway, Pl. XXXI) from abundant conidial heads during the first two weeks (Pl. IA, IVC, and fig. $41 \mathrm{~A}$ ); perithecia developing fron the center of the colony outward after the first few days, separately produced, often abundant (Pl. IVC); sectoring occasional; reverse of colony in varying shades of purplish-red during the growing period, becoming very dark in age. Heads short, columnar, ranging from 40 to $80 \mu$ by 25 to $40 \mu$, commonly 60 to $70 \mu$ by 30 to $35 \mu$ (Pl. IB and fig. $4 \mathrm{C}$ ); conidiophores commonly sinuous, with walls smooth, in shades of cinnamon brown (Pl. IC), ranging from 60 to $130 \mu$, commonly 75 to $100 \mu$ in length, about 2.5 to $3 \mu$ near the foot, increasing to 3.5 to $5 \mu$ below the hemispherical vesicle (fig. $39 \mathrm{~A}$ ); vesicle 8 to $10 \mu$ in diameter; sterigmata in two series, primary 5 to $6 \mu$ by 2 to $3 \mu$ and secondary 5 to $6 \mu$ by 2 to $2.5 \mu$; conidia globose, rugulose, 3 to $3.5 \mu$ in dianeter, green in mass.

Perithecia developed separately within or upon the conidial layer (Pl. IA), globose, ranging from 100 to $175 \mu$ in diameter, commonly 125 to $150 \mu$, with outer layer a yellowish to cinnamon colored envelope of scattered hyphae bearing hülle cells up to $25 \mu$ in diameter; wall composed of one layer of cells, dark reddish-purple; in ripening becoming a mass of 8-spored asci which break down quickly leaving the ascospores free. Ascospores purple-red, lenticular, smooth-walled with 2 equatorial crests (Pl. ID and fig. $43 \mathrm{~A}$ ), spore bodies about 3.8 to $4.5 \mu$ in length by 3.5 to $4 \mu$ in breadth, equatorial crests pleated with margin sinuous and entire ranging from 0.5 to $1 \mu$ in width (Table 1 ).

Diagnosis based primarily upon culture NRRL No. 187 (Thom No. 4640.5) obtained from the Bainier collection in Paris. Other strains assigned to Eidam's species included many isolations from American soil and decaying vegetation, as well as cultures from European contributors. Common.

The range of ascospore measurements found in a representative group of cultures is shown in the accompanying table.

In assigning Eidam's species name to the members of this series it is obvious that there are discrepancies. He described the perithecium as 


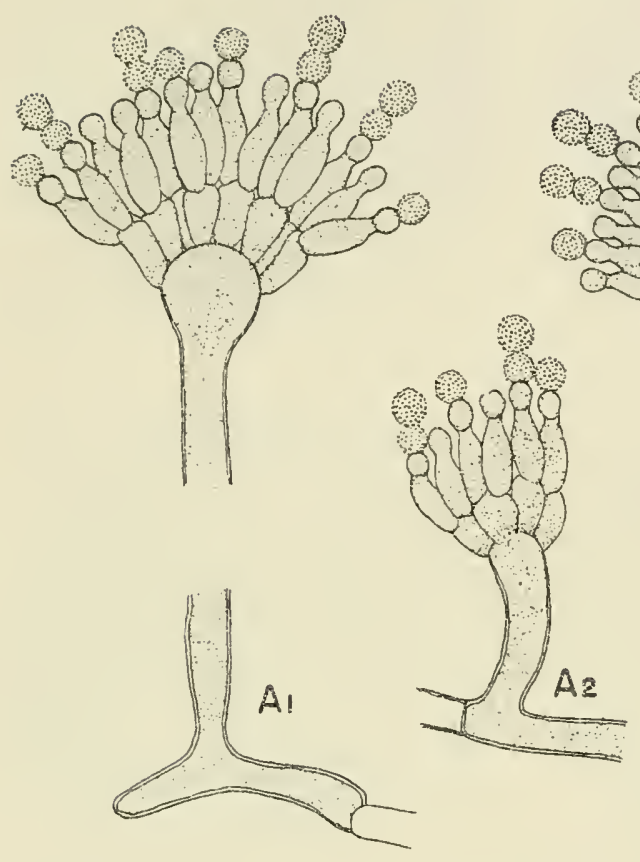

$\sqrt{3}$

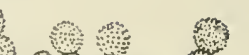

2. ON.

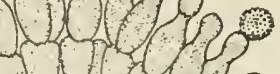

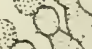

FIG. 39. Conidial structure in the Aspergillus nidulans group, $\times 900: A_{1}$, Typical conidial head of $A$. nidulans, NRRL $N o .18 \overline{7} ; A_{2}$, Diminutive head of the same strain; $B$, Typical head of A. caespilosus, NRRL No. 1929.

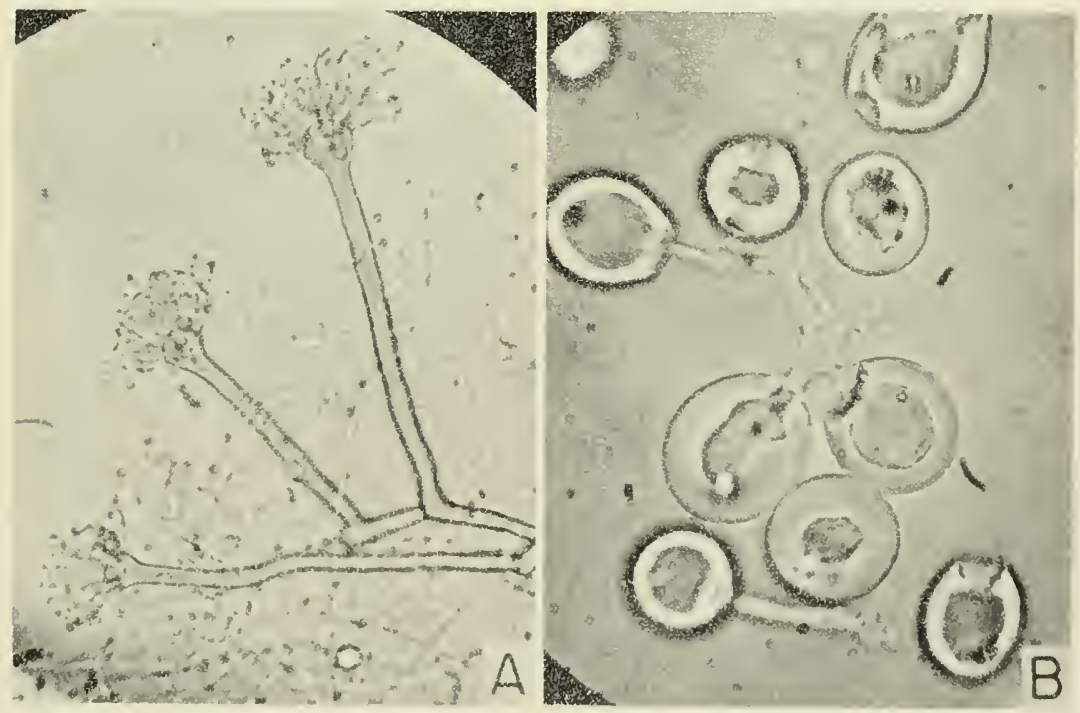

FIG. 40. Aspergillus nidulans. $A$, Conidial lıeads, $\times 370$. Conidiophores arise either from the substratum or from aerial hyphae. $B$, Hiulle cells, $\times 740$. 

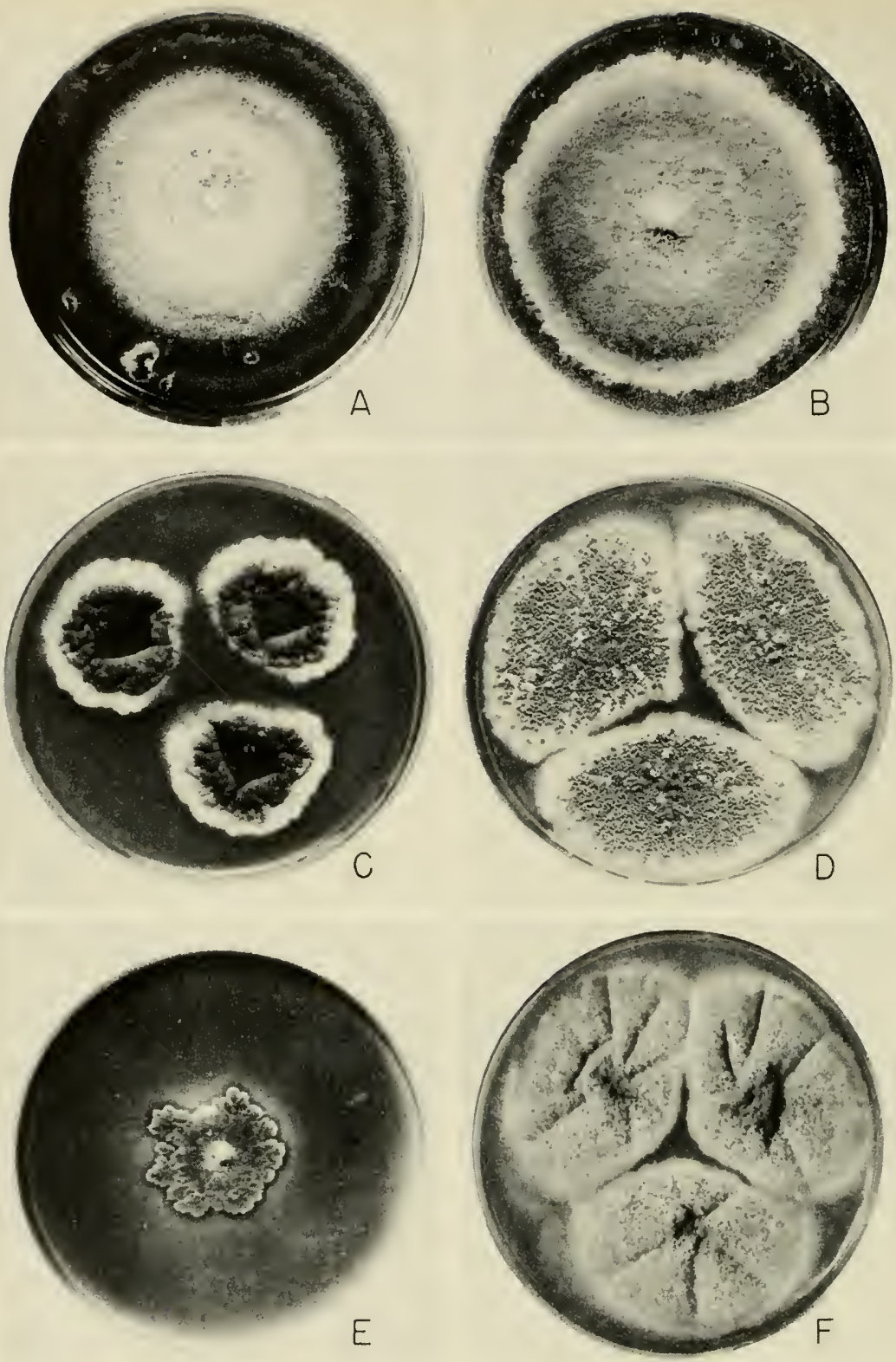

FIg. 41. Aspergillus nidulans group; different species growing upon Czapek's solution agar at room temperature. $A, A$. nidulans NRRL No. 194, typical strain producing very abundant dark green conidial heads. $B$, Naturally occurring mutation characterized by white heads, isolated from the preceding strain by Edward Yuill and described as $A$. nidulans mut. alba. $C, A$. rugulosus NRRL No. 207, characterized by heavy perithecium production, an almost complete absence of conidial structures, and a tendency of colonies to split in central areas as shown. $D, A$. variecolor NRRL No. 1954, characterized by the production of abundant large perithecia. $E, A$. unguis NRRL No. 216, characterized by an absence of perithecia and hülle cells. F, A. caespitosus NRRL No. 1929, characterized by the production of masses of hülle cells suggesting abortive perithecia. 
having a firm almost sclerotioid wall, whereas the wall is found to contain but one layer of cells. He figured the asci as few and scattered in a mycelial matrix within which ascospore production occupied many weeks. One isolated strain in our collection produces asci in this manner. For it, the varietal name, $A$. nidulans var. latus, was proposed by Thom and Raper (1939) on account of very broad crests on the ascospore in contrast to the usual types in A. nidulans which come much more closely to those indicated in Eidam's figures.

TABLE 1

Ascospore variation in strains of Aspergillus nidulans

\begin{tabular}{|c|c|c|c|}
\hline Culture number & Overall dimension of spores & Width of crests & Dimensions of spore bodies \\
\hline NRRL 193. & $6.2-6.6 \times 3.6-3.8 \mu$ & $1.0 \mu \pm$ & $4.2-4.6 \times 3.6-3.8 \mu$ \\
\hline NRRL 188. & $6.0-6.6 \times 3.6-3.9 \mu$ & $1.0 \mu \pm$ & $4.0-4.6 \times 3.6-3.9 \mu$ \\
\hline NRRL 189. & $6.0-6.4 \times 3.6-3.8 \mu$ & $1.0 \mu \pm$ & $4.0-4.4 \times 3.6-3.8 \mu$ \\
\hline NRRL 194. & $5.4-5.8 \times 3.6-3.8 \mu$ & $0.6-0.8 \mu$ & $4.0-4.4 \times 3.6-3.8 \mu$ \\
\hline NRRL $195^{*}$. & $5.4-5.8 \times 3.6-3.8 \mu$ & $0.6-0.8 \mu$ & $4.0-4.4 \times 3.6-3.8 \mu$ \\
\hline NRRL $191 \ldots$ & $5.4-5.8 \times 3.6-3.9 \mu$ & $0.6-0.8 \mu$ & $4.0-4.4 \times 3.6-3.9 \mu$ \\
\hline NRRL $187 \ldots$ & $5.4-5.8 \times 3.6-3.8 \mu$ & $0.7-0.8 \mu$ & $3.9-4.3 \times 3.6-3.8 \mu$ \\
\hline NRRL 192.. & $5.0-5.4 \times 3.6-3.8 \mu$ & $0.5-0.6 \mu$ & $4.0-4.4 \times 3.6-3.8 \mu$ \\
\hline NRRL $198 \ldots$. & $4.8-5.4 \times 3.6-3.8 \mu$ & $0.5-0.6 \mu$ & $3.8-4.4 \times 3.6-3.8 \mu$ \\
\hline NRRL $199 \ldots$. & $4.8-5.2 \times 3.6-3.8 \mu$ & $0.5 \mu \pm$ & $3.8-4.2 \times 3.6-3.8 \mu$ \\
\hline
\end{tabular}

* White mutant from culture NRRL No. 194; described by Yuill as Aspergillus nidulans mut. alba.

A. nidulans mut. alba Yuill, in Jour. of Botany (London)

$$
\text { 175, pl. 618. } 1939 .
$$

Colonies of the variety on Czapek's solution agar differ from the species in the entire absence of green color (fig. $41 \mathrm{~B}$ ). The ascospores have the characters of the species.

Culture obtained by Yuill as a mutant from a normal green strain of $A$. nidulans under investigation in his laboratory. Our record number is NRRL 195; the normal and parent strain is NRRL 194.

Aspergillus nidulans var. latus Thom and Raper. Mycologia 31:657. 1939.

Colonies on Czapek's solution agar differing from the species in colony development characterized by a felt of predominantly sterile mycelium; few conidial heads; fairly abundant perithecia developed in the mycelial felt and each surrounded by a thick covering of hülle cells (fig. $42 \mathrm{C}$ ), very slowly ripening and containing few and scattered asci in abundant sterile mycelium. Ascospore bodies smooth-walled, purple-red, 3.8 to $4.5 \mu$ by 3.5 to $4 \mu$, with crests 1.5 to $1.8 \mu$ in width.

Type culture NRRL No. 200 received from the Centraalbureau in 1909 and remaining constant in culture since that time. Its antecedent history is not known. 
Aspergillus quadrilincatus 'Thom and Raper. Mycologia 31: 660, figs. 24. 1939 .

Colonies on Czapek's solution agar spreading, plane or slightly wrinkled, with tendency toward flocesity, central area gray with a definite purplish tinge, and olive-green conidial areas toward the margin, oceasionally as sectors; perithecia developing separately but abundantly throughout the colony; reverse purplish-red; heads short columnar, green, mostly 60 to $70 \mu$ by 30 to $35 \mu$, occasionally larger or smaller; conidiophores sinuate, smoothvalled, dull brownish in color, 50 to $75 \mu$ in length by 3.5 to $4.5 \mu$ wide, broadenng to 7.5 to $9 \mu$ at the hemispherical vesicular areas; primary sterigmata 5 to $6 \mu$ by 2 to $3 \mu$, secondary sterigmata 5 to $7 \mu$ by 2 to $2.5 \mu$; conidia globose, pale yellow-green, rugulose, 3 to $4 \mu$ in diameter; perithecia enveloped by hülle cells, light brownish in color, spherical, partialiy embedded in the mycelial felt (fig. $42 \mathrm{D}$ ), about 125 to $150 \mu$ in diameter including the enveloping hülle-cell layer, with perithecial wall 1-cell layer in thickness, ripening quickly and with ripe asci breaking down to leave the ascopores free; ascospores purple-red, lenticular, with smooth wall, with spore body 4 to $4.8 \mu$ by 3.4 to $3.8 \mu$, and with two pleated equatorial crests about $0.5 \mu$ in width paralleled by a secondary narrower pair (fig. $43 \mathrm{~B}$ ) which are sometimes indistinct.

Type NRRL No. 201 (Thom No. 4138.N8) from New Jersey soil and kept in culture since 1916. Other strains examined include isolations from Texas, Colorado, Louisiana, and Maryland.

Aspergillus rugulosus Thom and Raper, Mycologia 31: 660-663, fig. 4. 1939.

Colonies on Czapek's solution agar slowly and restrictedly growing (fig. $41 \mathrm{C}$ ), buckled or wrinkled in a mass 2 to $3 \mathrm{~mm}$. deep, enveloping abundant perithecia at different depths, often eventually splitting in the central area, purple-gray to purple-brown in age, with green heads sparsely produced and hence not generally evident, occasionally seen as small groups and marginal extensions into drying media; reverse in shades of deep purplered; conidial heads, short columnar, 75 to $100 \mu$ by 30 to $40 \mu$; conidiophores sinuous, smooth-walled, pale brownish in color, 50 to $80 \mu$ long, slender, varying up to $5 \mu$ in width, then enlarging to vesicular hemispheres 8 to $10 \mu$ in diameter; primary sterigmata 7 to $8 \mu$ by 3 to $3.5 \mu$, secondary sterigmata 6 to $7 \mu$ by 2.5 to $3 \mu$; coniclia globose, green, rugulose, 3 to $4 \mu$.

Perithecia very abundant, of ten imbedded in the mycelium as 2 or 3 layers and each surrounded by hyphae and dark brown hülle cells (fig. $42 \mathrm{E}$ ), globose, 225 to $350 \mu$ in diameter including mycelial coverings, with dark reddish-purple walls of one cell thickness, quickly ripening and breaking down to leave ascospores free; asci 10 to $11 \mu$ in long axis; ascospores purplered, lenticular, walls conspicuously rugulose (fig. $43 \mathrm{C}$ ), with spore bodies 4 
to $4.4 \mu$ by 3.6 to $3.8 \mu$, and with 2 pleated equatorial crests with sinuate and entire margins about 0.5 to $0.6 \mu$ in width.

\section{A: A. nidulans}

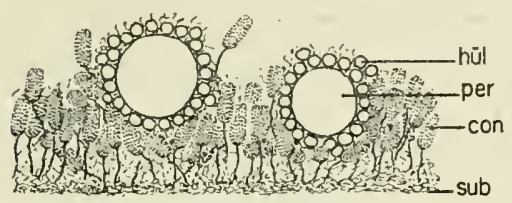

C: A. nidulans var. latus

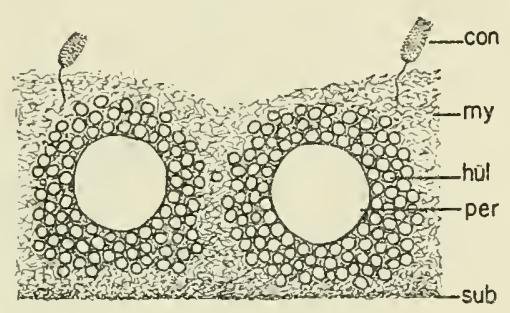

B: A. unguis

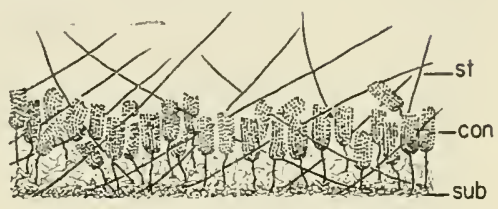

D: A. quadrilineatus

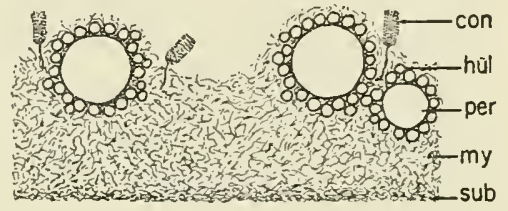

$\mu \int_{100}^{0} F:$ A. variecolor
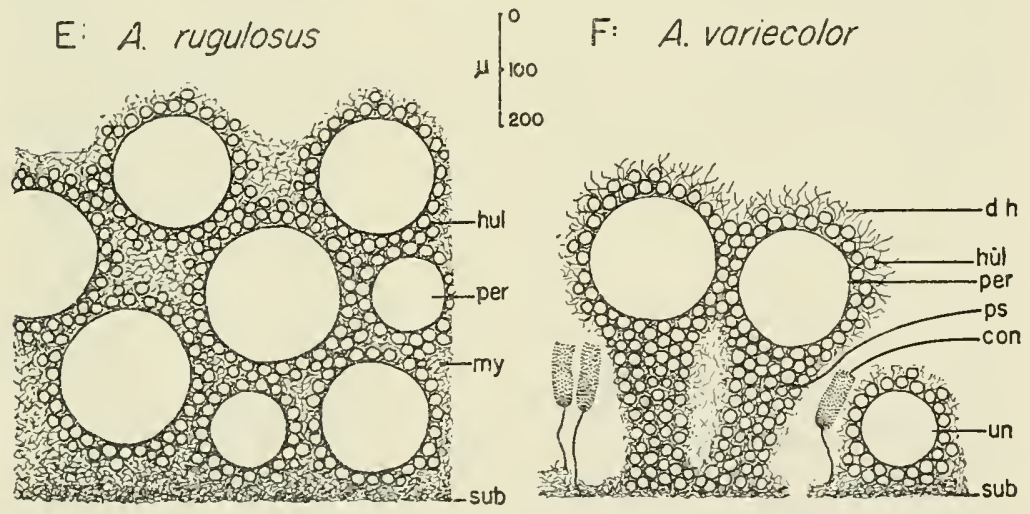

FIG. 42. Diagrammatic representations of cross sections of colonies of different species of the Aspergillus nidulans group showing the relative abundance of conidial heads and perithecia and the manner in which these structures are borne:con, conidial heads; d.h., mantle of divergent hyphae; hul, hülle cells; my, mycelial felt; per, perithecia; $p s$, pseudostalk of hülle cells and sterile hyphae; st, long, thick walled, sterile hyphae; sub, substratum; and un, unstalked perithecium. Scale approximate. (Reprinted from Thom and Raper, "The Aspergillus nidulans Group," Mycologia 31: 653-669. 1939.)

Cultures studied included Type NRRL No. 206 (Thom No. 4138.T11) from New Jersey soil, as discussed by Thom and Church in The Aspergilli, p. 138, and also isolates from Washington, D. C., Texas,_Nebraska, and California. Very common in soil. 


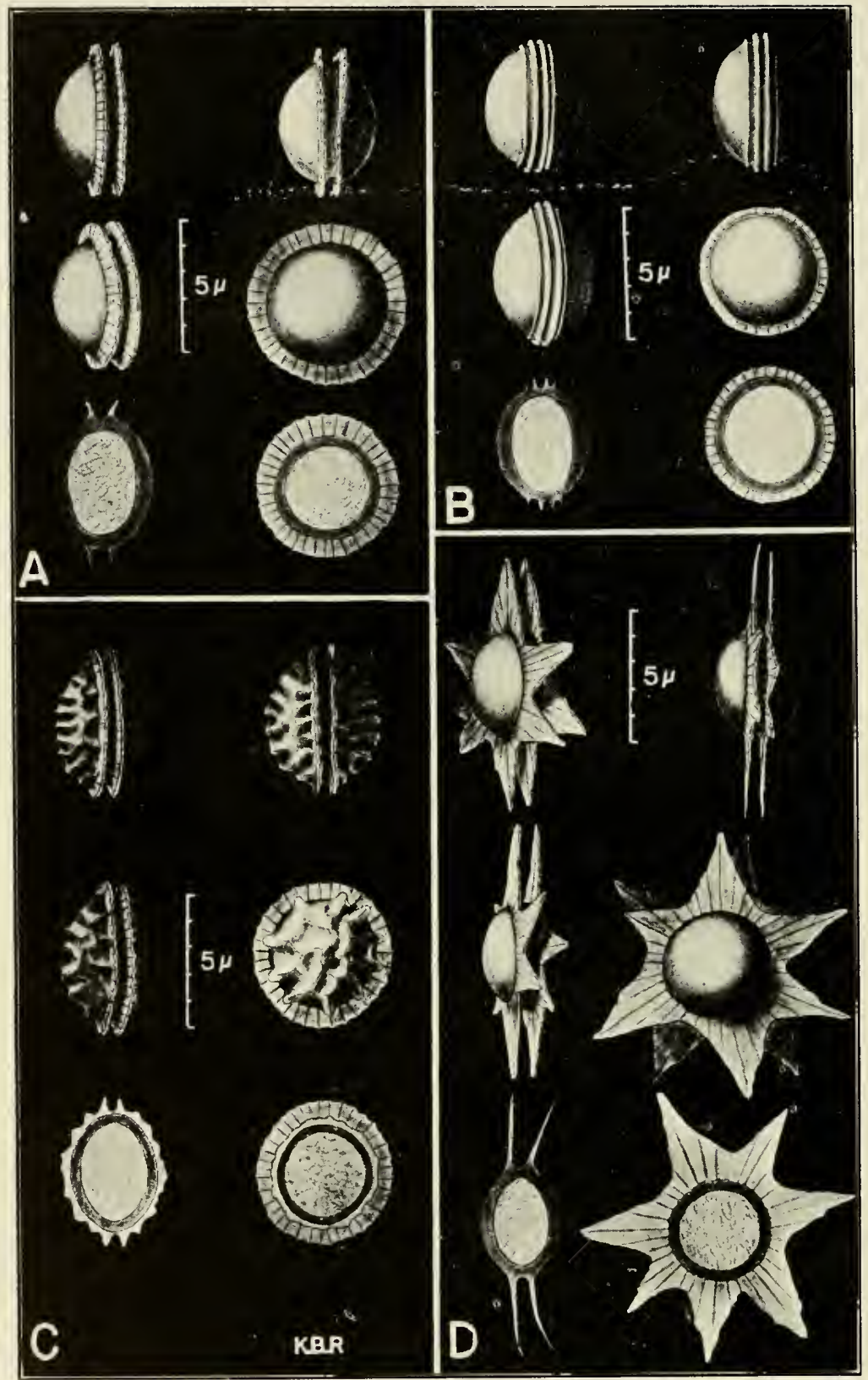

FIG. 43. Ascospores of different species of the Aspergillus nidulans group. $A, A$. nidulans. $B, A$.quadrilineatus. $C, A$.rugulosus. $D, A$. variecolor. In each species upper left and right and center left spores represent surface, profile views; center right, surface in face view; lower left, optical section in profile; and lower right, optical section in face view. (Reprinted from Thom and Raper, "The Aspergillus nidulans Group," Mycologia 31: 653-669. 1939.) 
Culturally and microscopically the above strains present similar pictures, with the exception of the strain recently received from Bliss in California (NRRL No.211). In contrast to the others, this culture produces abundant conidial heads and relatively fewer perithecia. The ascospores and conidial structures, however, duplicate those of the typical strains; hence we do not at present feel warranted in designating this as a variety, or otherwise separating it from the species $A$. rugulosus.

Aspergillus variecolor (Berk. and $\mathrm{Br}$.) Thom and Raper, in Mycologia 31: 663-667. fig. 4D and fig. 5. 1939.

Synonyms: Emericella variecolor Berk. and Br. in Berkeley, Introd. Crypt. Bot. p. 340-341; fig. 76. 1857. See Patouillard, Bull. Soc. Myc. Fr. $7: 43-49$. pl. 4. fig. 6-12. 1891.

Inzengaea erythrospora Borzi, Jahrb. Wiss. Bot. (Pringsheim)

16: 450-463. pl. 19, 20. (1884) 1885.

Emericella medias Chowdhury and Mathur, Ann. Myc. 36: 61-63. 1938.

Aspergillus stellatus Curzi, Rend. Acad. Naz. Lincei 19: 424-428. fig. 1. 1934.

Colonies on Czapek's solution agar with vegetative mycelium largely submerged, sparse, spreading slowly in the agar, producing green heads freely in the center of the colony, less abundantly in the outer areas, large gray perithecia produced in clusters in colony center and at the margin in some strains, with smaller perithecia scattered through the intervening thinner areas of the colony (fig. $44 \mathrm{~A}$ ), in other strains producing large perithecia abundantly throughout the colony (Pl. IVD); reverse color in shades of purple-red. Conidial heads green, columnar (fig. $44 \mathrm{D}$ ), relatively long, mostly 100 to $200 \mu$, occasionally up to $300 \mu$ by 30 to $40 \mu$; conidiophores arising directly from submerged hyphae, straight with smooth walls, cinnamon-brown in color, mostly 140 to $200 \mu$ long by 3 to $5 \mu$ in diameter, broadening gradually to become hemispherical vesicles about 8 to $10 \mu$ in diameter; primary sterigmata 7 to $8 \mu$ by 3 to $4 \mu$, secondary sterigmata 8 to $9 \mu$ by 2.5 to $3 \mu$; conidia globose, rugulose, 3 to $3.5 \mu$; perithecia when clustered (fig. $44 \mathrm{Aa}$ ) 300 to $400 \mu$ in diameter surrounded by a felt of hyphae and hülle cells and supported by masses of hyphae and hülle cells forming false stalks (fig. $42 \mathrm{~F}$ ), giving the structures a pyriform appearance (fig. $44 \mathrm{~B}$ ); scattered perithecia much smaller (fig. 44 C) and with envelope of supporting cells often much reduced in mass; hülle cells abundant and essentially like those of the species $A$. nidulans.

Perithecial wall when stripped of enveloping cells purple-red, brittle, composed of a single layer of cells; asci quickly ripening and breaking down to leave the cavity filled with ascospores; ascospores purple-red, with spore bodies lenticular and 3.6 to $4 \mu$ by 2.8 to $3 \mu$, with two prominent equatorial 


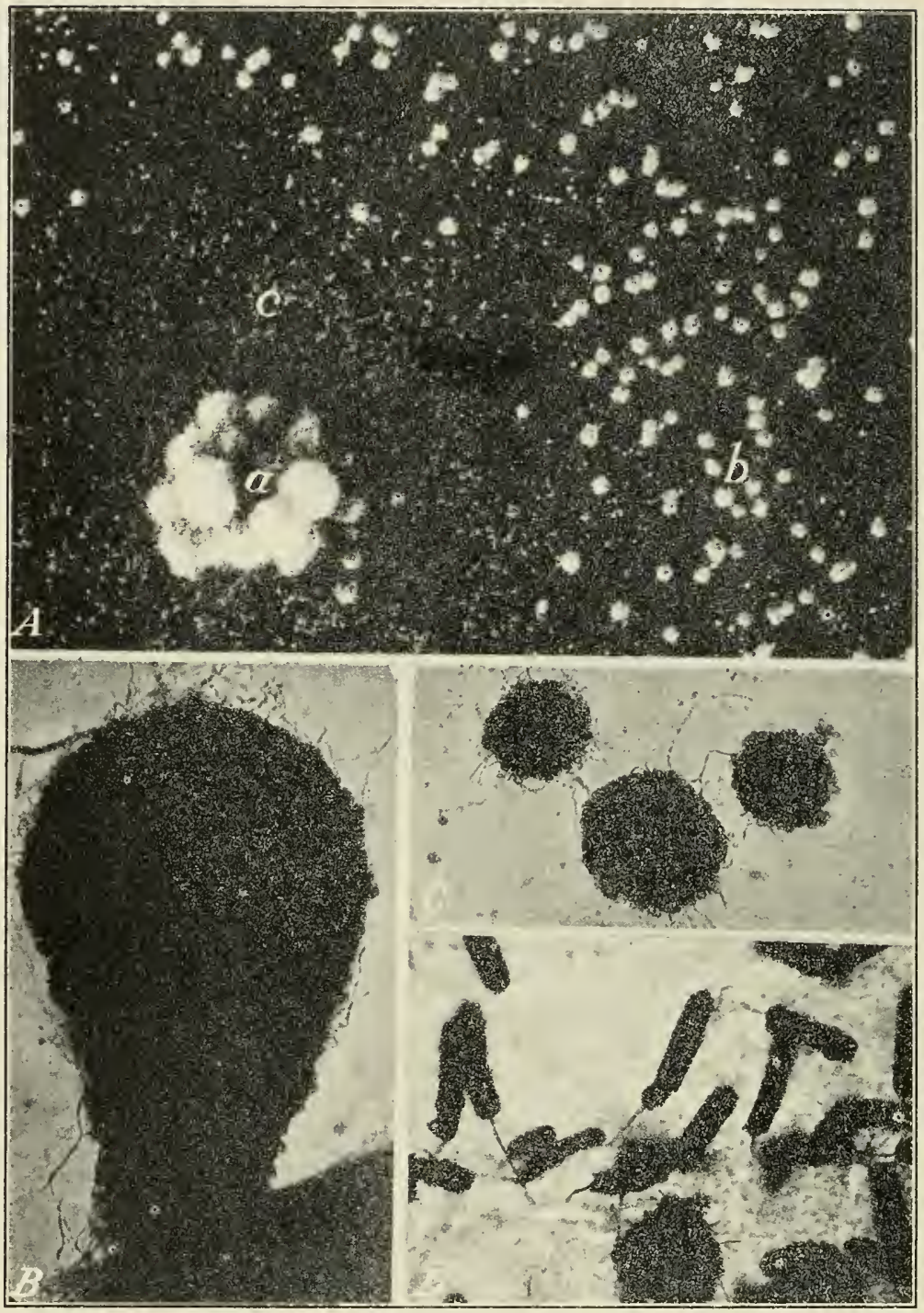

Fig. 44. Aspergillus variccolor. A, Central area of colony; a, cluster of large pseudostalked perithecia; $b$, scattered, smaller, unstalked perithecia (see also figure $42 F) ; c$, scattered conidial heads, $\times 6$. B, Enlarged view of large, pseudostalked perithecium, $\times 6.5 . C$, Enlarged view of small, unstalked perithecia, $\times 65 . D$, Enlarged view of conidial heads, $X$ 65. (Reprinted from Thom and Raper, "The Aspergillus nidulans Group," Mycologia 31: 65.3-669. 1939.)

crests, up to $3.5 \mu$ in width, pleated and cut to give a stellate appearance to the ascospores (fig. $13 \mathrm{D}$ ). 
The above description is based primarily upon a culture received from Prof. Verona in Italy and carried in our collection as NRRL No. 212 (Thom No. 5602.3).

Bliss forwarded a culture (NRRL No. 214) isolated from date fruits in California which differs from the above in the following particulars: (1) Conidial heads are produced abundantly, (2) the mycelium is not predominantly submerged, and (3) the colonies in reverse are deep purple. However, the ascospores of the two strains are strikingly similar in size and pattern, the perithecia of each appear pyriform in shape, and the conidial structures of the two are essentially alike. More recently a strain has been isolated from Arizona soil (NRRL No. 1954) which produces abundant large "stalked" perithecia but very few small perithecia or conidial heads. There is, thus, evidence of considerable natural variation among members of this species. All of these strains have the same type of stellate ascospore.

Since the type of ascospore described here had already been assigned to Emericella and Inzengaea, consideration of the literature of these genera is necessary.

Emericella variecolor, genus and species new, was described by Berkeley and Broome in 1857 as doubtfully a Gasteromycete or possibly a lichen. The perithecium with a mass of stellate spores was considered as gastromycetous in character while what we now recognize as the "hülle" cells of Eidam (figured) suggested to them the possibility of an algal associate. Berkeley's material was also examined by Montagne and part of it deposited in the Museum d'Histoire Naturelle de Paris. This was reexamined by Patouillard in 1891 and its ascomycetous nature determined. No conidial apparatus was found by either Berkeley or Patouillard.

Inzengaea erythrospora as the type species of a new ascomycetous genus was figured and described by Borzi in 1885, showing stellate red ascospores, and the hülle cells of Eidam. Borzi's figure showed a coremium-like conidial apparatus which was designated Coremium Borzianum by Saccardo. Ed. Fisher in 1893 transferred the species to Emericella of Berkeley and placed the genus next to Aspergillus in the "Pflanzenfamilien." Saccardo (in Syll. 9:610) on the other hand accepted Inzengaea and dropped Emericella because of the errors in description and placement by Berkeley. Borzi figured the spores of $A$. variecolor (Inzengaea erythrospora) correctly but obviously misinterpreted the germination of the ascospores since he showed them splitting as if turned $90^{\circ}$, bringing the crest perpendicular to the center of the valve instead of attached to its edges.

There the taxonomic situation stood until Vuillemin in 1927 concluded that the ascosporic apparatus, the stellate red ascospores and the cells of Eidam, as clearly shown in their figures and material, showed the identity 
of Emcricella and A. nidulans. He therefore transferred A. nidulans to Emericella as the oldest established genus and apparently did not even consider Inzengaea.

Ciferri (1938) has recently completed a study of Emericella variecolor embracing cultural investigations together with a review of the literature of the genus. He did not recognize the close relationship of this fungus to Aspcrgillus nidulans, and was apparently unmindful of the likeness of their conidial structures and the essential similarity of their perithecia and ascospores.

In 1934 Curzi described as Aspergillus stellatus a fungus characterized by hülle cells and red, stellate ascospores. However, he apparently did not know of either Berkeley's or Borzi's earlier designation of a similar fungus. It is unfortunate that his exceedingly descriptive binomial must be reduced to synonymy.

Fortunately for this discussion, cultures NRRL Nos. 212, 214, and 1954 present both the stellate spores and apparently stalked perithecia figured by Berkeley, Patouillard, and Borzi. (Compare figures 42, F, and 44, $\mathrm{B}$ with Berkeley's figure 76a, Patouillard's figures 7 and 8 , plate 4 , and Borzi's figure 10, plate 19.). This made possible a restudy of the whole morphologic situation from fresh material. The perithecial body itself was found not to be stalked but to rest upon a sterile mass of hülle cells and mycelium giving the superficial appearance noted by earlier observers.

The coremium of Borzi remains unaccounted for. Obviously in Borzi's discussion the material was rotten olives and very old. No cultures were made. The conidia-producing apparatus (figured) differs essentially in type from the conidial apparatus of the Aspergillaceae with which Fischer correctly placed Emericella because of its perithecia and ascospores. We are convinced that the coremia belonged to some other fungus.

It is not possible to separate the perithecium of this fungus from that of the other species in the $A$. nidulans group nor are there characters to take this type of perithecium out of a genus with the yellow perithecium of the great $A$. glaucus series of species which are widely known. Consistent with the policy of keeping the Aspergilli in one group, both Emericella and Inzengaca are dropped for purposes of this discussion.

Aspergillus caespitosus Raper and Thom, in Mycologia 36: 563-565, fig. 4. 1944.

Colonies varying markedly upon different media; upon Czapek's solution agar rather slow growing, attaining a diameter of 6 to $8 \mathrm{~cm}$. in three weeks at room temperature, plane or somewhat furrowed, mycelium largely submerged and extremely tough, tearing with difficulty, producing numerous dark green, hemispherical to loosely columnar heads in central colony areas, 
characterized particularly by clusters of irregular ovoid to elliptical, thickwalled hülle cells, at first colorless becoming reddish-purple in age, scattered
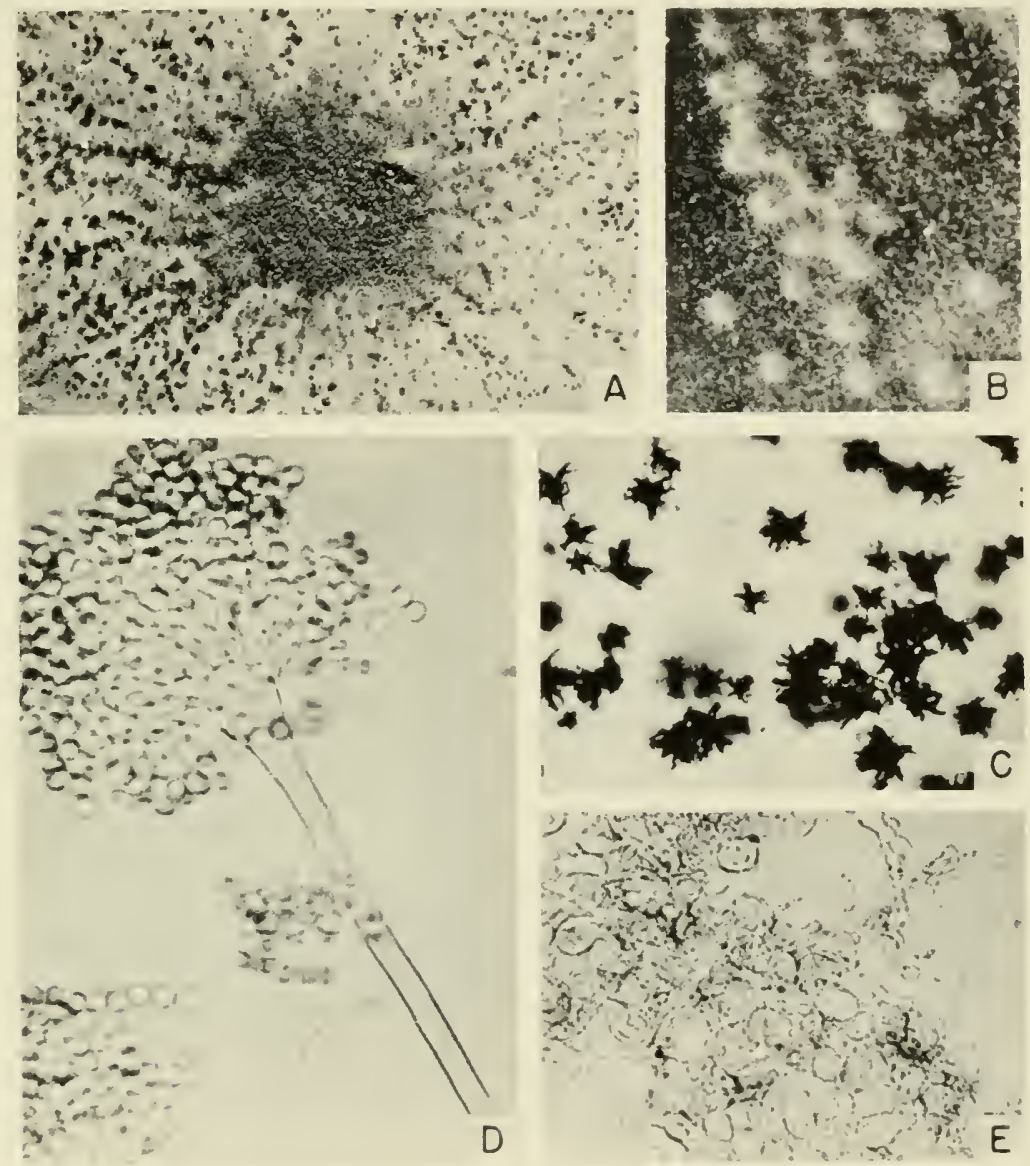

FIG. 45. Aspergillus caespitosus; NRRL No. 1929. A, Portion of colony on Czapek's solution with 1 percent liver extract, showing crowded conidial heads in central portion and scattered hülle cell masses in surrounding areas, two weeks old, incubation at room temperature, $\times 1.8$. $B$, Portion of colony enlarged showing hülle cell masses, $\times 10$. $C$, Silhouettes of conidial heads developed on hay infusion agar, $\times 48$. D, Typical conidial head showing form of vesicle and arrangement of sterigmata, $\times 600$, $E$, Portion of hülle cell mass showing irregular size and form of component elements, $\times 265$. (Reprinted from Raper and Thom, "New Species of Aspergilli from Soil," Mycologia 36: Nov.-Dec. 1944.)

unevenly (fig. $45 \mathrm{~A}$ and B) or arranged in irregular concentric zones; reverse colorless at first, becoming dark reddish-purple in age, particularly beneath the hülle masses; odor none. Conidial heads dark dull yellow-green to 
empire green (Ridgway, Pl. XXXII), generally hemispherical to loosely columnar, mostly 75 to $125 \mu$ in diameter. Conidiophores straight or slightly sinuous (fig. $45 \mathrm{D}$ ), mostly 250 to $325 \mu$ in length, occasionally up to $350 \mu$ by 5.0 to $6.5 \mu$ in diameter, of approximately uniform diameter throughout, relatively thick-walled, (1.2 to $1.5 \mu$ in basal portion to 0.8 to $1.0 \mu$ in terminal area), smooth, tan to light brown in color. Vesicle slightly elongate, the upper hemisphere loosely covered by sterigmata (fig. $45 \mathrm{D}$ ), the lower half sterile and often lightly colored, mostly 15 to $20 \mu$ in diameter. Sterigmata in two series (fig. $45 \mathrm{D}$ ), primaries normally 6.5 to $8.5 \mu$ by 3.5 to $5.0 \mu$, secondaries 6.5 to $8.0 \mu$ by 3.0 to $4.5 \mu$, typically bottle form but commonly much swollen and of ten quite irregular in form and dimensions. Conidia globose, spinulose, green, mostly 3.5 to $4.5 \mu$, rarely larger, hülle cells very abundant, thick-walled, irregularly globose, ovoid or elliptical (fig. $45 \mathrm{E}$ ), ranging from 12 to $18 \mu$ in globose cells to 12 to $15 \mu$ by 25 to $30 \mu$ in the most elongate bodies, forming compacted masses of indefinite size, extremely tough and in age becoming almost sclerotioid, at first colorless but in age characterized by an abundant reddish-purple intercellular pigmentation.

Colonies upon malt agar characterized by a dense stand of erect conidiophores bearing hemispherical to radiate or loosely columnar heads of dark green color approximately empire green (Ridgway, Pl. XXXII) and the complete absence of hülle cells; reverse in light brown shades; odor none. Details of morphology as upon Czapek's solution agar.

Colonies upon hay infusion agar like those upon malt except less heavily sporing.

Strains include NRRL No. 1929 (type) isolated from Arkansas soil and other isolations from Arizona and Texas soils.

This species is of particular interest because of its apparent transitional position between the $A$. nidulans group and $A$. ustus. In the character of its conidiophores, its reddish-purple pigmentation, and in the general color and markings of its conidia it retains the characters of $A$. nidulans and closely related species. In the absence of fertile perithecia and ascospores, the predominantly hemispherical shape of its conidial heads, and in the variable and irregular form of its hülle cells, it is strongly suggestive of the $A$. ustus series. While we are convinced of its.intermediate position between the $A$. nidulans group and $A$. ustus, we place it with the former since we believe it is most closely allied to this group. It is believed significant that superficially, cultures of Aspergillus caespitosus and Aspergillus variecolor (Berk. and Br.) Thom and Raper (1939) are strikingly similar upon Czapek's solution agar. This similarity is particularly marked when plates are viewed in reverse since an intense pigmentation marks the under surface of perithecia in the latter case and the under surface of older hülle masses in the former. 
Aspergillus unguis (Emile-Weil and Gaudin) Emend. Thom and Raper, Myc. 31, p. 667, fig. 6. 1939.

Synonyms: Sterigmatocystis unguis Emile-Weil and Gaudin, Arch. Med. Expt. Anal. Path. Paris 28: 463-465, fig. 4, 1919.

A. loakiashanensis Shih, Lingnan Sci. Jour. 15 (3): 369. p. 16, fig. 2. 1936.

Colonies on Czapek's solution agar restrictedly growing, plane, spreading at the margin as irregular lobes (fig. $41 \mathrm{E}$ ), yellowish-green, green to dark green becoming brown in age; without perithecia or hülle cells. Mycelial preparations show striking sterile, thick-walled hyphae with walls in brown

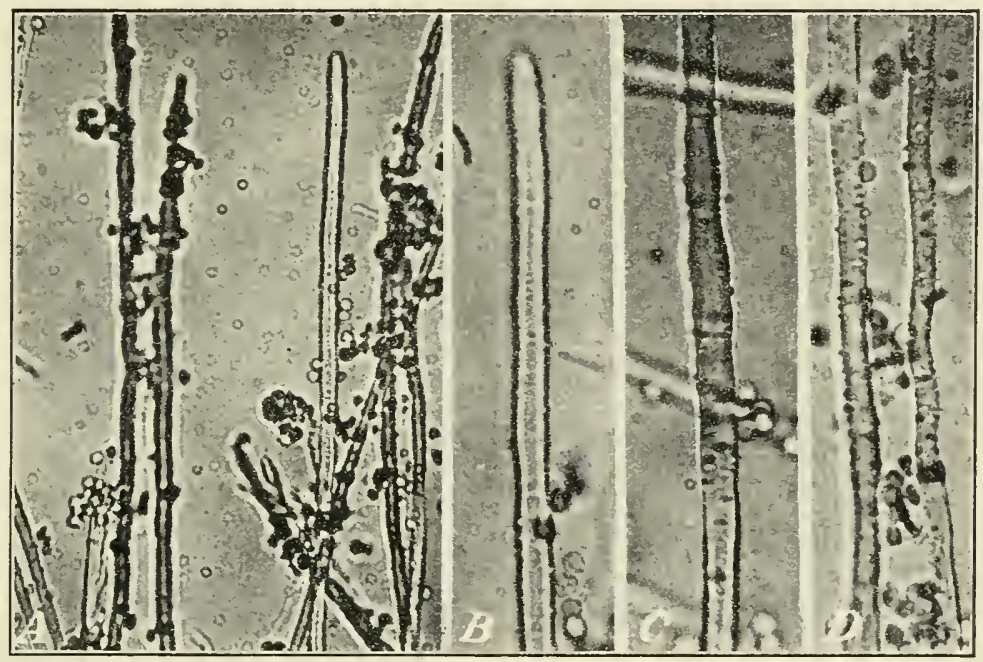

Fig. 46. Sterile spicule hyphae of Aspergillus unguis. A, Cluster of sterile hyphae, $\times 370$. $B$, Apex of sterile hypha, $\times 740 . C$ and $D$, Mid-portions of sterile hyphae showing thick roughened walls, $X 740$. (Reprinted from Thom and Raper, "The Aspergillus nidulans Group," Mycologia 31: 653-669. 1939.)

shades, irregularly roughened (fig. 46), tapering to a blunt point, arising sometimes from foot-cells suggesting the origin of conidiophores, sometimes apparently from mycelial cells, of ten up to $1,000 \mu$ or more in length, slanting upward but usually rising only slightly above the conidial area (fig. $42 \mathrm{~B})$.

Conidial heads columnar, 75 to $150 \mu$ by 40 to $50 \mu$; conidiophores smoothwalled, dull brown in color, mostly 45 to $65 \mu$ in length by 3 to $5 \mu$ in diameter, enlarging to vesicular hemispheres 9 to $12 \mu$ in diameter; primary sterigmata 5 to $6 \mu$ by 2.5 to $3 \mu$, secondary sterigmata 5 to $6 \mu$ by 2 to $2.5 \mu$; conidia globose, rugulose, dull green, 2.5 to $3.5 \mu$ in diameter.

Cultures of $A$. unguis are obtained frequently from medical laboratoies apparently as more or less active pathogens but occasionally isolated from 
soil and decaying organic matter. The question whether the nonascosporic members of the group have merely dropped the ascogenous phase or constitute a separate species was answered when more complete examination showed the sterile or spicule hyphae to be regularly produced in the non-ascosporic, but never found in ascosporic series.

\section{Pathogenicity}

Aspergillus nidulans in some of its forms and variants has been demonstrated as a parasite in human nails (onychomycosis), often enough to establish its pathogenicity. A. nantae Pinoy (1927) probably belongs here although the data are mainly pathological, hence not adequate for definite identification of the organism. A. nidulans forme cesarii Pinoy (1915) isolated from a mycetoma of the lung of a donkey, and A. nidulans var. nicollei Pinoy (1906) isolated in Tunis from a case of mycetoma, or madura foot, represent additional strains which were at least secondary pathogens. The nearly related $A$. unguis is usually the more common form isolated from human material. A. Brodeni (Mattlet) Dodge (1935) from a bronchomycosis in Africa might have been close to $A$. unguis. $A$. nidulans and $A$. unguis are both widely distributed as saprophytes; hence are constantly encountered as components of dirt reaching the extremities by contamination. Infection of the air passages is comparatively rare.

\section{Occurrence and Economic Importance}

In addition to their role as occasional disease producing agents, members of the $A$. nidulans group are believed to be significant in decomposition processes. They are among the molds most commonly isolated from soil, and very frequently appear in considerable abundance upon vegetable material undergoing slow decomposition. A spergillus rugulosus, A. quadrilineatus, and $A$. caespitosus occur most frequently in soils from the comparatively dry, warm soil of Texas, Arizona, and adjoining areas. Aspergillus variecolor has been isolated from olives in Italy, date fruit in California, and from Arizona soil. Aspergillus nidulans is abundant and cosmopolitan in its distribution. 


\section{Chapter XII \\ THE ASPERGILLUS USTUS GROUP}

\section{Outstanding Characters}

Colonies more or less floccose, at first white but becoming dull in age, in most members varying from olive-gray through reddish-brown to fuscous, as conidial structures develop.

Conidiophores in yellow-brown shades; smooth.

Heads irregular in form, ranging from more or less radiate to hemispherical to loosely columnar.

Vesicles hemispherical; sterigmata in two series, loosely arranged.

Conidia roughened, 3.0 to $5.0 \mu$, varying from echinulate to marked with conspicuous color bars, and ranging in color from pale blue-green through olive-green shades to deep brown (fuligineus).

Hülle cells regularly present, thick-walled, elongate, often more or less curved and twisted.

Included here are representatives of a most abundant and widespread group of fungi, especially common in soil and upon decaying vegetation.

\section{Group Key}

Colonies predominantly floccose, heavy sporing, hülle cells not aggregated in small clusters.............................. ustus (Bain.) Thom and Church Colonies more or less floccose, light sporing, hülle cells aggregated in small clusters

A. granulosus Raper and Thom

Aspergillus ustus (Bainier) Thom and Church, in The Aspergilli, p. 152. 1926.

Synonym: Sterigmatocystis usta Bainier, in Bul. Soc. Bot. France 28: 78. 1881.

Colonies upon Czapek's solution agar spreading broadly, plane, sulcate, or umbonate, rarely zonate, more or less felted or floccose; at first white, becoming olive-gray, yellow-brown, fuscous or russet to purplish vinaceous with the development of mature conidial structures (Pl. IV E, and figs. $48 \mathrm{~A}$ and $\mathrm{B}$ ); generally heavy sporing, with some conidiophores arising from the substratum but more abundantly from aerial hyphae; reverse in shades of yellow, orange, and brown to almost black in age; odor not pronounced. Heads radiate to irregularly hemispherical, sometimes loosely columnar, commonly splitting into more or less well-defined columns in age, very variable in size, ranging in color from dull green or olive-gray, through gray- 
ish-brown to fuscous or fuligineus. Conidiophores arising from submerged hyphae (fig. $47 \mathrm{~A}$ ) ranging up to $500 \mu$ long by 3 to $6 \mu$, aerially borne conidiophores, ranging from very short (fig. $47 \mathrm{~A}_{1}$ ) up to $125 \mu$ by 2 to $5 \mu$, sinuous, sparsely septate, with walls rather thin, smooth, and uniformly colored some shade of brown. Vesicles hemispherical to subglobose, 8 to $20 \mu$ in diameter, smaller in some strains (fig. $47 \mathrm{~A}$ ). Sterigmata colorless or
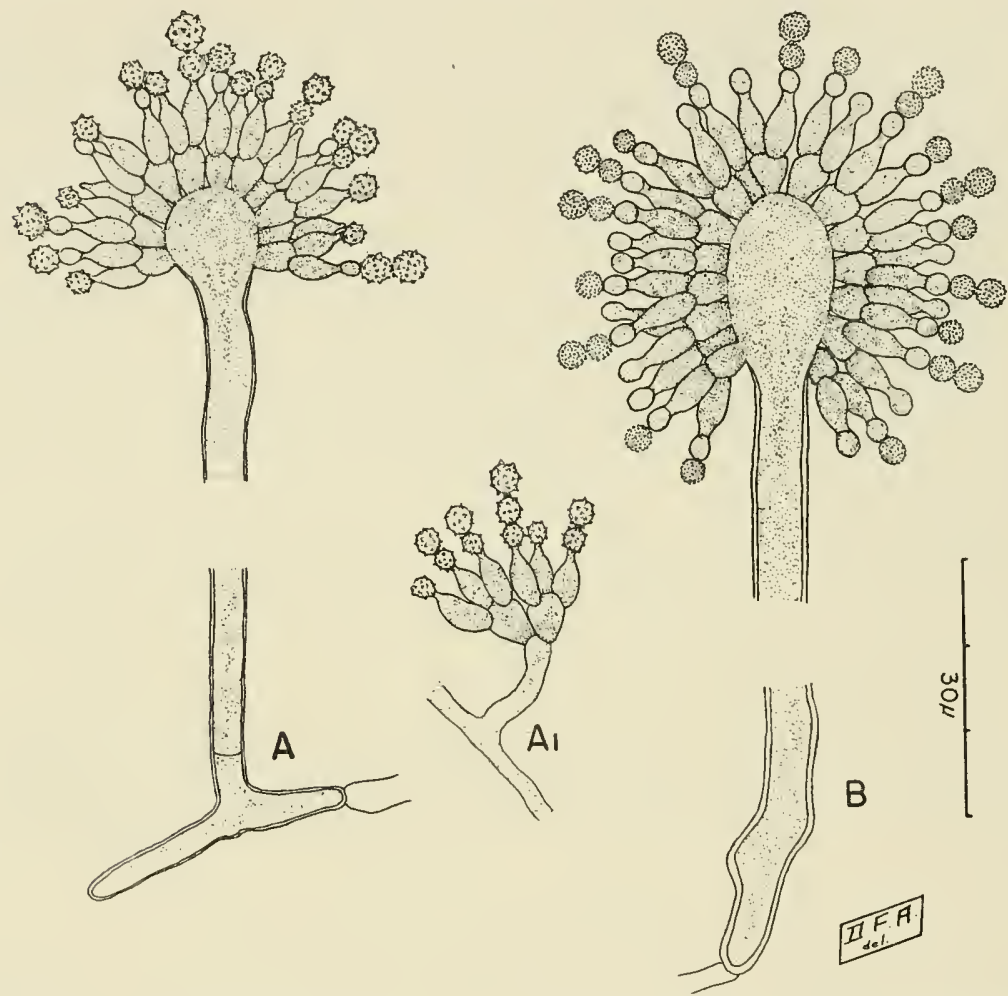

$\mathrm{F}_{1 \mathrm{G}}, 47$. Aspergillus ustus group, $\times 840$. A, Typical conidial head showing comparatively loose sterigmata in two series and conspieuously roughened conidia, strain NRRL No. 278. $A_{1}$, Diminutive head, as often seen in strain NRRL No. $275 . B$, Typical head of Aspergillus granulosus, NRRL No. 1932.

colored, semi-radiate, loosely arranged into two series, primary sterigmata 4 to $7 \mu$ by $3 \mu$, secondary sterigmata 5 to $7 \mu$ by 2.0 to $2.5 \mu$. Conidia globose, 3.5 to $5.0 \mu$, roughened, echinulate to marked with conspicuous color bars, ranging from greenish through olive-gray to yellow-brown or fuligineus. Many strains producing thick-walled hülle cells (fig. $49 \mathrm{E}$ ) ranging in form from irregularly ovate or elongate in some strains, to serpentine, helicoid, or twisted in others, essentially as in Aspergillus flavipes. 
Upon malt agar, colonies frequently heavier sporing and conidial heads generally tending to run to dull gray-green shades rather than brown.

Very common in soil and decaying vegetation. Species diagnosis represents a composite based upon many isolations from this country and abroad.
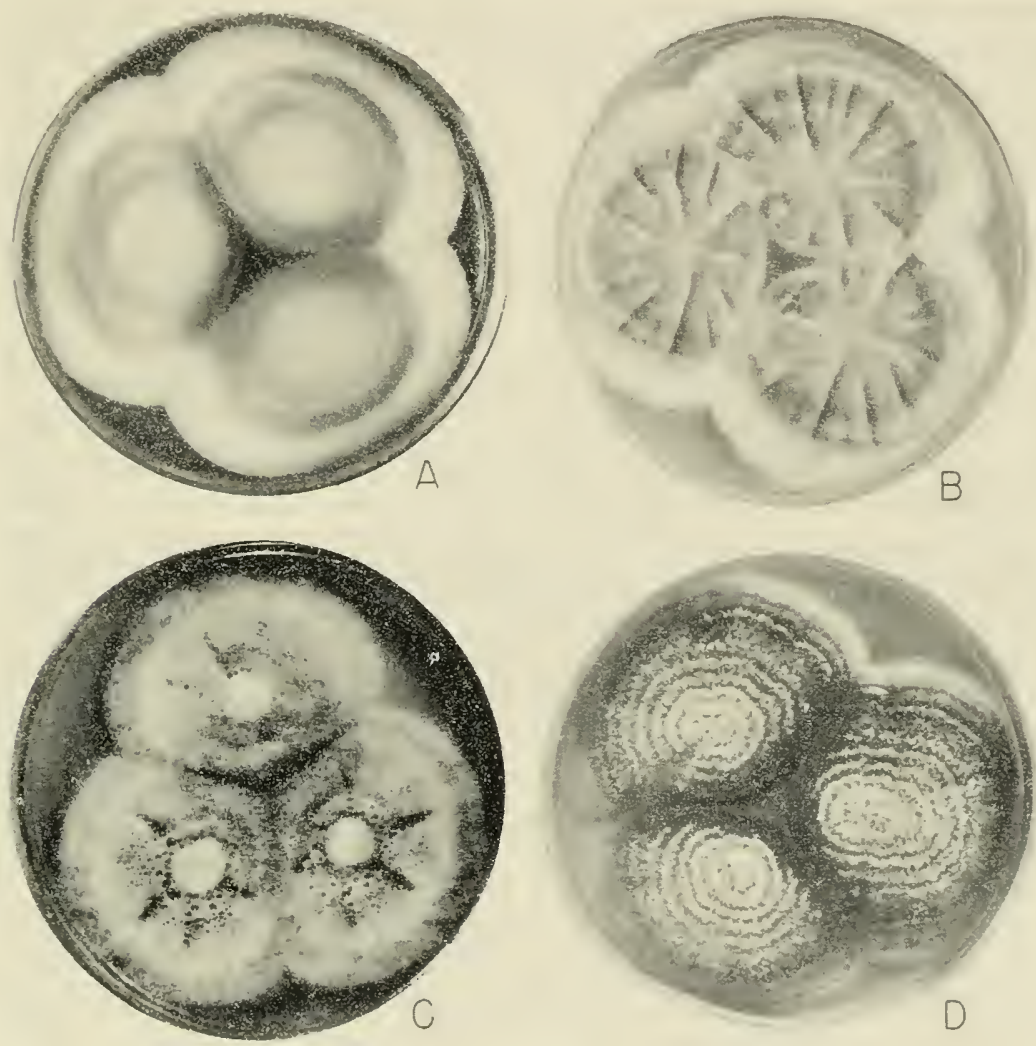

FIg. 48. Aspergillus ustus: cultures growing upon Czapek's solution agar at room temperature, 10 days. A, Strain NRRL No. 275 characterized by loose floccose colonies and moderate sporulation. B, Strain NRRL No. 278 characterized by heavier spore production and the presence of abundant hülle cells. $C, A$. ustus var. laevis, NRPL No. 1852, characterized by loose floccose colonies and conidial heads often near brick red in color. D, Strain NRRL No. 1974 characterized by the production of very abundant hülle cells in concentric zones.

Individual strains differ markedly in their general habit and colony coloration, in the color of their fruiting structures, in the marking and coloration of their conidia, in the presence or absence of hülle cells, and in the form of these structures when present. By a deliberate selection of strains, one can find sufficient difference to warrant the assignment of specific designa- 
tions to particular cultures. Yet all possess the characters noted above and so constitute a well-defined group whose variations are matters of detail. Such differences as occur, moreover, tend to become bridged as comparative cultural and microscopic studies of many isolations are made; hence the use of these differences becomes of questionable value for diagnostic purpose. We have considered it desirable, therefore, to include the whole series under one name as a single species aggregate, Aspergillus ustus, and to call attention to some of the major differences which one may expect to encounter among the members of this species. Although Bainier was not sufficiently explicit in his description of Sterigmatocystis usta to enable us to identify with certainty the form with which he worked, his usage is accepted upon (1) the basis of priority, and (2) the receipt of a culture (Thom No. 4640. 488) from his laboratory labeled Sterigmatocystis usta, which possessed the basic characters of the group as herewith set forth.

Long after the publication of Sterigmatocystis usta, Bainier described a second species belonging to this group, Sterigmatocystis insueta (Bul. Soc. Mycol. France 24: 85-87, Pl. VIII, Fig. 1-13. 1908), and emphasized the fuligineus character of its colonies, the predominant origin of brown fruiting structures from aerial mycelium, the larger size and the darker color of its conidia, which were characterized by the presence of pronounced color bars. Strains showing these characters probably represent the type most commonly encountered among miscellaneous isolations of forms belonging to this group. Recognition of these forms as constituting a distinct species has been considered, but in the absence of any clearly definable line of separation from $A$. ustus it is believed desirable to leave them within the somewhat extended framework of this species. Cultures possessing these characteristics very commonly exhibit hülle cells varying in different strains from ovoid to irregularly elongate, to serpentine, helicoid or otherwise twisted. One strain showing much-twisted hülle cells was isolated and contributed by Thaxter under the manuscript name $A$. helicophorus. Typically, the conidiophores of these forms are grayish-brown, commonly quite dark. The vesicle and sterigmata are likewise frequently colored. Conidial color as seen under high magnifications varies with age from pale to olive green to fuligineus, and conidial markings from fairly coarse echinulations to intensively colored bars and tubercles.

Possibly unaware of the existence of Sterigmatocystis usta and S. insueta (since no mention is made of either species), Abbott (1926) subsequently described Aspergillus minutus. His description indicated that he was dealing with a strain essentially similar to those considered by Bainier as $S$. insucta. This is confirmed by examination of his type culture, NRRL No. 283 (Thom No. 4894.2). A second culture, NRRL No. 285 (Thom No. 4894.1), received from Abboit under the manuscript name A. humus likewise represents a member of this series and differs from the more 
common forms only in its more floccose habits and in the production of somewhat smaller conidial heads.

Among the more striking members of the Aspergillus ustus series examined are two isolations from soil collected in Panama and Mexico, respectively, which upon Czapek's solution agar normally produce strongly zonate colonies consisting of alternating areas of crowded conidial heads and heavy hülle cell development (fig. $48 \mathrm{D}$ ). Except for this difference in colony appearance, however, these strains appear to be typical of the species as described above.

A single strain, NRRL No. 1852, isolated from Louisiana soil, possesses conidial heads of a dull brick red color (Ridgway, Pl. XXXIX: russet vinaceous to sorghum brown) and abundant, much-twisted hülle cells. While Blochwitz's description is too inadequate to permit of detailed comparison, it is suggested that this strain may represent his Aspergillus ustus var. laevis (Ann. Mycol. 32(1/2): 4. 1934), which was described as characterized by "red conidia and crooked hülle cells." In strain NRRL No. 1852, the conidia are conspicuously reddish en masse but appear only slightly colored when viewed with high magnifications. In contrast to most strains of the Aspergillus ustus group, the conidia are finely echinulate rather than coarsely roughened. It is suggested that this form (fig. $48 \mathrm{C}$ ) may represent a transition in the direction of Aspergillus flavipes since the latter species is likewise characterized by much-twisted hülle cells, brown conidiophore walls, and conidia which may show a reddish color in some strains but are smooth in all.

Aspergillus granulosus Raper and Thom, in Mycologia 36: 565-568, fig. 4. 1944.

Colonies upon Czapek's solution agar growing well, attaining a diameter of 8 to $10 \mathrm{~cm}$. in two to three weeks at room temperature, plane or irregularly furrowed, predominantly floccose, uneven in texture, buff to dull brown in color from felted sterile mycelia; conidial heads few in number and generally arising from the substratum direct, less often from aerial hyphae, commonly appearing in clusters, pale blue-green in color; colonies characterized particularly by abundant small, colorless clusters of irregularly globose, ovoid, or elliptical thick-walled hülle cells which superficially suggest perithecial initials and which in mass give to the colony a semi-granular appearance (fig. $50 \mathrm{~B}, \mathrm{E}$, and $\mathrm{F}$ ); reverse in shades of dull yellow and brown; slight mushroom odor. Conidial heads few in number, commonly clustered in small groups, most abundant at colony margin, sometimes occurring on tufts of aerial hyphae, hemispherical to radiate, 75 to $125 \mu$ in diameter, very loose, consisting of comparatively few divergent spore chains (fig. $50 \mathrm{C}$ ), approximately pale niagara green in color (Ridgway, Pl. XXXIII). Conidiophores erect, straight, nonseptate, mostly 350 to 


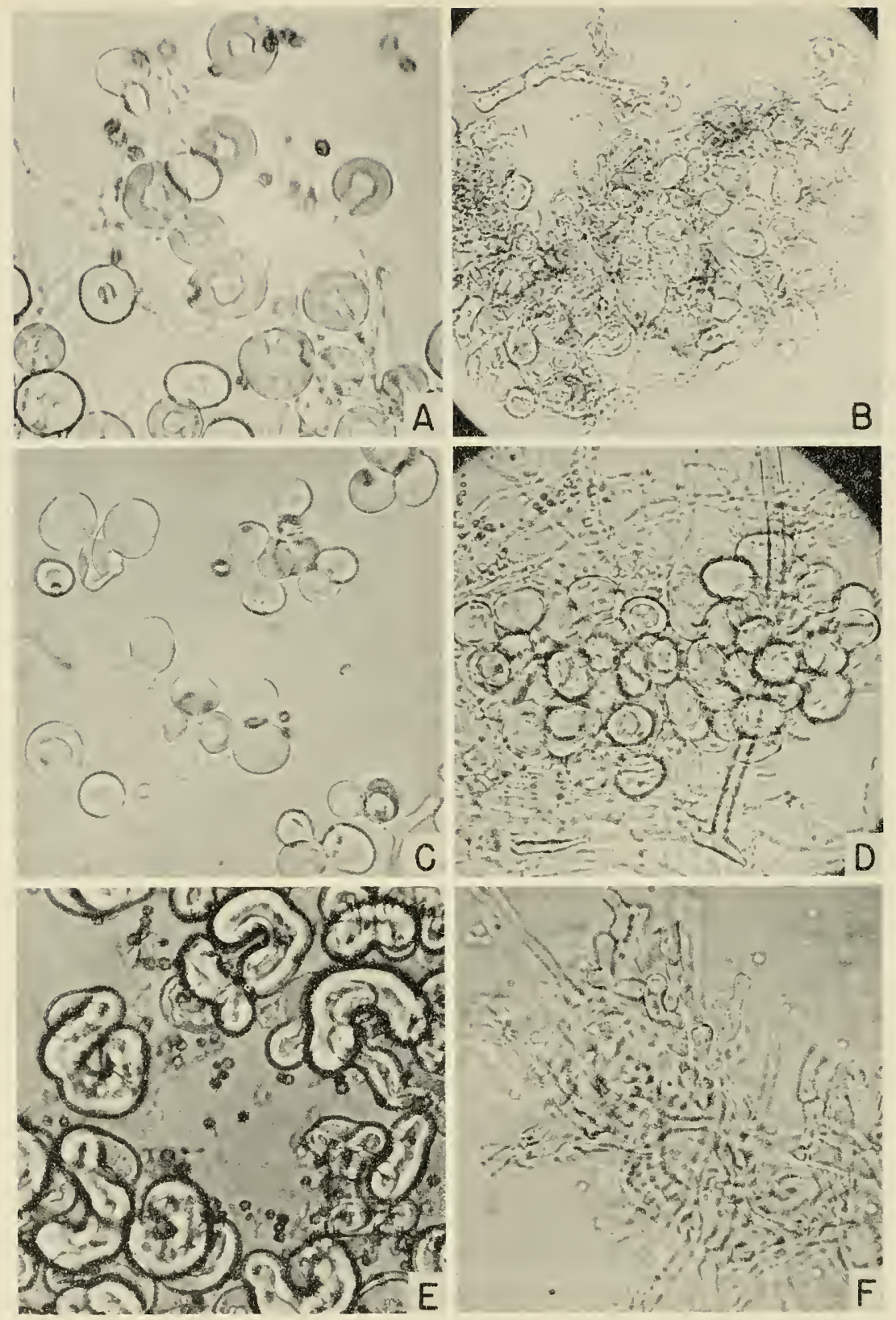

FIg. 49. Hülle cells. A, Characteristic thick-walled, globose to subglobose hülle cells of the Aspergillus nidulans group, A. variecolor, NRRL. No. $1954, \times 450 . \quad B$, Aspergillus caespitosus, strain NTRL No. 1930, hülle cells irregular in form and often poorly developed but of the same general pattern as $A$. nidulans, $\times 275$. C , Aspergillus janus, NRRL No. 1787, hïlle cells approximately globose and of the same basic type as in $A$. nidulans, $\times 450$. D, Aspergillus granulosus, NRRL No. 1932, hülle cells globose to ovoid, somewhat intermediate between $A$. nilulans and A. ustus, $\times 275$. E, Aspergillus ustus, NRRL No. 280, characteristic hülle cells, elongate and much twisted, $\times 450$. Hülle cells in Aspergillus flavipes are of the same pattern. F, Aspergillus carneus, NRRL No. 1926, heavy walled, strongly septate mycelium suggestive of hülle cells, $\times 275$. 
$500 \mu$ in length, 5.5 to $8.0 \mu$ in diameter, approximately uniform in width throughout, thin-walled, smooth, tan to light brown in color, of ten slightly
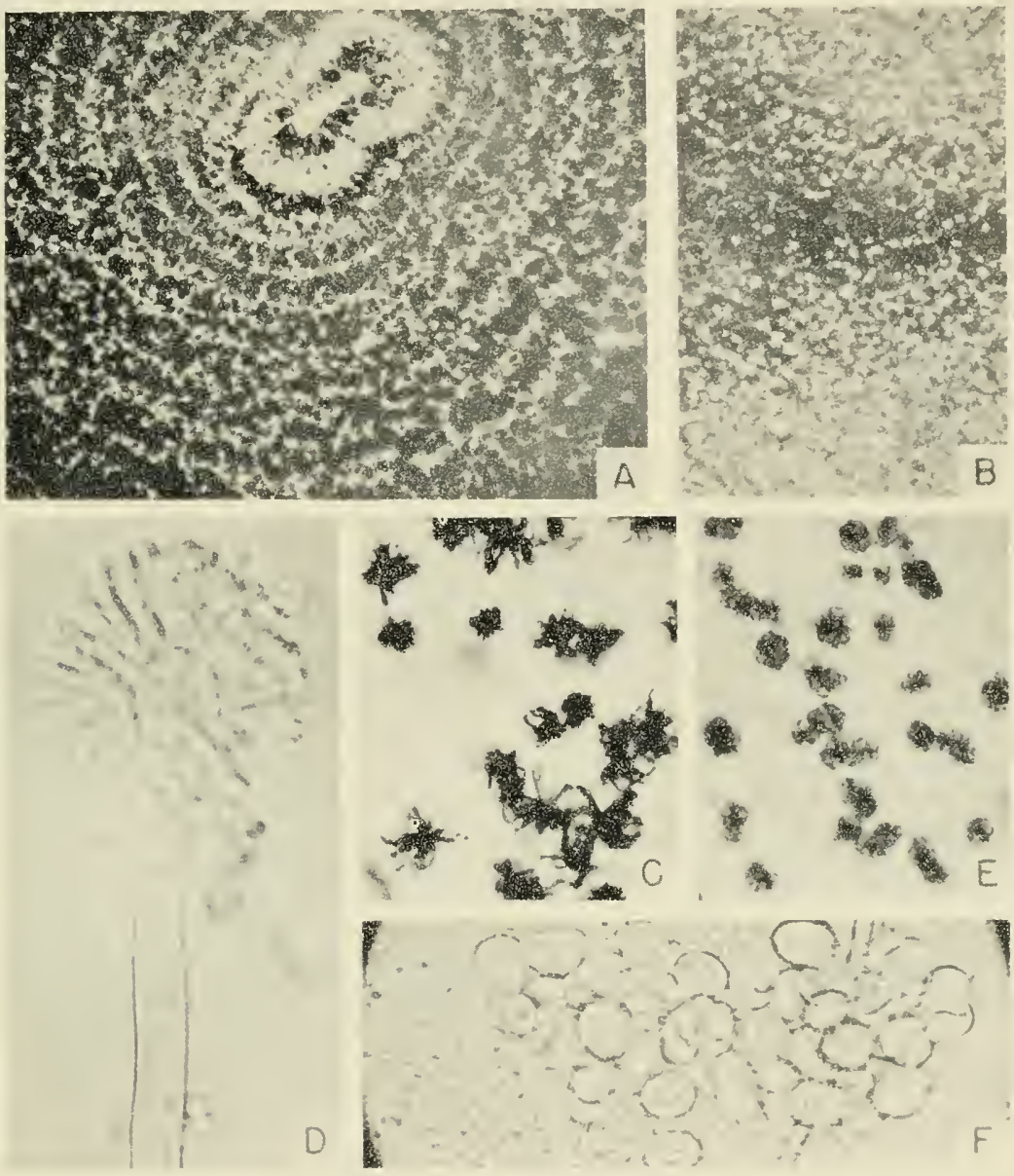

Fig. 50. Aspergillus granulosus; NRRL No. 1932. A, Portion of colony on hay infusion agar showing clusters of conidial structures and small masses of hülle cells, two weeks old, incubation at room temperature, $\times 1.5$. $B$, Portion of marginal area of two colonies on malt extract agar showing characteristic granular appearance resulting from numerous small clusters of hülle cells, $X 1.5$. C , Silhouettes of conidial heads, $\times 48$. $D$, Conidial head showing slightly elongate vesicle, sterigmata in two series, and constriction in conidiophore just beneath the vesicle, $\times 750$. $E$, Small clusters, or "granules", of hülle cells, $\times 48, F$, Double cluster of hülle cells, much enlarged, $\times 265$. (Reprinted from Raper and Thom, "New Species of Aspergilli from Soil," Mycologia 36: Nov.-Dec. 1944.)

constricted just beneath the vesicle (figs. $47 \mathrm{~B}$ and $50 \mathrm{D}$ ). Tesicle orate to elliptical, thin-walled and easily broken, largely covered by sterigmata, 12 to $18 \mu$ in diameter by 15 to $25 \mu$ in length. Sterigmata in two series, 
both comparatively short and stout; primaries 3.5 to $5.0 \mu$ by 3.0 to $4.0 \mu$; secondaries 4.0 to $5.5 \mu$ by 3.0 to $3.5 \mu$, commonly bottle-form. Conidia globose, pale green, delicately echinulate. Mostly 4.8 to $5.5 \mu$ in diameter, rarely larger. Hülle cells abundant, irregularly globose, ovoid or somewhat elongate, commonly 12 to $30 \mu$ in long axis, walls heavy, 4 to $5 \mu$ in thickness, borne primarily in small, colorless clusters which are quite conspicuous at colony margins and lend to them a characteristic granular appearance.

Colonies upon malt extract agar showing an accentuation of hülle cell development (fig. $50 \mathrm{~B}$ ) and a reduction in conidial heads. Otherwise duplicating the cultural picture presented upon Czapek's solution agar.

Colonies upon hay infusion agar (fig. $50 \mathrm{~A}$ ) thin but broadly spreading, characterized by scattered clusters of hülle cells and erect conidial fructifications giving to the culture a sparsely granular appearance.

Type culture NRRL No. 1932 was isolated in 1942 from a sample of soil collected in Fayetteville, Arkansas, and contributed by Mr. F. R. Earle. Additional strains have been isolated from soils collected in Texas, Arizona, and Costa Rica. It is believed common in soils where the temperature remains at a high level during part or all of the year.

Different strains vary materially in the number of conidial heads produced upon common laboratory media such as Czapek's solution and malt extract agars, ranging from abundant heads in some to only widely scattered heads in others. All fruit reasonably well, however, upon hay infusion agar, the medium upon which original isolations were made.

The brown color of the conidiophores, the presence of ovoid to somewhat irregular hülle cells, and the green color of its conidia place this species in the group with $A$. ustus. It differs markedly from the more common representatives of this group, however, in the lighter and persistently green color of its conidia, the small clusters rather than irregular masses of hülle cells, and in possessing somewhat more elongate vesicles. In this latter character it suggests Aspergillus flavipes but is in turn excluded from this group by the green color of its spores.

\section{Occurrence and Economic Importance}

Representatives of the Aspergillus ustus group are perhaps the most abundant of all aspergilli in soil. They regularly occur in large numbers and in considerable variety. The common species, A. uslus, occurs alike in cultivated and forest soils and in approximately equal abundance in soils from southern and from north temperate areas. A. granulosus, on the contrary, has been isolated only from southern sources. Members of the group are not known to be particularly active agents of decomposition, but their great abundance in nature is believed indicative of a significant role in many decay processes.

Their biochemical activities and potentialities are almost completely unknown. 


\section{Chapter XIII}

\section{THE ASPERGILLUS FLAVIPES GROUP}

\section{Outstanding Characters}

Conidiophores smooth, in some shade of yellow, with color often confined to the outer layer.

Heads barrel-form to columnar when well developed, white or slowly becoming some shade of vinaceous-buff to avellaneous (Ridgway, Pl. XL).

Vesicles subglobose to elliptical.

Conidia colorless, smooth, thin-walled.

Hülle cells generally present, helicoid or variously twisted.

The range of variation presented within the group has led workers with only a few representatives before them to offer names and descriptions for the strains under observation. However, when large numbers of strains are brought together and cultivated upon a considerable range of substrata the continuity of the group as a natural and related series of strains becomes apparent. Further study of this whole group may lead to separation upon lines accounting for certain published descriptions not at present identifiable. For purposes of the present manual, however, it is believed desirable to broaden the description of Aspergillus flavipes (Bain. and Sart.) Thom and Church sufficiently to include a closely related series of organisms rather than restrict it to the particular strains studied by Bainier.

Aspergillus flavipes (Bain. and Sart.) Thom and Church, The Aspergilli, p. 155. 1926.

Synonym: S. flavipes Bainier and Sartory (Bul. Soc. Myc. France 27: 90-96, Pl. III., fig. 1-6. 1911).

Colonies upon Czapek's solution agar rather slow growing, becoming 3 to $5 \mathrm{~cm}$. in diameter in about 10 days; mycelium yellowish, dull buff, commonly becoming brownish in age; heads pale to dull buff in some strains to avellaneous or even very light cinnamon in others (Pl. IV F and fig. $51 \mathrm{~A}$ ), submerged mycelium persistently colorless in some strains, developing many shades of yellow, orange, orange-brown to red (Madder-brown of Ridgway, Pl. XIII) or almost black in reverse of colonies in others; in some strains producing at the surface of the agar closely woven yellow to orange masses of hyphae enmeshing numerous helicoid or variously twisted, thick-walled hülle cells; occasional strains showing dark masses 
suggestive of sclerotia; aerial mycelium more or less abundant, colorless or yellow to orange; producing in many strains numerous large drops of transpired fluid, pale to yellow or orange-red (acting as an indieator, changing from yellow with acid to orange-red with alkali); commonly
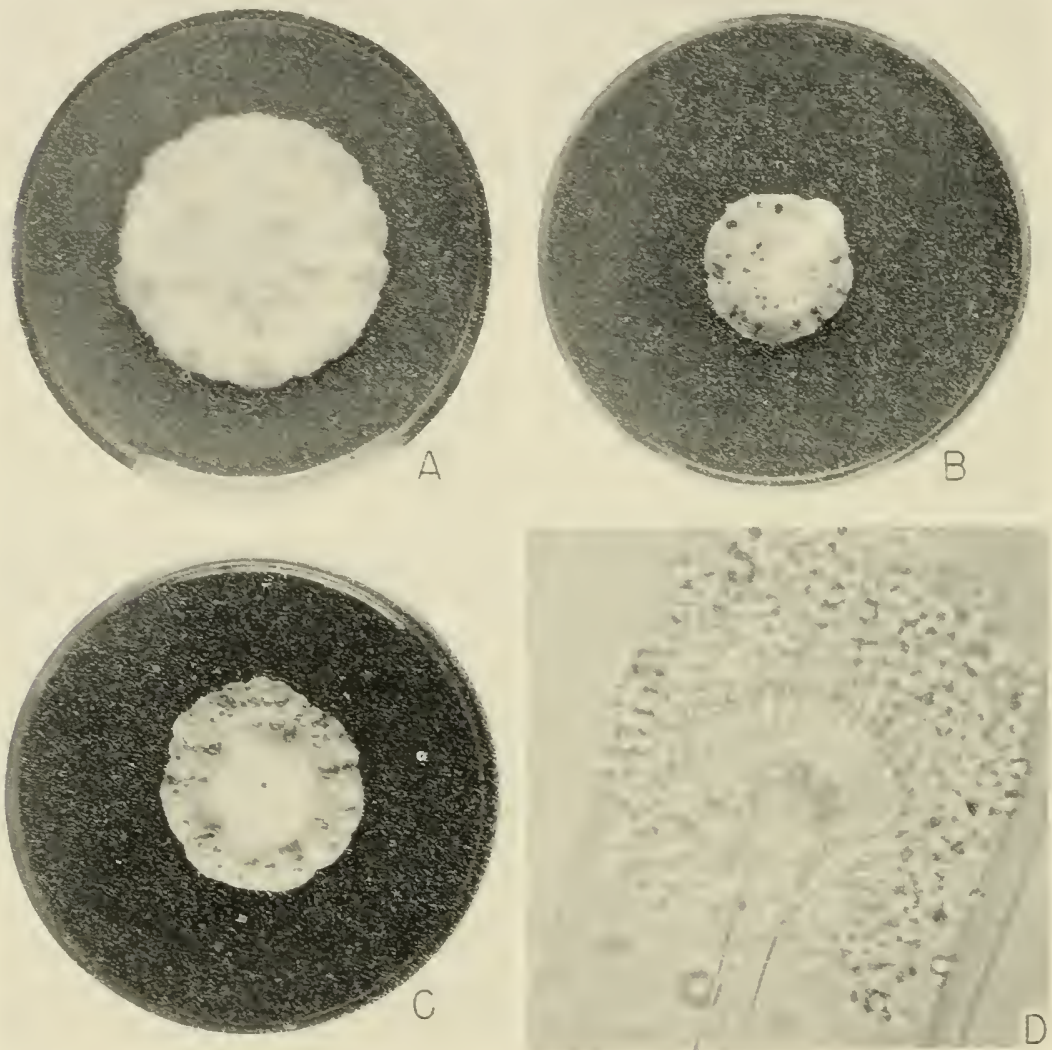

FIG. 51. Aspergillus faripes group. A, A. flaripes NRRL No. 295 growing on Czapek's solution agar, 2 weeks, room temperature. $B$, Strain NRRL No. 287 of the same species, found by White (19t3) to produce a penicillin-like substance. $C$, Strain NRRL No. 1959 characterized by the production of an excessive amount of exudate. $D$, Photomicrograph of a typical head showing the elongate vesicle and crowded sterigmata in two series, $X \div 50$.

characterized by a disagreeable odor approaching putridity. Heads typically becoming columnar masses, shading from persistently white, through shades of pale to deep avellaneous as in A. terreus; but usually in rather sharp contrast to the color of the mycelium. Conidiophores from 300 to $500 \mu$ by + to $5 \mu$ in crowded areas, up to 2 to $3 \mathrm{~mm}$. in length and 
8 to $10 \mu$ in diameter in some strains and under some conditions of culture, walls more or less yellow under the microscope, with color mostly localized in the outer layers of the cell-wall and occasionally as disk-like concretions on the surface of walls otherwise smooth. Vesicles subglobose to elliptical (fig. $51 \mathrm{D}$ ), up to 30 by $40 \mu$ in the largest forms, usually with diameter twice that of the conidiophore in smaller forms. Sterigmata in two series, colorless or nearly so, closely packed over the apex of the vesicle in small heads, and covering the vesicle in large heads, primary sterigmata about 6 or $8 \mu$ by 2 to $3 \mu$, secondary sterigmata 5 to $8 \mu$ by 1.5 to $2 \mu$. Conidia 2 to $3 \mu$, smooth, subglobose, colorless or nearly so under high magnification, with chains aggregated to form columns as seen with the handlens in old cultures.

Cosmopolitan in distribution and particularly common in soil and upon decomposing organic materials.

Historically, the name applied to the series is taken from strains 4640.474 and 4640.402 (Thom Collection) obtained through daFonseca from the Bainier collection in Paris in 1922. These strains showed smooth yellow con $s$ diophores 300 to $400 \mu$ by 3 to $4 \mu$, contrasting with heads that were rath er persistently white and possessed the general morphology of the series as described above. Hülle cells were not found. Nevertheless, the close relationship of these organisms to a great series of cultures obtained from many sources in which these structures are regularly found justifies us in broadening the use of the name.

Culture No. 4640.486 (Thom) received from the Bainier collection as S. rubescens (NRRL No. 291) shows deep floccose colonies with few heads and scattered dark hyphal masses in age. Hülle cells have not been seen in this strain.

Among forms commonly obtained from soils collected from widely scattered areas in this country and abroad, a series of isolates seems to comply with the description given by Blochwitz for Aspergillus archiflavipcs (Ann. Mycol. 32(1/2): 84. 1934). This species as described represents an extreme development toward radiate heads, abundant conidia, conidiophores 2 to $3 \mathrm{~mm}$. in length and the development of deep brown or actual red shades of color in the mycelium. Several strains observed in culture approach this description (fig. $51 \mathrm{C}$ ). Heads are at first globose, then become slowly barrel-form, i.e., short stocky columns. Large brown drops of transpired fluid are commonly seen which become yellow when acidified and return to reddish shades when alkali is added. Recognition of the species does not appear warranted since no clearly definable character exists which distinguishes these isolates from the less colored forms generally considered as representing Aspergillus flavipes in a more restricted sense. 


\section{Antibiosis}

Working with a culture of Aspergillus flavipes from Thom (No. 4303.46), NRRL No. 287, White (1943) has recently demonstrated the production of an antibacterial substance which in its action against Staphylococcus aureus strongly resembles penicillin. The substance was produced in greatest amount in a medium containing 5 to 10 percent corn steeping liquor as the sole nutrient. The addition of sugar is reported as definitely deleterious.

\section{Occurrence and Economic Importance}

Members of the Aspergillus flavipes group like hose of the A. ustus, versicolor, and terreus groups are cosmopolitan in distribution, and are especially common in fertile soil and upon decaying vegetation. They are not known to be active agents of decomposition but are capable of growing in the presence of a limited amount of water, hence are probably significant in initiating or continuing processes of decay where most micro-organisms are incapable of growing. Little is known of the biochemical activities or products of these forms. 


\section{Chapter XIV}

\section{THE ASPERGILLUS VERSICOLOR GROUP}

\section{Outstanding Characters}

Conidial heads hemispherical to almost globose, in many different shades but usually showing green or blue-green.

Conidiophores smooth, colorless, more or less sinuous.

Vesicles globose to ovate or elliptical with radiate sterigmata borne over the upper half to three-fourths of the surface.

Sterigmata in two series.

Spores globose or subglobose, echinulate.

Hülle cells found in occasional strains, globose.

Members of the Aspergillus versicolor group are cosmopolitan in distribution. They occur regularly in soil, upon decaying vegetation, upon stored grains, upon cured meats, and upon a multitude of other products exposed to occasional moist air or undergoing slow decomposition. The morphology of all strains (with the exception of Aspergillus janus) is basically alike, but different strains vary tremendously in their cultural appearance. This is especially true of Aspergillus versicolor where conidial heads in different strains vary in color from dark blue-green through green to yellow-green, to yellow and orange, and finally in some strains to yellowish-cream, buff or flesh color.

\section{Group Key}

I. Heads typically globose, less commonly hemispherical; blue-green in color with the blue element dominant; colony reverse and substratum usually in red or maroon shades ................................. sydowi series A. Conidial heads always blue-green, globose to radiate

A. sydowi (Bain. and Sart.) Thom and Church

B. Conidial heads of two types: (a) blue-green in color, vesicles subglobose to elongate, borne on short conidiophores, and, (b) white, clavate, borne upon long conidiophores.................. janus Raper and Thom

II. Heads hemispherical or nearly globose at times; in green shades without blue admixture, buff to orange-yellow or occasionally flesh colored; colony reverse usually in pink, yellow-red or purple-red shades, rarely almost colorless

A. versicolor series A. Conidia echinulate.................... versicolor (Vuill.) Tiraboschi B. Conidia smooth................... humicola Chad. and Sach. 
Aspergillus sydowi (Bain. and Sart.) Thom and Church, The Aspergilli, p. 147. 1926.

Synonym: S. sydowi Bainier and Sartory, in Ann. Mycol. 11: 25-29, Pl. III, 1913. Compare Pl. IV, cultures No. 4235.17 and K22, Thom and Church, The Aspergilli. 1926.

Colonies upon Czapek's solution agar growing well at room temperature, in most strains close-textured and velvety from crowded conidiophores and heads arising from the substratum (fig. $53 \mathrm{~A}$ ), in other strains more or less
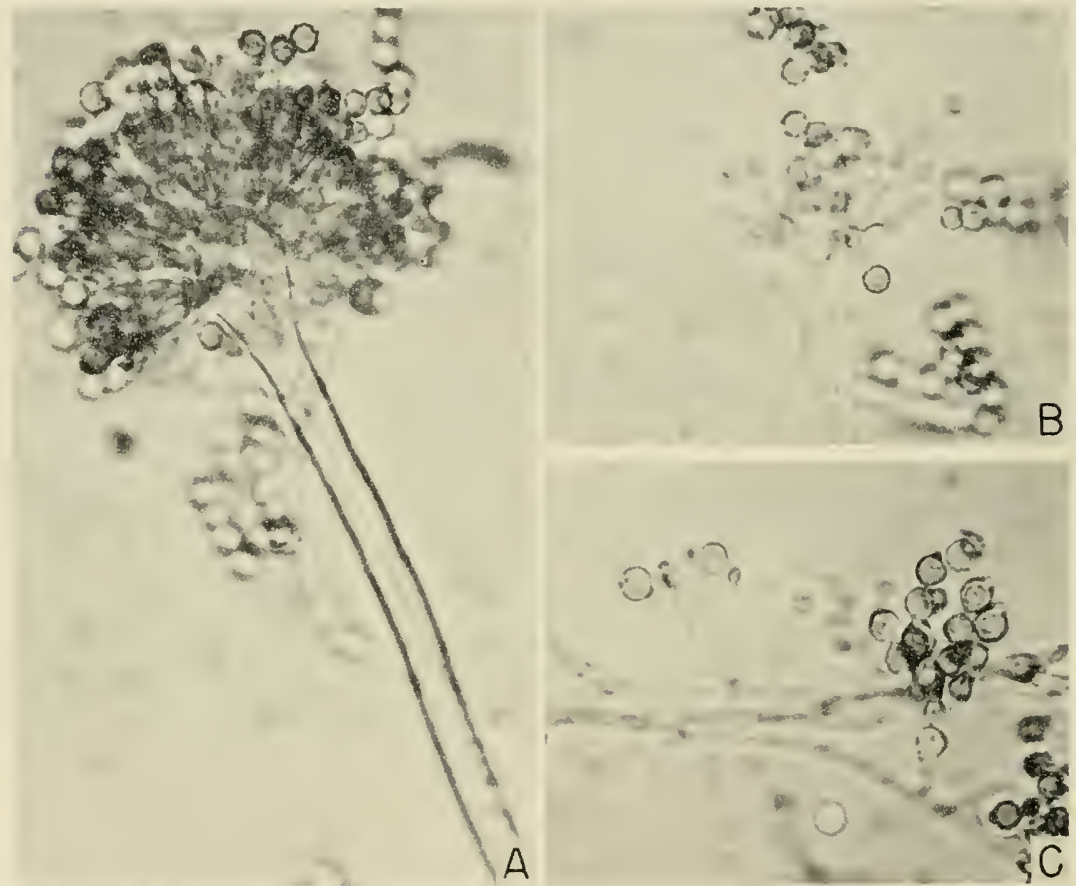

FIG. 52. Conidial structures in Aspergillus sydowi, NRRL No. 250, $\times 800 . A$, Typical, large conidial head borne upon an erect conidiophore arising from the substratum. $B$, Small head, borne as a lateral branch on an aerial hypha, consisting of a small cluster of double sterigmata. $C$, Minute heads in which sterigmata appear in a single series only.

floccose (fig. 53 B) from interlacing and trailing aerial hyphae bearing conidial heads ranging from large, well-formed structures to minute fruits consisting of chusters of simple sterigmata bearing few conidial chains, bluegreen in color, approximately Delft blue or deep Delft blue (Ridgway, Pl. XLII) with the blue effect especially marked in young fruiting areas (Pl. $\mathrm{V} \mathrm{A}$ ), reverse usually in shades of red, from coral red to maroon (Ridgway, 


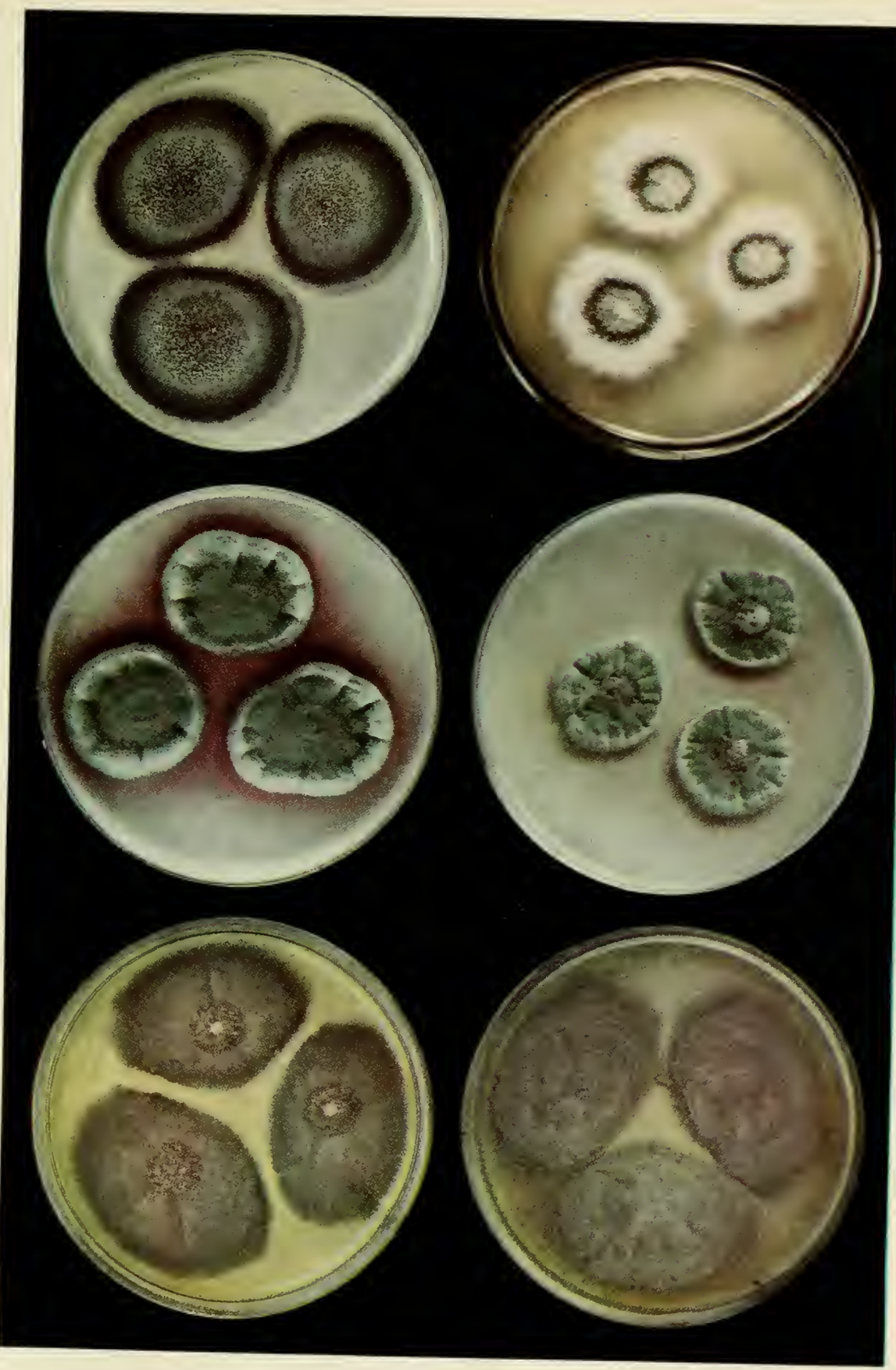

Plate $Y$

16 (upper left), Aspergillus sydou' (Bain. and Sart.), Thom and Church, NRRL No. 250, $B$ (upper right) Aspergillus jamus Raper and Thom, NRRL No. 175\%. C (center left), Aspergillus rersicolor (Vight) lower left), Aspergillus terreus Thom, TRRL Aspergillus versicolor (Vuill.) Tiraboschi, NRRL No. 233. E This objective could not be at tained in NRL No. 265. (The colony colors should be in cinnamon shades. (v. Tiegh.) Blochwitz, NRRL No in making the color reproductions. F (lower right), Aspergillus carneus were encountered in reproducing the colors of this colors should be in shades of vinaceous fawn. Difficulties all other cultures growing upon (zapeh's solution figure.) Figure $B$, cult ure growing on malt extract agar Research Laboratory. Reproduced through co-operation (Color photographs ly Haines, Northern Regional 

Pls. XIII and I) to almost black in some strains in age. Conidial heads typically radiate to nearly globose (fig. $52 \mathrm{~A}$ ), ranging from 100 to $150 \mu$, but often reduced to small penicillate clusters of sterigmata especially in marginal colony areas and upon aerial hyphae (fig. $52 \mathrm{~B}$ and C). ('onidio-
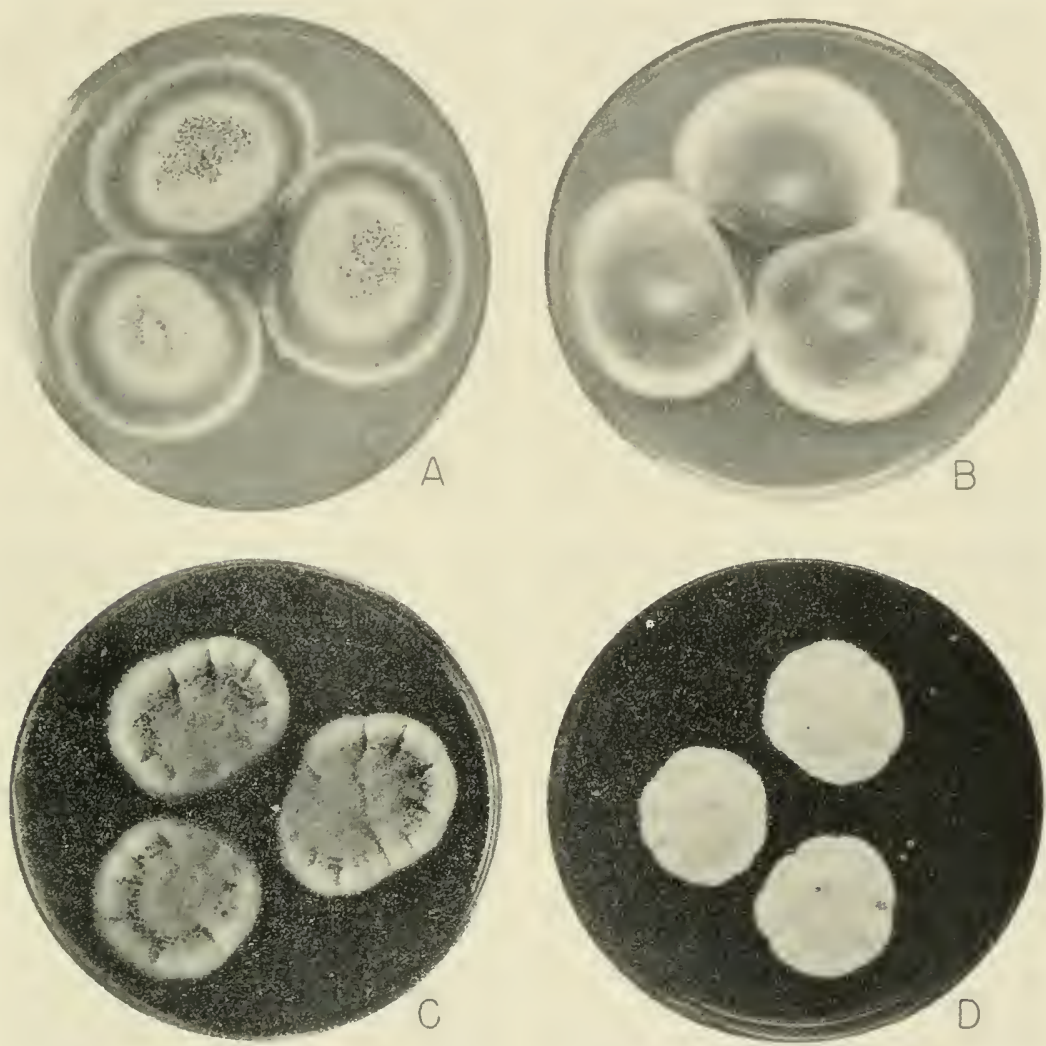

Fig. 53. Colony types in the Aspergillus versicolor group, on Czapek's solution agar, 10 days, room temperature. A, Typical heavy sporing strain of A. sydowi. $B$, Floccose, lighter sporing strain of the same species. C.A. versicolor, NRRL No. 239 , characterized by abundant green conidial heads and the production of deep red exudate. I) A. versicolor, NRRL No. 227, characterized by flesh-colored conidial heads.

phores mostly arising from submerged hyphae, up to $500 \mu$ in length by 5 to $8 \mu$ in diameter, colorless, smooth, comparatively thick-ralled. Vesicles nearly globose, fertile over almost the entire surface, up to $20 \mu$ in diameter. Sterigmata in two series; primary 6 to $7 \mu$ by 2 to $3 \mu$, secondary 7 to $10 \mu$ by 2 to $2.5 \mu$. Conidia globose 2.5 to $3.0 \mu$ (Bainier), in our culture up to $3.5 \mu$ in diameter, conspicuously spinulose, green en masse. Globose hülle cells 
closely resembling those of the $A$. nidulans group have been seen in oceasional strains. No sclerotia or perithecia are found, but clamydospores in solid substrata are reported.

A typical member of our series of isolations was sent to Bainier who concurred in our interpretation of his species.

Reduced conidial apparatus appears in varying degree in all strains of A. sydowi examined. In typical strains, primary sterigmata, with their clusters of secondaries each bearing a chain of conidia, are found singly or variously grouped along trailing aerial hyphae. In other strains there is a progressive development of aerial mycelium, in the form of trailing hyphae either single or in ropes, coupled with a reduction in the number of typical A. sydowi heads. Strains are even occasionally seen in which only a few A. sydowi conidiophores and heads are found in what is otherwise a penicillium-like colony. Thus a series of strains exists which shows a fairly complete gradation from Bainier and Sartory's Sterigmatocystis sydowi to Penicillium restrictum of Gilman and Abbott. We are led to believe that the latter species should probably be assigned to this section of the genus Aspergillus.

While morphologically very close to A. versicolor, A. sydowi is easily presumptively recognized by the characteristic blue-green color of its conidial heads and the red colors in the substratum. A partial list of the sources of the isolations studied includes soil in Washington, D. C., Illinois, Manitoba, Florida, and Ceylon; moldy silk from a stocking factory; concentrated sugar products from Louisiana; dried fish in Japan; and bee-hives in Michigan. It is wolld-wide in distribution and vely adaptable to substrata of widely different nature.

\section{Probable Synonyms}

Aspergillus tiraboschii Carbone (Atti d. Inst. Bol. Univ. Pavia Ser. II. Vol. XIV, p. 320,1914 ) is described in the colors of $A$. versicolor but with the head of $A$. sydowi. It would appear to be more or less intermediate but, unless reisolated, must be dropped because of incomplete data.

Sterigmatocystis tunetana Langeron (Bull. Soc. Path. Exot. 17 : 345-347, text fig., 1924). This mold was recorded as isolated from an ulcer of the hand but failed to produce lesions in animal tests; as described the colonies were blue-green as in A. sydowi.

A. sydowi var. achlamydosporus Nakazawa, Simo and Watanabe (Jour. Agr. Chem. Soc. Japan 10(2): 178-179. 1934). The absence of chlamydospores (hülle cells ?) in a strain of $A$. sydowi is hardly a sound basis for separation.

Stcrigmatocystis cyaneus Mattlet (Ann. Soc. Belg. Med. Trop. 6: 32, 1926) was described without data to separate it from $A$. sydowi.

S. cameleo Sartory, Sartory, and Meyer (Ann. Mycol. 29 : 360-361, Pl. III, figs. 7-8. 1930) by description must have been some strain of $A$. sydowi although the very small smooth conidia do not agree. It may possibly represent a form near $A$. humicola as described by Chaudhuri and Sachar (see p. 193). 
A spergillus janus Raper and Thom, in Mycologia 36:556-561, fig. 1. 1944.

Species characterized by conidial heads of two distinct types, (1) large white heads borne upon long conidiophores terminating in strongly elavate vesicles and (2) smaller, dark green heads borne upon short conidiophores with typically ovate vesicles (Pl. I E and $\mathrm{F}$, and $\mathrm{V} \mathrm{B}$ ).

Colonies varying greatly in color and in texture depending upon the substratum and the temperature of incubation. Upon Czapek's solution agar at $24^{\circ} \mathrm{C}$. (fig. $54 \mathrm{~A}$ ) colonies spreading irregularly, usually consisting of a central floccose mass 1 to $2 \mathrm{~mm}$. deep, pale yellow-buff in color, bearing few and seattered fruiting structures surrounded by an irregular zone of crowded fructifications with dark green heads oeeurring in a dense stand adjacent to the substratum (fig. $54 \mathrm{D}$ ) and with numerous long-stalked white heads projecting above this layer (fig. $54 \mathrm{C}$ ); reverse in dull yellow to light brown shades. When incubated at $20^{\circ} \mathrm{C}$., colonies more restrieted, less floceose and consisting almost exclusively of a dense stand of longstalked white heads with small green heads absent or developing only in age, and arising from trailing aerial byphae entwined among the white fruiting structures. When incubated at 30 to $32^{\circ} \mathrm{C}$. colonies ciose-textured, predominantly green but with central area commonly showing irregular patches of massed hülle cells, buff to dull yellow in color. Conidial heads abundant and consistently dark green in color. Reverse in dull brown shades.

White conidial heads loose in texture (fig. 54 C.), consisting of radiating and divergent chains of conidia, commonly 150 to $200 \mu$ in diameter, oceasionally larger. Conidiophores long, thin, mostly 2 to $2.5 \mathrm{~mm}$. in length by 8 to $10.5 \mu$ in diameter, occasionally larger, ereet, essentially uniform in diameter throughout but of ten marked by numerous and irregularly spaced constrictions, walls smooth, colorless, approximately 1 to $1.4 \mu$ in thickness. Vesicles thin-walled, clavate (fig. $54 \mathrm{G}$ ), mostly 45 to $60 \mu$ by 15 to $18 \mu$ with individual structures larger or smaller, entire surface loosely covered by sterigmata as a rule, but often showing barren areas which may oceupy any part of the sterigmatic surface. Sterigmata in two series, primaries 7 to $10 \mu$ by 3.5 to $4.5 \mu$; secondaries 6 to $8 \mu$ by 2.5 to $3 \mu$. Conidia smooth, colorless, globose to subglobose, mostly 2 to $2.5 \mu$, with maximum about $2.8 \mu$.

Green conidial heads compact, radiate when young, becoming columnar in age and often spreading into two divergent columns. Heads at first in blue to blue-green shades near dark gobelin blue (Ridgway, Pl. XXIV), becoming dark olive-gray in age (Ridgway, Pl. LI), in size commonly ranging from 60 to $75 \mu$ in diameter to 200 to $300 \mu$ in length. Conidiophores ereet, commonly 300 to $400 \mu$ in length, by 6.5 to $8 \mu$ in diameter, of uniform thickness throughout, walls smooth, colorless or very faintly green, approximately 1 to $2 \mu$ thick, enlarging rather abruptly into an ovate vesicle. Vesicle thin-walled, variable in form and dimensions, but commonly oroid (fig. 

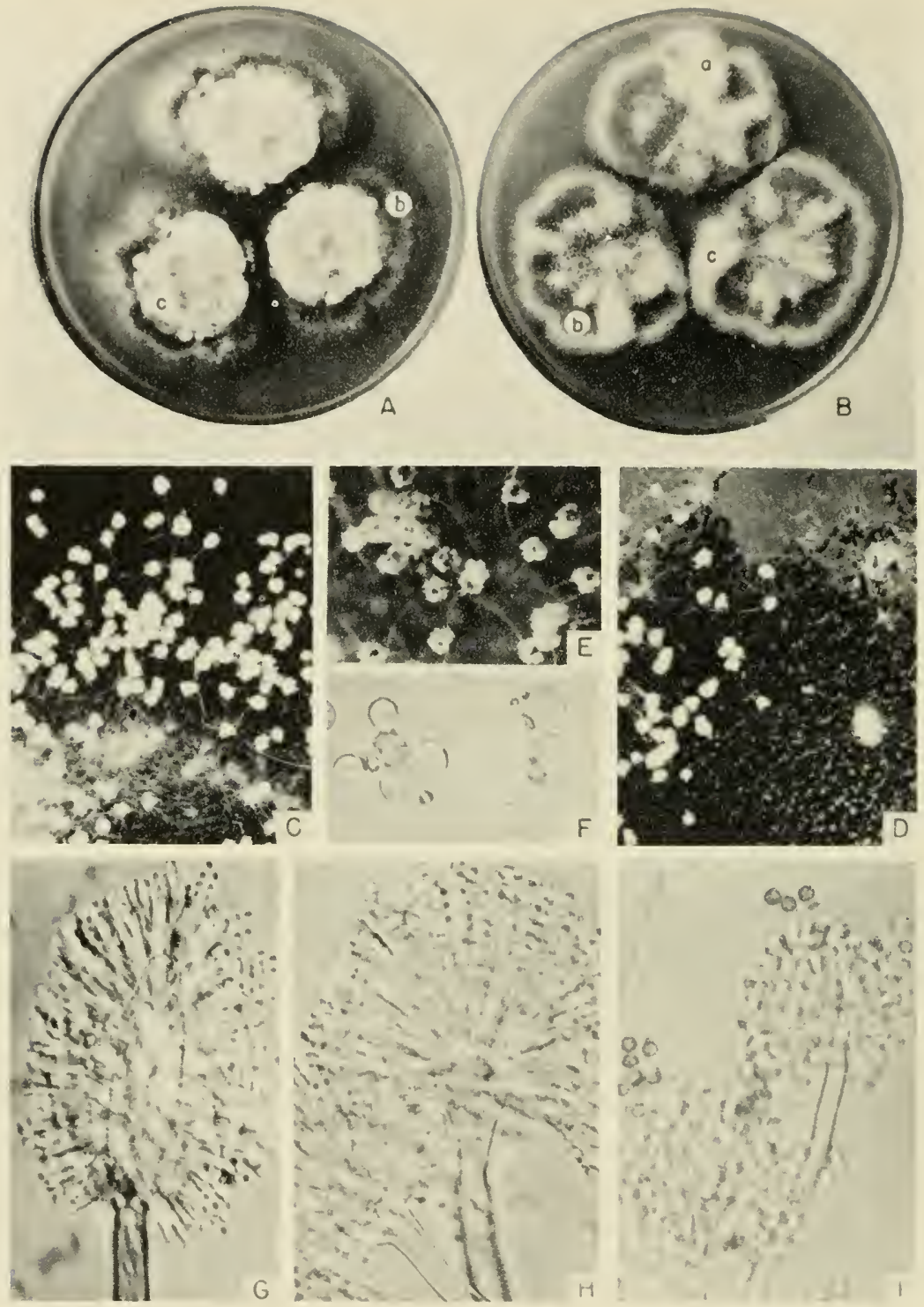

Fig. 54. Aspergillus janus; NRRL No. 1787. A and B, Colonies on Czapek's solution agar and malt agar, respectively, showing $a$, scattered white conidial heads, $b$, crowded green heads, and $c$, massed hülle cells, two weeks old, incubation at $24^{\circ}$ C., $\times \frac{1}{2}$. C , Characteristic white heads borne on long thin conidiophores, $\times 7.5$. $D$, Massed green heads in dense stand adjacent to the substratum, with white heads projecting from the left, and with localized development of hülle cells in two limited areas at right, $\times 7.5$. E, Heads of mixed character with older (outer) conidia white and younger (inner) conidia green, $\times 24 . F$, Hülle cells, $\times 350 . G$, Typical white head showing clavate vesicle, double sterigmata and small, smooth conidia, $\times 450$. $H$, Head of mixed character, at this stage (early) producing small white, smooth conidia, $\times 450$. I, Typical green head, showing small, nearly globose vesicle, double sterigmata and larger, echinulate, green conidia, $\times 450$. (Reprinted from Raper and Thom, "New Species of Aspergilli from Soil," Mycologia 36: Nov.-Dec. 1944.) 
54 I) and occasionally conspicuously elongate, typically fertile over the entire area, ranging in size from 20 to $30 \mu$ by 12 to $18 \mu$. Sterigmata in two scries, rather loosely arranged, primaries 7 to $10 \mu$ by 4 to $4.5 \mu$; secondaries 6 to $8.5 \mu$ by 2 to $2.8 \mu$. Conidia dark green in mass, conspicuously spinulose (fig. $5+\mathrm{I}$ ), globose, mostly 2.5 to $3.5 \mu$, occasionally larger or smaller.

Conidial heads of mixed character, containing both white and green spores, commonly encountered (fig. $54 \mathrm{E}$ ), usually borne upon long conidiophores approaching and often equalling in length those of white heads, vesicles clavate (fig. $54 \mathrm{H}$ ), sterigmata at first bearing colorless smoothwalled conidia, but subsequently bearing dark green spinulose conidia. At temperatures of $24^{\circ} \mathrm{C}$. and above, thick-walled hülle cells abundant, irregular in form (fig. $5+\mathrm{F}$ ), commonly globose to subglobose, not infrequently elongate, commonly more or less curved and often lobed.

Colonies upon malt extract agar growing luxuriantly (fig. $5+\mathrm{B}$ ), generally loose in texture with aerial mycelium prominent, conidial heads normally more abundant than upon Czapek agar, the proportion of white to green heads varying with the temperature of incubation.

Colonies upon hay infusion agar spreading broadly, consisting of a thin submerged mycelium from which develop erect, white and green conidial structures, the relative proportion of these types being dependent upon the temperature of incubation; since comparatively meager growth occurs upon this medium, and since there is a minimum of aerial vegetative hyphae, it constitutes a very favorable substratum upon which to observe the formation of the contrasting fruiting structures characteristic of the species.

The binomial Aspergillus janus was selected for this species because of the contrasting types of conidial heads produced-it is literally a "two-faced" mold.

Type culture NRRL No. 1787 was isolated in February 1942 from Panama soil collected during the summer of 1941 by John T. Bonner of Harvard University. Three additional isolations by members of the Northern Regional Research Laboratory staff have since been made from Panama soils subsequently collected by Mr. Benjamin T. Coghill.

It is believed that this species represents a normal component of the microflora of Panama. Additional evidence in support of this view is furnished by the fact that in 1925 Professor Roland Thaxter sent to Thom under the label "white Panama Aspergillus" a representative of this species. The form was never described by Thaxter, and viable cultures of it were lost from our collection some time prior to 1930. As the correspondence of the time is remembered, Thaxter was plagued by the presence of a small green mold which repeatedly appeared in his cultures as a "contaminant." Fortunately, the original tube received from Thaxter has been preserved, 
and re-examination of this culture leaves no question but that he was dealing with a strain of the species here described, and that the green form which troubled him so much was not, in fact, a contaminant but a different plrase of the same fungus.

Aspergillus jamus var. brevis Raper and Thom, in Mycologia 36: 561-563, fig. 2. 1944.

The variety differs from the species in a number of particulars, foremost among which are (1) the reduced length of the conidiophores bearing both white and green heads and (2) a consistent tendency for white and green conidial structures to develop in approximately pure stands and to appear as contrasting radial sectors.

White conidial heads are of the same general pattern and form as in the species, but are of somewhat smaller dimensions, are borne upon conidiophores generally less than $2 \mathrm{~mm}$. in length by 6 to $8 \mu$ in diameter, and are characterized by elongate but not strongly clarate resicles measuring 20 to $25 \mu$ by 14 to $18 \mu$; conidia are smooth-walled, colorless, globose to subglobose, 2.2 to $2.8 \mu$ in diameter. Green conidial heads are compact, globose to somewhat columnar, borne upon conidiophores 75 to $125 \mu$ by 4 to $6 \mu$ with globose to subglobose vesicles measuring 8 to $15 \mu$ by 10 to $18 \mu$; conidia are dark blue-green, strongly echinulate, and 3.5 to $4.5 \mu$ in diameter.

The vesicles of white heads in the variety brevis are of approximately the same size and form as the vesicles of green heads in the species itself, whereas the conidiophores bearing each type of head are approximately one-half the length of those bearing the same type of hear in the species. The most striking character distinguishing the variety, however, is the manner in which areas of white and green heads are sharply separated along radial lines. Conidial heads of mixed character are produced and usually can be found along the frontier between white and green sectors.

Type culture NRRL No. 1935 was isolated in July 1942 from a sample of soil collected in Alameda in southern Mexico, and forwarded to us in June by Mr. William B. Roos.

Aspergillus versicolor (Tuill.) Tiraboschi, in Ann. Botan. (Rome) 7:9. 1908 .

Synonym: S. versicolor Vuillemin. Mirsky, B., in Thèse de Méd. Nancy, no. 27, p. 15 et seq. 1903 . See Thom and Church, The Aspergilli, p. 142, 1926.

Colonies upon Czapek's solution agar rather slow growing, compact, in some strains velvety and consisting almost entirely of closely crowded conidiophores arising from the substratum, in other strains showing a marked development of floccose hyphae bearing more or less abundant 
conidiophores as short aerial branches, in still others a combination of both growth types with colony centers initially floccose and outer areas almost relvety, at first white, passing through shades of yellow, orange-yellow, tan,

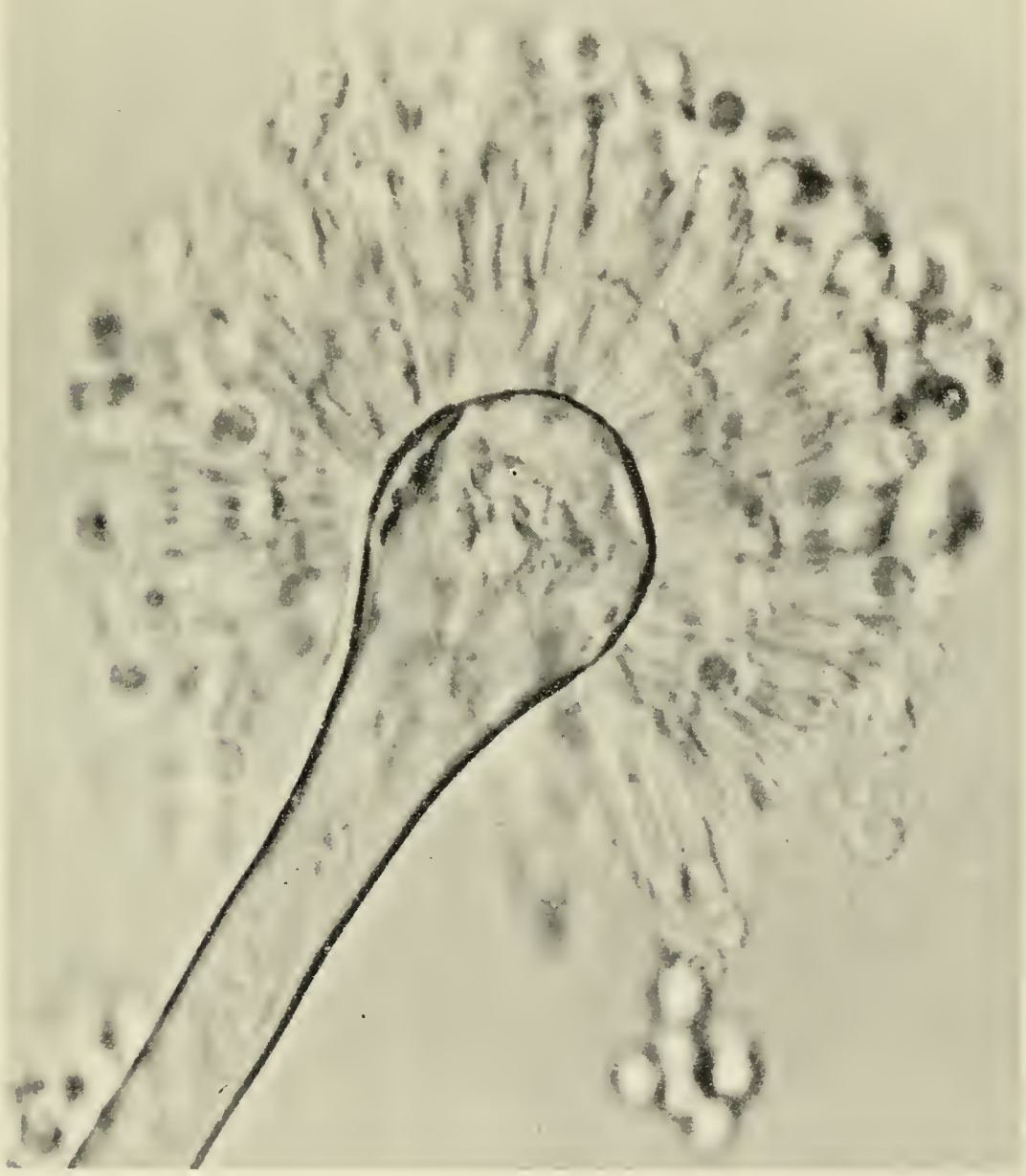

FIG. 55. Aspergillus versicolor, strain NRRL No. 227. Photomicrograph showing details of head structure. Note particularly the double series of sterigmata, $\times 2100$.

to yellowish-green shades such as pea green or sage green (Ridgway, Pl. XLVII) depending upon the strain (Pl. V C and D, and fig. $53 \mathrm{C}$ and $\mathrm{D}$ ) and conditions of culture, occasionally with the green colors almost or com- 
pletely lacking; reverse and substratum occasionally colorless or nearly so, mostly passing through shades of yellow to orange then rose, purple-red or red, the particular shade and intensity of color normally persisting as a strain or varietal character. Heads roughly hemispherical, radiate, up to 100 to $125 \mu$ in diameter; rarely approaching columnar. Conidiophores colorless, smooth, up to 500 or even $700 \mu$ by $5 \mu$ or approaching $10 \mu$ near the vesicles. Vesicles 12 to $20 \mu$ in diameter, fertile area hemispherical or semielliptical (fig. 55) passing almost imperceptibly into the funnel-like enlarged apex of the conidiophore. Sterigmata in two series, primary commonly 8 to $10 \mu$ by $3 \mu$, occasionally less, secondary 5 to $10 \mu$ by 2 to $2.5 \mu$. Conidia globose, usually delicately echinulate, mostly 2.5 to $3 \mu$, occasionally $3.5 \mu$ or $4 \mu$, usually borne in loosely radiating chains.

In occasional highly colored strains vesicles and sterigmata may be colored.

Hülle cells of the Aspergillus nidulans type are occasionally seen.

Neither perithecia nor sclerotia have been found.

The diagnosis is drawn broadly enough to cover a common and very abundant series of organisms which vary greatly in colony appearance. In some strains aerial growth is composed of conidiophores and heads only; in others it is made up of floccose felts or ropes of hyphae bearing short conidiophores and small heads which retain the characteristic arrangement of vesicles, sterigmata, and conidial chains.

The wide range of colony structure and coloration of strains, ${ }^{1}$ and the varied conditions under which these have been collected probably account for the appearance of many names in the literature which refer to cultures of the group but which cannot be safely separated and identified by the descriptions given.

The following list of species are believed to represent synonyms:

S. ambari Beauregard (Ann. de Micrographie 10: 255-278, pl.1. 1898). Probably one of the series, but never identified again.

S. bicolor J. Ray (Rev. Gen. Bot. 9: 193-212, 245-259, 282-304, Pl. 12-17. 1897) is probably one of this series. Primary sterigmata were reported filled with red coloring matter and conidia globose, spinulose, up to $2.5 \mu$ in diameter.

Sterigmatocystis brodeni Mattlet (Name only in Ann. Soc. Belge Med. Trop. 6: 31, 1926; discussion, ibid 4: 167-171, figs. 1, 2. 1924). Apparently a member of this series.

A. flavo-viridescens Hanzawa (Jour. Coll. Agr. Tohoku Imp. Univ. Sapporo 4: 232-3, pl. 21, figs. 1-4. 1911). Culture No. 4291.10 (Thom) received from Hanzawa under this name belongs to the $A$. versicolor group.

A. versicolor var. glauca Blochwitz (Ann. Mycol.32(1/2):86. 1934). This variety has the color of the $A$. glaucus group which is more deeply blue than $A$. versicolor. Isolated in the skin clinic at Kiel upon human skin showing "ringworm."

${ }^{1}$ For a more complete discussion of the range of cultural types found in this series, the reader is referred to Thom and Church, The Aspergilli, pp. 142-145, 1926. 
S. glauca Bainier (Bul. Soc. Bot. France 27: 29-30, pl. 1, fig. 3, 1880, ibid. 28: 77, 1881). From extract of henbane, dregs of wine, casks, and corks.

A. globosus Jensen (N. Y. Cornell Agr. Exp. Sta. Bul. 315, p. 482, 1912). The type culture received from Whetzel (Thom No. 2705) is certainly a member of this series, and is characterized by yellowish-green to olive-green conidial areas, with colony reverse in yellowish-orange to wine red.

S. polychroma Ferraris (Fl. Ital. Crypt. Hyph. p. 640). Syn. A. versicolor, fide Tiraboschi, in Ann. de Botanica (Rome) 7: 9, 1908.

S. spuria Schroeter (Cohn Krypto. Fl. von Schlesien 3: 2 Hälfte, Lief. 1, p. 218, 1893). Position in doubt. May represent a form of $A$. versicolor similar to the flesh colored forms discussed by Thom and Church in The Aspergilli, p. 145, 1926, or may belong with $A$. carneus (See p. 201).

A. tabacinus Nakazawa, Simo, and Watanabe (Jour. Agr. Chem. Soc. Japan 10(2): $177-178,1934)$. The detailed figures given in contrast to their own strain of $A$. versicolor showed differences which disappear when large numbers of isolations are studied.

Aspergillus humicola Chaudhuri and Sachar, in Ann. Mycol. 32: 97. 1934.

\section{Characterization after Chaudhuri and Sachar}

Colonies on Czapek's solution agar, at first white passing through shades of olive-gray (Ridgway, Pl. XLVI. 20. ${ }^{\circ-y y}$ ) velvety at margin, floccose toward the center; reverse and substratum in shades of yellow, heads radiate. Conidiophores arising directly from the substratum, up to $300 \mu$ in length by 4 to $5.4 \mu$ in diameter, or as short branches, about $70 \mu$ long, from aerial hyphae; walls smooth and almost colorless. Vesicles 9 to $15 \mu$ in diameter, colorless, flask-shaped, with sterigmata radiating from the whole surface of the larger heads, or only borne in the upper third in small heads; primary sterigmata 3.6 to $5.4 \mu$ by 1.8 to $2 \mu$; secondary sterigmata 3.6 by $1.8 \mu$. Conidia globose, smooth, 2 to $3 \mu$ in diameter, in radiating chains.

Neill (1939) is believed to have correctly placed this organism with $A$. versicolor and its allies despite the smoothness of its conidia. The "almost" colorless conidiophore suggests relationship to Aspergillus ustus; in this group certain forms (e.g., Blochwitz's A. ustus var. laevis) apparently have spores smooth or nearly so (see p. 175).

\section{Pathogenesis}

Aspergillus sydowi is not reported by name as a parasite, but strains described in terms which musi be interpreted as placing them with $A$. sydowi includes $A$. tunetanus (Langeron) Dodge from fleshy lesions on a hand in Tunis: A. Vancampenhouti (Mattlet) Dodge also from tropical Africa; $A$. cyaneus (Mattlet) Dodge from the same region. The evidence at hand links $A$. sydowi more closely with $A$. nidulans than was formerly supposed, although such relationship is strongly indicated by the identical character of their hülle cells. The fragmentary descriptions commonly given for 
individual pathogenic molds isolated by persons unfamiliar with the literature are rarely definite enough to separate nearly related forms.

An occasional culture of $A$. versicolor is obtained from apparently pathogenic sources. Thus far experimental work has not shown evidence of actual lesions in human flesh. Little colonies bearing green heads and conidia were drawn with a breast pump from an inflamed mammary gland and kept in culture for many years but failed to grow in laboratory media at blood heat. Aspergillus versicolor var. glauca Blochwitz was isolated from human skin showing "ringworm" at the skin clinic in Kiel, but apparently pathogenicity was not proved experimentally. Strains of A. versicolor are frequently observed upon dried salted lean beef, thus showing its caparity to grow in and upon meat products, but not giving direct evidence of narticipation in any pathological process.

\section{Occurrence and Economic Importance}

Members of the Aspergillus versicolor group appear widely distributed in soil, on spoiling and drying food stuffs, breads, cereals, old cheese, dried meats, cured India rubber, musty vegetable products, and other substrata. characterized by a moderately low water content or containing factors toxic: to most organisns. They are reported as capable of decomposing certain paraffins. The production of proteolytic enzymes by most strains is shown by the digestion of milk and the liquefaction of gelatin. Aspergillus sydowi, in particular, is a characteristic component of all soil examined.

Fat production by A. sydowi has been studied quite extensively by Professor Peterson and associates at the University of Wisconsin. For reference to this work see the various papers listed in the Topical Bibliography under the heading "Chemistry of Mold Tissues." 


\section{Chapter XV \\ THE ASPERGILLUS TERRELS GROUP}

\section{Outstanding Characters}

Heads columnar, in cinnamon, pale buff or light flesh colors.

Conidiophores smooth, colorless, rarely exceeding $250 \mu$ in length.

Vesicles hemispherical, with upper half to two-thirds covered by sterigmata.

Sterigmata in two series, generally crowded.

Conidia smooth, globose to slightly elliptical, small.

Included here are members of a variable and cosmopolitan group of Aspergilli especially common in soil. They differ markedly in color and in colony appearance and to a lesser degree in the texture of their conidial heads. The group may be separated as follows:

\section{Group Key}

I. Conidial heads in cinnamon or orange-brown shades, compact, uniform in diameter throughout............................... terreus series A. Colonies velvety, conidiophores mostly in a dense stand arising from the substratum.

1. Conidial heads in dull cinnamon shades........... A.terreus Thom

2. Conidial heads orange-brown near xanthine orange (Ridgway)

A. terreus var. boedijni (Bloch.) n. var.

B. Colonies floccose, conidial heads arising from aerial hyphae.

1. Mycelium colorless, heads light pinkish-cinnamon in color

A. terreus var. floccosus Shih

2. Mycelium yellow, heads developing late, in cream or light tan shades A. terreus var. aureus n. var.

II. Conidial heads white or flesh colored, loose textured not strictly uniform in diameter.

A. carneus series

A. Colonies in light flesh colors, ranging from near white to vinaceous-fawn

(Ridgway). Thick-walled hyphae suggestive of hülle cells are generally

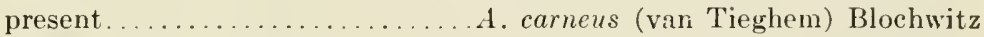

B. Colonies persistently white or becoming dull ivory in age. Thick-walled cells often present........................... niveus Bloch.

Aspergillus terreus Thom, in Turesson, Gote, Svensk Botanisk Tidskrift 10: 5, 1916, without description; diagnosis Thom and Chureh, in Amer. Jour. Bot. 5: 85-6. 1918.

Synonym: A. galeritus Blochwitz, in Ann. Mycol. 27(3/t): 205. Taf. III. 1929 .

Colonies upon Czapek's solution agar growing well at room temperature and up to $37^{\circ} \mathrm{C}$., spreading, plane or marked by shallow radial furrows, 

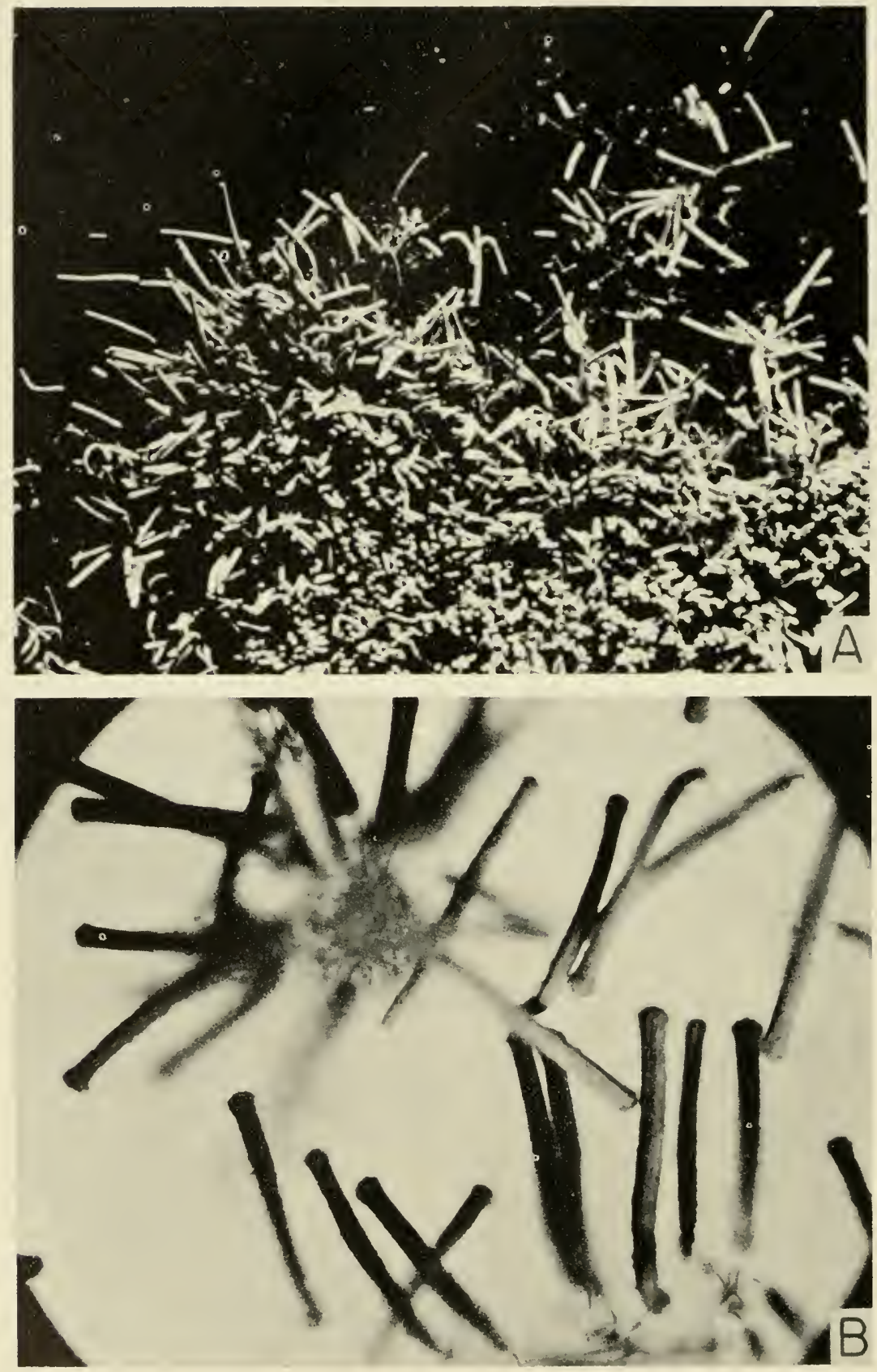

Frg. 56. Aspergillus terreus. A, Colony margin of a typical strain, NRRL No. 265 , showing crowded, long columnar heads, $\times 14$. $B$, Scattered but typical conidial heads of an ultra-violet light-produced mutation unable to utilize $\mathrm{NO}_{3}$ nitrogen, $\times 50$. Both cultures on Czapek's solution agar, 2 weeks. 
velvety (fig. $58 \mathrm{~A}$ ) or in some strains showing tendency toward floccosity in central colony areas, heavy sporing throughout with massed columnar heads giving to colonies their characteristic color and texture, in color ranging through various cinnamon shades (Ridgway, Pl. XXIX) depending upon the abundance and maturity of the heads (Pl. V E), amber exudate produced in some strains, odor transient to none, reverse in dull yellow to brown shades. Conidial heads long columnar (fig. $56 \mathrm{~A}$ ), with conidial chains compacted together, of uniform diameter throughout entire length, commonly ranging from 150 to $500 \mu$ or more in length by 30 to $50 \mu$ at maturity, ranging from cinnamon-buff through cinnamon to Sayal brown (Ridgway, Pl. XXIX). Conidiophores more or less flexuous, smooth, colorless, commonly ranging from 100 to $250 \mu$ by 4.5 to $6.0 \mu$, approximately uniform in width throughout (fig. $57 \mathrm{~A}$ and $19 \mathrm{~A}$ ). Vesicles hemispherical, dome-like, commonly 10 to $16 \mu$ in diameter, merging almost imperceptibly into the supporting conidiophore. Sterigmata in two series, primaries crowded (fig. $57 \mathrm{~A}$ ), parallel, 5.0 to $7.0 \mu$ by 2.0 to $2.5 \mu$, secondaries closely packed 5.5 to $7.5 \mu$ by 1.5 to $2.0 \mu$. Conidia globose to slightly elliptical, commonly 1.8 to $2.4 \mu$ in diameter.

Species description based upon type strain NRRL No. 255 (Thom No. 144) and innumerable additional isolations from soils and other sources in this country and abroad. The species is especially abundant in warm and comparatively dry arable soils.

The great majority of isolates belonging to this series fall within $A$. terreus in its strictest sense, and duplicate in all essential particulars the description given above for this species. Nevertheless, wide natural variation among strains is encountered when great numbers are isolated from widely separated sources. Some of these are quite striking in appearance and have apparently furnished the bases for species and varietal description by other workers.

\section{Aspergillus terreus var. boedijni (Bloch.) n. var.}

Blcchwitz, in Ann. Mycol. 32(1/2): 83, 1934, described Aspergillus boedijni as a new species differing from Aspergillus terreus primarily in the color of its conidia. These were reported as pure yellow at first, becoming pure brown or ochraceous-brown in age, and brighter than A. galeritus Blochwitz. ${ }^{1}$ In our experience strains are occasionally encountered which are characterized by a bright, orange-brown color instead of the dull cinna-

${ }^{1}$ A. galeritus Blochwitz (Ann. Mycol. 27(3/4):205, Taf. III, 1929) is a redescription of $A$. terreus Thom. Blochwitz acknowledged having Thom's type at hand when he renamed this species. No reason was given beyond the claim that he had had the organism in culture for some years before Thom's description $0^{*} A$. terreus was published. 
mon shades typical of the species. Among cultures currently under examination, this character is noted in an isolate from Argentine soil, is somewhat more marked in NRRL No. 680 flom Dr. G. A. Ledingham, Ottawa, Canada, and is particularly striking in NRRL No: 1913 isolated by Dr. .C W. Emmons from Arizona soil. In all of these strains the basic morphology is that of a typical A. terreus, hence Blochwitz's separation is reduced to varietal rank.

Aspergillus terreus var. floccosus Shih, in Lingnan Sci. Jour. 15: 372, Pl. 16, fig. 3, 1936.

Strains characterized by deep floccose colonies in which conidial heads are less abundant, develop late, and are borne almost entirely upon aerial hyphae, are frequently encountered (fig. $58 \mathrm{~B}$ ). While the conidial structures of certain of these strains appear entirely normal, in the majority of isolates the heads are somewhat less compact and generally lighter in color. This color difference is particularly marked among isolates from soils collected in Texas, Central America, and Cuba with color commonly light pinkish-cinnamon (Ridgway, Pl. XXIX) to vinaceous-buff (Pl. XL) in age. No sharp line of separation can be drawn between typical strains of $A$. terreus and the floccose forms under consideration since isolates of intermediate character are encountered; nevertheless, these strongly floccose cultures occur with sufficient frequency to warrant recognition of Shih's varietal designation if his interpretation is somewhat broadened. The variety is considered by the writers as a strongly floccose Aspergillus terreus in which the head is commonly less compact and lighter in color, but with the basic morphology of the conidial apparatus remaining that of the species proper. This variety is represented by such strains as NRRL Nos. 1920 and 1921, isolated from Cuban soil contributed by Professor J. M. Osorio, University of Havana; No. 1922, isolated from Texas soil, collected and sent to us by Dr. F. E. Clark from Greenville, Texas.

Other strains examined almost completely bridge the gap between the pale colored strains of $A$. terreus var. floccosus and the light flesh colored forms characteristic of Aspergillus carneus.

\section{Aspergillus terreus var. aureus n. var.}

This new and striking variety differs from the species in a number of particulars: colonies upon Czapek's solution and malt extract agars are comparatively slow growing, floccose, ranging up to 3 to $4 \mathrm{~mm}$. deep, and are bright golden yellow in color. Conidial structures are produced tardily and in limited numbers. Conidiophores are appreciably longer than those of the species, often becoming $500 \mu$ or more in length, and bear columnar heads, generally loose in texture, ranging in color from cream or light buff 
to light pinkish-cinnamon. Microscopically, the conidial structures approximate those of the species itself. Separation as a new variety is based primarily upon the characteristic coloration of the growing colony.
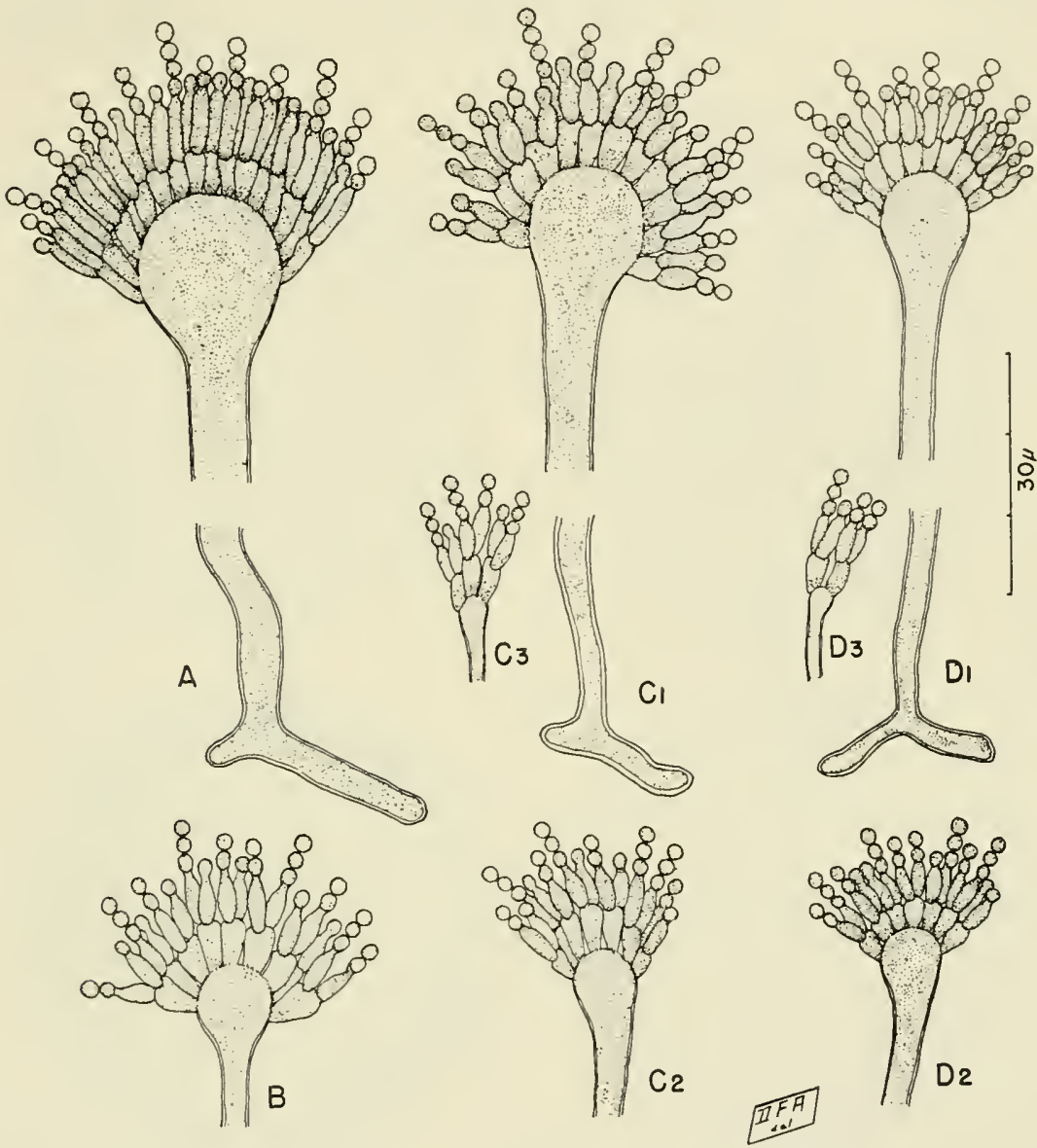

Fig. 57. Conidial structures of members of the Aspergillus terreus group, $\times 840$. A, A.terreus, type strain NRRI. No. 255 (Thom No. 164). B, A.terreus var, aureus, NRRL No. 1923. $C_{1}, C_{2}$, and $C_{3}, A$ carneus, NRRL No. 1928, conidial heads vary greatly in size. $D_{1}, D_{2}$, and $D_{3}, A$. niveus, NRRL No. 515, conidial heads of varying dimensions.

Type strain NRRL No. 1923 (fig. 16 D) was isolated from Texas soil contributed by Dr. F. E. Clark. Additional strains showing approximately the same cultural and morphological characteristics have been isolated from soils collected in Arkansas and Arizona. In A. terreus var. aureus the yellow coloring matter is lodged in the vegetative mycelium, and there are no suggestions of hülle cells; in $A$. carneus, however, approximately the same 
yellow tints are developed through the massing in localized colony areas of thick-walled hyphae suggestive of hülle cells (see p. 201).

At least two other species have been described which are believed to represent probably synonyms of Aspergillus terreus:

Aspergillus fuscus Amons (Archief, voor de Suikerindustrie in Nederlandsch. Indie Jaarg. 29, Deell, pp. 8-10,1921) by description is obviously a form closely related to, if not identical with, A. terreus.
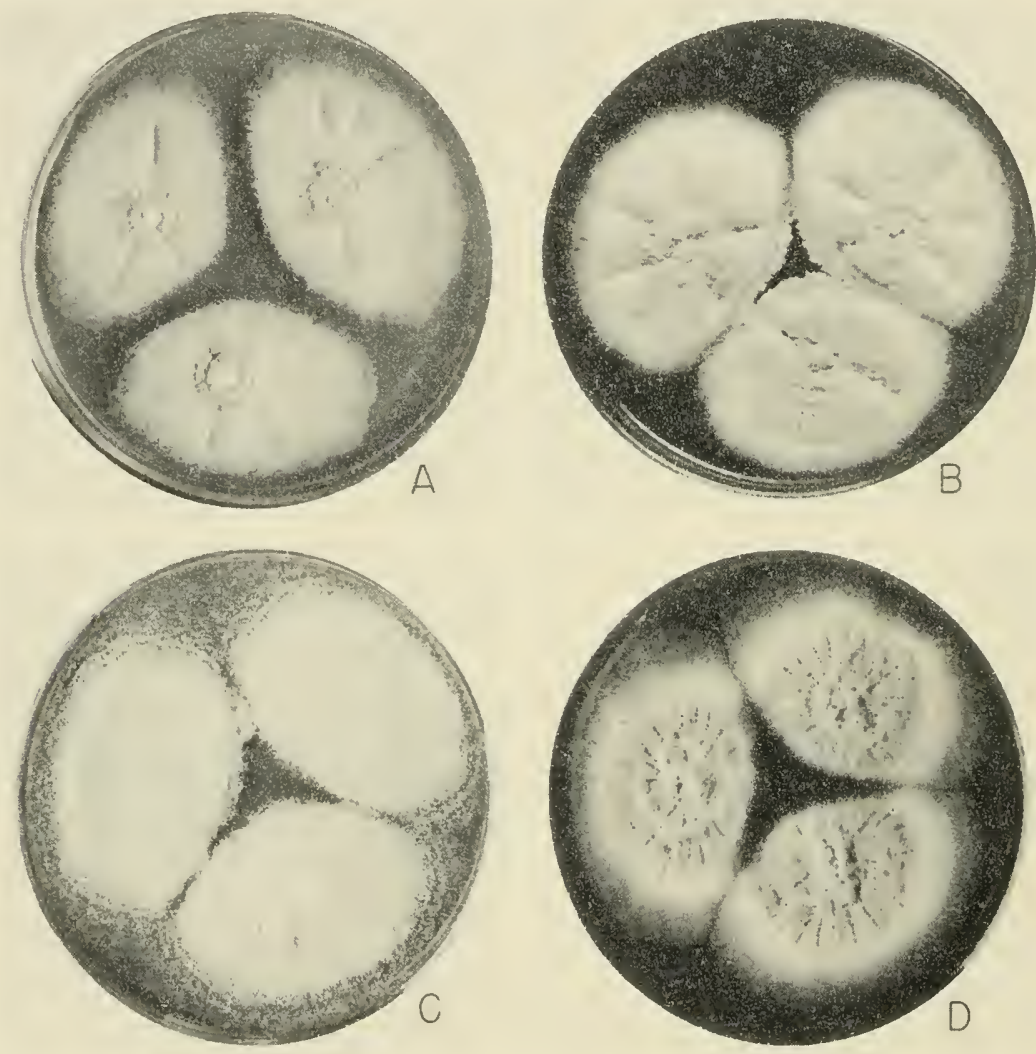

FIG. 58. Aspergillus terreus group; different species growing upon C'zapek's solution agar at room temperature. A, Typical, heavy sporing strain of $A$. terreus, NRRL No. 265. B, A. terreus var. floccosus, characterized by loose floccose colonics and limited spore production. C, A cameus, NRRL, No. 1928, heavy sporing and characterized by flesh-colored conidia. D, A. niveus, NRRL No. 515, characterized by white conidial heads.

Aspergillus connamominus (Weiss) Dodge, in Med. Mye., p. 627. 1935.

Synonym: S. cinnamominus Weiss, in Ann. Parasitol. Hum. Conp. 8: 189-193, 5 figs. 1930.

Characterization from Dodge: Hyphae septate and branched; conidiophores simple, $5 \mu$ in diameter, vesicle 12 by $9 \mu$. Primary phialides cylindric. $4 \mu$ long, bearing 
2 to 3 , sometimes 4 secondary phialides, 5 to $6 \mu$ long. Conidia spherical, 1 to $2 \mu$ in diameter, brown. Other spores, borne laterally on short branches (phialides ?), 3 to $4 \mu$. Chlamydospores occasional. Under some conditions, only monstrous phialides are formed, with a "pleomorphic" mycelium resulting. Described as present in lesions of pityriasis versicolor flava. Inoculation into the human skin reproduced the original disease. From the description given the organism is some strain of the $A$. terreus group.

\section{Aspergillus carneus (v. Tiegh.) Blochwitz}

Synonyms: Sterigmatocystis carnea v. Tiegh., in Bull. Soc. Bot. France 24: 103. 1877. Cited also in Saccardo Sylloge 4: 74, and in Wehmer's Monograph, p. 127 (Mem. Soc. Phys. Hist. Nat. Gen. pp. 1-157. 1899-1901).

Sterigmatocystis spuria Schröter as a bibliographic change in Cohn Krypt. Fl. Schleisen 3 : 2 Helfte Lief. 1, p. 218. 1893. Aspergillus carneus Blochwitz, in Ann. Mycol. 31(1/2): 81. 1933.

Colonies upon Czapek's solution agar growing well at room temperature spreading, plane or radially furrowed, more or less floccose, at first white but becoming pale vinaceous-fawn to vinaceous-fawn (Ridgway, Pl. XL) with the development of mature fruiting structures (Pl. V F, and fig. $58 \mathrm{C}$ ), ranging from 1 to $2 \mathrm{~mm}$. or more deep in central areas to very thin and spreading at colony margin, comparatively heavy sporing throughout with fructifications arising from both aerial and submerged mycelium, some strains showing limited areas yellow in color from an underlying felt of heavy-walled sterile hyphae suggestive of hülle cells (fig. $49 \mathrm{~F}$ ); odor often pronounced, somewhat putrid; reverse in orange-yellow, bright orange, to deep brown shades; conidial heads loosely columnar, averaging 150 to $200 \mu$ by 25 to $35 \mu$, but commonly somewhat larger, varying in color as the colony. Conidiophores variable in length, mostly 250 to $400 \mu$ but ranging up to 1 $\mathrm{mm}$., occasionally bearing secondary fruiting structures as short and irregularly placed branches, smooth, sinuous, uncolored, mostly 3.5 to $6.0 \mu$ in diameter. Vesicles hemispherical, ranging from 5.5 to $9 \mu$, rarely as much as $10 \mu$ (fig. $57 \mathrm{C}_{1}$ ). Sterigmata in two series, primary 5.5 to $6 \mu$ by 2 to $2.5 \mu$, secondary 5 to $5.5 \mu$ by 1.8 to $2 \mu$, commonly very few primary sterigmata present. Conidia globose to subglobose, thin-walled, averaging 2.4 to $2.8 \mu$, with maximum rarely exceeding $3.2 \mu$.

Colonies upon malt extract agar growing more restrictedly, heavier sporing, with pigmentation generally more pronounced and with conidial heads averaging slightly larger than on Czapek's solution agar, otherwise duplicating the above description.

This species is represented by strains NRRL No. 527, isolated as an air contaminant in Washington; NRRL No. 298, isolated from Kiansas soil; and other soil isolations from different parts of the United States, Mexico, 
Cuba, and Central America. Strains differing from the above in the absence of any yellow, thick-walled hülle-like cells are occasionally isolated from soils. NRRL No. 1928, isolated from Arkansas soil, is representative. In the absence of any yellow component, the color of these strains is more accurately described as pale to light grayish-vinaceous-fawn (Ridgway, PI. XXXIX).

The name Aspergillus carneus is revived to cover the forms under consideration since their most obvious identifying characteristic is the pale flesh color of their massed conidial heads. It is the belief of the writers that van Tieghem probably had in hand some member of this series when he proposed the name S. carnea, although, due to the inadequacy of his description, it is now impossible to establish this point with certainty. In any case, the name is excellently descriptive of strains commonly encountered, hence its application in this connection.

In describing Aspergillus carneus as a new species, Blochwitz (1933) acknowledged the earlier use of this specific name by van Tieghem but disregarded its validity. He undoubtedly applied it to a member of the species as it is considered by us since he noted that it differed from $A$. terreus (A. galeritus) principally in the flesh to rose color of its conidia. It is believed that Blochwitz's A. niveus var. nubila (1934) likewise represented a strain of $A$. carneus characterized primarily by conidia of darker rose, a condition which in older cultures is frequently suggested by NRRL No. 1928 cited above.

Gilman and Abbott in their "Summary of Soil Fungi" (i927) called attention to the repeated isolation, from Louisiana soils, of forms with the "general morphology of the Aspergillus candidus group but producing bright pink conidial heads." While the writers think the affinities of these forms lie more with Aspergillus terreus (columnar heads, coloriess conidiophores) and Aspergillus flavipes (elongate, irregular hülle cells) than A. candidus, we have every reason to believe they were dealing with forms similar to those here designated A. carneus.

S. albo-rosea Sartory, Sartory, and Meyer (Ann. Mycol. 28: 358-359, Pl. III, fig. 1-6, 1930) apparently represents a member of this series. This is indicated by the described coloration of colonies and more particularly by the detailed measurements cited for it.

Aspergillus niveus Blochwitz, in Ann. Mycol. 27(3/4): 205-6, fig. 2, Taf. III. 1929.

Synonym: A. eburneus Biourge, name attached to a culture received by Thom (No. 5402.1: NRRL No. 515).

Colonies upon Czapek's solution agar white, plane or radially furrowed, rather slow growing, forming a dense felt of mycelium and conidiophores up 
to $600 \mu$ to $1 \mathrm{~mm}$. deep, thinning toward the margin (fig. $58 \mathrm{D}$ ) and commonly spreading in unevenly radiating lines, commonly producing abundant amber to brown exudate; reverse in dark yellow shades through greenish to brownish-black; odor slight. Conidiophores smooth with walls colorless, sinuate, more or less septate, slender, 4 to $6 \mu$ in diameter, enlarging to a hemispherical resicle 8 to $15 \mu$ in diameter or sometimes larger at the apex, commonly 300 to $600 \mu$ in length, occasionally up to $1000 \mu$ long, and on other substrata sometimes longer. Conidial heads showing chains of conidia in comparatively loose columns, most frequently 20 to $30 \mu$ in diameter but in large heads up to $60 \mu$, with the general appearance of a snow white $A$. terreus. Vesicular area hemispherical (fig. $57 \mathrm{D})$. Sterigmata in two series, primary sterigmata 5 to $8 \mu$ by 2.5 to $3.0 \mu$, secondary sterigmata 5 to $7 \mu$ by 2 to $2.5 \mu$. Conidia 2.0 to $2.5 \mu$, rarely more, smooth, thin-walled, colorless.

Represented in the NRRL collection by Nos. 515 (Thom No. 5102.1), 288, and 1955. Repeatedly isolated from soil but less common than Aspergillus carneus and the ubiquitous A. terreus.

Typically the conidial apparatus is that of a white, loosely columnar form of A. terreus. As described by Blochwitz, it does not show yellow in culture. This is true of strain NRRL No. 515, although in age this culture reaches dull ivory to pale buff on agar slants. In other strains, such as NRRL No. 1955 from Dr. Timonin in Ottara, Canada, limited areas may become yellow from the development of massed thick-walled hyphae as in A. carneus.

Sterigmatocystis pusilla Peyronel (I germi atmospherici dei funghi con micelio. Thesis. Padova. 1931, p. 21) probably represents a synonym of $A$. niveus Blochwitz. It was described as follows: Colonies white, very thin; sterile hyphae creeping, sparingly branched, falsely septate, hyaline, 1.5 to $5 \mu$ in diameter; conidiophores erect, unseptate, hyaline, 60 to $80 \mu$ by 2.5 to $3 \mu$, with apical vesicles hyaline, obovoid or subglobose, 7 to $10 \mu$ in diameter, sterigmata radiate, in two series, with primaries 5 to $7 \mu$ by 2 to $2.5 \mu$ and secondaries 3 to $5 \mu$ by $2 \mu$ in groups of 2 to 3 ; conidia globose 2 to $2.5 \mu$, hyaline, smooth. Habitat: from air in northern Italy at altitude of 1,700 meters. From description this would appear to represent a short-stalked member of A. niveus.

In their most typical manifestation, the conidial heads of Aspergillus niveus are loosely columnar; vesicles are dome-like, and fertile over the upper one-half to two-thirds only. They thus stand out in sharp contrast against the typically globose heads and completely fertile vesicles of $A$. candidus. But all heads of $A$. candidus are not globose, and all vesicles are not fertile over their entire surface. In most strains small columnar heads, not particularly different from those of $A$. niveus, can be found (fig. $60 \mathrm{D}$ ). Considering this character, together with (1) the snow-white heads, (2) the smooth colorless conidiophores, and (3) the small smooth conidia of both species, one can readily imagine that we are here dealing with two interlock- 
ing groups. One basic character separating the two is the production of sclerotia. Characteristic reddish-purple to black sclerotia are commonly found in white-spored strains producing wholly or in part large globose heads ( $A$. candidus); they have never been seen in white-spored strains producing only loose columnar heads ( $A$. niveus). Until additional intermediate forms are isolated and studied, the relationship between these white-spored forms must remain a matter of conjecture, although in this manual they are placed adjacent in what we believe to represent a natural placement of the different groups.

\section{Pathogenesis}

Strains of $A$. terreus grow under a wide range of temperature, including $37^{\circ} \mathrm{C}$. It is not surprising, therefore, that an occasional member of the series is reported as a human parasite. One of these was re-described as Sterigmatocystis hortai by Langeron (1922). This culture, NRRL No. 274 (Thom No. 5071.1), received from France, was originally isolated from a human ear in Brazil. It is believed to be type and represents a characteristic strain of $A$.terreus. Another was found in metastatic lesions on a cornhusker's hand and forearm in Nebraska. Recently a strain was isolated from an aborted fetus from a cow in Maryland; the culture was entirely typical of $A$. terreus.

\section{Occurrence and Economic Importance}

Members of the Aspergillus terreus group are typically soil organisms, hence are most abundant in soil and upon decaying regetation. They frequently occur, however, upon a great variety of materials useful to man, including grains in storage, straw and forage products, cotton and other fibrous materials not adequately protected from excessive moisture, etc. Aspergillus terreus and A spergillus carneus are especially widespread in warm arable soils, and have been isolated in great abundance from soils collected in southern and southwestern United States. They are generally less common in forest than in cultivated soils, and are rarely found in acid forest soils from the colder temperate zone. There is little evidence that these forms are especially active agents of decay, but their great abundance in nature indicates that they undoubtedly play a significant role in the slow decomposition of organic materials. Aspergillus terreus and A. carneus grow well at temperatures of 35 to $37^{\circ} \mathrm{C}$, a character which possibly accounts for their great abundance in southern soils and their relative scarcity in soils from northern areas.

Aspergillus terreus has become of special biochemical interest since the discovery in 1939 by Calam, Oxford, and Raistrick that certain strains of this species are capable of producing itaconic acid from sugars. Extensive 


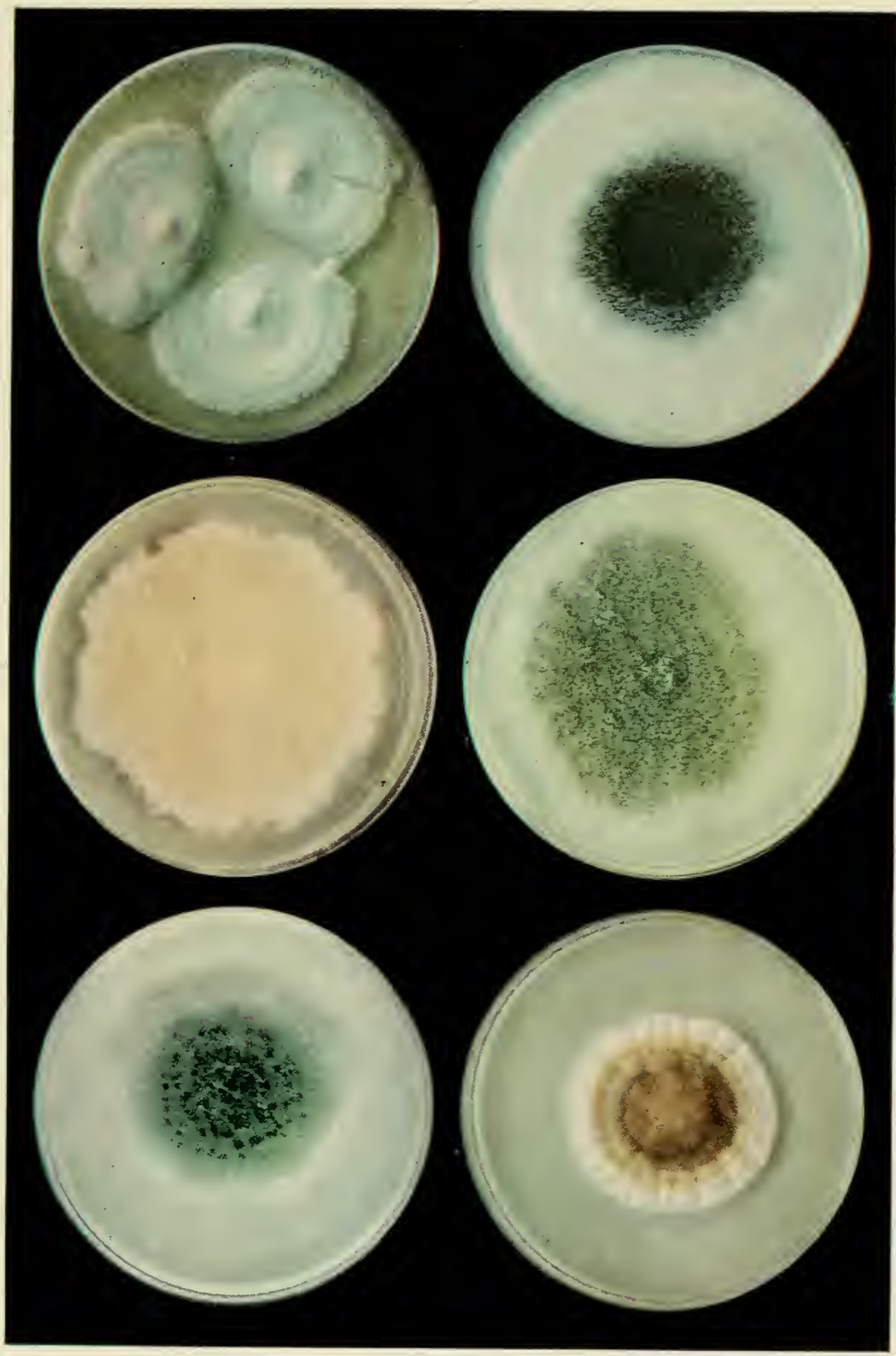

Plate Vi

A (upper left), Aspergillus candidus Link, NRRL No.308. B (upper right), Aspergillus niger group, strain NRRL No. $67, C$ (center left), Tan-spored mutant of strain 67 produced by ultraviolet radiation. $D$ center right), Aspergillus avenaceous Smith, NRRL No.517. E (lower left), Aspergillus alliaceus Thom and Church, NRRL No. 315, F (lower right), Aspergillus wentii Wehmer, NRRL No, 375. All cultures growing unon Czapek's solution agar. (Color photographs by Haines, Northern Regional Research Laboratory. Reproduced through co-operation of Chas. Pfizer \& Co., Inc.) 

investigations on this fermentation have been conducted in the Fermentation Division of the Northern Regional Research Laboratory, and papers reporting these studies are currently in press. More than 300 strainshave been tested and the most productive cultures selected for intensive study. Improved nutrient solutions have been developed and critical environmental factors explored with the result that substantial yields of itaconic acid are now obtained. It is believed that this process may become of industrial importance within a reasonable period of time. (See Lockwood, Raper, Moyer, and Coghill; Lockwood and Reeves; Lockwood and Ward; Moyer and Coghill; and Raper, Coghill, and Hollaender, in the Topical Bibliography under "Itaconic Acid.")

Timonin (1942) reported the production of citrinin by a white-spored Aspergillus identified by him as one of the A. candidus group. Careful examination and comparison of his culture with representative strains of $A$. candidus, $A$. carneus, and $A$. niveus show its true relationship to be with $A$. niveus in the terreus group, as it is considered here, rather than with $A$. candidus. 


\section{Chapter XVI \\ THE ASPERGILLUS CANDIDUS GROUP}

\section{Outstanding Characters}

Conidial heads persistently white or becoming yellowish cream in age; typically globose, but approaching columnar in small heads.

Conidiophores smooth, colorless or slightly yellowed in terminal areas. Sterigmata in two series, with primaries often much enlarged, sometimes varying greatly in size within the same head.

Conidia globose or subglobose, smooth.

Sclerotia present in some strains, dark, approaching purple to black when mature.

Grouping by color lead Thom and Church (1926) to establish their Section IX, or the so-called "White-spored Aspergilli." Only vaguely did they indicate that they had included a heterogeneous lot rather than described a natural group. Further study of the strains included in the "white" section showed the tardy development of colors approaching avellaneous or even carneus. The continued comparison of great numbers of strains in all groups has revealed such wide variations in color that the authors have come to regard whiteness, or lack of color, as a character of secondary importance in the allocation of strains to particular groups. This viewpoint is supported by the appearance, under controlled conditions, of white variants or mutants in a number of colored series. Yuill (1939) observed and isolated such colorless mutants from Aspergillus fumigatus and $A$. nidulans; Steinberg and Thom (1940) reported the same type of mutant for the former species; while Raper, Coghill, and Hollaender (in press) have succeeded in producing white mutants in Aspergillus terreus by irradiating spores with ultra-violet. Colorless members of the Aspergillus glaucus group, represented by $A$. niveo-glaucus, have been isolated by Blochwitz, Thom and Raper, and other investigators. Long before the work of Yuill, Schiemann (1916) had developed two color variants of Aspergillus niger, A. schiemanni (Schiemann) Thom (1926, p. 172) and A. cinnamomeus Schiemann (1912) which differed only from the parent strain by a progressive reduction of the amount of coloring substance, presumably the aspergilline of Linossier (1891). Steinberg and Thom (1940), working with a strain of $A$. niger, again produced variants approximating those of Schiemann, and Whelden (1940) secured the same by irradiating spores of $A$. niger with cathode rays. In cultures 
collected from nature from world-wide sources, strains characterized by heads approaching white are occasionally observed in other groups. It is apparent, then, that the capacity to produce a coloring substance, while ordinarily inherited or passed on in successive colonies of an organism, is not always uniformly maintained.

In the present treatment, the writers have sought to include within the Aspergillus candidus group only such forms as are clearly and closely related to it. The group is thus limited, essentially, to a single series containing only one clearly definable species, $A$. candidus. Different isolations vary materially in their general cultural appearance and in the details of their microscopic structure. Nevertheless, all possess the typically globose, white to dull buff or light gray conidial heads, the smooth colorless conidiophores, and the small, smooth, colorless conidia.

To facilitate recognition of members of the Aspergillus candidus group and to assist in the proper assignment of other white or light-colored species and strains, a general key covering all of these forms is presented.

\section{GENERAL KEY OF WHITE ASPERGILLI}

A. Heads (large ones) globose or radiate; conidiophores smooth-walled, colorless or yellowed toward the vesicle only; sclerotia occasionally seen.A. candidus group

B. Heads white, hemispherical to columnar; conidiophores smooth-walled, colorless

C. Heads initially white, tending to be columnar; conidiophores smooth-walled, showing some shade of yellow; contorted hülle cells usually found

A. flavipes, series, see p. 179

D. Heads white, borne upon long, smooth-walled, colorless conidiophores terminating in clavate vesicles, sterigmata in two series

White-spored phase of $A$. janus, see p. 187

E. Strains of white Aspergilli possessing the basic characters of their colored counterparts also occur as mutations in the A.fumigatus, A. nidulans, A. terreus, and glaucus groups.

Aspergillus candidus Link, Obs. p. 16, 1809. Thom and Church, The Aspergilli, p. 157.1926.

Colonies upon Czapek's solution agar persistently white, or becoming cream or yellowish-cream in age (Pl. VI A and fig. 59), often thin, vegetative mycelium often largely submerged, surface growth usually consisting of conidiophores and heads, and with scanty sterile mycelium or anastomosing ropes of hyphae bearing short-stalked fruiting structures; sclerotia produced in occasional strains; reverse usually uncolored. Heads white, globose, radiate, varying in the same culture from large globose masses 200 to $300 \mu$ in diameter to small heads often less than $100 \mu$ in diameter, commonly more or less elongated in heads with incomplete development of sterigmatic surface. Conidiophores varying with the strain, 
in short or dwarf races less than $500 \mu$ long, in other strains ranging up to 500 to $1000 \mu$ or longer, varying from $5 \mu$ in diameter in dwarf forms to 10 to $20 \mu$ in long-stalked forms, with walls thick, smooth, colorless or slightly yellowed near the vesicle in certain strains in age. Vesicles typically
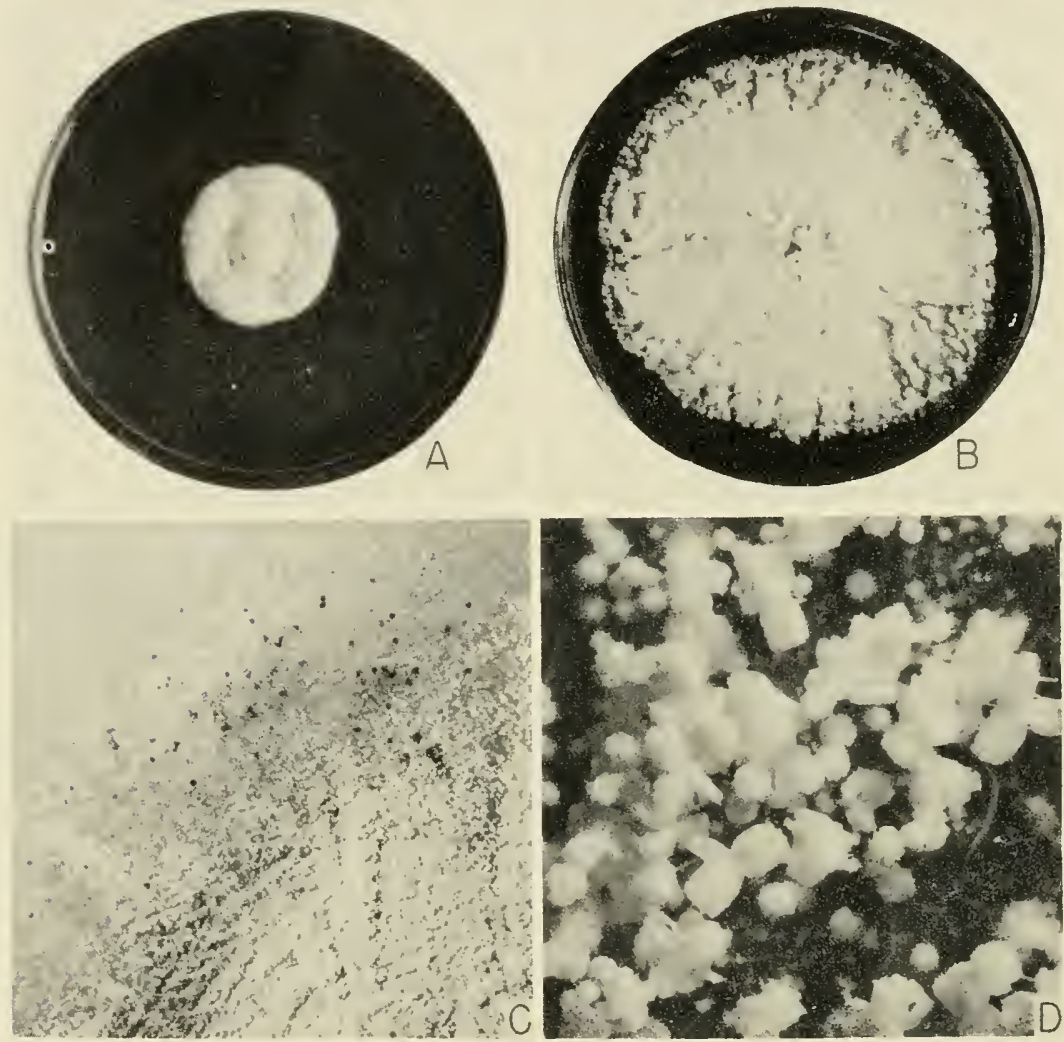

FIg. 59. Aspergillus candidus. A, Strain NRRL No. 305 on Czapek's solution agar at room temperature, 10 days. $B$, Strain NRRL No. 314 on Czapek's solution agar at room temperature, three weeks. Note contrast between compact colony of No. 305, consisting of crowded conidiophores, and loose spreading colony of No. 314 in which production of conidial heads is very irregular. $C$, Strain NRRL No. 312 , portion of colony showing scattered black sclerotia, $\times 3$. D, Strain NRRL No. 308 , conidial heads, $\times 18$.

globose, ranging from $40 \mu$ in diameter in very large heads (fig. $60 \mathrm{~A}$ ), and typically fertile over the whole surface, to small globose heads (fig. $60 \mathrm{~B}$ ), often very much reduced to support simple groups of sterigmata appearing almost penicillate (fig. $60 \mathrm{D}$ ). Sterigmata typically in two series, usually colorless, primary varying greatly in different strains, in different heads of 


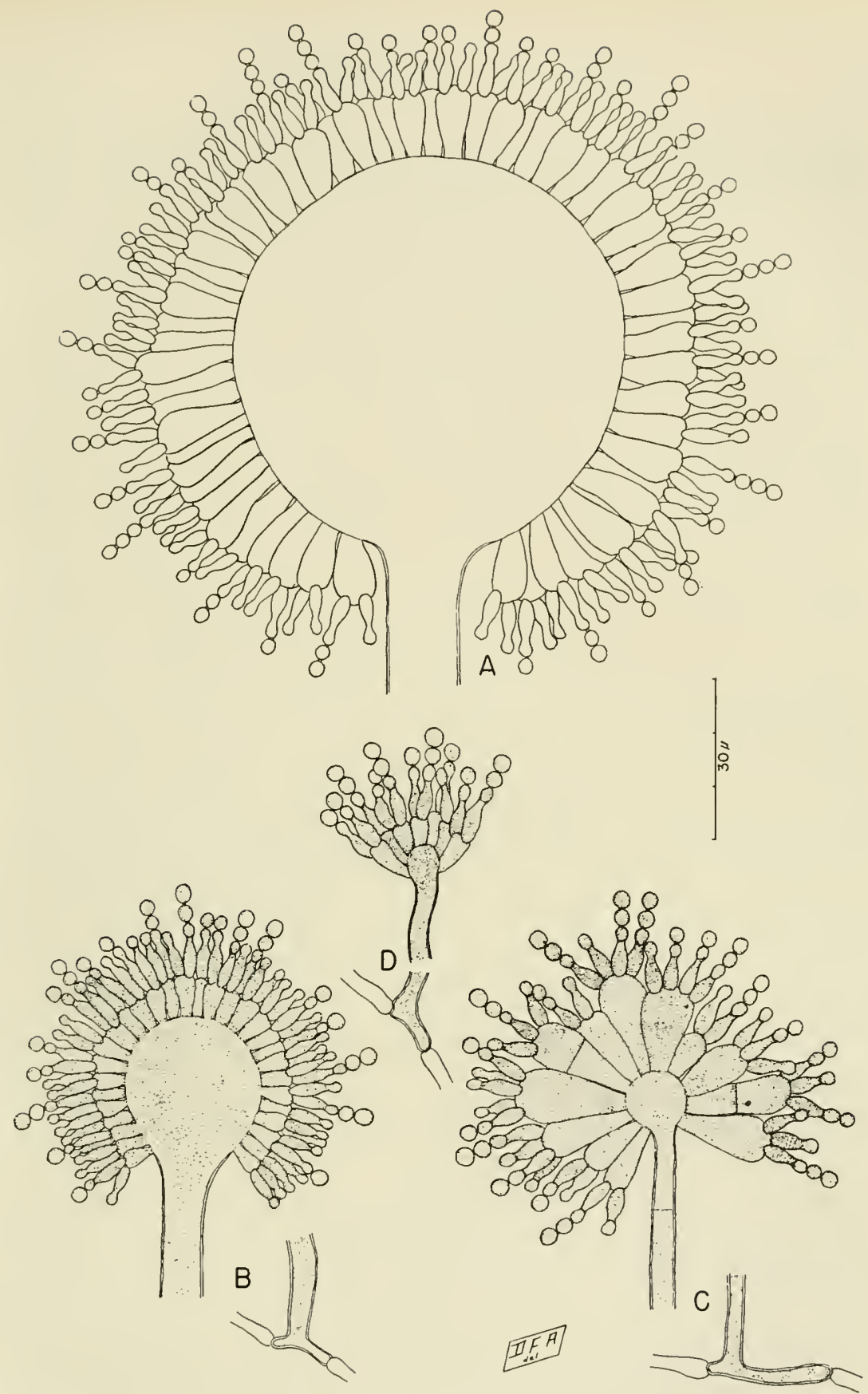

FIg. 60. Conidial structure of Aspergillus candidus, $\times 900: A$, Large globose head showing large primary sterigmata, strain NRRL No. $312 ; B$, Simaller globose head showing small primary sterigmata, strain NRRL No. 308; $C$, Head showing primary sterigmata of variable size, some septate, strain NRRL No. $312 ; D$, Diminutive head, same strain. Great variation in dimension is characteristic of the fruiting apparatus of most $A$. candidus strains. 
the same strain, and occasionally in the same head (fig. $60 \mathrm{C}$ ), ranging from $5 \mu$ in length in some cases to 15 or $20 \mu$ and even $30 \mu$ under other conditions, commonly septate; secondary sterigmata usually uniform in all beads, from 5 to $8 \mu$ by 2 to 2.5 or $3 \mu$. Conidia colorless, globose or subglobose in most strains to elliptical or barrel-form in others, thin-walled, 2.5 to $3.5 \mu$ or occasionally $4 \mu$, smooth.

Reddish-purple to black sclerotia, consisting of thick-walled, parenchymalike cells, occur in many strains (fig. 59 C).

As there is no possibility of determining which of the white Aspergilli was in Link's possession, the name is here used to cover a whole series of strains which are found everywhere but are most frequent in the later stages of decay in vegetation and are especially characteristic of moldy grain. Included within the series as we consider it are two rather different cultural entities. The first of these is characterized by thin colonies in which the mycelium is largely submerged and only fruiting structures, commonly arranged in concentric zones, rise above the level of the substratum (fig. 59 B). In other strains, colonies are rather floccose and somewhat felted and often attain a depth of $2 \mathrm{~mm}$., with fruiting structures arising from aerial as well as submerged mycelia. Sclerotia, generally in purple or black shades, regularly and consistently develop in many strains including representatives of both of the above colony types. Conidiophores vary greatly in size and characteristically reach their greatest dimensions upon the drier portion of agar slants, or upon slightly moistened grain and other comparable products low in water content. The sterigmata in organisms of this group warrant particular attention since the two series commonly, but not consistently, differ tremendously in size. In many strains the primary sterigmata are characteristically wedge-shaped and reach dimensions of 25 to $30 \mu$ by 10 to $12 \mu$ (fig. $60 \mathrm{~A}$ and C); such structures are commonly septate. In other heads from the same culture the primary sterigmata may be relatively small and measure 6 to $\delta \mu$ by 2.5 to $3.5 \mu$ (fig. $60 \mathrm{~B}$ ). Secondary sterigmata are consistently small and of the dimensions indicated in the species description.

"White" Aspergilli regularly constitute a normal element in the micropopulation from moist or improperly dried grains and of comparatively dry vegetation undergoing slow decay. From such material, many investigators have described molds characterized by white heads which obviously belong in this group, but without supplying sufficient critical data to permit subsequent verification of the exact types under study. Some of these descriptions were based upon molds growing in culture; more of them were not. A few of the more tangible of these probable synonyms will be briefly considered in this connection; others will be found in the general species index (pp. 331-?). 
S. alla Bainier (Bull. Soe. Bot. France 27: 30. 1880) was isolated from oatmeal, and while incompletely described, obviously represents a member of the $A$. candidus series. Several publications present elaborate eomparative tables to separate strains aceepted as $A$. candidus and $A$. albus, but the many strains obtainable vary into each other so completely that little or no basis for separation exists in fact.

A. albus Wilhelm (Beitr. z. Kenntn. d. Pilzgattung Aspergillus, Inag. Diss. St rassburg, p. 69, 1877) was described with characters which clearly ally it with $A$. candidus but without sufficient differences to separate it from other members of this group.

$S$. blanc-jaune Bainier nomen nudum-A culture from Bainier's collection reeeived by Thom under this name (No. 4640.490) represents a somew hat diminutive but otherwise typical member of this series.

S. albo-lutea Sartory and Meyer (Cited by Blochwit z in Ann. Myeol. 31: 73. 1933). Conidia were reported as turning yellowish in age. This character is eommon to many members of the group and has been so noted by Wehmer (1889-1901), Thom and Church (1926), and others. Retention of the speeies name is not warranted.

A. basidiosepta Sartory, Sartory and Meyer (Ann. Mycol. 27: 317-320, Pl. 7. 1929) apparently represents a member of the $A$. candidus series with comparatively long $(28$ to $30 \mu$ ) primary sterigmata which in age are characteristically septate. This character, which appears also in some members of the $A$. niger and $A$. ochraceus groups, however, is not sufficiently unique to warrant specific separation.

A. niveus var. major Blochwitz (Ann. Mycol, 32(1/2): 86. 1934). Described as showing vesicles globose, rarely oboval, or pear-shaped entirely covered with radiating sterigmata which are rarely absent toward the base; closely growing conidiophores 2 to $2.5 \mathrm{~mm}$. high. These characters suggest relationship with $A$. candidus rather than $A$. niveus.

A. okazakii Okazaki, in Centralb. f. Bakt. etc., 2 abt., 19, p. 481-484, taf. I. 1907; see also Centralb. f. Bakt. etc., 2 abt., 42, p. 225. 1914. This is eited by Saccardo in Syll. 22: 1260. 1913, as S. okazakii Saito but apparently without adequate ground for attributing the name or description to Saito.

Colonies described as white to sulphur yellow; conidiophores hyaline, straight or sinuate, smooth or asperulate, 200 to $500 \mu$ by 8 to $12 \mu$ figured as undulate, especially toward the base, with walls 2 to $3 \mu$ thick; heads 80 to $100 \mu$ in diameter; vesicles 12 to $40 \mu$ in diameter; primary sterigmata 15 to $20 \mu$ by 6 to $8 \mu$, secondary 8 to $14 \mu$ by 2.5 to $4 \mu$; conidia globose, hyaline, 2.5 to $5.4 \mu$, smooth, with conneetives. In the event that continued study of these white forms reveals the existence in nature of strains with more or less roughened conidiophores and conidial heads ranging to yellows, recognition of $A$. okazakii as a separate species would be warranted. Based upon current information, however, we believe it preferable to consider it synonymous with $A$. candidus Link.

A. sachari of Chaudhuri and Sachar (Ann. Mycol. 32: 95. 1934) is more or less arbitrarily left where the authors put it-as one of the $A$. sulphureus series near $A$. quercinus in the $A$. ochraceus group. The heads are pale yellow, the sclerotia are near the colors of that group, but the conidiophore is described as colorless and smooth which would put it in $A$. candidus.

A. sterigmatophorus Saceardo, in Mycologicae Venetae Speeimen. Atti d. Soe. Ven. Trent. d. Sci. Nat. 2, fasc. 2: 232. Tab. XVII, fig. 5-8. 1873; Syn. S. italica Saec. in F. italiei no. 109,1881 ; changed to $S$. italica Sacc. as a note only in Michelia 1:91. 1877; Latin diagnosis of $S$. italica in Saccardo Sylloge 4:72. 1886. Described from decaying corn kernels (Zea mays): white, sparse, with conidiophores unbranched, 2 to 3 septate above; with vesicles globose; sterigmata described as dichotomously or trichotomously branched with ultimate cells bearing conidial chains; 
conidia globose about $6 \mu$ in diameter, with connectives. The description repeats observations as to occurrence and appearance that are frequently seen. No one has since reported a member of the $A$. candidus group with conidia $6 \mu$ in diameter. Whether the organism described by Saccardo was a large-spored mutant, not since isolated, remains open to question.

\section{OTHER WHITE ASPERGILLI}

Other Aspergilli characterized by white heads but differing basically in morphology from the $A$. candidus group occur in the Aspergillus glaucus, A. nidulans, A. fumigatus, and $A$. terreus groups. Except for an absence of spore color, these duplicate the morphology of the groups to which assigned. In fact, in all cases except that of $A$. niveo-glaucus in the $A$. glaucus group, they represent colorless mutations produced experimentally from typical parent strains (Yuill, 1939; Steinberg and Thom, 1940; Raper, Coghill, and Hollaender, in press). While A. niveo-glaucus was isolated from nature and hence its parentage is not known, it is suspected that this represents a mutation of some form close to Aspergillus echinulatus. A. halophilus of Sartory et al (Ann. Mycol. 28: (3/4) pp. 362-3, Pl. 3, 1930) similarly belongs in the A. glaucus group. Attention has been called earlier to the fact that $A$. candidus differs from $A$. niger primarily in the absence of color and in possessing smooth spores. The question may arise whether we are not here dealing with a whole series of mutations from colored forms. While this is possible, no proof is at hand. The fact that they constitute such a typical and abundant element of the micropopulation of soil, decaying vegetation, etc., demands that they be considered along with other major groups of the Aspergilli quite aside from any questions of possible origin.

\section{Sclerotia}

In this arrangement of the Aspergilli, sclerotia, as compact globose or subglobose bodies composed of thick-walled pseudo-parenchyma, are not found in the groups characterized by the production of perithecia and ascospores, and only rarely, if at all, in groups characterized by the presence of hülle cells. In the great groups beginning with $A$. candidus, sclerotia appear with sufficient frequency to be morphologically significant as indicative of class relationship. Fundamentally, the typical A. candidus strain differs little from the black Aspergilli except for the absence of the dark color and rough spores.

\section{Group Relationships}

While there is much evidence of relationship with the black Aspergilli (smooth-walled conidiophores, globose vesicles and heads, and the presence of sclerotia), there are also certain indications of relationship to Aspergillus niveus. Typically both are characterized by snow-white conidial 
heads, and in all strains of Aspergillus candidus there are more or less abundant small heads which bear few and loosely arranged sterigmata in a manner strongly suggestive of typical conidial structures of A. niveus. Although it is our belief that these similarities in structure do not of necessity reflect close relationship between $A$. candidus and $A$. niveus, we do feel that there is need for additional study of strains which appear to be more or less transitional between the two groups.

\section{Occurrence and Economic Importance}

Members of the Aspergillus candidus group are very widely distributed in nature and occur with reasonable frequency upon vegtation in the later stages of decay. They are especially common upon moldy grains and are obviously able to grow in the presence of a very limited amount of moisture.

The biochemical and physiological activities of these form have not been studied extensively. A. okazakii was employed by Okazaki (1907 and 1914) for the production of a proteolytic enzyme preparation, "digestin," and is the basis of a Japanese patent, No. 11461, covering this process. Recently Timonin (1942) has employed a strain reported as belonging to the $A$. candidus group for the production of citrinin. Upon examination, however, this strain is found more nearly to represent $A$. niveus than $A$. candidus in the sense it is considered here. 


\section{Ch.ıpter XVII \\ ASPERGILLLS NIGER GROLP}

\section{Outstanding Characters}

Conidial heads carbon black, brownish-black or purple-brown; in mutants, shading toward colorles but not actually white.

Heads typically large and globose: but small heads produced in some strains, in extreme cases consisting of only a few sterigmata and chains of conidia.

Conidiophores smooth, colorless or tinged with rellow-brown colors in the upper one-third or less, splitting lengthwise into strips and shreds when broken.

Vesicles globose in large heads, fertile orer the entire surtace: in small heads often reduced to dome-iike apices of short conidophores ("fumigatiform").

Conidia rough, mostly showing bars or bands of brown-black coloring matter.

Sclerotia characteristic of many strains, more or less irregular, ranging from buff through gray to almost black.

The designition "-Aspergillus niger"l is commonly used to cover a great aggregate of Ispergilli differing in details of morphology, but having in common the production of conidial heads which are black, brownish-black, purplish-brown, or in some strains lighter in color but retaining the general appearance of the group.

When examined by the hundred as they are isolated from natural sources, the rast majority of cultures studied show the general morphology of Aspergillus niger van Tieghem, if a reasonable allowance be made for strain variation which is characteristic of all of these groups of cosmopolitan molds. At the same time, other organisms scarcely distinguishable in general cultural appearance often show marked differences in microscopic characters, hence have formed the basis of species descriptions. Other species have been segregated by various authors in the belief that they bore an obligate relation to the particular substata from which isolated (e.g., A. strychni, A. ficuum), but such specificity has not proved dependable. There are, in addition, a considerable number of probable rariants whose

${ }^{1}$ Biourge in his last manuscript proposed the use of the name Pulli for the black Aspergilli in recognition of the belief that Micheli (1:29) had one of them before him as his "Aspergillus capitatus capitulo pullo." 
origin is not known, and which may or may not have gonetic connection, but which do show superficial resemblances at least. Sorno of these have been described as species and can be more or less readily identified. Altogether, there is a considerable list of names which, at one time or another, have been applied to the black Aspergilli. It is now quite impossible to interpret many of these descriptions, or to find out exactly what type of organisms the rescribers had under observation. Pealizing the futility of attempting to separate all of these species, we have endeavored to include in the group key only such forms as possess well-marked characters, and those which the mycologist may reasonably expect to encounter in laboratory culture.

\section{Group Key}

I. Sterigmata in two series.

A. Conidia mostly less than $j \mu$ in diameter.......Aspergillus niger series

1. Conidia strongly colored, rough, definitely marked with echinulae, tubercles or color bars.

a. Primary sterigmata mostly under $20 \mu$ in length.

1.' Colonies with a penetrating artinomyces-like odor

2.' Colonies without such odor.

A. foetidus n. sp. (=A. aureus Nak.)

a. Colonies blark or deep brown to black.

1." Heards abundant over the entire colony

A. auamori Iakazawa

2." Heads few, scattered at margins of colonies

A. miyakrensis Nakazawa

b.' Colonies yellow-brown

Occasional Isolates. See also A. wentii group.

b. Primary sterigmata mostly 20 to $30 \mu$ long

A. niger van Tieghem series and species.

c. Primary sterigmata mostly 40 to $60 \mu$ long

d. Primary sterigmata up to 100 to $120 \mu$ long

A. phocricis (Cda.) Thom

A. pulverulentus (McAlpine) Thom

2. Conidia with color almost suppressed or diffused, leaving smorth walls with colorless spinules.

a. Conidia almost colorless, in mass very pale cimmamon

A. niger mut cinnamomeus n. comb.

b. Conidia with color diffused, sometimes a trace of roughening

A. niger mut. Schiemanni n. comb.

B. Conidia more than $5 \mu$ in diameter...........Aspergilius carbonarius series

1. Primary sterigmata less than $20 \mu$ in length.

a. Conidia purplish-black, 6 to $10 \mu$ in diameter

A. atropurpureus Zimmerman

b. Conidia brown, 5 to 7 or $\delta \mu$ in diameter. A. fumaricus Wehmer

2. Primary sterigmata 20 to $4.5 \mu$ in length. Conidia 6 to $8 \mu$ in diameter A. fonsecaeus n. sp. (= S. fusca Bainier)

3. Primary sterigmata up to 100 or $120 \mu$. Conidia 5.5 to $10.5 \mu$ 
II. Sterigmata in one series (Secondary sterigmata occasional in some strains) Aspergillus luchuensis series

A. Colonies black or black-brown.

1. Sterigmata 6 by $3 \mu$ (very short)............ luchucnsis Inui

2. Sterigmata about 15 to $20 \mu$ conidia 3 to $3.5 \mu .$. A. nan us Montagne

B. Colonies in reddish-brown shades.

and/or A. subfuscus Johan-Olsen

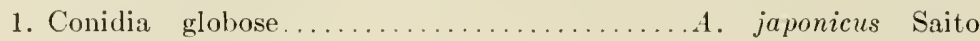

2. Conidia elliptical ................... violacco-fuscus Gasperini

Because of their great abundance in nature, the series most closely related to and including van Tieghem's species, based upon strains which satisfy his original description in a somewhat broadened sense, will be discussed first.

ASPERGILLUS NIGER SERIES

Species Characterized by Comparatively Small Primary Sterigmata and Small Conidia

Aspergillus niger van Tieghem, in Ann. Sci. Nat. Bot., s. 5, t. 8, p. 240. 1867.

Synonym: Sterigmatocystsis nigra van Tieghem, in Bul. Soc. Bot. France 24: 102-103. 1877. See also Thom and Currie, Jour. Agr. Res. 7: 1-15. 1916; and Thom and Church, The Aspergilli, p. 167. 1926.

Characterization: Colonies rapidly growing with abundant submerged mycelium, colorless, or in some strains with more or less yellow color in the hyphae and in the substratum, with aerial hyphae usually scantily produced, but abundant in age in certain strains. Conidial heads fuscous, blackish-brown, purple-brown, in every shade to carbonaceous black (Pl. VI B), varying in intensity with the quantity of coloring matter produced; typically globose or radiate (fig. $63 \mathrm{C}$ ), commonly up to 300,500 , or occasionally $1000 \mu$ in diameter with periphery variously splitting into radiating columns of conidia; small heads, more or less columnar and consisting of a few conidial chains often borne on trailing hyphae or short conidiophores near the substratum. Conidiophores mostly rising directly from the substratum, uncolored or yellow to brown near the vesicle only, smooth, with walls thick, frequently uneren on the inner surface and splitting lengthwise into strips when broken (fig. $64 \mathrm{~B}$ and $\mathrm{C}$ ), linseptate or with occasional thin septa, varying greatly in length and diameter in different strains and in colonies on different media or even in sections of the same colony, thus ranging from strains with conidiophores 200 to $400 \mu$ by 7 to $10 \mu$ to forms with conidiophores several millimeters long and $20 \mu$ or more in diameter. Vesicles globose or subglobose, thick-r alled, com- 

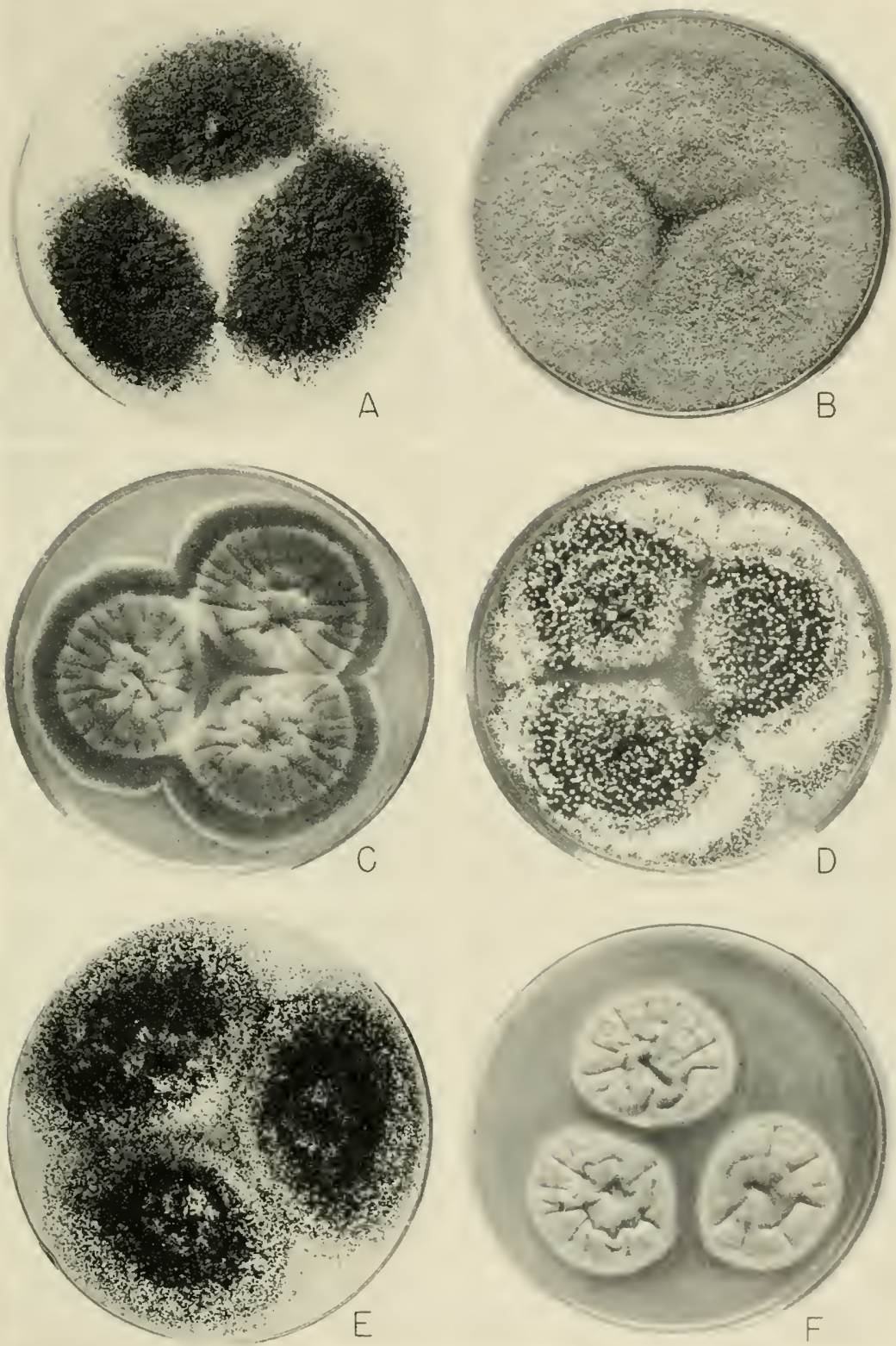

FIg. 61. Aspergillus niger group: cultures growing upon Czapek's solution agar at room temperature, 10 days. $A, A$. niger, NRRL No. 334 , typical strain. $B, A$. niger mut. schiemanni characterized by colonies light brown in color. $C, A$. foetidus, NRRL No. 341, characterized by a yellowish vegetative mycelium and a strong actinomyces-like odor. $D, A$. niger, NRRL No. 346, characterized by the production of abundant sclerotia. E, A. phoenicis, NRRL No. 1956, characterized by long uncrowded conidiophores. $F$, A. violaceo-fuscus, NRRL No. 360, characterized by compact, close-textured colonies and small heads with uniseriate sterigmata. 

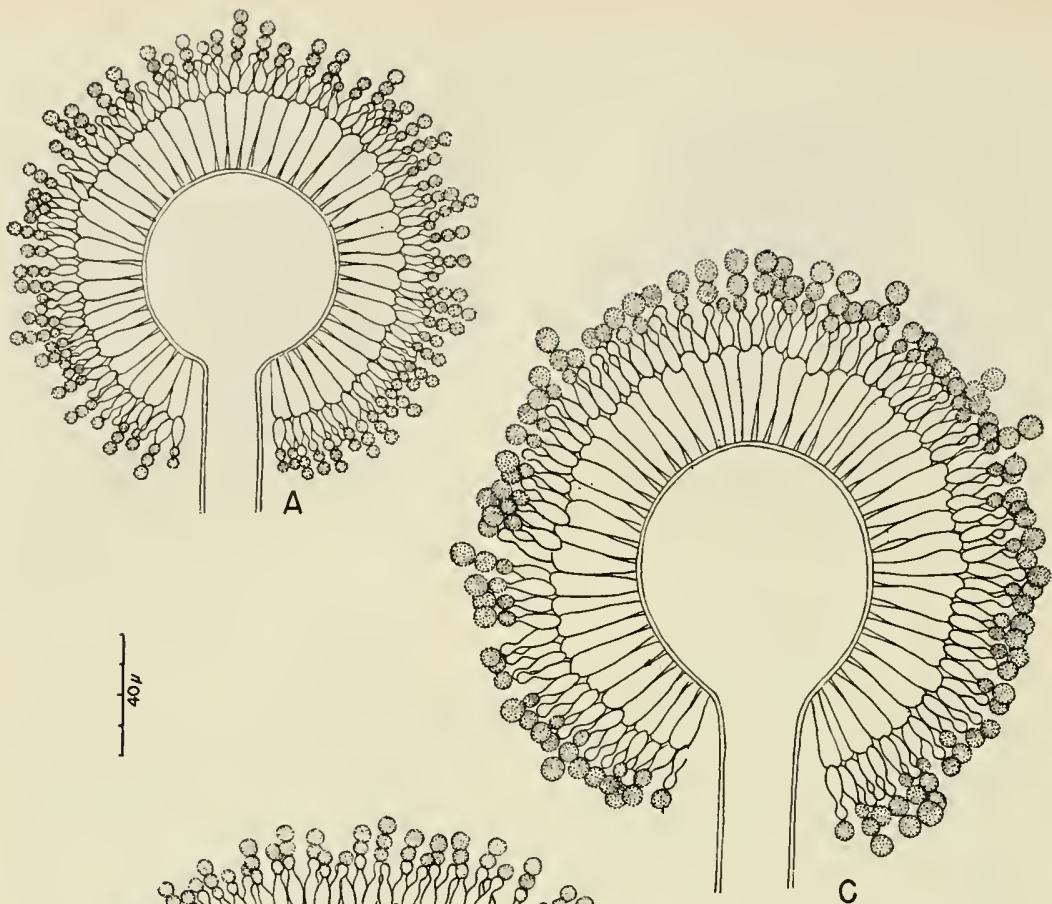

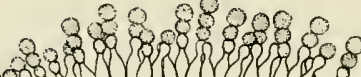

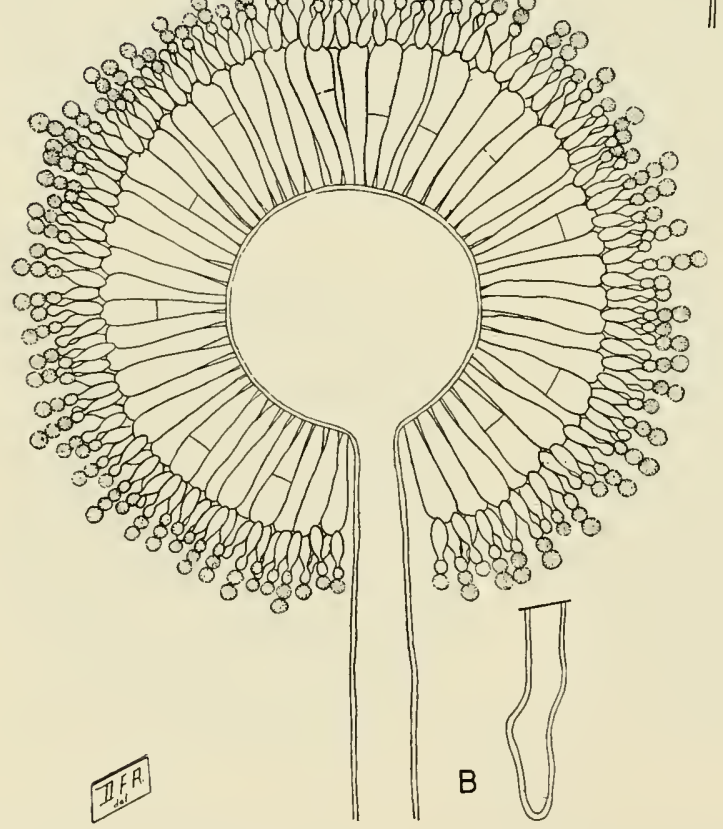

C

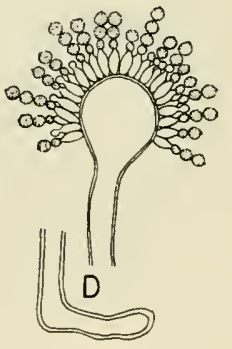

FIG. 62. Conidial structures in the Aspergillus niger group, $\times 500: A, A$. niger, NRRL No. 326, typical head showing globose vesicle and primary sterigmata about twice the length of secondaries; B, A. phoenicis, NRRL No. 1956, characterized by long and occasionally septate primary sterigmata; $C, A$. carbonarius, NRRL No. 369 , characterized by large primary sterigmata and large conidia; $D, A$. violaceofuscus, NRRL No. 360, characterized by small heads and sterigmata in a single series. 
monly 20 to $50 \mu$, occasionally up to $100 \mu$ in diameter (fig. $62 \mathrm{~A}$ ), colorless or more conmonly more or less intensely yellow-brown. Sterigmata in one series in young colonies and in small heads, but typically in two series, colorless at times, usually more or less intensely brown, even carbonaceous, primary sterigmata closely packed, covering the vesicle, varying greatly in size in the same colony but usually 20 to $30 \mu$ in length by 6 to $8 \mu$ in diameter at the outer end; secondary sterigmata more uniform, ranging usually from 6 to $10 \mu$ by 2 to $3 \mu$ (fig. $62 \mathrm{~A}$ ), both series often more or less brown to almost black. Conidia globose when ripe, with walls at first smooth with diffused brown or fuscous color (Ridgway, Pl. XLVI), then rough or spinulose from coloring substance deposited as tubercles, bars or loops between the outer primary wall and the inner, or secondary, wall, mostly 2.5 to $4 \mu$, occasionally up to $5 \mu$ in diameter.

Sclerotia globose, superficial, regularly produced by certain strains (fig. $61 \mathrm{D}$ ), sporadically by some, and not found in many others.

A. niger approximating the description of van Tieghem furnishes the most common morphological entity among the black Aspergilli. A few of the substrata and locations found in our record include chronic irritants in the human ear, pin-point colonies in the human lung, spoiling raw sugar, rancid butter and other fats, floating and submerged mycelium in many chemical solutions. It is abundant in soil cultures from every part of the world, and apparently especially so in the tropics. Molds under this name have been used in literally hundreds of biochemical investigations.

The introduction of a complete description from culture for each member of the series typically represented by van Tieghem's A. niger, but which vary from it in detailed measurements, calls for repetition of many common characters. In place of such descriptions the names and citations of the forms selected, either as unique or as representing sections of the series often encountered, are presented with the more important differences which furnish the bases of separation.

Aspergillus foctidus $\mathrm{n}$. sp.

Synonym: A. aureus Nakazawa, in Inst. Gov't. Res. Formosa, Rept. Vol. 1, 1907.

Not: A. aureus Berkeley, in English Flora Vol. 5, p. 346. 1836. Not $S$. aurea Greco, in Origine des Tumeurs et Mycoses Argentines, Buenos Aires, pp. 671-694, fig. 418-428. 1916.

Colonies upon Czapek's solution agar rather slow growing, producing a floccose basal mat of mycelium with abundant but uncrowded black heads above a mass of pale orange mycelium which is deeply orange in reverse. Heads up to $225 \mu$ in diameter are borne on conidiophores about $500 \mu$ 
long; vesicles comonly 20 to $30 \mu$ in diameter, occasionally much larger. Primary and secondary sterigmata are both 7 to $10 \mu$ by 2 to $4 \mu$; conidia are globose, spinulose, up to 4 or $4.5 \mu$.

Colonies have a penetrating actinomyces-like odor (also like Penicillium biforme noted in Thom, The Penicillia, p. 320. 1929), unlike any other species of Aspergillus. Numerous experiments over several years failed to justify the belief that the culture was contaminated with some actinomycete. The species is known only from Nakazawa's isolates which have maintained the odor and orange color for many years.

In the Awamori fermentation, $A$. foetidus (A. aureus Nakazawa) was $\beta$, the unfarorable organism which gave a yellow color to the "Koji" used. Nakazawa, Simo, and Watanabe (Jour. Agr. Chem. Soc., Japan, No. 144, pp. 931-974, illustr., 1936) listed five varieties of $A$. aureus as follows: var. minor, var. murinus, var. acidus, var. pallidus, and var. brevis.

Aspergillus awamori Nakazawa, in Inst. of Gov't. Res. Formosa, Rept. Vol. 1, 1907 and Vol. 2, 1912.

The measurements given are only slightly different from those of $A$. foetidus $(=A$. aureus). The unique odor and the yellow color in the mycelium and substratum were lacking. Colonies blackish-brown (deep chocolate) when heads were fully developed, conidiophores 1 to $2.5 \mathrm{~mm}$. by 9 to $15 \mu$; resicles globose, 30 to $45 \mu$ in diameter; primary sterigmata 9 to $12 \mu$ by 3.5 to $5.5 \mu$, and secondary 4.5 to $8 \mu$ by 1.5 to $3.5 \mu$; conidia globose or somewhat elliptical 3 to $5 \mu$ in long axis, fairly spinulose.

Nakazawa, Simo, and Watanabe (Jour. Agr. Chem. Soc. Japan, No. 1t4, pp. 931-97t, illust., 1936) studying the fermentation industries of Formosa found two general types of Aspergilli in the Awamori fermentation, $\alpha$ and $\beta$. Type $\alpha$ was A. awamori for which they described the following varieties: var. minimus, var. piceus, var. ferrugineus, var. fuscus, var. fumeus. This "awamori" series produced the more desirable type of product; citric acid was present, as well as alcohol due to the yeast used in the inoculum.

Aspergillus niger var. fermentarius Nakazawa, Simo and Watanabe, in Jour. Agr. Chem. Soc. Japan, 10(2) 1934. pp. 171-172, summarized on p. 181. Reported as a variety with "conidiophores 1037 to $2438 \mu$ by 13.1 to $16.0 \mu$; vesicles 22.6 to $73.6 \mu$; primary sterigmata 12.7 to $16.1 \mu$ by 3.3 to $7.2 \mu$, secondary 6.8 to $9.8 \mu$ by 3.3 to $4.6 \mu$; and conidia globose 2.3 to $4.6 \mu$ in diameter." This form obviously belongs close to $A$. awamori.

Aspergillus miyakoensis Nakazawa, Simo, and Watanabe, in Agr. Chem. Soc. Japan, Jour. 12(9): 963-4, fig. on 973. 1936.

The colonies figured by the authors show a cottony mycelium with long-stalked heads in a broad zone near the margin. The measurements 
differ from $A$. foetidus as follows: primary sterigmata are reported to be 12 to $20 \mu$ by 4.4 to $9 \mu$ in contrast to secondaries 7 to $10 \mu$ by 2.5 to $5 \mu$, whereas

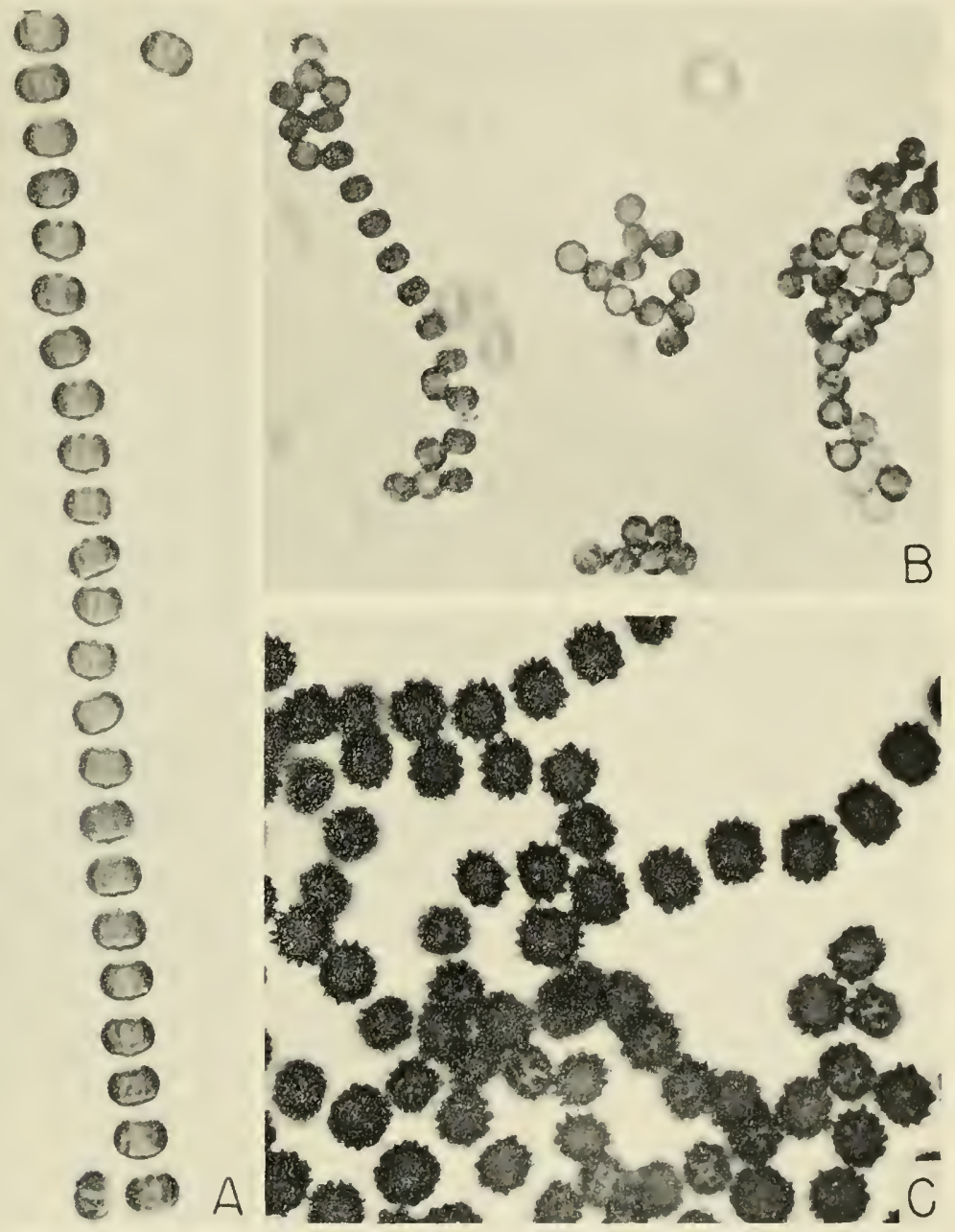

FIG. 63. Conidia, Aspergillus niger group. A, Single long chain of conidia, $\times 1000$ (Photograph by Edward Yuill). B, Conidia of a typical strain of $A$. niger, NRRL No. $344, \times 700$. C , Conidia of the citric and gluconic acid producing strain, NRRL No. $67, \times 700$. The large size and coarsely roughened walls are characteristic of the conidia of the latter strain.

in $A$. foetidus both series measure 7 to $10 \mu$ by 2 to $4 \mu$; conidia are globose 3.7 to $5.6 \mu$ in diameter.

The species repeats the colony appearance of certain mutants produced by means of chemical stimulants by Thom and Steinberg (1939) from the latter's standard strain of $A$. niger. 
A. hennebergi Blochwitz, in Ann. Mycol. 33: 238-9. 1935.

Colonies described as showing the colors and general aspect of $A$. tamarii or $A$. wentii but with conidiophores browned as in the upper part of the conidiophores of the A. niger group, and with "red" sclerotia; relationship doubtful. See also the A. wentii group.

Aspergillus niger van Tieghem, in Ann. Sci. Nat. Bot., s. 5, t. 8, p. 240. 1867.

Van Tieghem's strain is not fully verifiable among organisms now maintained in culture, although Biourge (personal communication) believed he had it. We believe the name can be most appropriately used to cover
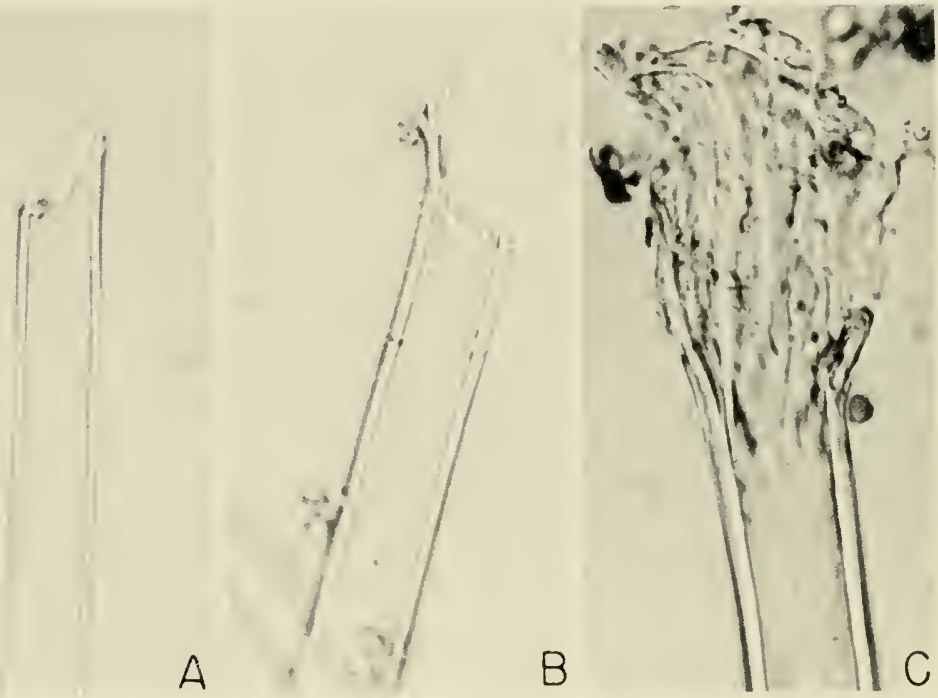

FIG. 64. Broken conidiophores of Aspergillus niger, NRRL No. $326, \times 700 . A$, Conidiophore showing a clean break suggesting a glass tube. $B$, Broken conidiophore showing fibrous wall structure. $C$, Portion of conidiophore crushed and further revealing the fibrous structure of the wall.

the exceedingly abundant isolates having the approximate measurements of sterigmata and conidia noted in van Tieghem's description. The reader can assume, therefore, that in the opinion of the authors any possible description for the species itself would read essentially like that already given for the series on pages $216-219$.

Spccies Characterized by Large Primary Sterigmata and Small Conidia

A. phoenicis (Corda) Thom, in The Aspergilli p. 175. 1926. In describing the black Aspergillus found upon dates, Patouillard and Delacroix (Bul. Soc. Myc. France 7: 118-120. 1891) compared their material to specimens 
in the museum labeled "Cstilago phoenicis" and attributed to Corda, thus establishing the identity of the organism of Corda, which was not recognizable from any previous references. A. ustilago Beck (1892) is described with the same measurements. The measurements of sterigmata place A. phocnicis in the section of the group with primary sterigmata 50 to $60 \mu$ in length; it was first described as C'stilago phoenicis by Corda (Icones Fung. IV., p. 9, pl. 3, fig. 26. 18t0) and transferred by Patouillard and Delacroix, as noted above, to S. phoenicis (Corda) Patouill. and Delacr. If we accept the use of "phoenicis" attributed to Corda as correct, this species becomes the type of the section of the black Aspergilli with primary sterigmata of intermediate length and conidia not over $4 \mu$ in diameter (fig. $61 \mathrm{E}$ and $62 \mathrm{~B}$ ). This was cited by Thom and Currie as A. phoenicis (Corda) Pat. and Delacr., and continued recognition of the species, to cover a group of strains occasionally encountered, appears warranted.

Aspergillus pulverulentus (McAlpine) Thom, in Jour. Agr. Res. 7:10-11. 1916.

Synonym: S. pulverulenta McAlpine, in Agr. Gaz. N. S. Wales (1896)

7: 302. 1897. See also Thom and Church, The Aspergilli, p. 179.1926.

McAlpine's data include: "White to dirty yellow mycelium;" heads 155 to $340 \mu$ in diameter, radiate with chains mostly separate; conidiophores erect, stiff, up to $7 \mathrm{~mm}$. by $20 \mu$ with walls up to $5 \mu$ thick; vesicles 70 to $170 \mu$ in diameter, globose or nearly so; primary sterigmata up to $144 \mu$ long by $8 \mu$, secondary 14 to $18 \mu$ long; conidia globose, rough, about $t \mu$ in diameter. Colonies with these general characters have been studied in culture at least twice and maintained for long periods; one came from Spain, the other from Texas. A. strychni Lindau (Hedwigia Bd. 43, Rept. 5, p. 306-7. 1904) is one of this series.

\section{Light Colored Forms}

Aspergillus niger mut. cinnamomeus (Schiemann) n. comb.

Synonym: A. cinnamomeus Schiem., in Ztschr. Induktive Abstan. u. Vererbungslehre, Bd. 8, Heft 112, pp. 1-35, 16 fig., 2 pl. (1 col.) 1912. See also Thom and Church, The Aspergilli, p. 164.1926.

Colonies upon Czapek's solution agar at room temperature, rapidly growing and spreading, producing an aerial growth of conidiophores and heads reaching a pale cinnamon upon maturity. Reverse only slightly 
colored in the same shade. Conidial heads not crowded, globose. Conidiophores smooth, thick-walled, with upper portion more or less brown, about $1.5 \mathrm{~mm}$. in length by 12 to $20 \mu$ in diameter. Vesicles up to 40 to $50 \mu$ in diameter, crushing readily. Sterigmata in two series; primary about 15 to $20 \mu$ by 3 to $5 \mu$, sometimes larger, secondary about 8 by 2 to $3 \mu$. Conidia 3 to $4 \mu$, thin-walled, globose or subglobose, smooth or nearly so, almost colorless when viewed singly, pale yellowish to cinnamon in mass.

Diagnosis based upon culture NRRL No. 348 (Thom No. 3534b) received from.Schiemann as a mutation induced by introducing potassium bichromate into the culture medium. Approximately the same mutant appeared in Steinberg and Thom's series of induced mutations $(1939,1940)$. Occasional cultures close to A. cinnamomeus have ben obtained from unknown sources in nature.

Aspergillus niger mut. Schiemanni (Schiemann) n. comb.

Synonyms: A. Schiemanni (Schiemann) Thom, Jour. Agr. Res. 7: 13. 1916.

A. fuscus Schiemann, in Ztschr. Induktive Abstam, u. Vererbungslehre, Bd. 8, Heft 1/2, p. 1-35, 16 fig. 2 pl. (1 col.) 1912.

Not A. fuscus Bonorden (Bot. Ztg. Jahr. 19: 202. 1861);

Not S. fusca Bainier (Bul. Soc. Bot. France 27: 29, Pl. 1, fig. 5. 1880)

Colonies upon Czapek's solution agar at room temperature, rapidly growing and spreading, developing a surface growth of conidiophores and heads forming a crowded fruiting area 2 to $3 \mathrm{~mm}$. deep in slanted tubes, becoming a shade of brown near fawn color (Ridgway, Pl. XL); reverse yellowish (fig. 61 D). Conidial heads large, fairly crowded. Conidiophores coarse, $2.5 \mathrm{~mm}$. or more long by 15 to $25 \mu$ wide. Vesicles up to 50 to $60 \mu$ in diameter. Sterigmata in two series; primary, 15 to $40 \mu$ by 4 to $6 \mu$, sometimes larger, secondary 7 to $8 \mu$ by 2 to $3 \mu$. Conidia thin-walled, smooth except sometimes a trace of markings, 3.5 to 4.5 or $5 \mu$ in diameter.

Culture NRRL No. 361 (Thom No. 3534C) was received from Schiemann. Culture NRRL No. 362 was received from Biourge under the same name but shows a much deeper brown color (near Natal brown, Ridgway, Pl. XL).

The mutant, A. niger mut. Schiemanni, is distinguished from the parent type of $A$. niger by the color and smoothness of its spores. The name, A. fuscus Schiemann, is invalidated by the prior usage of the names $A$. fuscus by Bonorden and S. fusca by Bainier.

Mutations of approximately this same type have been secured from cultures of strain NRRL No. 67 (PI. VI C) by ultra-violet irradiation 
(Raper, Coghill, and Hollaender); from cultures of other black Aspergilli through Cathode ray irradiation (Whelden 1939); by cultivation in the presence of various chemicals (Steinberg and Thom: 1939, 1940); and finally by the appearance in plate culture under normal conditions of a lightspored sector in an apparently typical culture of Aspergillus niger. See discussion Chapter VI. Two isolates labeled A. awamori Nakazawa which resemble this mutant in color have been received from Japan, one from Hanzawa, the other from Nakazawa. Both produce primary sterigmata less than $20 \mu$ in length but otherwise are close to Schiemann's mutant.

\section{Probable Synonyms}

Many species have been described by investigators working at different periods and at widely separated stations which obviously belong within the Aspergillus niger series as it is here considered. Some of these will be briefly noted since they were reported to present unique cultural or morphoological features, or since they account for interesting or important biochemical reactions.

A. giganteus Mattlet (Ann. Soc. Belge Med. Trop. 6:36. 1926) was described as a new species with conidiophores 2 to $6 \mathrm{~mm}$. long; conidia 4 to $5 \mu$ in diameter, rough, black-brown; but without other marks of separation. It would seem to have been a rather extreme variant in the two characters.

A. atropurpureus Blochwitz (Ann. Mycol. 32(1/2):86. 1934.) Not A. atropurpureus Zimmermann (Centralb. f. Bakt. etc., 2 Abt., 8, No. 5/7, p. 218. 1902). Blochwitz proposed to use the name $A$. atropurpureus for the purple-brown members of the $A$. niger group as he obtained them from the tropics. He gave conidial measurements as 2.5 to $3.5 \mu$ in diameter, and added that chemical differences in the coloring substances warranted separation from the black, or blacker forms.

A. ficuum (Reich) Hennings, in Hedwigia, 34, p. 86. 1895; and Reichardt, in Verhandl. K.K. Zoll. Bot. Gesell. Wien, 17: 335, 1867. Regarded as A. niger by Wehmer, in Centralb. f. Bakt. etc., 2 Abt., 18, No. 13/15, p. 394-395. 1907. See also Thom and Church, The Aspergilli, p. 174. 1926.

Slight differences in morphology between this and $A$. niger $\mathrm{v}$. Tieg. are reported by Hennings, but disregarded by Wehmer. Culture NRRL No. 364 (Thom. No. 142), received in 1909 from Westerdijk under this name, was reported by Thom and Currie (1916) as the most rapid producer of oxalic acid of all strains of $A$. niger tested. There is no morphological basis for separating this strain from $A$. niger, and it is distinguished in culture only by the tendency, after many years in laboratory culture, to produce rather floccose colonies.

A. batatae Saito, in Centralb. f. Bakt. ete., 2 Abt., 18, No.1/3, p. 34. 1907. Saito's organism as described is close to $A$. niger: colonies reach brownish-black through yellow shades; conidiophores 2 to $4 \mathrm{~mm}$. by 12 to $20 \mu$, smooth, thick-walled, brown in upper portion; vesicles 35 to $50 \mu$, globose; primary sterigmata 24 to $40 \mu$ long by $8 \mu$ at apex, secondary 10 by $3.2 \mu$; conidia globose, brown, finely roughened, 4 to $5 \mu$ in diameter. A culture under this name from Formosa (NRRL No. 363) presented the 
comparatively large conidia described by Saito but possessed no other marks which would assist in identification.

A. luteo-niger (Lutz) Thom and Church, in The Aspergilli, p. 166. 1926. Syn.: S. luteo-nigra Lutz, in Bull. Soc. Bot. France 53: 48-52. 1907. Thom and Church (1926) found one or more black Aspergilli in which the conidia appeared smooth in ordinary laboratory slide amounts in which they were treated with alcohol followed by lacto-phenol or other mounting fluids. Fragments floating in the mounting medium led to the test of such conidia in dry mounts, in oil, and in pure glycerine. Under these conditions, the conidia showed the typical marks of the A. niger series. Since smooth conidia were the sole definite contrast between A. luteo-niger Lutz and A. niger v. Tiegh., the strains studied were accepted as Lutz' organism. Since that time, it has been shown that many black Aspergilli, ranging widely among the variant forms encountered, pass through a physiological stage in which the outer wall and color hars, when subjected to alcohol or other fluid causing active osmotic currents, break in pieces and float away leaving the conidia smooth and colorless, or only partly shaded toward the dark color of the group. It thus appears that $A$. luteo-niger may be dropped as failing to designate any definite group of strains and as failing to present any character of diagnostic importance.

\section{ASPERGILLUS CARBONARIUS SERIES}

Species Characterized by Conidia in Excess of $5.0 \mu-$ Sterigmata in Two Series

Aspergillus atropurpureus Zimmermann, in Centralb. f. Bakt., etc., 2 Abt., 8, No. 5/7, p. 218.1902.

Conidiophores hyaline or somewhat brownish in age, up to 800 by $16 \mu$ to $20 \mu$; vesicles 60 to $80 \mu$ in diameter, hyaline to brown; sterigmata, primary 16 by $6 \mu$, secondary 3 to $4 \mu$ by 1.5 to $2.0 \mu$; conidia globose, rough, with prominent warts, purplish-black, 6 to $10 \mu$ in diameter. Isolated from Coffea liberica, in Java. Culture not studied by us. The species is possibly valid, and is presented as representative of occasional forms characterized by small sterigmata and large purple-black spores.

Aspergillus fumaricus Wehmer, in Ber. Deut. Chem. Gesell. 51, No. 14: 1663-1668, figs. 1-6. 1918.

This species was named, but not fully described by Wehmer, and was not distributed by him. Its biochemical activity as a producer of fumaric acid is covered in the paper cited above, together with the admission that it belonged to the $A$. niger group. Culture No. 4668.2 (C. Thom) received from Neuberg under this name presents a variant strain of the group which may be described as follows: Colonies producing a mass of yellow mycelium; conidiophores scantily and tardily produced up to 1,2 , or $3 \mathrm{~mm}$. long, by 20 to $22 \mu$ in diameter, smooth, bearing large radiate, yellow-brown heads. Tesicles up to $60 \mathrm{or}$ even $100 \mu$ in diameter, with walls thin, easily crushed. Sterigmata in two series, primary sterigmata up to $15 \mu$ by 4 to 
$5 \mu$, secondary sterigmata rather coarse, some growing out into aborted hyphae. Conidia commonly $5 \mu$, occasionally 7 to $\delta_{\mu}$ in diameter, with lengthwise color bars as in $A$. niger but paler in color.

Culture No. 4668.2 (Thom) is accepted as correctly named. By description, A. fumaricus Wehmer differs little from A. atropurpureus Zimm. except for the production of yellow-brown rather than purple-black conidial heads. Differences in color definition and discrimination by different workers tend to leave both species in doubt.

\section{Aspergillus fonsecaeus $\mathrm{n}$. sp.}

Synonym: S. fusca Bainier, in Bul. Soc. Bot. France 27 : 29, Pl. 1, fig. 5, 1880. (Bainier's material grown upon moist bread in Toulouse in 1880, was preserved in Roumeguère's Fungi Gallici Exsiccati No. 995)

Bainier described S. fusca in terms closely parallel to ran Tieghem's A. niger, except that the conidia were about double that species in size; Bainier reported these as rarely exceeding $9.4 \mu$ in diameter, while our examination of the exsiccati showed them to be mostly 5 to $8.5 \mu$ in diameter and marked as in A. niger and A. carbonarius. Da Fonseca, in Rio de Janeiro, contributed a culture (Thom No. 4707.878, NRRL No.67, and strain No. 67 of Herrick, May, and associates) possessing approximately these spore measurements, which has retained its characteristic features for more than 20 years and which has proved industrially useful. We believe, therefore, that recognition of a species with sterigmata of intermediate length and conidia about double the dimensions of A. niger van Tieghem is warranted. The name A. fonsecaeus is proposed since the binomial A. fuscus had already been used for an Aspergillus by Bonorden in 1861 (Bot. Ztg. Jahrg. 19: No. 29, p. 202) and has been used at least twice subsequent to this. The species would then include strains characterized by large, subglobose, coarsely roughened conidia ranging from 5.5 or $6.0 \mu$ to 8.5 or $9.0 \mu$ (fig. $63 \mathrm{C}$ ), including the strain " 67 " used by Herrick, May, Wells, Moyer, et al. of the Industrial Farm Products Research Division, U. S. Department of Agriculture, Arlington Farm, Virginia, for the production of citric and gluconic acids (see literature citations pp. 290-295).

The following description is based primarily upon strain NRRL No. 67:

Colonies upon Czapek's solution agar growing rapidly at temperatures from $24^{\circ}$ to $30^{\circ} \mathrm{C}$., attaining a diameter of 7 to $8 \mathrm{~cm}$. in eight to ten days, consisting of a basal regetative mycelium that is largely submerged and colorless, and abundant conidial structures commonly arranged in more or less conspicuous concentric zones; heads carbon black or brownish-black, imparting to the colony a like coloration ; reverse colorless in young colonies, 
commonly darkening in age and often becoming almost black after 2 to 3 weeks. Conidial heads large, globose, radiate or with chains of conidia massed in an indefinite number of loose divergent columns, commonly 300 to $500 \mu$ but of ten attaining a diameter up to $1 \mathrm{~mm}$. Conidiophores varying in length from 1.5 to $3.5 \mathrm{~mm}$. but averaging about 2.0 to $3.0 \mathrm{~mm}$., mostly 20 to $30 \mu$ in diameter with walls 2.0 to $3.0 \mu$ thick, smooth, often colored in dark shades in the region beneath the vesicle. Vesicles globose, fertile over the entire surface, commonly 50 to $75 \mu$ in diameter (fig. $62 \mathrm{C}$ ), usually in brown shades, often quite dark. Sterigmata in two series, usually brown, often dark: primary sterigmata variable in different heads and in different cultures, ranging from 15 to $20 \mu$ by 6 to $8 \mu$ in some to 35 to $45 \mu$ by 10 to $13 \mu$ in others; secondary sterigmata ranging from 8 to $14 \mu$ by 5 to $6.5 \mu$ but averaging about 9 to $10 \mu$ by 5 to $6 \mu$. Conidia large (fig. $63 \mathrm{C}$ ), globose, conspicuously roughened with prominent color bars, ranging from 5.5 to $8.5 \mu$.

Since strain " 67 " appears in the industrial fermentation literature as Aspergillus niger and has been consistently distributed under this name over a period of several years, it is not our purpose here to challenge this designation, for this binomial is often used in a very general sense to cover any black member of this group. We do wish to emphasize, however, that this strain does not represent the common type of black Aspergillus usually isolated in routine examination of soil and moldy materials in general. The fact that it possesses large spores is of the greatest value in checking its purity and further commends it for use in industrial operations. This strain was originally received from Rio de Janeiro, and recently Dr. Dorival M. Cardoso of Sao Paulo, Brazil, has reported (personal communication) that he has repeatedly isolated large-spored forms apparently closely related to it. It would, therefore, appear likely that this large-spored form represents a type of organism much more abundant in South America than in this country.

Aspergillus pulchellus (Speg.) Thom and Church, in The Aspergilli, p. 181. 1926.

Synonym: Aspergillopsis pulchellus Speggazzini, in Myc. Arg. V. in Ann. Mus. Nac. Buenos Aires Ser. 3, t. 13: 436.1911.

This species was described with colonies intensely black; conidiophores 1 to $2 \mathrm{~mm}$. by 18 to $20 \mu$, with walls darkened; vesicles 50 to $60 \mu$ in diameter; primary sterigmata 10 by $30 \mu$; and conidia 8 to $10 \mu$ in diameter and rough. The species appears to differ from van Tieghem's $A$. niger principally in its very large conidia, and it is extremely doubtful if it could be separated from $A$. fonsecaeus as described above.

A. dipus of Ferdinandsen and Wing (Bot. Tids. 30:220, fig. 6. 1910) represents another organism undoubtedly close to the forms under consideration. The presence of conspicuous foot cells, upon which character the species was based, is common to all members of the group, hence, is not a valid basis for separation. 
Aspergillus carbonarius (Bainier) Thom, in Jour. Agr. Res. 7: 12.1916.

Synonym: S. carbonaria Bainier, in Bul. Soc. Bot. France 27:27-28. 1880.

Colonies grown upon Czapek's solution agar show vegetative mycelium white or with some yellow in submerged areas, broadly spreading, more or less zonate; sclerotia produced upon the surface of the substratum in old cultures; fruiting areas carbon-black. Conidiophores colorless below, yellow to yellow-brown toward the apex, 4 to $6 \mathrm{~mm}$. or more in length and up to $25 \mu$ in diameter, with walls smooth, sometimes as much as $4 \mu$ in thickness. Heads globose, varying in diameter up to $500 \mu$. Vesicles up to $90 \mu$ in diameter, fertile over the entire surface, commonly with contents yellow-brown to black and in old heads forming with the primary sterigmata a hard, brittle, carbonaceous mass. Sterigmata in two series, primary sometimes one septate, from 20 to $40 \mu$ long in young or small heads, and up to $120 \mu$ long in large heads by 5 to $13 \mu$ in diameter at the apex, secondary, 8 to $14 \mu$ by 3 to $6 \mu$. Conidia at first smooth, becoming rough when ripe, 5.5 to $10.5 \mu$ in diameter. Colonies grow well upon all culture media used, with temperature optimum below $37^{\circ} \mathrm{C}$. A culture from Dr. A. F. Blakeslee (NRRL No. 369, Thom No. 4030.1) reproduces in detail the morphology recorded by Bainier. A. carbonarius has also been received in culture from the Gold Coast of Africa.

The same morphology was also found in one of Dr. Farlow's specimens, S. acini-uvae Caballero (Bol. R. Soc. Esp. Hist. Nat. 28: 429. 1928). This was described as it appeared upon rotting grapes as follows: vesicles 75 to $105 \mu$ by 73 to $98 \mu$; primary sterigmata 59 to $80 \mu$ by 13 to $22 \mu$ and secondary 15 to $20 \mu$ by 5 to $7 \mu$; conidia globose, rough, 6 to $10 \mu$. Blochwitz in "Die Aspergillaceen" (Ann. Mycol. 27(3/4): 204, 222, and 232. 1929), with part of the type specimen before him, declares it to be $A$. carbonarius, apparently without cultivating it. Mosseray (LaCellule 43: 222. 1934) places Caballero's organism in Aspergillus pulchellus.

We cannot agree with Blochwitz (idem. p. 221, 222, fig. 12) in describing the vesicle in $A$. carbonarius as subglobose, and figuring the head as hemispherical. Bainier's original figures show the vesicle fertile to the very base. This is characteristic of the strains observed from various sources.

ASPERGILLUS LUCHUENSIS SERIES

Species Normally Showing One Series of Sterigmata, Occasionally Two. Aspergillus luchuensis Inui, in Jour. Col. Sci. Imp. Univ. Tokyo 15: 469, Pl. 22, fig. 1-8. 1901. See also Usami, in Centralb. Bakt. etc., 2 Abt., 43, p. 250. 1915; The Aspergilli, p. 171. Pl. IV. 1926.

Colonies upon Czapek's solution agar spreading rapidly, producing abundant conidiophores and conidial heads which give a purple-black color 
to the whole colony, reverse in pale yellow shades. Conidial heads globose, up to 250 to $300 \mu$ in diameter, splitting in age into short columns of spores. Conidiophores up to $1500 \mu$ by $10 \mu$, smooth, yellow toward the vesicle. Vesicles yellow, up to $40 \mu$ in diameter, fertile over the entire surface. Sterigmata mostly in one series, $6 \mu$ or a little larger by $3 \mu$; branched sterigmata occasionally appear. Conidia globose, 3.5 to $4 \mu$, roughened with spines rather than bars of coloring substances.

Representatives of the species have been received from Japan (NRRL No. 356; Thom No. 4291.3), from West Africa, from Bermuda, and from various points in the United States. While not as abundant as forms with double sterigmata, isolates with this kind of head are not uncommon.

Inui also described A. perniciosus (Jour. Coll. Sci. Tokyo 15, p. 473, T. XXI, figs. 9-12. 1901) with color data closer to $A$. wentii than $A$. luchuensis. Its morphology, however, seems to belong here. It has not been rediscovered and discussed adequately in relation to either group. Culture No. 4707.757 (Thom), received from da Fonseca in Brazil, possibly represents this species: colonies in center at least transiently greenish, but without true green color; conidiophores not crowded, sinuous, apparently smooth when observed with low magnifications but with traces of pitting evident when examined with an oil-immersion objective, up to $1000 \mu$ by 10 to $15 \mu$; primary sterigmata 10 to $16 \mu$ by 3 to $4 \mu$, secondary up to $8 \mu$ by 2 to $3 \mu$; conidia 4 to $5 \mu$ in diameter, with yellow markings in the form of loops and bars.

A. luchuensis var. rubeolus Shih (Lingnan Sci. Jour. 15(3): 374. 1933) differs from the species by becoming chocolate brown rather than black. This would suggest careful comparison with A. japonicus Saito.

Aspergillus japonicus Saito, in Bot. Mag. (Tokyo) 20: 61, 5 figs. 1906.

Colonies upon Czapek's solution agar growing rapidly and spreading evenly, characterized by its purple-brown heads and the presence of few to many light brown sclerotia; reverse colorless or nearly so. Conidiophores 500 to $1000 \mu$ by 12 to $15 \mu$, figured as showing concretions on the surface, with walls more or less brown. Vesicles globose, fertile over the whole surface, witk walls brown and marked by the bases of sterigmata. Sterigmata in one series, 7 to $9 \mu$ by 5 to $6 \mu$, commonly falling away in mounts irom old cultures. Conidia globose, echinulate, 4 to $5 \mu$ in diameter. Sclerotia scattered throughout the colony, 650 to $1000 \mu$ in diameter, white to pale yellow in color and of ten overgrown by mycelium and conidiophores.

Type material has not been seen. The diagnosis is based upon two strains contributed by Dr. A. F. Blakeslee (NRRL No. 358: Thom No. 4030.3, and NRRL No. 359: Thom No. 4030.5) which come fairly close to 
Saito's description. A purple-brown strain (Thom No. 5362.7) collected by Manns in Honduras showed slightly darker colors and slightly different measurements. A strain received from Raistrick as coming from Blochwitz through the Centraalbureau in Baarn labeled A atropurpureus Zimmermann resembled the two Blakeslee cultures quite closely. It certainly did not comply with Zimmermann's description (see p. 226). A. luchuensis var. rubeolis Shih is probably closely related if not identical with $A$. japonicus Saito.

Aspergillus violaceo-fuscus Gasperini, in Atti. Soc. Toscana Sci. Nat. Pisa, Mem. 8, fasc. 2, p. 326. 1887.

Colonies upon Czapek's solution agar, comparatively slow growing (fig. $61 \mathrm{~F}$ ), purplish-brown with a faint violet shade, passing to purple drab in age, reverse colorless to dark purplish. Conidial heads purplish-brown, globose, not crowded, 100 to $150 \mu$ in diameter. Conidiophores mostly less than $1 \mathrm{~mm}$. in length but sometimes reaching $2 \mathrm{~mm}$., 12 to $18 \mu$ in diameter. Vesicles globose, varying up to $60 \mu$ in diameter. Sterigmata generally in one series (fig. $62 \mathrm{D}$ ), 5 to $8 \mu$ by $3 \mu$, occasionally in two series with secondaries 2 to $4 \mu$ long. Conidia elliptical, 3.5 to $5 \mu$ by 5 to $6.5 \mu$, at first hyaline, becoming violaceous, somewhat roughened.

By description this is a variant member of the great group of black Aspergilli characterized by short sterigmata and elliptical conidia.

Gasperini's material has not been seen but three cultures have been studied which are believed to differ from his species only in having somewhat smaller conidia. The first of these was received in 1914, from Puerto Rico (NRRL No. 360: Thom No. 3522.30), while the others were subsequently obtained from Jamaica and from Professor Raistrick in England.

In cultivating black Aspergilli an occasional strain produces heads at first showing a single series of sterigmata; then, as the colony becomes older, heads with both primary and secondary sterigmata dominate the culture. Upon careful examination many strains show both large heads with sterigmata in two series and, on shorter conidiophores mixed among the larger ones, small heads with simple sterigmata only or with both types mixed. Colonies of this kind probably accounted for A. nanus Montagne, A. subfuscus Johan-Olsen (Sopp.) and are known to account for A. pyri English.

Aspergillus nanus Montagne, in Syllog. Generum Specierumque Cryptogamarum, p. 300, No. 112, Paris, 1856. Sace. Syll. 4: 71. 1886. Species reported as a member of the black group with a single series of sterigmata about $15 \mu$ in length and spores $3 \mu$ in diameter. This may have represented young fruiting structures of a typical A. niger.

Aspergillus subfuscus Johan-Olsen, in Meddelelser fra Naturh. forening i Liris- 
tiania, 1885. Described as showing smooth globose spores, 3.0 to $3.5 \mu$ and a single series of sterigmata in culture, up to $20 \mu$ in length, but with some secondary sterigmata seen in the original material. The separation of this from $A$. niger appears questionable.

Aspergillus pyri English n. n., in Doctoral Thesis, State College of Washington, Pullman, Wash. pp. 76-78, 1940; cited in abstract. A form described as showing a single series of sterigmata 14.4 to $19.2 \mu$ by $3.6 \mu$ and spinulose conidia 3.6 to $4.8 \mu$ in diameter. In personal correspondence of June 1943, English stated that as a result of continued study of this strain and the finding of double sterigmata, he questioned the desirability of maintaining the spccies designation.

\section{SYNOPSIS OF SPECIES PROPOSED BY MOSSERAY}

Biourge, who was a discriminating collector, accumulated 63 strains of "black" (or related) Aspergilli for each of which he could see sufficient individuality to warrant preservation. Mosseray, working in Biourge's laboratory, studied these strains, attempted to establish species lines among them, and proposed new specific names for all forms which he believed undescribed. With the whole 63 strains in parallel culture, all bearing the names applied by Mosseray, Biourge and Simonart were inclined to withdraw part of Mosseray's new species names, a position with which the writers heartily agree. Nevertheless, to present one concept of the range of variation confronted by the student of this group, Mosseray's synopsis ${ }^{2}$ has been translated with minor emendations, and is herewith presented.

\section{Mosseray's Synopsis}

A. Conidia 6 to $10 \mu$ in diameter, rough; vesicles subglobose; primary sterigmata often $100 \mu$ or more in length; colonies jet-black.

a. Sporulation more or less dense; heads large; reverse fumose or very dark olive with mycelium more or less wrinkled.

Conidiophores 2, 4, or even $6 \mathrm{~mm}$. long; sclerotia present in "natural" media A. carbonarius (Bainier) Thom and Currie Conidiophores 1 to $2 \mathrm{~mm}$. long; sclerotia not reported.....A. pulchellus (Speg.) Thom and Church, Syn. S. acini-uvae Caballero

b. Sporulation less dense; heads small, also with very small heads with single

2 Translated and emended from Mosseray, Raoul, Les Aspergillus de la section "niger" Thom and Church, in La Cellule XLIII: 271-273. 1934. All data are based upon colonies grown in slanted test tubes using Biourge's formula of "neutral Raulin agar" of the following composition:

$\begin{array}{lc}\text { Water }(\text { distilled) } \ldots \ldots & 1000 \mathrm{cc} . \\ \text { Sucrose } \ldots \ldots \ldots \ldots \ldots & 50 \mathrm{~g} . \\ \text { Tartaric acid } \ldots \ldots \ldots & 0.40 \mathrm{~g} . \\ \mathrm{MgCO}_{3} \ldots \ldots \ldots \ldots \ldots & 0.250 \mathrm{~g} . \\ \mathrm{NH}_{4} \mathrm{NO}_{3} \ldots \ldots \ldots \ldots & 2.500 \mathrm{~g} . \\ \mathrm{K}_{2} \mathrm{CO}_{3} \ldots \ldots \ldots \ldots \ldots & 0.400 \mathrm{~g} .\end{array}$

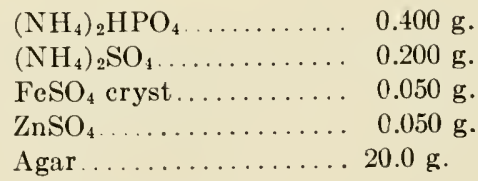

Sterilized at $120^{\circ} \mathrm{C}$. for 20 minutes. 
sterigmata and on very short conidiophores among them; mycelium grayyellow; reverse dark reddish-brown and mycelium much wrinkled

A. pseudo-carbonarius (Bainier nn.) Mosseray

B. Conidia $2.5-4$, even to $5 \mu$ in diameter, mostly more or less rough and more or less colored but some smooth or nearly so; vesicles normally globose; primary sterigmata mostly $20 \mu$ long or longer, sometimes up to $100 \mu$.

a. Colonies deep purple-brown or clear purple-brown.

I. Conidiophores short (up to $2 \mathrm{~mm}$. on an average).

$\alpha$. Wrinkles in reverse shallow, transverse (in tubes),

Heads small; conidiophores short (up to $1 \mathrm{~mm}$.) 8 to $15 \mu$ in diameter, primary sterigmata 5 to $20 \mu$; reverse cream colored

A. microcephalus Mosseray

Heads "medium"; conidiophores up to $2.5 \mathrm{~mm}$.; primary sterigmata up to $45 \mu$; reverse dark reddish-brown, often spotted and becoming very dark.................. phoenicis (Corda) Thom

Syn. A. longobasidia (Bainier n.n.) Mosseray

Syn. A. bainieri Mosseray 1934

$\beta$. Wrinkles in reverse of mycelium fairly numerous, occasionally anastomosing.

Appearance "mealy", deep purple-brown; conidiophores very short up to $0.5 \mathrm{~mm}$. and 7 to $13 \mu$ in diameter; primary sterigmata 4 to $12 \mu$; reverse deep olive-brown......... pseudo-citricus Mosseray Appearance sub-granilar; brown-purple, at times clear brown to umber; conidiophores up to $1.5 \mathrm{~mm}$. by 7 to $20 \mu$; conidia smooth or nearly so; growth rapid. Reverse slightly colored:

Reverse cream to pale brown; primary sterigmata 8 to $20 \mu$

A. fuliginosus Peck

Syn. S.fuliginosa Bainier

Syn. A. praecox Mosseray 1934a

Reverse very deep brown; primary sterigmata up to $50 \mu$; sclerotia occasional................. sclerotifer Mosseray

Reverse uncolored to citron yellow; primary sterigmata 8 to $20 \mu$

A. citrino-niger Mosseray

$\gamma$. Wrinkles sharp and numerous (reticulated). Reverse becoming rapidly dark, almost black.

Aspect mealy; color purple-brown; conidiophores up to $1 \mathrm{~mm}$; drops not colored; primary sterigmata 12 to $30 \mu$; conidia smooth or nearly so..................... densus Mosseray

Aspect mealy to granulate, deep purple-brown; drops numerous, bronze; primary sterigmata 12 to $30 \mu$...A. rutilans Mosseray

Aspect subgranular, brown-purple; drops numerous, black, some of them very large; primary sterigmata 12 to $30 \mu$

Reverse not so dark, sometimes olive or colorless.

A. guttifer Mosseray

Reverse deep olive, often spotted; primary sterigmata 15 to $30 \mu$

A. Buntingii Mosseray

Reverse not colored; commonly showing areas sterile or free from spores; mycelium slightly yellow at first; primary sterigmata 20 to $50 \mu \ldots \ldots \ldots \ldots \ldots \ldots$. variegatus Mosseray 
II. Conidiophores up to $3 \mathrm{~mm}$., rarely $4 \mathrm{~mm}$. long.

$\alpha$. Sporulation normal.

Reverse uncolored or slightly olive; drops few or none; primary sterigmata 20 to $40 \mu$; mycelium of ten yellow at first

A. niger Van Tiegham

Reverse orange-brown or purple-brown; drops large, black; primary sterigmata 8 to $25 \mu$; mycelium colorless or rarely yellow

A. Biourgei Mosseray

Reverse pale reddish-brown; drops very few; heads very small; primary sterigmata 15 to $30 \mu$; mycelium colorless or sometimes rose; sporulation very slow............ Churchii Mosseray

Reverse golden yellow; drops none; primary sterigmata 20 to $40 \mu$; mycelium reddish-yellow at first

A. luteo-niger (Lutz) Thom and Church

Reverse dark olive-brown; conidiophores up to 1 to $2 \mathrm{~mm}$.; primary sterigmata very variable; vesicles subglobose

A. anomalus Mosseray

Reverse cream to brown, spotted slightly, wrinkled with a dark band in center, sporulation scattered, pale, conidiophores up to $4 \mathrm{~mm}$. long............... tubingensis (Schober) Mosseray

$\beta$. Sporulation abnormal.

Sporulation absent in patches and in the margin, with a granular appearance, and proliferation of mycelium throughout the conidial area .

A. granulatus Mosseray

Sporulation massed at the thin end of the agar, less dense toward the bottom of the tube; mycelium wooly.

Mycelium wooly, gray-white or yellowish-gray, carrying on the thin areas numerous simple heads ("fumigatiformes") and some normal heads. Reverse cream, slightly wrinkled

A. velutinus Mosseray

Mycelium less wooly, white; conidiophores 1 to $3 \mathrm{~mm}$. long, very abundant at the thin end of the agar, the remainder sterile. Reverse much wrinkled, cream....A. ficuum (Reich.) Hennings

III. Conidiophores tall, up to $1 \mathrm{~cm}$; sporulation scanty, mostly toward the thin end of the agar.

Heads large, splitting into divergent columns; conidiophores up to $1 \mathrm{~cm}$. by $30 \mu$; primary sterigmata up to $100 \mu$; reverse cream or slightly

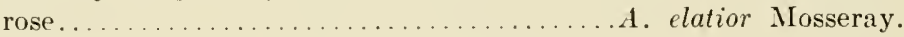

Heads small, mycelium gray-rose; conidiophores rarely up to $1 \mathrm{~cm}$., more often 2 to $6 \mathrm{~mm}$.; very numerous little "fumigatiform" heads, on short conidiophores at the surface; reverse clear rose or salmon

A. pseudo-elatior Mosseray

IV. Conidiophores very irregular from less than $1 \mathrm{~mm}$. to $3 \mathrm{~mm}$. with much larger heads; reverse clear reddish-brown; mycelium yellow at first then dark brownish-red; sporulation delayed. A . pseudo-niger Mosseray

b. Colonies not purple-brown but clear brown; morphology of A. niger; conidia mostly smooth or nearly so.

I. Colonies clear brown or sepia; conidiophores up to $1 \mathrm{~mm}$; reverse olive or lighter; with reticulated wrinkles; vesicles 20 to $50 \mu$; primary sterigmata 12 to $50 \mu$; conidia smooth........A. olivaceo-fuscus Mosseray 
II. Colonies umber; conidiophores 1 to $3 \mathrm{~mm}$; reverse reticulated, dark reddish-brown; conidia smooth or nearly so....A. Schiemanni Thom

Syn. A. fuscus Schiemann

III. Colonies reddish salmon; conidiophores 1, 2, or even $3 \mathrm{~mm}$; reverse slightly wrinkled, uncolored, or pale cream; conidia smooth

A. cinnamomeus Schiemann

C. Conidia 3 to $5 \mu$, globose, smooth, slightly colored; vesicles globose; conidiophores up to $1 \mathrm{~mm}$., slender; primary sterigmata 12 to $20 \mu$; colonies appearing somewhat granular, deep brown; reverse olive passing to bronze; mycelium sulphur yellow

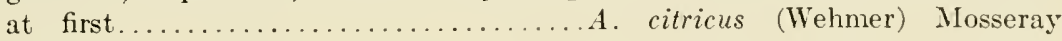

D. Conidia 3 to $5 \mu$, globose or elliptical, smooth or slightly rough; colonies violaceous. a. Sterigmata in two series; vesicles globose; colonies purplish-violet or mauve; reverse violet-brown; conidia 3 to $5 \mu$, globose, smooth, uncolored

A. auamori Usami

b. Sterigmata in one series, short; vesicles subglobose; colonies in violaceous shades to mauve.

Colonies mauve ("violet livide"), reverse uncolored, wrinkled; conidia globose or obovate, smooth 3 to $5 \mu$ in long axis; sporulation slow; narrowly

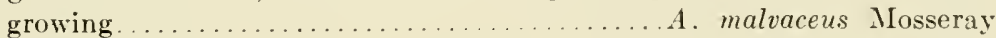

Colonies violaceous or dark violet slate; reverse dark yellow or orange, slightly wrinkled; conidia globose and rough, 3 to $4.5 \mu$; sporulation very rapid, and colonies broadly spreading, with sclerotia common on rice or other "natural" substrata, rare upon sugar media......A. japonicus Saito

Colonies violet-brown; reverse purplish-brown, wrinkled; conidia globose, rough, 3 to $5 \mu$ in long axis; sporulation slow and more or less incompletely covering the surface; dwarf heads abundant. A. atro-violaceus Mosseray

Colonies dark brown or carob brown, with a mealy or granular appearance, reverse dark brown, or olive at the margins, wrinkled; conidia smooth, globose, 2.5 to $4 \mu$; vesicles globose or pyriform; primary sterigmata 3.5 to

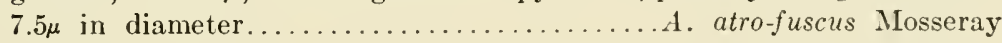

If the material available for study were limited to Biourge's 63 cultures, identification to strain, variety, or species might be possible. When Mosseray subsequently returned to Brussels as Mycologist at the Jardin Botanique de l'Etat and was confronted by hundreds of other strains largely from the Belgian Congo, many of which presented further variation, he began to see the impossibility of describing them all in terms which would permit subsequent identification. Biourge at that point (personal conference) withdrew his support from many of the diagnostic features accepted in Mosseray's mémoire and agreed to the proposal that specific names in black Aspergilli could only be serviceable as bringing together aggregates of strains showing common and fairly dependable morphological characters. This was the attitude held by Thom and Church in The Aspergilli (1926) based upon the examination of many hundreds of black Aspergilli, and it remains the position of the writers at this time.

Since variation is characteristic of the whole genus-not one group 
alone, the subject is covered in the chapters on Morphology and on Variation.

\section{Coloration}

Color has been emphasized in most of our attempts to separate these strains in culture. Linossier (1891) extracted from his strain of A. niger a coloring substance soluble in hot water which he called aspergilline. This substance is readily demonstrated. Blochwitz (1929, p. 219) reports this material to be soluble in " $\mathrm{NH}_{3}$ and alkalies," and we have extracted it with hot water. Microscopic comparison of conidiophores, heads, and separate conidia from numerous strains leads to the conclusion that the same substance may give a brown color to the upper one-third of the conidiophore, to the vesicle and its contents, to the walls of the sterigmata, and in $A$. carbonarius so fill the whole vesicle and sterigmatic area with a brittle mass as to justify Bainier in calling the head carbonaceous. Conidia in a few strains have appeared to possess smooth, uniformly brown cell walls. As a group, however, the black aspergilli have globose conidia with a firm, uncolored or slightly colored inner wall, a very thin outer wall, and between the two the coloring substance, presumably aspergilline, deposited as granules, warts, or bars running lengthwise of the cells and presenting a pattern characteristic of the whole group. When pale-colored variants (mutants?) such as $A$. cinnamomeus (p. 223), or darker ones such as $A$. schiemanni (p. 224), or the variously brown to deep carbon black ones like $A$, carbonarius are compared, the relative quantities of coloring matter seem to account for the progressive darkening of the head colors as described in the series.

In the "reverse", or underside, of the mycelium and in the culture substratum, a range of shades from colorless to yellows to reddish-brown, and even very dark shades, is reported for particular species growing upon specified substrata. The chemical reactions back of these colors are not known for the black aspergilli. Blochwitz has reported extractions of color from various species of Aspergillus and other molds, and observed that variations in these colors were readily obtained with reagents. Some of the extracted materials were reported to act as indicators. It is, therefore, doubtful whether the succession of colors present in such a related series of organisms represent substances differing fundamentally one from another, or merely successive steps in the transformation and reactions of the same general type of product.

\section{Occurrence and Economic Importance}

The black aspergilli are probably more common than any other representatives of the genus. They are world-wide in distribution and occur 
in and upon the greatest variety of substrata, including grains, forage products, spoiled fruits and vegetables, exposed cotton textiles and fabrics, leather, dairy products and other protein-rich substrata, and decaying vegetation in the field. They are abundant in all soils examined; and from studies which have been made by the authors and other investigators, it would appear that they are particularly abundant in soils from tropical and sub-tropical areas.

With the possible exception of the A. flavus-oryzae group, which is of great economic importance in the Orient, the black aspergilli are undoubtedly more widely used in industry than any other group of molds. Since the present volume is primarily a manual designed to assist the worker in the study, diagnosis, and maintenance of the aspergilli that come into his hands, no attempt will be made to discuss the various fermentations and other biochemical activities of the black aspergilli. However, these fermentations are of great importance and will be briefly noted. For the reader who is interested in these fermentations, or perchance, is actually conducting them, a fairly complete list of references is presented for each in the "Topical Bibliography" (Chapter XXII). In each case it has been our aim to present sufficient references to provide the reader with a reasonably comprehensive guide to the literature of the field.

Gallic Acid: Raulin and his coworkers in Paris during the early 1860's identified the organism active in the production of gallic acid by the fermentation of gallnuts and other tannin-bearing substances. The species was first discussed as Ascophora nigrans. Van Tieghem in 1867, named and described the organism correctly as $A$. niger. Recurrent investigations have been conducted on this fermentation from that period to the present time (see Topical Bibliography, p. 294).

Citric Acid: In 1917 Currie published his fundamental studies on the formation of citric acid by strains of $A$. niger and thereby established the basis for one of the most important of all industrial mold fermentations. Subsequent to this, investigators in the United States and abroad have made many additional and important contributions. While no attempt will be made to cite all of these, the works of Bernhauer, Wehmer, Doelger and Prescott, and the U. S. Department of Agriculture group, including Wells, May, Moyer, Herrick, and Ward, are considered to be outstanding. A selected list of references to the citric acid fermentation is presented on pages $290-293$.

Fumaric Acid: Certain strains of the A. niger group produce appreciable amounts of fumaric acid, and it was to one of these forms that Wehmer in 1918 applied the name $A$. fumaricus. At the present time, however, fumaric acid is produced in industry by fermentation with species of Rhizopus rather than strains of the black aspergilli (see p. 293). 
Gluconic Acid: Selected strains of A. niger are used industrially for the production of gluconic acid. This process, like the citric fermentation, has been investigated by many workers. Outstanding contributions have been made by Molliard, Bernhaner, and the U. S. Department of Agriculture group, including Herrick, May, Wells, Moyer, Gastrock, Porges, and others (see pp. 295-297).

Oxalic Acid: Under certain conditions some strains of $A$. niger produce appreciable quantities of oxalic acid. While it is usually avoided rather than encouraged, this fermentation has been investigated by Wehmer (1891 and 1892), Raistrick and Clark (1919), Jacquot (1938), and other's (see pp. 295-299).

The production of these various acids in quantities sufficient to have economic importance represents to an appreciable degree specific strain characteristics, and the greatest possible care must be exercised in maintaining these strains in a state of high productivity. It has been found, however, that in certain cases the same strains can be made to produce substantial yields of two or more of these acids by varying the composition of the nutrient solution and certain environmental factors. The reader is referred to the extensive literature on this subject.

Enzymes: While they are not commonly cultivated for the production of enzymes as such, as are members of the A. flavus-oryzae group, the black aspergilli produce a number of enzymes in appreciable quantities. Beginning with Fernbach in Germany (1890) and Bourquolet (1893) in France, various authors have devoted considerable study to their formation. A number of papers relative to enzyme production by members of the group are cited in the "Topical Bibliography" under the subtitle "Enzymes of Aspergillus niger" (pp. 294-295).

Fat Production: The mycelium of $A$. niger contains appreciable fatty materials (Pontillon, 1932; Bernhauer and Patzelt, 1935; Schmidt, 1935; and others). The waste mycelium from the citric acid fermentation is reported to provide a satisfactory source of sterols for irradiation in the production of Vitamin D.

Soil Testing: A. niger has been successfully employed as an assay organism for determining mineral deficiency of soils, particularly deficiencies in phosphorus and potassium. Papers on the so-called A. niger method of soil testing were first published by Kiessling and Schmidt and by Schlots, Smith, and Brown in the same year (1932), to be followed by more elaborate studies by Stock (1933), Niklas and associates (1933), and others. Additional references to this method are presented in the "Topical Bibliography" (pp. 313-314). The strain employed by Niklas is maintained in the culture collection of the Northern Regional Research Laboratory as No. 323. 
Hildew: Strains of $A$. niger represent a common cause of mildew on exposed wood surfaces and cotton fabrics. Partansky and McPherson (1940) used a strain of this species successfully for testing the moldresistant properties of oil paints. Aspergillus niger is commonly included in the mixtures of miscellaneous molds used for testing the effectiveness of mildew- and rot-proofing agents when impregnated in textiles and fabrics. Where pure cultures are employed, species of Chactomium and Metarrhizium are generally used.

\section{Mold Physiology}

Members of the Aspergillus niger group have been used extensively in investigations on mold physiology, probably more than any other form. As early as 1909 Latham studied nitrogen assimilation by $A$. niger ( $S$. nigra) to be followed in 1911 by Dox studying phosphorus assimilation by the same species. Beginning in 1918 and continuing up to the present time, Steinberg has published a succession of papers on the physiology of $A$. niger, with special reference to the rôle of heavy metals in its nutrition. A single strain of $A$. niger which is carried in the NRRL collection as No. 334 (Thom No. 4247) has been used throughout these investigations. Studies of a somewhat similar character have been conducted by Bortels (1927), Levy (1932), Gollmick (1936), and others. Citation of these papers, together with many additional references are presented in the Topical Bibliography under the subtitle "Physiology". An attempt has been made to present sufficient references to serve as a point of entrance to the literature of the field.

\section{Pathogenesis}

Members of the Aspergillus niger group are commonly isolated from the external ear of man, this being the source of the classic Sterigmatocystis antacustica of Cramer. Other species reported to have been isolated from cases of otomycosis include $A$. niger van Tieghem, $A$. phoenicis (Cda.) Thom and Church (with long primary sterigmata), A. giganteus (Mattlet) Dodge, and A. Macfici Dodge. A. Macfiei showed no signs of pathogenicity when tested on experimental animals. There is no morphology to distinguish either of the latter two forms from the ubiquitous saprophytic types, and it appears probable that many strains can become established in the auditory canal under certain favorable and probably temporary conditions.

Once entrenched in the flesh about the auditory canal, the mycelium has been found to be very persistent. One case is known in which occasional abscesses have occurred over a period of 25 years during which desultory treatment has quieted the inflammation but has not destroyed the parasite. 
In the same way, extensive development of these forms as points of infection in the lungs may give rise to conditions diagnosed as pseudotuberculosis which may persist for long periods without resulting either in death or complete recovery of the patient. Spores of $A$. niger are air borne and hence are commonly drawn into the respiratory tract. They are occasionally reported as causative agents in allergic reactions.

\section{Single Strain Cultures}

The great importance of some of the black aspergilli as fermentative agents makes punctilious preservation of the actual strain employed the only means either of insuring a process against breakdown or of introducing it in a new locality. Even when such a culture is maintained with the utmost care, natural variation sometimes occurs. Induced variation, such as that described by Steinberg and Thom in a series of papers (1939-40) presents a further hazard in processes where cultures are grown at extreme $\mathrm{H}$-ion concentrations, or in the presence of chemical or other substances which may actually be toxic at the concentrations employed. In a process in one laboratory a strain appeared that presented a colony aspect in which only a few typical black heads developed in a background of dwarfed and fractional conidial fruiting structures. In another case, a mutant appeared which differed so radically from $A$. niger in its secondary sterigmata and spore chains that the Yuills described it as a new genus and species, Cladosarum olivaceum (Trans. Brit. Myc. Soc. 22 : 194-200, Pls. 11-13. 1938).

The user of one of these Aspergilli, then, needs to know his organism in cultural aspect, in microscopic details, and in essential reactions in standardized and reproducible substrata. Furthermore, he must be able to maintain it free from contamination with other species, and likewise free from such variation, either natural or induced, as will interfere with consistent and controlled results. 


\section{Chapter XVili \\ THE ASPERGILLUS WENTII GROUP}

\section{Outstanding Characters}

Conidial heads large, typically globose, often splitting irregularly in age-varying in color from dull yellowish to ecru-olive, and from light to dark brown depending upon the species and strain.

Conidiophores smooth-walled or nearly so, but often appearing finely roughened when examined in dry mounts.

Vesicles globose, fertile over the entire surface. Sterigmata in two series.

Conidia commonly elliptical, smooth or somewhat roughened depending upon the species.

Sclerotia present or lacking, dark brown to black, characteristically white-tipped when young.

The Aspergillus wentii group as presented here is recognized as somewhat artificial, in comparison with such strictly natural groupings as the $A$. glaucus, $A$. nidulans, and $A$. clavatus groups. The degree of relationship between the species included is open to question. Yet all of the forms possess certain characteristics in common: (1) conidiophores are smoothwalled, or nearly so; (2) conidial heads are large and strictly globose, at least when young; and (3) all appear to occupy taxonomic positions somewhat intermediate between the Aspergillus niger group on the one hand and the Aspergillus flavus or Aspergillus ochraceus groups on the other.

\section{Group Key}

I. Conidia smooth-walled.

A. Sclerotia lacking; vegetative mycelium and young conidiophores reddish in color.

1. Conidial heads light brown, near wood brown (Ridgway Pl. XL)

A. panamensis Raper and Thom

B. Sclerotia present, dark brown to black, vegetative mycelium colorless, and young conidiophores colorless or pale yellow.

1. Conidial heads dull yellow to ochraceous; sclerotia globose or nearly so A. alliaceus Thom and Church

2. Conidial heads in yellow-green shades near ecru-olive (Ridgway Pl.

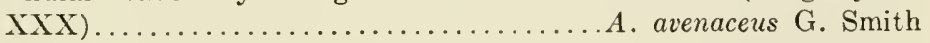

II. Conidia more or less echinulate.

A. Sclerotia present or lacking, depending upon the strain and the substratum; conidial areas in orange-brown to brown colors.

1. Conidiophores colorless; colonies often conspicuously floccose

A. wentii Wehmer

2. Conidiophores brown (See A. niger)........A. hennebergi Blochwitz 
Aspergillus panamensis Raper and Thom, in Mycologia 36: 568-572, fig. 5. 1944.

Colonies on Czapek's solution agar at room temperature very thin, consisting of a sparise and transparent growth of vegetative hyphae, almost wholly submerged, bearing widely scattered, erect conidial structures with radiate heads, light brown in color. Colonies upon malt extract agar at room temperature growing well and fruiting luxuriantly, reddish brown in color, consisting of a dense basal nycelium, predominantly red, from which develop massed condial structures in broken or continuous concentric zones (fig. $65 \mathrm{~A}$ ), many conidiophores abortive and sterile, fertile conidiophores bearing globose to radiate heads, light brown in color, near wood brown (Ridgway, Pl. XL); these, together with red-colored sterile structures and aerial hyphae, give the colony its characteristic appearance and color; in age, colonies tending to develop a loose floceose overgrowth, more or less obscuring the abundant conidial heads; reverse dull brown; odor none.

Conidial structures arising directly from the substratum, scattered or abundant, depending upon the culture medium employed (fig. $65 \mathrm{~B}$ ). Heads typically globose, in age characterized by loosely radiating chains of conidia, less commonly by few to several roughly columnar masses variable in size, commonly ranging from 250 to $450 \mu$ in diameter, occasionally up to $500 \mu$, varying in color from avellaneous to wood brown (Ridgway, Pl. XL) to Saceardo's umber (Ridgway, Pl. XXIX). Conidiophores straight, mostly 600 to $900 \mu$ in length by 9 to $12 \mu$ in diameter, occasionally larger, with walls smooth, comparatively heavy, ranging from 3 to $3.5 \mu$ thick in the basal area to 1.5 to $2 \mu$ in the terminal area, approximately uniform in diameter throughout except for a limited reduction immediately beneath the vesicle. Vesicle colorless, comparatively thin-walled, globose or slightly elongate, mostly 25 to $30 \mu$ in diameter, fertile over the entire area (fig. $65 \mathrm{C}$ ). Sterigmata in two series, closely packed, primaries 5.5. to $6.5 \mu$ by 2.4 to $2.8 \mu$, secondaries 5 to $6 \mu$ by 1.5 to $2 \mu$. Conidia light yellowishbrown in mass, globose to subglobose, smooth-ivalled, mostly 2.2 to $2.6 \mu$ in diameter, oecasionally $2.8 \mu$.

Type culture NRRL No. 1785 was isolated in January 1942, from Panama soil eollected by Mr. John T. Bonner. A second culture, NRRL No. 1786, differs from the above strain in minor details but clearly belongs with it. This was isolated from a second sample of Panama soil collected by Bonner.

The species is considered to represent a form somewhat intermediate between the Aspergillus niger group and A. wentii. Superfieially, at least, there is evidence of relationship with Aspergillus niger mut. cinnamomeus (syn. Aspcrgillus cinnamomcus Schiemann) and Aspcrgillus niger mut. Schicmanni (syn. A. fuscus Schiemann). It bears a certain 

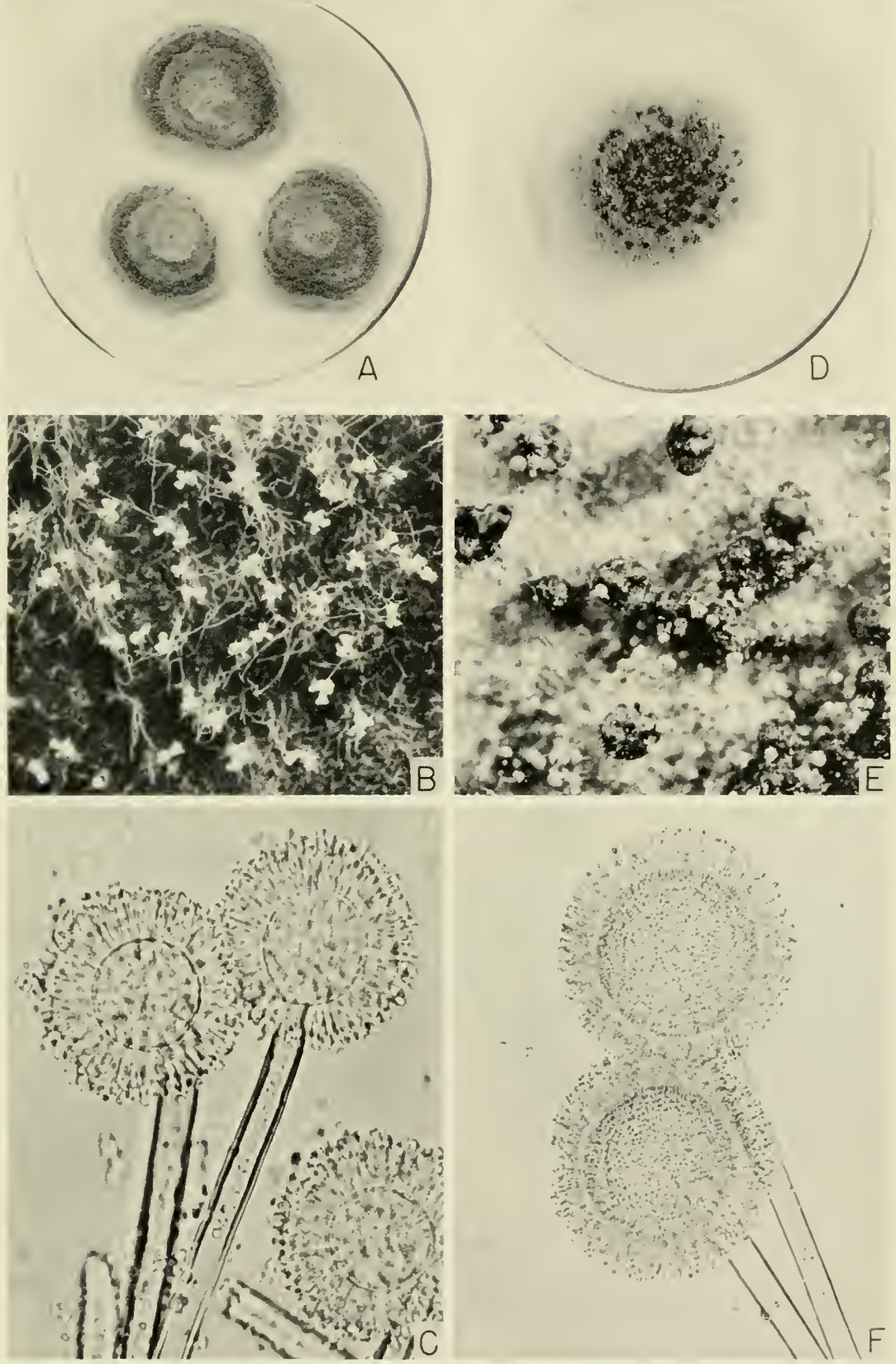

FIG. 65. A-C, Aspergillus panamensis NRRL No. 1785: A, Colonies upon malt extract agar, 10 days; $B$, Conidial heads, showing tendency to split into loose divergent columns, $\times 9 ; C$, Conidial heads showing globose vesicles and sterigmata in two series, $\times 500$. $D-F$, Aspergillus avenaceus NRRL No. 517: $D$, Single colony on Czapek's solution agar showing numerous large sclerotia; $E$, Portion of above colony showing greater detail of heads and sclerotia, $\times 6 ; F$, conidial heads showing large globose vesicles, $\times 160$. 
resemblance to these forms in the comparatively light color of its conidial heads and in the smallness and general character of its conidia, but differs from these forms in three very striking particulars. (1) It characteristically develops an extensive red-colored aerial mycelium upon media such as malt-extract agar where it attains its maximum growth; (2) it grows rery sparsely upon Czapek's solution agar, upon which the above noted forms grow luxuriantly; and (3) it possesses very small primary sterigmata, measuring 5.5 to $6.5 \mu$ by 2.4 to $2.8 \mu$ in contrast to 13 to $15 \mu$ by 3 to $5 \mu$ for mut. cinnamomeus and 15 to $40 \mu$ by $4.6 \mu$ for mut. Schiemanni. Whether or not the species actually represents a naturally occuring mutation from A. niger can only be guessed. The smallness of its conidia and primary sterigmata would hardly support this hypothesis. In cases where mutations have been obtained from known cultures, the dimension of specific structures in such mutations generally agree very closely with those of the same structures in the parent strain; and black Aspergilli with the dimensions of $A$. panamensis are rarely, if ever, encountered in nature. The possibility of this representing a mutation is not excluded, but until additional evidence supporting such origin is fortheoming, the writers feel warranted in maintaining as a distinct species this unique form which obviously is able to maintain itself in the soils of Panama.

The correct taxonomic position of this species remains in doubt. It is included with Aspergillus wentii, although we realize that this placement is not entirely satisfactory. As continued isolations are made from tropical soils and other sources it is our hope that additional forms may be found, which will furnish evidence of a more exact relationship.

The very sparse development of $A$. panamensis upon Czapek's solution agar, containing sucrose, results from an invertase deficiency; when dextrose is substituted as a carbon source the fungus grows luxuriantly and fruits abundantly.

Aspergillus alliaceus Thom and Church, in The Aspergilli, p. 163. 1926.

Discussed without name as Thom No. 4660 by Walker and Lindegren, in Jour. Agr. Res. 29: 507-514. 1924; and by Walker, Lindegren, and Bachmann, in Jour. Agr. Res. 30: 175-187. 1925.

Colonies on Czapek's solution agar rapidly and broadly spreading, with loosely floccose aerial sterile mycelium, bearing scattered ochraceous heads among abundant dark to almost black sclerotia (Pl. VI D and fig. 66 A) in some strains, predominantly floccose with limited conidial heads and few sclerotia in others (fig. $66 \mathrm{~B}$ ); reverse uncolored. Conidial heads dull yellow to ochraceous, strictly globose when young and remaining radiate or splitting irregularly in age (fig. $66 \mathrm{D}$ ), up to $300 \mu$ in diameter, often more abundant in cultures after many transfers. Conidiophores up to $150 \mu$ 
long by $15 \mu$, with walls up to $1.5 \mu$ in thickness, appearing smooth in liquid mounts, but showing rudimentary markings or pits when examined dry. Vesicles globose to subglobose, up to 40 to $50 \mu$ in diameter, with walls about $2 \mu$ in thickness, and showing pores where sterigmata are attached. Sterigmata in two series, primary 7 to $9 \mu$ or even $12 \mu$ by 3 to $4 \mu$, secondary
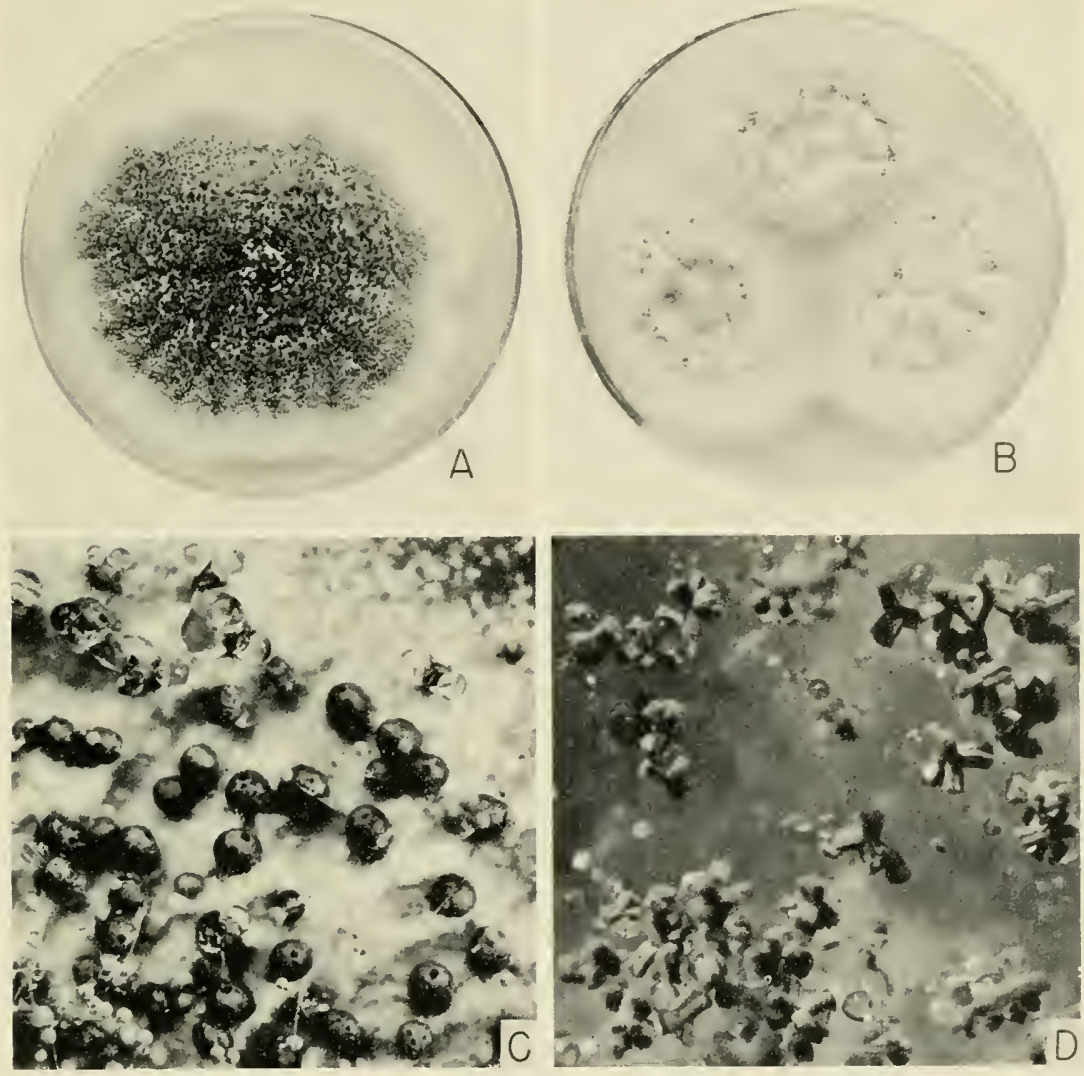

FIG. 66. Aspergillus alliaceus. A, Heavy sclerotium-producing strain, NRRL No. 315, on Czapek's solution agar, 10 days. B, Light sclerotium-producing strain, NRRL No. 318, growing under similar condition. $C$. Portion of $A$ showing greater detail of sclerotia and conidial heads, $\times 9 . \quad D$, Conidial heads from old culture (strain NRRL No. 318) showing tendency to split into divergent columns, $\times 18$.

7 to $8 \mu$ by $2 \mu$. Conidia elliptical to globose, yellowish, 2.5 or $3 \mu$ in diameter. Perithecia not found. Sclerotia often very abundant (fig. $66 \mathrm{C}$ ) at first white but quickly becoming black or nearly so, in many strains dominating the character of the colony.

Strains compared include NRRL No. 315 (Thom No. 4656) from a dead 
blister beetle (Macrobasis albida), NPRL No. 316 (Thom No. 4660) isolated from onion bulbs, and others with characteristic heads and sclerotia have been obtained from garlic bulbs, from cactus plants, and from soils, particularly from the general region of Texas, Arizona, and Mexico. Strains have also been isolated from soils collected near Calcutta.

The large, globose, pale yellow to ochraceous heads of the species strongly suggest relationship to the $A$. ochraceus group. The smooth-walled conidiophores and black sclerotia, however, more closely ally it with Aspergillus wentii and the other species grouped with it. It may, however represent a form somewhat transitional between the great groups represented by $A$. niger on the one hand and $A$. ochraceus on the other.

Aspergillus avenaceus Geo. Smith, in Brit. Mycol. Soc. Trans. 25: 24-27, Pl. 1, figs. 1-3. 1943.

Colonies on Czapek's solution agar (with sucrose) at room temperature spreading rapidly, more or less conspicuously zonate (fig. $65 \mathrm{D}$ ), slightly floccose, white at first, then dull yellow to ecru-olive (Ridgway, Pl. XXX) as shown in Pl. VI E, with, at times, a greenish tinge without becoming truly green; reverse pate dirty pink. Conidial heads large, globose 400 to $600 \mu$ in diameter, or up to $1,000 \mu$, splitting into columnar masses of conidial chains. Conidiophores up to $5 \mathrm{~mm}$. long, 18 to $30 \mu$ in diameter, with walls 2.5 to $4 \mu$ thick, smooth in fluid mounts; but appearing finely roughened when examined dry. Vesicles globose or slightly flattened, thick-walled, up to $185 \mu$ in long axis, sterigmata in two series, primary 22 to $50 \mu$ by $6 \mu$, secondary 11 to $13 \mu$ by $4 \mu$ (fig. $65 \mathrm{~F}$ ). Conidia ellipsoid, smooth, 4 to 6 or $6.5 \mu$ by 3.2 to $4 \mu$. Sclerotia dark grayish-brown to black (fig. $65 \mathrm{E}$ ), elongate, irregularly flask-shaped, sometimes with the "neck" forked, apical portion white to gray during development, 2 to $3 \mathrm{~mm}$. in long axis, scattered in concentric zones after 7 to 10 days.

On Czapek agar with glucose, sclerotia are more abundant and larger. On wort, or potato agar, conidial heads are abundantly produced but sclerotia are delayed for several weeks and are few in number.

Species characterization adapted from George Smith's description.

This very distinctive species (NRRL 517: Thom 5725) is represented by a single isolation from seed peas made in 1938 by Dr. G. E. Turfitt of the London School of Hygiene and Tropical Medicine, University of London.

Aspergillus wentii Wehmer, in Centralbl. f. Bakt. etc., 2 Abt., 2, p. 150. 1896. See also The Aspergilli, Thom and Church, p. 183. 1926.

Colonies on Czapek's solution agar, rapidly growing and broadly spreading, floccose with white or yellowish aerial hyphae which in some strains pile up in the plate (PI. VI F, and fig. $67 \mathrm{~A}$ ) or fill the test tubes for several 
centimeters (fig. $67 \mathrm{C}$ ), but remain inconspicuous in other strains (fig. $67 \mathrm{~B}$ ); with developing heads at first white through yellow shades to olive-brown, medal bronze or snuff brown (Ridgway, Pls. IV, XVI, XXX, column 19, and XXIX, column 15 K.), or, according to Wehmer, coffee brown to chocolate brown; reverse becoming reddish-brown in old cultures. Conidial heads large, globose, generally remaining radiate in age (fig. $67 \mathrm{D}$ ), ranging up to $500 \mu$ in diameter, changing from yellow shades to brown. Conidiophores up to several millimeters in height by 10 to $25 \mu$ in diameter, with walls colorless up to $4 \mu$ in thickness, studded with droplets in growing colonies and often appearing slightly roughened when examined dry, but uniformly smooth in fluid mounts. Vesicles globose or nearly so (fig. $67 \mathrm{E}$ ), varying up to $80 \mu$ in diameter, fertile over the entire surface. Sterigmata usually in two series, primaries 10 to $20 \mu$ by 3 to $5 \mu$, occasionally much larger, secondaries 6 to $8 \mu$ by $3 \mu$. Conidia borne in long chains, more or less elliptical, ranging from 3.5 to $6 \mu$ in long axis, but mostly 4 to $5 \mu$, double wall clearly evident, ranging from almost smooth to marked by ridges sometimes suggestive of $A$. niger, again more closely resembling the $A$. flavus series. No perithecia reported. Sclerotia of ten encountered (fig. $67 \mathrm{~F}$ ), dark brown to black, ovate with long axis vertical.

Culture description based upon strain NRRL No. 375 (Thom No. 116) obtained in 1909 from the Centraalbureau as Wehmer's original organism, as well as numerous isolations from soils and other materials collected by the authors in the United States, and other strains contributed by investigators from all over the world.

Numerous strains with the general aspect of Wehmer's species have been seen from Java, China, South America, Japan, the Straits Settlements, British Guiana, and Brazil. In our experience it has been isolated from cottonseed cake, from olives, from soil, and from numerous other sources. It is to be regarded as very widely distributed and to be common on many types of decaying vegetable products.

The variations in colony aspect in different strains run from an extreme of mycelial growth filling the test tube that is characteristic of cultures such as the Wehmer organism, to colonies forming a crowded surface growth of conidiophores only and distinguishable from $A$. tamarii only by a lack of greenish color in the early fruiting period and in the characteristic smooth conidiophores and finely roughened conidia.

Aspergillus archaeoflavus Blochwitz (Ann. Mycol. 31(1/2): 73-83. 1933) represents a non-floccose form which is hardly separable from $A$. wentii. In our examination of the type strain (NRRL No. 382: Thom No. 5346), received in 1933 from Baarn, measurements were somewhat less than those cited by the author. The absence of conidial markings, to which Blochwitz called attention, would not bar it from $A$. wentii since in some strains conidia are almost entirely smooth, in others finely roughened, while in still others they are conspicuously echinulate. 

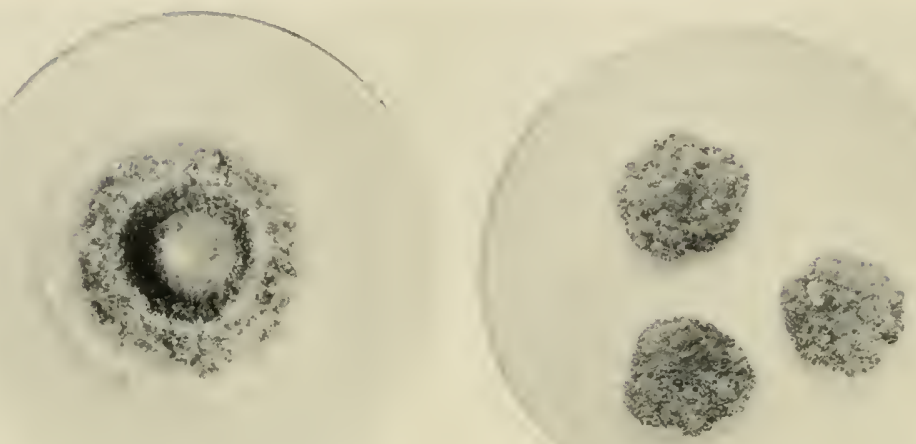

A
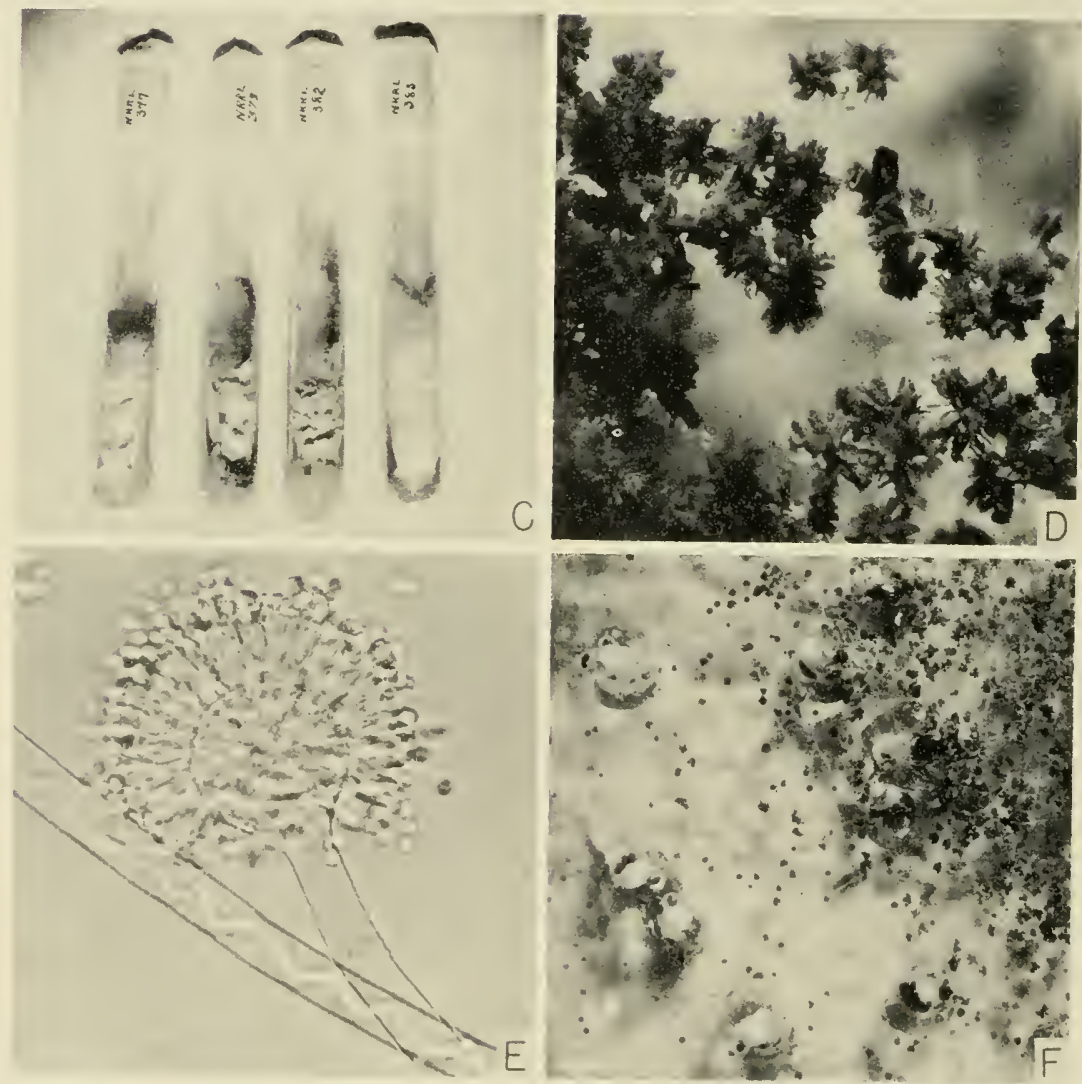

FIg. 67. Aspergillus wentii. $A$ and $B$, colonies of strains NRRL, No. 375 and No. 385 , respectively, upon Czapek's solution agar, 10 days. $C$, Tube cultures of four representative strains. $D$, Conidial heads from old culture, $\times 18 . E$, Single head showing smooth conidiophore, globose vesicle, and sterigmata in two series, $\times 500$. $F$, Detail of colony margin in strain NRRL No. 379 showing sclerotia and abundant conidial heads, $\times 9$. 


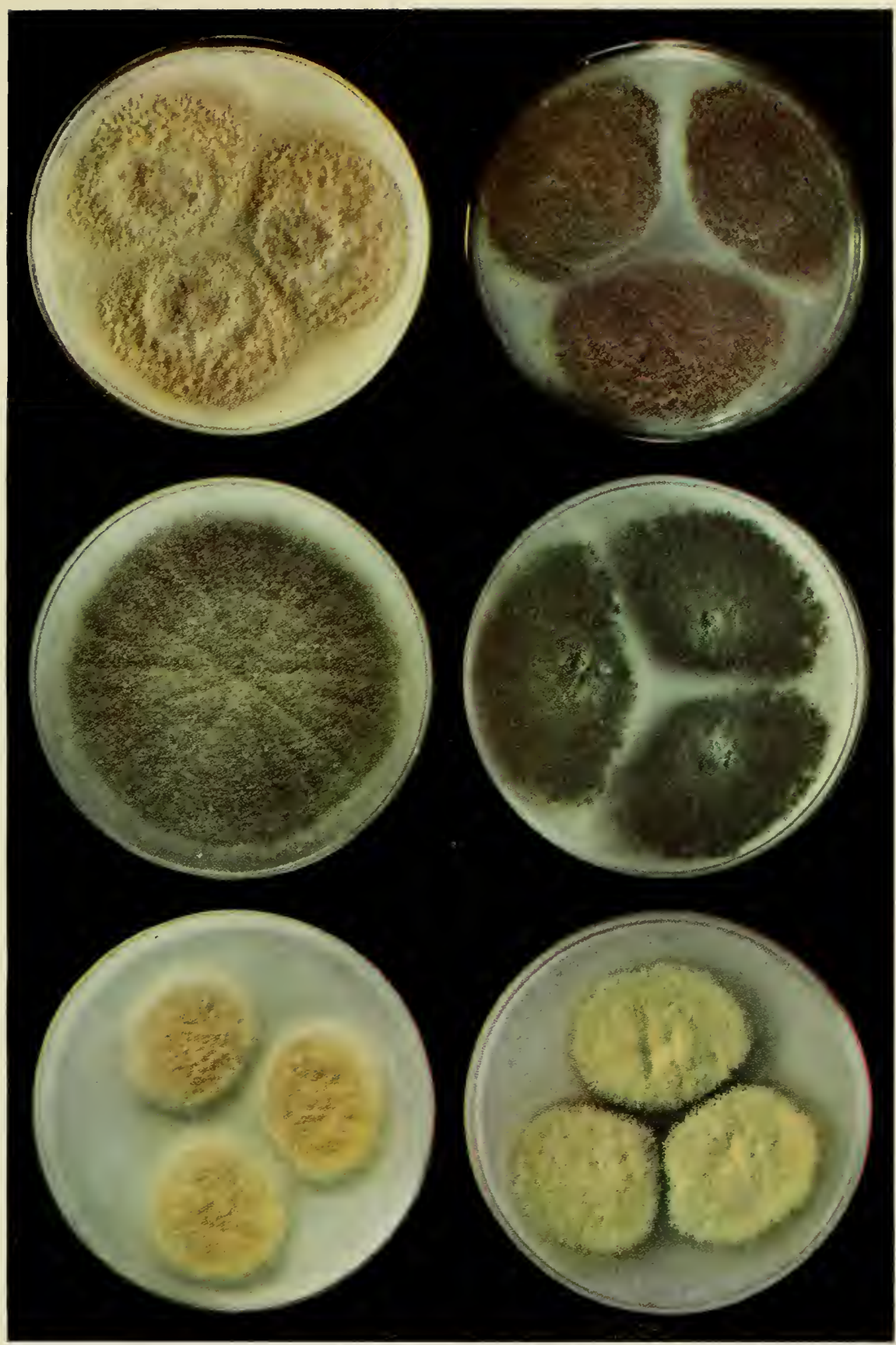

Plate VII

A (upper left), Aspergillus terricola var americana Marchal, NR RL No. 424. B (upper right), Aspergillus tamarii Kita, NRRL No. 427. C (center left), Aspergillus oryzae (Ahb.) Colin, NRRL No. 458 . D (center right), Aspergillus flavus Link, NRRL No. 1957. E (lower left), Aspergillus quercinus (Bain.) Thom and Church, NRRL No. 394. F (lower right), Aspergillus ochraceus Wilhelm, NRRL No. 395 . All cultures growing upon Czapek's solution agar. (Color photographs by Haines, Northern Regional Research Laboratory: Reproduced through co-operation of Chas. Pfizer \& Co., Inc.) 

Aspergillus wentii var. minimus Nakazawa, Takeda, Okada, and Simo (Jour. Agr. Chem. Soc. Japan $10(2): 176-177$. 1934) shows measurements varying somewhat from those of the species and from those given by Blochwitz, but it is not sufficiently marked to warrant separation.

Sterigmatocystis aerea Bainjer (Bull. Soc. Bot. France 27:2S. 1SS1) may have been a member of this series but was not sufficiently described to permit positive identifieation.

Aspergillus hennebergi Blochwitz, in Ann. Mycol. 33: 23S-239. 1935. The species is deseribed as having the aspect and colors of a non-floccose A. wentii or an A. tamarii with red sclerotia but with conidiophores browned as in the partially browned conidiophores of the A. niger group.

An albino "variant" of A. wentii was isolated by Mosseray (Ann. Soc. Sci. Brux. 54:161-189. 1934) from a normal culture of this species, and was found to retain its distinctive characters through repeated transfers in laboratory culture. No name was given to this mutant.

\section{Occurrence and Economic Importance}

Aspergillus wentii is a cosmopolitan species that is fairly common in soils, upon moist grains and other vegetable matter undergoing slow decomposition, and may be isolated less frequently from a wide variety of other materials collected from nature. It is apparently world-wide in distribution. In the Orient, it is often included with A spergillus tamarii, A. flavus, and A. oryzae, all under the latter name as a rule, in the "Koji" preparations used in the manufacture of various soy products. Likewise, it has been investigated with these same species in connection with the production of various mold enzymes. At the same time, it has been included with the black Aspergilli in studies on the production of organic acids by molds. Recently Karow (1942) has reported one strain of this species to give substantial yields of citric acid in submerged culture. Yabuta (1912) reports Koji acid production by A. wentii. On the whole, strains of the species appear to be somewhat less active biochemically than either the black Aspergilli or members of the A. flarus-oryzae group. It is, nevertheless, a vigorously growing species with definite biochemical possibilities and should not be overlooked in any program relating to mold fermentation.

Aspergillus alliaceus appears periodically upon alliaceous bulbs and occasionally upon cacti as at least a secondary parasite. It is not infrequently isolated from soils, and appears to be fairly common in the southwestern states of Texas, New Mexico, and Arizona. It has also been isolated from soils of other areas including Southern Mexico and India. Nothing is known regarding its biochemical possibilities.

Only the type strains of $A$. avenaceus and $A$. panamensis are known and neither has been shown to have any economic importance. 


\section{Chapter XIX \\ THE ASPERGILLUS TAMARII GROEP}

\section{Outstanding Characters}

Conidial heads radiate, generally loose-textured, hemispherical to globose, yellow-brown to olive-brown in color with green shades entirely lacking or only transiently produced in early stages.

Conidiophores colorless, typically roughened throughout a part or all of their length.

Vesicles subglobose to globose, fertile over the upper half to the entire surface.

Sterigmata in one or two series depending upon the species and strain, often showing both conditions within the same head.

Conidia heavy-walled, rough, elliptical, pyriform or subglobose, depending upon the species.

Sclerotia commonly present, purple to reddish-purple or black, whitetipped when young.

The Aspergillus tamarii group represents a collection of more or less closely related forms that are believed to be intermediate between the $A$. wentii and $A$. flavus-oryzae groups. Relationship to the latter group is unquestionable since there are intergrading forms which almost completely bridge the gap from one group to the other. The group embraces two principal series: one, typified by A. terricola, is characterized by dull yellow-brown conidial heads which never show any trace of green; the other, typified by A. tamarii, possesses dark brown conidial heads which commonly show transient shades of olive-green during the period of rapid growth.

\section{Group Key}

I. Conidia strongly elliptical, lemon-shaped. A. citrisporus von Höhnel

II. Conidia not strongly elliptical.

A. Conidial heads light yellow-brown when mature, showing no green color at any stage.

1. Colonies predominantly floccose, conidia with prominent projecting tubercles........................... lutescens Bainier

2. Colonies not predominantly floceose.

a. Heads large, conidia coarsely roughened with flattened bars and tubereles.......................... terricola Marchal

b. Heads small, conidia finely roughed

A. terricola var. americana Marchal 
B. Conidial heads dull dark brown when mature.

1. Green shades commonly evident, but confined to early stages

A. tamarii Kita

2. Green color persisting for several days, but eventually disappearing

Bronze series

Aspergillus citrisporus von Höhnel, in Sitzungsber. K. Akad. Wiss. Wien, Math. -Naturw. Kl. III, I Abt., p. 987, 1902. See The Aspergilli, Thom and Church, pp. 191-2. 1926.

Colonies on Czapek's solution agar, at room temperature, spreading fairly rapidly as submerged mycelium, producing a sparse aerial growth of conidiophores only; conidial areas at first yellow then gold and finally orange-brown (fulvus of Saccardo's Chromoiaxia, approximately Mikado brown of Ridgway); reverse colorless. Conidial heads up to $500 \mu$ in diameter, globose, radiate. Conidiophores 1 to $2 \mathrm{~mm}$. long by 20 to $25 \mu$ in diameter, with walls thin, about $1 \mu$ in thickness, turgid and studded with granules when examined dry, finely roughened, of ten collapsing in age. Vesicles 30 to $50 \mu$ in diameter, nearly globose, fertile over the whole surface. Sterigmata in one series, 8 to $12 \mu$ by 3 to $4 \mu$, producing loosely radiating chains of conidia, at first yellow or golden then brown. Conidia lemonshaped, 5 to $9 \mu$ by 5 to $6 \mu$, rough from irregularly branching ridges of coloring substance between the inner and outer walls. Sclerotia or perithecia verbally reported by Thaxter but not seen by us.

Diagnosis based on Thaxter's isolate (Thom No. 4181.10). Thaxter gave no description. He obtained it several times from caterpillar dung. It grows and fruits more abundantly on Sabouraud's agar but it is difficult to keep viable in stock cultures. Additional collections include strains from caterpillar dung in Ann Arbor, Michigan, and Hanover, New Hampshire.

Aspergillus lutescens Bainier nomen nudum; described by Thom and Church, in The Aspergilli, p. 193. 1926.

Colonies upon Czapek's solution agar rapidly growing and broadly spreading, floccose-woolly, at first white, becoming rusty-yellow as conidial formation begins and develops unevenly over the surface (fig. $68 \mathrm{~A}$ ), finally becoming chestnut-brown when conidial areas are mature; reverse of colony pale yellow. Conidial heads radiate, hemispherical to subglobose, approximately buckthom brown to Dresden brown (Ridgway, Pl. XV), comparatively small, ranging from 100 to $300 \mu$ in diameter. Conidiophores 12 to $15 \mu$ in diameter, varying greatly in length, mostly short, arising from the substratum, or as branches of aerial hyphae with walls pale yellowish and with pitting present but not conspicuous, not giving a rough appearance. Vesicles globose to subglobose (fig. $68 \mathrm{~B}$ ), 20 to $40 \mu$ in diameter. Sterigmata 

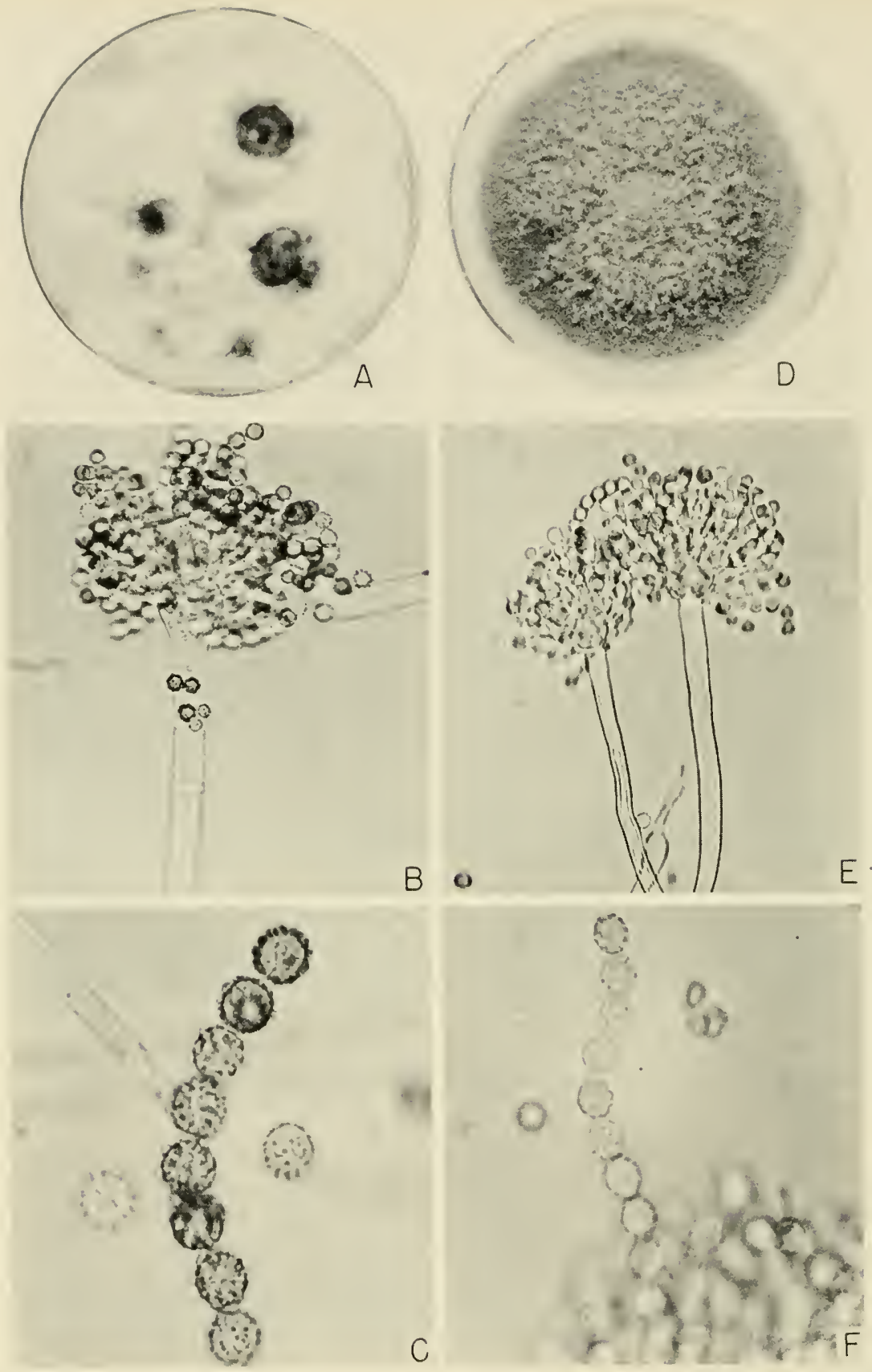

Frg. 68. A-C, Aspergillus lutescens NRRL No. 426: A, Colonies on Czapek's solution agar showing extreme floccose habit and light sporulation, 10 days; $B$, Single conidial head, $\times 500 ; C$, Conidia, $\times 1200$. D $-F$, A spergillus terricola NRRI 424: D. Single colony on Czapek's solution agar showing somewhat floccose but heavily sporing colony, 10 days; $E$, Conidial heads showing sterigmata in a single series, $\times 500 ; F$, Conidia, $\times 1200$ 
in one series in smaller and crowded heads, up to 15 to $20 \mu$ by 4.0 to $5.0 \mu$; sterigmata often in two series in larger heads with primaries about 15 to $18 \mu$ by 4 to $5 \mu$, secondaries 12 to $14 \mu$ by + to $5 \mu$. Conidia subglobose, varying from 5 by $7 \mu$ to $\delta$ by $9 \mu$, conspicuously roughened with prominent tubercles of color (fig $68 \mathrm{C}^{\circ}$ ).

The species is known only in the type culture from the Bainier collection, NRRL No. 425 (Thom No. 4640.478), and as a second strain, NRRL No. 426 , isolated in the Soil Microbiology Laboratory, Bureau of Plant Industry, Washington, D. C., about 1939.

Aspergillus terricola Marchal, in Rev. Mycologique 15, No. 59: 101-103. 1893.

Colonies umbrinus; mycelial hyphae 3 to $5 \mu$ in diameter, without anastomoses; conidiophores hyaline, continuous or septate in age, 600 to $1,000 \mu$ by 7 to $10 \mu$ (whole depth of colony growth); vesicles subglobose, hyaline, 39 to $50 \mu$, radiately covered with sterigmata; sterigmata in one series, 12 to $15 \mu$ by 4 to $7 \mu$; conidia umber (Sacc.), ovate or elliptical then globose, rough, with colorless connectives.

Description, from Marchal, of a culture isolated from soil in Belgium; not reported elsewhere, see variety below.

Aspergillus terricola var. americana Marchal, cultural description by Thom and Church, in Am. Jour. Bot. 8: 125. 1921.

Colonies on Czapek's solution agar growing rapidly at room temperature, often somewhat floccose in central colony areas (fig. $68 \mathrm{D}$ ), ranging from shades near yellow ochre (Ridgway, Pl. XV) when young, to Dresden brown or mummy brown in age, near Saccardo's umbrinus (Pl. VII A); aerial growth largely consisting of crowded conidiophores; reverse uncolored. Conidial heads radiate, hemispherical to subglobose, loose in texture, consisting of comparatively few divergent chains of conidia, up to $200 \mu$ in diameter. Conidiophores 300 to $600 \mu$ in length by 6 to $8 \mu$ in diameter, with walls pitted. Vesicles globose to subglobose (fig. $68 \mathrm{E}$ ), up to $25 \mu$ in diameter, fertile over the upper two-thirds or three-fourths. Sterigmata in one series, 7 to $10 \mu$ by 2 to $4 \mu$. Conidia tuberculate (fig. $68 \mathrm{~F}$ ) from the presence of color bars variously distributed between the outer and inner wall, orate to nearly globose, from 3 by $5 \mu$ up to 5 by $7 \mu$, usually about $5.5 \mu$, occasionally 5 to $8 \mu$ in diameter.

Type culture NRRL No. 424 (Thom No. 4838) isolated by F. M. Scales from redland soil in Georgia and discussed by Scales, in Jour. Biol. Chem. 19: $459-472,1914$, under the name A. terricola Marchal. Scales' culture was submitted to Marchal, who designated the form as A. terricola var. americana Marchal, distinguished as follows: "The dimensions of the 
vesicles 14 to $20 \mu$ instead of 30 to $50 \mu$; of the sterigmata 5.6 to $10.5 \mu$ by $2.2 \mu$ instead of 12 to $15 \mu$ by 4 to $7 \mu$; the spores only very delicately verrucose, separate your fungus from A. terricola."
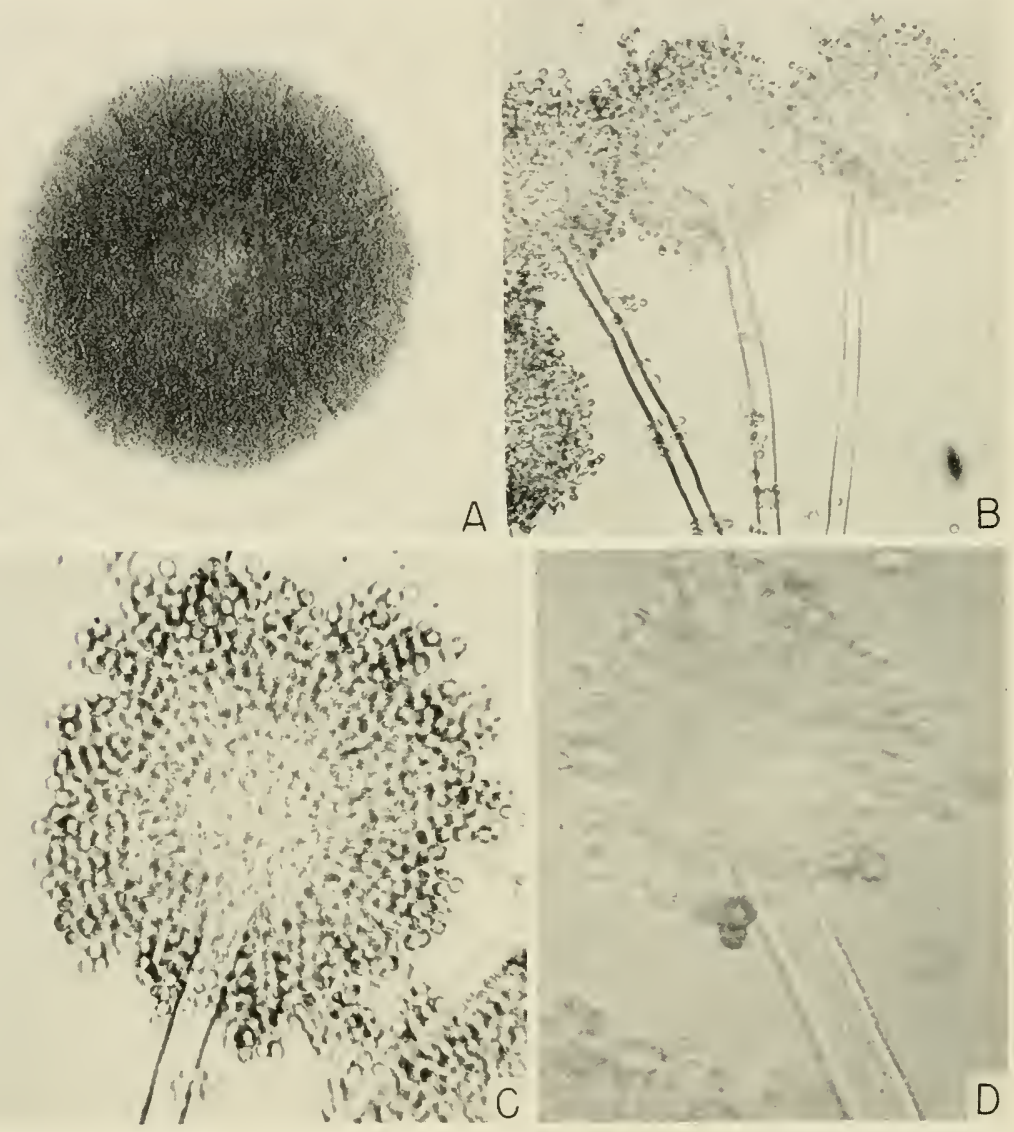

FIg. 69. Aspergillus tamarii. A, Colony growing on Czapek's solution agar characterized by heavy conidial production, strain NRRL No. 427, 10 days. $B$, Conidial heads, $\times 160$. C. Single head, $\times 300$. D, Single head further enlarged showing rough walls of conidiophore, and sterigmata in two series, $\times 500$.

Aspergillus tamarii Kiita, in Centralb. f. Bakt. etc. 2 Abt., 37, No. 17/21, pp. 433-452. 1913. Characterization of A. tamarii Kita given by Thom and Church in Am. Jour. Bot. 8: 118. 1921. See also Thom and Church, The Aspergilli, p. 194. 1926.

Colonies on Czapek's solution agar spreading broadly at room temperature (fig. $69 \mathrm{~A}$ ), with regetative hyphae mostly sulmerged, fruiting areas 
at first colorless, then passing through orange-yellow shades to brown in old colonies, variously Isabella color, light brownish-olive, buffy-citrine, medal bronze, or raw umber (Pl. VII B) (Ridgway, Pls. XXX, XVI, and IV, Column 19, and Pl. III, Column 17); not showing true green, but of ten presenting a suggestion of green that is transient and limited to areas of young heads; reverse uncolored or occasionally pinkish. Conidial heads varying greatly in size in the same fruiting area, from more or less columnar to nearly, but not completely, globose and up to $300 \mu$ in diameter, with radiating chains and columns of conidia. Conidiophores arising from submerged hyphae, up to 1 to $2 \mathrm{~mm}$. in length, colorless, with walls becoming abruptly thinner at the base of the resicle, frequently showing irregular thickenings within, as a rule markedly rough or pitted throughout part or all of their length (fig. $69 \mathrm{D}$ ), sometimes appearing smooth or nearly so when examined in liquid mounts, but consistently rough or pitted when examined dry. Vesicles globose to subglobose, 25 to $50 \mu$ in diameter (fig. $69 \mathrm{C}$ ), with fairly thin walls which frequently crush in mounts, fertile over almost the entire surface. Sterigmata, in one series in small heads, in two series in large heads; primary sterigmata commonly 7 to $10 \mu$ by 3 to $4 \mu$, becoming 20 to $35 \mu$ long in gigantic heads, secondary sterigmata 7 to $10 \mu$ by $3 \mu$. Conidia ranging from more or less pyriform, through subglobose to globose, conspicuously roughened from prominent tubercles and bars of orange-yellow coloring matter deposited between the loose outer wall and the firm inner wall, commonly ranging from 5.0 to $6.5 \mu$ in diameter, occasionally up to $8 \mu$. Sclerotia produced by many strains, usually purplish or reddish-purple, globose to pyriform with apex white.

Species characterization is based upon Thom and Church's culture No. 4235.12 (NRRL No. 429) which was submitted to and identified by Kita as A.tamarii (see Thom and Church, Am. Jour. Bot. 8: 118. 1921).

The organism described by hita proved to be one of a great series represented in our collection. It is common among cultures examined from North and South America, from Japan, China, India, and from Europe. The species has been found to be quite common in soil collected from many areas in the United States.

The outstanding characters are orange-yellow to brown colonies; coarse, colorless conidiophores, usually roughened but with this character sometimes obscure; large, radiate, loose-textured, conidial heads; sterigmata in one or two series, commonly with single and double sterigmata in the same head; conidia more or less pyriform 5 to $8 \mu$ in long axis with tubercles or bars of orange-yellow coloring matter between the inner and the outer cell walls.

In preparing the manuscript for "The Aspergilli" (1926), Thom and Church introduced into their general key, without name, on page 248 under No. 279, a series of forms with morphology and general appearance 
bridging the gap between $A$. tamarii Kita and the A. flavus group. Without publication they referred to these as "The Bronze Series." These strains have the yellow-green color of $A$. flavus during the early stages of their development, but subsequently develop the yellow to brown colors of $A$. tamarii. Recognition of this border group is necessary since some strains of A. tamarii do not assume a definitely green color at any state in their development, while others show green as a transient character. Strains of $A$. flavus, on the other hand, are typically characterized by the green to yellow-green colors. Furthermore, the conidia of A.tamarii are typically quite roughened, showing prominent tubercles or bars of coloring matter deposited between the outer and inner walls. In contrast, the conidia of A. flavus are less coarsely roughened and show more numerous and smaller tubercles or echinulations, as well as a greater tendency for the coloring substance to be generally diffused throughout the spore envelope. The forms under consideration show, in some degree, the coloration and spore characters of both groups and are believed to be truly intermediate between $A$. tamarii and A. flavus.

The fact that we received from Baarn in 1933, as Blochwitz's A. luteovirescens Bloch. (Ann. Mycol. 31: 73-83. 1933) a culture (Thom No. 5345 ) which represented satisfactorily this intermediate series is not accepted as justifying the assignment of this name to the series, since the morphological characters displayed by the strain were so completely at variance with the original description, and since Blochwitz considered his species to be close to $A$. ustus. We question whether any sharp line of separation can be drawn between the two series because of the repeated appearance of intermediate forms. While we do not feel justified in assigning to these forms any specific designation, we do feel obligated to continue to call attention to their existence. We have at times considered the desirability of moving the whole $A$. tamarii complex over into the $A$. flavus-oryzae group, but this course has been abandoned since it was felt that to do so would introduce into an otherwise perfectly integrated group, a series of organisms whose relationship to them, while strongly suggested, is not proved, and which in its typical form would introduce discordant features.

The species listed below are believed to represent probable synonyms:

Biourge attached the manuscript name $A$. vulpinus to a member of the A.tamarii series (Thom No. 4733.146) and contributed it to our collection, but it does not seem sufficiently different from the species to warrant separation.

One strain of A. tamarii was found in the Bainier collection (Thom No. 4640.397) as A. cacao, nomen nudum; another under the same name came from Pribram.

A. gigas Spegazzini, Myc. Argent. V, in An. Mus. Nac. Buenos Aires Ser. B. Tome 13: 42t. 1911, was described from decaying coffee leaves in terms that suggest its relationship to $A$. tamarii. 
A. spadix Amons, in Archief voor de Suikerindustrie in Nederlandsch-Indie Jaarg. 29, Deel 1, pp. 12-14. Jan.-June 1921. From the description this is a synonym of A. tamarii Kita. Colonies described as yellow-brown to deep brown, growing well one ommon laboratory media, without aerial mycelium; in reverse colorless; ricecolored to light violet at first, then light fuscous brown; conidiophores up to 2 to 3 $\mathrm{mm}$. by 8 to $9 \mu$ with walls about $0.9 \mu$ thick and pitted or rough; vesicles globose, up to $50 \mu$ in diameter, or almost clavate in small heads; conidia 5.5 to $7.2 \mu$, rough.

Culture: Amons. Not studied by us.

A. erythrocephalus B. and C., in Jour. Linn. Soc. (London), Bot. 10: 362. 1869. (See Fungi Cubensis Wrightiana, 1868; Type No. 612 in Curtis Herbarium deposited in the Cryptogamic Herbarium of Harvard University, bears Wright's No. 764. Part of the original material was removed by Dr. Farlow and given to Thom for study.)

Microscopic examination of this type specimen gives measurements as follows: conidiophores 45 to $70 \mu$ in diameter, up to $2 \mathrm{~mm}$. in length, with walls very heavy 5 to $12 \mu$ thick, varying from 5 to $6 \mu$ in the broader part to 10 to $12 \mu$ at the narrower base, pitted or roughened; vesicles up to $100 \mu$ in diameter, nearly globose, fertile all over; head washed free from spores about $150 \mu$ in diameter; sterigmata in two series, primary 8 to $10 \mu$ in length, secondary 8 to $9 \mu$ in length; conidia commonly 8 by $6 \mu$, ranging up to 8 to $12 \mu$ by 5 to $9 \mu$, finely pitted or roughened with rather thin walls. Colors in the material are questionable on account of the age of the collection.

Cultures: None. Type material only known. Placed between the A. tamarii and A. flavus groups. The amended description is offered due to the existence of a type specimen with very conspicuous characters under a name only very briefly described in 1869. When grown upon natural substrata such as grains, et cetera, conidiophores and heads of $A$. tamarii become very much larger than those ordinarily produced in culture media. This might account for this specimen which bears the name $A$. erythrocephalus $\mathrm{B}$. and $\mathrm{C}$.

\section{Occurrence and Economic Importance}

Of the species included in this group of brown-spored Aspergilli, only A. tamarii is in any sense widely distributed or common in nature. Aspergillus lutescens is known only as the type culture and as a second isolation made in Washington, D. C., many years later. Aspergillus terricola has not been positively identified since its description, although the form with smaller heads and less coarsely roughened spores designated A. terricola var. americana by Marchal is occasionally encountered. Aspergillus tamarii is, however, a cosmopolitan mold upon vegetable material undergoing slow decomposition and can be isolated from almost all soils examined. Like $A$. niger and $A$. flavus, it is more frequently recovered from warm and semi-tropical soils than from cool, temperate soils, although it occurs in the latter. The species commonly appears with A. flavus and A. oryzae as a constituent part of the "koji" used in the fermentation industries of the Orient. Certain strains apparently produce appreciable amounts of diastatic and proteolytic enzyme, while other strains are known to produce 
kojic acid. Gould reported this in 1938, and it was subsequently confirmed by A. J. Moyer (unpublislred notes) for a strain isolated from Panama soil at the Northem Regional Research Laboratory in 1941.

Kita's culture was isolated from a soybean sauce termed "Tamari", hence the species name. Tamari is made by a shorter fermentation process than soy sauce or shoyu, and differs from it in flavor. Kita believed that where it was made empirically, it owed its individuality to the particular aspergillus which he isolated and described. 


\section{Chapter XI}

\section{THE ASPERGILLUS FLAVUS-ORYZAE GROUP}

\section{Outstanding Characters}

Colonies varying from very light greenish-yellow to deep yellow-green (Iry Green).

Conidiophores rough or pitted, colorless.

Heads hemispherical to columnar to subglobose.

Sterigmata in one or two series, often varying in the same head.

Vesicles variable in form, from hemispherical to dome-shaped in small heads to globose in large heads.

Conidia more or less roughened, varying in color as the colony.

Sclerotia characteristic of many strains, generally grayish-brown to black, entirely lacking in others.

Two species names are widely used for members of this cosmopolitan group. Aspergillus oryzae is applied quite generally, without regard to morphology, to the strains used by the Japanese and Chinese in the fermentation of rice and soy products. Although purified cultures are used in many places, the nomenclature is based more upon utilization than upon morphology. There appears, however, in these industries, a series of strains with long conidiophores, radiate heads, mostly greenish-yellow, with the green often fading completely in old cultures. These strains appear to be most commonly used in the production of the diastatic type of ferments and to be distributed in the great culture collections as Aspergillus oryzae (Ahlb.) Cohn. Such strains seem to be mostly oriental or tropical in origin. Strains with shorter conidiophores and yellowish-green heads, on the other hand, appear wherever fermenting or decaying materials are examined microscopically, or by cuiture. Aspergillus flavus Link has been accepted as a species aggregate for this second array of forms from which the segregation of sections for description as separate species has been found difficult, if not almost impossible. If one wishes to perpetuate species names as roughly covering aggregates of closely related but varying strains, bearing always in mind that no sharp lines of differentiation exist, certain applications of names may be made arbitrarily about as follows:

\section{Group Key}

I. Sterigmata mostly in one series, double sterigmata also present.

A. Conidiophores long, 1 -several $\mathrm{mm}$., heads radiate, greenish-yellow; conidia pyriform, more or less roughened, variable in size up to 6,8 , or even $10 \mu$ in long axis

A. oryzae (Ahlburg) Cohn 
B. Conidiophores 600 to $1700 \mu$; heads radiate, hemispherical, pale greenishyellow; conidia smooth, globose 3.0 to $4.6 \mu \ldots .$. A. micro-virido-citrinus

Costantin and Lucet

C. Conidiophores mostly less than $500 \mu$; heads deep yellowish-green (Ivy Green); described as a parasite of the mealy bug of cane, occasionally

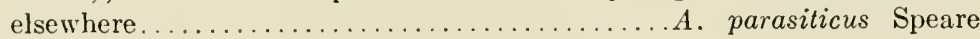

II. Sterigmata mostly in two series but single series common and often in same head, small heads usually showing single series only.

A. Conidiophores very variable in length, mostly 400 to $1000 \mu$; heads in various yellowish-green shades........................ flavus Link

B. Conidiophores mostly borne as short branches from trailing hyphae forming an uneven cottony mass; heads white to yellow with traces of green only

A. effusus Tiraboschi

Literally hundreds of strains of this group have been collected and compared. Many of them have been isolated from fermentation investigations in the laboratory and from industrial processes. No correlation of colony appearance, conidiophore or head morphology, color or microscopic detail, with actual utilization has been proved. A culture labeled $A$. oryzae (Ahlburg) Cohn, NRRL No. 447 (Thom No. 113) has been preserved for over 30 years without apparent change in morphology. It is probably derived from Cohn's organism. When, however, we scrutinize the Aspergilli obtained from the rice or soy fermentations of the Orient, cultures of the type represented by this strain are not the most common. The preeminently useful strains usually have the aspect of forms intermediate between A. flavus and A. oryzae. The dwarf green A. parasiticus of Speare isolated from dead mealy bugs of sugar cane in Hawaii proved no more parasitic to the same species of insects in the Barbados than other A. flavus strains sent with it. Teizo Takahashi contributed his series of strains under the letters used in his publication (1913). These are discussed at some length in Thom and Church's paper on A. flavus, A. oryzae and associated species (1921), and also in "The Aspergilli" (1926, p. 202). It is sufficient to say that they vary all the way from almost white with few lightly colored heads to rich yellow-green in which heads are very numerous and fairly dark. They vary likewise in the length and diameter of their conidiophores. Characters of color and conidiophore length are not always correlated, a:though it is generally true that the darker conidial masses are borne upon shorter stalks. The collections contributed by Oshima, Kita, Hanzawa, and others from Japan as well as those isolated from commercial "Koji" (sold as inoculum for fermentation industries) showed mainly the A. flavus morphology. Strains of this series appear constantly where cultures are made from soil or from decaying vegetation. A. flavus and its allies appear in collections from every correspondent who contributes Aspergilli. It is debatable whether the worker will be benefited or confused by the introduc- 
tion of some of the species names applied to members of the group. It must not be forgotten that any variant from the dwarf and deep green $A$. parasiticus to the longest stalked and palest greenish-yellow A. oryzae may be found if we look for it.

Aspergillus oryzae (Ahlburg) Cohn, in Jahresb. Sehles. Gesell. Vaterl. Cultur (1183) 61: 226 Breslau. 1884.

Synonym: Eurotium oryzae Ahlb. The name E. oryzae with an incomplete deseription for the saké organism was published by Korschelt, in Dingler's Polytechnisches Jour. 230: 330. 1878 , as taken from a letter from "Herr Ahlburg." See also Thom and Church, Amer. Jour. Bot. Bot. 8: 106. 1921, and The Aspergilli, p. 198. 1926.

Colonies on Czapek's solution agar rapidly spreading with regetative hyphae mostly submerged and forming a white to gray mycelial layer in the form of a tough felty mass (fig. $70 \mathrm{~A}$ ); developing pale greenish-yellow shades with the production of ripening conidial areas, varying from lime green to mignonette green (Ridgway, Pl. XXXI, column 25) with the green disappearing later and the general color shifting to yellowish-brown shades; myeclium and agar uncolored. Conidial heads predominantly large, abundant, globose, radiate, with chains of conidia separate rather than adhering (fig. $70 \mathrm{D}$ ), giving the pale yellow shades of the colonies. Conidiophores 2 to several $\mathrm{mm}$. long by up to 20 to $25 \mu$ in diameter with walls rather thin, definitely pitted or rough (fig. $70 \mathrm{~F}$ ), eolorless. Vesicles globose to subglobose, less often hemispherical, up to 50 or even $70 \mu$ with walls 1 to $1.5 \mu$. Sterigmata commonly in one series up to 15 or $20 \mu$ long by 3 to $5 \mu$; or in two series with primary sterigmata up to 12 by $5 \mu$, and secondary sterigmata 10 to $12 \mu$ by $3.5 \mu$ (fig. $70 \mathrm{~B}$ ). Conidia more or less pyriform (fig. $70 \mathrm{~F}$ ), varying greatly in size in the same culture and in different strains, 3 by $4 \mu, 4$ by $5 \mu, 5$ by $6 \mu$ or up to $9 \mu$ or $10 \mu$ in long axis oceasionally, rather thin-walled, roughened, becoming coarsely and deeply roughened in some strains. Sclerotia dark, few and not forming clumps, produced sporadieally under undefined conditions.

Diagnosis based primarily on culture NRRL No. 447 (Thom No. 113) received from the Centraalbureau at Baarn and believed to be derived from Cohn's original strain.

While the above description is believed to conform closely to the original coneeption of $A$. oryzae, strains possessing the essential morphology described but which are heavier sporing and somewhat darker in color are more commonly encountered. Culture NRRL No. 458, obtained from Dr. Oshima as strain AoOld and shown in Pl. VII C, and Fig. $70 \mathrm{C}-\mathrm{F}$, is representative of these forms. 

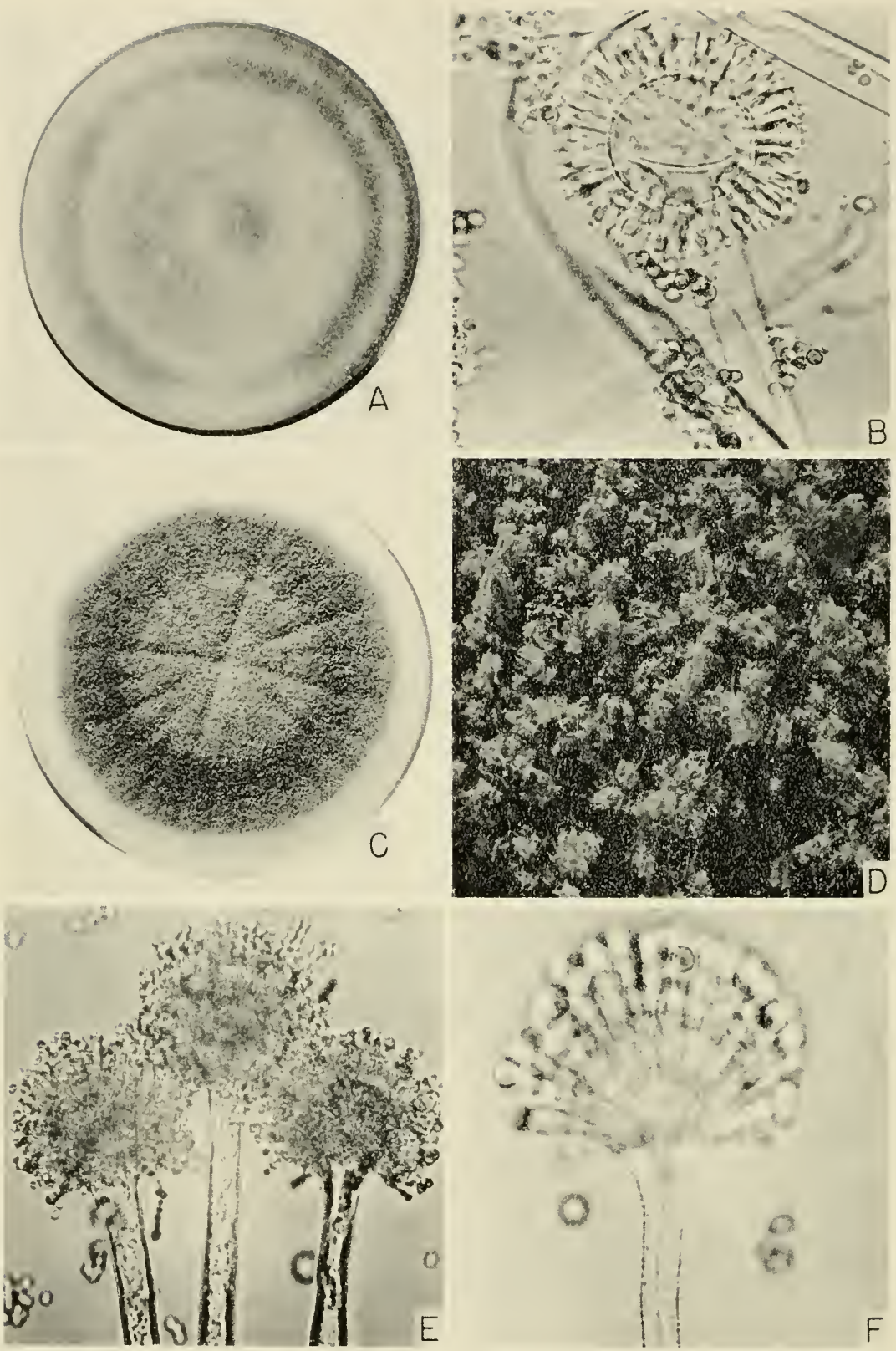

FIG. 70. Aspergillus oryzae. A and $B$, Strain NRRL No. 147 (Thom No. 113): $A$, Single colony on C'zapek's solution agar, loose-textured, conidiophores long and limited in number, 10 days; $B$, Details of single head, vesicle thin-walled, sterigmata mostly in two series, $\times$ 480. C- $F$, Aspergillus oryzae NRRL No. 458: $C$. Single colony on Czapek's solution agar, comparatively heavy sporing. 10 days; $D$, Conidial heads of the same, $\times 18 ; E$, Conidial heads further enlarged, $\times 270 ; F$, Single head showing vesicle, sterigmata in a single series, and roughened conidiophore, $\times 775$. 
The production of perithecia by members of this series was reported by Bezssonoff (1919) without adequate description and by Zikes (1922) whose culture, as received from him, belonged in the A. glaucus group. No ascosporic form is verifiable for the group thus far.

\section{Aspergillus micro-virido-citrinus Costantin and Lucet, in Ann. Sci. Nat.}

\section{Bot. (IX) 2: 158.1905.}

The appearance of colonies and measurements of conidiophores, heads, and spores indicate a form intermediate between $A$. flavus and $A$. oryzae except for its small conidia. The description is very nearly satisfied by Takahashi's culture "P" (NRRL No. 480). It was found to grow between $15^{\circ}$ and $45^{\circ} \mathrm{C}$. and to be pathogenic to rabbits. Colonies were greenishyellow to predominantly yellow but contained some definitely green admixture in contrast to $A$. oryzae, which often lacks green color entirely. Conidiophores 600 to $1700 \mu$ in length, up to $21 \mu$ in diameter near the vesicle, uncolored, "granular" (= pitted) above, smooth toward the base. Vesicles $2+$ to $62 \mu$ in diameter. Sterigmata varying in size and arrangement with the size of the heads examined. Conidia globose, smooth, 3 to $4.6 \mu(3.1 \mu$ as a minimum to occasional diameters of $5.5 \mu$ ).

An occasional culture shows the morphological characters described by Costantin and Lucet. No actual identity has been proved.

Aspergillus flavus Link, in Obs. p. 16. 1809; also in Sp. Plant. 6: 66. 1824 , cited as synonym of Monilia flava Persoon, Myc. 1, p. 30.

Synonym: Eurotium A spergillus flavus DeBary and Woronin, in Beitrage zur Morphologie und Physiologie der Pilze, III Reihe, p. 380. 1870. Exsiccati by Brefeld preserved in Rabenhorst, Fungi Europaei Edit. Nov. ser. II, No. 2135; one packet in the collection of the New York Botanical Garden.

Colonies on Czapek's solution agar spreading rapidly, with floccosity limited to scanty growth of sterile hyphae in older and dryer areas among crowded conidiophores; conidial areas range in color in various strains from sea-foam yellow through chartreuse yellow, citron green, lime green, to Kronberg's green (Pl. VII D and fig. $72 \mathrm{~A}$ ), or even to ivy green, (See Ridgway, Pl. XXXI, column 25), yellow-green colors are either persistent or, in old colonies, altered by the disappearance of the green factor leaving shades of yellow-brown; reverse yellowish at first, passing over into brown shades in age. Conidial heads vary from small with a few chains of conidia to large radiate (fig. $72 \mathrm{E}$ ) or columnar masses in the same culture and varying mixtures of different types and sizes of head. Conidiophores mostly arising from submerged hyphae, commonly 400 to $1000 \mu$ long by 5 to $15 \mu$ in diame- 

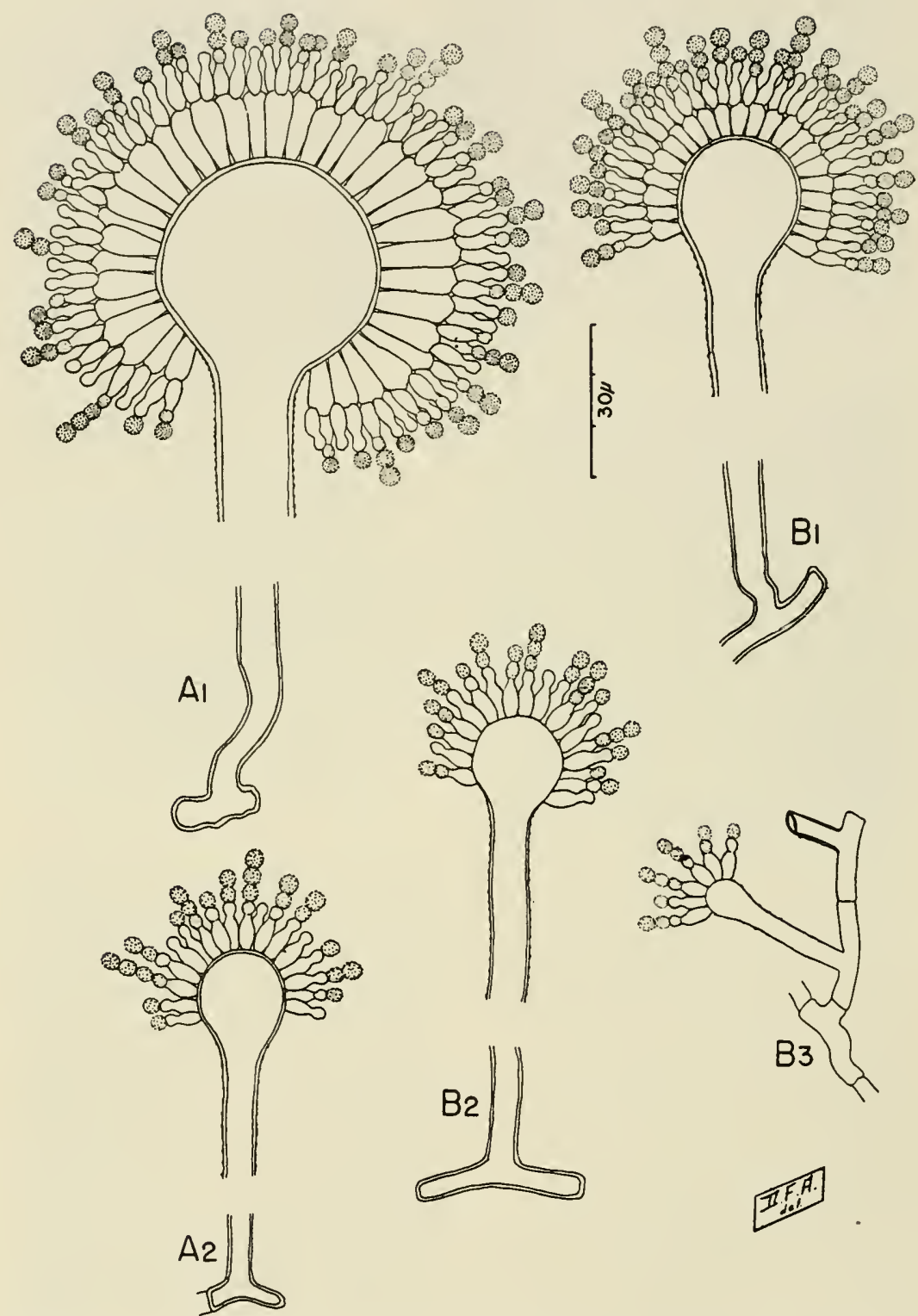

FIg. 71. Conidial structures in the Aspergillus flavus-oryzae group. A, A. flavus, NRRL No. 482: $A_{1}$, typical, large, radiate to globose head showing sterigmata in two series; $A_{2}$, small, loosely columnar head showing single series of sterigmata. $B, A$. effusus, NRRL No. $506: B_{1}$, large radiate head showing double series of sterigmata; $B_{2}$, smaller head showing sterigmata in a single series; $B_{3}$, diminutive head borne upon one of a chain of foot cells. In this group single and double sterigmata often occur in the same head (not illustrated). 

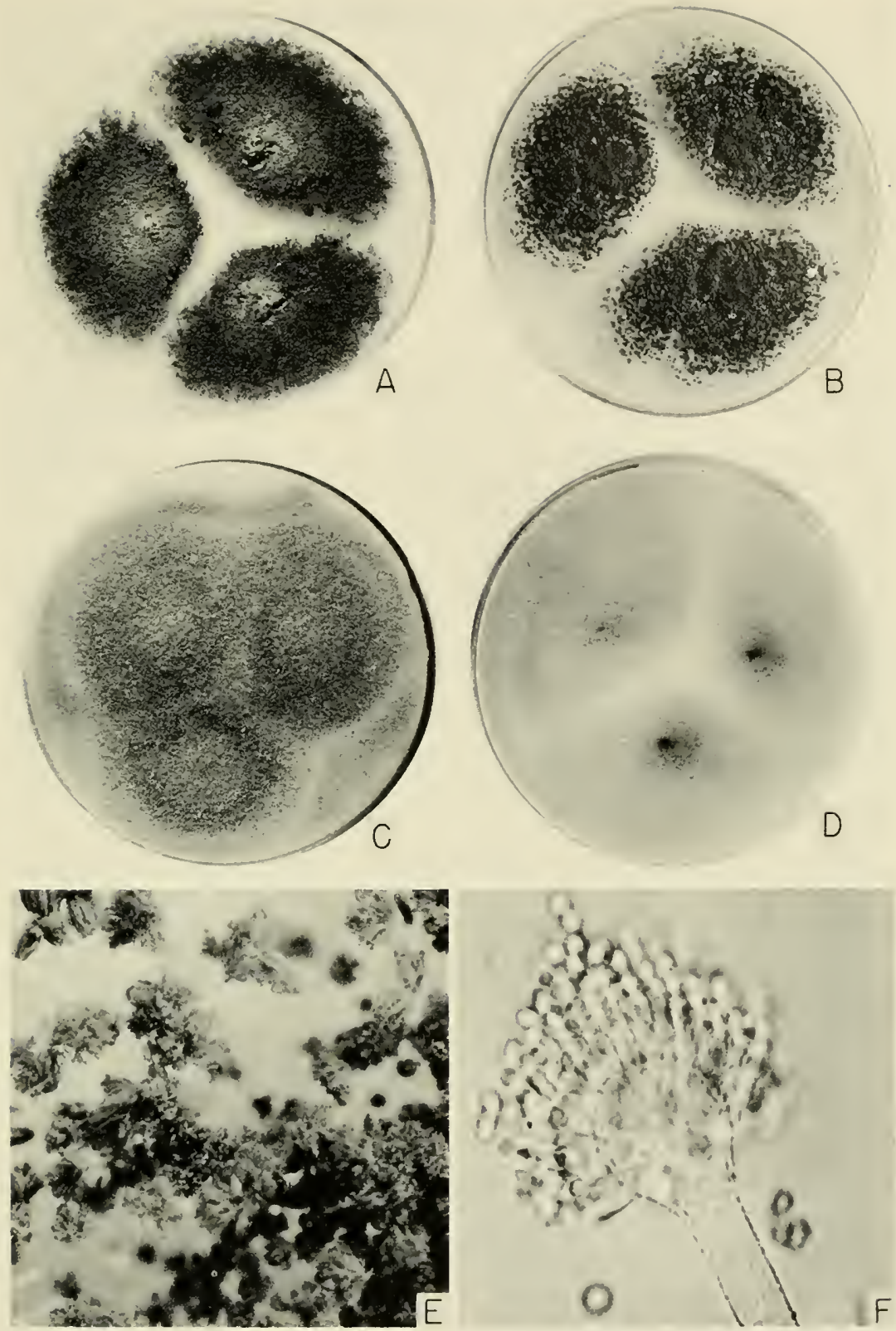

FIG. 72. Aspergillus flavus. A, Typical strain, NRRL No. 1957, showing crowded conidial heads and occasional black sclerotia. $B$, Heavy sclerotium producing strain, NRRL No. 500. C , Strain used for the production of kojic acid, NRRL No. 484 (This approaches the character of A. oryzae). D, Dr. White's strain of A. flavus (NRRL No. 501) showing characteristic thin growth and sparse sporulation. $E$, Typical conidial heads, $\times 18 . \quad F$, Single head showing vesicle, sterigmata, conidia, and the roughened conidiophore, $\times 780$. All cultures on Czapek's solution agar, 10 days. 
ter, with walls pitted, rough (fig. $72 \mathrm{~F}$ ) almost spiny in appearance, broadening upward and gradually enlarging into vesicles 10 to 30 or $40 \mu$ in diameter, dome-like in the smaller heads, flask-shaped in larger heads (fig. $72 \mathrm{~F}$ ). Sterigmata in a single series in many smaller heads (fig. $71 \mathrm{~A}_{2}$ ), or both single and double series on the same vesicles in large heads (fig. $71 \mathrm{~A}_{1}$ ), varying from single sterigmata only 10 to $15 \mu$ by 3 to $5 \mu$, to primary sterigmata 7 to $10 \mu$ by 3 to $4 \mu$ and a secondary series 7 to $10 \mu$ by 2.5 to $3 \mu$. Conidia pyriform to almost globose, nearly colorless to definitely yellowish-green, varying from $3 \mu, 3$ by $4 \mu, 4$ by $5 \mu$, or even larger and marked variously with pits, echinulations, or irregularly winding color bars and ridges to give a roughened effect of varying intensity. Sclerotia, when found, at first white then brown, hard parenchymatous, and a few strains white tipped, produced by some strains regularly and abundantly (fig. $72 \mathrm{~B}$ ), scantily by others under undefined conditions. Perithecia not found.

Description originally based upon culture NRRL No. 482 (Thom No. 108) from the Centraalbureau at Baarn, Holland, but supplemented by observation of many hundreds of cultures from many substrata and all parts of the world.

Unless segregation under a specific name is supported by adequate morphological and reproducible cultural data, there is no way to identify the organisms intended. Applying these criteria, no reasons are seen for the use of the following specific designations:

A. wehmeri Constantin and Lucet, in Ann. Sci. Nat. Bot. (IX) 2: 162 . 1905.

A. variabilis Gasperini, in Atti. Soc. Toscana Nat. Sci. Pisa Mon. 8, fasc. 2: 326. 1887.

A. pseudo-flavus Saito, in Centralb. Bakt. etc., 2 Abt., 18, No. i/2, p. 34, figs. 15-18. 1907, or its synonym S. pseudo-flava (Saito) Sacc., in Syll. 22: 1260. 1913.

A. siebenmanni Constantin and Lucet, in Ann. Sci. Nat. Bot. (IX) 2: 162. 1905, is a bibliographic species based upon an organism isolated from the human ear and diagnosed by Siebenmann (Zeitsch. f. Ohrenheilk 12:1883) as A. flavus. The describers regarded it as a separate species based only upon the description given by Siebenmann.

A. gymnosardae Yukawa, in Jour. Col. Imp. Univ. Tokoyo 1: 362, Pl. 18, figs. 1-7. 1911. A member of the A. flavus-oryzae group with measurements intermediate between more typical representatives of the two species.

A. thomii Graff, nomen nudum, a heavy sclerotium-producing strain distributed by Graff but never described. No diagnostic basis for the name was presented.

A. pollinis Howard, in Am. Bee Jour. 36:577-578. 1896, was discussed as an organism causing "pickled brood and bee paralysis"' (See also idem. 38: 530-531. 1898). Turesson (Svensk Bot. Tidskr. 11:30. 1917) decided the mold was A. flavus.

Aspergillus parasiticus Speare, in Hawaiian Sugar Planters' Exp. Sta., Path. and Physiol. Ser. Bul. 12, p. 38, pl. 3-4. 1912. See Thom and Church, The Aspergilli, p. 203. 1926.

Colonies on Czapek's solution agar with sucrose spreading rapidly, forming a surface growth of crowded conidiophores with very few sterile 
hyphae (fig. $73 \mathrm{~A}$ ), in deeper yellow-green shades near iry green (Ridgway, Pl. XXXI); reverse uncolored or yellowish. Conidial heads radiate, abundantly produced and giving color to the colony. Conidiophores given by Speare as 300 to $700 \mu$ long, commonly under $400 \mu$, with walls colorless, prominently rough or pitted, enlarging from $3 \mu$ at the foot up to 10 to $12 \mu$, and passing into vesicles up to $35 \mu$ in diameter (fig. $73 \mathrm{~B}$ ). Sterigmata in one series, 7 to $9 \mu$ by 2.5 to $3 \mu$, closely packed over the vesicular surface, yellow. Conidia pyriform to globose, very rough, \pm to $5 \mu$, occasionally $6 \mu$ in long axis, green. No sclerotia or perithecia reported.

Described as parasitic upon the mealy bug of sugar cane (Pscudococcus calceolariae Mask.) in Hawaii. Type culture NRRL No. 502 (Thom No. 3509) received from Speare. Cultures with the same morphology were isolated from infected mealy bugs from Demerara by Thom, and in Louisiana by Kopeloff. Johnston, working in Puerto Rico, considered that he had proved infectivity to be a strain function among organisms of the A. flavus series rather than associated with morphology. Blochwitz (Ann. Mycol. 32(1/2): 86. 1934) has called another nearly allied form A. flavus var.viridis, but gives no adequate data for separation. Cultures with these characters are occasionally obtained from sources not known to be associated with disease of insects. Shih has likewise deseribed from China as Aspergillus chungii (Lingnan Sci. Jour. 15(3): 378. 1933) a strain which apparently duplicates A. parasiticus.

Aspergillus effusus Tiraboschi, in Ann. di Bot. (Rome) 7: 16, fase. 1. 1908. See also Thom and Church, in Am. Jour. Bot. 8: 109-110. 1921, and Thom and Church, in The Aspergitli, p. 208. 1926.

Colonies on Czapek's solution agar rapidly and broadly spreading, floccose or piled cottony white (fig. $73 \mathrm{C}$ ), becoming dirty yellowish or, in restricted areas, pale greenish-yellow, then passing over into dull buff or tan shades as heads mature; reverse and agar yellowish. Conidial heads usually more or less columnar, mostly small, a few of them fairly large, many of them upon short conidiophores (fig. $71 \mathrm{~B}_{3}$ and $73 \mathrm{E}$ ), often less than $100 \mu$ long and 5 to $10 \mu$ in diameter, arising from the trailing floccose hyphae, quickly losing their yellow-green color. Conidiophores with walls pitted or roughened, sometimes bearing granules (produced by drying droplets of exuded fluid). Vesicles mostly under $20 \mu$ in diameter (fig. $71 \mathrm{~B}_{2}$ ). Sterigmata in one series in small heads, in either one or two series in large heads (fig. $71 B_{1}$ ), approximating the $A$. flaus type. Conidia pyriform to globose varying from 3 by $4 \mu$ to 5 by $7 \mu$. No sclerotia or perithecia reported. The species was deseribed originally from rotten corn (Zea Mays).

Culture description as given centers around eulture NRRL No. 506 (Thom No. 130) isolated by Dr. B. F. Lutman, Burlington, Vermont. Other 

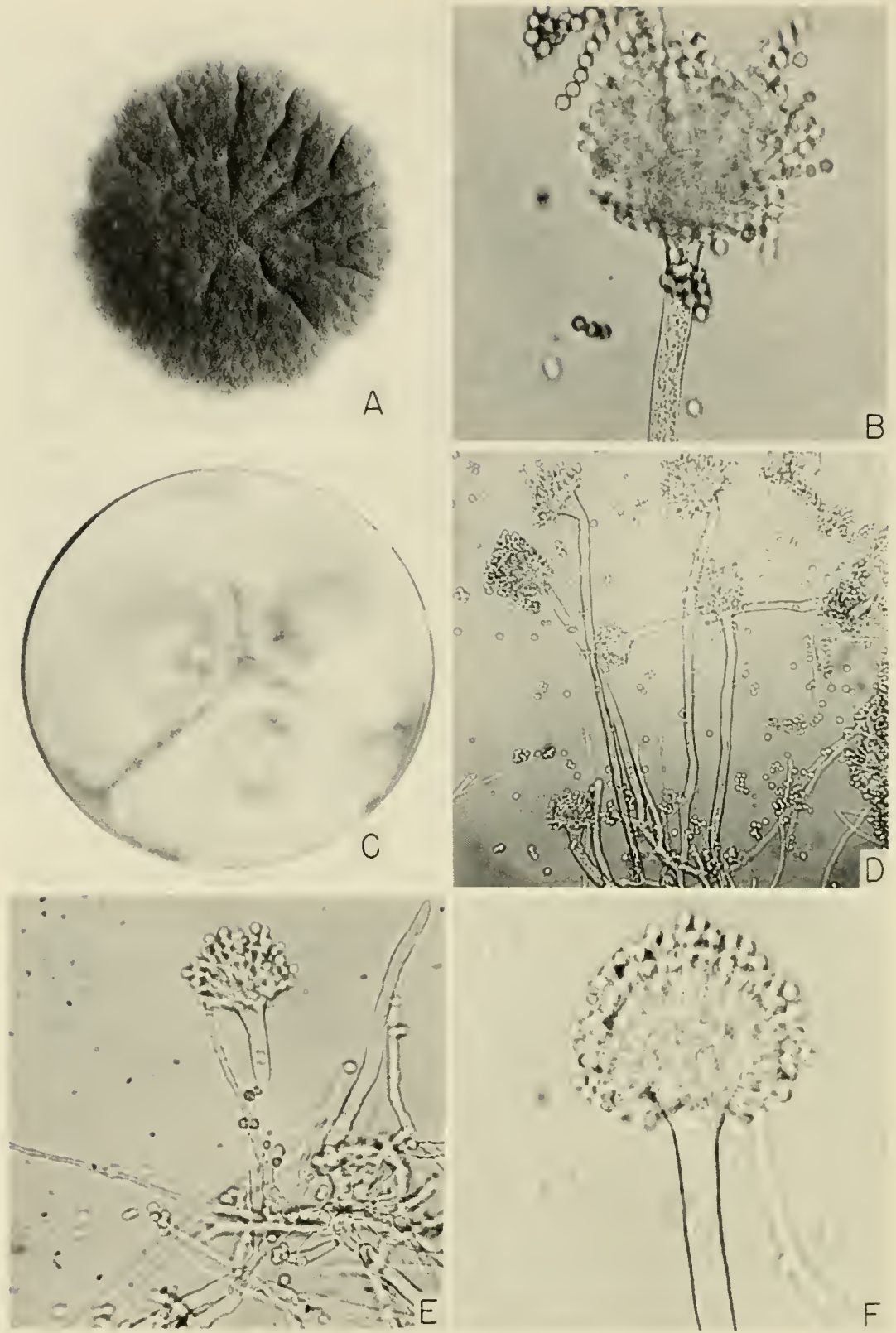

Fig. 73. $A$ and $B$, Aspergillus parasiticus, NRRL No. 465: A, Colony on Czapeks' solution agar showing heavy production of short-stalked conidial structures, 10 days; $B$, Single conidial head showing single series of sterigmata and roughened conidiophore, $\times 500$. C-F, Aspergillus effusus, NRRL No. 506:C, Colony on Czapek's solution agar showing characterisite floccose habit and light sporulation, 10 days; $D$, Conidial structures arising from aerial hyphae characteristic of species, $\times 165$; $E$, Single short-stalked fruiting structure arising from aerial hyphae, $\times 300 ; F$, Conidial head showing sterigmata mostly in two series, roughened surface of conidiophore not apparent, $\times 500$. 
strains studied include isolations from cornmeal from Indiana and from mealy bugs in Puerto Rico. Superficially this speeies bears little relation to A. flavus. Detailed microseopic examination of heads and spores, however, reveals true and elose relationships. Selective transfer from original colonies permitted changes in the predominance of the sterile areas over the fruiting areas without changing the general habit or nature of the colony. Blochwitz (Bot. Centralb. Beiheft Abt. Anat. Phys. 48: 176-182. 1931) regarded $A$. effusus as a floceose type of $A$. flavus. This position can be supported, but the authors feel that the species should be maintained sinee it is strikingly different from A. flavus in its general colony appearance, and since it is repeatedly, although infrequently, isolated from nature.

Whether Sterigmatocystis lutea Bainier (Bul. Soc. Bot. France 27: 27. 1880) was one of these can only be guessed from culture NRRL No. 508 (Thom No. 4640.473 ) received from the Bainier collection under this name. The strain is close to A. effusus. Bainier did not claim identity with $S$. lutea van Tieghem (Bul. Soc. Bot. France 24: 103. 1877), which was entirely undescribed.

Aspergillus jeanselmei Ota, in Ann. de Parasit. 1(2): 137-146. 1923, as received from Baarn in 1939 (NRRL No. 507: Thom No. 5665) represented a member of the A. flavus series with close affinities to A. effusus.

\section{Occurrence}

Members of the A. flavus-oryzae group are among the most abundant of all the Aspergilli. They are world-wide in distribution and are omnivorous in the substrata upon which they are able to grow and develop. They have been isolated from the widest variety of sources including: the fermentation industries of the Orient, grains and cereal products from different parts of the United States, various types of forage, egg noodles, bread and other bakery products, leather goods, dried dates, eured meats, dairy products, nut meats, soy sauce, home-canned fruits and vegetables, textiles, paper pulps, insects, tannin inoeulum, feces, sputum, the lung of a bird, and from the duodenum of man. They are very abundant in soil, and have been observed in almost all samples examined. They appear to be particularly eommon in the warm soils from tropical and sub-tropical areas. They vary greatly in eultural appearance and in the detailed measurements of their fruiting structures, and to a limited degree these differences can be correlated with the sourees from which they are obtained. Soil isolates commonly show conidial heads near yellow-green in color which are borne upon comparatively short eonidiophores. Isolates from the rice and soy fermentations of the Orient often show conidial heads pale yellow-green in color that are borne upon long, thin-walled eonidiophores. Exeeptions to this very general statement are common. 


\section{Kojic Acid}

The ability of members of the $A$. flavus-oryzae group to produce kojic acid has been recognized for more than three decades. It is only within the past fifteen years, however, that serious attention has been given to this fermentation. Beginning with the work of Challenger, Klein, and Walker in 1929 and 1931, and continuing with that of May, Herrick, Moyer, Ward, and Wells in 1931 and 1932, the proper nutrients and cultural conditions necessary for its production were defined. Subsequent contributions have been made by Kluyver and Perquin (1933) and by Barham and Smits (1936). In all of the early reports the responsible cultures were cited as $A$. oryzae, whereas in more recent ones the cultures employed have generally been identified as Aspergillus flavus. It is of interest to note that the strain studied by May and associates (NRRL No. 484: Thom No. 3538) was a thoroughly typical A. flavus when first isolated by Thom in 1914, but during the long period that it has been maintained in artificial culture it has gradually changed until today it more nearly resembles $A$. oryzae in its general habit and coloration (fig. $72 \mathrm{C}$ ). Its capacity to produce kojic acid remains undiminished, however. The culture employed by Barham (NRRL No. 625) likewise fails to satisfy the typical cultural picture of A. flavus, although it is discussed under this name. In contrast to these cultures, other strains belonging to this group have been under continuous laboratory cultivation for more than 30 years without apparent change in appearance or behavior. The above and additional references to the kojic acid fermentation are presented in the Topical Bibliography, pp. 297-298.

\section{Enzymes}

Members of the A. flavus-oryzae group produce diastatic and proteolytic enzymes abundantly. For this reason they have been much studied, and an extensive literature regarding mold enzymes has developed around the use of these fungi. In large measure the alcoholic and soy food industries of the Far East are based upon these molds and their enzymes. In the production of alcoholic beverages, the diastatic enzymes produced by an Aspergillus (regularly identified as $A$. oryzae) are employed to hydrolyze the rice starch. Alcohol is then produced from the resultant sugars by the addition of a fermentative yeast. In the soy industries, closely related molds, or even the same strains, are used as a source of proteolytic enzymes. In 1894 Takamine secured a series of U. S. patents covering the production of diastatic enzymes and the making of alcoholic liquors (see Topical Bibliography, p. 302). Subsequent to this, other investigators, mostly Japanese, published a number of papers in this field. Oshima in 1922 and 1928 reported on the production of protease by members of the A. flavus- 
oryzae group. Today considerable quantities of diastatic enzymes, proteolytic enzymes, and mixed diastatic and proteolytic preparations are being manufactured from these molds for use in the textile and tanning industries particularly. Within recent years considerable attention has been given to "moldy bran" (bran seeded with seleeted strains of $A$. oryzae) as a possible substitute for malted barley as a saccharifying agent in the production of industrial alcohol (Underkofler, Fulmer, and Schoene, 1939; Schoene, Fulmer, and Underkofler, 1940; Hao, 1942; Hao, Fulmer, and Underkofler, 1943; Christensen, 1943).

References to papers dealing with the production of enzymes by molds belonging to this group are presented in the Topical Bibliography, pp. 302-304. No attempt has been made to present a complete bibliography of the subjeet, but it is believed that sufficient citations are listed to introduce the reader to the extensive literature of the field.

\section{Pathogenesis (See Topical Bibliography pp. 307-310)}

Pathogenesis has been occasionally reported for strains identified as members of the $A$. flavus series. Observations reporting their presence in the external ear go back to Siebenmann (1882). Ota described $A$. jeanselmei as a parasite of human nails in Paris in 1923. Bereston and Keil (1941) described a case of infected nails in which the strain, as seen by us, proved to be a variant of $A$. flavus. There are ample reeords to show an occasional infection of the human being. There are no data as to the route of infection, and the question whether the parasite is a primary or a secondary (wound) parasite stands unanswered. The constant presence of members of this group in every human environment, together with a lack of evidence of ability to penetrate sound human tissue, leaves some doubt-not as to its ability to grow when once established, but whether it can actually "break and enter" as a direet agent of disease. Aspergillus flavus is commonly isolated from sputum. In birds, eases of lung involvement have been reported. There is no question but that the mold can persist for reasonable periods of time within the animal body. A. flavus is one of the more common air-borne molds, and oceasional allergic reactions are attributed to it, although instances where it is the sole responsible agent are not known to have been reported.

\section{Antibiosis}

The production of antibacterial substances by strains of A spergillus flavus has been observed by a number of workers during the past five years. White (1940) reported a eulture of $A$. flavus (found by the writers to be a somewhat atypical strain) to produce some substanee which was definitely bactericidal against some gram-negative and some gram-positive bacteria. 
A more detailed study of this strain was subsequently made by White and Hill (1943) and the name "aspergillic acid" was assigned to the active substance. The compound shows a comparatively high toxicity to laboratory animals. Utilizing the White strain, Jones, Rake, and Hamre (1943) have made additional studies on the biological properties of aspergillic acid. In the meantime, Glister (1941), at Oxford University reported the production of an antibacterial substance effective against gram-negative and gram-positive bacteria by a different strain of $A$. flavus (found by the writers to be wholly typical of the species). He noted the possibility of relationship between the substance with which he was working and that earlier reported by White.

Following the work of Jones, Rake, and Hamre (1943), McKee and MacPhillamy (1943) succeeded in demonstrating the production of a second and entirely different antibacterial substance. In certain chemical properties, and in its action on bacteria, this was found to resemble penicillin very closely, but actual identity was not proved. In a subsequent and more detailed report, McKee, Rake, and Houck (1944) defined more exactly its bactericidal action against various gram-negative and gram-positive bacteria and proved additional evidence of its penicillin-like characters. They designated the substance "flavicidin".

Concurrent with this work, Bush and Goth (1943a and 1943b), working at Vanderbilt University, succeeded in demonstrating the production of an antibacterial substance from still another strain of $A$. flavus which was strongly active against Staphylococcus and other gram-positive forms but comparatively inactive against gram-negative forms belonging to the $E$. coli group. The substance was termed "flavicin". Identity with flavicidin and with penicillin is possible but has not yet been proved. Cook and Lacey (1944) report the production of appreciable amounts of an antibiotic substance from a strain of $A$. parasiticus. This was provisionally designated "parasiticin", and its similarity to penicillin was noted. Identity with flavicin (Bush and Goth) and flavicidin (McKee, Rake, and Houck) was suggested. Since A. parasiticus is so closely related to A. flavus, it would seem probable that an antibiotic produced by it would be similar to that produced by the latter species under the same conditions.

Waksman and Bugie (1943) investigated a large number of strains belonging to the A. flavus-oryzae group. They found strains of A. oryzae to show little activity, whereas strains of $A$. flavus showed increased but varying amounts. Yields were markedly influenced by various nutritional and environmental factors. Two types of antibacterial substances were observed: aspergillic acid and a substance similar to, if not identical with, penicillin. When grown in submerged culture, one strain was found to produce amounts comparable to the best strains of Penicillium notatum tested. Unfortunately, yields were not quantitatively determined. 


\section{Chapter XXi \\ THE ASPERGILLUS OCHRACEUS GROUP}

\section{Outstanding Characters}

Conidial heads ranging from sulphur yellow to varying shades of ochraceous, depending upon the species and strain, showing a greenish tint only in the single species A. sparsus; heads globose or radiate with conidial chains commonly adhering into divergent columns.

Conidiophores normally showing shades of yellow in the outer layers of the wall which is rough or pitted, usually prominently but occasionally reduced to traces which are seen most readily in dry mounts.

Sterigmata in two series with the primary often quite large and septate. Conidia in some series thin-walled and smooth, in others showing definitely double walls, more or less roughened or echinulate.

Sclerotia present in most species and strains, often dominating the cultures; in others entirely lacking. When present, ranging in color from cream or buff through pink and orange shades to purplish-vinaceous.

Molds belonging to this group are common wherever organic matter is decomposing under natural conditions. In spite of great variation in superficial appearance, length of conidiophore, size of heads, intensity and shades of color, and sclerotium production, they fit together into a great natural group of related forms. Extreme variants in the several series can be easily considered separate species and have been so described, but collections of great numbers of such forms present so many gradations that identification by description becomes doubtful if not impossible. Allocation to series centered upon some described species gives a practical method of grouping together closely related members of the great aggregate.

The name "ochraceus", derived from the pigment ocher and attached to the most abundant series in the group, is an old mycological usage which was more or less definitely followed in Saccardo's use of it for a plaque in his Chromotaxia. Ridgway analyzed the colors more exactly, dropped the term ochraceus but included a plaque near to it as Pl. XV, Col. 15 YO, Ochraceous-Buff. The strains of this group, however, mostly show colors closer to the yellower tints in Ridgway's Plate XXX, column 19. It is important that relationship with the great group shall be quickly grasped whether exact identity with particular strains already known is claimed or not.

Group characterization: The colony appearance of this group of Aspergilli varies greatly with the presence or absence of sclerotia. Some species 
are typically ochraceous in color from abundant conidial heads and are not known to produce sclerotia. Others produce a few sclerotia, or clusters of sclerotia, under very special conditions but still are characterized by their abundant conidial heads. Others always produce sclerotia in suffieient number to dominate the colony. Sclerotia, when present, are globose or elliptical, $0.5 \mathrm{~mm}$. or more in long axis, and vary in color from shades of yellow to orange-vinaceous or even purplish. The submerged mycelium varies from colorless to yellow, orange, or purplish shades. Heads are globose or radiate and vary in color from bright yellow near citrine through pale yellows to varying shades of ochraceous but never appear in umber shades, and show green (near olivine, Ridgway, Pl. XXXII) only in $A$. sparsus. Conidiophores vary greatly in length and diameter but have walls yellow, especially in the outer layer, pitted or rough-in occasional dwarf strains with fairly thin walls the pitting becomes difficult to demonstrate, hence careful examination with a good oil immersion objective may be necessary. Vesicles are globose, or occasionally somewhat elliptical, varying greatly in size with walls usually colorless and much thinner than the conidiophore wall. Sterigmata are always in two series, with primary sterigmata varying, as in the $A$. niger group, from fairly short to very long, but commonly 15 to $30 \mu$ upon Czapek's solution agar and occasionally much longer on special substrata; secondary sterigmata differ less conspicuously than primary sterigmata, being commonly 7 to $10 \mu$ by 1.5 to $2.5 \mu$; both series of sterigmata are almost colorless when examined with high magnifications. Conidia are small, sometimes elliptical, but mostly globose•or subglobose, smooth in most strains, but in others very delicately wrinkled or spinulose without prominent tubercles or bars of color, mostly 2 to $t \mu$ in long axis, larger in occasional strains.

The following key is an attempt to arrange this group into a satisfactory order to bring nearly related organisms together. For this purpose specific names already in the literature with the particular distinguishing marks cited in their diagnoses are introduced as more or less definitely fixed points in the group with which new material or unknown material can be compared.

\section{Group Key}

I. Conidial heads in fairly pure yellow tints such as sulphureus and citrinus

The A. sulphureus series

A. Sclerotia usually not abundant; conidiophores up to $1000 \mu$ long; heads abundant, radiate; vesicles globose; conidia globose or subglobose, smooth, about $3 \mu \ldots \ldots \ldots \ldots \ldots \ldots \ldots \ldots$. sulphureus Fresenius

B. Sclerotia abundant, commonly characterizing the culture; heads scattered, or grouped in restricted areas, particularly at the drying margin of agar slant cultures; conidia smooth, up to $4.0 \mu$

1. quercinus (Bainier) Thom and Church

II. Conidial heads in pale to darker ochraceous shades... The A. ochraceus series 
A. Conidia thin-walled, smooth.

A. ochraceus sub-series glaber

1. Selerotia dominating the colony appearance; conidia small, about $3.0 \mu$.

a. Sclerotia abundant, buff to pink or purplish in color in age; heads hemispherical to columnar, cream-buff to pale ochraceous

A. sclerotiorum Huber

b. Sclerotia abundant, at first gray, quickly becoming black; heads globose or radiate; upon onions and other bulbs

A. alliaceus Thom and Church (A. ventii group, p. 241)

2. Sclerotia present but secondary to the crowded, honey-yellow conidial heads; conidia smooth

A. melleus Yukawa.

B. Conidia with firmer walls, echinulate or barred

A. ochraceus sub-series cchinulata

1. Conidia elliptical to subglobose.

a. Conidia 3.5 to $5.0 \mu$ in long axis; echinulate heads ochraceous, radiate, and splitting; sclerotia in type material

A. ochraceus Wilhelm

b. Conidia 3.0 to $3.5 \mu$, spinulose; sclerotia not found

A. elegans Gasperini

c. Conidia up to $6.3 \mu \mathrm{in}$ diameter, rough (Otherwise $A$. ochraceus)

$S$. butyracea Bainier

2. Conidia globose, echinulate, 7.0 to $8.0 \mu$ in diameter (Otherwise $A$. ochraceus)................. delacroixii (Sace.) Thom and Church

3. Conidia strongly clliptical or pyriform, spinulose, 4.0 to $6.0 \mu$ by 3.0 to $4.5 \mu \ldots \ldots \ldots \ldots \ldots \ldots \ldots \ldots \ldots \ldots \ldots \ldots \ldots \ldots$ ostianus Wehmer III. Conidial heads commonly showing a definite greenish tint, near olive-buff to

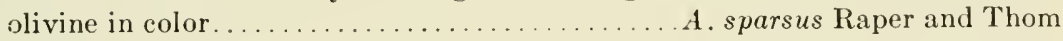

Aspergillus sulphureus (Fres.) Thom and Church, in The Aspergilli, p. 185. 1926.

Syn.: S. sulphurea Fresenius, in Beitr. zur Mykologie, Heft 3, p. 83, taf. XI, fig. 30-33. 1863. See also Sace. Sylloge 4: 73. 1886.

Colonies upon Czapek's solution agar at laboratory temperature, growing rapidly, forming a variously wrinkled to zonate felt, white or tinged with yellow to pink or purplish shades. Conidial heads irregularly produced in most eultures, in pale or sulphur yellor tints, mostly globose, but often splitting into spore eolumns in age. Conidiophores with walls firm, rough or pitted, yellow, varying greatly with the strain from very short and sparingly produced to elustered in areas and dominating the culture, or entirely covering the mycelium where sclerotia are absent. Vesicles typieally globose and fertile over the whole surface. Radiating primary sterigmata elosely packed on the vesicular surface, varying from 8 to $10 \mu$ by 3 to $5 \mu$ to several times larger in some big heads, secondary sterigmata usually uniform, phialiform about 8 to $10 \mu$ by 2 to $3 \mu$. Conidia small, globose or subglobose 2 to 3 or $3.5 \mu$ in long axis, thin-walled, smooth.

A. sulphureus as described by Fresenius had long eonidiophores and yel- 
low globose heads. Sclerotia were not reported. In our experience, however, they are seen not infrequently in cultures which otherwise fit the description of $A$. sulphureus as presented. In the sub-series, represented by $A$. quercinus (Bainier, Thom and Church), sclerotia are very abundant and heads comparatively few and scattered among the sclerotia. In addition to these two names which roughly designate sections or series of isolates as we find them, every gradation between these extremes may be anticipated.

The specics is fairly common in soils, on cereal grains, and upon decaying vegetation.

Several species have been described as having conidial heads sulphur yellow but doubtfully separable from $A$. sulphureus.

S. ochroleuca Spegazzini, in Myc. Argent V, p. 434, in Anal. Mus. Nac. Buenos Aires, Ser. 3, T. 13.1911.

S. auricoma Gueguen, in Bul. Soc. Myc. France 15: 171-187, figs. 1-48. 1899. This appears to have been a member of the series with primary sterigmata proliferating abundantly to form little conidiophores and secondary heads thus giving the head the appearance of bearing yellow hair. Such proliferation occasionally occurs in many species but has not again been found in this group.

S. vitellina Ridley, in Jour. Bot. (London) 34: 152, pl. 257, figs. 14-16. 1896, is described as producing bundles or coremia composed of partially adherent conidiophores. The organism does not appear to have been cultivated and has not since been reported.

Aspergillus quercinus (Bainier) Thom and Church, in The Aspergilli, p. 186, 1926.

Synonym: S. quercina Bainier, in Bull. Soc. Bot. France 28: 78. 1881. Sce also Sartory, Etude biologique du Sterigmatocystis quercina Bainier, in Bul. Soc. Myc. France 26: 349. 1910.

Colonies upon Czapek's solution agar spreading broadly, characterized by the presence of an aerial white mycelium and the abundant production of sclerotia over the whole area (Pl. VII, E and fig. $74 \mathrm{~A}$ ), or in sectors, or variously distributed; the mass changing from yellow to orange-yellow and finally to rufous or brick red shades with the ripening of the sclerotia; reverse in shades of yellowish-orange. Conidial heads in ycllow tints, near sulphureus, scattered among the sclerotia or occurring in long-stalked groups in the dryer areas of the culture tube or plate, mostly up to $200 \mu$ in diameter, but occasionally larger, up to 300 or even $400 \mu$ (fig. 74 C). Conidiophores with walls mostly pale yellow, especially in the outer layer, pitted (fig. $74 \mathrm{D}$ ), occasionally with abundant granules, about $2 \mu$ in thickness, varying from short and inconspicuous in crowded sclerotial areas to very long tufts in dryer areas, up to 2 or even several millimeters by 10 to $20 \mu$. Vesicles colorless, crushing easily, 35 to $45 \mu$ in diameter, fertile over 

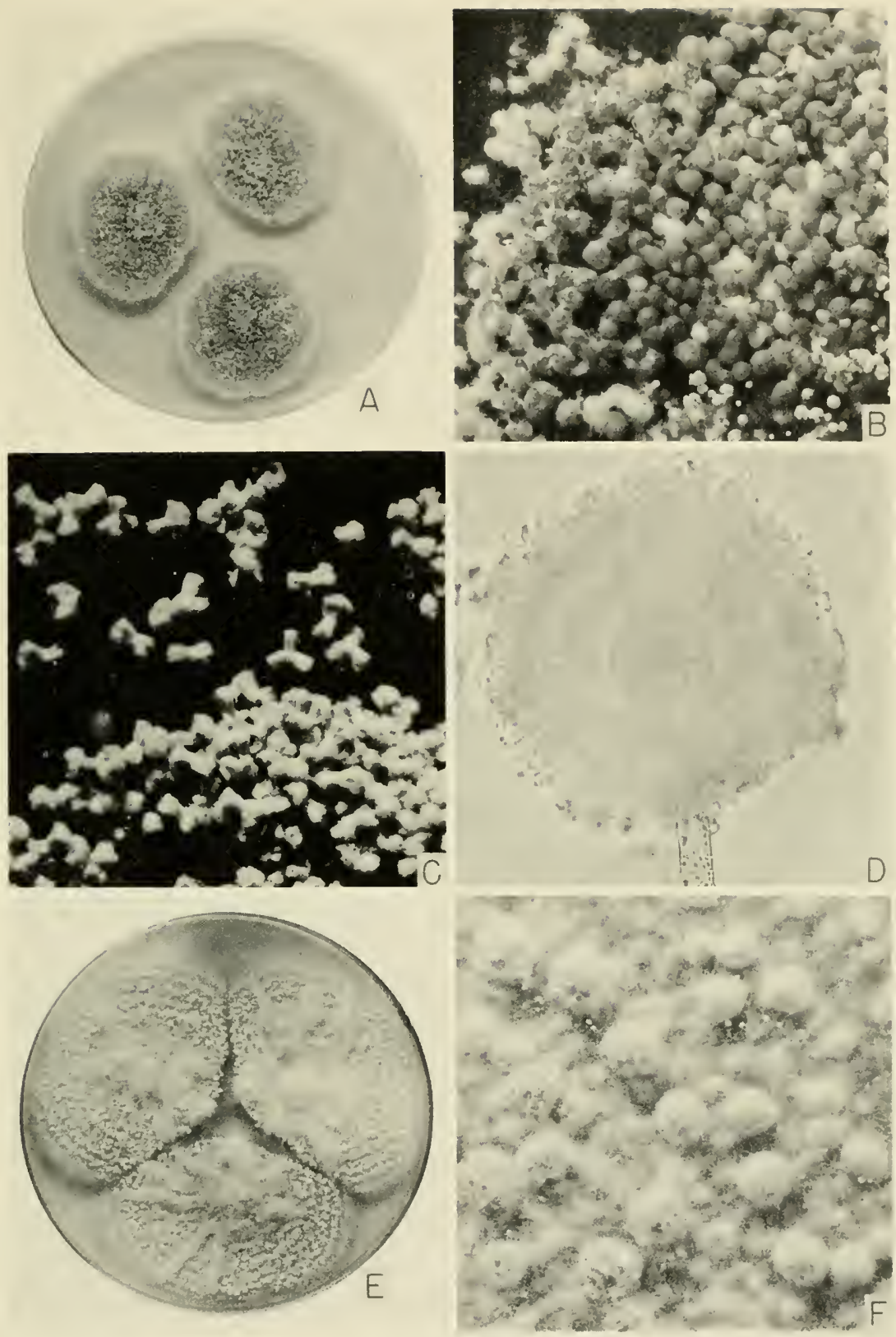

FIg. 74. A-D, Aspergillus quercinus, NRRL No. 394: A, Colonies on Czapek's solution agar, 10 days, predominantly selerotial but with localized areas of heavy conidial production in central areas. $B$, Marginal area of the same colony showing very abundant sclerotia, and seattered conidial heads in lower right-hand corner. $C$, Conidial heads on hay infusion agar plate, $\times 18$. D, Single head showing globose character, crowded sterigmata, and roughened conidiophore. $E$, Aspergillus sclerotiorum on Czapek's solution agar, 10 days. F, Portion of the same enlarged to show abundant large sclerotia and scattered comparatively small heads, $\times 6$. 
the entire surface. Sterigmata in two series: primary 10 to 20 or even $30 \mu$ in larger heads by 2 to 4 or $7 \mu$ at the tips; secondary 10 by 2 to $2.5 \mu$. Conidia 2.5 to $3 \mu$ by 3 to $3.5 \mu$ to almost globose, very nearly colorless in mounts, smooth, thin-walled. Sclerotia white to brick red, up to $500 \mu$ in diameter (fig. $7+\mathrm{B}$ ).

The original culture as described by Bainier in 1880 was not preserved. Neither was that of Sartory in 1910. However, cultures of organisms varying about as here described and complying fairly closely with Bainier's description are not infrequently encountered from widely separated regions.

Aspergillus sachari Chaudhuri and Sachar, in Amn. Myc. 32: 95. 1934.

This organism was placed by the describers near A. quercinus on the basis of the colors of the head and sclerotia. They ignored the colorless, smooth conidiophores which, if correctly observed, would make their placing untenable. It is left here arbitrarily until rediscovered and its characters verified. Compare: A. candidus group.

Species characterization based upon the original description follows:

Colonies on Czapek's solution agar naphthalene yellow, (Ridgway, PI. XYI. 23, yellow, f.) more or less floccose; reverse colorless at first then in yellow shades. Sclerotia scattered through the colony, quickly developed, hard, white through yellow shades to cinnamon, up to $2 \mathrm{~mm}$. in long axis with a depression in the center. Heads abundant, radiate, 50 to $85 \mu$ in diameter, or somewhat columnar in age up to 160 by $100 \mu$. Conidiophores 700 to $900 \mu$ by about $8 \mu$ with smooth colorless walls, about $1.5 \mu$ thick; vesicles globose, 20 to $30 \mu$ in diameter; sterigmata in two series, covering the whole vesicle, radiate; primary 5.4 by $2 \mu$, secondary 7.2 by $1.8 \mu$, conidia colorless, smooth, globose, 2 to $2.7 \mu$ in diameter. Found in alkaline soil.

\section{Aspergillus sclerotiorum Huber, in Phytopathology 23 (3): 306-8, Fig. I. 1933.}

Colonies on Czapek's solution agar rather slow growing, forming a smooth mycelial layer irregularly overgrown with loose tufts of sterile hyphae within which numerous sclerotia develop and often dominate the colony appearance (fig. $74 \mathrm{E}$ ), white to shades of yellowish or ochraceous; reverse cream. Conidial heads given as sulphur yellow by Huber, but in our cultures found in ochraceous shades such as cream-buff (Ridgway, Pl. XXX), mostly appearing as columnar masses of conidia up to $250 \mu \mathrm{long}$, sometimes splitting, 60 to $70 \mu$ at the base and up to $125 \mu$ at the apex. Conidiophores with walls yellow and pitted, commonly 200 to $400 \mu$ long by 7 to $10 \mu$ in diameter, but varying from very short, $50 \mu$ in length, to occasional groups up to $1200 \mu$; in old cultures, arising as branches from loose aerial hyphae. Vesicles subglobose, 15 to $20 \mu$ in long axis. Sterigmata in two series, primary usually 8 to $9 \mu$, occasionally much more but not above $20 \mu$ long by 3.5 to $4.5 \mu$ in diameter, secondary $\delta$ to $9 \mu$ by $2 \mu$. Conidia smooth, mostly 2 to $2.5 \mu$. Sclerotia abundant (fig. $74 \mathrm{~F}$ ), beginning to appear within 3 days, white to cream or pink, up to $1.5 \mathrm{~mm}$. in diameter, well scattered over the 
mycelium or vaguely in zones, giving the characteristic appearance to the colony, hence the name.

The type culture NRRL No. 415 (Thom No. 5351 received from Huber) was isolated from rotting apples in Oregon and proved capable of causing decay in apples under controlled conditions in which other strains failed to produce rot. It has not been discussed by others. Blochwitz (Ann. Mycol. 32(1/2): 88. 1934) considered A. sclerotiorum to be a synonym for A. elegans Gasperini, but this is not consistent with the description.

A spergillus melleus Yukawa, in Jour. Coll. Agr. Imp. Univ. Tokyo 1, No. 3, p. 366, Taf. 16. 1911.

Colonies on Czapek's solution agar rapidly growing and spreading, forming an aerial felt of sterile hyphae and conidiophores, and producing sclerotia with walls white to yellowish-brown, commonly a shade of yelloworange near "melleus" of Saccardo's Chromotaxia (Approximately Ridgway $\mathrm{Pl}$. XXX, 19", honey yellow; or warm buff, Pl. XV, 17'd); reverse reddish to brown shades. Conidial heads ranging from small to large and globose, commonly splitting into dense columnar masses, sometimes 250 to $300 \mu$ in long axis. Conidiophores with walls yellow, pitted, up to 500 or even $1000 \mu$ long, 15 to 20 or even $25 \mu$ in diameter. Vesicles up to 40 to $50 \mu$ in diameter, globose in large heads, more or less pyriform in small heads, fragile and readily crushed in mounting. Sterigmata in two series, primary 10 to $20 \mu$ by 2.5 to $4 \mu$, secondary commonly 3 to $10 \mu$ by 2 to $3 \mu$, with occasional larger sterigmata in either series. Conidia in long chains, almost colorless when mounted, very thin-walled, smooth, slightly elliptical, about $3 \mu$ or a little more in long axis. Sclerotia ranging from 400 to $700 \mu$ in diameter, in yellow-brown shades.

Hanzawa furnished Thom's culture No. 4291.6 (NRRL No. 416) as the type strain used by Yukawa. Other strains, including cultures from Formosa, South Africa, and the United States, indicate that the cultural aspect of Yukawa's species is carried by organisms widely distributed. Some related strain may have been described by Tiraboschi as A. ochraceus var. microspora (in Ann. di Botanica VII. 14. 1908) from corn bread in Italy. This name is used also by Nakazawa (Jour. Agr. Chem. Soc. Japan 10: English summary p. 185. 1934).

Aspergillus ochraceus Wilhelm, in Inaug. Diss. Strassburg, p. 66. 1877. Wilhelm's exsiccati exist as Rabh. Fl. Europaei no. 2361.

Colonies upon Czapek's solution agar spreading fairly broadly, usually plane, zonate in some strains, characterized by a tough submerged felt which may be colorless, yellow, orange, or purplish and, arising from this, more or less crowded conidial structures which give to the colony its characteristic 
color and appearance (Pl. VII F and fig. $75 \mathrm{~A}$ ); reverse ranges from colorless through orange to purplish shades.

Conidial heads when large mostly globose or variously splitting into masses of conidial chains (fig. $75 \mathrm{C}$ ), variously colored from very pale to deep ochraceous shades. Conidiophores vary greatly in length and diameter
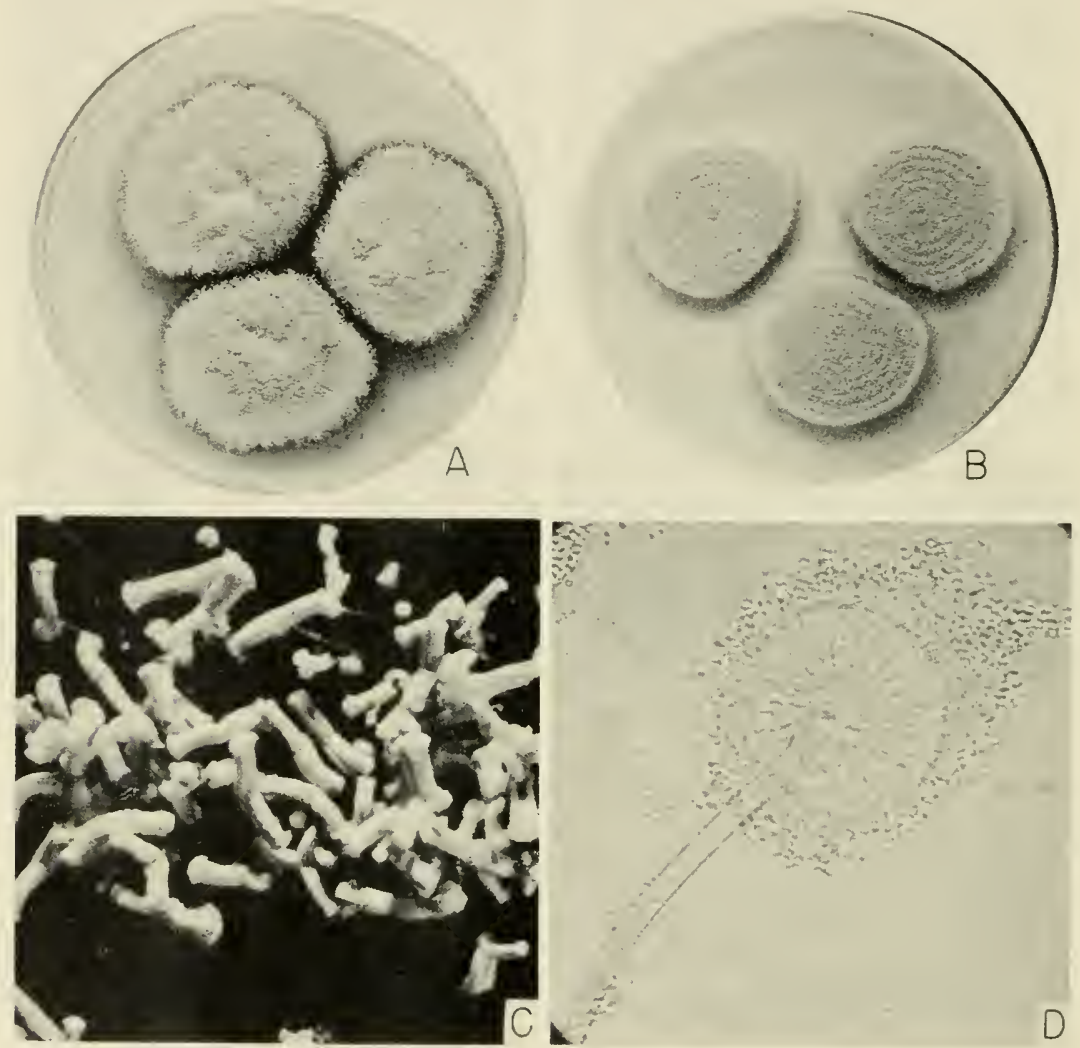

FIG. 75. Aspergillus ochraceus series. $A$ and $B, A$. ochraceus, NRRL No. 398 and No. 408, respectively, growing on Czapek's solution agar at room temperature, 10 days. C, Mature heads of strain No. 398; the tendency to split into divergent columns in age is characteristic. $D$, Photomicrograph of a single head showing globose vesicle, sterigmata in two series, and coarsely roughened conidiophore, $\times 325$.

in the various strains, but typically show a yellow color in the outer layers of the thick wall which shows characteristic pitting or roughening (fig. $75 \mathrm{D})$. Vesicles mostly globose or somewhat elliptical, and fertile over the whole surface (fig. 75 D). Sterigmata in two series: primary varying from small to very large, commonly 15 to $30 \mu$ in length; secondary fairly uniform, 
commonly 7 to $10 \mu$ by 1.5 to $2.5 \mu$. Conidia globose, subglobose, or somewhat elliptical, more or less rough or echinulate, ranging from 3.5 to $5.0 \mu$ in long axis. No perithecia reported. Sclerotia are present in Wilhelm's material preserved in exsiccati.

Among the great number of isolates belonging to this group a considerable number show approximately the characters as drawn from Wilhelm's description and his exsiccati. In the limited sense then, A. ochraceus Wilhelm can be interpreted to apply to those ochraceous strains which bear echinulate conidia from 3.5 to $5.0 \mu$ in diameter and produce sclerotia. Among the strains included, almost all are found to produce colonies of consistent aspect in continuous culture.

Aspergillus elegans Gasperini, in Atti. Soc. Toscana Sci. Nat. Pisa Mem., 8: 328, fasc. 2. 1887.

Synonym: S. elegans (Gasp.) Sacc., in Syll. 10: 525.

This species by description differs little from $A$. ochraceus Wilhelm except for the absence of sclerotia, and conidia which do not exceed $3.5 \mu$ in diameter. Thom and Church did not recognize it as a valid species in 1926, and one may be certain that no sharp line can be drawn separating A. elegans from A. ochraceus. Nevertheless, forms producing conidia consistently less than $3.5 \mu$ are commonly encountered among miscellaneous isolations from nature, and there is an argument for retaining a species to include such forms. The following species diagnosis, taken from Saccardo (10: 525) was presented by Thom and Church (1926):

"Mycelium white; stalks continuous, unbranched, hyaline then pale ochraceous, 1 to $6 \mathrm{~mm}$. long, by 5 to $12 \mu$ in diameter, delicately studded with drops; vesicle up to $70 \mu$ diameter, radiate, entirely covered with sterigmata; sterigmata, primary 4 to $26 \mu$ long, secondary 7 to $14 \mu$ long by 1 to $2 \mu$; conidia ochraceous, elliptical to globose, up to 3 to $3.5 \mu$, with wall very delicately verruculose, sclerotia not found."

Various other species have been described which are obviously closely related and belong to the $A$. ochraceus series. A partial list would include:

S. helva Bainier, in Bull. Soc. Bot. France 28: 78. 1881. Thom's culture No. $46+0.476$, received under this name, came from the Bainier collection.

A. alutaceus Berkeley and Curtis (in Grevillea 3, No. 25, p. 108. 1875) was the name proposed for a mold found upon corn. The specimen is preserved as No. 3793 in Curtis' Herbarium now in the Cryptogamic Herbarium of Harvard University. The specimen shows that this species was probably a strain of A.ochraceus.

A. ochraceus var. microspora Tiraboschi (in Ann. di Bot. [Rome] 7: 14. 1908) represents a strain in which all measurements were reported as reduced.

A. rehmii Zukal. A culture from Dr. Westerdijk, received under this name, is also a member of this series. 
Certain large-spored species of doubtful validity and relationship have been described that are believed to represent members of the $A$. ochraceus series. Although obviously rare, these species were described with sufficiently distinctive characters to warrant their retention. With continued search, it is possible that they may be reisolated.

Aspergillus delacroixii (Sace.) Thom and Church, in The Aspergilli, p. 190.1926.

Synonym: S. ochracea Delacroix, in Bull. Soc. Myc. France 7: 109, Pl. VII, fig. f. 1891. (Delacroix failed to recognize the previous use of the specific name.)

S. delacroixii Sacc., in Sacc. 10: 527.

This species is reported as having conidiophores pale yellow and rough, 500 to $1000 \mu$ in length; vesicle globose, thick-walled, punctate, yellow; sterigmata in two series, primary $39 \mu$ by $12 \mu$, secondary (from Delacroix's figures) about 8 to $10 \mu$ by 2 to $3 \mu$; conidia globose, finely roughened, 7 to $8 \mu$ in diameter. The yellow and roughened conidiophores ally this species with the A. ochraccus group; hence would justify the description, if a form with such large conidia should be found again. Otherwise, it is possible that some old material of a strain of $A$. oryzae might have furnished the type.

\section{Aspergillus butyracea (Bainier) n. comb.}

Synonym: S. butyracea Bainier, in Bull. Soc. Bot. France 27: 29. 1880.

Specimen attributed to Bainier in C. Roumeguere's Fungi

Gallici Exsiccati No. 995.

This is described as a large-spored strain belonging in this group. The specimen showed a black Aspergillus, as well as an ochraceous form with spores up to about $6 \mu$ and rough. Colonies butter yellow, including conidiophores, heads, and conidia; conidiophores yellow, in mounts finely punctate or pitted, 13 to $16 \mu$ in diameter; sterigmata primary up to $25 \mu$, secondary 10 to $12 \mu$ in length; conidia described as smooth $5.2 \mu$, but those found in the material were rough and up to $6.3 \mu$. It is entirely possible that this species may be found again, but is has not been reported since Bainier described it.

A. penicillopsis (Henngs.) Racib., P. Hennings Synonym Stilbothamnium penicillopsis P. Henn. and E. Nym. described in Fungi Monsunenses (Warburg. O. Monsunia, Bd. I, p. 37. 1900. Leipzig); exsiccati of type in Pathological Collections, U. S. Dept. Agr. Bureau of Plant Industry, as: Raciborski no. 87, in Crypt. Parasiticae Java.

This material shows an Aspergillus with the color and general appearance of A. ochraceus but of gigantic proportions. Measurements as follows: 
Conidiophore 50 to $70 \mu$ in diameter, $10 \mathrm{~mm}$. or more long with walls 7 to $12 \mu$ thick. Surface ragged and more or less pitted. Vesicle up to $175 \mu$ in diameter with walls $7 \mu$ thick, marked with a deep pit for each sterigma. Sterigmata, primary 50 to $90 \mu$, at times 120 by 8 to $10 \mu$ at the outer end, sometimes with one cross wall; secondary 15 to $25 \mu$ by 3 to $4 \mu$, clustered on the apex of the primary. Occasionally with a sterile cell interposed between secondaries and primaries. Conidia 8 to $12 \mu$ by 5 to $8 \mu$, elliptical, pitted, yellowish.

Gigantic forms such as this occasionally appear under field conditions, hence reach fungus herbaria. Whether these are really different from some of the usual species can only be determined by collection and laboratory cultivation. Until such study has been made, such forms as A. penicillopsis must be questioned.

Aspergillus ostianus Wehmer, in Bot. Centralb. 80: 449-461. 1899; also Monogr. pps. 117-119, Taf. II, No. 1. 1899-1901.

Colonies upon Czapek's solution agar growing fairly well, producing a surface growth of crowded conidiophores and conidial heads in yellowish to ochraceous shades, passing to shades of cinnamon in very old cultures with reddish-brown colors in reverse (Wehmer reported rusty-yellow, pale to deep brownish-yellow to cinnamon). Conidial heads globose, up to $200 \mu$ in diameter. Conidiophores yellow, coarsely roughened, mostly 500 to $700 \mu$ long in crowded areas, becoming 1 to $2 \mathrm{~mm}$. in margins of old colonies and usually 7 to $10 \mu$ in diameter, with walls heavy, up to 2 or $2.5 \mu$ in thickness. Vesicles commonly globose, about $40 \mu$ in diameter, occasionally much larger up to $70 \mu$, in growing heads thin-walled, colorless, crushing easily, leaving the funnel-like yellow tip of the conidiophore open. Sterigmata in two series: primary from 15 to $20 \mu$ long in smaller heads to $35 \mu$ by about $8 \mu$ at the tip in large heads, secondary 10 to $13 \mu$ by about $3 \mu$. Conidia commonly 3 to $4 \mu$ or even $5 \mu$ in long axis, varying from pyriform to elliptical, or at times subglobose, rough. Sclerotia occasionally present but not conspicuous.

This diagnosis was based upon Wehmer's description, and is reasonably well represented by strain NRRL No. 420 (Thom No. 4724.35) received from Raistrick in 1924 and reported by him to have come from Westerdijk as Wehmer's original strain. With conspicuous appearances suggesting relationship to the $A$. ochraceus group, the shape and ultimate color of the ripe spores suggest a border line position between $A$. ochraceus and $A$. tamarii.

Aspergillus sparsus Raper and Thom, in Mycologia 36: 572-5it, fig. 6. 1944 .

Colonies upon Czapek's solution agar at room temperature spreading broadly, dull grayish-brown in color, at first largely submerged, but later 
developing limited aerial growth, giving rise to widely scattered erect conidial struetures (fig. $76 \mathrm{~A}$ ) characterized by dull greenish-tan heads not affecting the color of the colony as a whole; reverse in brown shades; odor none. Colonies upon hay infusion agar spreading broadly, almost wholly submerged, giving rise to seattered but conspicuous conidial structures (fig. $76 \mathrm{~B}$ ), often in definite concentric zones; heads globose, radiate, in olive-
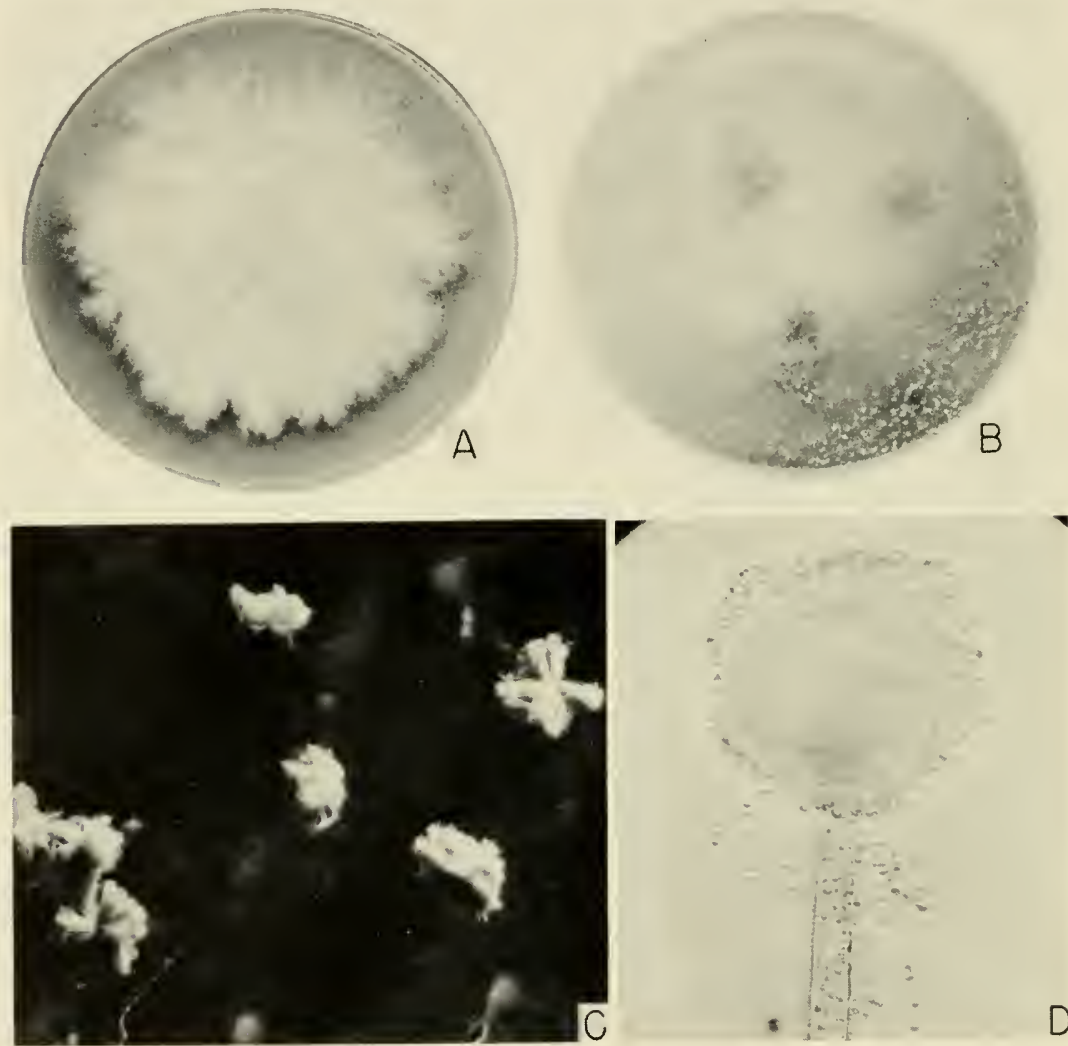

FIg. 76. Aspergillus sparsus: $A$ and $B$, Colonies on malt extract and hay infusion agars, respectively, 2 weeks; note seattered heads in $B$ and almost complete absence in $A$. C C Conidial heals, $\times 18$. $D$, Single conidial head showing globose vesicle and roughened conidiophore, $\times 425$.

buff shades (Ridgway, Pl. XL). Colonies upon malt extract agar spreading irregularly, floceose, 1 to $2 \mathrm{~mm}$. deep, cream-buff in color; conidial structures very few in number; heads globose, radiate, dull olive-yellow in color (Ridgway, Pl. XL). Conidial structures generally few in number, never abundant, erect, arising from a submerged mycelium; heads typieally globose becoming more or less radiate in age (fig. $76 \mathrm{C}$ ), mostly 200 to $250 \mu$ 
in diameter, occasionally as much as $500 \mu$, pale olive-buff to olive-buff (Ridgway, Pl. XL) upon Czapek and hay infusion agars to pale olivine or olivine (Ridgway, Pl. XXXII) upon malt extract agar. Conidiophores straight, mostly 1 to $1 \frac{1}{2} \mathrm{~mm}$. in length by 10 to $12 \mu$ in diameter, approximately uniform in diameter throughout, wall 1.2 to $1.5 \mu$ in thickness, conspicuously echinulate, typically arising from a foot cell enmeshed in a network of "feeder" hyphae, often tapering abruptly in the region immediately beneath the resicle. Vesicle comparatively thin-walled, globose (fig. $76 \mathrm{D}$ ), mostly 40 to $50 \mu$ in diameter, occasionally larger or smaller, bearing sterigmata over the entire surface. Sterigmata in two series, primaries crowded, comparatively short and stout, commonly 8 to $10 \mu$ by 3 to $5 \mu$, secondaries 6 to $8 \mu$ by 2.5 to $3.5 \mu$. Conidia pale yellowish in mass, individually showing slight coloration, subglobose to slightly elliptical, very finely roughened, mostly 3 to $3.5 \mu$ in long axis.

Type culture NRRL No. 1933 was isolated in February 1943, from soil collected in La Lima, Honduras, by Dr. L. A. Underkofler. A second strain which duplicates the type almost exactly was subsequently isolated from soil collected in Bixar County, Texas, by Sister Mary Clare of Our Lady of the Lake College, San Antonio, Texas.

The correct position of this species within the genus Aspergillus is open to question. The presence of a colored, coarsely roughened conidiophore indicates close relationship with Aspergillus ochraceus. This is likewise supported by the globose vesicle and head, although these characters are typical of other groups as well. The scarcity of fruiting structures upon all media, and more particularly the greenish tint of the spore masses, however, tend to set it apart from the common representatives of this great group. The general habit of the colonies together with the paucity of conidial structures is strongly suggestive of Aspergilius alliaceus, but this latter species does not show any trace of greenish color in its conidial heads; it does possess smooth, colorless conidiophores, and upon ordinary culture media regularly produces an abundance of black sclerotia which very often dominates and characterizes the culture. In the color and character of its conidial heads $A$. sparsus is somewhat suggestive of George Smith's new species, A. avenaceus (Trans. Bul. Mycol. Soc. 25: 24-27, Pl. I. 1943), but it differs from this, as it does from $A$. alliaceus, in possessing rough conidiophores and in its failure to produce sclerotia. Until additional related forms are isolated, we believe it best to consider this species as a member of the A. ochraceus group, realizing that it does not entirely fit this placement as the group has hitherto been considered.

\section{Occurrence and Economic Importance}

Members of the $A$. ochraceus group are widely distributed in nature and can be obtained from a variety of sources. They are especially common 
in soils and have been isolated from samples colleeted in many parts of the world. Within the group, strains approximating the species $A$. ochraceus are by far the most abundant, although heary sclerotium-producing strains approximating $A$.quercimus are not infrequently encountered. They are a common component of the microflora of deeaying vegetation, but there is little evidence that they play a very active role in processes of decomposition.

Huber (1933) found a member of the group, A. sclerotiorum, capable of rotting apples and pears. Members of the group are commonly found in musty or moldy cereal grains, but are not as characteristic of this substratum as is Aspergillus candidus and members of the A. glaucus group. In at least one instance $A$. ochraceus was reported as a human pathogen (Ceni, 1905).

In the Orient, $A$. ochraceus and allied species constitute a portion of the mold flora characteristically found on "Katsuobushi" and other fermented preparations made from fish (Yukawa, 1911). Aspergillus melleus Yukawa was isolated from such material. Beeause of the mixture of forms present, including members of the A. glaucus and A. flavus-oryzae groups, it is probably incorrect to say that any particular species or group of species is responsible for this fermentation. (See also Hanzawa, 1911).

A. ochraceus has been used to bring about desired changes in the flavor of coffee, and its use is covered by U. S. Patent No. 1,313,209. Samples of the fermenting coffee showed the organism used to be a strain of $A$. ochraceus indistinguishable from Wilhelm's species. Whereas A. niger, A. tamarii, and A. flavus were also capable of developing in the fermenting coffee, $A$. ochraceus alone of the species tried gave a satisfactory flavor.

As a whole, the $A$. ochraceus group corsstitutes a very abundant, but little studied, group of molds. Whether the scareity of published reports regarding biochemical activities indicates an absence of such, or whether it merely reflects a limited amount of investigation, ean at present only be guessed. 
PART III

REFERENCE MATERIAL 



\section{Chapter XXII TOPICAL BIBLIOGRAPHY}

In preparing this manual, the writers have considered it inadvisable to attempt to discuss the biochemical activities of the Aspergilli, since this subject is book-length in itself. Furthermore, the aim of the manual is to provide the mycologist and microbiologist with a means of identifying and interpreting Aspergilli as they are isolated from nature and to furnish the chemist, working with molds, with a guide which will enable him to maintain industrially important cultures in an optimum condition. Nevertheless, because of the increasing importance of the Aspergilli as agents responsible for industrial fermentations, as subjects for physiological and biochemical investigations, and now as possible sources of various antibiotics, it has seemed advisable to present a topical bibliography dealing with these and related subjects. We have not attempted to present a complete bibliography, but rather to present a sufficient number of references to provide the investigator and student with an entrée to the literature of particular fields. An attempt has been made to choose the more important papers for citation. Believing that more recent contributions will generally be of the greatest interest and value to the user, this topical bibliography is presented in chronological, rather than alphabetical, order. A list of subjects under which references are presented follows.

CONTENTS OF TOPICAL BIBLIOGRAPHY

Acid Production by Aspergilli................................... 290

Citric Acid ......................................... 290

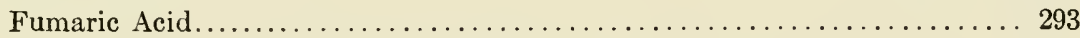

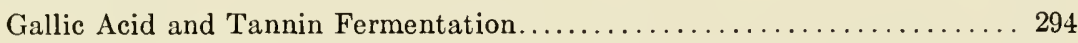

General Papers and Reviews.............................. 294

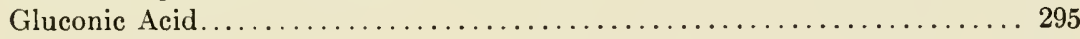

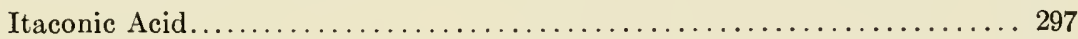

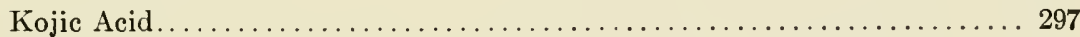

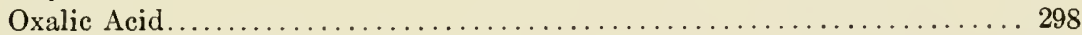

Miscellaneous Acids.................................... 299

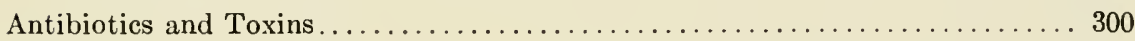

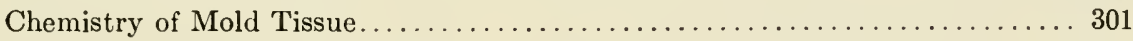

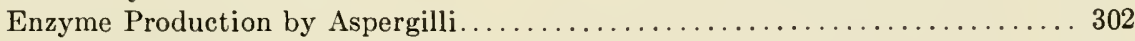

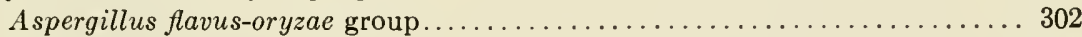

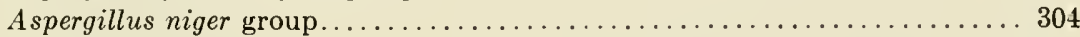

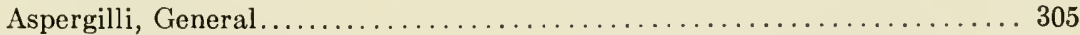

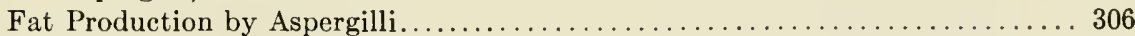

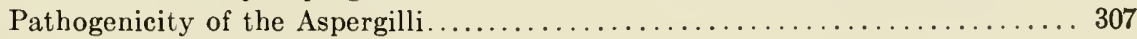

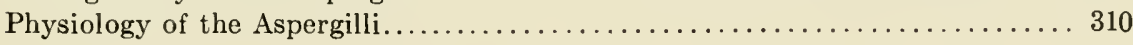


Pigments and Coloring Substances........................... 312

Soil Tests for Mineral Deficiencies............................ 313

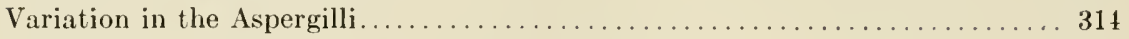

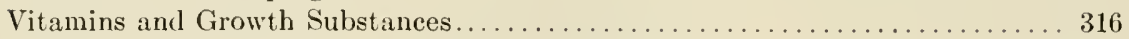

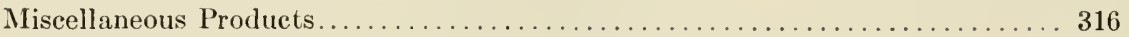

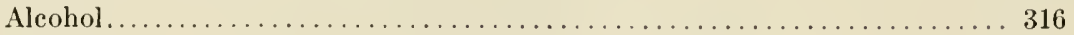

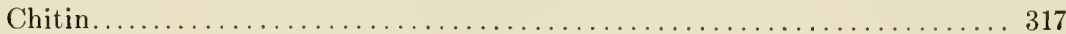

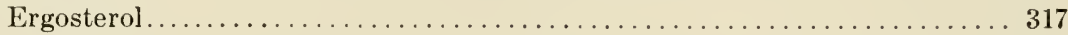

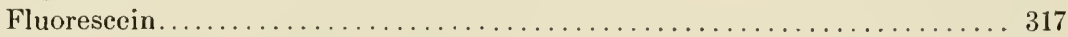

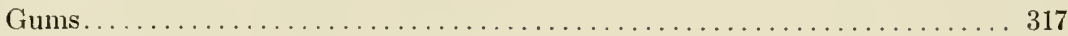

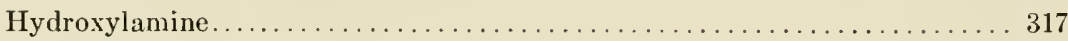

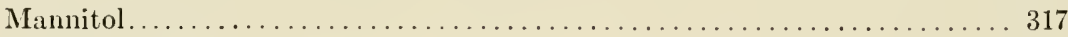

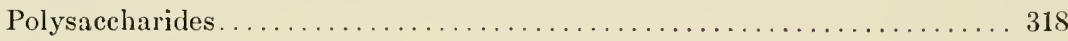

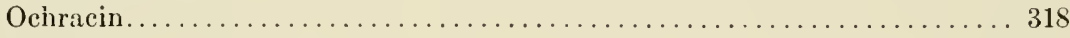

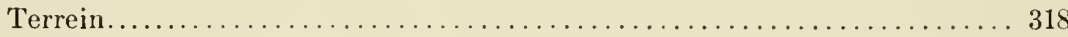

\section{Acid Production}

Citric Acid

Wehmer, C. 1893. Note sur la fermentation citrique. Bull. Soc. Chim. France 9: $728-730$.

Weнner, C. 1893. Préparation d'acide citrique de synthèse, par la fermentation du glucose. Compt. Rend. 117: 332.

Wehmer, C. 1894. Process of making citric acid. U. S. Patent 515,033. February 20 .

Wehmer, C. 1897. Über Zwei weitere freie Citronensäure bildende Pilze. Chem. Ztg. 21: 1022-1023.

Wenmer, C. 1912. Über Citronsäuergärung. Chem. Ztg. 36: 1106-1107.

Currie, J. N. 1917. The citric acid fermentation of Aspergillus niger. Jour. Biol. Chem. 31: 15-37, pls. 2, figs. 5.

Molliard, M. 1919. Production d'acide citrique par le Sterigmatocystis nigra. Compt. Rend. Acad. Sci. (Paris) 168: 360-363.

Butkewitsch, W. 1923. Über die "Citronensäuregärung." Biochem. Ztschr. 142: $195-211$.

Molliard, M. 1924. Influence de la nature des sucres sur la formation d'acides organiques par le Sterigmatocystis nigra en milieu déséquilibré. Compt. Rend. Soc. Biol. (Paris) 90: 1395-1397.

WeHmer, C. 1925. Bildung von citronensäure aus glykonsäure durch pilze. Ber. Deut. Chem. Gesell. 58: 2616-2619.

Bernhauer, I. 1926. Über die Säurebildung durch Aspergillus niger. III. Die Bedingungen der Zitronensäurebildung. Biochem. Zeitschr. 172: 324-49.

Henneberg, W. 1926. Handbuch der gàrungsbakteriologie, 2 v., illus. Berlin.

Amelung, H. 1927. Beiträge zur Säurebildung durch Aspergillus niger. Zeit. Physiol. Chem. 166: 161 .

Challenger, F., Subramanian, V. and Walker, T. K. 1927. The mechanism of the formation of citric and oxalic acids from sugar by Aspergillus niger. Jour. Chem. Soc. (London) 200-208.

Kostytschev, S. AND Tchesnokov, W. 1927. Bildung von Zitronensäure und Oxalsäure dureh Aspergillus niger. Planta, Abt. E. Zeits. Wiss. Biol. 4: 181-200. 
Walker, Th. K., Subramaniam, V. and Challenger, F. 1927. The mechanism of the formation of citric and oxalic acids from sugars by Aspergillus niger.

II. Jour. Chem. Soc., London, 3044-54.

Bernhauer, K. 1928. Zum Chemismus der Citronensäurebildung durch Pilze. I. Die Säurebildung aus verschiedenen Kohlenst off verbindungen. Biochem. Zeitschr. 197: 309-26.

Bernhauer, K. 1928. Zum Chemismus der Citronensäurebildung durch Pilze. II. Die Citronsäurebildung aus Gluconsäure. Biochem. Zeitschr. 197: $327-42$.

Bernhauer, K. and Schön, K. 1928. Zum Chemismus der Citronensäurebildung Durch Pilze. III. Mitteilung: Úber die Hypothesen Citronensäurebildung und das Auftreten von Acetaldehyd in den Pilzkulturen. Biochem. Ztschr. 202: 164-179.

Bernhauer, K. 1928. Beiträge zur Enzymachemie der durch Aspergillus niger Bewirkten Säurebildungsvorgänge, I. Ztschr. Physiol. Chem. 177: 86-106.

Bernhauer, K. 1928. Über die Säurebildung bei Aspergillus niger. IV. Die Bedeutung der Myzelent wicklung für die Säurebildung. Biochem. Zeitschr. 197: $287-308$.

Fernbach, A. And Yuill, J. L. 1928. Improvement in and relating to the processes for the production of citric acid. U. S. Pat. 1,691,965 and 1,691,966.

Filosfov, M. S. ANd Malinorskit, T. E. 1928. O limonno-kislom drozenii. Nauchnuie Zapiski 5: 235-239.

SzC̈cs, J. 1928. Process for producing citric acid by means of fermentation. Canad. Pat. 251,180. 1925, and U. S. Pat. 1,679,186.

CAHN, F. J. 1929. Perfectionnements à la fabrication de l'acide citrique par fermentation. Fr. Pat. 675,236 and 675,237.

Challenger, F. 1929. The production of citric acid by fermentation process. Ind. Chem. 5: 181-184.

Amelung, H. 1930. Wachstum und Säurebildung von Aspergillus niger unter Wasser. Chem. Ztg. 54: 118.

Chrzaszcz, T. AND Tiukow, D. 1930. Biochemische Umbildungen der Essigsäure durch Schimmelpilze und über den Chemismus der Citronensäurebildung. Biochem. Zeit. 229: 343.

Bernhauer, K., Siebenäuger, H. And Tschinkel, H. 1931. Zum Chemismus der Citronensäurebildung durch Pilze. IV. Über die Umwandlung der Zuckersäure. Biochem. Ztschr. 230: 466-474.

Bernhauer, K. and Siebenäuger, H. 1931. Zum Chemismus der Citronensäurebildung durch Pilze. V. Die Citronensäurebildung aus Essigsäure. Biochem. Zeit. 240: 232-44.

TAYlor, J. N. AND Boyd, R. G. 1931. United States citric acid industry now selfsufficient. U. S. Dept. Com., Bur. Foreign and Dom. Com., Com. Rpt. 4: $227-228$.

Bernhauer, K. 1932. Die oxydative Gärungen. Verlag Julius Springer, Berlin. MAY, O. E. ANd Herrick, H. T. 1932. Production of organic acids from carbohydrates by fermentation. U. S. Dept. Agr. Circular 216.

Porges, N. 1932. Citric Acid Production by Aspergillus niger. Am. Jour. Bot. 19: $559-567$.

Sakaguchi, K. ANd Yamaya, J. 1932. Production of citric acid by molds. III. Japanese black molds. Jour. Agr. Chem. Soc. Japan 8: 489-97.

Kostytschev, S. AND Berg, Y. 1933. Conditions of Biochemical Production of Citric Acid. Bull. State Inst. Agr. Microbiol. USSR 5: 8-27. 
Butkewitsch, W. S., Menzschinskaja, E. W. And Trofimova, E. I. 1934. Zur Biochemischen Herkunft von Citronen-und Oxalsäure. I. Utber die Bildung von Citronensäure aus Essigsäure. Biochem. Zeit. 272: 290. II. Die Mycelsubstanzen als ein Quelle der Säurebildung. Biochem. Zeit. 272: 364. Doelger, W. P. and Prescott, S. C. 1934. Citric Acid Fermentation. Ind. Eng. Chem. 26: 1142-49.

Sotnikov, E. 1934. Production of citric acid by Aspergillus niger. I. Production of citric acid in unchanged solutions. Compt. Rend. Acad. Sci. USSR 3(4): 273.

Sotnikov, E. 1934. Production of citric acid by Aspergillus niger. II. Production of citric acid in changed solutions. Compt. Rend. Acad. Sci. USSR 3(4): $279-83$.

Sotnikov, E. 1934. Production of citric acid by Aspergillus niger. III. Production of citric acid in often-changed solutions. Compt. Rend. Acad. Sci. USSR 3(7): 544-7.

Zubiova-Gitler, S. R. ANd Zalts, R. M. 1934. Preparation of Citric Acid from Wood-Pulp Hydrolysates. Lesokhimicheskaya Prom. 3: 4-7.

Butkewitsch, V. S. And Gaewskaya, M. S. 1935. Yield of citric acid from sugar as a basis for estimating the mechanism of its formation from the latter. Compt. Rend. Acad. Sci. U.S.S.R. (N.S.) 3: 405-408. Abstracted in Chem. Abstr.

CahN, F. J. 1935. Citric acid fermentation on solid materials. Ind. Eng. and Chem. 27: 201-204.

Chrzaszcz, T. And Perros, E. 1935. Optimale Bedingungen der Citronensäureanhaufung, sowie einiger Beobachtung $\mathrm{zu}$ Theorie der Citronsäurebildung. Biochem. Ztschr. 280: 325-336.

Giordani, M. 1935. Fermentazione citrica. La Chimica e 1'Industria 17: 77-81. Gudlet, M. A. 1935. Gasregime of Aspergillus niger as related to citric acid formation. Proc. Inst. Sci. Res. Food Ind. (Leningrad) 3, No. 1: 45-69. Abstracted in Chem. Abst.

Ivanov, N. N. 1935. Biochemical production of citric acid. Proc. Inst. Sci. Research Ind. (Leningrad) 3, No. 1, 3-4. Practical and theoretical studies.

Kresting, E. K. 1935. Effect of radium on citric acid formation by $A$. niger. Proc. Inst. Sci. Res. Food Ind. (Leningrad) 3(4/5): 130-145. No marked effect.

Bernhauer, Ḱ. And Iglauer, A. 1936. Über die Säurebildung aus Zucker durch Aspergillus niger. VI. Faktoren der Citronensäureanhaufung. Biochem. Ztschr. 286: 45-59.

Cahn, F. J. 1936. Citric Acid Manufacture. U. S. Patent 2,047,669. July 14.

Chrzaszcz, T. ANd M. ZaKomonny. 1936. Über die Bedeutung der Äpfelsäure bei der Umbildung der Essigsäure in Citronensäure durch verschiedene Schimmelpilze. Biochem. Zeit. 285: 348-355.

Clutterbuck, P. W. 1936. Recent developments in the biochemistry of moulds. Jour. Soc. Chem. Ind. 14: 55T-61T.

Ivanov, N. N. 1936. Biochemical production of citric acid. Proc. Inst. Sci. Research Ind. (Leningrad) 3, No. 4, 5. Laboratory and semiplant stages to commercial production.

Lamb, A.R. 1936. Utilization of (Cane) Molasses. Proc. Hawaiian Sugar Planters' Assn. 56th meeting, 42-3.

Wells, P. A., Moyer, A. J. And MaY, O. E. 1936. The chemistry of the citric acid fermentation. I. The carbon balance. Jour. Amer. Chem. Soc. 55: 555-558. 
Nakazawa, R., Takeda, Y and Nakano, M. 1937. Citric Acid Manufacture by Fermentation. I. Aspergillus awamori var. fumeus. Jour. Agr. Chem. Soc. Japan 13: 52-62.

L'vov, S. And Toupizina, G. M. 1938. Effect of sodium fluoride on the formation of citric and gluconic acids by the fungus Aspergillus niger. Compt. Rend. Acad. Sci. (USSR) 21: 307-11.

Wells, P. A. And Herrick, H. T. 1938. Citric Acid Industry. Ind. and Eng. Chem. 30: 255-262.

Zender, J. 1938. Production of Citric Acid. U. S. Patent 2,121,063. June 21.

Bernhauer, I. 1939. Gärungschemisches Praktikum. Zweite Ouflage. pp. 317. Verlag Julius Springer, Berlin.

Zhuravski1, G. I. 1939. The Gas Exchange in Aspergillus niger during the Formation of Citric Acid. Microbiology 8: 414-430. (USSR)

Berhauer, K., KNobloch, H. And Zippelius, O. 1940. Über die Säurebildung aus Zucker durch Aspergillus niger. VIII. Der Einfluss von Magnesium auf die Säurebildung. Biochem. Ztschr. 303: 300-307.

Das Gupta, G. C., Saha, K. C. And Guha, B. C. 1940. The fermentative production of citric acid and oxalic acid from molasses. J. Indian Chem. Soc., Ind. and News Ed. 3: 64-74.

Mel'nikova, A. A. And Trofinova, E. I. 1940. Activation of the process of citric acid accumulation by surface molds of Aspergillus niger. Microbiology (USSR) 9: 558-68.

Prescott, S. C. and Dunn, C. D. 1940. Industrial Microbiology. Chapt. XXV: The Citric Acid Fermentation, pp. 358-379. McGraw Hill, N. I.

WANG, Y. 1940. Citric acid metabolism of Aspergillus carbonarius. Jour. Shanghai Sci. Inst., Sect. IV. 5: 61.

Barinova, S. A. 1941. Considerations in the appraisal of the circulating-medium system of obtaining citric acid from cultures of Aspergillus niger. Microbiology 10: 854 . (USSR)

Bernhauer, K., Iglauer, A., And Knobloch, H. 1941. Über die Säurebildung aus Zucker durch Aspergillus niger. X. Der Einfluss der Zuckersorte auf die Citronensäurebildung. Biochem. Zeit. 307: 298.

Karow, Edward Otto. 1942. The Production of Citric Acid in Submerged Culture. Doctoral Thesis submitted to Faculty of Rutgers Univ., New Brunswick, N. J., March, 1942. Strain of Aspergillus wentii found to give good yields.

SzÜcs, Joseph. 1944. Method of producing citric acid by fermentation. U.S. Patent 2,353,771, July 18.

\section{Fumaric Acid}

Ehrlich, F. 1911. Über die Bildung von Fumarsäure durch Schimmelpilze. Ber. Deut. Chem. Ges. 44: 3737-3742.

Weнмек, C. 1918. Über Fumarsäure-Gärung des Zuckers. Ber. Deut. Chem. Gesell., 51: 1663-1668, figs. 6.

Ehrlich, F. 1919. Über Fumarsäure-Gärung des Zuckers. Ber. Deut. Chem. Ges. 52: $63-64$.

Schreyer, R. 1928. Säuerungsversuche mit dem Pilz Aspergillus fumaricus. Biochem. Zeit. 202: 131-56.

Tнгеs, W. 1930. Untersuchungen über den einflüss der bedingungen auf die säurebildung des schimmelpilzes Aspergillus fumaricus. Centralb. Bakt. (etc.)

82(2): 321-347. 
Thies, W. 1931. Decomposition of salts of organic acids by the mold fungus Aspergillus fumaricus. Ber. 64B : 214-8.

Chrzaszcz, 'T. and Zakoronorny, M. 1933. Biochemical Transformation of Sugar by Fungi. The Transformation of Fumaric Acid, the Accumulation of Formic Acid, and the Chemistry of Oxalic Acid Formation. Biochem. Zeit. 259: 156 .

\section{Gallic Acid and Tannin Fermentation}

Scheele, K. 1787. Über das wesentliche galläpfelsalz. Crell's Chem. Ann. 1: $3-12$.

Robiqcet, E. 1852. Recherches sur la fermentation gallique. Jour. Pharm. 22: 129-130.

LAROCQUE. 1852. Remarques à l'occasion d'une communication récente de M. Ed. Robiquet, sur la fermentation gallique. Compt. Rend. Acad. Sci. (Paris) 35: 221.

van Tieghem, Ph. 1867. Sur le fermentation gallique. Compt. Rend. Acad. Sci. Paris 65: 1091-1094.

Duclaux, E. 1883. Chimie biologique. In Frémy, E. Encyclopédie Chimique 9(1): 908 .

Fernbach, A. 1900. Sur la tannase. Compt. Rend. Acad. Sci. Paris 131: 12141215.

Pottevin, H. 1900. La tannase, diastase dédoublant l'acide gallotannique. Compt. Rend. Acad. Sci. Paris 131: 1215-1217.

Calmette, A. 1902. Verfahren zur Umwandlung von Tannin in Gallusäure. Ger. Pat. 129,164 .

Knudson, L. 1913. Tannic acid fermentation. I. Jour. Biol. Chem. 14: 159184 , illus.

Iinudson, L. 1913. Tannic acid fermentation. II. Effect of nutrition on the production of the enzyme tannase. Jour. Biol. Chem. 14: 185-202.

Dalvi, P. D. 1931. Biochemistry of tan-liquor fermentation. Jour. Ind. Inst. Sci. 13A: 173-92.

Nicholson, W. N. and Nierenstein, M. 1931: Action of tamnase on gallotannin. Biochem. Jour. 25: 752-5.

Deys, W. B. and Hiskman, M. J. 1937. Splitting off of gallic acid from tannin, especially from theotannin, by Aspergillus niger. Proc. Acad. Sci. Amsterdam 40: 518-25.

Chi-Laing Kuo. 1939. Researches on Fermentation for the Production of Gallic Acid. III. Effect of adding yeast. Whang-Hai 1(2): 11-19; cited C. A. 34: 2129. 1940.

Kwang-Chur Hsieh. 1939. Researches on Fermentation for the Production of Gallic Acid. IV. Optimum concentration of gallnut extract and rate of fermentation. Whang-Hai 1(3): 1-3; cited C. A. 34: 2129. 1940.

Wen-Ten Wei. 1939. Researches on Fermentation for the Production of Gallic Acid. V. Changes in tannin content, gallic acid content and total acidity in the fermentation liquor. Whang-Hai 1(3): 4-5; cited C. A. 34: 2130. 1940 .

\section{General-Acid Production}

Elfving, F. 1918-19. Über die Bildung organischer Säuren durch Aspergillus niger. Öfvers. Finska Vetensk. Soc. Förhandl., 61, Afd. A, No. 2, Art. 15, pp. 1-23. 
Falck, R. and Vax Beyma, Thoe Kingua. 1924. Methodisches und Prinzipielles zur Darstellung organischer Sauren auf biologischen Wege mit Hilfe von Fadenpilzen. Ber. Deut. Chem. Gesell. 57: 915-920.

Challexger, F., Slbramaxial, $\mathrm{Y}$. Axd Walker, T. K. 1927. Formation of organic acids from sugars by Aspergillus niger. Nature (London) 119: 674 .

Herrick, H. T. ANd MaY, O. E. 1929. Molds and Chemical Manufacture. Ind. Eng. Chem. 21: 61s.

May, O. E. Axd Herrick, H. T. 1930. Some minor industrial fermentations. Ind. Eng. Chem. 22: 1172 .

MAY, O. E. Axd Herrick, H. T. 1932. Production of organic acids from carbohydrates by fermentation. U. S. Dept. Agr. Bul. No. 216: 1-30.

SAKAgtchi, K. 1932. Acid and alcohol production by Aspergillus. VIII. The species of Aspergillus and the production of acids. Jour. Agr. Chem. Soc. Japan 8: $434-44$.

MaY, O. E. ANd Herrick, H. T. 1934. Some practical and theoretical aspects of mold metabolism. Jour. Bact. 28: 145-151.

Bervater, K. 1936. Gärungschemisches Praktikum. 249 pp., Verlag Julius Springer, Berlin.

Bershater, K. 1939. Gärungschemisches Praktikum. 317 pp., Verlag Julius Springer, Berlin.

Wells, P. A. ANd Ward, G. E. 1939. Fermentation Processes. Ind. Eng. Chem. 31: $172-177$.

\section{Gluconic Acid}

Molljard, M. 1922. Sur une nouvelle fermentation acide produite par le Sterigmatocystis nigra. Compt. Rend. Acad. Sci. Paris 174: 881-883.

Butкewtsch, W. 1924. Utber die Bildung der Glucon-mnd Citronensäure in den Pilzkulturen auf Zucker. Biochem. Zeitschr. 154: 177.

Molliard, M. 1924. Nouvelles Recherches sur la formation d'acides organiques par le Sterigmatocystis nigra en milieux deséquilibrés. Compt. Rend. Acad. Sci. (Paris) 178: 41-45.

Bershauer, K. 1926. Über die Säurebildung durch Aspergillus niger. I. Allgemeines und Methodisches bei der Untersuchung der Säurebildungsvorgänge. Biochem. Zeitschr. 172: 296-312.

Berviater, K. 1926. Über die Säurebildung durch Aspergillus niger. II. Die Bildung der Gluconsäure. Biochem. Zeitschr. 172: 313-23.

Bernhater, K. Axd Wolf, H. 1928. Zur Enzymchemie der Aspergillus niger bewirkten Säurebildungsvorgänge. II. Zeits. Physiol. Chem. 177: 270-279.

Müller, D. 1928. Studien über ein neues Enzym Glykoseoxydase. I. Biochem. Zeits. 199: 136-170.

Weнner, C. 1928. Abnahme des Säuregarungsvermögens und Änderung der Säure bei einem Pilz. (Gluconsäure statt Fumarsäure-Garung.) Biochem. Zeits. 197: $418-432$.

May, O. E., Herrick, H. T., Moyer, A. J. Axd Hellbach, R. 1929. Semiplant scale production of gluconic acid by mold fermentation. Ind. Eng. Chem. 21: 1198 .

Mïller, D. 1929. Studien über ein neues Enzym Glykoseoxydase. II. Biochem. Zeits. 205: 111-143.

Harrison, D. C. 1931. Glucose dehydrogenase; a new oxidizing enzyme from animal tissues. Biochem. Jour. 25: 1016-1027. 
SCHREYer, R. 1931. Comparative studies on the formation of gluconic acid by molds. Biochem. Zeit. 240: 295-325. A. wentii and A. luchuensis.

SAKAGUCHI, K. 1932. The production of acids and alcohol by Aspergillus. IV. The production of d-gluconic acid by Aspergillus oryzae. Jour. Agr. Chem. Soc. Japan 8: 264-265.

Angeletti, A. And Ponte, D. 1933. Arione de al cuni funghi zu soluzioni di aldosi e di altre materie zuccherine. Nota VI. Formazione di acido ossalico dall acido d'gluconico. Ann. Chim. Appl. 23: 33-37.

Currie, J. N., Kíne, J. H. and Finlay, A. 1933. Production of gluconic acid by fungi. U. S. Pat. 1,893,819.

KARDO-SYssojeva, E. 1933. The formation of gluconic acid by Aspergillus niger. Biochem. Zeit. 266: 337-351.

May, O. E., Herrick, H. T., Moyer, A. J. And Weld.s, P. A. 1934. Gluconic acid production by submerged mold growth under increased air pressure. Ind. Eng. Chem. 26: 575-578.

Bolcato, V. 1935. La reazione del mezzo e l'attivita dei feltri communi e preformati di un Aspergillo. Ann. Chim. Appl. 25: 423-432.

Herrick, H. T., Hellbach, R. and May, O. E. 1935. Apparatus for the application of submerged mold fermentations under pressure. Ind. Eng. Chem. 27: 681-683.

Kardo-Syssojeva, E. 1935. Biochemical Production of Calcium Gluconate. Prod. Inst. Sci. Res. 3: 83-94.

Moyer, A. J., Wells, P. A., Stubbs, J. J., Herrick, H. T. and May, O. E. 1937. Gluconic acid production. Development of inoculum and composition of fermentation solution for gluconic acid production by submerged mold growths under increased air pressure. Ind. Eng. Chem. 29: 777-781.

Wells, P. A., Lynch, D. F. J., Herrick, H. T. and May, O. E. 1937. Translating mold fermentation research to pilot plant operation. Chem. Met. Eng. 44: 188-190.

Wells, P. A., Moyer, A. J., Stubbs, J. J., Herrick, H. T. And May, O. E. 1937. Gluconic acid production. Effect of pressure, air flow, and agitation on gluconic acid production by submerged mold growths. Ind. Eng. Chem. 29: 653-656.

Gastrock, E. A., Porges, N., Wells, P. A. And Moyer, A. J. 1938. Gluconic acid production on pilot plant scale: effect of variables on production by submerged mold growth. Ind. Eng. Chem. 30: 782-789.

Lvoff, S. And Loupizina, G. M. 1938. Effect of sodium fluoride on the formation of citric and gluconic acid by the fungus Aspergillus niger. Compt. Rend. (Doklady) Acad. Sci. 21: 307-311.

Moyer, A. J., Umberger, E. J. and Stubbs, J. J. 1940. Fermentation of Concentrated Solutions of Glucose to Gluconic Acid: Improved Process. Ind. Eng. Chem. 32: 1379-1383.

Porges, N., Clark, T. F. and Gastrock, E. A. 1940. Gluconic Acid Production: Repeated use of Submerged A. niger for Semicontinuous Production. Ind. Eng. Chem. 32: 107-111.

KNobloch, H. and MaYeR, H. 1941. Uber die bildung von d-glucon-, d-mannonund d-Galaktonsäure durch Aspergillus niger in Schüttelkultur. Biochem. Zeit. 307: 285.

Porges, N., Clark, T. F. and Aronovsky, S. I. 1941. Gluconic Acid Production: Repeated Recovery and Re-use of Submerged Aspergillus niger by Filtration. Ind. Eng. Chem. 33: 1065-1067. 
Carrero, Jaime Gonzalez. 1942. Obtaining Gluconic Acid by the Fermentation Method. Ion. 2: 819. Abstracted in Chem. Abst. 37: 6816, 1943.

\section{Itaconic Acid}

Kinoshita, K. 1929. Formation of itaconic acid and mannitol by a new filamentous fungus. Jour. Chem. Soc. Japan 50: 583-93.

Kinoshita, K. 1931. Über die produktion von itaconsaure und mannit durch einen neuen Schimmelpilz, Aspergillus itaconicus. Acta Phytochimica 5: 271-287.

Kinoshita, K. 1937. Physiological and biochemical studies of Aspergillus itaconicus. Acta Phytochim. (Japan) 9: 159-87.

Calam, C. T., Oxford, A. E. And Raistrick, H. 1939. The biochemistry of microorganisms. LXIII. Itaconic acid, a metabolic product of a strain of Aspergillus terreus Thom. Biochem. Jour. 33: 1488-95.

Lockwood, L. B., Raper, Kenneth B., Moyer, A. J. and Coghill, R. D. The production and characterization of ultraviolet-induced mutations in Aspergillus terreus. III. Biochemical characteristics of the mutations. Am. Jour. Bot. (in press).

Lockwood, L. B. and Reeves, Max D. In press. Some factors affecting the production of itaconic acid by $A$ spergillus terreus.

Lockwood, L. B. ANd WARD, G. E. In press. Fermentation process for itaconic acid. Jour. Ind. Eng. Chem.

Moyer, A. J. And Coghill, R. D. In press. The laboratory-scale production of itaconic acid by $A$ spergillus terreus.

Raper, Kenneth B., Coghill, R. D. and Hollaender, Alexander. The production and characterization of ultraviolet-induced mutations in Aspergillus terreus. II. Cultural and morphological characteristics of the mutations. Am. Jour. Bot. (in press).

\section{Kojic Acid}

Saito, K. 1907. U̇ber die Säurebildung bei Aspergillus oryzae. Bot. Mag. (Tokyo) 21: $7-11$.

YABUtA, T. 1912. On Koji acid, a new organic acid formed by Aspergillus oryzae. Jour. Coll. Agric. Tokyo 5: 51-58. The paragraphs on Koji acid were contributed by Dr. J. F. Brewster.

YABUTA, T. 1924. The constitution of kojic acid, a $\gamma$-pyrone derivative formed by Aspergillus oryzae from carbohydrates. Jour. Chem. Soc. (London) 125: $575-587$.

TAmixa, H. 1927. Metabolism of Aspergillus oryzae. I. Acta. Phytochim. 3: $51-173$.

Challenger, F., Klein, L. And Walker, T. K. 1929. Production of Kojic Acid from Pentose by $A$. oryzae. Chemistry and Indus. 48: 602.

Katagiri, H. and Kitahara, K. 1929. The formation of kojic acid by Aspergillus oryzae. Part I. The formation of kojic acid from pentoses, sugar alcohols and gluconic acid. Bull. Agr. Chem. Soc. of Japan 5: 38.

Birkinshaw, J. H., Charles, J. H. V., Lilly, C. H. and Raistrick, H. 1931. Studies in the biochemistry of micro-organisms. VII. Kojic acid (5hydroxy-2-hydroxymethyl- $\gamma$-pyrone). Phil. Trans. Roy. Soc. London 220B: $127-138$.

Challenger, F., Klein, L. and Walker, T. K. 1931. The formation of Kojic Acid from sugars by Aspergillus oryzae. Jour. Chem. Soc. pp. 16-23. 
May, O. E., Moyer, A. J., Wells, P. A. And Herrick, H. T. 1931. The production of Kojic Acid by Aspergillus flavus. Jour. Am. Chem. Soc. 53: 77ł-782, illus.

MaY, O. E., WARD, G. E. AND Herrick, H. T. 1932. The effect of organic stimulants upon the production of kojic acid by Aspergillus flavus. Zentralbl. Bakt. Parasitenk. u. Infektionsk. II Abt. 86: 129-134.

Di CapUa, A. 1933. Action of iron on the formation of kojic acid by means of Aspergillus flavus. Gazz. Chim. Ital. 63: 296-302.

Katagiri, Hideo and Kitahara, Kakuo. 1933. Formation of kojic acid by Aspergillus oryzae. Mem. Coll. Agr. Kyoto Imp. Univ. No. 36, p. 1-29.

Klutyer, A. J. and Perquin, L. H. C. 1933. Conditions for the formation of kojic acid by Aspergillus flavus Link. Biochem. Zeit. 266: 82-95.

Barham, H. N. and Smits, B. L. 1934. Kojic Acid-A review. Trans. Kansas Acad. Sci. 37: 91-113.

Friedemann, T. E. 1934. Chemical and physiological properties of kojic acid. Science $80: 34$.

Barham, H. N. and Simts, B. L. 1936. Production of kojic acid from xylose by Aspergillus flavus. Ind. Eng. Chem. 28: 567-70.

Gould, B. S. 1935. The metabolism of Aspergillus tamarii Kita. Kojic acid production. Biochem. Jour. 32: 797-802.

Rayman, M. M. 1941. Production of chemicals by the fermentation of the acid hydrolyzate of oat hulls. Ia. St. Coll. Jour. Sci. 16: 122-124. Kojic acid from oat hulls by Aspergillus flavus.

\section{Oxalic Acid}

Duclaux, E. 1S59. Sur la nutrition intracellulaire. Ann. Inst. Pasteur 3: 97112 , illus.

WeHner, C. 1891. Entstehung und physiolgische Bedeutung der Oxalsäure in Stoffwechsel einiger Pilze. Bot. Ztg. 49: 233-246, and others.

WeHNER, C. 1891. Zur zersetzung der oxalsäure durch licht-und stoffwechselwirkung. Ber. Deut. Bot. Gesell. 9: 21S-229.

Wenмer, C. 1892. Über oxalsäure-bildung durch pilze. Liebigs Ann. Chem. 269: $383-389$.

WenмeR, C. 1897. Ḱleinere mykologische mitteilungen. Zur Oxalsäuregärung durch Aspergillus niger. Centralb. Bakt. (etc.) 2 Abt. 3: 102-108, 147-153.

Emmerling, O. 1903. Oxalsäurebildung durch Schimmelpilze. Centralb. f. Bakt. etc. 2 Abt. 10: 273.

Currie, J. N. and Thom, C. 1915. An oxalic acid-producing Penicillium. Jour. Biol. Chem. 22: 287-293.

Raistrick, H. AND ClaRk, A. B. 1919. On the mechanism of oxalic acid formation by Aspergillus niger. Biochem. Jour. 13: 329-344.

Walker, T. K., Subramanian, V. and Challenger, F. 1927. The mechanism of the formation of citric and oxalic acids from sugar by Aspergillus niger. II. Jour. Chem. Soc. (London )3044-3054.

Chrzaszcz, T. and Trukow, D. 1930. Oxalsäure in Schimmelpilzekulturen. Biochem. Ztschr. 218: 73-85.

HofuAn, Edward, 1931. Formation of Oxalic Acid by Aspergillus niger from Uronic Acid. Biochem. Zeit. 243: 423-8.

Bernhauer, K. and Slanina, F. 1933. The chemistry of the processes of acid formation induced by Aspergillus niger. X. The formation of oxalic acid from formic acid. Biochem. Zeit. 264: 109. 
Webrer, H. A. 1934. Production of Oxalic Acid from Cellulosic Agricultural Material. Ia. Eng. Expt. Sta. Bull. 118: pp. 55.

Hofmann, Edward. 1936. Acidification of the Culture Media by Aspergillus niger and the Formation of Oxalic Acid. Arch. Phys. Biol. 13: 84-87.

Allsopp, A. 1937. Formation of oxalic acid by Aspergillus niger. New Phytol. 36: $327-356$.

JAQU゚OT, R. 1938. Oxalic Acid Fermentation: Mechanism of Oxalicogenesis by Moulds. Ann. des Ferm. 4: 281-294.

JAQUot, R. 1938. Influence of $\mathrm{pH}$ on the Production of Oxalic Acid by Various Molds. Compt. Rend. Soc. Biol. 127: 1431-1432.

JAQuot, R. 1938. Production of Oxalic Acid by Molds; Energy Expenditure. Compt. Rend. Soc. Biol. 128: 69-70.

Mel'nikova, A. A. And Butketich, V. S. 1939. Biochemical formation of oxalic acid from sugar. Microbiology (USSR) 8: 818-26.

Simo, Mituo. 1939. Crystals occurring in the mold membrane in oxalic acid fermentation. Jour. Agr. Chem. Soc. Japan 15: 751-2.

\section{Miscellaneous Acids}

Bernhauer, K., Bocki, N. and Sichenauger, H. 1932. The formation of acid from sugar by Aspergillus niger. V. The formation of malic acid besides citric acid. Biochem. Zeit. 253: 37.

Bernhauer, I. AND Scheler, Z. 1932. The chemistry of the processes of acid formation induced by Aspergillus niger. VI. The formation of glycolic and glyoxylic acids from acetate salts. Biochem. Zeit. 253: 11.

Bernhauer, I. Anc Bocki, N. 1932. The chemistry of the processes of acid formation induced by Aspergillus niger. VIII. The transformation of aconitic acid into citric acid; and further notes on the degradation of acetic acid. Biochem. Zeit. 253: 25.

SAKAguchi, K. 1932. The production of acids and alcohol by Aspergillus. VI. The production of d-gluconic acid by Aspergillus oryzae. Jour. Agr. Chem. Soc. Japan 8: 264-5.

Kardo-Suisoeva, E. 1933. Formation of glucuronic acid by Aspergillus niger. Biochem. Zeit. 268: 337-51.

Nishikawa, H. 1933. Biochemistry of the filamentous fungi. II. A metabolic product of Aspergillus melleus Yukawa. Bul. Agr. Chem. Soc. Japan 9: 107-109. Mellein and melleic acid.

Nishikawa, H. 1933. Biochemistry of the filamentous fungi. III. A metabolic product of Aspergillus melleus Yukawa. Bull. Agr. Chem. Soc. Japan 9: 148-151. Mellein and melleic acid.

Sumiki, Y. 1933. Fermentation products of molds. X. Aspergillus glaucus. Jour. Agr. Chem. Soc. Japan 9: 714-16. Records formation of glaucic acid.

Glimin, E. ANd Nitzsche, M. 1934. Formation of malic acid from asparagine by fermentation at different $\mathrm{pH}$ values. Biochem. Zeit. 268: 444-50. A. niger and yeast employed.

Birkinshaw, J. H. 1937. Biochemistry of the lower fungi. Biol. Rev. Cambridge Phil. Soc. 12: 357 . Records production of aconitic acid by Aspergillus itaconicus.

Steinkerg, R. A. 1942. The process of amino acid formation from sugars in Aspcrgillus niger. Jour. Agr. Res. 64:615-33. 


\section{Antibiotics and Toxins}

Bodin, E. And Gautier, L. 1906. Note sur une toxine produite par l'Aspergillus fumigatus. Ann. Inst. Pasteur 20: 209-224.

BoAs, F. 1919. Auto-toxicity in Aspergillus niger. Ber. Deut. Bot. Gesell. $37(1): 63-65$.

Walker, J. C., Lindegren, C. C. and Bachmann, F. M. 1925. Further studies on the toxicity of juice extracted from succulent onion scales. Jour. Agr. Res. 30: 175-187.

Anslow, Winston K. And Raistrick, Harold. 1938a. The biochemistry of microorganisms. LVII. Fumigatin (3-hydroxy-4-methoxy-2,5-toluquinone) and spinulosin (3,6-dihydroxy-i-methoxy-2,5-toluquinone), metabolic products respectively of Aspergillus fumigatus Fresenius and Penicillium spinulosum Thom. Biochem. Jour. 32:687-96.

Anslow, Winston K. And Raistrick, Harold, 1938b. The biochemistry of microorganisms. LIX. Spinulosin (3,6-dihydroxy-1-methoxy-2,5-toluquinone) a metabolic product of a strain of Aspergillus fumigatus Fresenius. Biochem. Jour. 32: 2288-9.

Woolley, D. W., Berger, J., Peterson, W. H. and Steenbock, H. 1938. Toxicity of Aspergillus sydowi and its correction. Jour. Nutrition 16: 465-76.

White, EDwin C. 1940. Bactericidal filtrates from a mold culture. Science 92: 127. A spergillus flavus.

Glister, G. A. 1941. A new antibacterial agent produced by a mold. Nature 148: 470. Aspergillus flavus.

Oxford, A. E. ANd Raistrick, H. 1942. Anti-bacterial substances from moulds. IV. Spinulosin and fumigatin, metabolic products of Penicillium spinulosum Thom and Aspergillus fumigatus Fresenius. Chem. and Ind. 61: 128.

Timonin, M. I. 1942. Another mould with anti-bacterial ability. Science 96: No. 2500, p. 494. Aspergillus sp., white-spored.

Waksman, Selman A., Horning, Elizabeth S. and Spencer, Ernest L. 1942. The production of two antibacterial substances, fumigacin and clavacin. Science 96: 202. August 28 .

Waksman, S. A., Horning, Elizabeth S. and Spencer, Ernest L. 1942. Two antagonistic fungi, Aspergillus fumigatus and Aspergillus claratus and their antibiotic substances. Jour. of Bact. 45: 233-248.

White, E. C. And Hill, Justina H. 1942. Antibacterial filtrates from a strain of Aspergillus flacus. Jour. Bact. 43: 12.

Wiesner, B. P. 1942. Bactericidal effects of Aspergillus clavatus. Nature 149: 356. Wilkins, W. H. AND HaRRIs, G. C. M. 1942. Investigations into the production of bacteriostatic substances by fungi. I. Preliminary examination of 100 fungal species. Brit. Jour. Exper. Path. 23: 166.

Bush, M. T. And Goth, A. 1943. An antibacterial substance produced by an Aspergillus flarus. Federation Proc. 2: 75.

Bush, Milton T. ANd Goth, Andres. 1943. Flavicin: an antibacterial substance produced by an Aspergillus flarus. Jour. Pharm. and Exp. Ther. 78(2): 164-169.

Chain, E., Florey, H. W., Jennings, M. A. And Williams, T. I. 1943. Helvolic acid, an antibiotic produced by Aspergillus fumigatus mut. helvola Yuill. Brit. Jour. Expt. Path. 24: 108-118.

Crowfoot, D. M. AND Low, B. W. 1943. A note on the crystallography of helvolic acid and the methyl ester of helvolic acid. Brit. Jour. Expt. Path. 24: 120. 
Jones, H., Rake, G. ANd Hanre, D. M. 1943. Studies on Aspergillus flavus. I. Biological properties of erude and purified aspergillic acid. Jour. Bact. 45: $461-469$.

McKee, C. M. and MacPhillamy, H. B. 1943. An antibiotic substance produced by submerged cultivation of Aspergillus flavus. Proc. Soc. Exp. Biol. and Med. 53(No. 2): 247-248.

Philpot, Flora J. 1943. A penicillin-like substance from Aspergillus giganteus Wehm. Nature 152: 725.

Waksman, S. A. And Bugie, Elizabeth. 1943. Strain specificity and production of antibiotic substances. II. A spergillus flavus-oryzae group. Proc. Natl. Acad. Sci. 29: 282.

Waksman, S. A. And Geiger, W. B. 1943. The nature of the antibiotic substances produced by Aspergillus fumigatus. Jour. Bact. 47: 391-397.

Waksman, Selian A. and Horning, Elizabeth S. 1943. Distribution of antagonistic fungi in nature and their antibiotic action. Mycol. 35 (No. 1): $47-65$.

Waksman, Selman A. And Schatz, Albert. 1943. Strain specificity and production of antibiotic substances. Proc. Natl. Acad. Sci. 29: (No. 2) 74-79. Aspergillus clavatus group.

White, E. C. And Hill, J. H. 1943. Studies on antibacterial products formed by molds. I. Aspergillic acid, a product of a strain of Aspergillus flavus. Jour. Bact. 45: 433-444.

Cook, A. H. ANd LACEy, M. S. 1944. An antibiotic from Aspergillus parasiticus. Nature 153: 460 . April 15.

Hooper, I. R., Anderson, H. W., Skeld, P. and Carter, H. E. 1944. The identity of clavacin with patulin. Science 99: 16 .

McKee, C. M., Rake, G. And Houck, C. L. 1944. Studies on Aspergillus flavus. II. The production and properties of a penicillin-like substance-flavacin. Jour. Bact. 47: 187-197.

Menzel, Arthur E. O., Wintersteiner, O. And Hoogerheide, J. C. 1944. The isolation of gliotoxin and fumigacin from culture filtrates of Aspergillus fumigatus. J. Biol. Chem. 152: 419-429.

\section{Chemistry of Mold Tissue}

Norman, A. G., Peterson, W. H. And Houtz, R. C. 1932 . I. Soluble Carbohydrate Constituents. Biochem. Jour. 26: 1934-45. A. fischeri.

Nornan, A. G. And Peterson, W. H. 1932. II. The Resistant Cell-wall Material. Biochem. Jour. 26: 1946-53. A. fischeri.

Pruess, L. M., Eichinger, E. C. and Peterson, W. H. 1934. III. Composition of Certain Molds with Special Reference to the Lipid Content. Zentbl. Bakt., 2 Abt. 89, No. 17-20, pp. 370-377, figs. 2.

Strong, F. M. and Peterson, W. H. 1934. IV. The Lipids of Aspergillus sydowi. Jour. Amer. Chem. Soc. 56, No. 4, pp. 952-955.

Gorcica, H. J., Peterson, W. H. and Steenbock, H. 1934. V. Fractionation of the Nitrogen in the Mycelium of Aspergillus fischeri. Biochem. Jour. 28, No. 2, pp. 504-511.

Prill, E. A., Wenck, P. R. ANd Peterson, W. H. 1935. VI. Factors Influencing the Amount and Nature of the Fat Produced by Aspergillus fischeri. Biochem. Jour. 29, No. 1, pp. 21-33, fig. 1.

Kroeker, E. H., Strong, F. M. and Peterson, W. H. 1935. VII. The Lipids of Penicillium aurantio-brunneum. Jour. Amer. Chem. Soc. 57, No. 2, 354-356. 
Wenck, P. R., Peterson, W. II. and Greene, H. C. 1935. Vili. Innate Factors influencing Growth and Sterol Production of Aspergillus fischeri. Zentbl. Bakt. Parasitenk. II Abt. 92: 324-30.

Wenck, P. R., Peterson, W. H. and Fred, E. B. $1935 . \quad$ IX. Cultural factors influencing growth and sterol production of Aspergillus fischeri. Zentbl. Bakt. Parasitenk., II Abt. 92: No. 13-19, 330-338.

Woolley, D. W., Strong, F. M., Peterson, W. H. and Prill, E. A. 1935. X. The phospholipides of Aspergillus sydowi. Jour. Amer. Chem. Soc. 57, No. 12, pp. 2589-2591.

Woolley, D. W. and Peterson, W. H. 1936. XI. Isolation of leucine and isoleucine from Aspergillus sydowi. Jour. Biol. Chem. 114, No. 1, pp. 85-90.

Woolley, D. W. and Peterson, W. H. 1937. XII. Isolation of arginine, histidine, and lysine from Aspergillus sydowi. Jour. Biol. Chem. 118, No. 2, pp. 363-370.

Woolley, D. W. and Peterson, W. H. 1937. XIII. Isolation of some monoaminomonocarboxy and some monoaminodicarboxy acids from Aspergillus sydowi. Jour. Biol. Chem. 121, No. 2, pp. 507-520.

Woolley, D. W. And Peterson, W. H. 1937. XIV. Isolation of cyclic choline sulfate from Aspergillus sydowi. Jour. Biol. Chem. 122, No. 1, pp. 213218 , fig. 1.

Bohonos, N., Woolley, D. W., and Peterson, W. H. 1942. XV. (Not numbered.) The unautolyzable protein of Aspergillus sydowi. Archives of Biochem. 1 (No. 2): 319-324.

Bohonos, N. And Peterson, W. H. 1943. XVI. Isolation of fungus cerebrin from the mycelium of Aspergillus sydowi. Jour. Biol. Chem. 149: 295-300.

\section{Enzyme Production}

\section{Enzymes-Aspergillus Flavus-Oryzae Group}

Hoffmann. 1874. Über die Bereitung von Shoyu, Saké und Mirin. Mitt. Gesell. Ost. 1(6): 8-11.

Kellner, O., Mori, Y. and Nagaoka, M. 1888. Researches on the manufacture, composition and properties of "koji." Imp. College of Agriculture, Tokyo, Bull. 5: 9-33.

Kellner, O., Nagaoka, M. and Kurashima, Y. 1889. Researches on the manufacture and composition of "miso." Imp. Coll. Agr. Tokyo Bull. 6: 1-24.

TAKamine, J. 1894. Process of making diastatic enzyme, U. S. Patent 525,823, Sept. 11; other patents in the same diastatic series No. 525,825; 525,971; 562,$103 ; 826,699 ; 975,656 ; 991,560 ; 991,561 ; 1,054,626$; and $1,054,324$.

Takamine, J. 1894. Process for making alcoholic liquor. U. S. Patent 525,819; 525,821 ; and 525,822 .

Kellner, O. 1895. Ueber die Bereitung von Saké, Shoyu und Miso. Chem. Ztg. 19: $120-121$.

Prinsen Geerligs, H. C. 1896. Einige chinesische Sojabohnenpräparate. Chem. Ztg. 20: 67-69.

Kita, Gen-Itsu. 1913. Japanisehe Sojaindustric. Wochsch. Brau. 30: 549-552, $559-561,5$ illus.

Takamine, Јокicur. 1914. Enzymes of Aspergillus oryzae and the application of its amyloclastic enzyme to the fermentation industry. Chem. News 110: 215-218. In this paper Takamine summarizes the history of the studies of A. oryzae and its application to various fermentation industries. 
Takamine, J. 1920. Process for the making of enzyme extracts. U. S. Patent 152,792. Oct. 25 .

TAKamine, J., JR. AND Oshima, K. 1920. The properties of a specially prepared enzymic extract, polyzime, comparing its starch liquefying power with malt diastase. Jour. Amer. Chem. Soc. 42: 1261-5.

Osнina, Кокісні. 1922. Studies on the protease of the A. oryzae-flavus group and its rôle in shoyu brewing. Amer. Food Jour. 17(1): 30-31.

Church, M. B. 1923. Soy and related fermentations. U. S. Dept. Agr. Bull. 1152: $1-27,2$ pls., 6 figs.

Nishimura, S. 1925. Zur Kenntnis der Takadiastase. Chemie der Zelle und Gewebe 12: 202-216.

Oshina, K. 1928. Disinfectants for preserving the amylase solution of Aspergillus oryzae. Jour. Soc. Chem. Ind. 31: 750-3, 180-3. B.

Oshima, K. 1928. Protease and amylase of Aspergillus oryzae. Jour. Coll. Agr. Hokkaido Imp. Univ. 19: 135-244.

Harada, T. 1931. The preparation of Aspergillus oryzae enzymes. Ind. Eng. Chem. 23: 1424-7.

Iто, M. 1932. The nature of koji diastase. Jour. Fac. Agr., Hokkaido Imp. Univ. 30(5): 243-386.

WEI, N-S and Chin, K-S. 1934. The diastatic activity of Aspergillus. Science (China) 18: 1193-8. A. oryzae.

Kirsh, D. 1935. Lipase production by Penicillium oxalicum and Aspergillus flavus. Botan. Gaz. 97: 320-33.

Stuart, L. S. 1935. The production of lipolytic and depilating enzymes by the Aspergillus flavus-oryzae group. Jour. Bact. 29: 88-9.

Токчока, Y. 1938. Koji amylase. X. Effect of external conditions on $\gamma$ - and $\beta$ amylases and maltase. Jour. Agr. Chem. Soc. Japan 14: 429-438.

Toкuока, Y. 1938. Koji amylase. XI. Effect of Koji material on production of amylase and maltase. Jour. Agr. Chem. Soc. Japan 14: 839-842.

Babakina, B. G. AND Zamysolv, A. D. 1939. Enzyme preparation from Aspergillus oryzae in the leather industry. Biokhimiya 4: 316-26.

Nagatomo, Takeo. 1939. The optimum hydrogen-ion concentration for amylase of Aspergillus oryzae. Jour. Agr. Chem. Soc. Japan 15: 753-6.

O'tani, Yosio. 1939. Enzymes in young mycelia of Aspergillus oryzae. Bull. Agr. Soc. Japan 15: 59-64.

Proskuryakov, N. I. and Osipov, F. M. 1939. Enzymic cleavage by molds. Biokhimiya 4: 50-59. A. oryzae.

Токчока, Y. 1939. Koji amylase. XIII. Effect of the variety of A. oryzae employed in saké brewing upon the formation of amylase, maltase, and protease in Koji. Jour. Agr. Chem. Soc. Japan 15: 414-418.

Underkofler, L. A., Fulmer, E. I. And Schoene, Lorin. 1939. Saccharification of starchy grain mashes for the alcoholic fermentation industry. Use of mold amylase. Ind. and Eng. Chem. 31: 734-738. A. oryzae.

Schoene, Lorin, Fulmer, E. I. And Underkofler, L. A. 1940. Saccharification of starchy grain mashes for the alcoholic fermentation industry. Comparison of several saccharifying agents. Ind. and Eng. Chem. 32: 544-547. (Moldy bran, malt, and soybean meal.)

Hao, Lu Cheng. 1942. Fungal amylases as saccharifying agents in the ethanol fermentation of starchy materials. Doctoral Thesis Ia. St. Coll., Abstracted in Ia. St. Coll. Jour. Sci. 17(1): 71-73. 
Hao, Lu Cheng, Fulmer, E. I. and Underkofler, L. A. 1943. Fungal amylases as saccharifying agents in the alcoholic fermentation of corn. Ind. Eng. Chem. 35: 814-818. A. oryzae and A. flavus.

Christensen, L. M. 1943. U. S. Patent 2,325,368. July 27. Apparatus for facilitating the growth of molds on solid substrates as in the large-scale production of highly diastatic bran mold.

\section{Enzymes-Aspergillus Niger Group}

Bourquelot, E. 1886. Recherchés sur les propriétés physiologiques du maltose. Jour. Anat. et Physiol. (Paris) 22: 162-204.

Fernbach, A. 1890. Formation de sucrase chez l'Aspergillus niger. Ann. Inst. Pasteur 4: 1-24.

Bourquelot, E. 1893. Les ferments solubles de l" Aspergillus niger." Bul. Soc. Myc. France 9: 230.

Young, V. H. 1918. Some factors effecting inulase formation in Aspergillus niger. Plant World 21: 75-87, and 114-133.

Funke, G. L. 1922. Researches on the formation of diastase by Aspergillus niger van Tieghem. Rec. Trav. Botan. Neerl., XIX, 219-275, 14 figures. Abstracted in The Review of Applied Mycology, 2, Part 7:330-331, 1923.

Sснмidt, Donothea. 1925. Über die Pilzstärke (amylose) bei Aspergillus niger van Tieghem und einige Bemerkungen über ihren diastatischen Abbau. Biochem. Ztschr. 158: 223-252.

Funke, G. L. 1926. Researches on the formation of diastase by Aspergillus niger. Rec. Trav. Bot. Neerland. XXIII: 200.

Bernhauer, K. 1928. Beiträge zur enzymchemie der Durch Aspergillus niger bewirkten Säurebildungsvorgänge. I. Ztschr. Physiol. Chem. 177: 86-106.

Müller, D. 1931. Glucose oxidase. IV. Glucose oxidase from A. niger. Biochem Zeit. 232: 423.

Rossi, G. And Scandellari, G. 1932. Influence of salts on invertase formation in Aspergillus niger. Atti IV Congr. Naz. Chim. Pura Applicata 1933: 795-9.

Mrwa, T. And Yoshir, S. 1934. The formation of urease by Aspergillus niger. Science Repts. Tokyo Bunrika Daigaku I: 243-70.

Otani, H. 1934. Sulfopeptidase of molds. I. Aspergillus niger. Acta. Schol. Med. Univ. Imp. Kioto 17: 242.

Drboglav, M. A. and Krrsanova, B. A. 1935. A study of the invertase of Aspergillus niger. Proc. Inst. Sci. Res. Food Ind. (Leningrad). 3: 115-129.

Mezzadroli, G. and Amati, A. 1935. Action of various alkaloids on the sucrase produced by Aspergillus niger. Atti Accad. Lincei 21: 46-50.

Otani, H. 1935. The proteolytic enzymes of A. niger. Acta. Schol. Med. Univ. Imp. Kioto 17 : 317-322.

Rossi, G. And Scandellari, G. 1935. The influence of different salts contained in Wehmer's liquid medium on the quantity of invertase produced by Aspergillus niger growing in this medium. Biochim. Terap. Sper. 22: 87-91.

Drboglav, M. A. and Kirsanova, B. A. 1936. Peroxidase activity of Aspergillus niger. Proc. Inst. Sci. Res. 3: 103-110.

Drboglav, M. A. and Kirsanova, B. A. 1936. Amylase activity of Aspergillus niger. Proc. Inst. Sci. Res. Food Ind. (Leningrad) 3: 4, 112-15.

Pronin, S. I. And Blinnikova, E. I. 1936. The thermostability and regeneration of amylase from Aspergillus niger. Arch. Sci. Biol. USSR 41: 3, 11-14. 
Moor, J. C. 1939. The enzymes of Aspergillus niger which transform alanine and aspartic acid. Proc. Acad. Sci. Amsterdam 42: 195-200.

Müller, D. 1940. Glucose oxidase. Naturwissenschaften 28: 516. A. niger.

\section{Enzymes-Aspergilli, General}

Dox, A. W. 1910. The intracellular enzymes of Penicillium and Aspergillus. U. S. Dept. Agr., Bur. Anim. Ind. Bul. 120, 70 pp.

Hopffe, ANna. 1919. Ueber einen bisher unbekannten, celluloselösenden, im Verdauungstraktus vorkommenden Aspergillus, " $A$. cellulosae," seine Züchtung und seine Eigenschaften. Centralb. f. Bakt. etc., 1 Abt. 83: 531-537.

Conpron, A. 1921. Studies in the mechanism of enzyme action. I. Rôle of the reaction of the medium in fixing the optimum temperature of a ferment. Roy. Soc. London, Proc., Ser. B, 92, no. B642, pp. 1-6, figs. 3.

Waksman, S. A. 1922. Enzymes of Microorganisms. Abstr. Bact. 6: 265-299, 331-360.

WaksuAN, S. A. 1922. Use of enzymes in the clarification of jellies and fruit juices. Canner 54(18): 45-46.

Oshma, K. and Church, Margaret B. 1923. Industrial mold enzymes. Jour. Ind. Eng. Chem. 15: 67.

Owen, W. L. 1923. Summarizes his many years study on this subject in "Mold fungi in sugar inversion." Facts about Sugar 16: 519-521, 546-548.

Müller, D. 1928. Studien über ein neues Enzym Glykoseoxydase; frühere Untersuchungen über die Wirkung von Enzympräparaten aus Aspergilleen auf Monosaccaride und organische Säuren. Biochem. Zeitschr. 199: 136-70.

Ivanov, N. N. AND Avetissova, A. N. 1931. The enzymic transformation of guanidine to urea. Biochem. Zeit. 231: 67-78.

Eyre, J. C. 1932. Cultural studies on Aspergillus with special reference to lipase production of strains isolated from stored copra and cacao. Ann. Appl. Biol. 19: 351-69.

Hofmans, E. 1934. Enzymes from molds which hydrolyze glucosides and disaccharides. Biochem. Zeit. 273: 198-206.

Johnson, M. J. And Peterson, W. H. 1935. The proteinase and aminopolypeptidase of Aspergillus parasiticus. Jour. Bact. 29: 90. Abstract.

KIrsh, D. 1935. Factors influencing the activity of fungus lipase. J. Biol. Chem. 108: 421-30.

Mrhaeloff, S. 1935. Soluble enzymes secreted by Aspergillus fumigatus. Bull. Inst. Egypte 17: 163-95.

Otani, H. 1935. The Mold Enzymes Splitting Nucleic Acid. Acta. Schol. Med. Univ. Imp. Kioto. 17: 323-329. A. niger and A. oryzae.

Grassmann, W. and Rubenbauer, H. 1937. Enzymes from molds. U. S. Patent 2,102,315. December 14. C. A. 32, 1291.

Mrwa, Tomo and Mrwa, Ayako. 1940. $\alpha$-glucosidase. II. Enzymes of mold fungi. Jour. Chem. Soc. Japan 61: 1172.

Pearce, A. A. 1940. On the so-called "Iodide Oxidase," Mechanism of Iodide Oxidation by Aspergillus. Biochem. Jour. 34: 1493-1500. A. niger.

Underkofler, L. A. 1942. Microbial amylases-Their application to alcoholic fermentation. Brewers' Digest 17:29. A review with 25 references.

Ayers, G. B. And Tobie, W. C. 1943. The isolation of crude proteolytic enzymes 
from microörganisms. Jour. Bact. 45: 18. Abstract. Tests on twenty-six Aspergilli.

\section{Fat Production by Aspergilli}

Boun, P. R. 1931. Mechanisn of the synthesis of fats at the expense of sugars. Compt. Rend. 193: 441-2.

Pruess, L. M., Peterson, W. H., Steenbock, H. and Fred, li. B. 1931. Sterol content and antirachitic activatibility of mold mycelia. Jour. Biol. Chem. 90: $369-384$.

Rockweld, G. E. and O'Flaherty, F. 1931. Studies in the physiology of moulds. II. Composition and culture of moulds. Jour. Amer. Leather Chem. Assoc. 26: 216.

Pontrillon, Charles. 1932. A physiological study of the lipids of Sterigmatocystis nigra. Rev. Gen. Botan. 44: 417-49, 465-83, 526-60.

Pruess, L. M., Peterson, W. H. and Fred, E. B. 1932. Isolation and identification of ergosterol and mannitol from Aspergillus fischeri. Jour. Biol. Chem. 97: 483-489.

Pritess, L. M., Gorcica, H. J., Greene, H. C. and Peterson, W. H. 1932. Wachstum und Steringehalt gewisser Schimmelpilze. Biochem. Zeits. 246: 401-413.

Pruess, L. M., Eichenger, E. C. and Peterson, W. II. 1934. The chemistry of mold tissue. II. Composition of molds with special reference to the lipid content. Centralbl. Bakt. Parasitenk. u. Infektionsk. II Abt. 89: 370-377.

Strong, F. M. and Peterson, W. H. 1934. Chemistry of mold tissue. IV. The lipides of Aspergillus sydowi. Jour. Amer. Chem. Soc. 56: 952-955.

Bernhauer, Kontad and Patzelt, George. 1935. Mold sterols. I. Sterol formation by Aspergillus niger. Biochem. Zeit. 280: 388-93.

Prill, E. A., Wenck, P. R. and Peterson, W. H. 1935. The chemistry of mould tissue. VI. Factors influencing the amount and nature of the fat produced by Aspergillus fischeri. Biochem. Jour. 29: 21-33.

Sснмпт, C. F. (JR.) 1935. The formation of fatty acids by Aspergillus niger. Jour. Bact. 30: $445-46$.

Schmid, C. F. (JR.) 1935. The formation of fatty acids from glucose by Aspergillus niger. Jour. Biol. Chem. 110: 511-20.

Ward, G. E., Lockwood, L. B., Mar, O. E. And Herrick, H. T. 1935. Production of fat from glucose by molds. Cultivation of Pcnicillium javanicum van Beijma in large-scale laboratory apparatus. A. flavus mycelium contained 16 percent. Ind. Eng. Chem. 27: 318-322.

Wenck, P. R., Greene, H. C. And Fred, E. 13. 1935. Factors influencing the growth and sterol formation of Aspergillus fischeri. Jour. Bact. 29: 89-90.

Wenck, P. R., Peterson, W. H. and Fred, E. B. 1935. The chemistry of mold tissue. IX. Cultural factors influencing the growth and sterol production of Aspergillus fischeri. Zentralbl. Bakt. Parasitenk. u. Infektionsk. II. Abt. 92: 330-338.

Woolley, D. M., Strong, F. M., Peterson, W. H. and Prill, E. A. 1935. Chemistry of mold tissue. $\mathrm{X}$. The phospholipides of Aspergillus sydowi. Jour. Am. Chem. Soc. 57: 2589-91.

Bernhauer, K. and Posselt, G. 1937. Über Schimmelpilzlipoide. II. Mitteilung: Z/ie Zusammensetzung eines Aspergillus-niger-Fettes. Biochem. Zeit. 294: 215-20. 
Ruppol, E. 1937. Chemical composition of the fat from Aspergillus citromyces. Jour. Pharm. Belg. 19: 63-8.

Tauson, V. O. 1938. The conversion of energy by microörganisms. $\mathrm{X}$. The accumulation of fat in Aspergillus flavus. Microbiology (USSR) 7: 360-65.

\section{Pathogenicity of Aspergilli}

Dusch, Theodor yon and Pagenstrecher, A. 1857. Fall von Pneumomycosis Aspergillus pulmonum hominis. Arch. Path. Anat. Phys. (Virchow) 11: $561-566$.

Cramer, Carl. 1859. Über eine neue Fadenpilzgattung: Sterigmatocystis Cramer. Vierteljahrschr. Naturf. Ges. Zürich 4: 325-337, Pl. 2.

Green, John Orne. 1869. Two cases of parasitic growth (Aspergillus glaucus) in the external auditory meatus. Trans. Am. Otol. Soc. 1: 23-26.

Hallier, Ersst. 1870. Mittherling über die Ohrpilze, welche Herr Dr. R. Hagen in Leipzig zur mikroscopischen Untersuchungen Einsandte. Zeitschr. Parasitenk. 2: 259-284, Pls. 5 and 6.

Steudener, F. 1870. Zwei neue Ohrpilze nebst Bemerkungen über die Myringomycosis. Arch. Ohrenheilk. 5: 163-168, Pl. 1.

Leidy, Joseph. 1875. On a fungus in a flaningo. Proc. Acad. Nat. Sci. Phila. 27: 11-12.

Burnett, Charles H. 1878. The growth of the fungus Aspergillus in the human ear. Philadelphia Med. Times 8: 435-440, 3 figs.

Siebendann, F. 1883. Die Fadenpilze Aspergillus flavus, niger, fumigatus; Eurotium repens (und Aspergillus glaucus) und ihre Beziehung zur Otomycosis Aspergillina. Zeitschr. Ohrenheilk. 12: 124-161.

Mackenzie, John N. 1893. Preliminary report on Aspcrgillus mycosis of the antrum maxillare. Johns Hopkins Hosp. Bull. 4: 9, 10.

Renon, L. 1897. Etude sur l'Aspergillose chez les animaux et chez l'homme. Paris, pp. I-XII and 1-301.

WrNfield, James McF. 1897. A favus-like eruption of the oral mucous membrane caused by Aspergillus nigrescens. Jour. Cut. Genito-Urin. Dis. 15: 13-17, 3 figs.

Blumentritr, Fritz. 1901. Über einen neuen, in Menschen gefundenen Aspergillus (A. bronchialis) n. sp. Ber. Deutsch. Bot. Ges. 19: 442-446, Pl. 22.

Ceni, Carlo. 1905. P'otere patogeno dell'Aspergillus ochraceus e suo rapporto coll'etiologia e patogenesi della pellagra. Arch. Ital. Mal. Nerv. Mentali. 42: 231-244, Pl. 6 [Riv. Sperim. Freniatria 31].

Costantin, J. And Lucet, A. 1905. Recherches sur quelques Aspergillus pathogènes. Ann. Sci. Nat. Bot. IX, 2: 119-171, Pl. V.

Galli-Valerio, Bruno and Rochaz-De Jongh, J. 1905. Ueber die Wirkung von Aspergillus niger und A. glaucus auf die Larven von Culex und Anopheles. Centralb. f. Bakt., etc., 1 Abt. 38: 174-176.

Noyes, A. W. Finch. 1905. Aspergillus niger growth on the tongue. Intercolonial Med. Jour. Australia 10: 153, 154.

Neumann, G. 1908. Aspergillose des oiseux domestiques. Rev. Vét. (Toulouse) 33: 417-424, fig. 1 .

Bainier, G. And Sartory, Antoine. 1909. Etude d'un Aspergillus pathogène (Aspergillus fumigatoides nov. sp.). Bull. Soc. Myc. France 25: 111-118, Pl. 5 [see also C. R. Soc. Biol. 66: 22, 23, 1909].

Coupin, H. 1909. Atlas des champignons parasites et pathogènes de l'homme et des animaux. Paris. 
Guéguen, FErnand. 1909. Aspergillus fontoynonti nova sp., parasite probable des nodosités juxta-articulaires. Comp. Ren. Soc. Biol. 66: 1052, 1053; also 67: 10-12.

Gúfguen, Fernand. 1911. Abcès sous-dermiques à répétitions products par l'Aspergillus fontoynonti $\mathrm{n}$. sp. morphologique et biologique de cette espèce. Arch Parasitol. 14: 177-192.

Quevedo, José M. 1912. Sur une encephalo-myelite épizootique d'origine mycosique chez les chevaux. Premier Congr. Internat. Path. Comp. 2: 200-202.

Quevedo, J. M. 1912. Estudio de un Aspergillus patogeno. De Agronomia 3: 1-36.

Stout, A. B. 1912. A fungous infection of the ear. Jour. N. Y. Bot. Garden 13: $126-7$.

Denchweinitz, George E. 1915. Kerato mycosis. Ploc. Path. Soc. Phila. n. s. 18: 19 .

Hollande, A. Ch. And Beaunerie, J. 1916. Spirales de Curchmann et Asp. pulmonaire. Bul. Soc. Myc. France 32: 17-24.

Chable, Robert. 1917. Über die pathogene Wirkung des Aspergillus fumigatus in der Subkutis der Meerschweinchens. Arch. Derm. u. Syphilis, 124: 14.

Castellani, A. And Chalmers, A. J. 1919. Manual of Tropical Medicine, ed. 3, London, pp. 1026-1032.

Emile-Weil, P. and Gaudin, L. 1319. Contribution à l'étude des onychomycoses. IV. Onychomycoses causées par Sterigmatocystis unguis. Archives Med. Exp. Anat. Path. Paris 28: 463-465. illust.

Harmer, Douglas and Jockes, T. 1919. Specimens of Aspergillus fumigatus from nasal sinuses. Proc. Roy. Soc. Med. (London) 12: Laryngology: 187-188.

Sartory, Antoine and Flament, I. 1920. Étude morphologique et biologique d'un Aspergillus nouveau isolé d'expectorations d'un malade suspect de tuberculose pulmonaire. Compt. Rend. Soc. Biol. 93: 1114-1115.

Galavielle and Cazejust. 1922. Un cas d'otomycose aspergillaire. Bull. Sci. Pharmacol., XXIX, nos. 8-9, p. 438-439.

Langeron, Maurice. 1922. Sur un champignon d'une otomycose brésillienne Sterigmatocystis Hortai. Bull. Soc. Path. Exot. 15: 383, 384.

Church, Margaret B. And Buckley, John S. 1923. Laboratory feeding of molds to animals. N. Amer. Vet. 4: 7-13.

Gardey, F. 1923. Aspergillosis of the lung. Semana Medica, Buenos Aires, 1: 390 .

LyNCH, K. M. 1923. Aspergillus in scalp lesions following red-bug (Leptus) bites. Archives Derm. and Syph. 7: 599.

Ota, Masao. 1923. Sur une nouvelle espèce d'Aspergillus pathogène: Aspergillus Jeanselmei n. sp. Ann. Parasitol Hum. Comp. 1: 137-146.

Sartory, A. 1920 to 1923 . Champignons Parasites de l'Homme et des Animaux. In fascicles.

Sartory, A. AND Bailly, A. 1923. Les mycoses pulmonaires et leurs parasites, Paris, p. 335 .

Vincens, F. 1923. Sur l'Aspergillomycose des abeilles. Compt. Rend. Acad. Sci. (Paris) 177: 540-542.

Cleland, J. B. 1924. Aspergillosis of pleura with sclerotium formation. Med. Jour. Australia (Sydney), 1:634.

Langeron, Maurice. 1924. Un Sterigmatocystis nouveau, parasite de l'homme en Tunisie, S. tunetana n. sp. Bull. Soc. Path. Exot. 17: 345-347, 1 fig. 
MacFarlan, D. 1921. Fungus on Tongue. Jour. Amer. Med. Assn. 83: 1538.

Andersox, C. 1925. Sur un cas d'otomycose à Sterigmatocystis nigra. Arch. Inst. Pasteur Tunis 14: 93-96.

Van Leeuwex, W. Stori, Biex, Z., Kremer, W. and Varekamp, H. 1925. Ueber die Bedeutung kleinsporiger Aspergillus-Arten (Typus Aspergillus fumigatus) fur die Aetiologie des Asthma bronchiale. Ztschr. Immunitätsforchung. Bd. 44: 1-26.

LAPHAM, MARY E. 1926. Aspergillosis of the lungs and its association with tuberculosis. Jour. Am. Med. Assoc. 87: 1031-1032.

Sartory, Antolne, Sartory, R. and Meyer, Jacques. 1926. La formation des perithèces chez l'Aspergillus fumigatus Fresenius sous l'influence du radium. Compt. Rend. Acad. Sci. 183: 1360-1362.

Steele, Albert E. 1926. A case of infection with Aspergillus versicolor. Boston Med. Surg. Jour. 195: 536-538.

Pinoy, E. And Naxta, A. 1927. Aspergillose expérimentale chez le lapin. Compt. Rend. Soc. Biol., 97: 67-8.

Pinoy, E. ANd Nanta, A. 1927. Sur l'existence fréquente d'une mycose de la rate en Algérie. Compt. Rend. Acad. Sci., 184: 347-8.

Van Leeuwex, W. Storm, Einthoven, W. And Kremer, W. 1927. The allergenproof chamber in the treatment of bronchial asthma and other respiratory diseases. The Lancet. 212: 1287-9.

Vax Leeuwex, W.. Storm and Kremer, W. 1927. Schimmelpilzallergene als Krankheitsursachen. Klin. Wochenschrift 6(9): 1-9.

Gosset, A., Bertraxd, J. and Magrou, J. 1928. Recherches experimentales sur l'aspergillose splenique. Compt. Rend. Soc. Biol., 98: 769-70.

Hansex, K. 1928. Ueber Schimmelpilz-Asthma. Verhandl, d. Deutsch. Gesellsch. f. Inn. Med. Kong. 40: 204-6.

Keller, Рh. 1928. Epidermal Aspergillusymkose der Haut. Dermat. Wochenschr. 87: 1831-3.

MAtTa, Alfredo DA. 1928. Sterigmazocystis tropicalis n. sp. de fungo patogenico para o homen. Bol. Inst. Brasil Sci. 3: 51-54, 1 pl.

Nanta, A. Axd Chatellier, L. 1928. Aspergillose cutanee et splenomycose. Bull. Soc. Franç. de Dermat. et Syph. 35: 621-3.

WAHL, E. F. AND ERICkson, M. J. 1928. Primary pulmonary aspergillosis. Jour. Med. Assn. Georgia 17: 341-9.

Coccheri, P. 1929. Micosi pulmonare da Sterigmatocystis nigra van Tieghem. Atti Ist. Bot. R. Univ. Pavia IV, 1: 161-1S1, 8 figs.

Puestow, K. L. 1929. Maduromycosis, a contribution to the study of maduromycosis with report of a case of infection with Aspergillus nidulans. Arch. Derm. Syphilol. 20: 642-661.

Agostini, Axgela. 1930. Dermatomicosi dovuta a Eurotium rubrum Bremer. Atti. Ist. Bot. R. Univ. Pavia IV, 2: 65-79, 5 figs.

Bernton, Harry S. 1930. Asthma due to a mold-Aspergillus fumigatus. Jour. Am. Med. Assn. 95: 189-190.

DA Fonseca, Olympio. 1930. Mycetoma por "Aspergillus Amstelodami." Rev. Med.-Cir. Brasil 38: 415-430, Pl. 1, (1-19).

TAlice, R. V. AND MCKínyon, J. E. 1931. Aspergillus (Eurotium) montevidensis, n. sp. isole d'un cas d'otomycose chez l'homme. Soc. de Biol. (Paris) Compt. Rend. 108: 1007-1009.

Bernton, Harry S. and Thom, Charles, 1933. The importance of molds as allergic excitants in some cases of vasomotor rhinitis. Jour. Allergy 4: 114-122. 
Bernstein, Theodore B. and Feinberg, Samuel MI. 1942. Air-borne fungus spores. Jour. of Allergy (St. Louis) 13(3): 231-241.

Morrow, Marie B., Lowe, E. P. and Prince, Homer E. 1942. Mold fungi in the etiology of respiratory allergic diseases. I. A survey of air-borne molds. Jour. of Allergy 13(3): 215-226.

\section{Physiology of the Aspergilli}

Pfeffer, W. 1895. Ueber Elektion organischer Nährstoffe. Jahrb. Wiss. Bot. 28: 205 .

Latham, Marion E. 1909. Nitrogen assimilation of Sterigmatocystis nigra and the effect of chemical stimulation. Bul. Torrey Bot. Club 36: 235-244.

Dox, A. W. 1911. The phosphorus assimilation of Aspergillus niger. Jour. Biol. Chem. 10: 77-80.

Bornand, M. 1913. Influence des métaux sur le développement de l'Aspergillus niger cultivé sur liquide de Raulin. Centralb. f. Bakt., etc., 2 Abt. 39: $488-496$.

Zaleski, W. AND PJukow, D. 1914. Über Elektion der Stickstoffverbindungen durch Aspergillus. Ber. Deut. Bot. Gesell. 32: 479.

Duggar, B. M., Severy, J. W. and Schmitz, H. 1917. Physiology of the fungi. (V) Growth of certain fungi in plant decoctions. (A. niger) Ann. Missouri Bot. Garden 4: 280.

Steinberg, R. A. 1918. A study of some factors influencing the stimulative action of zine sulphate on the growth of Aspergillus niger. I. The effect of the presence of zine in the culture flasks. Memoirs Torrey Bot. Club 17: 287-293.

Bezssonof, N. 1919. Utber das Wachstum der Aspergillaceen und anderer Pilze auf stark zuckerhaltigen Nährboden. Ber. Deut. Bot. Gesell. 36: 646-648.

Sternberg, R. A. 1919. A study of some factors influencing the stimulative action of zine sulphate on the growth of Aspergillus niger. II. A comparison of two strains of the fungus. Bull. Torrey Bot. Club 46: 1-20, pl. 1.

Steingerg, R. A. 1919. A study of some factors in the chemical stimulation of the growth of Aspergillus niger. Amer. Jour. Bot. 6: 330-372.

Weвb, R. W. 1919. Studies in the physiology of the fungi. X. Germination of the spores of certain fungi in relation to hydrogen-ion concentration. Ann. Missouri Bot. Gard. 6: 201-222. Webb reviews the literature of the acidity of culture media quite fully in this paper.

Zeller, S. M. ANd Schuitz, H. 1919. Studies in the physiology of the fungi. VIII. Mixed Cultures. Ann. Missouri Bot. Garden 6: 183-192.

Pringsheim, H. and Lichtenstein, S. 1920. Versuche zur Anreicherung von Kraftstroh mit Pilzeiweiss. Cellulosechemie 1: 29-39.

Arnstrong, G. M. 1921. Studies in the physiology of the fungi. MIV. Sulphur nutrition, etc. Ann. Missouri Bot. Gard. 8: 237-281.

Molliard, M. 1924. Retentissement de la composition minerale du millieu nutritif sur la structure du Sterigmatocystis nigra. Compt. Rend. Acad. Sci. (Paris) 178: $1865-1867$.

Cook, S. F. 1926. The effects of certain heavy metals on respiration. Jour. Gen. Physiol. 9: 575-601. Aspergillus niger studied.

Bortels, H. 1927. Über die Bedeutung von Eisen, Zink und Kupfer fur Mikroorganismen. Biochem. Zeits. 182: 301-358. A. niger studied.

TAmiy A, H. 1927. Studien über die Stoffwechselphysiologie von Aspergillus oryzae. I. Acta Phytochim. 3: 51-173. 
Meyer, R. 1928. Effect of temperature on the course of growth in fungi. Biochem. Zeitschr. 198: 463-4ī. A. niger studied.

Roberg, M. 1928. Über die Wirkung von Eisen-, Zink-, und Kupfersalzen auf Aspergillen. Centralb. Bakt. 2 Abt. 74:333-370.

Bortels, H. 1929. Biokatalyse und Reaktionsempfindlichkeit bei niederen und höheren Pflanzen. Angew. Bot. 11: 285-332.

TAmiYa, Hiroshi AND MiWA, Y. 1929. Anaerobic respiration of Aspergillus. Zeitschr. Bot. (8) 21: $417-32.7$ fig.

Sakamura, T. 1930. Die resorption des ammonium- und nitratstickstoffs durch A spergillus oryzae. Planta 11: 765-814.

Iatznelson, R.S. 1931. The effect of olive oil on the metabolism of certain fungi. I. The effect of the addition of olive oil in the chemical composition of mycelia of the fungi and on their utilization of various foodstuffs. Arch. Sci. Biol. USSR 31: 385-98. Aspergillus flavus and Penicillium sylvaticum.

RoBerg, M. 1931. Weitere Untersuchungen über die Bedeutung des Zinks für Aspergillus niger. Centralb. Bakt. 2 Abt. 84: 196-230.

Yonemoto, S. AND Kato, H. 1931. Factors influencing the perithecium formation of Aspergillus galactus Link. Bull. Miyazaki Coll. Agr. Forestry 3: 59-66.

Hopkins, S. J. And Chibnall, A. C. 1932. Growth of Aspergillus versicolor on higher paraffins. Biochem. J. 26: 133-142.

Kiessling, L. E. ANd Schmidt, A. 1932. Influence of organic substances on the growth of Aspergillus niger. Arch. Pflanzenbau 9: 293-305.

Levy, G. 1932. Influence of aluminum on the development of Sterigmatocystis nigra. Bull. Soc. Chim. Biol. 14: 745-57.

Mezzadroli, G. ANd Amati, A. 1932. The action of certain alkaloids on the development of Aspergillus niger. Atti. Accad. Lincei 16: 366-9.

Porges, N. 1932. Chemical composition of Aspergillus niger as modified by zinc sulphate. Bot. Gaz. 94: 197-205.

BACH, D. AND Desbordes, D. 1933. Direct transformation of nitrates into ammonia by the mycelium of lower fungi. Compt. Rend. 197: 1+63-5. Aspergillus repens studied.

Bousquet, Jean. 1934. The influence of the composition of the air on the development of cultures of Aspergillus niger. Bull. Sci. Pharmacol. 41: 28-34.

Pirschle, IARl. 1934. Vergleichende Untersuchungen über die physiologische wirkung der elemente nach wachstumversuchen mit Aspergillus niger (stimulation und toxizität). Planta 23: 177-224.

Steinberg, R. A. 1934. The so-called "Chemical Stimulation" of Aspergillus niger by iron, zinc and other heavy metal poisons. Bull. Torrey Bot. Club 61: 241-8.

Steinberg, R.A. 1935. Nutrient-solution purification for removal of heavy metals in deficiency investigations with Aspergillus niger. Jour. Agr. Res. 51(5): $413-424$.

Steinberg, R. A. 1935. The nutritional requirements of the fungus Aspergillus niger. Bull. Torrey Bot. Club 62: 81-90.

VASIL'Ev, G. 1935. Biochemical characterization of certain strains of Aspergillus niger on the basis of acid-forming capacity. Biochem. Zeit. 278: 226-34.

Golmirck, F. 1936. The influence of zinc, iron, copper and their combinations on the growth of Aspergillus niger. Centr. Bakt. Parasitenk, II Abt. 93: 421-42.

Iтzевотт, D. 1936. Über die bedingungen der stickstoffaufuahme, vor allem der nitrataufnahme, bei Aspergillus niger. Flora (Jena) 131 (n. s. 31): 60-\$6. 
SteinberG, R. A. 1936. Relation of accessory growth substances to heavy metals, including molybdenum, in the nutrition of Aspergillus niger. Jour. Agr. Res. 52: 439-48.

Steinberg, R. A. 1936. Effects of barium salts on Aspergillus niger and their bearing on the sulfur and zinc metabolism of the fungus in an optimum solution. Botan. Gaz. 97: 666-71.

Butkevich, V.S. AND Trofimova, E. I. 1937. Magnesium as an activator of biochemical conversions. Compt. Rend. Acad. Sci. (USSR) (In English) 17: $221-5$.

Steinberg, R. A. 1937. Rôle of molybdenum in the utilization of ammonium and nitrate nitrogen by Aspergillus niger. Jour. Agr. Res. 55: 891-902.

BATEs, JAmes C. 1938. Effects of certain alkaloids on the growth of Aspergillus niger and Rhizopus nıgricans. Univ. Kansas Sci. Bull. 25: 85-112.

Steinberg, R. A. 1938. The Essentiality of Gallium to Growth and Reproduction of Aspergillus niger. Jour. Agr. Res. 57: 569-574.

Steinberg, R. A. 1938. Correlation between Biological Essentiality and Atomic Structure of the Chemical Elements. Jour. Agr. Res. 57: 851-858.

Tausun, V. O. 1938. The conversion of energy by microörganisms. VIII. The amount of living and of dead cells in mold fungi. Microbiology (USSR) 7: 75.

Steinberg, R. A. And Bowling, J. D. 1939. Optimum solutions as physiological reference standards in estimating nitrogen utilization by Aspergillus niger. Jour. Agr. Res. 58: 717-732.

Steinberg, R. A. 1939. Effects of nitrogen compounds and trace elements on growth of Aspergillus niger. Jour. Agr. Res. 59: 731-748.

Steinberg, R. A. 1939. Relation of carbon nutrition to trace-element and accessory requirements of Aspergillus niger. Jour. Agr. Res. 59: 749-763.

Kauffmann-Cosla, O., Vasiliu, N. and Brull, R. 1940. Effect of ions on the germination and development of the spores of Aspergillus niger. Rev. Gen. Botan. 52: 97 .

Steinberg, R. A. 1940. Action of some organic compounds on yield, sporulation, and starch formation of Aspergillus niger. Jour. Agr. Res. 60: 765-773.

TAMIYA, H. AND UsAMI, S. 1940. Growth of Aspergillus oryzae with the addition of amino acids as the only source of carbon and nitrogen. Acta Phytochim. (Japan) 11: 261-98 (in German).

GarreaU, Y. 1941. Formation of sulfuric acid from some organic sulfur derivatives by Aspergillus niger. Compt. Rend. Soc. Biol. 135: 508.

Ivanov, N. N. AND Makrinova, N. A. 1941. The influence of acenaphthene on formation of organic acids in Aspergillus niger. Doklady Akad. Nauk USSR 30: $356-8$.

Steinberg, R. A. 1941. Sulfur and trace-element nutrition of Aspergillus niger. Jour. Agr. Res. 63: 109-27.

\section{Piguents and Coloring Substances}

Linossier, Georges. 1891. Sur une hématine végétale, l'aspergilline. Compt. Rend. Acad. Sci. (Paris) 112: 807-808.

KLöcker, A. 1916. Über die Bildung eines Fluoresein ähnliches Stoffes in Kulturen von A.glaucus. Zentbl. f. Bakt. 2 Abt. 46: 225-6.

Blochwitz, A. 1928. Farbenänderung, Verschiedenfarbigkeit, Farbenvariation bei Schimmelpilzen. Ber. Deuts. Bot. Ges. 48: 516 . 
Metz, O. 1930. Über Wachstum und Farbstoffbildung einiger Pilze unter dem Einfluss von Eisen, Zink und Kupfer. Arch. Mikrobiol. 1: 197-251.

Blochwitz, A. 1931. Die Farbstoffe der Schimmelpilze. Zentbl. f. Bakt., II, 80: 201.

Raistrick, H. 1932. Biochemistry of the lower fungi. Ergebnisse der Enzymforschung. 1: $345-363$.

Quilico, A. ANd Di CAPCA, A. 1933. Aspergilline, the pigment of Aspergillus niger spores I. Atti Accad. Lincei 17: 93-8.

Quilico, A. And Di CAPUA, A. 1933. Aspergilline, the pigment of Aspergillus niger spores II. Atti Accad. Lincei 17: 177-82.

Gould, B. S. ANd Raistrick, H. 1934. The biochemistry of microörganisms. XL. The crystalline pigments of species in the Aspergillus glaucus series. Biochem. Jour. 28: 1640-56.

Raistrick, H., Robinson, Robert and Todd, A. R. 1937. The chemistry of Aspergillus colouring matters. Part I. (London) Chem. Soc. Jour. 1937 : 80-88. Aspergillus glaucus group.

Cruickshank, J. H., Raistrick, H. and Robinson, Robert. 1938. The chemistry of Aspergillus colouring matters. Part II. (London) Chem. Soc. Jour. 1938: 2056-2064. Aspergillus glaucus group.

Lavollay, J. and Laborey, F. 1938. The circumstances of the appearance of yellow pigments in the liquid culture of Aspergillus niger. Compt. Rend. 206: $1055-6$.

Ashley, J. N., Raistrick, H. and Richards, T. 1939. The biochemistry of microorganisms. LXII. The crystalline coloring matters of species in the Aspergillus glaucus series. 2. Biochem. Jour. 33: 1291-1303.

Lavollay, J. ANd Laborey, F. 1939. Characterization of lactoflavin produced by Aspergillus niger $\mathrm{v}$. Tgh. partially deficient in magnesium. Compt. Rend. 208: 1056-8.

Knobloch, H. and Selliann, R. 1941. The formation of flavin-type pigments in liquid cultures of Aspergillus niger. Zentr. Bakt. Parasitenk 103: 277.

\section{Soll Tests for Mineral Deficiencies}

Schlots, F. E., Surth, F. B. And Brown, P. E. 1932. Aspergillus niger as an indicator of available phosphorus in the soil. Proc. Iowa Acad. Sci. (1931)38: 303-307.

Menlich, A., Truog, E. And Fred, E. B. 1933. The Aspergillus niger method of measuring available potassium in soil. Soil Science 35: 259.

Niklas, H., Poschenrieder, H. and Trischler, J. 1933. Urtiele und erfahrungen über die Verwendbarkeit und Brauchbarkeit der Aspergillus-Kalimethode und deren beurteilung nach dem Stand der bisherigen Forschungergebnisse. Ztschr. Pflanzenern. Dung. und Bodenk., 12: 3, 109-130.

Stock, Jurgen. 1933. Kulturversuche mit Aspergillus niger als Indikator für die Dungerbedurftigkeit. Bot. Arch. 35: 1-76.

Butkevich, V. S. And Naidina, O. G. 1934. Microbiological methods in determining the fertilizer requirements of soils. Chemisation Socialistic Agr. No. 4, 62-60.

Niklas, H. 1934. Bodenuntersuchungsmethoden. Die Aspergillus methode von Niklas und mitarbeitern. Ztschr. Pflanzenern. Dung. und Bodenk. 13: 64-65.

Suith, A. M. and Dryburg, A. 1934. The examination of soils by means of Aspergillus niger. Jour. Soc. Chem. Ind. 53: 250-25t. 
VARALlyaY, G. 1934. (Determination of the effect of $\mathrm{K}$ and $\mathrm{P}$ by a comparative method with Aspergillus). Ztschr. Pflanzenern. Dung. und Bodenk. 34A: 215-223.

Smith, F. B., Brown, P. E. and Millar, H. C. 1935. The assimilation of phosphorus by Aspergillus niger and Cunninghamella sp. Jour. Amer. Soc. Agron., 27: 12, 985-1000.

Sтоскі, A. 1936. Die mikrobiologischen Methoden zur Bestimmung des Dungerbedurfnisses der Boden. Sehweiz. Landw. Monatsh. 14(6) : 169-179.

Mooers, C. A. 1933. An evaluation of the Neubauer and the Cunninghamella and Aspergillus niger methods for the determination of the fertilizer needs of a soil. Soil Sci., 46: 211-227.

Mulder, E. G. 1939. The importance of eopper for the growth of microörganisms and a microbiological method of estimation of soil copper available to plants. Arch. Mikrobiol. 10: 72-86.

\section{VARIATION}

Schiemann, Elisabeth. 1912. Mutationen bei Aspergillus niger van Tieghem. Ztschr. Induk. Abstam. u. Vererbungslehre, Bd. S, Heft 1/2, pp. 1-35, 16 figs., 2 pl. (1 col.).

Schrami, R. 1914. Über eine bemerkenswerte Degenerationsform von A. niger. Myc. Centralb. 5: 20-27.

HaENicke, A. 1916. Vererbungsphysiologische Untersuchungen an Arten von Penicillium und Aspergillus. Zeitschr. Bot. 8: 225-352.

Wehrier, C. 1919. Verlust des Oxalsäure-Bildungsvermogens bei einem degenerierten Aspergillus niger. Centralb. f. Bakt., etc., 2 Abt. 49: 145-148.

Blochwitz, Adalbert. 1923. Eine allgemeine Ursache spontaner Verlustmutationen bei Schimmelpilzen. Ber. Deut. Bot. Gesell. 41: 205-208.

Blochwitz, Adalbert, 1925. Entehung von Aspergillus-varietäten mit verzweigten Conidienträgern. Ber. Deut. Bot. Gesell. 43: 103-10s.

Sartory, A., Sartory, R. ANd Meyer, J. 1927. La formation des périthèces chez l'Aspergillus fumigatus Fresenius sous l'influence du radium. Compt. Rend. Soc. Biol. 96: 276-278.

Sartory, A., Sartory, R. and Meyer, J. 1927. Les variations des appareils, vegetatifs et conidiens, de l'Aspergillus fumigatus Fresenius en cultures sur milieux dissociés et non dissociés sous l'influence des radiations du radium. Bull. Sci. Pharmacol., 34: 193-202.

BARnes, B. 1928. Variations in Eurotium herbariorum (Wigg.) Link, induced by the action of high temperatures. Ann. of Bot. 42: 783-812.

Sartory, A., Sartory, R. And Meyer, J. 1928. Contribution a l'etude biologique de l'Aspergillus fumigatus Fresenius issu de souches sexuées et asexuées. Compt. Rend. Soc. Biol., 98: 215-21.

Blochwitz, ADalbert. 1932. Variabilität und Vererbung bei Schimmelpilzen. Ber. Deut. Bot. Gesell. 50: 248-255.

Galloway, L. D. 1933. The stimulation by dilute antisepties, of sectoring in mould cultures. Brit. Mycol. Soc. Trans. 18(II): 161-162. Salicylanilide at concentration of 0.003 to 0.005 percent of the sodium salt produced sectoring.

Greene, H. C. 1933. Variation in single spore culture:3 of Aspergillus fischeri. Mycologia 25: 117-138.

Henrard, Paul. 1934. Polarite, Heredite, et Variation chez diverses espèces d'Aspergillus. La Cellule, tome XLIII, fasc. 3: 351-424. 
Mosseray, Raoul. 1934a. Les Aspergillus de la section niger Thom and Church. La Cellule, tome XIIII, fasc. 2. 203-285.

Mosseray, R. 1934b. Races naturelles et variations de culture chez divers Aspergillus. Ann. Soc. Sci. Bruxelles, ser. B, 54: 161-189.

Kresling, E. And Stern, E. 1936-37. Über die Wirkung von radium- und ultravioletten Strahlen auf die Entwicklung, die biochemischen Eigenschaften und die Rassenbildung des Aspergillus niger. Zentralblatt fur Bakt. Abt. II, 95: $327-40$.

Sartory, A., Sartory, R. And Meyer, J. 1936. Étude de l'action du radium sur l'Aspergillus fumigatus Fresenius en culture sur milieux dissociés et non dissociés. Compt. Rend. Acad. Sci., 183: $7 \bar{\imath}-79$.

Nakazawa, R. and Simo, M. 1938. Effect of irradiation on fermentative microorganisms. I. Morphological and biochemical characteristics of races of Aspergillus niger produced by radium treatment. Jour. Agr. Chem. Soc. Japan 14: 895-910.

Whelden, R. M. 1938. Changes observed in cultures of Aspergillus niger bombarded as spores with low voltage cathode rays. Mycologia 30: 265-268.

Yuill, Edward and Yulle, John L. 1938. Cladosarum olivaceum. A new hyphomycete. Trans. Brit. Mycol. Soc. 22: 194-200, illus.

Buchwald, C. E. ANd Whelden, R. M. 1939. Stimulation of growth in Aspergillus niger under exposure to low velocity cathode rays. Am. Jour. Bot. 26: 778784.

Nakazawa, Ryozi and Sino, M. 1939. The action of radium and x-rays on microorganisms. II. The production of acids by radium-irradiated Aspergillus niger. Jour. Agr. Chem. Soc. Japan 15: 547-52.

Thon, Charles and Steinberg, Robert A. 1939. The chemical induction of genetic changes in fungi. Proc. Nat. Acad. Sci. 25: 329-335.

YUill, EDWARD. 1939. Two new Aspergillus mutants. Jour. of Botany, 174-175, pl. 618. June.

Zahl, Paul A., Koller, L. R. and Haskins, C. P. 1939. The effects of ultraviolet radiation on spores of the fungus Aspergillus niger. Jour. Gen. Physiol. $22(6)$ : $689-98$.

Gossop, George Harold, Yuild, Edward and Yuill, John Lewis. 1940. Heterogeneous fructifications in species of Aspergillus. Trans. Brit. Myc. Soc. $24(3 / 4): 337-344$.

Simo, M. 1940. Experimentelle Untersuchungen uber die Wirkung von Radium und Röntgen-Strahlen auf die Garungsmikroorganismen. III. Utber die Bedingung der Citronensauregarung durch Aspergillus niger Radium rasse. Jour. Agr. Chem. Soc. Japan 16: 129.

Steinberg, R. A. And Thom, Charles. 1940. Chemical induction of genetic changes in Aspergilli. Jour. of Heredity 31: 61-63.

Steinberg, Robert A. And Thom, Charles. 1940. Mutations and reversions in reproductivity of Aspergilli with nitrite, colchicine and d-lysine. Proc. Nat. Acad. Sci. 26(6): 363-366.

Whelden, Roy M. 1940. "Mutations" in Aspergillus niger bombarded by low voltage cathode rays. Mycologia 32: 630-643.

Steinberg, R. A. 1942. Reversions in morphology of nitrite-induced "Mutants" of Aspergilli grown on amino acids. Jour. Agr. Res. 64: 645.

Thom, Charles. 1942. Chemical induction of genetic changes in Aspergilli. Jour. Franklin Inst. 233: 284. 


\section{Vitanins and Growth Substances}

Boysen-Jensen, P. 1931. Formation of a growth regulator by Aspergillus niger. Biochem. Zeit. 239: 243-9.

Schopmeyer, H. ANd Fulier, E. I. 1931. The production of yeast growth stimulants by the molds. I. Aspergillus niger, Trichoderma lignorum and Aspergillus clavatus. Jour. Bact. 22: 23-28.

Sakanura, T. And Yanagihara, T. 1932. The production of the growth substance by Aspergillus niger. Proc. Imp. Acad. Tokyo 8: 397-9.

Schopmeyer, H. 1932. Production of yeast-growth stimulants by molds on various media. Ia. St. Coll. Jour. Sci. 6: 471-2.

BünNisg, E. 1934. Growth and nitrogen assimilation in Aspergillus niger under the influence of growth regulators. Ber. Deut. Botan. Ges. 52: 423-44.

Bernhauer, K. And Gorlich, B. 1936. The formation of vitamin C-like substances by fungi and bacteria. I. Biochem. Zeit. 286: 60 .

Komarov, S. N. 1936. Methods of obtaining an antirachitic preparation from the waste products of citric acid manufacture from the mycelium of Aspergillus niger. Proc. Sci. Inst. Vitamin Res. USSR 1(2): 162-71.

Opfel, V. V. 1936. Chemistry and biochemistry of flavins. Vitamins and vitaminization. Proc. Sci. Inst. Vitamin Res. USSR 1(2): 5-52.

Scheunert, A. AND Schieblich, M. 1936. Vitamin production by Aspergillus oryzae. Biochem. Zeit. 286: 66-71.

Fukumoto, J. and Shinomura, H. 1937. Formation of Vitamin C-like substance by molds. Jour. Agr. Chem. Soc. Japan 13: 613-20.

Fitavin, G. S. 1939. Action of mercury salts on the formation of vitamin $\mathrm{B}_{2}$ in Aspergillus niger. Biokhimiya 4: 283-94.

Lavollay, J. AND Laborey, F. 1939. Characterization of lactoflavin produced by Aspergillus niger $\mathrm{v}$. Tgh. partially deficient in magnesium. Compt. Rend. Acad. Sci. 208: 1056-8.

SakuraI, Kiyura. 1939. Vitamin $B_{1}$ synthesis by microörganisms. I. Molds. Jour. Sci. Hirosima Univ. Ser. B. 2, 3: 191-200.

Krtavis, G. S. 1940. Crystalline riboflavin obtained through the action of mercuric salts on Aspergillus niger. Compt. Rend. Acad. Sci. USSR 28: 517-18.

SakuraI, K. 1940. Vitamin synthesis by microörganisms. II. Molds. Jour. Sci. Hirosima Univ. Ser. B, 2, 4: 1-6.

KNoblock, H. and Seliman, R. 1941. The formation of flavin type pigments in liquid cultures of Aspergillus niger. Zent. Bakt. Parasitenk II Abt. 103: $277-80$.

Eakin, R. E. ANd Eakin, E. A. 1942. Biosynthesis of Biotin. Science 96: 187. A. niger synthesizes biotin from biotin free media.

\section{Miscellaneous Products}

\section{Alcohol}

Molltard, M. 1916. Catalytic rôle of potassium nitrate in alcoholic fermentation produced by S. nigra. Compt. Rend. Acad. Sei. (Paris) 163: 570-572.

Yuile, J. L. 1928. Alcoholic fermentation by Aspergillus flavus Brefeld. Biochem. Jour. 22: 1504-7.

SAKagUChi, K. AND NAKANo, M. 1932. Alcohol fermentation by Aspergillus oryzae. Jour. Agr. Chem. Soc. Japan 8: 115-22.

Vyatkin, V. 1940. New process for making alcohol from chicory. Spirto-Vodochnaya Prom. 17(9): 13-14. 


\section{Chitin}

Norman, A. G. And Peterson, W. H. 1932. The chemistry of mold tissue. II. The resistant cell wall material. Biochem. Jour. 26: 1946-53.

\section{Ergosterol}

Pruess, L. M., Peterson, W. H. Axd Fred, E. B. 1932. Isolation and identification of ergosterol and mannitol from Aspergillus fischeri. Jour. Biol. Chem. 97: 483-9.

\section{Fluorescein}

KLoEcker, A. 1917. Formation of a substance resembling fluorescein in the cultures of Aspergillus glaucus. Compt. Rend. Tran. Lab. Carlsberg 11: 312313.

\section{Gum}

SANBorn, J. R. 1934. Microbiological film production. Ind. Eng. Chem. 26: 532-33.

SANBorn, J. R. 1935. Sheet material and method of manufacturing the same. U. S. Patent $2,026,253$.

Sanborn, J. R. 1936. Guns produced by fungi; industrial utilization. Ind. Eng. Chem. 28: 1189-1190.

\section{Hydroxylamine}

Lemoigne, M. AND Desveatx, R. 1935. Formation of hydroxylamine in cultures of Sterigmatocystis nigra in a medium rich in ammonium nitrate. Compt. Rend. 201: 239-41.

Lemotgre, M. And Desyetcx, R. 1936. Recherches sur la rôle biochimique de l'hydroxylamine. I. Fornation de l'hydroxylamine par le Sterigmatocystis nigra sur des millieux au nitrate d'ammonium. Bull. Soc. Chem. Biol. 16: $604-614$.

\section{Mannitol}

Braconxot, H. 1S13. Nouvelles recherchés analytiques sur les champignons pour servir de suite à celles qui ont été insérées dans les tons. LXXIX et LXXX des Annales de chimie. Ann. Chim. 87: 237-270. (Cited by Birkinshaw et al. 1931.)

Vauquelin. 1813. Experiences sur les champignons. Ann. Chim.85:1-25. (Cited by Birkinshaw et al. 1931.)

Birkinshaw, J. H., Charles, J. H. V., Hetherington, A. C. and Raistrick, H. 1931. Studies in the biochemistry of micro-organisms. IX. On the production of mannitol from glucose by species of Aspergillus. Trans. Roy. Soc. London 220B: 153-171.

Pruess, L. M., Peterson, W. H. And Fred, E. B. 1932. Isolation and identification of ergosterol and mannitol from Aspergillus fischeri. Jour. Biol. Chem. 97: 483-9.

Yamasake, I. ANd Shimonura, M. 1937. Formation of d-mannitol from glycerol by molds of the Aspergillus glaucus group. Biochem. A. 291: 340-8. 


\section{Polysaccharides}

Kostrchev, S. 1920. Formation of sugar by molds. Z. Physiol. Chem. 111: 236. Nomman, A. G., Peterson, W. H. and Houtz, R. C. 1932. The chemistry of mould tissue. I. Soluble carbohydrate constituents. Biochem. Jour. 26: 1934-45.

HIDA, T. 1934. Über die Stärkebildung von Schimmelpilzen. Jour. Shanghai Sci. Inst. 1: 85-116.

Clutterbuck, P. W. 1936. Recent developments in the biochemistry of the fungi. Jour. Soc. Chem. Ind. 55: 55T-61T.

\section{Ochracin}

YABUTA, T. AND SUmikr, Y. 1934. Chemical constitution of ochracin (a fermentation product of Aspergillus ochraceus). II. Jour. Agr. Chem. Soc. Japan 10: 703-14.

\section{Terrein}

Raistrick, H. ANd SMith, G. 1935. Biochemistry of micro-organisms. XLII. The metabolic products of Aspergillus terreus Thom. A new mold metabolic product-Terrein. Biochem. Jour. 29: 606-11. 


\section{Chapter XXIII}

\section{GENERAL BIBLIOGRAPHY}

Aввотт, E. V. 1926. Taxonomic studies on soil fungi. Iowa State Coll. Jour. Sci. 1: $15-36$.

Alsberg, C. L. And Black, O. F. 1913. Contributions to the study of maize deterioration. U. S. Dept. Agr., Bur. Plant Ind. Bul. 270: 1-18.

Amann, J. 1896. Conservirungsflüssigkeiten und Einschlussmedien fur Moose, Chloro- und Cyanophyceen. Zeitsch. f. Mikrosopie 13: 18-21.

Amons, W. J. Tн. 1921. Bijdrage tot de kennis van de flora van achteruitgaande suiker. Archief voor de Suikerindustrie in Nederlandsch-Indie 29: 4-19.

Anslow, Winston K. and Raistrick, Harold. 1938a. The biochemistry of microorganisms. LVII. Fumigatin and spinulosin, metabolic products respectively of Aspergillus fumigatus Fres. and Penicillium spinulosum Thom. Biochem. Jour. 32: 687-96.

Anslow, Winston K. and Raistrick, Harold. 1938b. The biochemistry of microörganisms. LIX. Spinulosin a metabolic product of a strain of Aspergillus fumigatus Fresenius. Biochem. Jour. 32: 2288-89.

Ashlex, Julius Nicholson, Raistrick, Harold and Richards, Taliesin. 1939. The crystalline colouring matters of species in the Aspergillus glaucus series. Part II. Biochem. Jour. 33: 1291-1303.

Bainier, G. 18s1. Sur quelques espèces de Sterigmatocystis. Soc. Bot. de France, pp. 76-79. February.

1908a. Myeothéque de l'école de pharmacie XXIV-XXVII. Bul. Soc. Mycol. France 24: 73-94. Plate VIII, figs. 1-13.

ANd Sartory, A. 1908b. Étude d'un Aspergillus pathogène, Aspergillus fumigatoides. Compt. Rend. Soc. Biol. 66: 22-23.

And Sartory, A. 1909. Étude d'un Aspergillus pathogène (Aspergillus fumigatoides n. sp.) Bul. Soc. Mycol. France 25: 111-118, illus. Pl. V.

and Sartory, A. 1911a. Étude d'une espèce nouvelle de Sterigmatocystis, Ster. flavipes nov. sp. Bul. Soc. Mycol. France 27:90-96. Plate III, figs. 1-6. AND SARtory, A. 1911b. Etudes biologiques et morphologiques de certains Aspergillus. Bul. Soc. Mycol. France 27: 98-102; 346-368.

AND Sartory, A. 1911e. Étude biologique et morphologique de certain Aspergillus à pigment (suite). Bul. Soc. Mycol. France 27: 453-468, illus.

And Sartory, A. 1912a. Etude de quelques Citromyces nouveaux. Bull. Soc. Mycol. France 28: 38-48.

And Sartory, A. 1912b. Etude biologique et morphologique de certains Aspergillus. Bul. Soc. Mycol. France 28: 257-269, illus. A. scheclci, A. umbrosus.

- and Sartory, A. 1913. Étude d'un espece nouvelle de Sterigmatocystis. Sterigmatocystis sydowi n. sp. Ann. Mycol. 11: 25-29. Plate III.

Barnes, B. 1928. Variations in Eurotium herbariorum (Wigg.) Link induced by the action of high temperatures. Ann. Bot. (London) 42: 783-812, illus.

Bary, A. De. 1854. Ueber die entwickelung und den zusammenhang von Aspergillus glaucus und Eurotium. Bot. Ztg. 12: 425-134, 441-151, 465-171, illus. 
1870. Eurotium, Erysiphe, Cicinnobolus. Nebst Bemerkungen über die geschlechtsorgane der ascomyceten. I. Eurotium. Senckenb. Naturf. Gesell. Abhandl. 7: 361-382, illus.

Berkeley, M. J. 1857. Introduction to eryptogamic botany. 604 pp., illust. Bailliere. London.

Bernhauer, K. 1929. Ueber die Charakterisierung der Stämme von Aspergillus niger auf Grund ihres biochemischen Verhaltens. I. Vergleichende Untersuchungen über die säurebildung durch verschiedene pilzstämme. Biochem. Zeitschr. 197: 278-86.

And Patzelt, George. 1935. Über Schimmelpilz-sterine. I. Mitteilung: Die Sterinbildung bei Aspergillus niger. Biochem. Zeit. 280: 388-93.

and Posselt, Grete. 1937. Uber Schimmelpilzlipoide. II. Mitteilung: Die Zusammensetzung eines Aspergillus niger Fettes. Biochem. Zeit. 294: 215-20.

Bernton, Harry S. 1930. Asthma due to mold-Aspergillus fumigalus. Jour. Am. Med. Assn. 95: 189-190.

Bezssonoff, N. 1919. Über das Wachstum der Aspergillaceen und anderer Pilze auf stark zucherhaltizen Nähroböden. Ber. deut. Bot. Gesselsch. 36: 646648.

Blakeslee, A. F. 1915. Lindner's roll tube method of separating cultures. Phytopathology 5: 68-69, Pi. VII.

Brourge, Ph. 1923. Les moisissures du groupe Penicillium Link. Étude Monograph. La Cellule 33: 1-330, Col. Pl. 13, figs. 1-137.

1939. Brevis conspectus generis Aspergillus Link. Manuscript prepared for presentation at the Third International Microbiological Congress in New York. Unpublished: Copy at the Northern Regional Research Laboratory, Peoria, Illinois.

Blochwitz, AdAlBert. 1925. Entstehung von Aspergillus-varietäten mit verzweigten conidienträgern. Ber. deut. Bot. Ges. 43: 105-108.

1928. Farbenänderung, Verschiedenfarbigkeit und Farbenvariation bei Schimmelpilzen. Ber. deut. Bot. Ges. 46: 516-24.

1929a. Die gattung Aspergillus. Neue Spezies. Diagnosen. Synonyme. Ann. Mycol. 27: 205-240, illus.

\section{9b. Die Aspergillaceen. Ann. Mycol. 27: 185-240, illust.}

1930. Standorte und geographische Verbreitung der Schimmelpilze. Ann. Mycol. 28(3/4): 241-268.

1931. Luftmyzelbildungen bei Schimmelpilzen. Bot. Centralb. Beiheifte Abt. Anal. und Phy. 48: 176-182.

1932a. Perithecien, Sklerotien und Eidamsche Blasen der Aspergillaceen. Beih. z. Bot. Centr., Abt. I, 49: 262-292.

1932b. Die Urformen der Aspergillen. Hedwegia 72: 173-174.

1932c. Variabilität und vererbung bei Schimmelpilzen. Deut. Bot. Gesell. Ber. 50: 248-256.

1933. Die Gattung Aspergillus. Neue Spezies, Synonyme und Nachträge. Ann. Myc. 31: 73-83.

1934. Die Gattung Aspergillus. III. Neue Spezies, Varianten und Mutanten der Konidienfarbe, Synonyme und interessante Standorte. Ann. Mycol. 32: 83-89.

1935. Die Urformen der Aspergillen. II. Bot. Centralb. Beihefte Abs. Morph. and Physiol. 63: 48-50.

Boediun, Ki. B. 1928. Notes on some Aspergilli from Sumatra. Ann. Mycol. 26: 69-84. 
BонN, P. R. 1931. Mechanism of the synthesis of fats at the expense of sugars. Compt. Rend. 193: 441-2.

Borzi, A. 1884. Inzengaea, ein neuer Askomycet. Pringsheim Jahrb. Wiss. Bot. 16: 450-463, pi. 19, 20.

Brefeld, O. 1874. Botanische untersuchungen über Schimmelpilze Heft 2. Leipzig.

1875. Neue kulturmethoden fur die untersuchung der pilze. Sitzungsber. d. Ges. naturf. Freunde zu Berlin. 125-33.

1876. Mycologische untersuchungen. Sitzungsber. der Versamml. deut. Naturforscher und Aerzte zu Hamberg im September. Bot. Ztg. 35 (1877): 77-80.

Buller, A. H. Reginald. 1933. Researches on fungi, V. pt. 1. pp. 1-167.

Bush, M. T. AND GoTh, A. 1943a. An antibacterial substance produced by an Aspergillus flavus. Federation Proc. 2: 75.

Bush, M. T. And Goth, A. 1943b. Flavicin: an antibacterial substance produced by an Aspergillus flavus. Jour. Pharm. and Exp. Ther. 78(2): 164-169.

Calam, C. T., Oxford, A. E. and Raistrick, H. 1939. The biochemistry of microörganisms. LXIII. Itaconic acid, a metabolic product of a strain of Aspergillus terreus Thom. Biochem. Jour. 33: 1488-1495.

Ceni, C. 1905. Potere patogene dell'Aspergillus ochraceus e suo rapporto coll'etiologia e patogenesi della pellagra. Riv. sper. d. freniat. Reggio-Emilia, 31; also Arch. Ital. Mal. Nerv. Ment. 42: 595-617, P. 11.

Centraalbureau Voor Schimmelcultures. 1939. List of cultures. 132 pp., Baarn, Netherlands.

Chaudhuri, H. and Sachar, G. S. 1934. A study of the fungus flora of Punjab soils. Ann. Mycol. 32: 90-100.

Chowdhury, H. P. And Mathur, R. S. 1938. On a new species of Emericella found in Lucknow. Ann. Myc. 36: 61-63.

Ciferri, R. 1938. Ritrovamento e cultura dell'Emericella variecolor Berkeley (Eurotiaceae). Nuovo Giorn. Bot. Ital. 45: 159-173. pl. 6.

Cook, A. H. ANd LaCeY, M. S. 1944. An antibiotic from Aspergillus parasiticus. Nature 153 (April 15) : 460.

Conda, A. C. I. 1842. A. glaucus var. repens. Icones Fungorum 5: 53, illus.

Costantin, J. And Lucet, A. 1903. Sur le Sterigmatocystis pseudo-nigra. Bull. Soc. Myc. France 19: 33.

Costantin, J. And Lucet, A. 1905. Recherches sur quelques Aspergillus pathogènes. Ann. Sci. Nat. Bot. IX. 2: 119-180, pl. 5.

Cramer, C. 1859. Vierteljahresschrift der Naturforsch. Gesellschaft Zurich 4: 325.

Cruickshank, J. H., Raistrick, H. and Robinson, Robert. 1938. The chemistry of Aspergillus colouring matters. Part II. (London) Chem. Soc. Jour. 1938: 2056-2064.

Curzi, M. 1934. Un' aspergillacea ad ascospore stellate. Rend. Accad. Naz. Lincei 19: 424-428. fig. 1.

Czapek, F. 1902. Untersuchungen uber die stickstoff gewinnung und Eiweissbildung der Pflanzen. Beitrage zur Chemischen Physiologie und Pathologie Band 1. Heft 10-12, pp. 540-560; also Band 3, pp. 47-66, 1903.

Dale, E. 1909. On the morphology and cytology of Aspergillus repens DeBary. Ann. Mycol. 7: 215-225.

1912. On the fungi of the soil. Ann. Mycol. 10: 452-477.

1914. On the fungi of the soil II. Ann. Mycol. 12: 33-62. 
Dangeard, P. A. 1907. L'origine du périthèce chez les ascomycètes. Le Botaniste, 10: 1-385.

Delacroix, G. 1893a. Espèces nouvelle observées au Laboratoire de Pathologie végetale. Bul. Soc. Mycol. France 9: 184-188.

1893b. Champignons parasites nouveaux. Bul. Soc. Mycol. France 9: $26+268$.

Dezmazieres, J. 1834. Descriptions et figures de six Hyphomycetes inédites à ajouter a la Flore française. Ann. d. Sci. Nat., 2 Ser. Bot. 2: 69-73.

Donge, C. W. 1935. Medical Mycology, 900 pp. illus., Mosby and Company, St. Louis.

Dox, A. W. 1910. The intracellular enzymes of Penicillium and Aspergillus with special reference to those of $P$. camemberti. Bul. U. S. Dept. Agr. Bur. Animal Ind. 120.

Eidam, E. 1883. Zur Kenntniss der Entwicklung bei den Ascomyceten. III. Sterigmatocystis nidulans n. sp. Cohn Beitr. Biol. Pflanzen 3: 392-411, pl. 20-22.

Elser, W. J., Thomas, R. A. and Steffen, G. I. 1935. The desiccation of sera and other biological products (including microörganisms) in the frozen state with the preservation of the original qualities of products so treated. Jour. Inmun. 28: 433-473.

Enile-Weil, P. and Gaudin, L. 1934. Contribution à l'Étude des onychomycoses. IV. Onychomycoses causées par Sterigmatocystis unguis. Arch. Med. Exp. Anat. Path. Paris 28: $463-465$, illust.

Exgler, A. And Prantl, K. 1897. Die naturlichen Pflanzenfamilien etc. I. Teil Abt. 1: 1-3.

Ferdinanden, C. C. F. and Winge, O. 1910. Fungi from Prof. Warming's expedition to Venezuela and the West Indies. Kob. Bot. Tids. 30: 20S-222. A. dipsus was one of a miscellaneous series described.

Da Fonseca, Olympio. 1930. Mycetoma por "Aspergillus amstelodami." Rev. Med. Cir. Brasil 38: 415-430, Pl. 1 (1-19).

Frazer, H. C. I. and Chambers, H. S. 1907. The morphology of A. herbariorum. Ann. Mycol. 5: 419-431, Pls. XI, XII.

Fresenius, G. 1850-53. Beiträge zur Mykologie. Frankfurt.

1863. Beiträge zur Mycologie. Heft 3. Frankfurt.

Fuluer, E. I. and Werkman, C. H. 1930. An index to the chemical action of microörganisms on the non-nitrogenous organic compounds. 198 pp. Springfield, Illinois, and Baltimore.

Galloway, L. D. 1930. The fungi causing mildew in cotton goods. Jour. Text. Inst. 21: T277-T286.

1933. The stimulation by dilute antiseptics of sectoring in mold cultures. (the action of Salicylanalide on A. terreus in flour agar). Brit. Mycol. Soc. Trans. 18(II): 161-162.

Gastrock, E. A., Porges, N., Wells, P. A. And Moyer, A. J. 1938. Gluconic acid production on pilot plant scale: Effect of variables on production by submerged mold growth. Ind. Eng. Chem. 30: 782-789.

Gilian, J. C. ANd Аввотt, E. V. 1927. A summary of the soil fungi. Ia. St. Coll. Jour of Sci. 1: 225-345.

Glister, G. A. 1941. A new antibacterial agent produced by a mold. Nature 148: 470. A. flavus.

Govld, B. S. 1938. The metabolism of Aspergillus tamarii Kita. Kojic acid production. Biochem. Jour. 32: 797-802. 
AND Raistrick, H. 1934. The biochemistry of microörganisms. XL. The crystalline pigments of species in the Aspergillus glaucus series. Biochem. Jour. 28: 1640-1656.

Greene, H. C. 1933. Variation in single spore cultures of Aspergillus fischeri. Mycologia 25: 117-138. 3 figs., 2 plates.

- ANd Fred, E. B. 1934. Maintenance of vigorous mold stock cultures. Ind. Eng. Chem. 26: 1297-98.

Gú́guen, Fernand. 1904. Champignons Parasites de l'homme et des animaux. Paris pp. 462.

1909. Aspergillus fontoynonti nova sp., parasite probable des nodosités juxta-articulaires. Compt. Rend. Soc. Biol. 66: 1052-53; also 67: 10-12.

Guéguen, Fernand. 1911. Abcès sous-dermiques à répétition produits par l'Aspergillus fontoynonti $\mathrm{n}$. sp. morphologique et biologique de cette espèce. Arch. Parasitol. 14: 177-192.

Haller, D. Alberti. 1742. Enumeratio Methodica Stirpium Helvetiae Indigenarum, etc.

1768. Historia stirpum indigenarum Helvetiae inchoata, etc.

Hallier, Ernst. 1870. Mittheilung über die Ohrpilze, welche Herr Dr. R. Hagen in Leipzig zur mikroscopischen Untersuchungen Einsandte. Zeitschr. Parasitenk. 2: 259-281, Pls. 5 and 6.

Hansen, H. N. 1938. The dual phenomenon in imperfect fungi. Mycologia 30: $442-455$.

- AND SMITh, R. E. 1932. The mechanism of variation in imperfect fungi: Botrytis cinerea. Phytopath 22: 953-964.

Hanzawa, Jun. 1911. Untersuchungen über die Pilze auf dem getrockneten Boniten oder "Katsuobushi." Jour. Coll. Agr. Tohoku Imp. Univ. Sapporo 4, pt. 4, p. 215, Taf. XIX-XXIII.

Henrard, P. 1934. Polarité, hérédité, et variation chez diverses espèces l'Aspergillus. La Cellule XLIII : $351-424$, Pls. I-V.

von Höhnel, F. 1902. Fragmente zur mykologie (I. Mittheilung) Sitzungsber. K. Akad. Wiss. Wien, Math.-Naturw. Kl. III. 1 Abt. p. 987.

Hooper, I. R., Anderson, H. W., Skell, P. and Carter, H. E. 1944. The identity of clavacin with patulin. Science 99: 16.

HopfFe, A. 1919. Ueber einen bisher unbekannten cellulose-lösenden im Verdauungstraktus vorkommenden Aspergillus, "A. cellulosae," seine Zuchtung und seine Eigenschaften. Centralb. f. Bakt., etc. 1, Orig. 83: 531-537.

Hopkins, S. J. And Chibnali, A. C. 1932. Growth of A. versicolor on higher paraffins. Biochem. Jour. 26(1): 133-142.

Huber, G. A. 1933. Aspergillus sclerotiorum n. sp., and its relation to decay in apples. Phytopathology 23: 306-308.

Jones, H., Rake, G. And Hamre, D. M. 1943. Studies on Aspergillus flavus. I. Biological properties of crude and purified aspergillic acid. Jour. Bact. 45: 461-469.

Karow, E. O. 1942. The production of citric acid in submerged culture. Doctoral Thesis, Rutgers Univ., New Brunswick, N.J.

KeItт, G. W. 1915. Simple technique for isolating single-spore strains of certain types of fungi. Phytopath. 5: 266-269.

Krnoshita, K. 1931a. Über eine neue Aspergillus-art, A. itaconicus nov. spec. (Secondary title under the Japanese title.). Bot. Mag. Tokyo 45(530): 45-61, figs. 1-9 and Plate IV. 
1931b. Utber die Produktion von Itaconsäure und Mannit durch einen neuen Schimmelpilzen, Aspergillus itaconicus. Acta Phytochimica 5(3): 271-287, figs. 5 .

Kita, G. 1913. Einige japonische Schimmelpilze. Centralb. f. Bakt. etc. 2 Abt. 37(17/21): 433-452. A. tamarii Kita.

KIтA, G. 1914. Einige japonische Schimmelpilze. II. Mitt. Über die Aspergillusarten aus "Katsuobushi" und Vergleichung von vier A. ochraceus-artigen Pilzen. Centralb. Bakt. etc., 2 Abt. 41: 351-363.

Iinier, H. 1928. Die sexualität der nideren Pflanzen, Differenzierung, Verteilung, Bestimmung, und Vererbung des Geschlechts bei den Thallophyten. Jena. A. flavus, A. herbariorum, A. nidulans, A. repens.

Konschelt, O. 1878. Ueber Sake, das alcoholisches Getränk der Japaner. Dingler's Polytechniches Journ. 230: 76-80; 172-181, 229-240; 330-335; 421-427. Eurotium oryzae.

LAMBERT, E. B. 1939. A spore isolator combining some of the advantages of the LaRue and Keitt methods. Phytopath. 29(2): 212-214.

Langeron, Maurice. 1922. Sur un champignon d'une otomycose brésilienne Sterigmatocystis hortai. Bull. Soc. Path. Exot. 15: 383-384.

Langeron, Maurice. 1922. Utilité de deux nouvelles coupures génériques dans les Périsporiacés: Diplostephanus n. gen. et Carpenteles n. gen. Compt. Rend. Soc. Biol. (Paris) 87: 343-345.

LaRue, C. D. 1920. Isolating single spores. Bot. Gaz. 70: 319-320.

LiNk, H. F. 1809. Observationes in ordines plantarum naturales. Gesellschaft Naturforschender Freunde zu Berlin, Magazin 3, 1809; this paper is usually cited as "Link Obs."

- 1824. Species Plantarum. Ed. 4, T. 6, pt. 1, p. 65.

Linossier, G. 1891. Sur une hémative végétate: l'aspergilline, pigment des spores de l'Aspergillus niger. Ann. Microgr. 3: 359-62; also Compt. Rend. Acad. Sci. 112: $489-492$.

Lockwood, L. B., Raper, Kenneth B., Moyer, A. J. and Coghill, R. D. The production and characterization of ultraviolet-induced mutations in Aspergillus terreus. III. Biochemical characteristics of the mutations. Am. Jour. Bot. (in press).

Ludwig, F. 1892. Lehrbuch der Niederen Kryptogamen, pp. 258. Stuttgart. Mangin, M. L. 1909. Qu'est-ce que l'Aspergillus glaucus? Étude critique et expérimentale des formes groupées sous ce nom. Ann. des Sci. Nat., Bot., Ser. 9, 10: 303-371, illus.

Marchal, E. 1893. Sur une espèce nouvelle du genre Aspergillus Mich. Aspcrgillus terricola. Revue Mycologique 15, no. 59: 101-103.

Martin, G. W. 1941. Outline of the fungi. Univ. Ia.: Studies in Nat. Hist. XVIII. Suppl. pp. 3-64.

Matta, A. DA. 1928. Sterigmatocystis tropicalis n. sp. de fungo pathogenico para o homen. Bol. Inst. Brasil Sci. 3: 51-4, 49.

Mattlet, G. 1924. Bronchomycoses dues à des Sterigmatocystis dans l'Urundi. Communication préliminaire, Ann. Soc. Belge. Med. Trop. 4: 167-176, 3 figs.

McKee, C. M. and MacPhillamy, H. B. 1943. An antibiotic substance produced by submerged cultivation of Aspergillus flavus. Proc. Soc. Exp. Biol. and Med. 53(2): 247-248.

Mehlich, A., Truog, E. And Fred, E. B. 1933. The Aspergillus niger method of measuring available potassium in soil. Soil Science 35: 259. 
Meissner, Richard. 1897. Ueber eine neue species von Eurotium Aspergillus. Bot. Ztg. 55: 337-344, 353-357, illus.

Micheli, Petro A. 1729. Nova plantarum genera. 234 pp., illus. Florentiae. Mirsky, B. 1903. Sur quelques causes d'erreur dans la determination les aspergillées parasites de l'homme. Thèse de Med., Nancy 27 (1903) No. 15.

Montagne, J. F. 1856. Sylloge generum specierumque Cryptogarum. Paris.

Mosseray, Raoul. 1934a. Les Aspergillus de la section "Niger" Thom et Church. La Cellule, tome XLIII, fasc. 2.

1934b. Sur la systématique des Aspergillus de la section "niger" Thom et Church. Ann. Soc. Sci. Bruxelles, 54, sér. B, 72.

Moyer, A. J., Wells, P. A., Stubbs, J. J., Herrick, H. T. and May, O. E. 1937. Gluconic acid production. Development of inoculum and composition of fermentation solution for gluconic acid production by submerged mold growths under increased air pressure. Ind. Eng. Chem. 29: 777-781.

Nakazawa, R. 1907. On the koji fungus, Aspergillus awamori. Inst. of Gov't. Research, Formosa, Rept. Vol. I, 1907; Vol. II, 1912. In Japanese.

, Simo, M. and Watanabe, H. 1936. Ueber die garungoörganismen in awamori-Bereitung. I. Aspergillus ustus. Gov't. Research Inst. Tawarii, Japan Rept. no. 177. (Reprinted from the Jour. Agr. Chem. Soc. Japan no. 144.)

——, Takeda, Y., Okada, T. and Shmo, M. 1934. Molds putrefying tobacco. I. Agr. Chem. Soc. Japan Jour. 10: 135-192, illus. (In Japanese. Abstract in Chem. Abs. 28: 3178. 1934.)

NeILL, J. C. 1939. The mould fungi of New Zealand. II. The genus Aspergillus. Roy. Soc. New Zeal. Trans. and Proc. 69: 237-264, illus.

Oкадакі, K. 1907. Eine neue Aspergillusart und ihre praktische Anwendung. Centralb. f. Bakt. abt. 2. 19: 481-484.

1914. Beiträge zur Affinität eines neuen weissen Fadenpilzes (A. okazakii). Centralb. f. Bakt. Abt. 2. 42: 225-240.

Ота, M. 1923. Sur une nouvelle espèce d'Aspergillus pathogène: Aspergillus jeanselmei n. sp. Ann. Parasit. Humaine et Comp. 1: 137-146.

Owen, W. L. 1923. Mold fungi in sugar inversion. Facts about Sugar 16: 519-521, $546-548$.

Oxford, A. E. And Raistrick, H. 1942. Antibacterial substances from moulds. IV. Spinulosin and Fumigatin, metabolic products of Penicillium spinulosum Thom and Aspergillus fumigatus Fresenius. Chen. and Ind. 61: 128.

Partansky, Alex M. And McPherson, Robert R. 1940. Testing mold-resistant properties of oil paints. Ind. Eng. Chem. 12: 443-445, illust.

Patoulllard, M. N. 1891. Remarques sur l'organisation de quelques champignons exotiques. II. Emericella variecolor Berk. et Br. Bull. Soc. Myc. Fr. 7: 45-47, pl. 4.

Perquin, L. H. C. 1938. Bijdrage tot de Kennis der Oxydatieve Dissimilatie van Aspergillus niger van Tieghem. Thesis, Delft.

Persoon, C. H. 1797. Tentamen dispositionis methodica fungorum.

1801. Synopsis methodica fungorum.

Peyronel, B. 1914. I germi atmosferici die funghi con micelio. Dissert. Padova.

Philpot, Flora J. 1943. A penicillin-like substance from Aspergillus giganteus Wehm. Nature 162: 725 .

Pontillon, Charles. 1932. A physiological study of the lipids of Sterigmatocystis nigra. Rev. Gen. Botan. 44: 417-49, 465-83, 526-60. 
Prill, E. A., Wenck, P. R. And Peterson, W. H. 1935. The chemistry of mould tissue. VI. Factors influencing the amount and nature of the fat produced by Aspergillus fischeri. Biochem. Jour. 29: 21-33.

Pruess, L. M., Eichenger, E. C. And Peterson, W. H. 1934. The chemistry of mold tissue. II. Composition of molds with special reference to the lipid content. Centralbl. Bakt. Parasitenk. u. Infe'ttionsk. II Abt. 89: 370-377. Gorcica, H. J., Greene, H. C. And Peterson, W. H. 1932. Wachstum und Steringehalt gewisser Schimmelpilze. Biochem. Zeits. 246: 401-413. Peterson, W. H. and Fred, E. B. 1932. Isolation and identification of ergosterol and mannitol from Aspergillus fischeri. Jour. Biol. Chem. 97: 483-489.

—_- Peterson, W. H., Steenbock, H. and Fred, E. B. 1931. Sterol content and antirachitic activatibility of mold mycelia. Jour. Biol. Chem. 90: 369-384.

Quevedo, José M. 1912. Sur une encephalomyelite épizoötique d'origine mycosique chez les chevaux. Premier Congr. Internat. Path. Comp. 2: 200-202.

Quilico, A. 1933. Sulla natura del pigments delle spore dell'Aspergillus niger. Note III. Sull'Aspergillina. Gazz. Chim. Ital. 63(1): 400-410.

Quilico, A. ANd DiCApuA, A. 1933. Sopra l'aspergillina, il pigments delle spore dell'Aspergillus niger. Atti R. Accad. Lincei VI. Rend. Cl. Sci. Fis. Mat. e Nat 17: 93-99.

Raistrick, H., Robinson, Robert and Todd, A. R. 1937. The chemistry of Aspergillus colouring matters. Part I. (London) Chem. Soc. Jour. 1937: 80-88.

Raper, Kenneth B., Coghill, R. D. and Hollaender, Alexander. The production and characterization of ultraviolet-induced mutations in Aspergillus terreus. II. Cultural and morphological characteristics of the mutations. Am. Jour. Bot. (in press).

Raper, Kenneth B. and Thom, C. 1944. New Species of Aspergillus from Soil. Mycologia 36: 555-575, illust.

Raulin, J. 1863. Etudes chimiques sur la végétation des Mucedinees. Compt. Rend. Acad. Sci. (Paris) 57: 228; also Ann. Sci. Nat. Bot. 5 ser., 11: 93. 1869.

Ridgway, Robert. 1912. Color Standards and Color Nomenclature. 43 pp., illus. Washington, D. C.

Ridley, H. N. 1896. The Dracaenas of the Malay Peninsula. Jour. Bot. (London) 34: 152 .

Rockwell, G. E. and O'Fraherty, F. 1931. Studies in the physiology of moulds. II. Composition and culture of molds. Jour. Amer. Leather Chem. Assoc. 26: 216.

Ruppol, E. 1937. Chemical composi⿺ion of the fat from Aspergillus citromyces. Jour. Pharm. Belg. 19: 63-8.

Sabet, Younis S. 1939. On some fungi isolated from soil in Egypt. Fouad I. Univ. Fac. Sci. Bull. No. 19.

SacCardo, P. A. 1891. Chromotaxia seu nomenclator colorum, etc., Patavii. This gives 50 colors with a synonymy in Latin, Italian, French, English, and German. This is cited as Sacc. Chromotaxia.

Saito, Ki. 1907. Mikrobiologische Studien über die Zubereitung des Batatenbranntweines auf der Insel Hachijo (Japan). (Asp. batatae, A. pseudoflavus n. sp.). Centralb. f. Bakt. Abt. 2, 18: 30-37.

Sakaguchi, Ii. And Kodana, K. 1934. Classification of Aspergillus by means of the fermentation of sugars. Jour. Agr. Chem. Soc. Japan 10: 581-83.

Sartory, A. 1913. Sur la présence d'Aspergillus fumigatus Fr. sur des cigares. Compt. Rend. Soc. Biol. (Paris) 74: 650-651. 
1920. Production de périthèces chez un Aspergillus sous l'influence d'une bactérie. Compt. Rend. Soc. Biol. (Paris) 83: 1113-1114.

AND Barlly, A. 1921. Du pouvoir agglutinant du sulfate de thorium sur les spores d'Aspergillus fumigatus Fr. Compt. Rend. Acad. Sci. (Paris) 172: 1257 .

and Flanent, L. 1920. Étude morphologique et biologique d'un Aspergillus nouveau isolé d'expectorations d'un malade suspect de tuberculose pulmonaire. Compt. Rend. Soc. Biol. 93: 1114-1115.

, Sartory, R. Huffschuit', G. And Meyer, J. 1930. Un cas d'onychomycose provoquée par un Eurotium nouveau, E. diplocyste n. sp. Compt. Rend. Soc. Biol. 104: S\$1-\$\$3.

, Sartory, R. and Meyer, J. 1926. La formation des périthèces chez l'Aspergillus fumigatus Fresenius sous l'influence du radium. Compt. Rend. Acad. Sci., 183: 1360-2.

, Sartory, R. ANd Meyer, J. 1927. Les variations des appareils vegetatifs et conidiens de l'Aspergillus fumigatus Fresenius en cultures sur milieux dissociés et non dissociés sous l'influence des radiations du radium. Bull. des. Sci. Pharm. 34(4): 193-202.

, Sartory, R. ANd Meyer, J. 1929. Un champignon nouveau de genre Sterigmatocystis (Sterigmatocystis basidiopseta n. sp.) à basides cloisonnées. Ann. Mycol. 27(6/6): 317-320.

, Sartory, R. And Meyer, J. 1930. Etude d'une nouvelle espèce de Sterigmatocystis: Sterigmatocystis albo-rosea. Ann. Mycol. 28(3/4): 358-359.

Sartory, R. And Meyer, J 1930. Etude d'une nouvelle espèce de Sterigmatocystis: Sterigmatocystis cameleo. Ann. Mycol. 28(3/4): 360-361.

, Sartory, R., and Meyer, J. 1930. Étude d'une nouvelle espèce d'Aspergillus: Aspergillus halophilus. Ann. Mycol. 28(3/4): 362-363.

, Sartory, R., and Mexer, J. 1936. Effect of pressure or partial vacuum on the biochemical behavior of some lower fungi. Compt. Rend. 203: 128991.

And Sydow, H. 1913. Etude biologique et morphologique d'un Aspergillus nouveau. Ann. Mycol. XI, no. 2, p. 156.

Schiemann, Elisabeth. 1912. Mutationen bei Aspergillus niger van Tieghem Ztschr. Induk. Abstam. u. Verubungslehre, 8: heft 1/2, pp. 1-35, fig. 16, 2 plates.

Schmidt, C. F. (JR.). 1935. The formation of fatty acids by Aspergillus niger. Jour. Bact. 30: 445-46.

1935. The formation of fatty acids from glucose by Aspergillus niger. Jour. Biol. Chem. 110: 511-20.

Schwartz, W. 1928. Entwichlungsphysiologische Untersuchungen über die Gattungen Aspergillus und Penicillium. I. Aspergillus-Arten. Flora, 23: $386-440$.

Sнгн, Y. K. 1936. A taxonomic study of the genus Aspergillus around Wuchang, Central China (Hyphomycetes). Lingnan Sci. Jour. 15(3): 365-37S; 15(4): 607-612.

Siebenmann, F. 1883. Die Fadenpilze Aspergillus flavus, niger, fumigatus; Eurotium repens (und Aspergillus glaucus) und ihre Beziehung zur Otomycosis aspergillina. Zeitschr. Ohrenheilk. 12: 124-161.

Smith, George. 1928. The identification of fungi causing mildew in cotton goods. The genus Aspergillıs-Part I. Jour. Text. Inst. 19: 92-100. 
1931. The identification of fungi causing mildew in cotton goods. The genus Aspergillus-Part II. Textile Inst. Jour. 22: T110-T116, illus.

1938. Introduction to Industrial Mycology, pp. 302, 127 figs. Edward Arnold, London.

1943. Two new species of Aspergillus. Brit. Mycol. Soc. Trans. 26(1/2): 25-27; plates II and III. A. avenaceus and $A$. proliferans.

Speare, A. T. 1912. Fungi parasitic upon insects injurious to sugar cane. Honolulu Exp. Sta. Hawiian Sug. Pl. Assn. Path. Physiol. Sci. Bull. 12: 1-62. A. parasiticus.

Spegazzini, C. 1899. Fungi Argentini. An. del. Mus. Nac. de Buenos Aires 6: 81-365. Frequently cited as F. Argent.

- 1911. Myc. Arg. V. An. Mus. Nac. Buenos Aires, Ser. 3, 13: 431.

Spieckermann, A. And Bremer, W. 1902. Untersuchungen über die veränderungen von futter- und nahrungsmitteln durch mikroorganismen. I. Untersuchungen über die veränderungen fettericher futtermittel bein Schimmelin. Landw. Jahrb. 31: 81-128, illus.

Steinberg, Robert A. And Thom, Charles. 1940a. Chemical induction of genetic changes in Aspergilli. Jour Hered. 31: 61-63.

- and Thom, Charles. 1940b. Mutations and reversions in reproductivity of Aspergilli with nitrite, colchicine and d-lysine. Natl. Acad. Sci. Proc. 26: 363-366.

Strong, F. M. And Peterson, W. H. 1934. Chemistry of mold tissue. IV. The lipides of Aspergillus sydowi. Jour. Amer. Chem. Soc. 56: 952-955.

Takahashi, T. And Yanamoto, T. 1913. On the physiological differences of the varieties of Aspergillus oryzae employed in the three main industries of Japan, namely, saké, shoyu, and tamari manufacture. Journ. Coll. Agr., Tokyo 5: 153-61.

Talice, R. V. and MacKinnon, J. E. 1931. Aspergillus (Eurotium) montevidensis, n. sp. isolé d'un cas d'otomycose chez l'homme. Soc. de Biol. (Paris) Compt. Rend. 108: 1007-1009.

TAmiYA, H. ANd Morita, S. 1929-1930. Bibliographie von Aspergillus 1729 bis 1928. Bot. Mag. Tokyo Bd. XLIII, Heft 506 bis Bd. XLIV, Heft. 524. Contains 2424 literature citations with index.

Tauson, V. O. 1938. The conversion of energy by microörganisms. X. The accumulation of fat in Aspergillus flavus. Microbiology (USSR) 7: 360-5.

Thom, C. And Church, M. B. 1918. A. fumigatus, A. nidulans, A. terreus n. sp. and their allies. Amer. Jour. Bot. 5: 84-104.

- ANd Church, M. B. 1921. Aspergillus flavus, A. oryzae, and associated species. Amer. Jour. Bot. 8: 103-126.

- and Church, M. B. 1926. The Aspergilli, pp. 1-272, illus. Baltimore. - and Currie, J. N. 1916. Aspergillus niger group. Jour. Agr. Res. 7: 1-15. 188.

and Raper, Kenneth B. 1939. The Aspergillus nidulans group. Mycologia 31: 653-669, illus.

- and RAPER, K. B. 1941. The Aspergilluš glaucus group. U. S. Dept. Agr. Misc. Pub. 426: 1-46, figures 14.

- and Steinberg, Robert A. 1939. The chemical induction of genetic changes in fungi. Natl. Acad. Sci. Proc. 25: 329-335.

van Tieghen, Ph. 1867. Recherches pour servir à l'histoire physiologiques des Mucédinées. Fermentation gallique. Ann. Sci. Nat. Bot. ser. 5, 8: 210-244. 
1877. Sur la développement de quelques ascomycètes. Bul. Soc. Bot. France 24: 96-105, 157-161, 203-206, 206-210.

Trmonin, M. I. 1942. Another mould with antibacterial ability. Science 96: 494. Tiraboschi, C. 1908. La proteolosi negli schizomiceti ifomiceti. Giornale d. Soc. de Igiene, Milano. Aspergillus effusus.

True, R. H. 1914. The molds of cigars and their prevention. U. S. Dept. Agr. Bul. 109: 1-8.

VAN DER BIJL, P. A. 1920. Studies on some fungi and the deterioration of sugar. Union of South Africa, Dept. Agr. Science Bul. 12, 31 pp.; also Science Bul. $18,19 \mathrm{pp}$.

Vuillemin, Paul. 1920. Nouvelles souches thermophiles d'Aspergillus glaucus. Soc. Mycol. de France, Bul. Trimest. 36: 127-136, illus.

1927. Sartorya, nouveau genre de Plectascinées angiocarpes. Compt. Rend. Acad. Sci. (Paris) 184(3): 136-137.

Wazsman, S. A. ANd Bugie, Elizabeth. 1943. Strain specificity and production of antibiotic substances. II. Aspergillus flavus-oryzae group. Proc. Natl. Acad. Sci. 29: 282.

, Horning, E. S. And Spencer, E. L. 1942a. The production of two antibacterial substances, fumigacin and clavacin. Science 96: 202-203.

, Horning, Elizabeth S. and Spencer, Ernest L. 1942b. Two antagonistic fungi, Aspergillus fumigatus and Aspergillus clavatus, and their antibiotic substances. Jour. Bact. 45: 233-248.

and Schatz, Albert. 1943. Strain specificity and production of antibiotic substances. Proc. Nat. Acad. Sci. 29: 74-79.

Ward, G. E., Lockwood, L. B., May, O. E. and Herrick, H. T. 1935. Production of fat from glucose by molds. Cultivation of Penicillium javanicum van Beyma in large-scale laboratory apparatus. A. flavus mycelium contained 16 percent. Ind. Eng. Chem. 27: 318-322.

Wевв, P. H. W. 1942. Studies on the elongation of the conidiophores of Aspergillus giganteus as affected by temperature, nutrients, and light. Thesis, George Washington Univ., Washington, D. C.

Wenmer, C. 1891. Entstehung und physiologische Bedeutung der Oxalsäure in Stoffwechsel einiger Pilze. Bot. Ztg. 49: 233-246, and numerous additional pagings in the same journal.

1897. Zur Oxalsäurebildung durch Aspergillus niger. Centralb. f. Bakt. etc. 2 Abt., 3: 102-108.

1899. Ueber einige neue Aspergillus arten. Bot. Centralb. 80: 449-461. A. varians, A. minimus, A. ostianus.

1901. Die Pilzgattung Aspergillus in morphologischer, physiologischer und systematischer Beziehung. Mémoires de la Société de Physique et d'Histoire Naturelle de Genève, Tome XXXIII, Seconde Partie, pp. 1-157. This reference is commonly cited as Wehmer's Monograph.

1907 Zur Kenntnis einiger Aspergillus arten. Centralb. f. Bakt. etc. II, Abt. Bd. 18: 385-395.

1924. Oxalsäure- und Zitronensäure-Entstehung in ihrer gegenseitigen Beziehung bei verschiedenen Rassen des Pilzes Aspergillus niger. Ber. Deut. Chem. Gesell. 67: 1659-65.

Weisner, B. P. 1942. Bactericidal effects of Aspergillus clavatus. Nature 149: 356-357.

Wenck, P. R., Greene, H. C., And Fred, E. B. 1935. Factors influencing the growth and sterol formation of Aspergillus fischeri. Jour. Bact. 29: 89-90. 
-—, Peterson, W. H. and Fred, E. B. 1935. The chemistry of mold tissue. IX. Cultural factors influencing the growth and sterol production of Aspergillus fischeri. Zentralbl. Bakt. Parasitenk. u. Infektionsk. II. Abt. 92: 330-338.

Whelden, Roy, M. 1940. Mutations in Aspergillus niger bombarded by low voltage cathode rays. Mycologia $32: 630-643$.

White, E. C. 1940. Bactericidal filtrates from a mold culture. Science 92: 127.

- ANd Hill, J. H. 1943. Studies on antibacterial products formed by molds. I. Aspergillic acid, a product of a strain of Aspergillus flavus. Jour. Bact. 45: $433-444$.

Wickerham, L. J. And Andreasen, A. A. 1942. The lyophil process: its use in the preservation of yeasts. Wallerstein Lab. Comm. 6(16): 165-169. 1 plate.

Wiggers, Fredericus Henricus. 1780. Primitiae Florae Holsaticae. 114 pp. Kiliae. (Facsimile ed. 23 by W. Junk. 1925.)

Wilhela, Ki. A. 1877. Beiträge zur Ǩenntniss der Pilzegattung Aspergillus. Inaug. Diss. Strassburg pp. 66.

Wilkins, W. H. ANd Harris, G. M. C. 1942. Investigation into the production of bacteriostatic substances by fungi. I. Preliminary examination of 100 fungal species. Brit. Jour. Exptl. Path. 23: 166.

Wilson, J. A. AND DAUB, G. 1925. Aspergillus niger, a common mold that causes black spots on leather. Jour. Amer. Leather Chemists Assoc'n 20: 400-405, figs. 12 .

Wolf, Frederick A. 1938. I. Fungal flora of Yucatan caves. Carnegie Inst. Wash. Pub. No. 491, p. 19-21.

Woolley, D. M., Strong, F. M., Peterson, W. H. and Prili, E. A. 1935. Chemistry of mold tissue. $\mathrm{X}$. The phospholipides of Aspergillus sydowi. Jour. Am. Chem. Soc. 57: 2589-91.

YABUtA, A. 1912. On Koji acid, a new organic acid formed by Aspergillus oryzae. Journ. Coll. Agr., Tokyo, 5: 51-8. Orig. Comm. 8th Intern. Congr. Appl. Chem., (Append.), 25 (1913) 45-62.

Үамамото, Y. 1927. Ueber drei Stämme von Aspergillus ventii (Japanisch.) Jour. Soc. Agr. Forest., Sapporo, 19: 128- 10.

Yuill, EDwaRd. 1939. Two new Aspergillus mutants. Jour. Bat. (London): $174-175$, illus.

AND Yuill, John L. 1938. Cladosarum olivaceum, a new Hyphomycete. Brit. Mycol. Soc. Trans. 22: 194-200, illus.

YUkawa, M. 1911. Zwei neue Aspergillusarten aus "Katsuobushi." Jour. Coll. Agr. Tokyo 1: 357-366.

Zikes, H. 1922. Uber die Perithecienbildung bei A. oryzae. Centralb. f. Bakt. etc. 2 Abt. 56: 339-343. 


\section{Chapter XXIV}

\section{CHECK LIST OF SPECIES AND GENERA}

\section{GENERIC NAMES FOUND APPLIED TO ASPERGILLI}

Alliospora G. Pim, in Proc. R. I. Acad. 1883, and Jour. Bot. 1883, p. 234; also Sacc. Syll. 18, No. 5216. 1906. A. Sapucaya represents a monotypic genus found upon putrifying Lecythis Sapucajo. From the description it was some member of the $A$. niger group. Saccardo's diagnosis only seen.

Ascophora. Ascophora nigrans was vaguely assigned mostly to Mucors but appears to have been used by Raulin's group studying the tannic acid fermentation until Van Tieghem described A. niger. . . .

Aspergillopsis Sopp, in Videnskapsselskapets Skr. I Mat.-Naturv. Kil. No.11, pp. 201-204, Taf. XX, fig. 149. 1912. Sopp probably had some members of the $A$. ustus group............. Aspergillopsis Spegazzini, in An. Mus. Nat. Buenos Aires Ser. 3, 13: 434. 1911. The black spored Aspergilli (essentially the $A$. niger group) were regarded as dematiaceous and assigned to a new genus in that group. Spegazzini's genus has not been accepted.

Aspergillus Micheli, in Nova Plantarum Genera p. 212, Pl.91. 1729. Compare Link, in Obs. p. 16, 1809, and Corda in Icones Fungorum 4: 31, Tab. VII, fig. 94. $1840 \ldots \ldots \ldots \ldots \ldots \ldots . . . .$.

Subgenus-Micraaspergillus Wehmer, in Monogr. 1901. (Mémoires de la Société de Physique et d'Histoire Naturelle de Genéve, Tome XXXIII, Seconde Parte, pp. 1-157. 1899$1901) \ldots \ldots \ldots \ldots \ldots \ldots \ldots \ldots . \ldots 16$

Subgenus-Macroaspergillus Wehmer in Monogr. 1901. The subgenera are not accepted here..................... 16

Cladosarum Yuill and Yuill, in Trans. Brit. Myc. Soc. 22: 194200, Pls. 11-13. 1938. Regarded as a laboratory mutant not encountered in nature. In A. niger group. See..........

Dimargaris van Tieghem, in Ann. Sci. Nat. 6 Ser. 1: 54. 1875. The name Dimargaris sp. has been seen upon living cultures in one of the great laboratory collections. These cultures were obtained from dog and squirrel dung. The organism so labeled belonged to the Aspergillus candidus group. The genus Dimargaris (type D. crystalligena $\mathrm{v}$. Tieghem) is regarded by Fitzpatrick as closely related to Dispira and as a synonym of Dispira by Zycha (Kryptogamenflora der Mark Brandenburg. Pilze II. Mucorineae. Band VIa: 1-264, 114 figs. 1935). In any case, it is only reported as a parasite of the mycelia of the Mucorineae, hence certainly was not an $A s$ pergillus...................

Diplostephanus Langeron, in Compt. Rend. Soc. Biol. Paris 87: 343345. 1922. This genus is proposed by Langeron to include ascosporic Aspergilli (Sterigmatocystis) with two series of 
sterigmata. A. nidulans Eidam is named as type. The proposal is rejected here...............

Emericella Berkeley and Broome, in Introd. Crypt. Bot. pp. 340-341, fig. 76. 1857. See also: $\mathrm{Pa}$ touillard in Bul. Soc Myc. France 7: 43-49, pl. 4, figs. 6-12. 1891. Based upon E. variecolor. Syn. A. variecolor q.v.......... 163

Euaspergillus Ludwig, in Lehrbuch der niederen Iíryptogamen $\mathrm{p}$. 258. Stuttgart 1892. The genus was proposed to cover the sclerotium producing Aspergilli such as $A$. niger, $A$. flavus, $A$. ochraceus. The name has not been accepted...............

Eurotium Link, in Obs. p. 31, Taf. 2, fig. 44. 1809. Link described Aspergillus glaucus as a conidial mold and Eurotium herbariorum for the ascosporic form, supposing them to be different fungi. His genus Eurotium has been widely accepted, but it is rejected here for reasons discussed elsewhere. Forms listed as Eurotium but having by description black perithecia are excluded. No species with aspergilloid conidial structures has black (Dematiaceous) perithecia.

Inzengaea Borzi, in Jahrb. Wiss. Bot. (Pringsheim) 16: 450-463, Pl. 19, 20. (1884) 1885. Type sp.: I. erythrospora Borzi: Syn. A. variecolor q.v...............

Mucor used by Wiggers (Wichers) in Primitiae Florae Holsaticae. 1780. (Republished in facsimile Edition No. 23 by W. Junk in 1925). "Mucor herbariorum sessilis luteus" is given as No. $1158 \ldots \ldots \ldots \ldots \ldots \ldots \ldots \ldots . . \ldots \ldots$

Rhodocephalus Corda, in Icones I: 21. 1839, and the figures given for $R$. aureus in Icones III, Taf. II, fig. 33 suggest $A$. terreus.

Sartorya Vuillemin, in Compt. Rend.
Acad. Sci. (Paris) 184: 136-317. 1927. Inadequately described as an ascosporic phase of $A$. fumigatus. It was not distributed in culture............... Sterigmatocystis Cramer, in Vierteljahresschrift der Naturforchungen Gesellschaft, Zurich 4: 325. 1859. Type: A. antacustica Cramer. Cramer proposed to include in his new genus all aspergilloid species showing both primary and secondary sterigmata. S. nigra (A. niger) was the organism under discussion, hence the type species. Many mycologists have agreed with Cramer; others, such as Fischer, Wehmer, and Thom, have rejected Sterigmatocystis....................

\section{CHECK LIST OF NAMED SPECIES}

S. acini-uvae Caballero, in Boletin R. Soc. Esp. Hist. Nat. 28: 429. $1928 \ldots \ldots \ldots \ldots \ldots \ldots \ldots \ldots . . \ldots 229$

Syn. A carbonarius fide Blochwitz, or A. pulchellus fide Mosseray....................

$S$. aerea Bainier, in Bul. Soc. Bot. France 28: 78. 1881. A.wentii group.................. 249

A. africanus Dur. et. M., in Fl. Alg., I.P. 342. 1849. Not an Aspergillus.

A. ageni This name is cited by Lindt, in Arch. Exp. Path. Pharm. 25: 265. 1859, as taken from Saccardo's Sylloge. Search for this reference leads to the conclusion that in this citation A. Hageni was made to read A.ageni.

S. alba Bainier, in Bul. Soc. Bot. France 27: 30. 1880. Some member of $A$. candidus group... . 211

S. alba-cyanogena Biourge, listed among cultures in the Biourge Collection. 1939.

S. alba-lutea Biourge, n. m., in MS. p. 9, among the niveus series. 
A. alba-roseus attached to a culture in the Bainier Collection and by inference in the Biourge Collection as near $A$, niveus Blochwitz. Thom's No. 4640.490.

S. alba-sclerotifera Biourge, nomen nudum, listed in his MS. as related to $A$. okazakii. It produces a violet or blue color in reverse.

S. alba-sulphurea Biourge, nomen nudum, listed in MS. as near A. okazakii. It produces a blue or violet reverse.

A. albidus Eichelbaum, in Verhandlungen d Naturw. Ver. Hamburg 3 Folge XIV; 35.1906.

Syn. E. albidum Sacc., Syll. 22: 1254. 1913.

A. albidus Speg. A culture under this label distributed by Biourge represents a strain of $A$. niveoglaucus q.v................... Bot. France 27: 30. 1880. This was a small pale yellow form, but no further data were given and it has not since been identified. Apparently close to A. carneus. S. albo-lutea Sartory, Sartory, and Meyer, cited by Blochwitz, in Ann. Mycol. 31: 73. 1933. Some member of the $A$. candidus

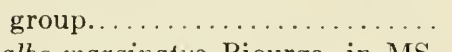

A. albo-marginatus Biourge, in MS. p. 2, as change of name from Penicillium albo-marginatum, figured only in the Penicillium Monograph in La Cellule tome XXXIII. 1 fase. fig. 129 . It was some member of the $A$. restrictus series, received and studied as Thom No. 4733.134a. Near A. gracilis in the A. glaucus group.

S. albo-rosea Sartory, Sartory, and Meyer, in Ann. Mycol. 28: 358359, Pl. III, figs. 1-6. 1930. Compare $S$. albo-rosea in Blochwitz Ann. Mycol. 31: 75. 1933. A. carneus series.
A. albus Wilhelm, Inaug. Diss. Strassburg, p. 69. 1877. Probably $A$. albus Haller, S. alba Sace., Monilia alba Persoon. In A. candidus group............ 211

A. alliaceus Thom and Church, The Aspergilli, p. 163. 1926. See A. wentii group............. 244

A. alternatus Berkeley, in Ann. Nat. Hist., Ser. 1, Vol. 1: 262. 1838. Not an Aspergillus.

A. alutaceus B. and C., in Grevillea 3: No. 25, p. 108 . 1875. In the A. ochraceus group, unidentifiable to strain or series........ 281

$S$. ambari (alternative spelling, ambaris) Beauregard, in Ann. de Micrographie 10: 255-278, 1 plate. 1898. Cited without description in Compt. Rend. Hebdom. des Séances de la Soc. de Biol. 28: Mai (1898). $A$. vesicolor group............... 192

A. amoenus Roburg, in Hedwigia 70: 138-9. 1930. Distributed by the Centraal-bureau voor Schimmelcultures, Baarn. Received by us on November 20, 1933, and determined as a member of the $A$. gracilis series, but the description allies it with A. versicolor.

A. amstelodami (Mangin) Thom and Church, in The Aspergilli, p. 113. 1926; also Thom and Raper, U. S. D. A. Misc. Publ. 426, pp. 22-26. 1941......... 122

Syn. E. amstelodami Mangin, in Sci. Nat. Bot. Ser. 9, 10: 360361. 1909. A.glaucus group.... 122 Incorrectly spelled $A$, amsterodami in Nakazawa, Jour. Agr. Chem. Soc. Japan 10(2): 1934.

Syn. E. repens var. amstelodami Vuill., in Soc. Mycol. de France, Bul. Trimest. 36: 131. 1920.

var. alophote Duche, cited by Dodge in his Med. Mycol. p. 630. 1935. Ascospore lacks the crest of the species. 
A. anomalus Mosseray, in La Cellule XLIII : 248-249, pl. 4, figs. 107112. 1934. Member of $A$. niger group................. 234

S. antacustica Cramer, in Vrtljschr. Naturf. Gesell. Zürich, Jahrg. 4, Heft. 4: 325. 1859. In $A$. niger group............... 239

Syn. A. phoenicis in A. niger group.

A. archaeoflavus Blochwitz, in Ann. Mycol. 31(1/2): 73-83. 1933. The type culture (Thom No. 2505346 ) was too close to $A$. wentii for satisfactory separation...... 247

A. archiflavipes Blochwitz, in Ann. Mycol. 32(1/2): 84. 1934. A form of $A$. flavipes characterized by deep brown to red shades of color in the mycelium.......... 181

A. argentinus Spegazzini, in Rev. Agr. Veter. LaPlata p. 245. 1896. A conidial form in the $A$. glaucus group; not identifiable.

A. argillaceus was distributed but not described by Biourge. It appears to be a non-ascosporic strain of the $A$. repens series....

A. atro-fuscus Mosseray, in La Cellule XLIII: 269-270, pl. 4, figs. 126-129. 1934. Member of A. niger group............. 235

A. atropurpureus Zimmermann, in Centbl. Bakt. [etc.] Abt. 2, Bd. 8, No. 5, p. 218. 1902. A. niger group.

A. atropurpureus Blochwitz, in Ann. Mycol. 32(1/2): $\quad 86.1934$. Blochwitz proposed this name to cover purple-brown series in $A$.

niger group................

A. atro-ruber Estienne

Syn. Penicillium roseo-cinnabarinum Biourge, in La Cellule 33: fasc. 11, 319-321, PI. XVII, fig. 97. 1923.

Syn. Physomyces heterosporus Harz, fide Biourge.

Syn. S. atro-rubra (Estienne) Biourge MS.
A. atro-violaceus Mosseray, in La Cellule XLIII: 268-269, pl. 4, figs. $122-125 . \quad 1934 \ldots \ldots \ldots \ldots .235$

Blochwitz in Ann. Mycol. 33: 240. 1935, identifies this with $A$. violaceo-fuscus Gasperini........ 231

A. atrovirens Karst, in Symb. 26:28; Sacc. Syll. 10: 524. Inadequately described for identification.

A. aurantiacus Berkeley, in British Fungi fasc. IV: 1843. Cited by Montagne in Ann. Sci. Nat. Bot. 3. Ser., 12: 299. 1819.

Syn. Nematogonium aurantiacum.

$S$. aurea Greco, in Origine des Tumeurs et Mycoses Argentines, Buenos Aires, pp. 671-694, figs. 418-428. 1916. Not since recognized. Some A ochraceus strain. A. aureoglaucus Roburg, in Hedwigia 70: 137. 1930. Culture some strain of $A$. repens contributed by Roburg. Noted as some member of $A$. glaucus group by Blochwitz in Ann. Mycol. 33: 240.1935.

A. aureus Berkeley, in English Flora 5: 346. 1836. The golden yellow, elliptical conidia reported by Berkeley suggest A. citrisporus but actual identification from the description is impossible................ 219

A. aureus Nakazawa, in Inst. Gov't. Res. Formosa Rept. Vol. 1. $1907 . . . \ldots \ldots \ldots . . . \ldots . . .219$

226 A. aureus varieties described by Nakazawa, Simo and Watanabe in Jour. Agr. Chem. Soc. Japan No. 144, 1936 (In Japanese). var. acidus.................. 220 var. brevis................. 220 var. minor................ 220 var. murinus............... 220 var. pallidus............... 220 comparison of figures and measurements given convince the authors that these varieties could not be safely identified by descriptive data. 
S. auricoma Gueguen, in Bull. Soc. Mycol. France 15: 171-187, figs. 1-48. 1899. In A. ochraceus group..

A. auriculaire Moquin-Tandon, in Elements Bot. Med. 2 ed., p. 466. 1866. This name was not accompanied by adequate description to separate the form intended from other species which are occasionally found in the ear.

A. aviarius Peck, in N. Y. State Mus. Rept. (1890), 44: 120. 1892. A strain of $A$. fumigatus isolated from a canary........ 151

A. arenaceus George Smith, in Brit. Mycol. Soc. Trans. 25: 24-27, Pl. 1, figs. 1-3. 1943......... 246

A. awamori Nakazawa, in Inst. of Gov't. Research, Formosa Rept. 1: 1907, and 2: 1912. A member of the $A$. niger group............

A. awamori varieties described by Nakazawa, Simo, and Watanabe in Jour. Agr. Chem. Soc. Japan, No. 144, 1936. (In Japanese). var.ferrugineus............... 220 var.fumeus.................. 220

var. fuscus . . . . . . . . . . . . 220

var. minimus............... 220

var. piceus............... 220

No doubt the describers had a series of industrially significant strains but it is doubtful if others could identify them from the description and figures given.

A. awamori Usami, in Myk. Centralbl. 4: 193. 1914. Species recognized by Mosseray in La Cellule 43: 264. 1934. With $A$. awamori Nakazawa cited as a synonym.

A. bainieri Mosseray, in Ann. Soc. Sc. Bruxelles 54, ser. B, p. 79 , $1934 \ldots \ldots \ldots \ldots \ldots \ldots \ldots \ldots$. . . . . . . Syn. A. longobasidia Bainier fide Mosseray in La Cellule XLIII (2): 227. 1934............ 233

A. barbae Castellani. Cited by Sartory, A., in Champignons parasites de l'homme et des animaux, p. 595. 1922. This organism was found in the beard of a native of Uganda and again from Ceylon. The only further information given is conidia 4 to $5 \mu$, globose, dark brown.

S. basidiosepta Sartory, Sartory, and Meyer, in Ann. Mycol. 27: 317320. Pl. VII. 1929. A variant of the A. candidus group........ 211

A. batatae Saito, in Centralb. f. Bakt. 2, Abt. 18(1/2): 34. 1907. In the $A$. niger group.............

A. belfanti Carbone, in Atti d. Inst. Bot. Univ. Pavia Serie II. Vol. XIV: 63, fig. 11. 1914. Probably in $A$. glaucus group.

A. (Sporodinia) bellomontii Montagne, in Ann. Sci. Nat. Bot. Ser. 4, V. 12: 181-182. 1859. Noted as related to $A$. maximus (Sporodinia). Not an Aspergillus.

S. bicolor J. Ray, in Rev. Sc., Ser. 4, V. 8: 176-177, 193-212. Lille, 1897. Probably in A. sydowi series.................... 192

A. biourgei Mosseray, in La Cellule XLIII : 241-242, pl. 4, figs. 8185. 1934. One of the $A$. niger group.

S. blanc-jaune Bainier, nomen nudum. A culture so labeled from the Bainier Collection represents a diminutive member of the A. candidus group.....

A. blochwitzii Biourge, nomen nudum, listed in Biourge MS (p. 3) among the A. claratus group as a synonym of A. clavatus var. gigantea Blochwitz.

A. boedijni Blochwitz, in Ann. Mycol. 32: 83-89. 1934.

Syn. A. terreus var. boedijni....... 197

$E$. bonariense Speg., in Anal. de la Soc. Cient. Argen. 10: 177. 1880. Some member of the $A$. glaucus group but data are inadequate.

Coremium Borzianum Saccardo.

See A. variecolor. 
A. bouffardi Brumpt (1905). Cited by Castellani and Chalmers in Man. Trop. Dis. p. 805. 1913.

Syn. Madurella Bouffardi (Brumpt) Dodge. Med. Myc. p. 685. 1935.

A. Brodeni (Mattlet) Dodge, in Dodge Med. Mycol., p. 635. 1935 .

Syn. S. Brodeni Mattlet, in Ann. Soc. Belge Med. Trop. 4: 167171, figs. 1, 2. 1924. Probably A. unguis.

var. Vancampenhouti (Mattlet) Dodge, in Dodge Med. Myc., 636. 1935.

A. bronchialis Blumentritt, in Ber. Deut. Bot. Ges. 19: 442-446, pl. 22, figs. 1-6. 1901; also ibid. 23: 419-427, pl. 19, figs. 1, 3, 6, 7, 8, 19, and 23. 1905. In the $A$. fumigatus group............. 151

A. brunneo-fuscus See, in Les Malades du papier piqué Paris, p. 29. 1919. Unidentified except as a member of the $A$. glaucus group.

A. brunneo-virens Delacroix, in Bul. Soc. Myc. France 13: 120, with text figure. 1897. Unidentified except as a member of the $A$. glaucus group.

A. brunneus Delacroix, in Soc. Mycol. Bul. France 9: 185, Pl. XI, fig. III. 1893. Delacroix described this as the conidial stage of A. echinulatus q.v...... 131

S. Buntingiana Biourge, nomen nudum, listed in Biourge's MS. as applied to a culture of a member of the $A$. candidus group received from Bunting.

A. Buntingii Mosseray, in La Cellule XLIII: 236-238, pl. 4, fig. 91-95. 1934. Member of A. niger group in Biourge Collection 1939..... 233

A. butyracea (Bainier) n. comb...... 282 Syn. S. butyracea Bainier, in Bull. Soc. Bot. France 27: 29. 1880. C. Roumeguere's Fungi Gallici Exsiccati No. 995 is recorded as Bainier's material. In the A. ochraceus group............ 282

A. byssoides Sprengel, in Sys. Veg., ed. 16, V. 4: 541. 1827. A fungus on rotting paper with globose fuscous heads but not identifiable.

A. cacao. This name appeared upon a culture in the Bainier collection (Thom No. 4640.397) and has been contributed also by Pribram (4777.4). The organism is a strain of $A$. tamarii..................... 256

A. caesiellus Saito, in Jour. Coll. Sci. Imp. Univ. Tokyo 18: 49, Pl. III, fig. 14. 1904. Regarded by Neill (in Royal Soc. New Zealand Trans. 69: 242. 1939) as A . restrictus G. Smith... 141

A. caespitosus n. sp. Raper \& Thom, in Mycologia 36: 563-565, fig. 3. 1944. A member of A. nidulans group................. 166

A. calyptratus Oudemans, in Arch. Neerl, II. 7: 283, pl. 13.1902. See: A.fumigatus group........ 151 var. italicus Ferraris, in Ann. Mycol. 10: 294. 1912.

S. cameleo Sartory, Sartory, and Meyer, in Ann. Mycol. 29: 360361, Pl. III. 1930. Some variant of A. sydowi............ 186

S. candida Sacc., in Michelia 1, p. 91. 1877. In the $A$. candidus group.

S. candidula Bainier, in Sacc. Syll. 4: 73.1886.

Syn. S. candida Bainier, in Bul. Soc. Bot. France 27: 30. 1880. A member of the $A$. candidus group.

A. candidus Link, in Obs. p. 16 . $1809 \ldots \ldots \ldots \ldots \ldots \ldots \ldots . \ldots 207$

var. thermophilus Nakazawa et al., in Jour. Agr. Chem. Soc. Japan 88: 17. 1932, (in Japanese) cited Blochwitz Ann. Mycol. 33: 244. 1935.

A. capitulo pullo Haller, in Historia Stirpum Indigenarum Helvetiae 
Inchoata, etc. 1768. Apparently taken from Micheli 1729.

S. carbonaria Bainier, in Bul. Soc. Bot. France 27: 27-28. 1880 .

See A. carbonarius (Bainier)

Thom. In A.niger group....... 229

A. carbonarius (Bainier) Thom, in Jour. Agr. Res. 7:12. 1916.... 229

A. carbonarius seu ater Meis and Parascandalo, in Gaz. Ospedali 16: 769-772. 1895. Cited by Dodge, in Med. Myc. 679, as not an Aspergillus.

A. carneolus Sacc., in Michelia 1, p. 77. 1877. And Fungi italici No. 18. In A. glaucus series.

A. carneus (van Tieghem) Blochwitz, in Ann. Mycol. 31(1/2): 81. 1933.

Syn. Sterigmatocystis carnea van Tieghem, in Bull. Soc. Bot. France 24: 103. 1877. See also Saccardo Sylloge 4: 74, and Wehmer's Monograph (Mem. Soc. Phys. His. Nat. Gen. pp. 1-157). 1899-1901. Species in A.terreus group.............. 201 var. subglobosa Blochwitz, in Ann. Mycol. 33: 243.1935.

var. opaca Blochwitz, in Ann. Mycol. 33: 249. 1935.

A. carnoyi Biourge, descr. Thom and Raper in U. S. Dept. Agr. Misc. Pub. 426, p. 34-5. 1941........ 134

S. castagnei Biourge, undescribed culture in the Biourge Collection, listed as near A. carbonarius in the $A$. niger group.

A. castanea Patterson, in Bul. Torrey Bot. Club 27: 284. 1900. In $A$. tamarii series.

A. cellulosae Hopffe, in Centralb. $\mathrm{f}$. Bakt., etc., 1 abt., 83: 531-37. 1919.

Syn. A.fumigatus.... . . . . . . . . 151

A. cervinus Massee, in Kew Misc. Bul. 4: 158. 1914. Neill considers A. gratioti Sartory to be a synonym but that is not probable.
A. chevalieri (Mangin) Thom and Church, in The Aspergilli, p. 111, 1926.

Syn. E. cheralieri Mangin, in Ann. Sci. Nat. Bot. Ser. 9, 10: 361362, fig. 12 . 1909. In the $A$. glaucus group.

A. chevalieri var. intermedius Thom and Raper in U. S. Dept. Agr. Mise. Pub. 426, p. 21, fig. 8B. 1941.

A. chevalieri var. multiascosporus Nakazawa, Takeda, Okada, and Simo, in Agr. Chem. Soc. Japan, Jour. 10: 135-192. 1934. Received from Baarn and appears as NRIL No. 88. A strain of A. chevalieri... .

E. chilense Montagne, in Syll. Crypt. No. 919, Fl. Chil. VII, p. 476 ; cited by Saccardo Syll. 1, p. 27. In the A. glaucus group.

S. chlorina Cooke and Massee, in Grevillea 18: 7. 1889. Probably a nonascosporic strain of the A. glaucus group.

A. chrysospermum Thaxter, nomen nudum, attached to a culture later shown to be $A$. citrisporus Van Höhnel, q.v.

A. chungii Shih, in Lingnan Sci. Jour. 15(3): 378. 1933. In the A. flavus group.

A. churchii Mosseray, in La Cellule XLIII : 242-244, pl. 4, figs. 76-80. 1934. Representative of the $A$. niger group. In the Biourge Collection 1939.

A. cimmerius Berkeley and Curtis, in Grevillea 3: 108. 1875. This specimen was identified by Dr. Farlow (Bibliographical Index I. pt. 1, page 277) as Periconia chlorocephala, Fres.

A. cinerescens Bainier and Sartory, in Bul. Soc. Mycol. France 27: 98-104, pl. III, figs. 6-12. 1911. In the $A$. glaucus group.

A. cinereus Spegazzini, in Anal. Soc. Cient. Argen. 10: 162-163. 1880. 
Not identifiable; believed in the A. glaucus group.

A. cinnamomeus Schiemann, in Ztschr. Induk. Abstam. u. Vererbungslehre, Bd. 8 , Heft 1/2: 1-35, 16 figs. $2 \mathrm{pl}$. (1 col.). $1912 \ldots \ldots$

Syn. A. niger mut. cinnamomeus. . . 223 A. cinnamominus (Weiss) Dodge, in Dodge Med. Myc., p. 627. $1935 \ldots \ldots \ldots \ldots \ldots \ldots \ldots \ldots .20 \ldots \ldots$ Syn. S. cinnamominus Weiss, in Ann. Parasitol. Hum. Comp. 8: 189-193, 5 figs. 1930. See A. terreus. 200

A. circinatus Mangin, in Bul. Soc. Mycol. France 15: 223, Pl. XI, figs. 5, 6, 7. 1899. Not recognizable-probably not an Aspergillus.

A. citricus (Wehmer) Mosseray, in La Cellule XLIII: 262-263, pl. 4, fig. 105-106. 1934. Member of A. niger group. In Biourge's Collection.

A. citrino-niger Mosseray, in La Cellule XLIII: 231-232, pl. 1, fig. 7. 1934. Member of $A$. niger group. In Biourge's Collection only....

A. citrisporus von Höhnel, in Sitzungsber. Kais. Akad., Wiss. Wien, Math.- Naturw. Kl. III, Abt. I: 987. 1902. In the A. tamarii group..

A citromyces E. Ruppel, in J. Pharm. Belg. 19: 63-8. 1937. C.A. 31: 6687.

A. clavatus Desm., in Ann. Sci. Nat. Bot. Ser. 11, 2: 71, pl. 2, fig. 4. 1834 .

A. clavatus var. gigantea Blochwitz. Cited by Biourge in unpublished MS.

A. clavatus Desm. mut. gigantca Blochwitz, in Ann. Mycol. XXVIII. $(3 / 4): 1920$ is used by Blochwitz on several occasions..

A. clarellus Peck, in N. Y. St. Mus. Rept. 34: 44, pl. 2, figs. 1-5. 1881.

Syn. A. clavatus Desm.
S. coerulea Blochwitz, listed in Biourge's MS. as one of the blue Aspergilli (A. sydowi).

A. concentricus Castellani, in Trans. Int. Derm. Cong. 6: 667-671, pl. 49, 50. 1907 .

Syn. Epidermophyton concentricum (Blanchard) Castellani and Chalmers.

A. condylomatae Greco. A name attached to a pathogen without data for identification.

A. conicus Blochwitz published by E. Dale in Ann. Mycol. 12: 38. 1914. Described as a Penicillium in Ann. Mycol. 10: 465. 1912. In the $A$. restictus group................. 140

A. conoideus Sprengel, in Sys. Veg. ed. 16, V. 4: 541. 1827. This is cited as Byssus conoidea Müll. in Flora Danica, Taf. 897, fig. 2 which is a myxomycete, hence this name may be dropped.

A. cookei Sacc., in Sylloge Fung. IV : 71. 1886 .

Syn. 1. mucoroideus Cooke, in Grev. 12: 9. 1883. Some member of the $A$. glaucus group.

233 E. coriorum Wallr., in Compendium Flora Germania 4: 330. 1833.

Syn. E. repens DeBary fide Mangin. Not recognizable.

S. corolligena Massee, in Bul. Royal Bot. Gard. Kew. No. 1, p. 5. 1910. Not recognizable.

S. coronata v. Tieghem, in Bull. Soc. Bot. France 24: 103. 1877. Unidentifiable.

S. coronella Costantin, in Muced. Simples p. 34, fig. 2, 1888 . Unidentifiable.

A. crocatus Berkeley and Curtis. Specimen only in the Curtis Collection. This type specimen has been identified as Chondromyces and is so reported in Farlow's Bibliographical Index I. pt. 1, page 277.

A. cucurbitaceus in the Curtis Collection (Harvard). Was identified by Farlow as Choanephora. 
A. curtisii Berkeley, in Ravenel Fungi Carolinense fase. IV. 1855. Farlow identified this in his Bibliographical Index as Rhinotrichum curtisii.

S. cyanca Bainier, nomen nudum, in Biourge's list of the nidulans group.

A. cyaneus (Mattlet) Dodge, in Dodge Med. Mycol. p. 636, 1935.

Syn. S. cyaneus Mattlet, in Ann. Soc. Belg. Med. Trop. 6(1): 32 . 1926. In A. sydowi series. . . . . 186

$A$. cyanogenes Biourge, nomen nudum. One of the $A$. conicus strains in A. restrictus series... . 140

S. dasytricha Ell. and Ev., in Jour. Mycol. 2: 104. 1886. Sacc. Syllog. X: 525. Not an Aspergillus.

A. delacroixii (Sacc.) Thom and Church, in The Aspergilli, p. 190. $1926 \ldots \ldots \ldots \ldots \ldots \ldots \ldots \ldots$. . . . . .

Syn. S. delacroixii Sacc, in Sylloge $10: 527$..

Syn. S. ochracea Delacroix, in Bul. Soc. Mycol. France 7: 109, pl. VII, fig.f. 1891.

A. delacroixii Sacc. and Sydow, in Sylloge Fungorum 14: 1044.

Syn. A. olivaceus Delacr., in Bul. Soc. Mycol. France 13: 118. 1897. Some unidentifiable member of the A. glaucus group.

A. densus Mosseray, in La Cellule XLIII : 232-234, pl. 4, figs. 99102. 1934. In the Biourge Collection............... 233

A. depauperatus Petch, in Trans. Brit. Mycol. Soc. XVI. p. 244, text fig. 1931. Parasite upon Lepidosaphis ulmi on Hawthorn in England, also on Aspidiotus in Ceylon. As described: conjdiophores up to $50 \mu$ by 2.5 to $3.5 \mu$, broadening to a fertile apex 4 to $5 \mu$ in diameter with a few chains of conidia attached directly to the vesicular surface; conidia 2 to $4 \mu$ by 1.5 to $2.5 \mathrm{u}$. Possibly some strain of the $A$. restrictus series not far from $A$. gracilis.
A. derxii Biourge, nomen nudum, listed among the $A$. versicolor group in unpublished MS. and represented in his collection in 1939.

E. desmazieri Castagne, Pl. Mars. II, Sup., p. 56. 1851. Sec also Berlese, Fungi Moricolae Appendice p. 11. 1889. As described by Berlese, this was a perithecial form probably belonging to the A. glaucus group, but not sufficiently described to identify.

A. desseyi Speg., in Physis., Rev. Soc. Argentina Cien. Nat. VIII: 115-117, 1 fig. 1925. Named A. dessyi Speg., in Rev. Appd. Mycol. 4: 542. 1925. Some form near $A$.fumigatus........ 151

A. dierckxii Biourge, nomen nudum, on culture distributed by Biourge; a strain of the $A$. repens series. See Thom and Raper U. S. D. A. Misc. Publ. 426, p. 12 . 1941 ...

A. diplocystis (Sartory, Sartory, Hufschmitt and Meyer) Dodge, in Dodge Med. Mycol. p. 625. $1935 \ldots \ldots \ldots \ldots \ldots \ldots \ldots \ldots . . . \ldots \ldots$

Syn. E. diplocyste Sartory, Sartory, Hufschmitt and Meyer, in Compt. Rend. Soc. Biol. 104: 881-883. 1930. The authors describe a mold with conidial head near $A$. nidulans and the ascospores of $A$. chevalieri.

E. diplocystis B. and Br., in Jour. Linn. Soc. (London), Bot. 14: 55-56, Tab. 10. 1875. Neither description nor figure would identify this with any definite Aspergillus, although the specimen may have been the perithecia of some member of the $A$. glaucus group.

S. dipus Ferdinandsen and Winge, in Bot. Tids. 30: 220, fig. 6. 1910 . Not separable from other largespored forms in this section of the $A$. niger group........... 
A. disjunctus Bainier et Sartory, in Bul. Soc. Myc. France 27:346-368, pl. X-XI. 1911. A member of the A.glaucus group. See A . cchinulatus.

A. diversicolor Waksman, in Soil Science 2: 126. 1916. The reference to an organism by Waksman under this name was a typographical error for A versicolor.

S. dubia (B. \& Br.) Sace, in Fungi italici pl. 902, and Sylloge 4: 72 . 1886.

Syn. A. dubius Corda, in Berkeley and Broome, in Ann. Nat. Hist. 2 Ser., 7: 100 (No. 520). 1851. Not thus far recognized.

A. dubiosus Lindau, in Rabh. Krypt. Fl. 8: 151. 1907. In the A.candidus group.

A. eburneus Biourge, nomen nudum, was attached to a culture received from Biourge and recorded as NRIRL 515. It is accepted as $A$. niveus ........ 202

A. echinosporus Sorokin. Abst. by Busch, in Ztschr. f. Pflanzenkrankheiten 3: 155. 1893. Ref. in Sacc. Sylloge 11: 592. 1895. The description suggests a Haplographium.

A. echinulatus (Delacr.) Thom and Church, in The Aspergilli, p. 107. 1926 .

Syn. E. echinulatum Delacr., Soc.

Mycol. de France, Bul. Trimest 9: 266, Pl. XIV, fig. III. 1893. Syn. E. verruculosum Vuill., in Soc. Mycol. de France, Bul. Trimest. 34: 83. 1918. In A. glaucus group

A. effusus Tiraboschi, in Ann. di Bot. 7 (fasc. 1): 16. 1908. See also Thom and Church, in Am. Jour. Bot. 2:109-110. 1921. Described originally from rotten corn (Zea Mays); belongs to the $A$. flavus-oryzae group.......... 267

A. elatior Mosseray, in Ia Cellule XLIII: 253-255, pl. 3, figs. 29-32. 1934. In the Biourge Collection. 234
A. elegans Gasperini, in Atti. Soc. Toscana Sci. Nat. Pisa, Mem. 8: (fasc. 2): 328. 1887. In $A$. ochraceus group............. 281

E. epixylon Kunze and Schm. (D. Schw. No. 83; Wallr. Fl. Crypt. No. 2078). In Compendium Florac Germanicae 4: 331. 1883. This was evidently some member of the A. glaucus group.

A. erythrocephalus Berkeley and Curtis, in Jour. Linn. Soc. [London], Bot. 10: 362. 1869. In A. tamarii group.......... 257

Inzengaea erythrospora Borzi, in Jahrb. Wiss. Bot. (Pringsheim) 16: 450-463, pls. 19-20. 1884. Manifestly A. variecolor...... 163

A. exiguus Hann.

Syn. A. conicus fide Blochwitz, in Ann. Mycol. 31: 73.1933.

S. ferruginea Cooke, in Grevillea VIII. (1879), 95., in Jour. Quekett Mic. Club 2 Ser. 2: 139. 1885. Not an Aspergillus. Republished in Veg. Wasps and Plant Worms, p. 184. 1892; see also Petch in Trans. Brit. Myc. Soc. XVI, p. 72. 1931, who examined type specimens as rustcolored patches on pupae; conidophores hyaline smooth, $12 \mu$ diam., vesicle globose $50 \mu$; primary sterigmata 30 by 4 to $12 \mu$, secondary $14-18$ by $7-9 \mu$, conidia from 9 by $5 \mu$ to 6 to $9 \mu$ subglobose, brown, coarsely rough; a brown member of the $A$. niger group.

A. ferrugineus Fuckel, in Fungi rhenani No. 157, also Symbolae Mycologicae in Jahr. d. Nassauischen Vereins fur Naturkunde Jahrg. XXIII and XXIV: 358. 1869-70. Not identified. A. ferrugineus Link, in Sp. Plant, Ed. 4, 6: pt. 1, p. 68. 1824. Also in Fries. Sys. Myc. 3, p. 387. 1829. Both authors seem to have had members of the A. glaucus group. A. ficuum (Reich.) Hennings...... 225 
Syn. Sterigmatocystis ficuum (Reich.) P. Henn., in Hedwigia 34, Heft 2: 86.1895.

Syn. U'stilago ficuum Reichardt, in Verhandl. K. K. Zool. Bot. Gesell. Wien. 17: 335. 1867. A culture distributed under this name (Thom 142) is a rapid oxalic acid producing organism. In the A. niger group.

A. fiemonthi Sopp, cited as a name on a culture by Biourge in MS. p. 19 , in the A. flavus group.

A. fimetarius Peck, in N. Y. State Mus. Bot. Rept. 42, p. 128. 1889. Probably A. candidus group.

A. fimeti Sacc., in Michelia 2: 543 . 18s2. Inadequately described.

A. fischeri Wehmer, in Centralb. f. Bakt. II abt. 18(12/15): 390-392, figs. 5. 1907. In the A. fumigatus group....

Syn. A. fumigatus-ascosporic, see Thom and Church, in Am. Jour. Bot. 5: 91-92. 1918.

A. flacaureus Biourge, nomen nudum, listed in his MIS. as a member of the A. flavus-oryzae group with yellow-orange reverse, as shown by a culture in his collection.

A. flavescens Wreden, in Compt. Rend. Acad. Sci. Paris 65: 368 . 1867. Also St. Petersb. Med. Zeitschr. 13: 133. 1867. Identifications have not been satisfactory. Possibly in $A$. nidulans group.

A. flavidus Berkeley and Broome, in Jour. Linnean Soc. (London), Bot. 14: 101. 1875. Cited by Saccardo in Fungi of Ceylon, London 1871-73, No. 913 S. Probably a member of the $A$. flavus group but not more closely identifiable.

A. flavipes (Bainier and Sartory) Thom and Church, in The Aspergilli, p. 155.1926 .
Syn. S. flaripes Bainier and Sartory, in Bul. Soc. Mycol. France 27: 90-96, pl. III, figs. 1-6. $1911 \ldots \ldots \ldots \ldots \ldots \ldots \ldots \ldots . \ldots 179$

A. flavo-viridescens Hanzawa, in Journ. Coll. Agr. Tohoku Imp. Univ. Sapporo 4: 232-3, Pl. 21, figs. 1-4. 1911. In A. versicolor group.................. 192

A. flavus Link, in Obs. p. 16, 1809. (H. F. Link Observations in Ordines plantarum naturales, Gesellschaft Naturforschender Freunde zu Berlin, Magazin 3, 1809-commonly cited Link Obs.).................... 263

A. flavus forma Maydis Ciferri, R., in Ann. Mycol. 20: 46. 1922.

A. flavus var. japonica. cited by Blochwitz in Ann. Mycol. 31: 74. 1933, without description.

A. flavus var. viridis Blochwitz, in Ann. Mycol. 32: 86. 1934. Is vaguely designated as a nontropical strain apparently duplicating A. parasiticus Speare... 267

A. flavus mut. rufa Blochwitz, in Ann. Mycol. 27(3/4): 201. 1929. A mutation which shows gradations to pure brown from the green form.

A. flavus mut. fusca Blochwitz, in Ber. d. Bot. Ges. 48: 576. 1928. Eurotium Aspergillus flavus DeBary and Wor. Terminology employed by DeBary and Woronin (Beitr. z. Morph. u. Physiol. d. Pilze, III Reihe, p. 380. 1870) to designate relationship of the asexual species, A. flavus, with species characterized by a perfect stage and included in Eurotium..................... 263

A. foetidus n. sp................ 219

Syn. A. aureus Nakazawa. A black Aspergillus with a strong musty odor .................. 219

A. fonsecaeus n. sp.

Syn. S. fusca Bainier, in Bul. Soc. Mycol. France 27: 29, pl. 1, fig. 5 . 1850. A black Aspergillus on 
rubber, from Fonseca in Rio de Janeiro . . . . . . . . . . . . . . . 227

A. fontoynonti Gueguen, in Archives de Parasitologie 14: 177-192, 2 plates, 36 figs. 1910. See also Compt. Rend. Soc. Biol. Paris 66: 1052. 1909. The figures and description place this form unquestionably in the A. glaucus group

A. freisingianus Biourge, nomen nudum, listed with the A. claratus group as "Trés liquifiant, comme celle de Blochwitz." In the Biourge Collection 1939.

E. fructigenum Link, in Sp. Pl. Ed. 4, 6: 80 . 1824. Some unidentifiable member of the $A$. glaucus group.

S. fuliginosa Bainier, in Bul. Soc. Bot. France 28: 79. 1881. Some variety of $A$. niger.

A. fuliginosus Peck, in Bul. Buffalo Soc. Nat. Sci. 1:69. 1873. Also in Ann. Rep. N. Y. St. Mus. Nat. Hist. 26: 79. 1874. Some strain of the $A$. niger group. Mosseray applied it to a culture in Biourge's Collection.........

E. fulvescens Cooke, in Grevillea 8: 11. 1880 .

Syn. Badhamia fulvescens Cooke. Change of generic designation only, from the same material. Not identifiable from descriptions.

A. fulvus Montagne, in Ann. Sci. Nat. Bot. Ser. 3, Vol. XII : 298. 1849.

Syn. S. fulva Sacc. Syll. 4:74. Not identifiable. Described from diseased silk worms in Southern France, but not since identified. A. fumaricus Wehmer, in Ber. Deut. Chem. Gesell. 51(14): 1663-68, figs. 1-6. 1918. A unique member of the $A$. niger group

Sartorya fumigata Vuillemin, in Compt. Rend. Acad. Sci. (Paris) 184: 136-137. 1927. Possible synonym of $A$. fischeri q.v..... 153
A. fumigatoides Bainier and Sartory, in Bull. Soc. Mycol. France 25: 112, pl. 5. 1909. Compt. Rend. Soc. Biol. [Paris] 66: 22. 1909. See also Sartory, A., Champignons parasites de l'homme et des animaux, p. 573. 1922 .

Syn. E. fumigatoides (B. \& S.) Sacc. Syll. 22: 1255. 1913. Probably close to $A$. fischeri.

A. fumigatus Fresenius, in Beiträge zur Mykologie, p. 81, pl. 10, figs. 1-11 Frankfurt. 1850-53 ..... 148 var. Alpha, Sion and Alexandrescu, in Compt. Rend. Soc. Biol. 64: 288-289. 1908..... 151 var. cellulosae Bäumli, cited by Sartory, Sartory, and Meyer, in Compt. Rend. 203: 1289-91. 1936. See C. A. 31: 2249. 1937. Mut. helvola E. Yuill, in Jour. Bot. (London) 1939. p. 174. A buff-colored mutant which appeared in culture. .

var. maguus Nakazawa, Simo, and

Watanabe, in Jour. Agr. Chem. Soc. Japan No. 144. 1936. (In Japanese.)

var. minimus Surtory, in Bul. Acad. Med. Paris Ser. 3, 82: 304. $1919 \ldots \ldots \ldots \ldots \ldots \ldots \ldots \ldots 151$

var. tumescens Blumentritt, in Ber.

Deut. Bot. Gesell. 23: 419-427, Pl. 19, fig. 5, 6, 18-21. 1905... 151 var. subglobosus Blochwitz, in Ann. Nycol. 33: 244. 1935.

Aspergillopsis fumosus Sopp, in Videnskapselskapets Sks. I Mat.-Naturv. Kl. No. 11, pp. 202-204, Taf. XX, fig. 149. 1912. Not identified but suggested as some strain near A. ustus.

A. fungoides Greco, in Origine des tumeurs et mycoses Argentines, Buenos Aires pp. 505-509, figs. $297,298,299.1916$. The description is inadequate for identification.

S. fusca Bainier, in Bull. Soc. Bot. France 27: 29, pl. I, fig. 5. 1880. Compare Roumeguere Fungi 
gallici exsiccati, No. 995 grown upon moist bread in Toulouse, 1880. Some member of the $A$. niger group.

\section{7}

A. fusco-cinereus Ellis and Morgan, M.S. name attached to Morgan's specimen No.67t. Evidently a member of the A. claratus group but not since seen. . . . . . . . .

E. fuscum Pruss, in Linnaea 25: 78. 1852. Some unidentifiable member of the A. glaucus group. A. fuscus Amons, in Archief voor de Suikerindustriein NederlandschIndië 29, Deel 1, January-June pp. 8-10. 1921. Not A. fuscus of Bonorden, Bainier, or Schiemann. See: A. terreus group. 200

A. fuscus Bonorden, in Bot. Ztg. Jahrg. 19: 202. 1861. Not identifiable

A. fuscus Schiemann, in Ztschr. Induk. Abstam. u. Vererbungslehre. Bd. 8(1/2): 1-35, 16 figs., 2 pl. (1 col.). 1912 .

Syn. A. schiemanni (Schiemann)

Thom q.v..

A. galactus Link, cited by Yonemoto and Kato, in Bull. Miyazaki Coll. Agr. Forestry 3: 59-66. 1931. Perithecia formed between $15^{\circ}$ and $28^{\circ} \mathrm{C}$.

A. galeritus Blochwitz, in Ann. Mycol. 27(3/4): 205, Taf. III. 1929. Syn. of A.terreus Thom ...... 197

A. gallomyces Calmette, Alb. Kaiserliches Patent Amt. Klasse 12 q. N. 129164 August 12 (1900). Apparently a variant of $A$.niger.

$A$ giganteus (Mattlet) Dodge, in Dodge Med. Mye., p. $629 . \quad$ 1935. 225 Syn. S. gigantea Mattlet, in Ann. Soc. Belge. Med. Trop 6: 35 . 1926. Not A. giganteus Wehmer. Some member of the $A$. niger group.

A. giganteus Wehmer, in Centralb. f: Bakt. 2 abt., 18(13/15): 385. 1907. See the A.clavatus group. A. giganto-sulphureus Saito, in Jour. Coll. Sci. Imp. Univ. Tokyo 18:
48, pl. III, fig. $12 \mathrm{a}$ to d. 1904 Apparently a non-ascosporic member of the A. glaucus group.

A. gigas Speg., in Ann. Mus. Nae. Buenos Aires Ser. 3, V. 13: 434 1911. Probably one of the $A$. tamarii series

$A$. glaber Dale, nomen nudum attached by Biourge to a culture belonging with $A$. conicus.

S. glauca Bainier, in Bul. Soc. Bot.

France 27: 29, Pl. I, fig. 3. 1880.

Also Bul. Soc. Bot. France 28:

77. 1881. Probably belongs in A. versicolor group. .

A. glaucoides Spring, in Bull. Acad. Sci. Belg. 19: 560-572. 1852.

Probably A. fumigatus.

A. glaucus Link, in Obs. p. 16. 1809. See $A$. glaucus group.

mut. alba Blochwitz, in Deut. Bot.

Gesellsch. Ber. 50: 248-256. 1932. See: A. niveo-glaucus Thom and Raper.

var. albida Speg., in An. Mus. Nac.

Buenos Aires T. VI: $332 . \quad 1899.135$

var. minimus Hanzawa, in Jour.

Coll. Agr. Tohoku Imp. Univ.

Sapporo 4: 220. 1911.

var. oblongispurus E. \& W. Field

Mus. Bot. 1: S8. 1896.

var. olivascens Saccardo, in Miche-

lia 2: 543 . 1878. Distributed by Sace. as No. 1376 Myco. ital. var. subolicaceus Ferraris, in Fl. ital. Crypt. P. I, fasc. 13, p. 911. 1914.

var, repens Corda, in Icones 5: 53 . 1842.

Syn. A. repens DeBary q.v...... 103

A. globosus Jensen, in Cornell Agr. Exp. Sta. Bul. 315, p. 482. 1912. The strain studied by Jensen (No. 2705) was clearly A. versicolor. .

A. globosus Link

Syn. Sporodinia aspergillus Schröter, in Sace. Syll. 7: 207.

A. godfrini Sartory and Roederer, in Assoc'n. Française pour l'avancement des Sciences, 42nd 
Session, Tunis, pp. 601-603. 1913. Some one of the A. glaucus group.

A. gracilis Bainier, in Bul. Soc. Mycol. France 23: 92, PI. IX, figs. 11-14. 1907. In $A$. restrictus series.

var. exiguus Bainier \& Sartory, in

Bull. Soc. Mycol. France 28: 47, pl. 2. 1912. According to the description this variety differs in physiological characters slightly from A. gracilis Bainier....... 14

A. granulatus Míosseray, in La Cellule XLIII : 249-50, pl. 3, figs. 2528. 1934. A member of the $A$. niger group; in the Biourge Collection...

A. granulosus Raper and Thom, in Mycologia 36: 565-568, fig. 4. 1944. In the A. ustus group.... 175

A. gratioti Sartory, in Compt. Rend. Acad. Sci. (Paris) 170: 523-524. 1920. Also in Champignons parasites de l'homme et des animaux, pp. 578-579. 1922. Probably some member of the $A$. fumigatus group........... 154

A. Greconis Dodge, in Dodge Med. Myc., p. 634.1935

Syn. S. aurea Greco. q.v.

A. griseus Link, in Sp. Plant. Ed.4, 6(1):69. 1824. Incorrectly cited by Bonorden and Wehmer as Link Obs. 1: 69. 1809. Name also used by Fries and by Bonorden. Not identified.

A. guegueni was figured in Biourge's monograph of the Penicillia (La Cellule t. 33, fasc. 1, pp. 7-330. 1923) as $P$. guegueni Plate $X X$. He afterward distributed it as $A$. guegueni. In A. restrictus series 139

A. guttifer Mosseray, in La Cellule XLIII : 235-236, pl. III, fig. 5357. 1934. In the Biourge Collection................... 233

A. gymnosardae Yukawa, in Jour. Coll. Agr. Tokyo I: 362, Pl. 18, figs. 1-7. 1911. This fungus was found by Yukawa under the name "awokabi" and is described by him as essential to the ripening of the tunafish preparation, "katsuobushi." The dimensions given are intermediate between those of $A$. flavus and $A$. oryzae, and closely approximate those of A. pseudo-flavus. Although we have cultures related to these forms, we have not been able to identify these intermediates except as members of the $A$. flavus-oryzae group.......... 266

A. hageni Hallier, in Cattaneo Mico. Corp. Um. p. 123, Pl. 6, fig. \&. Florentin 1892 . 146 Syn. Otomyces Hageni Hallier, in Zeitschr. Parasit. 1: 195. 1869. 2: 22, 233, and 259, Pl. 5. 1870. Not identifiable.

A. halophilus Sartory, Sartory, and Meyer, in Ann. Mycol. XXVIII: 362-363, PI. III, figs. 11-14. 1930. Probably some nonascosporic member of the $A$. glaucus group.................. 118

$A$. helicophorus nomen nudum attached by Thaxter to a culture that was found to belong with $A$. ustus ..................... 174

S. helva Bainier, in Bul. Soc. Bot. France 28: 78. 1881. In $A$. ochraceus group............ 281

A. hennebergi Blochwitz, in Ann. Mycol. 33: 238. 1935. Regarded by Neill as one of the A. ochraceus group. See A. wentii group. 249

A. herbariorum, species name seems to appear first as Mucor herbariorum in Primitiae Florae Holsaticae by Fredericus Henricus Wiggers, 1780, republished in Facsimile edition No. 23 by W. Junk. 1925. See A. glaucus group................. 100

Eurotium herbariorum ser. minor Mangin, in Ann. des Sci. Nat., Bot. (Ser. 9) 10:365. 1909. See A. mangini.............. 127

Eurotium herbariorum ser. major Mangin, in Ann. Sci. Nat. Bot. 
(Sel. 9) 10:365. 1909. A. glaucus group characterized by large ascospores. Would fall within A. echinulatus q.v.

A. heterocephalus Spring, in Bull. Acad. Sci. Belg. 19: 568. 1852. This name was given to colonies in a hen's egg which showed small heads globose and large heads columnar. Since no adequate figure or description was offered, it may be discarded as a nomen nudum.

Physomyces heterosporus Harz. See A. atro-ruber.

P. (Micro-aspergillus) Hickeyi, figured by Biourge in La Cellule XXXII, fase. 1: 1-331. 1923 (Penicillium Monograph), proved to be indistinguishable from A. gracilis q.v..

A. hispidulus Sprengel, in Sys. Veg. Ed. 16, 4: 541. 1827. There is no suggestion of an Aspergillus in the description given.

A. holmiensis Biourge, nomen nudum, listed among the A. versicolor group in Manuscript and in culture in his collection in 1939 .

A. hortai (Langeron) Dodge, in Dodge Med. Mycol., p. 628. $1935 \ldots \ldots \ldots \ldots \ldots \ldots \ldots \ldots$ Syn. S. hortai Langeron, in Bul. Soc. Path. Exotique 15: 383-384, figs. 1-3. 1922

Syn-A terreus Thom ........... 195

A. humicola Chaudhuri and Sachar, in Ann. Mycol. 32: 97. 1934.

Cited by Neill as A. versicolor. 193

A. humus Abbott, in Ia. St. Coll. J. Sci. 1: 15-36. Fig. 2a-e. 1926. See also Louisiana Station Bul. 194. One of the A. ustus series. 174

A. hypojanthinus Biourge, was published as Penicillium hypojanthinum in Biourge Monogr. La Cellule 33: fasc. 1, pl 321-2, Pl. XXII, fig. 130. 1923. Afterward he transferred it to Aspergillus. A. restrictus series.... 139
A. incrassatus Spring, in Bull. Acad. Roy. Belg. 19: 559, fig. 2. 1852. Not identifiable.

E. insigne Winter (exsiccati in Rabh. Fungi No. 1732), in Hedwigia 1873 and Rabh. Krypt. Fl. Aufl. 1, Abt. 2, 2: 61. 1887. Probably not an Aspergillus.

S. insueta Bainier, in Bull. Soc. Mycol. France 24: 85-87, Tab. VIII, figs.1-13. 1908. A.ustus series. 174

A. intermedia Speg., in Myc. Arg. V. in An. Mus. Nac. Buenos Aires Ser. 3, T. 13: 435. 1911. In the A. niger group.

A. itaconicus Kinoshita, in Botan. Mag. Tokyo 45: 60-61. 1931. Species diagnosis and figure reprinted in Acta Phytochim. (Tokyo) 5(3): 271-287. 1931. See $A$. glaucus group..

S. italica Sacc., in F. italici No. 109. 1881. Also in Michelia 1: 91. 1877.

Syn. A. sterigmatophorus Sacc., in Atti. d. Soc. Ven.-Tren. d. Sc. Nat. 2, fasc. 2: 232, Table XVII, fig. 5-8. 1873. Some member of the A.candidus group...... 211

$A$. janus n. sp. Raper and Thom, in Mycologia 36: 556-561, fig. 1. $1944 \ldots \ldots \ldots \ldots \ldots \ldots \ldots .187$ var. brevis Raper \& Thom, in Mycologia 36: 561-563, fig. 2. 1944. 190

A. japonicus Saito, in Bot. Mag. Tokyo 20: 61-63. 1906. In $A$. niger group .............. 231 var. capillatus Nakazawa, Takeda, and Suematu. Culture available in Centraalbureau. 1939. var. or mut. grisea Blochwitz, in Ann. Mycol. 33: 240. 1935. It was a change of name only, $A$. malvaceus Mosseray.

A. javanicus cited by Takahashi, and Sakaguchi, in Jour. Agr. Chem. Soc. Japan 1: No. 10, 1925, only in parenthesis after $A$. fumaricus Wehmer and apparently deemed a synonyn. In $A$, niger group. 
A. jeanselmei Ota, in Ann. de Parasitologie 1(2): 137-146. 1923.

A. flarus group

A. keratitis Ball, in Amer. Med. 2: 31. 1901. This organism was found in an ulcer in the human cornea. Not identifiable..... 147

A. koningi Oudemans, in Arch. Neerl. Ser. II, V. 7: 284, Tab. XIV. 1902. Not since identified.

A. laneus Link Obs. p. 16.1809. Syn. Botrytis lanea (Bonorden) Sace, in Syll. 4: 74 .

A. laneus in Schweinitz in Syn. Am. Bor. is syn. for Rhinotrichum curtissii Berk. in Grevillea 3: 108. 1875.

A. laokiashanensis Shih, in Lingnan Sci. Jour. 15(3): 368. 1933. Near $A$. unguis in the $A$. nidulans group

E. lateritium Montagne, in Century VI. No. 35, Ann. Sci. Nat. Bot. 3 Ser. XI: 154. 1849. Sylloge p. $25 \bar{r}$. One of the $A$. glaucus group with ascospores 7 to $10 \mu$ in diameter.

A. Lepidophyton Wehmer.

Syn. Epidermophyton concentricum fide Dodge, Med. Nyc. 490. 1935.

A. lignieresi Cost and Lucet, in Ann. Sci. Nat. IX. Bot. 2: 137, Pl. 5, figs. 19-23. 1905. This culture from the lung of a penguin differs in cultural details from typical A. fumigatus especially by the presence of swollen groups of cells in the mycelium

A. longobasidia Bainier, nomen nudum; a culture so labeled (Thom $46+0.477$ ) was received from the Bainier Collection through daFonseca. This was the type of A. bainieri Mosseray, in Ann. Soc. Sc. Bruxelles 54, ser. B, p. 72. 1934. Member of the $A$. niger group characterized by very long primary sterigmata.
A. lovanicnsis Biourge, nomen nudum; culture distributed by Biourge and appearing in NRRL Collection as No. 76. See Thom and Raper U. S. D. A. Mise. Publ. 426, p. 18. 1941 ...... 117

A. luchuensis Inui, in Jour. Col. Sci. Imp. Univ. Tokyo 15: 469, Pl.22, figs. 1-8. 1901

var. rubeolus Shih, in Lingnan Sci. Jour. 15(3): 374.1933

S. lutea Bainier, in Bul. Soc. Bot. France 27: 27. 1880. A. flavus group. See also Sartory and Jourde, in Compt. Rend. Acad. Sci. (Paris) 146: 548-549. 1908. . 269

A. luteus (van Tieghem) Dodge, in Dodge Med. Myc., p. 625. 1935. Syn. S. lutea van Tieghem, in Bul. Soc. Bot. France 24: 103. 1877.

A. luteo-niger (Lütz) Thom and Church, in The Aspergilli, p. 166. 1926

Syn. S. luteo-nigra Lutz, in Bul. Soc. Bot. France 53: 48-52. 1907. Collected by A. Chevalier at San Thome, Africa, in fermenting seeds of Theobroma cacao. In the A. niger group. . 226

A. luteo-niger van Luijk, nomen nudum, in Biourge's MS. p. 15. Probably attached to a culture of some black Aspergillus.

A. luteo-virescens Blochwitz, in Ann. Mycol. 31(1/2): 73-83. 1932. In $A$. tamarii series.......... 2

A. lutescens Bainier, nomen nudum, described by Thom and Church in The Aspergilli, p. 193, 1926. In $A$. tamarii series

A. lutricolor Biourge, nomen nudum, listed in MS. among the $A$. ochraceus group; in the Biourge Collection.

A. Macfici Dodge, in Dodge Med.

- Mycol. p. 269.1935.

Syn. Sterigmatocystis sp. Macfie, in Ann. Trop. Med. Parasitol. 15:279-281. 1921. Pathogenic? In the A, niger group ......... 239 
A. macrosporus Bonorden, in Handbuch d. allg. Myk. 1851, fig. 193. Not identifiable.

A. malignus Lindt, in Arch. Exp. Path. Pharm. 25: 256-271, figs. 1-11. 1889. In A. fumigatus group .

A. malraceus Mosseray, in La Cellule XLIII: 265-266, Pl. 4, figs. 134135. 1934. A member of the $A$. niger group. In the Biourge Collection

A. mangini

Syn. A. minor (Mangin) Thom and Raper, in U. S. D. A. Misc. Publ. 426, p. 27.1941.

Syn. E. herbariorum ser. minor Mangin, in Ann. Sci. Nat. Bot. (Ser. 9) 10: 365. 1909.

Member of the $A$. glaucus group with ascospores of intermediate size

A. maximus Link, in Obs., p. 16. 1809. Not an Aspergillus.

A. maydis Quevedo, in De Agronomica Nos. 8 and 9, Buenos Aires, 1912. See also Sartory, in Champignons parasites de l'homme et des animaux p. 532, 1922. A species probably belonging to the $A$. glaucus group described in connection with disease in horses.

Emericella medias Chowdhury and Mathur, in Ann. Mycol. 36: 6163. 1938. Probably A. variecolor q.v................. 163

A. medius Meissner, in Bot. Ztg. 55: 336-344, 354-357. 1897. See Thom and Raper, U. S. D. A. Misc. Publ. 426, p. 33, $1941 \ldots 133$

A. melleus Yukawa, in Jour. Coll. Agr. Tokyo 1(3): 366, Taf. XVII. 1911. In A. ochraceus group.

A. mencieri Sartory et Flament, in Compt. Rend. Soc. Biol. Paris 83:114-115. 1920. See also Sartory et Bailly in Les Myocoses Pulmonaires et leur Parasites
(Paris) pp. 180-181. 1923. A. glaucus group; not since reported. 146 A. michelii Preuss, in Linnaea 25: 76. 1852. Not recognized.

A. microcephalus Mosseray, in La Cellule XLIII, p. 225-227, Pl. 3, fig. 42-48. 1934. Member of the $A$. niger group. In the Biourge Collection. ........... 233

A. microsporus Böke. The description and figures given by Cattaneo and Oliva in Arch. Lab. Bot. Critt. Garovaglio 5: 123, Pl. 6, fig. 9, 1888, have been seen. Not recognizable.

A. micro-virido-citrinus Costantin \& Lucet, in Ann. Sci. Nat. Bot. IX (2): 158. 1905. In A. flavus group ................. 263

A. minimus Wehmer, in Bot. Centralb. 80: 449-461. 1899. See also Wehmer's Monogr. p. 79, 1899-1901. Wehmer's culture was lost. It has not been identified since.

S. minor Bainier, in Bul. Soc. Bot. France 27: 30. 1880. Not recognizable. Possibly A. sydowi?

A. minor (Mangin) Thom and Raper in U. S. Dept. Agr. Misc. Pub. 426 , p. 27-29. 1941. See $A$. mangini............... 127

147 A. minutus Abbott, in Louisiana Sta. Bul. 194. 1926. Also in Iowa St. Coll. Jour. Sci. 1: 15-36, figs. a, b, c, and d. 1926. See $A$. ustus group.

A. miyakoensis Nakazawa, Sino, and Watanabe, in Agr. Chem. Soc. Japan Jour. 12(9): 963-964. 1936. See $A$. niger group

A. mollis Bainier and Sartory, in Bul. Soc. Mycol. France XXVII: 453, Pl. XVI. 1911. Not A. mollis Berkeley. See A. glaucus group .

A. mollis Berkeley, in English Flora V, pt. 2, p. 340. 1836. Not identifiable. 
A. mongolicus Biourge, nomen nudum. Biourge distributed under this name a culture essentially like $A$. echinulatus (q.v.). It appears in the NRRL Collection as No. 137. See Thom and Raper U. S. D. A. Misc. Publ. 426, p. 33. $1941 \ldots \ldots \ldots \ldots \ldots .132$

A. montevidensis Talice and MacKinnon, in Soc. Biol. [Paris] Compt. Rend. 108: 1007-1009. 1931. See Thom and Raper U. S. D. A. Misc. Publ. 426, p. 26. 1941. In A. glaucus group..... A. mouthoni Biourge, nomen nudum, listed by Biourge among his Les "Eurotium" in unpublished Manuscript. Probably in culture in his collection.

A. mucoroides Corda, in Icones fungorum II: 18, fig. 76. 1837. Probably some member of the A. glaucus group-unidentifiable.

A. mucoroideus Cook, in Grevillea XII: 9. 1883. See A. coolei Sace.

A. mülleri Berkeley, in Jour. Linn. Soc. XIII: 175. 1873. Not identifiable.

A. mutabilis Bainier et Sartory, in Bul. Soc. Mycol. France 27: 458, pl. XVII. 1911. In $A$. glaucus group................

A. mycetomi Villabruzzi and Gelonesi, in Annali Med. Nav. Colon.

33: 283-308, 8 figs. 1927.

Syn. Madurella $s p$. ; undeterminable from the data available.

A. mycobanche Link, in Sp. Plant Ed. 4, 6: 65. 1824. A fungus from rotting Peziza-not identifiable.

A. nantae Pinoy, in Compt. Rend. Soc. Biol. 97(19): 67-68. 1927. This was probably $A$. unguis in the $A$. nidulans group. Listed by Biourge as in his collection, 1939 .
A. nanus Montagne, in Sylloge Generum Specierumque Crypt. p. 300, No. 1112. Paris. 1856. Also in Saccardo Sylloge Fungorum 4: p. 71. Patavii 1886. In the $A$. niger group..... 231

A. nanus Oudemans, in Nederl. Kruidk. Arch. Ser. 3, 2: 1121. 1904. Not A, nanus Mont. q.v. Probably some conidial form in the A. glaucus group.

E. nebulosum Fries, in Sys. Myc. 3: 334. 1832. No data are given to link this with Aspergillus.

A. nicollei Pinoy, cited by Biourge in 1939 manuscript. He has apparently raised to species rank $S$. nidulans var. nicollei Pinoy, q.v.............. 170

A. nidulans (Eidam) Wint., in Rab. Krypt.-Fl. 12:62. 1884,..... 156 Syn. S. nidulans Eidam, in Cohn, Beitr. Biol. Pfeanzen 3: 392-411, Pl. 20-22. 1883.

forme Cesarii Pinoy, in Bul. Soc.

Path. Exot. 8: 11. 1915....... 170

mut. coerulea Blochwitz, nomen nudum upon a culture in the Biourge Collection, 1939. Listed in Biourge MS.

mut. alba E. Yuill, in Jour. Bot. (London) 1939, p. 175, pl. $618 \ldots \ldots \ldots \ldots \ldots \ldots \ldots \ldots$

129 var. latus Thom and Raper, in Mycologia 31(6): $657-9$. $1939 \ldots \ldots \ldots \ldots \ldots \ldots \ldots . \ldots \ldots$ var. Nicollei Pinoy, in Compt. Rend. Acad. Sci. Paris 144: 396. 1907. This variety was found fruiting within luman tissue in a subject affected with "Madura-foot." Listed in Biourge's Collection, 1939, as S. nicollei Pinoy.............. 170 Syn. S. nidulans var. Nicollei Pinoy, in Archives d. Parasitologie 10: 437-458, Pl. XI. 1906.

Diplostephanus nidulans (Eidam) Langeron, in Compt. Reud. Soc. Biol. Paris 87: 343-345. 1922. 
Syn. for A. nidulans Eidam q.v.................. 156

A. niger van Tieghem, in Ann. Sci. Nat. Bot. Ser. 5, V. 8(4): 240 . 1867. See also Thom and Currie, in Jour. Agr. Res. 7: 1-15. 1916.

Syn. S. nigra van Tieghem, in Bul. Soc. Bot. France 24: 102-103. 1877.

Syn. Aspergillopsis nigra (v. Tieghem) Speg., in Myc. Arg. $V$, in Ann. Mus. Nac. Buenos Aires (ser. 3) 13: 435. 1911.

mut. cinnamomeus n. comb. See:

A. cinnamomeus Schiemann.... 223

var. laeris Blochwitz, in Ann.

Mycol. 33: 249. 1935. Proposed for smooth-spored strains.

A. niger var. fermentarius Nakazawa, Simo, and Watanabe, in Jour. Agr. Chem. Soc. Japan 144: 1712 and 181.1936 (in Japanese)... mut. fusca Blochwitz, in Ann. Mycol. 32: 87. 1934. In the A. niger group. Such a separation based upon conidial color is of doubtful validity.

mut. Schiemanni n. comb..

See: A. schiemanni (Schiemann) Thon.

forma Tuebingen Schober, in the Centraalbureau, is apparently the strain used in the researches of Schober.

A. niger citricus Wehmer, name on a culture received from Neuberg (C. T. No. 4668.4) but without description.

Syn. A . citricus Mosseray q.v..

S. nigra Bainier. Syn. for S. phoenicis (Corda) Patouillard and Delacroix, in the $A$. niger series.

A. nigrescens Robin, in Histoire Naturelle des Vegetaux Parasites (Paris) p. 518, pl. 5, fig. 2. 1853. No success has been made in interpreting Robin's organism.............. 151
A. nigricans Wreden, in Compt. Rend. Acarl. Sci (Paris) 65: 368. 1867. Probably A. niger group.

A. nigriceps $\mathrm{B}$. and $\mathrm{C}$., in the Curtis Collection. Specimen collected by Charles Wright (No.927) in Cuba. Cited by Cooke, M. C. in Grevillia 17: 21, Sept. 1888. A slide from this material prepared by Bullard and preserved in the Harvard collection shows a characteristic organism of the $A$. niger series, not separable from $A$, niger van Tieghem.

A. niveocandidus Lindau, in Rabh. Krypt. F1. 8: 151. 1907. In the A. candidus group.

A. niveo-glaucus Thom and Raper, in U. S. Dept. Agr. Misc. Pub. 426, p. 35-36. 1941

A. niveus Blochwitz, in Ann. Mycol. 27(3/4): 205-206, Taf. III, fig. 2. 1929. A member of the $A$. terreus group.............. 202

var. major Blochwitz, in Ann. Mycol. 32(1/2): 86. 1934. Apparently belongs in the $A$. candidus group.............. 211

var. nubila Blochwitz, in Ann. Mycol. 32(1/2): 85. 1934. See A. carneus............... 202

A. Nölting Hallier, in Zeitschr. Parasit. (Not found) cited by Cattaneo and Oliva in Arch. Lab. Bot. Critt. Garovaglio 5: 122. 1888. Not recognizable.

A. novus, nomen nudum attributed to Wehmer; culture in the Centraalbureau at Baarn was identified by Thom and Raper as $A$. pseudo-glaucus............... 111

E. obliteratum Schw. Syn. fungorum in Amer. Bor. N. 2725. Some unidentifiable member of $A$. glaucus group.

A. oblongisporus E. and E., No. 760 in Nuttall's Flora of Fayette County, West Virginia. Listed apparently by Millspaugh in "The Living Flora of West Virginia" in W. Va. Geol. Survey 
5(A): 32. 1913. as A. glaucus var. oblongisporus. E. \& W. Examination of this material shows it to be a mixture of $A$. flavus and $A$. repens.

S. ochracea Bainier, in Bul. Soc. Bot. France 28: 75. 1881. Some member of the $A$. ochraceus group.

S. ochracea Delacroix, in Bul. Soc. Mycol. France 7: 109, Pl. VII,

fig. f. $1891 \ldots \ldots \ldots \ldots \ldots \ldots . . \ldots .282$

Syn. S. delacroixii Sacc., in Sylloge 10: 527. Delacroix described his organism without recognizing the previous use of the specific name........ 282

S. ochracea (Wilhelm) Schröter. See: A. ochraceus Wilhelm.

A. ochraceo-ruber Sacc., in Michelia I: 77. 1877. (Saccardo, P. A. No. 1063 in Myco. Veneta, on bark of Walnut, 1876, see fig. 17 in Fungi italici). Some conidial form of the $A$. glaucus group.

A. ochraceus Wilhelm, Inaug. Diss.

Strassburg. p. 66. 1877.

var. microspora Tiraboschi, in

Annali di Botanica 7: 14.

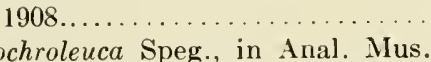

S. ochroleuca Speg., in Anal. Mus.
Nac. Buenos Aires, Ser. 3, t.

13: 434. 1911. In the A. ochraceus group..................

A. ochroleucus Haller, in Enum. Method. Stirp. Helvet. Indig. $t$. I: p. 6. 1742. Hist. Stirp. t. III. 1768. Cited by Gasperini as questionable syn. for $A$. elegans.

A. okazakii Okazaki, in Centralb. f. Bakt. 2 abt. 42(10/14): 225 .

1914. A. candidus group..

This is cited in the Sylloge 22: 1260 as S. okazakii (Saito) Saccardo.

A. oligosporus Corda, in Icones III, Tab. II. Not an Aspergillus.

S. olivacea van Tieghem, in Bull. Soc. Bot. France 24: 103. 1877. Not identified with $A$. olivaceus
Preuss, 1852. Not identifiable.

A. olivacco-fuscus Mosseray, in La Cellule XLIII : 25S-259. pl. 3, fig. 49-52. 1934. A member of the $A$. niger group in the Biourge Collection............ 234

Cladosarum olivaceum Yuill and Yuill, in Trans. Brit. Myc. Soc.

22: 194-200, Pl. 11-13. 1938....

A. olivaceus Delacroix, in Bul. Soc. Mycol. France 13: 118-120, text fig. 1897

Syn. A. delacroixii Sacc. and Sydow q.v. Not A. olivaceus Preuss. 1852. Probably in $A$. glaucus group.

A. olivaceus Preuss, in Linnaea 25: 77. 1852. Not identifiable.

A. Oniki on a culture from Okunuki in the Centraalbureau collection. No description found. Blochwitz cites it in Ann. Mycol. 33: 240. 1935. As A. ochraceus.

A. oösporus Wallroth, as Flora Cyptogamica Germaniae No. 1928, in Compendium Florae Germanicae 4: 296. 1833. Some one of the A. glaucus group.

281 A. oriolus nomen nudum attributed to Biourge. Distributed by Biourge Collection and appears as NRRL No.87. It was identified as a strain of $A$. chevalieri by Thom and Raper in U. S. D. A. Misc. Publ. 426, pl. 21. 1941. 120

A. oryzae (Ahlburg) Cohn, in Jahresb. Schles. Ges für Vaterl. Cultur 21: 226. 1883. In the A. flavus-oryzae group....

Syn. E. oryzae (Ahlb.) Korschelt. Name with incomplete description of saké organism published in Dingler's Polytech. Jour.

230:330. 1878........... 261

A. oryzae var. basidiferens Constantin and Lucet, in Ann. Sci. Nat. Bot. IX (2): 167. 1905. In the A. flavus-oryzae group.

Syn. A. oryzae var. basidifer Sacc. Syll. 22. 
A. ostianus Wehmer, in Bot. Centralb. 80: 449-461. 1899. Monogr. pp. 117-119, Taf. II. No. I. 1899-1901. In the $A$. ochraceus group.

A. ovalispermus Link, in Obs. II, p. 37,1816 ; also in Sp. Plant Ed. IV. Vol. VI(1): 66. 1824. Cited as synonym of $A$. oösporus Wallr. (1833) without evidence of identity or reason for redescription. A. panamensis Raper and Thom, in Mycologia 36: 568-572, fig. 5 . 1944.

A. parasiticus Speare, in Hawaiian Sugar Planters Exp. Sta., Path. \& Physiol. Ser. Bul. 12: p. 38, Pl. 3 and 4. 1912. In the $A$. flavus group.

Syn. A. flavus var, viridis Blochwitz, q.v......

A. penicillatus Greville, in Scottish Cryptogamic Flora 1: 32, plate 32. 1823.

Syn. A. glaucus (conidial); not identifiable to species.

A. penicillatus Link, in Sp. Plant Ed. 4, 6(1): 69. 1824. The description given by Link is not sufficient to identify any form, but since he probably intended to cover the organism of Greville, it may be assumed to be conidial A.glaucus. Sturm cites it as Briarea elegans without specifying his reasons.

A. penicilloides Spegazzini, in Rev. Agrar. Veter. La Plata. p. 245. 1896. In the $A$. restrictus series..

A. penicillopsis (Hennings) Racib. P. Hennings as Stilbothamnium penicillopsis P. Henn. \& E. Nym. described in Fungi Monsunensis (Warburg-Monsunia, Bd. I: p. 37 (Leipzig). 1899. Exsiccati of type in Pathological collections U. S. Dept. Agr. Bur. of Plant Industry as: Raciborski No. 87, in Crypt. Paras. Java.
See also Paras. Alg. u Pelz. Javas II : 7. $1900 \ldots \ldots \ldots \ldots \ldots \ldots 282$

A. periconioides Sacc, in Ann. Mycol. 11: 320. 1913. No. 195 in Sydow Fungi exotici exsiccati collected by P. W. Graff on leaves of Carica in Luzon, 1912. Not irlentifiable.

A. perniciosus Inui., in Jour. Coll. Sci. Imp. Univ. Tokyo 15: 473. 1901. Inui recorded a yellowish greenish color in the mycelium of this species which is regarded as related to $A$. luchuensis and $A$. niger.

A. pertardus is a nomen nudum on a culture distributed by Biourge. The organism was close to $A$. restrictus series................ 142

A. petiolatus Haller, in Historia stirpum indigenarum Helvetiae inchoata, etc. 1768 .

A. phaeocephalus Durieu and Montagne, in FI. Alg. p. 342. 1819.

Syn. S. phaeocephala (Dur. et Mont.) Saccardo, Fungi italici fig. 903 and No. 1244 in Sacc. Myc. Veneta. 1877. One of the A. niger group.

A. phoenicis (Corda) Thom, in The Aspergilli, p. 175. $1926 \ldots \ldots \ldots .222$ Syn. S. phoenicis (Corda) Patouill. and Delacr., in Bul. Soc. Mycol. France 7: 119, P1. 9. $1891 \ldots \ldots .223$ Syn. Ustilago phoenicis Corda, in Icones Fungorum 4: 9, pl. 3, fig. 26. 1840. One of the $A$. niger group...

A. pictor Blanchard, cited by Castellani \& Chalmers in Man. Trop. Med. p. 806. 1913.

Syn. Trichophyton pictor Blanchard, in Traite Path. Gen. II : 919. 1896. The production of polychromatic cultures suggests relationship to $A$. versicolor.

A. pollinis Howard, in Am. Bee Jour. 36: 577-578. 1896. See also idem. 38: 530-531. 1898. This was $A$. flavus fide Turesson 
in Svensk Bot. Tidskr. 11: 30 .

$1917 \ldots \ldots \ldots \ldots \ldots \ldots \ldots . \ldots \ldots$

S. polychroma Ferraris, in Fl. It. Crypt. Hyph. p. 640. 1906.... 193

Syn. A.versicolor q.v........... 190

A. polychromus De Mello, in Jour.

Indian. Bot. 1(5): 158-161.

1920. The data given are not sufficient to distinguish whether he had $A$. nidulans or $A$. sydowi.

A. polychromus Sartory, Sartory, and Meyer. Cit. Syn. of $A$. versicolor fide Blochwitz in Ann. Mycol. 31: 73.1933.

A. polymorphus Moquin-Tandon, in Elements Bot. Med. 2 Ed., 469. 1866. Not identifiable.

A. pouchetii Montagne, in Ann. Sci. Nat. Bot. 4 Ser. 6(12): 182-183. 1859. As described this was one of the mucors, noted as having resemblances to $A$. maximus (Sporodinia).

A. praecox Mosseray, in La Cellule XLIII: 229. 1934. Cited as synonym of $A$. fuliginosus Peck..................... 233

S. prasina Bainier, in Bull. Soc. Bot. France 27: 31. 1880. Not identifiable.

A. profusus Hann, nomen nudum. Cited by Thom and Raper in discussing $A$. scheelei Bainier and Sartory (Bul. Soc. Myc. France 28: 257. 1912) as nearly related to $A$. scheelei. See Thom and Raper, U. S. D. A. Misc. Publ. 426, p. 13, 1941. Syn. of A. pseudoglaucus........... 111

A. proliferans Geo. Smith, in Brit. Mycol. Soc. Trans. 26(1/2): 26, Pl. III. 1943. In the $A$. ruber section of the $A$. glaucus group................. 117

A. pseudo-carbonarius (Bainier) Mosseray, in La Cellule XLIII: 224-225, Pl. 3, fig. 7-13. 1934... 233

Syn. S. pseudo-carbonaria nomen nudum on culture (Thom 4610.482) from the Bainier collection.
A. pseudo-citricus Mosseray, in La Cellule XLIII: 228-229, pl. 4, figs. 103-104. 1934. Member of the A. niger group; in the Biourge Collection........... 233

A. pseudo-clavatus Purjewicz, in Schrift. Naturforch. Gesell. Kiev. 16, 2, p. 309, 1900. See: Saccardo Sylloge Fung. 16, p. 1028. In A. clavatus group......

A. pseudo-clatior Mosseray, in La Cellule XLIII: 255-256, pl. 3, figs. 33-37. 1937. Member of the $A$. niger group; in the Biourge Collection 1939........ 234

A. pseudoflaus Saito, in Centralb. f. Bakt. 2 abt. $18(1 / 3): 34$, figs 15-18. $1907 \ldots \ldots \ldots \ldots \ldots \ldots 266$

Syn. S. pscudoflava Sace. Sylloge Fungorum 22: 1260-1266. The morphology given indicates that A. pseudoflavus is one of the intermediate forms which bridge the gap between typical A. flavus and $A$. oryzae.

A. pseudoglaucus Blochwitz, in Ann. Mycol. 27:207. 1929. Emended description by Thom and Raper in U. S. D. A. Misc. Publ. 426, p. 12, 1941. A. glaucus group... 110

S. pseudo-nidulans Vuillenin, Arch.

Parasitologie 8: 540-542. 1904. Vuillemin transfers the ascosporic form described by Grijns as A. fumigatus in Centralbl. Bakt. II, 11: 330. 1903, to this specific name, emending Grijns's description by indicating the double nature of the band by which he separates his form from A. nidulans as described by Eidam. This discussion by Vuillemin tallies with the commonest of our American soil forms of $A$. nidulans but not with the description by Grijns.

A. pseudo-niger Mosseray, in La Cellule XLIII: 256-258, Pl. 4, figs. 113-117. 1934. A member of the $A$. niger group; in the Biourge Collection. 1939...... 234 
S. pseudo-nigra Costantin and Lucet, in Bul. Soc. Mycol. France 19: 33-44. 1903. In the $A$. niger group.

A. pseudo-Schiemanni Biourge, nomen nudum, cited from Centraalbureau catalogue 1931, as an actively diastatic organism represented in the Biourge Collection.

A. pulchellus (Speg.) Thom and Church, in The Aspergilli, p. 181. $1926 \ldots . .$.

Syn. Aspergillopsis pulchella Speg., in Myc. Arg. V, An. Mus. Nac. Buenos Aires, Ser. 3, T. 13: 436. 1911. In the $A$. niger group................

E. pulcherrimum Winter, in Rabh. Krypt. Fl. 2, aufl. 1 Abt. 2: 60. 1887. Noted there as also in Herbarium of Winter and in Hansen, Fungi fimicoli Danici 1041 of Sep. Abdr. A coprophilus form from the dung of foxes in Leipzig and dogs in Denmark, by Hansen; not an Aspergillus.

A. pulmonum hominis Welcker. Discussed by von Dusch, in Virchow's Archiv. (N. F. 1) 11: 561-566. 1857. Apparently A. pulverulentus (McAlpine) Thom, in Jour. Agr. Res. 7: 10-11. 1916.

Syn. S. pulverulenta McAlpine, in Agr. Gaz. N. S. Wales 7: 302. 1896. In the A. niger group.... 223

A. pulvinatus B. and C. original collection as far as seen appears to be in the Curtis Collection from Society Hill, S. C. 1855; also one marked F. cub.-Wright No. 642 in the Curtis Collection; another series of specimens of $\mathrm{B}$. and C. No. 1648 in Ellis and Everhart N. A. Fungi collected on dead twigs at Newfield, N. J. 1885. Also No. 2306 in the Ellis Collection. Not an Aspergillus.
S. purpurea van Tieghem in Bul. Soc. Bot. France 24: 101-103. 1877. Possibly A. nidulans.

A. purpureofuscus Fries, in Sys. Myc. 3: 388. 1829. Probably the same as $A$. purpureofuscus of Schweinitz.

A. purpureofuscus Schweinitz, in Synopsis fungorum in America boreali media degentium. Secundum observationes. In Trans. Amer. Phil. Soc., N. S. 4: 282, No. 2680. 1834. Also in Saccardo Sylloge Fungorum 4: 68, Patavii. 1886. Not an Aspergillus.

A. purpureus Haller. Historia stirpum indigenarum Helvetiae inchoata, etc. 1768. Not recognizable.

S. pusilla Peyronel, in I genu atmospherici dei funghi con micelio. Thesis. Padova p. 21. 1914. See: the A. niveus series........ 203

A. pusillus Massee, in Kew. Bull. Misc. Inf. 4: 158. 1914. From Soil, Sudan. Not identifiable.

A. pyri English, nomen nudum, name published in Research Studies of the State College of of Washington VIII (3): 127. 1940. (Doctoral Thesis: Taxonomic and pathogenicity studies of the fungi which cause decay of pears in Washington.) Subsequent work by English led to recognition of the name as a synonym of $A$.niger.......... 231

A. quadrifidus Link, in Obs. 2: 36 . 1816. Probably not an Aspergillus.

A. quadrilineatus Thom and Raper, in Mycologia 31(6): 660, fig. 3D and $4 \mathrm{~B}, 1939 \ldots \ldots \ldots \ldots \ldots .160$

A. quercinus (Bainier) Thom and Church, in The Aspergilli, p. 186-187. 1926............. 276 Syn. S. quercina Bainier, in Bul. Soc. Bot. France 28: 78 . $1881 \ldots \ldots \ldots \ldots \ldots \ldots \ldots \ldots \ldots$ 
A. quininae Heim., in Bul. Soc. Myc. France 9: 239. 1894. The culture was found upon quinine solution but the description given will not separate it from A. fumigatus.

A. racemosus Persoon, in Neues Mag. Bot. 1: 121. 1794. Also in Tentamen Disp. Meth. Fung. p. 41. 1797. Not recognizable.

A. ramosus Hallier, in $\mathrm{Ztschr}$. f. Parasit. 2: 266-269, Pl. 6, figs. 1-6. 1870. The figures and descriptions evidently represent a strain of A. fumigatus..

A. raulini, nomen nudum, in Biourge's table, probably attached to a culture in his collection.

A. rehmii Zukal, in Oesterr. Bot. Zeitschr. 43: 160, Pl. II, figs. 1-10. 1893. 'Zukal regarded this form as close to $S$. sulphurea Fresenius, but his description of perithecia and ascospores excludes the $A$. ochraceus group. Blochwitz evidently believed that Zukal had a mixed culture; hence, the name would be untenable. Cultures belonging to the A. ochraceus group have been distributed under the name but without proving their authority. 281

A. repandus Bainier and Sartory, in Bul. Soc. Myc. France 27: 463, Pl. XVIII. 1911. A. glaucus group.

A. repens (Cda.) DeBary, in Abhandl. I. Senkenberg. Natürf. Gesellsch. 7: 379.1870.

A. repens DeBary and Woronin, in Beitrage zur Morphologie und Physiologie der Pilzen p. 379. 1866.

Syn. A. glaucus var. repens Corda, in Icones 5: 53, Taf. II, fig. 24. 1842 ..

E. repens var. amstelodami Vuill., Soc. Mycol. de France, Bul. Trimest. 36:131. 1920..
A. restrictus G. Smith, in Jour. Text. Inst. 22: T. 115, fig. 5. $1931 \ldots \ldots \ldots \ldots \ldots \ldots \ldots \ldots 141$

A. restrictus var. B., G. Smith, in Jour. Text Inst. 22: T. 115, figs. 4, 6, and 8. 1931. See A. restrictus series in $A$. glaucus group................ 141

A. roseus Batsch, in Elenchus Fungorum, p. 183, No. 58, fig. 58.

- 1783. Cited by Link, Spec. Pl., ed. IV, t. VI, pt. 1, p. 68.1824. Also by various authors for a rosy or flesh-colored organism, and by Corda as Haplotrichum roseum in Pracht-flora, Pl. XI.

A. roseus Link, in Sp. Plant, ed. IV, t. 6, part 1, p. 68 . 1824. Link took the name "roseus" used descriptively, but not nomenclatorially, by Batsch (El. Fung. p. 183 , no. 58 , fig. 58 . 1783) for a mold presumed to have been an Aspergillus, by later authors. The name appears as No. 2724 in the Curtis Collection (1849) for a member of the $A$. candidus group. Neither descriptions nor specimen dating back to these authors fix this name for any definite series.

A. rubens Green, in Boston Soc. of Med. Sc. 1868. Not identifiable.

A. ruber (Bremer) .............. 114

Syn. A. ruber (Spieckernann and Bremer) Thom and Church, in The Aspergilli 112. 1926...... 114

Syn. Eurotium rubrum Bremer, in Zeitschr. f. Untersuch. d. Nahrung. und Genussmittel IV. 1901, p. 72 ; also in Die fettverzehr. Organismen in Nahr. u. Futtermitteln, Dissert. Munster. $1902 \ldots \ldots \ldots \ldots \ldots \ldots \ldots \ldots . . \ldots \ldots$

Syn. E. rubrum Spieckermann and Bremer, in Landw. Jahrb. 31: 81-128. 1902............... 114

A. ruber Estienne

Syn. Physomyces heterosporus Harz. 
Syn. S. rubra (Estienne) Biourae. Syn. Monascus purpureus; certainly not an Aspergillus.

S. rubescens nomen nudum on a culture in the Bainier Collection (Thom No. 4640.487). A. flavipes group......

A. rufescens Berlese, in Fungi Moricolae Fasc. VII. No. 4, Tav. 54, figs. 12-17. 1889. Probably conidial strain of $A$. glaucus group.

A. rugulosus Thom and Raper, in Mycologia 31(6): 661-2; fig. $3 \mathrm{E}$ and $4 \mathrm{C}, 1939$.

A. rutilans Mosseray, in La Cellule XLIII. 234-235, Pl. 4, fig. 8690. 1934. A member of the $A$. niger group; in the Biourge Collection 1939

$E$. sacchari Spegazzini, in Anales del Museo Nacional de Buenos Aires, 6, Ser. 2, 3: 244. 1899. Some insufficiently described member of the $A$. glaucus group.

A. sachari Chaudhuri and Sachar, in Ann. Mycol. 32: 95. 1934. Blochwitz in Ann. Mycol. 33: 240,1935 , leaves this species in A. guercinus................

A. salmoneus Biourge, nomen nudum, cited by Henrard in La Cellule XLIII: fase. 2, p. 353. 1934 , as one of the series "glauci." On p. 370 , idem, he records that single ascospores required 3 months for germination and that this species was found to be homothallic. Identification to species within the $A$. glaucus group is not possible without more information.

Alliospora Sapucaya Pin, in Proc. R. I. Acad. 1883 and in Jour. Bot. 1883. p. 234. One of the $A$. niger group causing rot in alliaceous bulbs.................

A. sartoryi nomen nudum was attached by Biourge to a culture of an organism close to $A$. gracilis in the $A$, restrictus series................... 139

A. sartoryi Sydow, in Ann. Mycol. 11: 156-160, Pl. VIII. 1913. Possibly in A. tamarii group.

A. schcelei Bainier and Sartory, in Bul. Soc. Myc. France 28: 257262, Pl. X. 1912. See Thom and Raper in U. S. D. A. Misc. Publ. No. 426, p. 12. 1941. A. glaucus group............... 107

A. scheelei Bainier and Sartory var. $B$., idem. See $A$. repcns series in A. glaucus group............ 107

160 A. schiemanni (Schiemann) Thom, in Jour. Agr. Res. 7: 13. 1916. See also A. fuscus Schiemann; name changed because previously used.... . . . . . . . . . . . 221

S. schneggiana Biourge, nomen nudum, in Biourge's MS; listed in the $A$. candidus group as applying to a culture received from Schnegg.

A. sclerotifer Mosseray, in La Cellule XLIII (fasc. 2) : 247-248. 1934. A member of the $A$. niger group................... 233

A. sclerotiorum Huber, in Phytopathology 23(3): 306-8, fig. 1 . 1933. A heavy sclerotiumproducing member of the $A$. ochraceus group........... 278

A. sejunctus Bainier et Sartory, in Bul. Soc. Myc. France 27: 346-368, Pls. X-XI. 1911. An ascosporic form of the $A$.glaucus group.

E. semi-immersum Marchal, in C. R. Soc. Roy. Bot. Belg. 33: 128, Pl. II, fig. 3. 1895. Not an Aspergillus.

A. siebenmanni Costantin and Lucet, in Ann. Sei. Nat. Bot. 9, 2, p. 162. 1905. This name is based upon Siebenmann's description of an organism from the human ear identified by Siebenmann as A. flarus, but regarded by the describers as a separate species 
based upon the description given by Siebenmann.......... 266

A. simplex Persoon, in Tent. Disp. Meth. Fung. p. 41. 1797. Tradition calls it a Penicillium. A. soya on a culture from Okunuki in the Centraalbureau collection. No description found; cited by Blochwitz in Ann. Mycol. 33: 240. 1935 , as $A$. flavus.

S. skottsbergii Bresadola and Vestergren no. 250 in Vestergren, Micromycetes rariores selecti Rossia baltica: ins. Osilia, Kielkond in silva abiegna prope Kattiel in foliis vivis Aqulegiae vulgaris. 1899. Distributed without description; examined in collaboration with Professor Thaxter at the Harvard University Cryptogamic Herbarium; did not prove to be an Aspergillus.

A. spadix Amons, in Arch. v. Suikerindustrie in Nederlandsch Indie 29: 12-14. 1921. This is one of the A.tamarii series........... 257

A. sparsus Raper and Thom, in Mycologia 36: 572-574, fig. 6 . 1944. 283

A. sphaerospermus Corda, in Icones II : 18. 1854. No description.

A. spiralis Grove, in Journal of Bot.

23: 164, tab. 257, fig. 5. 1885. A conidial organism of the $A$. glaucus group.

A. spirius cited by Amons in Arch. v. d. Suikerindustrie in Nederlandsch-Indie 29: 14. 1921. Not identifiable.

S. spuria Schroeter, in Cohn, Kryptogamen Flora von Schlesien 3: 2 Hälfte, Lief. 1, p. 218.

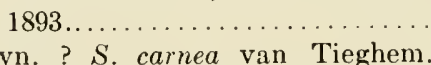
1877. See: A. carneus (van Tieghem) Blochwitz......... 201

A. stellatus Curzi, in Rend. Acad. Naz. Lincei 19: 424-428. fig. 1. 1934. Culture in Centraalbureau list 1939 .

Syn. A. variecolor...............
E. stercoraria Hansen, in Meddelelser fra den Naturhist. Foren. i. Kjöbenhavn p. 310. 1876.

Syn. Anixiopsis stercoraria Hansen, in Bot. Ztg. 55: 127-131, Tab. II, fig. 8. 1897.

A. stercoreus Sacc., in Michelia 1: 78. 1877. Also Fungi italici no. 19. An unidentifiable member of the A. glaucus group.

A. sterigmatophorus Saccardo, in Atti Soc. Ven.-Tren. Sci. Nat. 2, fasc. 2: 232, Tab. XVII, fig. 5-8.

$1873 \ldots \ldots \ldots \ldots \ldots \ldots \ldots . \ldots \ldots$

Syn. S. italica q.v............. 211

A. strychni Lindau, in Hedwigia Bd. 43, Heft 5: 306-307. 1904... 223 Syn. A. pulverulentus McAlpine, 1896. In the A. niger group.... 223

A. subfuscus Johan-Olsen, in Meddelelser fra Naturh. forening i Kristiania. 1885. Cited also in $O$. Johan-Olsen, Videnskapselkabet i Kristiania p. 21. 1886. Some member of the $A$. niger group.

A. subgriseus Peck, in Bul. Torrey Bot. Club, 22, 5: 210. 1895.

Syn. E. subgriseum Peck, in Rept.

N. Y. State Mus. Bot. p. 30.

1910. Also in N. Y. State Mus.

Bul. 150. 1911. There is no way to identify Peck's species.

A. sulphureus (Fres.) Thom and Church, in The Aspergilli, p. 185-186. 1926.............. 275

Syn. S. sulphurea Fresenius, in

Beitr. Z. Mykologie, Heft 3: p. 83, Tab. XI, fig. 30-33. 1863. Also Sylloge 4: 73.1886 . In $A$. ochraccus group............ 275

A. sydowi (Bainier and Sartory) Thom and Church, in The Aspergilli, p. 147-148. 1926...

Syn. S. sydowi, Bainier et Sartory, in Ann. Mycol. 11: 25-29, Pl. III.

1903. A. sydowi series. 184

var. achlamydosporus Nakazawa,

Simo, and Watanabe, in Jour. Agr. Chem. Soc. Japan 10(2): 178-179. 1934. The absence of 
Hülle cells is inadequate for separation................ 186

A. syncephalis Gueguen, in Champ. Parasit. Hom. Anim. p. 165, fig. 6. 1904. Probably A. fumigatus.................. 151

S. szurakiana Moesz, in Bot. Közlem.

19: 44-66, 13 figs. 1921. One of the $A$. candidus group.

A. tabacinus Nakazawa, Simo, and Watanabe, in Jour. Agr. Chem. Soc. Japan 10(2): 177-178. 1934. Appears to have been a strain of the $A$. versicolor series..................... 193

A. tamarii Kita, in Centralb. f. Bakt. etc., 2 Abt. 37, No. 14/16, pp. 432-452. 1913. A tamarii series....................

A. tardior Biourge, nomen nudum, listed among his cultures of the A. restrictus group (Biourge's Sec. I. Microaspergilli).

A. terreus Thom, in Turesson, Göte, Svensk Botanisk Tidskrift 10: 5. 1916. Without description; diagnosis Thom and Church Amer. Jour. Bot. 5: 85-86. 1918. The A. terreus series............ 195 var. aureus, n. var............. 198 var. Boedijni (Bloch.) n. comb. Thom and Raper.

Syn. A. Boedijni Blochwitz, Ann. Mycol.32(1/2):83. 1934_.... 197 var. floccosus Shih, in Lingnan Science Jour. 15: 372, pl. 16, fig.

3. 1936 198

var. subfloccosus Shih, in Lingnan

Science Jour. 15: 371, pl. 16, fig. 4. 1936 .

A. terricola Marchal, in Rev. Mycol. 15(59): 101-103. 1893. In $A$. tamarii series.

var. Americana Marchal in Thom and Church, Am. Jour. Bot. 8: 125. 1921. Also in Thom and Church The Aspergilli, p. 192. 1926 .

A. thomii. This was an undescribed sclerotium-producing strain of A. flavus sent by P. W. Graff to
Amer. Type Cult. Collection.................. 266

A. tiraboschii Carbone, in Atti d. Inst. Bot. Univ. Pavia Ser. II Vol. XIV: 320. 1912. In the A. sydowi-versicolor group...... 186

A. tokelau Wehmer, in Centralbl. Bakt. I, 35: 140. 1903. Cited by Dubreuihl in Jour. Med. Bordeaux 32:312. 1902. Wehmer's culture was one of the $A$. glaucus group but not the pathogenic organism causing the disease "tokelau." Dodge, Med Mye. 490. 1935, calls it Epidermophyton.

S. tropicalis Matta, in $\mathrm{Bol}$. Inst. Brasil. Sci. 3: 51-54, 2 figs. 1927. Probably A. sydowi but not separable from other strains.

A. tubingensis (Schober) Mosseray, in La Cellule XIIII : 245-247, pl. 3, fig. 58-60. 1934. Member of the $A$. niger group; in the Biourge Collection 1939........ 234

A. tunetanus (Langeron) Dodge, in Dodge Med. Mycol., p. 635. 1935.

Syn. S. tunetana Langeron, in Bul. Soc. Path. Exot. XVII : 345-347, illust. 1924. See A. sydowi series.................... 186

A. umbrinus Patterson, in Bull. Torrey Bot. Club 25: 284. 1900. In the $A$. tamarii series.

A. umbrosus Bainier and Sartory, in Bul. Soc. Mrycol. France 28: 267, Pl. XII. 1912. In the $A$. glaucus group............... 129

A. unguis (Emile-Weil and Gaudin) Emend. Thom and Raper in Mycologia 31(6): 667-8, fig. 6 . $1939 \ldots \ldots \ldots \ldots \ldots \ldots \ldots . \ldots \ldots$

Syn. S. unguis Emile-Weil and Gaudin, in Arch. Med. Exp. Anat. Path. (Paris) 28: 463-465. 1919. See Thom and Raper, The A. nidulans group, Mycologia 31: 667. 1939.......... 169

A. ustus (Bainier) Thom and Church, in The Aspergilli, p. 152-153. $1926 \ldots \ldots \ldots \ldots \ldots \ldots \ldots \ldots \ldots$ 
Syn. S. usta Bainier, in Bul. Soc. Bot. France 28: 78. 1881. A. ustus group.

var. laevis Blochwitz, in Ann. Mycol. 32(1/2): 84. 1934.

A. ustilago Beck, in Itin. Prin. S. Coburgi. 2: 148 (Wien). 1888. Saccardo, in Sylloge Fungorum. 10: 526 (Patavii). 1892.

Syn. A. phoenicis in the $A$. niger group.

A. Vancampenhouti Mattlet, in Ann. Soc. Belge Med. Trop. 4: 167-176. 1924. Name only ibid. 6: 31. 1926. In the $A$. versicolor group.............. 1

S. varia Bainier, in Bul. Soc. Bot. France 27: 30. 1880. Probably some nonascosporic $A$. nidulans, or $A$. unguis.

A. variabilis Gasperini, in Atti Soc. Toseana Nat. Sci. Pisa, Mem. 8, fasc. 2, p. 326. 1887. From the description, this was probably some strain of the A. flacus group.

A. rarians Ceni, in Rivista sperimentale di Freniatria, p. 31. 1905. Certainly identified by Tiraboschi in Annali di Bot. 7: 9-10, 1908, as A versicolor.

A varians Wehmer, in Bot. Centralb. 80: 460-1. 1899. Also in Wehmer Monogr. pp. 77-79, taf. I. 1899-1901. If Wehmer's description is correct, his species is not known in culture now. The authors have believed that $A$. varians is identical with $A$. itaconicus.................. 142

A. variecolor (Berk. and Br.) Thom and Raper, in Mycologia 31: 663667, fig. 4D and fig. 5. 1939 ..

Syn. Emericella variecolor Berk. and Br., in Introd. Crypt. Bot. p. 340-341, fig. 76. 1857. See Patouillard in Bull. Soc. Myc. Fr. 7: 43-49, pl. 4, fig. 6-12. 1891.

Syn. Inzengaea erythrospora Borzi, Jahrb. Wiss. Bot. (Pringsheim)
16: 450-463, pl. 19, 20 (1884). 1885 .

Syn. Emericella medias Chowdhury

\& Mathur. Ann. Myc. 36: 61-63. 1938 .

Syn. A. stellatus Curzi, Rend. Acad. Naz. Lincei 19, p. 424-428, fig. 1. 1934 .

A. v'ariegatus Mosseray, in La Cellule XlIII : 238-239, pl. 4, fig. 72-75. 1934. Nember of the $A$. niger group; in the Biourge Collection. 1939.

A. velutinus Mosseray, in La Cellule XLIII : 252-253, pl. 3, fig. 38-41, 1934. Nember of the $A$. niger group; in the Biourge Collection. 1939.

S. veneta Massalongo, in Bul. Soc. Bot. Ital. No. 7-8, p. 159.1900. Not cultivated and not identifiable by description, although Werkenthin (Phytopathology 6: 24i-249. 1916) used A. venetus for strains now known to be $A$. terreus.

E. verruculosum Vuillemin, in Bul. Soc. Myc. France 34: 83. 1918. See $A$. echinulatus in $A$. glaucus group.

A. versicolor (Vuillemin) Tiraboschi, in Ann. Bot. (Rome) 7:9. 1908. 190 Syn. S. versicolor Vuillemin, cited by Mirsky, in Thèse de Med. Nancy No. 27, p. 16. 1903. A. versicolor series............. 190

mut. coerulea Blochwitz, in Ann. Mycol. 27(3/4): 201. 1929. Represents a pure blue strain arising from a green strain. Probably A. sydowi.

var. fulvus Nakazawa, Takeda and Suematu, culture available from the Centraalbureau, 1939.

var. glauca Blochwitz, in Ann. Mycol. 32: 86. 1934. A strain greener than typical for the species, from human skin accompanying a Trichophyton. Similar green strains have been observed by us . . . . . . . . 192 
A. violaceo, cited by Biourge in his MS. p. 16 , but no data offered.

A. violacco-fuscens (Was this $A$. violaceo-fuscus?) mut. grisea Blochwitz, in Ann. Myeol. 32: 87. 1934. The grounds of separation are vague.

A. violaceo-fuscus Gasperini, in Atti soe. Toseana Sei. Nat. Pisa, Mem. 8, fasc. 2, p. 326. IS87. In A. niger group.

A. virens Link, in Obs. Ord: Pl. Nat. p. 16, 1809; also Sp. Pl., Ed. 4, 6: pt. 1, p. $6 \overline{7} .1824$. This has never been surely identified.

A. virens (Link?) Eichelbaum, in Verh. Naturw. Ver. Hamburg 3 Folge. XIV. p. 34. 1906. This might have been elose to $A$. fischeri.

Syn. E. virens (Eichelb.) Saee. Saccardo cites deseription of preeeding under this name in Sylloge Fung. 22: 1255. 1913.

1. viridans nomen nudum attached by Biourge to a culture distributed by him. One of the $A$. restrictus series.

A. riridis apparently undeseribed, appears only on a specimen in the Curtis Collection now in the Cryptogamic Herbarium of Harvard Iniversity; the label is "A. riridis Sehw. in herb., $A$. virens Lk. in Syn.: U. S., Herb. Sehw." No Aspergillus could be found in the specimen.

A. rirido-griseus Costantin and Lueet, in Ann. Sci. Nat. Ser. IX, 2: 140. 1905. One of the A. fumigatus group

S. ritellina Ridley, in Jour. Bot. 34: 152, Plate 357, figs. 14-16. 1896. No one has sinee reported lidley's organism with certainty but a culture belonging in the $A$. ochraceus group appears under the name in C. B. S. list. Blochwitz regarded it as a synonym for A. flarus.......

A. culpinus nomen nudum was distributed by Biourge. It belonged to A. tamarii Kita

S. welwitschiae (Bresadola) Hennings. Cited by Wehmer in Centralb. f. Bakt. etc., 2 Abt. 18: 394-395. 1907. Examination of material from Hennings by Wehmer furnished no basis for separating the form from $A$, niger. Examination of similar speeimen in the Farlow Herbarium shows primary sterigmata up to 50 by $12 \mu$ which would place it near $A$. phoenicis.

Syn. Ustilago welwitschiae Bresadola.

A. wehmeri Constantin and Lucet, in Ann. Sei. Nat. Bot. Ser. IX, 2: 162. 1905. The name is proposed by Costantin and Lucet for the organism which Brefeld and Wehmer deseribed as $A$. $f a-$ rus Link and which is so used in this paper. The uneertainties in the identification of Link's species do not seem important enough to justify the ehange of name. A. wehmeri is to be regarded as a synonym of $A$. flacus q.v. ............... 266

A. wentii Wehmer, in Centralb]. Bakt. 2 Abt., 2: 150. 1895. See also Wehmer, Die Pilzgattung Aspergillus, etc., in Mem. Soc. Phys. d'Hist. Nat. Geneva 33: part 2, p. 119. 1899-1901.

var. minimus Nakazawa, Takeda, Okacla, and Simo, culture available from the Centraalbureau in 1939

A. westendorpii Sace. and March, in Rev. Mycol. 7: 149. 1885. In A. clatatus group............ 98 


\section{Chapter XXV}

\section{ACCEPTED SPECIES, VARIETIES, AND MUTATIONS}

A. alliaceus Thom and Church

244

A. amstelodami (Mang.) Thom and Raper. 122

A. atropurpureus Zimmerman ..... 226

A. avenaceus G. Smith .......... 246

A. awamori Nakazawa .......... 220

A. butyracea Bainier............ 282

A. caespitosus Raper and Thom.... 166

A. candidus Link.............. 207

A. carbonarius (Bain.) Thom...... 229

A. carneus (v. Tiegh.) Bloch., emend. 201

A. carnoyi (Biourge) Thom and Raper.

A. cheralieri (Mang.) Thom and Church.

A. chevalieri (Mang.) Thom and Ch. var. intermedius Thom and Raper

A. citrisporus von Höhnel.

A. clavatus Desm.

A. conicus Blochwitz.

A. delacroixii (Sacc.) Thom and Church. 140

A. echinulatus (Delacr.) Thom and Church.

A. effusus Tiraboschi 131

A. elegans Gasperini 267

A. fischeri Wehmer..

A. flavipes (Bain. and Sart.) Thom and Church................ 179

A. flavus Link................ 263

A. foetidus n. sp.............. 219

A. fonsecaeus n. sp............ 227

A. fumaricus Wehmer............ 227

A. fumigatus Fresenius........... 148

A. fumigatus (Fres.) mut. helvola Yuill

A. giganteus Wehmer........... 95

A. gracilis Bainier.............. 138

A. granulosus Raper and Thom..... 175

A. humicola Chaudhuri and Sachar. 193

A. itaconicus Kinoshita . . . . . . . . 142

A. janus Raper and Thom....... 187
A. janus var. brevis Raper and Thom 190

A. japonicus Saito............ 231

A. luchuensis Inui.............. 230

A. lutescens (Bain.), Thom \& Church 251

A. mangini n. comb............ 127

A. medius Meissner............. 133

A. melleus Yukawa............ 279

A. micro-virido-citrinus Cost. and

Lucet................... 263

A. miyakoensis Nak., Simo \& Wat.. 220

A. montevidensis Talice and MacKinnon.................... 125

A. nidulans (Eidam) Wint........ 156

A. nidulans (Eidam) Wint. mut. alba Yuill ...................... 159

A. nidulans (Eidam) Wint. var.

latus Thom and Raper....... 159

A. niger van Tieghem.......... 216

A, niger v. Tiegh. mut. cinnamomeus

(Schiem.) n. comb.......... 223

A. niger v. Tiegh. mut. schiemanni

(Schiem.) n. comb. . . . . . . . 224

A. niveo-glaucus Thom and Raper. 135

A. niveus Bloch., emend.......... 202

A. ochraceus Wilhelm.......... 279

A. oryzae (Ahlburg) Cohn........ 261

A. ostianus Wehmer............ 283

A. panamensis Raper and Thom... 242

A. parasiticus Speare.......... 266

A. penicilloides Speggazzini ...... 142

A. penicillopsis (Hennings) Racib.. 282

A. phoenicis (Cda.) Thom......... 222

A. proliferans G. Smith ......... 117

A. pseudoglaucus Bloch.......... 110

A. pulchellus (Speg.) Thom and

Church.................. 228

A. pulverulentus (McAlpine) Thom 223

A. quadrilineatus Thom and Raper 160

A. quercinus (Bain.) Thom and Church................. 276

A. repens (Cda.) DeBary......... 103

A. restrictus G. Smith............ 141 
A. ruber (Brem.)................ 114

A. rugulosus Thom and Raper..... 160

A. sclerotiorum Huber ........... 278

A. sparsus Raper and Thom........ 283

A. sulphureus (Fres.) Thom and

Church.................. 275

A. sydowi (Bain. and Sart.) Thom and Church................. 184

A. tamarii Kita................ 254

A. terreus Thom............... 195

A. terreus Thom var. aureus n. var.. 198

A. terreus Thom var. boedijni n. var. 197

A. terreus Thom var. floccosus Shih 198
A. terricola Marchal .

253

A. terricola var. americana Marchal 253

A. umbrosus Bainier and Sartory... 129

A. unguis (Emile-Weil and Gaudin)

Thom and Raper........... 169

A. ustus (Bainier) Thom and Church 171

A. ustus (Bain.) Thorn and Ch. var.

laevis Blochwitz, n. comb...... 175

A. variecolor (Berk. and Br.) Thom and Raper.............. 163

A. versicolor (Vuill.) Tiraboschi .... 190

A. violaceo-fuscus Gasperini ....... 231

A. wentii Wehmer.............. 246 



\section{INDEX}

Abbott, E. V., 174, 175, 319

Accepted species, 360

Acid production

Aconitic, 299

Amino, 299

Aspergillic, 272

Citric, 237, 249, 290-293

Fumaric, 237, 293-294

Gallic, 237, 294

General, 294-295

Glaucic, 299

Gluconic, 238, 295-297

d-Gluconic, 299

Glucuronic, 299

Glycolic, 299

Glyoxylic, 299

It aconic, 143, 204-205, 297

Kojic, 249, 258, 270, 297-298

Malic, 299

Melleic, 299

Oxalic, 238, 298-299

Aconitic acid, 299

Actinomycetes, 42, 31

Agar slant cultures, 51

Albino forms, 206, 212

Alcohol, 316

Alcoholic fermentations, 270

Alexander, D. F., ix

Alkaline reaction by $A$. clacatus, 95

Allergy, 154, 271

Alliospora, 9

Alphabetical check list of species and genera, 330

Amann, J., 47, 319

Amino acid formation, 299

Anastomoses, 65

Anslow and Raistrick, 154, 319

Antibiotics, 98

from $A$. clavatus, 98

from $A$. giganteus, 99

from A. flavipes group, 182 from A. flavus-oryzae group, 272

from A. fumigatus, 154

Arlington Farm, 227

Ascogone, 27

Ascomycetes, 6

Ascophora nigrans, 9

Ascospore, 28

Color in A. nidulans group, 28, 156

Germination, 28, 29

Markings, 108, 130, 152, 162

in A. fischeri, 162

in A. glaucus group, 108,130

in A. nidulans group, 162

Aspergillaceae, 6

Aspergilleae, 6

Aspergillic acid, 272

Aspergilline, 22

Aspergillopsis Sopp, 9

Aspergillopsis Spegazzini, 8

Aspergillosis, in birds, 148, 154

Aspergillus Micheli, 6, 7

Aspergillus, Generic diagnosis, 7

Aspergillus species, *see also Check list of species, p. 331

A. alliaceus Thom and Church, $244^{*}, 246$, $\mathbf{2 4 5}, 249$

A. amstelodami (Mangin) Thom and Church, 122*, 124, 106, 108, 123

A. archiflavipes Bloch., 181

A. atropurpureus Zimm., 226 *

A. arenaceus Geo. Smith, 246* 243, 249

A awamori Nakazawa, $220^{*}$

A. butyracea (Bainier) n. comb., 282*

A. caespitosus Raper and Thom, 166*168,167

A. candidus Link, 207*-212, 208, 209

A. capitatus ochroleucus Micheli, 3

A. capitulo pulla Micheli, 3, 214

A. carbonarius (Bainier) Thom, 229*$230,217,218,236$

* Names are cited in this index for recognized or historically important species only. For a complete list of published names for the Aspergilli, see the Check List of Species, Chapter XXIV. 
A. carneus (van Tiegh.) Bloch., 201*202, 199, 200

A. carnoyi (Biourge) Thom and Raper, $134^{*}-135$

A. chevalieri (Mangin) Thom and Church, 118*-120, 104, 106, 108, 119

A. chevalieri (Mangin) var. intermedius Thom and Raper, 121*, 119

A. citrisporus von Höhnel, 251*, 51

A. clavatus Desm., 92*-95, 93, 94

A. conicus Blochwitz, $140^{*}$

A. delacroixii (Sacc.) Thom and Church, $282^{*}$

A. echinulatus (Delacr.) Thom and Church, 131*-132, 128, 130, 106, 109

A. effusus Tiraboschi, $267^{*}-269,268,69$

A. elegans Gasperini, 281*

A. fischeri Wehmer, $151^{*}-153,149,152$

A. flavipes (Bainier and Sartory) Thom and Church, $179^{*}-181,180$

A. flavus Link, 263*-266, 264, 265, 69

A. foetidus n. sp., $219^{*}, 217,40$

A. fonsecaeus n. sp., $227^{*}-228,76,221$

A. fumaricus Wehmer, $226^{*}$

A. fumigatus Fresenius, 68, 148*-151, 149

A. fumigatus (Fres.) var. helvola, 65, 72, 150

A. giganteus Wehmer, 95*-97, 96, 239

A. glaucus Link, 3, 4, 101

A. gracilis Bainier, 138*-139

A. granulosus Raper and Thom, $175^{*}-$ $178,176,177$

E. herbariorum, 4

A. humicola Chaudhuri and Sachar, 193*

A. itaconicus Kinoshita, 142*-143, 109

A. janus Raper and Thom, 45, 92, 187*$190,188,46$

A. janus var. brevis Raper and Thom, $190^{*}$

A. japonicus Saito, $230^{*}$

A. luchuensis Inui, 230*

A. lutescens (Bain.) Thom and Church, $251^{*}-253,252$

A. mangini (Mangin) n. comb., $127^{*}-129$, 128

A. medius Meiss., 133*-134, 46, 128

A. molleus Yukawa, 279*

A. micro-virido-citrinus Cost. and Lucet, $263^{*}$

A. miyakoensis Nak., Simo, and Wat., 220-221
A. montevidensis Talice and MacKinnon, $125^{*}, 123$

A. nidulans (Eidam) Wint. 156*-159, $157,158,161,162$

A. nidulans mut. alba Yuill, 65, 72, 158, $159^{*}$

A. nidulans var. latus Thom and Raper, $159^{*}$

A. niger van Tieghem, $216^{*}, 217,218,221$, 222, 18, 72, 239

A. niger mut. cinnamomeus (Schiem.) n. comb., 73, 74, 206, 223*-224, 244, 242, 236

A. niger mut. schiemanni (Schiem.) n. comb., 73, 74, 206, 224*-225, 217, 244, 242,236

A. niveo-glaucus Thom and Raper, $135^{*}-$ $137, \mathbf{1 9}, \mathbf{1 2 8}, 136$

A. niveus Bloch., emend. 202*-204, 199, 200

A. ochraceus Wilhelm, 279*-281, 280

A. oryzae (Ahlburg) Cohn, $261^{*}-263,262$, 264, 69

A. ostianus Wehmer, 283*

A. panamensis Raper and Thom, 242*244, 243, 249

A. parasiticus Speare, $266^{*}-267,268,69$

A. penicilloides Speg., 142*

A. penicillopsis (Hennings) Racib., 282*283

A. phoenicis (Cda.) Thom, 222*, 223, 218, 239

A. proliferans G. Smith, $117^{*}$

A. pseudo-glaucus Bloch., $110^{*}-111,105$

A. pulchellus (Speg.) Thom and Church, $228^{*}$

A. pulverulentus (McAlpine) Thom, 223*

A. quadrilineatus Thom and Raper, $160^{*}$ 161,162

A. quercinus (Bainier) Thom and Church, 276*-278, 277

A. repens (Cda.) De Bary, 103*-109, 104, 105, 106, 108, 109

A. restrictus G. Smith, $141^{*}, 136,139,109$

A. ruber (Bremer) n. comb, 114*-117, 104, $106,108,113,115$

A. rugulosus Thom and Raper, $160^{*}-163$, $158,161,162$

A. sclerotiorum Huber, $278^{*}-279,277$

A. sparsus Raper and Thom, 283*285,284 
A. sulphureus (Fres.) Thom and Church, $275^{*}-276$

A. sydowi (Bain. and Sart.) Thom and Church, $184^{*}-186,185$

A. tamarii Kita, $254^{*}-257$

A. terreus Thom, 66, 195*-197, 196, 199, 200

A. terreus var. aureus n. var., 66, 67, 198*, 199

A. terreus var. boedijni (Bloch) n. comb., $66,67,197^{*}$

A. terreus var. floccosus Shih, 66, 67, 198*

A. terricola Marchal, 253*

A. terricola var. americana Marchal, $253^{*}-254,252$

A. umbrosus Bain. and Sart., 129*-131, 128,130

A. unguis (Emile-Weil and Gaudin) Thom and Raper, 169*-170

A. ustus (Bainier) Thom and Church, $171^{*}-175,172,173,176$

A. ustus var. laevis Bloch., $175^{*}, 173$

A. variecolor (Berk. and $\mathrm{Br}$.) Thom and Raper, $163^{*}-166,158,161,162,164$

A. versicolor (Vuill.) Tiraboschi, 190*193, 185, 191

A. violaceo-fuscus Gasperini, 231*,217, 218

A. wentii Wehmer, $246^{*}-247,248,249$

Assumptions, Basic, 10

B

Baarn, Holland,-See Centraalbureau

Bacillus anthracis, 154

Bacteria in Aspergillus cultures, 59

Bactericidal agents--See Antibiotics

Bainier, G., vii , 4, 174, 227, 229, 236, 278

Bainier and Sartory, 107, 319, 114, 129, 133

Barham and Smits, 270, 298

Barnes, B., 74, 144, 145

Barthel, C., 56

Bary, A. De, vii , 4, 101 , 26, 28, 7, 27, 103

Basidia, 23

Berkeley, M. J., 163, 165

Bernhauer, K. I., 237, 293, 295, 299, 320, $291,238,306$

Bernton, H.S., 154, 320

van Beyma, F. H., viii
Bibliographies, types included, viii

Check list of species with bibliographic references, 331-359

General, viii, $319-330$

Topical, viii, 289-318

Bichloride of Mercury, 61

Binocular, wide-field, 40, 45, 51

Biourge, Ph., ix, 4, 135, 139, 214, 232, 235

Bisby, G. R., ix

"Black Aspergilli"-see A, niger group

Blakeslee, A. F., 41, 320, 229, 230

"Blase", 22

Blochwitz, Adalbert, ix, 4, 64, 66, 137, $175,197,202,203,206,229,231,236$, $256,267,279$

Bonner, J. T., 242

Borzi, A., 165, 163, 321

Brant, Nancy, ix

Brefeld, O., 4

Bulb disease, 246, 249

Buller, A. H. R., 65, 321

Bush and Goth, 272, 300

\section{C}

Calam, Oxford and Raistrick, 204, 321

Candidus group, 206-213

Group relationships, 212

Occurrence, 213

Other white Aspergilli, 206, 207, 212

Outstanding characters, 206

Sclerotia, 212

Variation in head size, 209

Capitulum, 3

Carbon dixoide ice, 53

Cardozo, D. M., 228

Cathode rays, 75

Cellulose, decomposition, 150

Centraalbureau voor Schimmelcultures, viii, $231,247,261,166$

Chaetomium, 239

Challenger et al., 270, 197

Characters, Diagnostic, 82

Chemistry of Mold Tissue, 301-302

Chitin, 317

Chlamydospores, 28

Chowdhuri and Mathur, 163, 321

Christensen, L. M., 271, 304

Chromotaxia, Saccardo, 242, 273

Church, M. B., ix

Ciferri, R., 166, 321 
Citric acid, 237, 249, 290-293

from A. niger, 237. See also 290-293

from A. wentii, 249

Citrinin, 205

Cladosarum olivaceum Yuills, 9, 65, 73, 240

Barnes "Creamy", 74, 145

Conidium formation, 73

Morphology of , 73

Nuclear behavior, 73,74

Clarke, F. E., 198, 199

Classification, 6

Clavacin, 98

Clavatin, 99

Clavatus group, 92-99

Antibiotics, 98-99

Occurrence, 98

Outstanding characters, 92

Synonyms, 98

Coffee fermentation, 286

Coghill, R. D., ix, 75, 326

Colony characters, 11-16

Colony types, 11-13, 12

Color, 13

in conidial walls, 14

in conidiophores, $14,22,148,153,171$, 179,273

as group characters, 13

in the mycelium, 13

influence of $\mathrm{pH}, 14$

in the substratum, 15

Color photographs of Aspergilli, Plates I-VII

of A. alliacéus Thom and Church, Plate VI, D

of A. amstelodami (Mang.) Thom and

Church, Plate III, E

of A. arenaceus Smith, Plate VI, E

of A. candidus Link, Plate Ví, A

of A. carneus (v. Tiegh.) Blochwitz, Plate V, F

of A. claratus Desm., Plate III, A

of A, flavipes (Bain. and Sart.) Thom and Church, Plate IV, F

of A. flavus Link, Plate VII, D

of A. fumigatus Fres., Plate TV, B

of A. giganteus Wehmer, Plate III, B

of A. janus Raper and Thom, Plate I,

$\mathrm{E}$ and $\mathrm{F}$; Plate $\mathrm{V}, \mathrm{B}$

of A. nidulans (Eidam) Wint., Plate

I, A-D; Plate IV, C
Color photographs of Aspergilli-Cont'd. of $A$. niger group, N.R.R.L. 67, Plate VI, B

of $A$. niger group, tan-spored mutant Plate VI, C

of A. niveo-glaucus Thom and Raper, Plate III, F

of A. ochraceus Wilhelm, Plate VII, F of A. oryzae (Ahlb.) Cohn, Plate VII, C of A. quercinus (Bain.) Thom and Church, Plate VII, E

of A. repens (Cda.) De Bary, Plate III, $\mathrm{C}$

of $A$. restrictus Snith, Plate IV, A

of A. ruber (Bremer), Plate III, D

of A. sydowi (Bain. and Sart.) Thom and Church, Plate $\mathrm{V}, \mathrm{A}$

of A. tamarii Kita, Plate VII, B

of A.terricola var. americana Marchal, Plate VII, A

of A. terreus Thom (unirradiated), Plate II, A; Plate V, E

of A.terreus ( $\mathrm{C}-\mathrm{V}$ mutants), Plate II, $\mathrm{B}-\mathrm{F}$

of A. ustus (Bain.) Thom and Church, Plate IV, E

of A variecolor (Berk. and $\mathrm{Br}$.) Thom and Raper, Plate IV, D

of A. versicolor (Vuill.) Tiraboschi, Plate $\mathrm{V}, \mathrm{C}$ and $\mathrm{D}$

of A. wentii Wehmer, Plate VI, F

Conidia, 18, 19, 24

Coloration, 13

Endogenous, 25

Formation, 23, 24

Germination, 29

Nuclear behavior, 23

Conidiophore, or stalk, 17, 18, 19

Color, 22

Surface, 19

Connective, 25

Contaminants, $5 \bar{i}-62$

Bacteria, 59

Elimination of , 41-42, 58,59

Mold disease, 59,60

Other molds, $5 \mathrm{~s}$

Recognition of, $5 \mathrm{~S}$

Cook and Lacey, 272, 301

Corda, A. C. I., 3, 223

Coremia, 13

Cramer, C., 4, 239, 321 
Culture Collections, 50

American Type Culture Collection, Washington, 50

Centraalbureau voor schimmelcultures, Baarn, Holland, 50

National Type Culture Collection, Iondon, 50

Northern Regional Rescarch Laboratory, Peoria, Ill., 50

Thom Collection, 50

Culture media, See Media

Culture, purification of , 41,42

Cultures, types of, 38

Dilution cultures, 40

Hanging drop, 45

Single-colony inoculation, 39,40

Single spore cultures, 42

Spot inoculation, 39

Streak cultures, 41

Three-point inoculation, 39,40

Currie, J. N., 237, 328

Curzi, M., 163, 166, 321

Czapek's solution agar, 32

\section{$\mathrm{D}$}

Dale, Elizabeth, 27, 101, 321

Dangeard, P. A., 27, 322, 28, 30

De Bary, A., See Bary, A. De

Descriptive sheet, 82

Descriptive terns, 11

Desiccation of molds, vacuum, 53

Dewar flask, 53, 54

Diastatic enzymes, 259, 257

"Digestin", 213

Dilution cultures, 40

Dimargaris, 9

Diplostephanus Langeron, 8

Disjunctor, 25

Dodge, B. O., 111

Dodge, C. W., 5, 154, 322

Doelger and Prescott, 237

Dox, A. W., 239, 322, 32

Dried cultures-See Lyophil preservation of molds

Dried specimens, 62

Drierite, 53

Dual character of $A$. janus, 188, 189

E

Earle, F. R., 178

Ecads, 64
Eidam, E., 155, 156, 322

"Eidamsehe blasen", 28

Elser, W. J., et al., 53, 322

Emericella variecolor Bcrk. and Br., 9, 163

Emmons, C. W., 198

Endogenous conidia, 25

Engler and Prantl, 6

Enzymes, 238, 249, 213, 257, 270-271, 194, 302-306

Enzyme production

by $A$. flavus-aryzae group, 270-271, 302304

by $A$. niger group, 238, 304-305

by $A$. okazakii, 213

by $A$. tamariz group, 257

by $A$. wentii group, 249

Ergosterol, 194, 317

Escherichia coli, 154

Euaspergillus Ludwig, 8

Eurotiaceac, 6

Eurotiales, 6

Eurotium Link, 7, 101

Eurotium herbariorum, 101, 125, 127

F

Fat production, 194, 238, 306-307

Ferdinandsen and Winge, 17, 228, 322

Fermentation Division, N.R.R.L., ix, 205

Fernbach, A., 238, 304

Fish, fermented, 286

Fisher, Ed., 165

Flavicidin, 272

Flavicin, 272

Flavipes group, 179-182

Antibiosis, 182

Color reactions, 180

Occurrence, 182

Outstanding characters, 179

Flavus-oryzae group, 259-272, 69, 68

Antibiosis, 271-272

Enzyme production, 270-271

Kojic acid, 270

"Moldy bran", 271

Occurrence, 269

Outstanding characters, 259

Pathogenesis, 271

Variation in, 68-69, 260

da Fonseca, Olympio, 227, 230, 322 
Foot-cell, 17, 19

of A. effusus, 264

Frazer and Chambers, 27, 322

Fresenius, G., vii, 4, 275, 148

Fumaric acid, 237, 293-294

Fumigacin, 154

Fumigatin, 154

Fumigatus group, 148-154

Allergy, 154

Antibiosis, 154

Economic importance, 153

Occurrence, 153

Outstanding characters, 148

Pathogénicity, 154

Thermophilic habit, 153

Fungi Imperfecti, 6

\section{G}

Gallic acid, 238, 294

Galloway, L. D., 75, 322

Gene mutation, 63

General bibliography, 319-330

Generic diagnosis, 6

Gilman and Abbott, 202, 322

Glaucic acid, 299

Glaucus group, 100-147

Economic importance, 146

Group relationships, 102

Historical considerations, 101

Laboratory cultivation, 101

Occurrence, 146

Outstanding characters, 100

Pathogenicity, 146

Pigment formation, 15

Temperature relations of, 45, 133, 134

Variation in, $143-145$

Glister, G. A., 272, 300

Gluconic acid, 238, 295-297

d-Gluconic acid, 299

Glucuronic acid, 299

Glycolic acid, 299

Glyoxylic acid, 299

Gould, B. S., 258, 298

Gould and Raistrick, 15, 323

Graphic key to groups, 87

Greene, H. C., 65, 323

Greene and Fred, 56, 323

Group keys

A. candidus group, 207

A. clavatus group, 92
A. flavipes group, 179

A. flavus-oryzae group, 259-260

A. fumigatus group, 148

A. glaucus group, 102

A. nidulans group, $155-156$

A. niger group, 215-216

A. niger group (Mosseray's), 232-235

A. ochraceus group, 274-275

A. tamarii group, 250-251

A. terreus group, 195

A. ustus group, 171

A. versicolor group, 183

A. wentii group, 241

Growth substances, 316

Guegen, F., 4, 323

Gum, 317

\section{$\mathrm{H}$}

Haines, R. W., ix, Plates I-VII

Haller, D. A., 3

Hanging drop culture, 45

Hansen, H. N., 65, 323

Hansen and Smith, 65, 323

Hanzawa, J., ix, 225

Hao, L. C., 271, 303, 304

Hay-infusion agar, 35

Head, 16, 18-21

Henrard, P., 27, 323

Herbarium specimens, 62

Herrick, H. T., ix, 237, 238, 227

Heterothallism, 27

High sugar Czapek's agar, 101

Historical introduction, 3

Hollaender, A., 75, 326

Homothallism, 27

Hooper, et al., 99, 323

Huber, G. A., 278, 286, 323

Hulle cells, 28

in A carneus, 176, 202

in $A$. flavipes group, 179

in A. nidulans group, 157, 167

in A. ustus group, 176

in A. versicolor group, 188, 192

Hydrogen-ion concentration, 14, 34, 35, 95

Hydroxylamine, 317

Hyphomycetes, 6

\section{I}

Identification of Aspergilli, 81-91

Incubation of stock cultures, 51 
Incubators, 48

Induced variation, 74

Industrial Farm Products Research Division, 227

Inoculating needles and loops, 47

Intermediate species, 86

Interpretation of Descriptions, 10

Inui, T ., 230

Invertase deficiency in A. panamensis, 244

Inzengea erythrospora Borzi, 9

Isolation of single spores, 43,44

Itaconic acid, 204-205, 143, 297

\section{J}

Jardin Botanique de l'Etat, 235

Jones, Rake, and Hamre, 272, 301

\section{K}

Karow, E. O., 249, 293, 323

"Katsuobushi", 286

Keitt, G. W., 43, 323

Keys, 86-91

based on color, $88-89$

based on morphology, 90-91

Graphic, 87

to groups, 86-91

to species-see group keys

Kinoshita, K., 142, 143, 323, 324

Kita, G., ix, 255, 260, 324

Kluyver and Perquin, 270, 298

Koji, 38, 249, 257, 260

Kojic acid, 249, 258, 270, 297-298

$\mathrm{L}$

Lacto-phenol, 48

Lambert, E. B., 43, 324

Langeron, M., 204, 324

La Rue, C. D., 43, 324

Ledingham, G. A., ix, 198

Link, H. F., 3, 101, 210

Linossier, G., 206, 324, 15, 236

Lockwood, et al., 76, 324, 205, 297

Loops, transfer, 47

Ludwig, F., 8, 324

Lutz, L., 226

Lyophil preservation of bacteria, 53

Lyophil preservation of molds, 53 Advantages of, 56
Apparatus, $\mathbf{5 4}$

Methods, 53, 55

Recultivation, 55

Viability, 53

\section{M}

Ma, Roberta, ix

Macroaspergilli, 16

Macy, H., ix

Maintenance of cultures, 51-57

Malic acid, 299

Malt extract agar, 35

Mangin, M. L., 118, 122, 127, 143

Mannitol, 317

Manual, use of, 81-91

Marchal, E., 253, 324

Martin, G. W., 6

Mary Clare, Sister, 285

May, O. E., ix, 227, 237, 238, 270

McCoy, Prof. Elizabeth, 56

McKee and MacPhillamy, 272, 301

McKee, Rake, and Houck, 272, 301

Mealy bugs, 267, 260

Media, culture, 31

Czapek solution agar, 32

Hay infusion agar, 35

High-sugar Czapek agar, 101

Influence of, $\mathbf{3 6}$

Malt extract agar, 35

Mosseray's Raulin's solution, 34

Neutral Raulin's solution, 33

Sporulation, 37, 38

Steep-liquor Czapek's agar, 35

Steinberg's solution, 34

Melleic acid, 299

Metarrhizium, 239

Methyl cellosolve, 53

Micheli, P. A., vii, 3, 100

Microaspergilli, 16

Microbiological Congress, Third International, 5

Microscopes, 48

Mildew, 146, 239

Mites, 60

Damage caused by, 61

Elimination of, 61-62

Poison, 61

Mixed cultures, 57

Mold Disease of $A$. niger, 59,60

Mold Tissue, Composition of, 301-302 
"Moldy bran", 271

Molliard, M., 238, 295

Monilia, 3

Monograph, 5

Monospore Cultures, 42

"Monster", 74, 85

Montagne, J. F., 3

Morphology and Description, 10

Morrow, Marie B., ix

Mosseray, Raoul, ix, 70, 75, 83, 229, 232, 235

Mosseray's Synopsis of Species, 232-234

Mounting fluid, 47

Moyer, A. J., 37, 227, 237, 238, 258, 270

Moyer and Coghill, 205, 297

M-type and C-type, 65

Mucidinaceae, 6

Mucidineae, 6

Mucoraceae, 42

Mucor herbariorum, 7

Mutant, definition of, 63

Mutation, 63

Mutations, deficiency, 76

Mutations, induced, 74

by cathode rays, 75

by chemicals, 74,75

by heat stimulation, 74

by ultra-violet radiation, $75,76,77$

in A. glaucus group, 74

in A. niger, 75

in A. terreus, 75

Mutations, "injury", 75

Mutations, morphological, 76, 77

Mutation, natural, 71

in A. fumigatus, 72

in A. glaucus group, 73

in $A$. nidulans, 72

in $A$. niger, $\mathbf{7 2}, 73$

Mutations, physiological, 78

Mutation, taxonomic usage, 84

Nakazawa, R., ix, 225

Nakazawa, Simo, and Watanabe, 220

Natural groups, 82,87

Natural Mutation, 71

Natural relationship of groups, 87

Needles, transfer, 47

Neill, J. C., 5, 69, 325, 83, 143, 193

Neutral Raulin's solution, 33
New species, 84

Bases for description, 85

Recognition of, 84

Nidulans group, 155-170

Oecurrence, 170

Outstanding characters, 155-156

Pathogenicity, 170

Niger group, 214-240

Citric Acid, 237, 290-293

Coloration, 236

Conidiophore structure, 19, 222

Enzyme production, 238, 304-305

Fat production, 238, 306

Fumaric Acid, 237, 293-294

Gallic Acid, 237, 294

Gluconic Acid, 238, 295-297

Industrial strains, preservation, 240, $50-62$

Mildew, 239

Mosseray's Synopsis of Species, 232234

Occurrence, 236

Outstanding characters, 214

Oxalic acid, 238, 298-299

Pathogenesis, 239-240, 307-310

Physiology, 239, 310-312

Soil analysis, 238, 313-314

Strain " 67 ", 227

Variation in head structure, 218

Variation in spore size, 221

Niklas, H., 238, 313

Northern Regional Research Laboratory vii, 50

\section{$\mathrm{O}$}

Ochraceus group, 273-286

Fermentations, 286

Occurrence, 285-286

Outstanding characters, 273

Ochracin, 318

Odor

Actinomyces-like in A. foetidus, 219

foetid in A. clavatus, 95

foetid in A. flavipes, 180

Okazaki, K., 213, 211, 325

Oryzae group-See Flavus-oryzae group

Oshima, K., ix, 260, 303, 261, 270

Ota, M., 271, 308

Overgrowths, 58

Oxalic acid, 238, 298-299

Oxford and Raistrick, 154, 325 
Parasiticin, 272

Parasitism of Aspergilli, 59

Partansky and McPherson, 239, 325

Pathogenicity, 307-310

in A. flavus-oryzae group, 271

in A. fumigatus group, 154

in A. glaucus group, 146-147

in $A$. nidulans group, $169-170$

in A. niger group, 239-240

in A.terreus group, 204

in A. versicolor group, 193-194

Patouillard and Delacroix, 222, 223

Patulin, 98

Penicillin, 99, 182

Penicillin-like substances

in A. flavipes, 182

in A. flavus, 272

in A. giganteus, 66

Penicillium chrysogenum Thom, 57

Penicillium notatum Westling, 272

Penicillium patulum West., 99

Penicillium restrictum Abbott, 67, 186

Penicillium rugulosum Thom, 59

Penicillium spinulosum Thom, 154, 300

Perithecia, 26

in A. fischeri, $149,151-153$

in A. glaucus group, 27, 100-137, 106, 115

in $A$. nidulans group, 155-166, 161, 164

Persoon, C. H., 3

Pfizer (Chas.) and Co. Inc., ix, Pls. I-VII

Phialids, 23

Philpot, F. J., 99, 301

Photographic equipment, 49

Physiology, 239, 310-312

Pigments, 15, 143, 236, 312-313

Plectascineae, 6

Plug poison, 61

Poison, cotton plugs, 61

Polysaccharides, 318

Pontillon, C., 238, 306

Preservation of Cultures, 51

in agar slants, 51-52

in lyophil form, $53-56,52$

in soil, $56-57,52$

on vegetable substrata, 57

Industrial strains, 240, 50-62

Progressive variation, 68
Proteolytic enzyme, 213, 257, 271

Pulli, 214

Quilico and Di Capua, 15, 313

$\mathrm{R}$

Raistrick, Harold, ix, 111, 117, 143, 231, 238,283

Raistrick, Robinson and Todd, 15, 313

Rami, 23

Raper, Coghill, and Hollaender, 75, 326, $205,206,212,225$

Raper and Thom, 166, 175, 187, 190, 242, 283

Raulin, J., 4, 237

"Raulin neutre-gelose", 70, 33

Raulin's solution, 34

Recultivation of dried cultures, 55

Reduced structures in $A$. sydowi, 184

Rejected species-See Check list of species

Resting nuclei, 73

Ridgway, Robert, 13, 326

Ridley, H. N., 13, 64, 326

$\mathrm{S}$

Saccardo, P. A., 11, 165, 326

Saccharification, 271

Saito, K., 231, 297

Salmonella typhi-murinum, 154

Saltant, 64

Sartory, A., 4

Sartory and Meyer, 212, 327

Sartorya fumigata, 9, 153

Scales, F. M., 253

Schiemann, Elizabeth, 74, 223, 224, 327, 206

Schmidt, C. F., Jr., 238, 306

Schwartz, W., 30, 327

Sclerotia, 30, 212

in A. candidus group, 212, 208

in A. flavus-oryzae group, 259, 265

in A. niger group, 214, 217

in A. ochraceus group, 273,277

in A. tamarii group, 250

in A. wentii group, 241, 243

significance of, 212

structure of, 30

Secondary growth, 58 
Sectors, 64

Septa, 17

Shih, Y. K., ix, 198

Short-lived species, 51

Siebenmann, F., 271, 307

Simonart, Paul, ix, 232

Single-spore cultures, 42, 44

Smear cultures, 41

Smith, George, ix , 5, 117, 102, 137, 139, $141,246,285$

Sodium lauryl sulfonate, 37

Soil analysis, 238, 313-314

Soil Fungi, Summary of, 202

Soil Preservation of Molds, 56

Directions, for, 56

Viability, 56

Sopp, O. J. O., 9

Soy products, including soya sauce, 249 , 258,270

Speare, A. T., 260, 266, 328

Species

Accepted, 360-361

Check list of, viii, 331-359

Definition of, 83

Diagnosis of, 82

Keys to, see group keys

New, 84

Rejected-See Check list of species

Transitional, 86

Specimens, identification from, 81

Spegazzini, C., 8, 229, 328

Spinulosin, 154

Spores

Ascospores, 28, 29, 152, 162, 130, 108

Conidia, 23-25, 24, 221

Sporodinia, 3

Sporulation media, 37,38

Sprays, 62

Stalk-See Conidiophore, 17

Staphylococcus, 53, 98, 99

Staphylococcus aureus, 154, 182

Steep liquor Czapek's agar, 35

Steinberg, R. A., 239, 310, 311, 312

Steinberg's nutrient solution, 34

Steinberg and Thom, 75, 328, 206, 212, $224,225,240$

Sterigmata, 22

Primary, 19, 23

Secondary, 19, 23

Sterigmatocystis Cramer, 8

"Sterile hyphae" in A. unguis, 169
Stock cultures, 51-57

Agar slants, 51

Cultivation, 51

Incubation, 51

Periodic transfer, 51

Storage, 51

Streak cultures, 41

Streptococcus viridans, 154

Substrata, see Media

Swift, Marjorie E., 111

Synonyms

in $A$. candidus group, 211

in $A$. chevalieri series, 120,121

in A. clavatus group, 98

in A. echinulatus, 133

in A. flavus-oryzae group, 266, 269

in A. luchuensis series, 232

in $A$. niger series, $225-226$

in A. niveo-glaucus, 137

in $A$. ochraceus series, 281

in $A$. repens series, 107,110

in $A$. restrictus series, 139

in $A$. ruber series, 117

in A. sulphureus series, 276

in $A$. sydowi series, 186

in A tamarii group, 256-257

in $A$. terreus group, 197,200

in $A$. umbrosus series, 131

in A. versicolor series, 192,193

in $A$, wentii group, 247-248

Synonymy, 6

"System und Phylogenie", 5

\section{$\mathrm{T}$}

Takahashi, T., 260, 328

Takamine, J., 270, 302

"Tamari", 258

Tamarii group, 250-258

Economic importance, 257-258

Occurrence, 257

Outstanding characters, 250

Tamiya and Morita, 4

Tannic acid, 237, 294

Tannin, 237, 294

Taubenhaus, J. J., ix

Temperature, 45, 46

Temperature, effect of, on A. giganteus, 45,97

in A. glaucus group, 45

on $A$. janus, 45,187

on A. medius, 45, 46, 134 
Temperatures, optimum, 45

Terminology, descriptive, 11

Terrein, 318

Terreus group, 195-205

Antibiotics, 205

Itaconic acid, 204, 205, 297

Mutations, 75

Occurrence, 204

Outstanding characters, 195

Pathogenesis, 204

Variation in, 66, 67, 197

Tessar lenses, 49

Thaxter, R. T., 50, 189, 251

Thermophilic species, 45

Thom, C., vii, 5, 195, 328

Thom and Church, vii, 4, 5, 102, 137, 206, $235,255,260$

Thom and Raper, 74, 328, 102, 168

Thom and Steinberg, 75, 328, 144, 150, 212,221

van Tieghem, Ph., vii , 4, 237

Timonin, M. I., 203, 205, 329, 213

Topical bibliography, 289-318

Transfer needles and loops, 47

Transfer of stock cultures, 51

Transitional species, 86

Trichoderma, 42

Turfitt, G. E., 246

"Type" culture collections, 50

\section{$\mathrm{U}$}

Ultra-violet radiation, $75,76,77$

Underkofler et al., 271, 303, 285

Ustilago phoenicis Cda., 223

Ustus group, 171-178

Hulle cells, types, 176

Occurrence, 178

Outstanding characters, 171

\section{V}

Vacuum desiccation of molds, 53

Vacuum tester, 55

Variant, 64

Variation, natural, 63-78

in A. fischeri, 65

in A. flavus-oryzae group, 68,69

in A. fumigatus, 68

in $A$. niger group, 70,71

in A. sydowi, 67

in A. terreus group, 66,67

Intra-group, 68
Intra-species, 66,67

Intra-strain, 65

Variation, references; $314-315$

Variety, taxonomic usage, 81

Vegetable substrata, 57

Versicolor group, 183-194

Occurrence, 194

Outstanding characters, 183

Pathogenesis, 193

Strain variation, 192, 186

Vesicle, 18, 19, 22

Vestigial characters, 82

Vibrio cholorae, 154

Vitamin D, 238

Vitamins, 316

Vuillemin, P., 165, 329

\section{W}

Waksman and Bugie, 272, 301

Waksman et al., 98, 300, 99, 301, 154

Ward, G. E., 237, 270, 329

Webb, P. H. W. , 97, 329

Wehmer, C., vii, 4, 226, 237, 238, 247, 283

Weisner, B. P., 98, 300

Wells, May, Moyer, Herrick, et al., 227, 237,270

Wentii group, 241-249

Economic importance, 249

Occurrence, 249

Outstanding characters, 241

Westerdijk, Johanna, viii, 110, 142, 283

Whelden, R. M., 75, 330, 206, 225

White; E. C., 182, 330, 271, 300, 272

White mutants, 206

Wickerham, L. J., 55

Wickerham and Andreasen, 53, 330

Wiggers, Fredricus Henricus, 7, 100

Wilhelm, K. A., vii, 4, 281, 330

Wilkins and Harris, 99, 300

Wire, nichrome, 47

platinum-iridium, 47

Wolf, F. A., 97, 330

Yabuta, T., 249, 297, 330

Yuill, Edward, 65, 150, 159, 206, 212, 221

Yuill, John and Edward, ix, 73, 145, 240

Yukawa, M., 279, 286, 330

$\mathrm{Z}$

Zea Mays, 267

Zonation, 11-13, 12 




